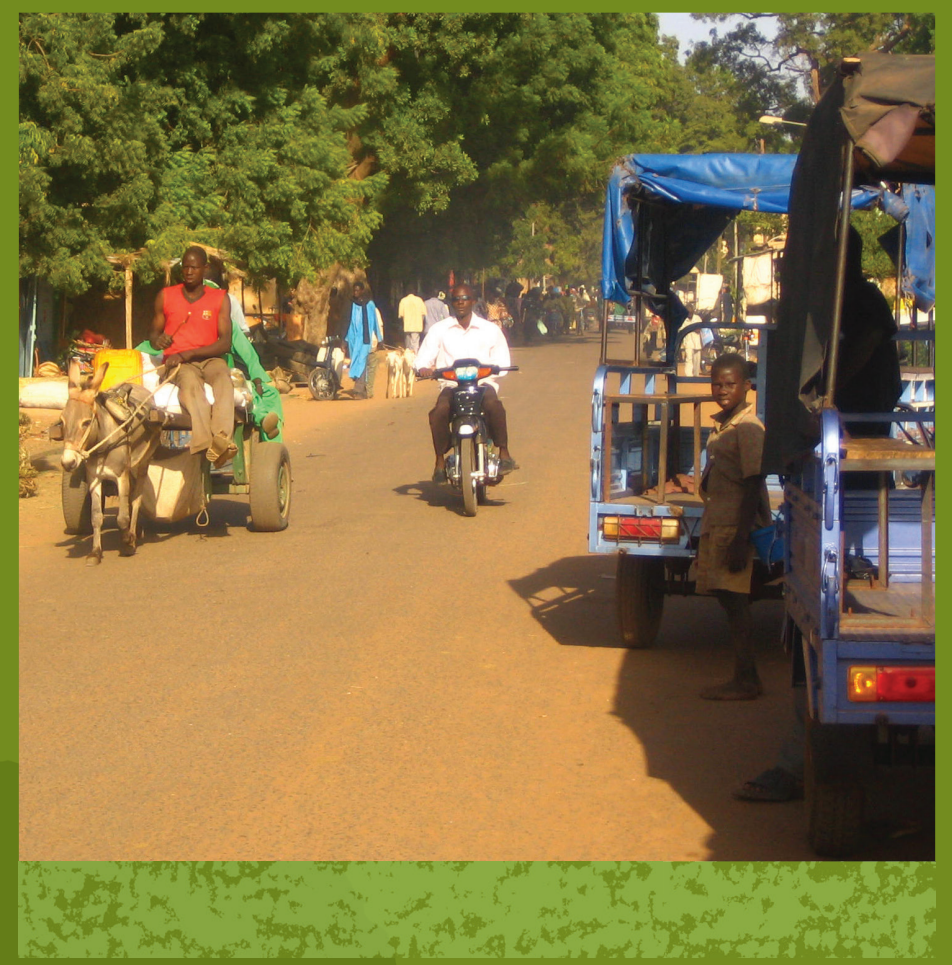

\title{
Transformations rurales
} \section{et développement}

Les défis du changement structurel dans un monde globalisé

Bruno Losch, Sandrine Fréguin-Gresh, 
Transformations rurales et développement 



\section{Transformations rurales et développement}

\section{Les défis du changement structurel dans un monde globalisé}

Bruno Losch

Sandrine Fréguin-Gresh

Eric Thomas White 
This work was originally published by The World Bank in English as Structural Transformation and Rural Change Revisited. Challenges for Late Developing countries in a Globalizing World in 2012. In case of any discrepancies, the original language will govern.

La version originale de cet ouvrage a été publiée en anglais par la Banque mondiale en 2012, sous le titre Structural Transformation and Rural Change Revisited. Chalenges for Late Developing countries in a globalizing World. En cas de divergence, le texte en langue originale prévaut.

\section{[Avertissement]}

The findings, interpretations, and conclusions expressed herein are those of the author and do not necessarily reflect the views of the Executive Directors of The World Bank or the government they represent or of the Agence Française de Développement.

The World Bank and Agence Française de Développement do not guarantee the accuracy of the data included in this work. The boundaries, colors, denominations, and other information shown on any map in this work do not imply any judgment on the part of The World Bank or the Agence Française de Développement concerning the legal status of any territory or the endorsement or acceptance of such boundaries.

Les analyses et conclusions de ce livre sont formulées sous la responsabilité de son auteur. Elles ne reflètent pas nécessairement le point de vue de la Banque mondiale, de ses administrateurs ou des États qu'ils représentent, ni celui de l'Agence Française de Développement.

La Banque mondiale et l'Agence Française de Développement ne garantissent pas l'exactitude des données citées dans cet ouvrage. Les frontières, les couleurs, les dénominations et toute autre information figurant sur les cartes du présent rapport n'impliquent de la part de la Banque mondiale ou de l'Agence Française de Développement aucun jugement quant au statut juridique d'un territoire quelconque et ne signifient nullement que l'institution reconnait ou accepte ces frontières.

Traduction : Christine Mercier avec le concours de Bruno Losch.

Photographie de couverture : Bruno Losch, Une rue à Ségou, Mali.

Maquette de couverture : Debra Naylor, Naylor Design, Inc.

Transformations rurales et développement. Les défis du changement structurel dans un monde globalisé. Copyright $\odot 2013$ Banque internationale pour la reconstruction et développement (BIRD)/Banque mondiale.

Publié par Pearson France

74 , rue de Lagny

93100 Montreuil

France

Réalisation PAO : Graph'im64

ISBN : 978-2-326-00022-3 


\section{Collection L'Afrique en développement}

Créée en 2009, la collection «L'Afrique en développement » s'intéresse aux grands enjeux sociaux et économiques du développement en Afrique subsaharienne. Chaque numéro de cette collection dresse l'état des lieux d'une problématique et contribue à alimenter la réflexion sur l'élaboration des politiques locales, régionales et mondiales. Décideurs, chercheurs et étudiants, y trouveront les résultats de travaux de recherche les plus récents, mettant en évidence les difficultés et les opportunités de développement du continent.

Cette collection est dirigée par l'Agence Française de Développement et la Banque mondiale. Pluridisciplinaires, les manuscrits sélectionnés émanent des travaux de recherche et des activités de terrain des deux institutions. Ils sont choisis pour leur pertinence au regard de l'actualité du développement.

En travaillant ensemble sur cette collection, l'Agence Française de Développement et la Banque mondiale entendent renouveler les façons d'analyser et de comprendre le développement de l'Afrique subsaharienne.

\section{Membres du comité consultatif :}

\section{Agence Française de Développement}

Rémi Genevey, directeur de la stratégie

Alain Henry, directeur de la recherche

\section{Banque mondiale}

Shantayanan Devarajan, chef économiste, région Afrique

Santiago Pombo-Bejarano, directeur éditorial, bureau des publications 


\section{L'Afrique subsaharienne}

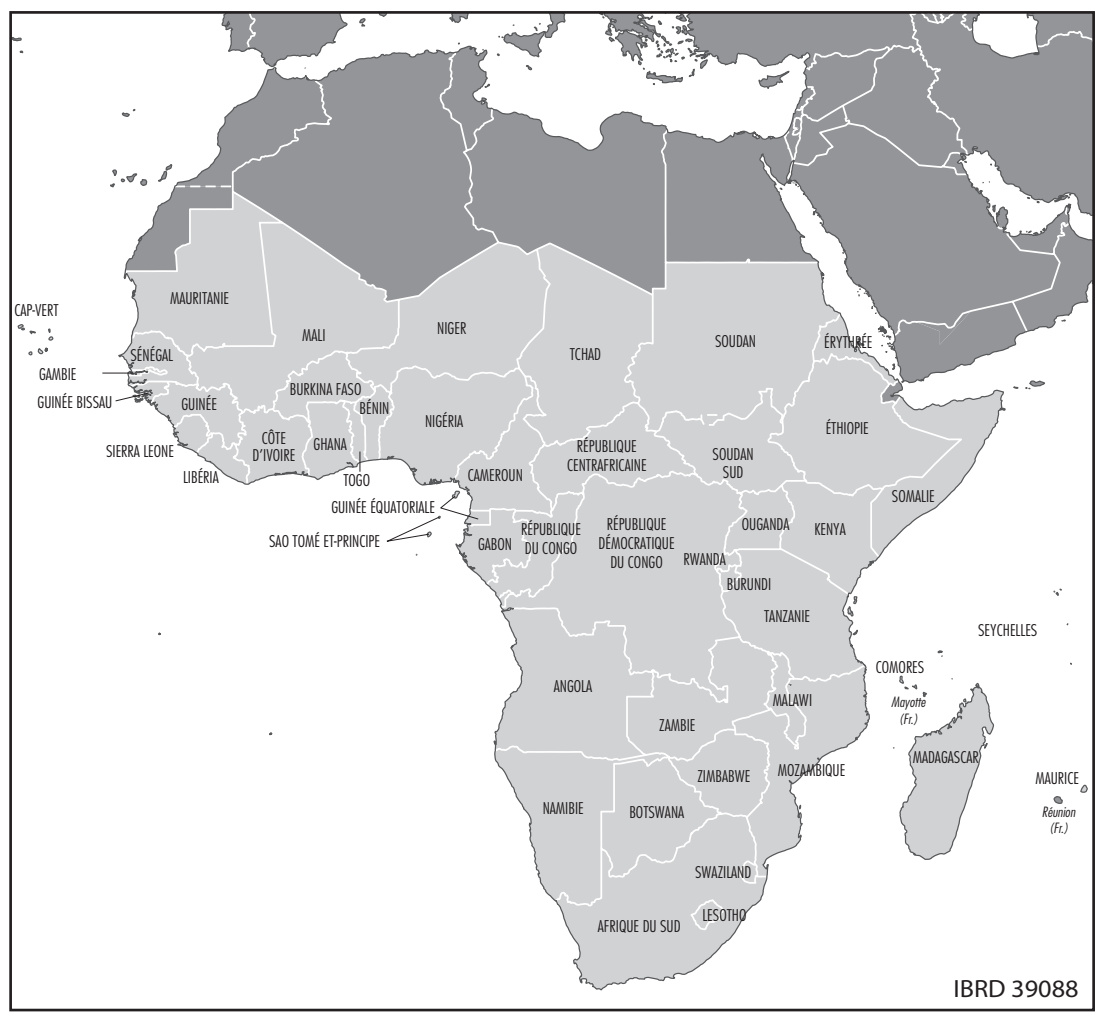




\section{Titres déjà publiés dans la collection}

\section{En français}

Vivian Foster et Cecilia Briceno-Garmendia, Infrastructures africaines, une transformation impérative, Pearson, 2010

Bernard Dafflon et Thierry Madiès, L'Économie politique de la décentralisation dans quatre pays d'Afrique subsaharienne, AFD, 2011

Thierry Paulais, Financer les villes africaines, Pearson, 2012

Nancy Benjamin et Ahmadou Aly Mbaye, Les Entreprises informelles de l'Afrique de l'Ouest francophone Pearson, 2012

Hinh T. Dinh, Vincent Palmade, Vandana Chandra et Frances Cossar, L'Industrie légère en Afrique, Pearson, 2012

François Roubaud et Philippe de Vreyer, Les Marchés du travail urbains en Afrique subsaharienne, IRD-AFD, 2013

Bruno Losch, Sandrine Fréguin-Gresh et Eric-Thomas White, Transformations rurales et développement, Pearson France, 2013

\section{En anglais}

Vivian Foster and Cecilia Briceno, Africa's Infrastructure: a Time for Transformation, World Bank, 2010

Jorge Arbache, Alexandre Kolev and Ewa Filipiak, Gender Disparities in Africa's Labor Market (2010), World Bank, 2010

Jean-Claude Devèze (ed), Challenges for African Agriculture, World Bank, 2010

Hinh Dinh, Vincent Palmade, Vandana Chandra and Frances Cossar, Light Manufacturing in Africa: Focused Policies to Enhance Private Investment and Create Productive Jobs, World Bank 2012

Nancy Benjamin and Ahmadou Aly Mbaye, Informal Sector in Francophone West Africa: Firm Size, Productivity and Institution, World Bank, 2012

Thierry Paulais, Financing Africa's Cities, World Bank, 2012

Bruno Losch, Sandrine Fréguin-Gresh and Eric-Thomas White, Structural Transformation and Rural Change Revisited, World Bank, 2012

Bernard Dafflon and Thierry Madies (eds), The Political Economy of Decentralization in Subsaharan Africa, World Bank, 2013

Mary Hallward-Driemeier and Tazeen Hazan, Empowering Women: Legal Rights and Economic Opportunities in Africa, World Bank, 2013

Mary Hallward-Driemeier, Enterprising Women: Expanding Economic Opportunities in Africa, World Bank, 2013

Frank F. K. Byamugisha, Securing Africa's Land for Shared Prosperity, World Bank, 2013

Tous ces titres sont téléchargeables sur les sites de l'AFD et de la Banque mondiale: http://www.afd.fr/home/publications/travaux-de-recherche/

publications-scientifiques/co-editions-scientifiques

https://openknowledge.worldbank.org/handle/10986/2150 


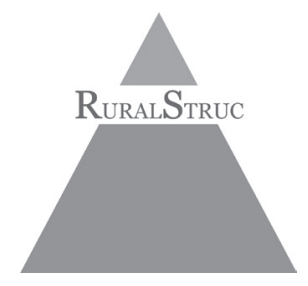

Le Programme RuralStruc sur les dimensions structurelles de la libéralisation pour l'agriculture et le développement rural est une initiative conjointe de la Banque mondiale, de la coopération française (Agence Française de Développement, ministère de l'Agriculture et de la Pêche, ministère des Affaires étrangères et européennes, Centre de coopération internationale en recherche agronomique pour le développement - CIRAD) et du Fonds international de développement agricole. Il est géré par la Banque mondiale.

Mis en œuvre sur une durée de sur cinq ans (2006-2010), ce programme visait un double objectif : analyser les processus de libéralisation et d'intégration économique et leurs impacts sur l'agriculture et le secteur rural des pays en développement, et préciser la situation des économies rurales en termes de revenus, de diversification et de transformation globale. Les résultats obtenus permettent d'améliorer le dialogue entre les partenaires nationaux et internationaux et de donner des orientations aux débats sur les politiques agricoles et rurales.

Le programme a fait appel à un dispositif comparatif regroupant sept pays - Mexique, Nicaragua, Maroc, Sénégal, Mali, Kenya et Madagascar - correspondant à différents stades de transition économique et démographique. Les travaux ont été menés par des équipes nationales d'experts et de chercheurs et structurés en deux phases : la première était destinée à dresser l'état des lieux des dynamiques en cours dans chaque pays (2006-2007), la seconde portait sur des études de cas sectorielles et régionales s'appuyant sur des enquêtes auprès de ménages ruraux (2007-2010).

http://www.worldbank.org/afr/ruralstruc

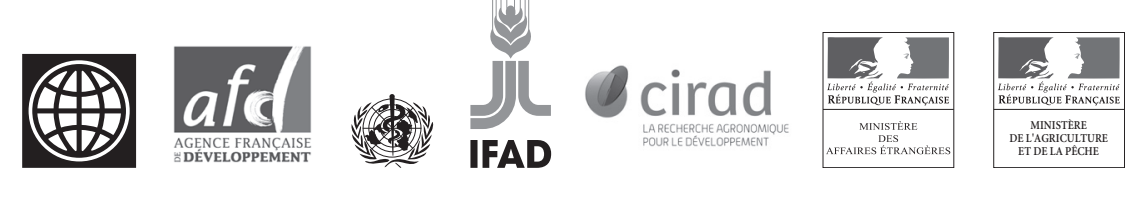




\section{Sommaire}

Avant-propos

xvii

Remerciements

xix

A propos des auteurs xxiii

Acronymes $\quad x \times v$

$\begin{array}{ll}\text { Aperçu } & 1\end{array}$

Du débat de l'OMC à la crise des prix alimentaires - un manque de vision structurelle à long terme 2

Le programme de recherche 4

Le rôle persistant de l'agriculture et l'ampleur de la pauvreté rurale 6

Production agricole, marchés et processus de différenciation 7

Diversification des activités et reconfiguration de l'économie rurale 10

La relation diversification-revenu et la transformation rurale 13

Principales leçons pour les politiques publiques $\quad 15$

1 Du contexte aux choix d'analyse

Un contexte mondial déroutant, en rapide mutation 24

Principaux objectifs et hypothèses du programme : reconnecter les débats 31

Structure générale du programme : études de cas nationales, enquêtes régionales et processus collaboratifs 34

Synopsis de l'ouvrage 45

Notes 48

2 Les défis du changement structurel

Différences régionales et positionnement des pays du programme 54 
Le changement structurel dans une économie mondiale ouverte 63

Notes 74

3 Les réalités rurales : agriculture et pauvreté

Le rôle central de l'agriculture dans les différents contextes régionaux 79

Une pauvreté rurale endémique 86

Les stratégies d'activités des ménages 108

Notes 116

4 Les multiples facettes de la diversification rurale et l'économie rurale non agricole

La question de la diversification rurale 119

Les réalités des activités off-farm dans les régions étudiées 123

Diversification off-farm et transformation rurale 153

Notes 155

5 À la recherche des nouvelles formes de la spécialisation agricole

Le grand chambardement 160

Une nouvelle agriculture insaisissable 173

Spécialisation agricole et transformation rurale 196

Notes 197

6 Des profils régionaux de transformation rurale aux recommandations politiques

201

Profils régionaux de diversification - spécialisation des revenus 201

Quelles recommandations pour l'élaboration des politiques

publiques? 227

Notes 242

Annexe

Cartes des pays étudiés 247 


\section{Encadrés}

1.1 RuralStruc : qu'y a-t-il derrière ce nom ?

1.2 Le Rapport sur le développement dans le monde 2008 (RDM08) et ses trois mondes

1.3 Libéralisation ou mondialisation?

1.4 Les trois hypothèses du programme RuralStruc 33

1.5 Rural et urbain : quelle définition pour chaque pays? 40

1.6 Le projet Activités rurales génératrices de revenus (RIGA) 41

3.1 Densité, distance et division : les trois grandes caractéristiques géographiques du développement économique selon le RDM09

3.2 Urbanisation, réseaux de transports et moyens d'existence des ruraux 85

3.3 Le paradoxe de Sikasso... et de Koutiala 94

4.1 Zones franches industrielles et travail salarié non agricole au Nicaragua

4.2 Les maquilas du secteur textile à La Fuente, Tequisquiapan, Mexique 137

4.3 Caractéristiques des migrations internationales dans les pays du programme RuralStruc

4.4 Migrer à Dakar pour y vendre des cartes téléphoniques : une voie illusoire de sortie de la pauvreté

4.5 Inégalités d'accès aux transferts publics dans la région du Sotavento, Mexique

5.1 La restructuration du secteur du maïs mexicain 168

$\begin{array}{lll}5.2 & \text { La révolution des supermarchés } & 169\end{array}$

$\begin{array}{lll}5.3 & \text { Regoverning Markets } & 171\end{array}$

5.4 La success story des exportations horticoles kényanes : qui est concerné?

5.5 L'exception du Sotavento : spécialisation des exploitations et effondrement de l'autoconsommation

5.6 La grande distribution alimentaire : un développement contrasté dans les pays du programme RuralStruc

5.7 Lecofruit : les petits producteurs malgaches vendent sur les marchés européens

6.1 Les clés des exceptions au profil en U inversé

6.2 Des moyennes de diversification du revenu à l'écart de diversification 


\section{Figures}

1.1 Comment naviguer dans le livre

2.1 Comparaison du PIB par habitant et de son évolution entre pays et régions, 1960-2009

2.2 Part de l'agriculture dans le PIB et la population active, 1965-2005 56

2.3 Écart structurel et PIB par habitant, 1965-2005 56

2.4 Évolution de la structure du PIB : comparaison ASS - Asie, 1965-2005

2.5 Ratio d'activité par grandes régions, 1950-2050

2.6 Cohortes d'entrants sur le marché du travail par grandes régions, 1955-2050

2.7 Cohortes annuelles entrant sur les marchés du travail rural et urbain et part de la population rurale en Afrique subsaharienne, 1955-2050 71

3.1 Part des ménages ruraux interrogés ayant une exploitation agricole 81

3.2 Part moyenne des revenus issus de l'exploitation agricole et des revenus hors exploitation, par région

3.3 Temps de trajet jusqu'à la ville de 50000 habitants la plus proche dans les quatre pays d'ASS

3.4 Revenu annuel moyen par habitant dans les régions étudiées $\quad 87$

3.5 Revenu annuel médian par habitant dans les régions étudiées 91

3.6 Distribution des ménages par classe de revenus dans les pays d'ASS 96

3.7 Distribution des ménages par classe de revenus dans les pays étudiés hors ASS 96

3.8 Revenus par quintiles de ménages dans les régions d'ASS 98

3.9 Revenus par quintiles de ménages dans les régions étudiées hors ASS 99

3.10 Taille des ménages dans les régions étudiées 101

3.11 Point de vue des chefs de ménages sur leur sécurité alimentaire 110

3.12 Stratégies d'activités des ménages dans les régions étudiées selon la typologie du RDM08 111

4.1 Classification des activités et des revenus des ménages ruraux 122

4.2 Participation aux activités off-farm dans les régions étudiées 123

4.3 Valeur moyenne et part relative des revenus off-farm dans les régions étudiées

4.4 Distribution des tailles d'exploitation par actif familial 129

4.5 Rémunération théorique et effective du travail agricole salarié dans les régions étudiées

4.6 Participation à l'emploi salarié non agricole et niveau de rémunération dans les régions étudiées 
4.7 Distribution des ménages par classes de revenu non agricole, Kenya et Mexique

4.8 Types d'activités d'auto-emploi au Sénégal

4.9 Revenus des activités d'auto-emploi dans les régions étudiées

4.10 Importance de la migration par région étudiée

4.11 Destination des migrants par région étudiée

5.1 Nouvelles configurations et tendances des systèmes agroalimentaires résultant de la libéralisation et de la mondialisation

5.2 Structure générale des revenus des exploitations par région étudiée 175

5.3 Principaux produits des exploitations par région étudiée

5.4 Part de l'autoconsommation selon les régions étudiées

5.5 Répartition de la production des exploitations entre autoconsommation et principaux types de produits vendus

5.6 Types de commercialisation dans les zones étudiées

6.1 Structure des revenus par région et par quintile

6.2 Indice de diversification par région et par quintile

6.3 Représentation stylisée de la configuration en U inversé

6.4 La diversification au sein et entre les ménages et la courbe en U inversé

6.5 La relation entre revenu et écart de diversification

6.6 La relation revenu/écart de diversification selon les régions étudiées (ASS et hors ASS)

6.7 Distribution des ménages par groupe cible et par région 


\section{Tableaux}

1.1 Sélection d'indicateurs pour les pays du programme RuralStruc

1.2 Principales filières analysées dans les études nationales RuralStruc 42

1.3 Régions d'enquêtes sélectionnées dans les pays RuralStruc

2.1 Dynamique comparée de la croissance régionale du PIB par habitant, 1960-2007

2.2 Population mondiale par région en valeur absolue et en pourcentage, 1960-2050

2.3 Population des pays du programme RuralStruc, 1960-2050

2.4 Entrants sur le marché du travail dans les pays du programme RuralStruc

2.5 Taux d'urbanisation par grandes régions, 1960-2050 70

2.6 Population rurale par grandes régions, 1960-2050 70

3.1 Niveaux de revenu annuel dans les régions étudiées 88

3.2 Structure des ménages et revenu par équivalent adulte 102

3.3 Revenus des ménages avec et sans exploitation agricole 103

3.4 Proportion, taille et revenu annuel des ménages dont le chef de famille est une femme $\quad 105$

3.5 Revenu total du premier quintile en US\$ PPA et en kilocalories 107

3.6 Distribution des ménages par classes de revenus exprimés en kilocalories

3.7 Stratégies d'activités des ménages dans les régions étudiées selon la typologie du RDM08

4.1 Principaux revenus et activités off-farm 127

4.2 Niveau d'instruction des ménages enquêtés 135

4.3 Principales configurations migratoires dans les régions étudiées 148

5.1 Les restructurations dans le secteur agricole et agroalimentaire des pays du programme RuralStruc (hors ASS)

5.2 Les restructurations dans le secteur agricole et agroalimentaire des pays du programme RuralStruc (en ASS)

5.3 Classification des produits utilisée pour l'analyse des données 174

5.4 Participation des ménages maliens aux marchés alimentaires 179

5.5 Importance des arrangements contractuels formels et informels dans les régions étudiées

6.1 Résultats des régressions au niveau régional 204

6.2 Résultats des régressions agrégées au niveau national 206

6.3 Variables les plus étroitement associées au revenu, par région 211 
6.4 L'écart de diversification dans les régions étudiées

6.5 Distribution des ménages de l'échantillon RuralStruc par groupe cible

Pour des raisons éditoriales, les annexes détaillées ne sont pas présentées dans ce livre.

Elles sont accessibles en ligne sur : http://www.worldbank.org/afr/ruralstruc. 



\section{Avant-propos}

Après un long délaissement, les questions structurelles retrouvent une nouvelle vigueur dans l'économie du développement. L'analyse des structures, qui était centrale après la Seconde Guerre mondiale, a été pratiquement bannie à compter de la mise en ouvre du Consensus de Washington dans les années 1980. Toutefois, la nouvelle théorie du développement qui prend forme aujourd'hui adopte un point de vue plus nuancé ; sans nier l'importance des marchés, elle affirme la nécessité d'approches plus compréhensives du développement qui reconnaissent à l'État un rôle plus actif et reflètent mieux les réalités économiques - c'est ce que j'ai appelé la Nouvelle économie structurelle.

Transformations rurales et développement est une contribution minutieuse et importante à ce renouveau des approches structurelles. Ce livre, qui améliore considérablement notre compréhension des économies rurales et des processus de changement, arrive à point nommé.

L'ouvrage s'appuie sur les résultats du programme RuralStruc, mis en œuvre dans le cadre d'un solide dispositif collaboratif réunissant sept pays et coordonné par Bruno Losch. Lancé en 2006 à une époque où le dialogue international sur la politique agricole, enlisé dans les questions commerciales, perdait de vue le phénomène plus général de la transformation rurale, ce programme prévoyait qu’il serait indispensable de réinvestir dans les questions structurelles. Ses résultats renforcent la valeur du Rapport sur le développement dans le monde 2008, qui a été le premier à rappeler le rôle de l'agriculture dans le développement. Ils redonnent vie à un ancien paradigme pour proposer une nouvelle perspective, qui remet en question une part importante de la pensée actuelle sur le développement rural, en particulier pour les pays qui amorcent leur transformation structurelle.

Reposant sur une méthodologie articulant la collecte de données microéconomiques et un point de vue macrostructurel, le programme a conduit d'importants travaux de terrain afin d'examiner les stratégies économiques et les moyens d'existence des ménages ruraux et a associé les résultats à une parfaite compréhension du changement structurel. L'ouvrage souligne les profils récurrents de diversification et de spécialisation qui accompagnent ce processus. En outre, rétablissant le lien avec une vision plus générale, il souligne les difficultés des pays 
dont le développement s'est amorcé tardivement, où les ménages disposent de peu d'alternatives de diversification. C'est le cas en particulier en Afrique subsaharienne, où une transition économique faiblement engagée et la transition démographique en cours engendrent à la fois une forte croissance de la population active et des difficultés considérables pour la jeunesse du sous-continent.

À partir de leur analyse, les auteurs tirent une série d'enseignements pour les politiques publiques. Ils pointent à juste titre qu'il est important que les États reconstituent leurs propres capacités d'élaboration de stratégies de développement global. Ces capacités sont indispensables pour surmonter les principales contraintes, définir les priorités et garantir une coordination adaptée des interventions. Mais surtout, les auteurs montrent qu'en Afrique subsaharienne, un réinvestissement volontariste dans l'agriculture (outre la mise à profit des opportunités de développement de l'industrie manufacturière et des services) sera l'instrument politique le plus important des vingt prochaines années pour progressivement augmenter les revenus, atténuer les risques et favoriser l'innovation et la demande rurale, principal moteur de la diversification rurale - une étape cruciale de la transformation structurelle. Ils soulignent aussi le rôle de l'État dans la fourniture des biens publics, dans la conception de mesures incitatives appropriées et dans l'exploitation du levier que constitue le développement des petites villes pour le changement rural. Tous ces éléments sont des rappels de bon sens et utiles à la communauté des donateurs, aux États et aux parties prenantes des débats au niveau local; ils représentent une contribution importante au rôle de l'agriculture dans le développement.

Justin Yifu LiN

Premier VICE-PRÉSIDENT ET ÉCONOMISTE EN CHEF

BANQUE MONDIALE 


\section{Remerciements}

Ce livre est dédié à la mémoire de Jacques Faye (1946-2010), sociologue rural et membre fondateur de l'IPAR (Initiative Prospective Agricole et Rurale, Sénégal).

Cet ouvrage est une des nombreuses productions issues du programme de la Banque mondiale consacré aux « Dimensions structurelles de la libéralisation pour l'agriculture et le développement rural » (RuralStruc). Au cours des quatre années sur lesquelles ont été mises en ouvre ses deux phases et d'une cinquième année consacrée à la production d'une synthèse et aux premières activités de diffusion des résultats, une collaboration étroite a été instaurée avec de nombreux experts et chercheurs des sept pays participants au programme.

Les activités du programme ont été conduites sous la direction de Bruno Losch (chef de projet, Banque mondiale et CIRAD). Le travail de terrain a été réalisé avec l'appui d'une équipe de coordination formée de Bruno Losch, Sandrine Fréguin-Gresh et Thierry Giordano (ministère français des Affaires étrangères et européennes), respectivement basés à l'université de Pretoria (Postgraduate School of Agriculture and Rural Development) et à la Banque de développement de l'Afrique australe (DBSA).

À la Banque mondiale, le programme a bénéficié de l'appui sans réserve de John Mclntire, directeur Afrique du réseau ESSD. Les évaluateurs du document de projet étaient Louise Cord et Robert Schneider, et Camilla Toulmin (IIED). La mise en œuvre du programme a bénéficié des conseils de Karen Brooks, responsable de secteur (AFTAR) et de Stephen Mink, conseiller senior (AFTSN), qui a été associé à chaque étape du processus. Shanta Devarajan, économiste en chef pour la région Afrique, et Justin Yifu Lin, Premier vice-président et économiste en chef, ont apporté un important soutien à la diffusion des résultats.

Au sein de l'Agence Française de Développement, des ministères français de l'Agriculture et de la Pêche, et des Affaires étrangères et européennes, du Centre de coopération internationale en recherche agronomique pour le développement, et du Fonds international de développement agricole, Bernard Esnouf et 
Didier Simon, Philippe Beyries et Isabelle Ouillon, Florence Lasbennes, Patrick Caron, Jean-Philippe Audinet et Philippe Rémy, ont apporté un suivi et un soutien sans faille, dans la durée.

Le rapport final du programme dont est issu cet ouvrage a été établi par Bruno Losch, Sandrine Fréguin-Gresh et Eric Thomas White (Banque mondiale), avec d'importantes contributions de Thierry Giordano et Jean-François Bélières (CIRAD). L'évaluation par les pairs a été assurée par Derek Byerlee et Don Larson (Banque mondiale) et André Pouillès-Duplaix (AFD).

Les résultats présentés dans les pages qui suivent s'appuient sur deux ensembles de sept rapports nationaux et sur les données produites par les équipes nationales dans les deux phases du programme RuralStruc. Les auteurs et contributeurs aux rapports nationaux sont les suivants :

Kenya : Lilian Kirimi, Mary Mathengue, John Olwande et Betty Kibaara (Tegemeo Institute) ; Paul Gamba, Sam Onyuma et Job Lagat (Egerton University).

Madagascar : Alain Pierre-Bernard, Rivo Ramboarison, Lalaina Randrianarison, Nicole Andrianirina et Lydia Rondro-Harisoa (APB Consulting).

Mali : Amadou Samake, Bino Teme, Ousmane Sanogo, Manda Sadio Keita et Aly Ahamadou (IER) ; Jean-François Bélières, Pierre-Marie Bosc, Christian Corniaux, Jacques Marzin, Denis Gautier et Kako Nubukpo (CIRAD) ; Nango Dembele, John Staatz et Valerie Kelly (Michigan State University) ; El Hadji Oumar Tall ${ }^{\dagger}$ et Bakary Sékou Coulibaly (CEPIA).

Maroc : Najib Akesbi et Driss Benatya (Institut Agronomique et Vétérinaire Hassan II) et Noureddine El Aoufi (Université Mohammed V) pour la phase 1 ; le bureau Icon2e pour la phase 2.

Mexique : Fernando Saavedra, Fernando Rello, Hector Robles, Christian Muñoz et Claudio Gonzalez (FLACSO) ; Virginie Brun (IRD/CEMCA) ; Éric Léonard (IRD) ; Rafael Palma Grayeb (Universidad Veracruzana).

Nicaragua : Arturo H. Grigsby Vado, Francisco J. Perez, Ligia I. Gómez, Edna S. García, Miguel A. Alemán et Yuri L. Marín (Universidad Centro-americana, Instituto Nitlapán).

Sénégal : Cheikh Oumar Ba, Jacques Faye ${ }^{\dagger}$ Ibrahima Hathie, Pape Nouhine Dièye, Bocar Diagana, Adama Faye, Madické Niang et Mamadou Dansoko (IPAR et ASPRODEB).

Jean Coussy (Ceri-Sciences Po) et Jean-Jacques Gabas (Université Paris X-Orsay et CIRAD) ont participé à la préparation du programme et à son atelier de lancement au Sénégal (avril 2006), ainsi qu'à plusieurs événements de diffusion des résultats. Jean-Jacques Gabas a également animé deux ateliers d'étudiants de Sciences Po Paris (2006 et 2007) et a participé à la préparation de plusieurs fiches de synthèse du programme. Éric Léonard (IRD) et Jean-Pierre Rolland (ministère français des Affaires étrangères et européennes) ont apporté leur soutien au déroulement du programme respectivement au Mexique à Madagascar. 
Emmanuelle Benicourt (consultante) et Véronique Meuriot (CIRAD) ont également contribué au projet. Émilie Losch a dessiné le logo du programme et Erin O'Brien s'est chargée de la mise en forme des rapports nationaux et des versions préliminaires du rapport final.

Au sein de la Banque mondiale, les personnes suivantes ont contribué au programme : Bruno Losch (chef de projet), Patrick Labaste et Michael Morris (responsables successifs du fonds fiduciaire multibailleur de RuralStruc), Eric Thomas White, Malick Antoine, Angela Lisulo, Benjamin Billard, James Keough, Beatriz Prieto-Oramas et Ingrid Mollard. Hawanty Page et Germaine Mafougong-Ethy ont assuré le support administratif du programme. Jeff Lecksell et Bruno Bonansea ont établi les cartes sur la base des informations communiquées par les équipes nationales.

Le programme a bénéficié des conseils de son comité scientifique d'orientation, présidé par C. Peter Timmer (Cabot Professor of Development Studies, emeritus, Harvard University) et constitué par Kirsten Albrechtsen de Appendini (Colegio de México), Pierre-Marie Bosc (CIRAD), Peter Gibbon (Danish Institute for International Studies), Catherine Laurent (Institut national de la recherche agronomique), Jean-Luc Maurer (Institut des hautes études internationales et du développement), Sandra Polaski (Carnegie Endowment for International Peace), Marc Raffinot (Université Paris-Dauphine) et Sibiri Jean Zoundi (Club du Sahel et de l'Afrique de l'Ouest, OCDE).

Enfin, il a également bénéficié du suivi attentif d'un comité de pilotage formé de ses bailleurs de fonds (Agence Française de Développement, ministères français des Affaires étrangères et européennes et de l'Agriculture et de la Pêche, Centre de coopération internationale en recherche agronomique pour le développement, Fonds international de développement agricole et Banque mondiale) et successivement présidé par Florence Lasbennes (ministère des Affaires étrangères et européennes) et Marie-Cécile Thirion (Agence Française de Développement).

La version française a été préparée par Christine Mercier avec le concours de Bruno Losch. 



\section{À propos des auteurs}

Bruno Losch est économiste senior à la Banque mondiale et directeur de recherches au CIRAD (Centre de coopération internationale en recherche agronomique pour le développement - UMR Art-Dev). Dans le cadre de ses fonctions actuelles, il a dirigé le programme RuralStruc, une initiative conjointe de la Banque mondiale, du FIDA et du gouvernement français. Au CIRAD, il a été en charge du programme Agricultures familiales, puis a rejoint l'université de Berkeley en qualité de chercheur invité. Titulaire de masters en sciences politiques et en géographie et d'un doctorat en économie, il a beaucoup publié dans le domaine des études rurales, des politiques publiques et de l'économie politique du développement.

Sandrine Fréguin-Gresh est titulaire d'un master en agronomie et en géographie et d'un doctorat en économie agricole. Elle est chercheur au CIRAD (UMR ArtDev), où elle a axé ses travaux sur l'agriculture contractuelle et la transformation rurale en Afrique, en Amérique centrale et aux Caraïbes. Après avoir travaillé à l'université de Pretoria, Afrique du Sud, en qualité de chercheur invité, elle a été détachée à l'Institut de recherche et d'études du développement - Nitlapán - de l'Université centraméricaine à Managua, Nicaragua, où elle dirige des projets de recherche sur le marché du travail, les migrations et la transformation agraire.

Eric Thomas White est consultant auprès de la Banque mondiale et directeur associé du cabinet de conseil en développement international INTEGRA LLC, où il dirige les activités sur les technologies de l'information et de la communication (TIC) pour l'agriculture. Il a notamment effectué des missions de conseil auprès de la Banque africaine de développement et du Millennium Challenge Account en Zambie. Auparavant, il a servi comme lieutenant dans la marine américaine. Il est titulaire d'un master d'administration publique en développement international de la Harvard Kennedy School et d'une licence d'économie de l'Université de Virginie. 



\section{Acronymes}

AFD

ALE

ALENA

ASS

CAFTA-DR

CEA

CER

CIRAD

CNUCED

EqA

FAO

FIDA

GAFSP

GIEC

$\mathrm{H} 1$, etc.

IAASTD

IDE

IFOAM

$\mathrm{iHH}$

ILC

kcal

LSMS
Agence Française de Développement

Accord de libre-échange

Accord de libre-échange nord-américain

Afrique subsaharienne

Accord de libre-échange entre Amérique centrale - République dominicaine

Commission économique des Nations unies pour l'Afrique

Communauté économique régionale

Centre de coopération internationale en recherche agronomique pour le développement

Conférence des Nations unies sur le commerce et le développement

Équivalent adulte

Organisation des Nations unies pour l'alimentation et l'agriculture

Fonds international pour le développement agricole

Programme mondial pour l'agriculture et la sécurité alimentaire

Groupe d'experts intergouvernemental sur l'évolution du climat Hypothèse 1, etc.

Évaluation internationale des connaissances, des sciences et des technologies agricoles pour le développement

Investissement direct à l'étranger

Fédération internationale des mouvements de l'agriculture biologique

Indice de Herfindahl-Hirschman

Coalition foncière internationale

kilocalorie

Études de mesure des niveaux de vie 
LSMS-ISA Enquêtes LSMS intégrées sur l'agriculture

MdPM Moyenne des parts des ménages

NEPAD Nouveau partenariat pour le développement de l'Afrique

OCDE Organisation de coopération et de développement économiques

OIT Organisation internationale du travail

OMC Organisation mondiale du commerce

OMD Objectifs du Millénaire pour le développement

ONU-Habitat Programme des Nations unies pour les établissements humains

ONUDI Organisation des Nations unies pour le développement industriel

PEA Population économiquement active

PDD Programme de Doha pour le développement

PDDAA Programme détaillé de développement de l'agriculture africaine

PdMR Part des moyennes régionales

PIB Produit intérieur brut

PIB agr. Produit intérieur brut agricole

PMA Pays les moins avancés

PNB Produit national brut

PPA Parité de pouvoir d'achat

RDM Rapports annuels de la Banque mondiale sur le développement dans le monde

RIGA Projet Activités rurales génératrices de revenus

RNFE Économie rurale non agricole

RuralStruc Programme sur les dimensions structurelles de la libéralisation pour l'agriculture et le développement rural

TIC Technologies de l'information et de la communication

UNRISD Institut de recherche des Nations unies pour le développement social

WDI Indicateurs du développement dans le monde de la Banque mondiale

ZFI Zone franche industrielle 


\section{Aperçu}

Les quarante dernières années de l'histoire mondiale ont été marquées par de profondes transformations. La population de la planète s'est accrue de 3,2 milliards d'individus - un quasi-doublement - et, pour la première fois, la population urbaine est plus nombreuse que la population rurale. L'avènement d'une économie globale, stimulée par le progrès technique et l'importance croissante des économies émergentes dans le paysage politique international, a considérablement modifié les perspectives du développement mondial.

Dans les quarante prochaines années, la population mondiale s'accroîtra encore de 2,3 milliards d'individus et l'urbanisation touchera $70 \%$ de l'humanité. Ce changement démographique abrupt, opéré en moins d'un siècle, pose la question de la soutenabilité : l'épuisement des ressources naturelles, les conséquences du changement climatique et les risques élevés associés aux inégalités de développement économique entre les différentes régions du monde remettent en cause la trajectoire de croissance mondiale.

Le débat international déjà ancien autour des multiples défis posés par un monde peuplé de 9 milliards d'individus (le moyen de les nourrir n'étant pas des moindres) gagne aujourd'hui en intensité. Cependant, les chiffres globaux tendent à détourner l'attention de phénomènes d'égale importance concernant la dynamique de croissance démographique et la distribution de la population. Ainsi, la marginalisation progressive du monde "développé " (c'est-à-dire des pays ayant atteint le plus haut niveau de richesse matérielle) dans la population mondiale constitue une évolution majeure : en 2050, l'Asie demeurera la région la plus peuplée, mais l'Amérique du Nord et l'Europe réunies ne représenteront plus que $15 \%$ de la population mondiale. À cette date, le poids démographique relatif de l'Afrique subsaharienne et de l'Europe, qui était respectivement de $10 \%$ et $20 \%$ en 1960, sera inversé. Cette redistribution de la population accentuera les inégalités d'accès aux ressources.

Pourtant, malgré l'urbanisation continue, 2,8 milliards de personnes résideront encore en zone rurale en 2050. Cette masse de ruraux vivra principalement de l'agriculture. Mais les différents rythmes régionaux d'urbanisation auront une forte incidence sur la distribution des populations : l'Asie du Sud et l'Afrique subsaharienne concentreront près des deux tiers de la population rurale mondiale et l'Afrique subsaharienne sera la seule région où le nombre de ruraux continuera d'augmenter. 
Ces tendances sont particulièrement importantes car elles remettent en question les perspectives de développement d'une grande partie du monde. Ainsi, l'évolution de l'agriculture imprimera sa marque sur le changement économique, social et environnemental car, au-delà de la production d'aliments, elle représente l'activité centrale et la principale source de revenus de milliards de ruraux. La situation est particulièrement délicate pour l'Afrique subsaharienne, où la faible diversification de l'économie - illustrée en particulier par le taux d'industrialisation anémique de la région - limite les perspectives d'emploi hors des secteurs agricole et informel. Dans les quinze prochaines années, sous l'effet de la dynamique démographique, 330 millions de jeunes entreront sur le marché du travail - un chiffre à peu près équivalent à la population actuelle des ÉtatsUnis ; 195 millions d'entre eux vivront en zone rurale où ils devront trouver des emplois. À défaut, ils migreront dans les villes ou dans des pays voisins, où ils contribueront aux difficultés économiques, sociales et politiques engendrées par la méga-urbanisation et les migrations de masse.

Même si la diversification économique et la gestion de la croissance urbaine demeurent essentielles, les économies caractérisées par une forte population rurale et une industrialisation lente devront s'efforcer de créer des emplois ruraux. Cette configuration explique que l'évolution de la politique de développement agricole et rural dans les vingt prochaines années jouera un rôle décisif dans la lutte contre la pauvreté, le développement économique et la stabilité politique.

\section{Du débat de l'OMC à la crise des prix alimentaires - un manque de vision structurelle à long terme}

Le programme RuralStruc a été lancé en 2005 dans le contexte d'un intense débat international autour de la libéralisation des marchés agricoles et de ses conséquences pour l'agriculture des pays en développement. Son objectif premier était de porter un nouveau regard sur l'agriculture et son rôle dans le développement, et plus particulièrement de reconnecter les questions liées à la libéralisation du commerce avec la discussion plus générale de la transformation et de l'évolution des économies rurales dans un contexte de mondialisation accélérée.

Le programme a été exécuté sur cinq ans (2006-2010) pendant lesquels le champ du débat international sur l'agriculture s'est radicalement transformé. Trois grandes questions ont affecté ce débat : (1) les préoccupations croissantes quant aux conséquences du changement climatique mondial, qui ont culminé avec le Sommet de Copenhague en 2009 ; (2) la crise mondiale des prix produits alimentaires ; et (3) la crise financière mondiale et ses conséquences pour la croissance. Ces questions demeurent d'actualité et l'agriculture reste un domaine d'attention internationale, comme en témoigne la mise en place d'un G20 agricole.

Au sein du débat international, le " Rapport sur le développement dans le monde 2008 : L'agriculture au service du développement» (RDM08) offrait un rappel fort et solidement argumenté du rôle central de l'agriculture dans le pro- 
cessus de développement et de sa contribution importante à la réduction de la pauvreté. La crise des prix des produits alimentaires et les questions plus pressantes à court terme qui l'ont accompagnée ont retardé la prise en compte de ses recommandations dans les politiques publiques, mais sa contribution au débat n'en est pas moins précieuse.

L'originalité du RDM08 résidait dans l'adoption d'une approche régionale reposant sur les différents stades du processus de transformation structurelle. Il introduisait l'idée de "trois mondes de l'agriculture ", correspondant aux fonctions de l'agriculture aux différentes phases du développement d'un pays. Le premier monde, qui comprend la majeure partie de l'Afrique subsaharienne, correspond aux pays dont la croissance économique et l'emploi sont très dépendants de l'agriculture ("pays à base agricole»). Le deuxième monde correspond aux " pays en mutation ", pour la plupart en Asie du Sud et de l'Est, où le rapide creusement des écarts entre les revenus ruraux et urbains et la persistance d'une extrême pauvreté rurale sont parmi les principales sources de tensions sociales et politiques. Enfin, le troisième monde désigne les « pays urbanisés », au rang desquels figurent la plupart des pays d'Amérique latine, où l'agriculture peut aider à réduire la pauvreté rurale résiduelle par une meilleure intégration aux marchés alimentaires modernes et le développement de services à l'environnement.

Bien que la conceptualisation de ces trois mondes ait aidé à formuler des recommandations de politique publique, certaines des difficultés auxquelles les différents pays sont confrontés ont été oubliées. Il s'agit notamment des questions démographiques (en particulier la croissance démographique et ses conséquences pour l'emploi) et des asymétries de compétitivité résultant de la mondialisation.

Ces difficultés remettent en cause la viabilité de la trajectoire historique de transformation structurelle, à savoir le glissement progressif de l'agriculture vers l'industrie, puis vers les services. La dynamique sous-jacente à cette transition économique (composante essentielle de la transformation structurelle) est l'augmentation de la productivité agricole, qui favorise le changement technique et permet au travail et au capital de s'orienter vers d'autres activités économiques. Simultanément, une recomposition spatiale d'ensemble s'opère lorsque la maind'œuvre se reporte d'activités éparses (agriculture) vers des activités plus concentrées (industrie) et que le processus d'urbanisation s'accélère. Ce processus de changement se traduit par une augmentation des revenus et de la richesse et une amélioration des conditions de vie qui, alliées aux progrès de la médecine, amorcent la transition démographique (diminution progressive et échelonnée des taux de mortalité et de natalité). Il en résulte une première phase de croissance rapide de la population, suivie d'une stabilisation.

Cette vision évolutionniste, confirmée statistiquement par les caractéristiques des transitions passées, est remise en cause par les dynamiques du monde actuel, un constat qui rappelle la nécessité d'une perspective historique plus contextualisée pour comprendre les changements structurels en cours. La prise en compte du «moment » est essentielle à la compréhension des processus, car les opportunités, les contraintes et l'équilibre des pouvoirs ont évolué constamment tout au long de l'histoire mondiale. 
Ainsi, trois caractéristiques des transitions passées doivent être gardées à l'esprit. Tout d'abord, les transitions intervenues en Europe occidentale et en Amérique du Nord au XIX $x^{\mathrm{e}}$ siècle et dans la plus grande partie du $\mathrm{Xx}^{\mathrm{e}}$ ne peuvent être isolées de l'hégémonie politique européenne et américaine, qui a réduit ou éliminé la concurrence et créé des marchés captifs très lucratifs. L'accès à ces marchés a fortement favorisé la spécialisation économique et l'industrialisation. Ensuite, la transition européenne a bénéficié d'un mouvement d'émigration sans égal qui a facilité l'ajustement des économies européennes et la gestion des excédents de main-d'œuvre. En effet, entre 1850 et 1930, près de 60 millions d'Européens ont émigré vers les "nouveaux mondes », 35 millions rien qu'aux ÉtatsUnis. Enfin, les transitions d'Amérique latine et d'Asie se sont amorcées dans la période très particulière de développement national autocentré qui a caractérisé l'ordre international entre la crise de 1929 et l'époque actuelle de mondialisation, engagée à la fin des années 1970. Ce régime de développement s'est caractérisé par la substitution aux importations, le protectionnisme et une forte intervention de l'État, qui ont tous contribué à la modernisation économique. En Amérique latine, la transition économique s'est amorcée entre les deux guerres mondiales ; en Asie, elle a démarré dès les années 1950. Ces deux régions ont en outre bénéficié de programmes d'assistance massifs mis en œuvre pendant la guerre froide.

Aujourd'hui, la situation des pays en développement qui se situent aux premières étapes de leur transformation structurelle est profondément différente. L'Afrique subsaharienne - la dernière région du monde à s'engager dans ce processus - doit simultanément affronter les défis d'une transition économique à son stade initial et d'une transition démographique inachevée dans le contexte d'une économie mondiale ouverte et des contraintes du changement climatique.

Les pays en retard de développement - la plupart des pays d'Afrique subsaharienne - ont certains avantages sur leurs prédécesseurs : ils peuvent bénéficier des progrès techniques et de l'expérience acquise et profiter des nouvelles opportunités d'accès aux marchés mondiaux. Mais ils sont aussi confrontés à de nouvelles contraintes, comme les colossales asymétries de productivité, l'intensification de la concurrence internationale (en particulier des grands pays émergents) et la dégradation de l'environnement. Ces défis de la période et l'instabilité du contexte international réduisent considérablement leur marge de manœuvre pour gérer leur transformation structurelle, surtout lorsqu'il s'agit d'améliorer les conditions de vie de populations en forte croissance.

\section{Le programme de recherche}

Ces défis ont pesé sur la conception du programme RuralStruc, dont l'objectif était triple : (1) contribuer aux connaissances analytiques sur les processus de changement et leurs effets sur l'agriculture et l'économie rurale des pays en développement, (2) alimenter et améliorer les débats nationaux et internationaux en promouvant et en réintroduisant la question du changement structurel dans la définition des politiques agricoles et rurales et (3) proposer des orientations 
pour l'élaboration des politiques publiques. La devise du programme était : «Une meilleure compréhension pour de meilleures politiques ».

La conception du programme reposait sur trois hypothèses articulées. La première $(\mathrm{H} 1)$ est que la restructuration des marchés agroalimentaires mondiaux et l'asymétrie grandissante des conditions de la concurrence internationale entraînent une différenciation croissante des structures agricoles, de commercialisation, de transformation et de distribution. La deuxième hypothèse $(\mathrm{H} 2)$ est que face à ces marchés mondiaux plus concurrentiels et plus exigeants, les sources de revenus et la structure d'activités des ménages ruraux se transforment au profit d'activités situées hors exploitation agricole. La troisième hypothèse (H3) est que les processus de marginalisation dans l'agriculture et les difficultés des ménages ruraux à s'adapter à ce nouveau contexte (surtout dans des situations caractérisées par l'absence d'alternatives réelles à l'activité agricole) conduisent parfois à des impasses dans le processus de transformation structurelle. Cette dernière hypothèse est particulièrement sensible pour le premier des trois mondes de l'agriculture, à savoir les pays à base agricole.

Une approche comparative a été adoptée pour tester ces hypothèses. Sept pays à différents stades de transformation structurelle et d'intégration économique ont été sélectionnés pour tirer les leçons des différents modes d'adaptation des économies rurales au changement. Le Kenya, le Mali, Madagascar et le Sénégal se situent au premier stade de la transition économique et font partie du premier des trois mondes de l'agriculture. Le Maroc et le Nicaragua se situent à un stade intermédiaire du processus de transformation : l'agriculture conserve une place très importante dans leur économie mais son rôle est en recul. Enfin, le Mexique, pays à revenu intermédiaire de la tranche supérieure, est bien plus avancé dans le processus de transformation : il est profondément intégré à ses voisins du Nord à travers l'Accord de libre-échange nord-américain (ALENA) et fait partie du «monde urbanisé » du Rapport sur le développement dans le monde (RDM08).

Les activités du programme ont été déployées suivant un processus collaboratif réunissant sept équipes nationales, qui ont été étroitement associées à chaque étape, notamment la préparation, la mise en œuvre, l'analyse, la diffusion et la discussion des résultats. La première phase du programme a été consacrée à la production d'une série de documents à caractère général, présentant l'état des connaissances sur les processus de transformation rurale dans chaque pays. Cet exercice a fait apparaître les faiblesses du corpus de connaissances empiriques sur les caractéristiques des économies rurales, en particulier les moyens d'existence et les activités génératrices de revenu des ménages. Les seules informations disponibles provenaient d'études de cas poursuivant des objectifs variés et réalisées selon des méthodologies différentes, qui empêchaient leur exploitation systématique.

À la suite de ces premiers résultats, il a été décidé, lors de la seconde phase, de recueillir des données primaires au moyen d'enquêtes de terrain. Début 2008 , près de 8000 ménages ruraux ont été enquêtés sur leurs activités et leurs revenus dans 26 régions des sept pays participants (il est à noter que ces enquêtes ont eu lieu avant que la crise des prix alimentaires ne prenne toute son 
ampleur). Le corpus de données ainsi constitué a fourni un instantané unique en son genre des structures de revenus ruraux que la méthodologie commune permettait de comparer entre les régions étudiées. Les enquêtes ayant été réalisées en un seul passage, il n'a pas été possible de conduire d'analyse dynamique. Cependant, comme elles employaient la même méthodologie avec une réalisation simultanée dans des régions situées à des stades différents de développement et d'intégration à l'économie mondiale, une interprétation dynamique des résultats entre régions et entre pays a été possible. Cette interprétation a permis d'étudier les facteurs de la transformation rurale et de contribuer au débat sur la transition économique et le changement structurel.

\section{Le rôle persistant de l'agriculture et l'ampleur de la pauvreté rurale}

L'analyse a fait apparaître un ensemble hétérogène de situations rurales qui partageaient néanmoins d'importantes caractéristiques communes, dont la première était la domination persistante de l'agriculture parmi les activités économiques. Quatre-vingt-quinze pour cent des ménages interrogés ont des activités agricoles : cultures, élevage ou transformation de produits à la ferme. Cependant, certaines régions sont nettement plus diversifiées, comme Tequisquiapan (État de Querétaro) au Mexique, où seulement $30 \%$ des ménages ont des activités agricoles, et le Souss au Maroc, où le pourcentage de ménages agricoles ne dépasse pas $75 \%$.

Outre les niveaux élevés d'activité agricole, les régions étudiées se caractérisent par une pauvreté généralisée, en particulier en Afrique subsaharienne. Le revenu médian estimé (qui donne une meilleure idée de la réalité rurale que les moyennes régionales) se situe entre 0,5 et 2 dollars américains (US\$) à parité de pouvoir d'achat (PPA) par personne et par jour en Afrique subsaharienne (excepté dans le district de Nakuru North au Kenya, où il atteint $3 \$$ ) contre 1,5 à 5,5 \$ PPA dans les autres régions. S’agissant du revenu moyen, $70 \%$ des ménages interrogés en Afrique subsaharienne gagnent moins de 2 \$ PPA par personne et par jour et $40 \%$ ont moins de $1 \$$ par jour, ce pourcentage pouvant atteindre $80 \%$ dans certaines régions, en particulier au Mali.

L'examen des $20 \%$ de ménages les plus pauvres d'une région exprime la réalité de la pauvreté de façon encore plus frappante que les valeurs régionales. Ce quintile inférieur dispose de moins de 1 \$ par jour dans toutes les régions de l'enquête hormis le Mexique, même dans des régions qui étaient initialement considérées comme « les mieux loties » en raison de leurs liens avec les marchés et de solides dotations en facteurs. En outre, le revenu moyen des quintiles supérieurs est généralement accru par un très petit nombre de ménages nettement plus aisés qui bénéficient de conditions socio-économiques très particulières.

Une conséquence de cette grande pauvreté est que les ménages sont exposés à des risques élevés qui limitent leur capacité d'investissement et d'innovation. Cette situation désespérante est aggravée pour les ménages qui sont également 
confrontés à l'insécurité alimentaire. Lorsque les revenus en dollars PPA sont convertis en kilocalories ( $\mathrm{kcal}$ ) sur la base des prix locaux, une proportion importante des ménages de toutes les régions étudiées peine à satisfaire ses besoins caloriques journaliers minimaux : dans 11 des 19 zones étudiées en Afrique subsaharienne, le quintile inférieur est en moyenne incapable de fournir $2450 \mathrm{kcal} /$ personne/jour. Deux régions au Nicaragua sont dans la même situation.

Ces résultats, qui mettent en évidence l'importance des revenus de l'exploitation agricole et une pauvreté généralisée, même dans les économies rurales plus diversifiées, ont donné un relief particulier aux trois hypothèses du programme. Dans le cas des pays d'Afrique subsaharienne, les données indiquent que même lorsque des processus de différenciation des exploitations ont été suscités ou renforcés par une intégration croissante aux marchés alimentaires mondiaux, aucun n'a été suffisamment profond pour avoir un effet structurant sur l'économie rurale des régions étudiées. Les données montrent également que quelles que soient les autres activités dans lesquelles les ménages ont pu se diversifier, dans un contexte marqué par le développement d'une économie rurale non agricole, peu d'entre eux ont pu totalement abandonner l'agriculture et peu de ceux qui sont restés en zone rurale ont échappé à la pauvreté. À l'autre extrémité du gradient de la transition, le cas du Mexique montre que même lorsque les processus de différenciation sont importants et que de nombreux ménages quittent l'agriculture, la pauvreté rurale peut demeurer significative. Dans ces économies, de nombreux ménages des quintiles inférieurs sont encore pauvres - leur revenu est en dessous du seuil de 2 US\$ par jour et parfois même de 1 US\$ par jour.

De même, les différences observées en comparant les zones rurales des sept pays, tant en termes de niveau que de distribution des revenus, éclairent les processus de changement structurel. En Afrique subsaharienne, l'écrasante majorité des ménages ruraux est pauvre, mais les inégalités entre eux sont faibles (les indices de Gini construits à partir de l'échantillon vont de 0,35 à 0,45$)$. Au Maroc et au Nicaragua, dont la transition est plus rapide, le revenu rural moyen est sensiblement plus élevé mais les inégalités sont très marquées (indices de Gini entre 0,6 et 0,7 ). $\mathrm{Au}$ Mexique, où les revenus ruraux médians sont les plus élevés de l'échantillon, les indices de Gini sont relativement bas $(0,4)$; le problème des inégalités en zone rurale y a été supplanté par celui des inégalités entre urbains et ruraux. Le problème au Mexique est la marginalisation croissante des zones rurales et le pays affiche en effet l'écart le plus marqué de l'échantillon entre le revenu des ménages enquêtés et le PIB national par habitant, avec un rapport allant de quatre à sept.

\section{Production agricole, marchés et processus de différenciation}

Les travaux en économie agricole de ces dix dernières années évoquent abondamment l'intégration des producteurs agricoles des pays en développement à l'économie de marché. De nombreuses études de cas décrivent comment les producteurs ont forgé de nouveaux liens avec les marchés de produits à forte valeur ajoutée, ont opéré une intégration verticale par le biais de contrats et ont recueilli 
les bénéfices de la « révolution des supermarchés ». Bien que ces processus soient en cours dans plusieurs régions du monde en développement, leur impact semble toutefois exagéré, surtout lorsqu'il s'agit de la proportion d'agriculteurs effectivement partie prenante de ce nouveau monde agricole. Il existe certes de nouvelles opportunités, mais elles sont souvent très localisées dans des régions particulières et surtout, elles touchent un nombre assez faible de producteurs. Dans un pays donné, alors que des milliers, voire des dizaines de milliers de ménages agricoles peuvent avoir bénéficié du développement de chaînes de valeur intégrées, des centaines de milliers, voire des millions de ménages restent enracinés dans des types d'agriculture plus traditionnels. Cette situation est illustrée par le succès bien connu de l'horticulture kényane (situées au deuxième rang des exportations de produits de base du pays, les exportations horticoles concernent moins de 50000 ménages agricoles au Kenya sur un total supérieur à 3,5 millions).

\section{Importance des denrées alimentaires de base et de l'autoconsommation}

L'importance des denrées alimentaires de base, une céréale généralement, est une caractéristique couramment observée de la production des ménages étudiés : le riz à Madagascar, au Mali et au Sénégal, auquel s'ajoutent le millet et le sorgho dans ces deux derniers pays ; le blé au Maroc ; le maïs au Kenya, au Mexique et au Nicaragua. Au total, $98 \%$ des ménages étudiés en Afrique subsaharienne et $76 \%$ dans les autres régions produisent des denrées alimentaires de base. Dans l'échantillon de RuralStruc, elles représentent en moyenne $62 \%$ de la production des exploitations agricoles. Ce chiffre atteint souvent $80 \%$ en Afrique subsaharienne tandis que la situation est plus diversifiée dans les autres pays, qui cultivent davantage d'autres produits. Ainsi la proportion se situe autour de $45 \%$ au Nicaragua et au Maroc, bien qu'elle ait été plus faible dans ce dernier pays où la sécheresse avait réduit la part relative du blé durant l'année de l'enquête. La spécialisation dans le maïs dans les régions étudiées au Mexique découle en revanche d'incitations spécifiques.

L'importance généralisée des denrées alimentaires de base tient au fait que les niveaux de risques, et parfois l'insécurité alimentaire, ont conduit une forte proportion de ménages d'Afrique subsaharienne à maintenir, au moins en partie mais significativement, une activité d'agriculture de subsistance. Ces ménages ne cultivent pas seulement des produits vivriers, ils consomment aussi une forte proportion de leur production. L'autoconsommation représente à peu près la moitié de la production, des extrêmes étant observés d'un côté au Mali (75 \% à Diéma ou Tominian) et de l'autre à Mekhé, au Sénégal (moins de $20 \%$ ). Hors d'Afrique subsaharienne, la part de la production autoconsommée est inférieure (20 à $30 \%$ ), bien qu'elle soit importante pour les quintiles inférieurs au Nicaragua (jusqu'à $60 \%$ ). Le très faible niveau de l'autoconsommation dans les régions mexicaines est la conséquence d'une restructuration de la filière maïs après l'entrée en vigueur de l'ALENA.

De manière générale, la part de l'autoconsommation est inversement proportionnelle à la richesse des ménages et de la région. Les ménages enquêtés en 
Afrique subsaharienne sont moins avancés dans ce processus parce qu'ils sont plus pauvres. L'importance de l'autoconsommation révélée par l'enquête illustre deux effets complémentaires qui limitent la participation des petits agriculteurs sur les marchés. Un effet d'offre qui renvoie aux stratégies de gestion des risques que les ménages déploient pour garder le contrôle de leurs approvisionnements alimentaires - réponse directe à une situation de marchés incomplets et imparfaits. Un effet de demande qui s'explique par le manque d'acheteurs, conséquence d'une faible accessibilité physique aux marchés et d'une médiocre intégration aux filières de commercialisation, ou de l'insuffisance des surplus de production, trop faibles pour attirer les commerçants.

\section{Commercialisation par les circuits traditionnels}

Ces observations expriment une réalité duelle. Les zones rurales - en particulier en Afrique subsaharienne - conservent une activité d'agriculture de subsistance même si la connexion aux marchés est aujourd'hui indéniablement meilleure. Ainsi les ménages qui ne vendent aucun produit font exception et la grande majorité d'entre eux achètent également des denrées alimentaires produites par d'autres.

Dans les régions d'Afrique subsaharienne étudiées, les circuits de commercialisation traditionnels persistent. La plupart des agents de collecte privés recourent à des stratégies informelles fondées sur la confiance pour obtenir la production des agriculteurs, et la contractualisation reste faible, même parmi les exploitations solidement intégrées aux marchés par des relations continues avec des grossistes ou des agro-industriels (c'est le cas avec les situations de monopsone comme pour le coton au Mali). Quelques agro-industries locales pratiquent toutefois la contractualisation (tomates dans le Haut Delta, Sénégal ; lait à Antsirabe et haricots verts à Itasy, Madagascar ; canne à sucre au Kenya) mais les systèmes de commercialisation modernes sont plus courants hors d'Afrique subsaharienne. Cependant, la contractualisation intervient rarement au niveau des producteurs et le plus souvent en aval, entre le grossiste, l'unité de collecte ou l'organisation de producteurs et l'entreprise de transformation ou le service d'achats des distributeurs (comme dans l'industrie laitière au Nicaragua).

Lorsqu'il y a eu diversification agricole, celle-ci s'est opérée sans aucun schéma évident. Les enquêtes ont révélé des exemples hétérogènes de diversification agricole qui se sont produits en réponse à des opportunités spécifiques de la région. Ce sont l'héritage d'une culture commerciale coloniale (coton au Mali, arachide au Sénégal, café au Kenya), un investissement réalisé par une entreprise étrangère (haricots verts produits par Lecofruit à Madagascar) ou l'émergence d'entrepreneurs locaux permise par un investissement public en infrastructures (comme la dynamique de production d'échalotes liée aux aménagements hydro-agricoles dans la zone de l'Office du Niger au Mali).

S’agissant de la première hypothèse du programme, la conclusion est que les ménages des enquêtes RuralStruc participent à des économies rurales qui n’ont pas été radicalement remaniées par l'intégration verticale et la révolution des 
supermarchés (la situation de la région du Sotavento au Mexique est une exception). La rareté des systèmes de production agricole marqués par les nouvelles formes d'intégration aux marchés n'est pas surprenante. Plus que de nouveaux types de liaisons marchandes, la différenciation entre exploitations traduit surtout des différences dans le patrimoine des ménages, qui correspond plus certainement et d'abord aux caractéristiques des systèmes agraires locaux.

\section{Importance du patrimoine des ménages}

Les analyses économétriques des déterminants du revenu agricole des ménages enquêtés corroborent cette conclusion. Les calculs de régression révèlent que les revenus agricoles des ménages dépendent davantage des déterminants traditionnels du revenu que de nouveaux facteurs. L'importance généralisée de la dotation en terres est particulièrement frappante (elle est récurrente dans 22 des 30 zones étudiées, ce qui en fait la variable la plus significative de l'enquête). Cela laisse à penser qu'il est souvent plus utile d'agrandir la surface cultivée que d'utiliser des engrais ou des semences améliorées.

Les deux autres déterminants du revenu par tête sont l'existence d'un grand cheptel et d'une petite famille, tandis que l'intégration au marché et l'utilisation d'intrants agricoles modernes (semences et engrais) semblent moins importantes. Bien que l'enquête n'ait pas recueilli d'informations détaillées sur les pratiques des agriculteurs et n'ait pas permis une compréhension fine des systèmes de production, une conclusion notable est que l'intégration au marché n'améliore pas nécessairement les revenus et que son impact sur les résultats est spécifique au contexte. Ainsi, les effets revenus de la contractualisation sont très variables et dépendent à la fois du niveau de revenus (les ménages pauvres peuvent être en situation de forte dépendance vis-à-vis de l'agro-industriel) et du contexte régional (notamment de l'existence ou non d'une concurrence entre opérateurs en aval de la production).

\section{Diversification des activités et reconfiguration de l'économie rurale}

Étant donné l'ampleur de la pauvreté observée par l'enquête, les risques auxquels les ménages sont exposés (qu'il s'agisse des risques liés au climat, aux maladies et aux nuisibles, aux prix ou à l'accès au marché) sont un problème majeur et un déterminant décisif de leurs stratégies d'activités et modes de vie. Les ménages qui encourent des risques élevés dans leurs activités agricoles recherchent souvent des opportunités hors de l'exploitation ; c'est pourquoi une grande majorité des ménages enquêtés ont des activités externes (75\% en moyenne). Les proportions sont plus élevées en Afrique subsaharienne (80 à $95 \%$ ) et plus faibles dans les autres régions, où l'on observe une plus forte spécialisation sur l'exploitation agricole.

Malgré ces tendances générales, le développement de l'économie rurale non agricole demeure inégal et les activités non agricoles se caractérisent souvent par 
l'importance de l'auto-emploi, la fourniture de menus services et la faiblesse de l'emploi salarié. Ainsi, l'image qui ressort des données d'enquête est très éloignée de cette économie rurale dynamique souvent décrite dans la littérature.

\section{Des opportunités de diversification inégales}

La diversification associe le plus souvent quatre grandes catégories de revenus : travail salarié agricole et non agricole, travail indépendant et transferts.

Travail salarié agricole. L'emploi salarié agricole est une activité courante (constatée pour un quart de l'échantillon) et peut aider les pauvres à compléter les revenus de leur exploitation. C'est notamment le cas entre les campagnes agricoles mais, en règle générale, les salaires ne sont pas très élevés. Les salaires observés (habituellement par référence à la haute saison, où la demande de main-d'œuvre est forte) vont de 2 à 4 US\$ PPA par jour dans les régions d'Afrique subsaharienne étudiées et de 10 à 15 US\$ PPA par jour ailleurs. Les emplois agricoles sont presque toujours saisonniers et procurent un très faible revenu sur l'année. Bien que de nombreux ménages ruraux effectuent ce type de travaux, cela reste un complément limité des revenus. Le seul cas où le travail salarié agricole peut aider les ménages à échapper à la pauvreté est lorsqu'un membre du foyer obtient un emploi permanent, qui peut rapporter 7 US\$ PPA par jour au Sénégal et jusqu'à 9 US\$ PPA par jour au Mexique. Mais ces opportunités sont trop rares pour offrir une solution durable au plus grand nombre.

Travail salarié non agricole. L'emploi salarié non agricole est une option assez rare, essentiellement circonscrite à des régions exceptionnellement dotées en ressources, en infrastructures et en services. Seulement $15 \%$ des ménages enquêtés exercent ce type d'activité et le pourcentage varie considérablement d'une région à l'autre. Les possibilités d'emploi salarié non agricole ne sont que sporadiques en Afrique subsaharienne et sont rencontrées principalement dans les autres pays. Les maquiladoras (unités industrielles à forte intensité de main-d'œuvre) de Tequisquiapan (Mexique) et Terrabona (Nicaragua), où une industrie du prêtà-porter s'est développée en zone rurale, en sont un exemple. Ce type d'emploi manufacturier est rare en Afrique subsaharienne, surtout en zone rurale, et les emplois salariés se résument surtout aux services. Ils sont en général peu rémunérés et relèvent du secteur informel, bien que des emplois formels existent également (par exemple dans l'administration ou le tourisme). Ce sont généralement les ménages déjà bien lotis et disposant d'un capital humain et social suffisant qui peuvent espérer les emplois les plus lucratifs.

Auto-emploi. Le travail indépendant généré par les auto-entrepreneurs (self-employment) est très répandu partout. C'est la source la plus courante de revenu hors exploitation dans la plupart des régions étudiées et la principale possibilité de diversification pour les ménages les plus pauvres. En Afrique subsaharienne et dans le Sotavento (Mexique), 40 à $80 \%$ des ménages interrogés exercent une activité indépendante. Au Maroc, au Nicaragua et dans la région de Tequisquiapan (Mexique), où les options économiques sont plus nombreuses (emplois salariés), 
la fréquence de l'auto-emploi est nettement plus faible (5 à $15 \%$ ). Les activités indépendantes sont presque toujours des micro-activités et consistent souvent à exécuter de menus travaux ou des « petits boulots ». On peut distinguer deux grandes formes d'auto-emploi : la diversification positive (en général une activité à temps plein), dans laquelle l'activité indépendante apporte une contribution importante au revenu du ménage, et la diversification neutre, dans laquelle les ménages les plus pauvres et les plus marginalisés déploient des stratégies d'adaptation ou de survie en exerçant des activités indépendantes mineures, dont ils tirent un très faible revenu. La diversification positive est surtout accessible aux ménages les mieux lotis - ceux qui ont de meilleurs actifs ou en ont davantage et qui peuvent réaliser un investissement initial (par exemple, un moulin, une machine à coudre, du matériel de soudure). On pourrait à juste titre considérer les autres catégories d'activité indépendante comme une forme de sous-emploi, surtout celles qui sont liées aux stratégies d'adaptation (par exemple, le petit commerce) ; elles n'offrent pas de véritable solution pour diminuer la pauvreté.

Transferts. Les transferts tiennent une place importante dans le revenu des ménages ruraux. Bien que les transferts publics sous forme de subventions agricoles et de filets de sécurité n'aient été observés qu'au Mexique, ils y constituent une part conséquente du revenu des ménages (entre 12 et $20 \%$ dans le Sotavento par exemple). Les transferts privés liés aux migrations (envois de fonds de travailleurs à l'étranger) sont plus courants mais difficilement quantifiables. Ils ont été mentionnés par $24 \%$ des ménages de l'échantillon, la plupart dans des régions historiquement marquées par les migrations. L'importance des fonds envoyés dépend du type de migration (courte ou longue durée) et de la destination (nationale ou internationale, dans des pays à revenu élevé ou des pays voisins). Cependant, ils ne tiennent une part importante dans le revenu total du ménage que dans une région : à Diéma, Mali (40\%). Dans les autres régions où ils sont observés, ils représentent généralement entre 5 et $15 \%$ du revenu (Maroc, Nicaragua et Sénégal), excepté au Kenya et à Madagascar, où ils sont insignifiants. Les ménages des quintiles pauvres migrent souvent pour une courte durée afin de réduire le nombre de bouches à nourrir pendant la saison sèche. Dans ce cas, les envois de fonds sont très faibles, voire inexistants, et les conditions de vie des migrants peuvent être très difficiles.

\section{Des processus d'adaptation rurale qui font écho aux recompositions structurelles globales}

Outre le bénéfice direct des envois de fonds des travailleurs à l'étranger, la migration peut aussi apporter un bénéfice indirect sous forme d'effet réseau. L'amélioration des infrastructures de transport et de télécommunications permet une nouvelle forme d'organisation dans laquelle les membres de la famille contribuent au revenu du ménage depuis d'autres lieux, où ils exercent différentes activités économiques. Ces « systèmes en archipel » favorisent la diversification et la gestion des risques, renforcent les opportunités économiques des ménages et offrent de nouvelles perspectives pour la transformation rurale. Cette configuration a été observée à plusieurs reprises dans l'échantillon de RuralStruc. 
S’agissant de la deuxième hypothèse du programme (H2), les caractéristiques générales de la diversification rurale illustrent des processus hétérogènes d'adaptation et de transformation rurale. Dans une certaine mesure, elles reflètent la transition économique globale : une première diversification très peu payante dans les premières phases de la transformation structurelle, suivie d'une diversification plus mature qui conforte le processus de transformation aux phases ultérieures. Ces caractéristiques rappellent que la proximité avec une zone à forte densité de population ne suffit pas à stimuler la croissance économique. Les modalités de l'urbanisation sont importantes, surtout le niveau des infrastructures, les biens publics et les services, qui sont essentiels pour resserrer les liens entre monde urbain et monde rural.

\section{La relation diversification-revenu et la transformation rurale}

Nombreux sont les résultats de l'enquête RuralStruc qui portent à réfléchir. En effet, la plupart des ménages enquêtés dans les pays d'Afrique subsaharienne, et des proportions significatives dans les trois autres pays, restent très pauvres et cantonnés dans une agriculture de subsistance. Pour les ménages dont les revenus se situent dans les quintiles les plus bas, la sécurité alimentaire est un défi majeur. Les processus d'intégration verticale et de contractualisation sont peu développés ; et les autres activités qu'ils peuvent espérer exercer sont très peu rémunératrices ou ne sont accessibles qu'aux individus déjà bien lotis.

Cependant, les enquêtes offrent également des résultats plus encourageants. Si le niveau de revenus varie d'un pays et d'une région à l'autre, de nombreuses données montrent qu'en dehors de l'Afrique subsaharienne, le revenu moyen augmente. La situation s'améliore pourtant aussi dans certaines régions africaines étudiées, par exemple dans le Bas Delta au Sénégal et dans le district de Nakuru North au Kenya. Au Maroc et au Nicaragua, la diminution des risques et l'amélioration des débouchés commerciaux ont permis à certains ménages d'entreprendre davantage de diversification sur l'exploitation agricole. Dans ces deux pays et au Mexique, le nombre croissant de possibilités offertes par l'économie a permis d'obtenir une meilleure rémunération des autres activités rurales. Cette tendance est particulièrement visible dans la région de Tequisquiapan (Mexique), où $70 \%$ des ménages ruraux n'ont plus d'activité agricole directe. Bien que cette tendance puisse engendrer une forme critique de marginalisation pour les ménages qui n'ont pas accès à l'emploi salarié, la situation du ménage moyen s'est améliorée. Dans cette région, parmi les ménages qui ont une exploitation agricole (30\% de l'échantillon), ceux dont un membre exerce une activité salariée ont le revenu par tête le plus élevé.

Afin d'explorer l'ampleur de ces processus de transformation, une attention particulière a été portée aux phénomènes de diversification et de spécialisation et à leurs liens avec le niveau de revenu. Deux indicateurs (l'indice de Herfindahl-Hirschman et la part des revenus hors exploitation dans le revenu) ont été utilisés pour illustrer la perte d'importance de l'activité agricole, à la fois dans 
le revenu des ménages ruraux et au niveau régional. Plusieurs tendances ont été identifiées. Premièrement, les ménages des zones étudiées dans les pays les plus riches, hors Afrique subsaharienne, tendent à avoir des niveaux de diversification rurale plus faibles. Ce constat est assez surprenant car on considère généralement que la transformation structurelle s'accompagne d'une augmentation du revenu et d'un recul de la part de l'agriculture dans celui-ci.

Deuxièmement, aucun schéma clair ne s'est dessiné au niveau infranational. Dans certains pays, les régions les plus riches étaient plus diversifiées, dans d'autres, elles l'étaient moins, et l'absence de tendance claire était également observée à l'intérieur des régions étudiées. Néanmoins, les écarts de niveaux de diversification entre les quintiles de revenus étaient assez marqués, indiquant une forte interaction entre ces deux facteurs, sans que le sens de cette relation soit pour autant clairement identifiable.

\section{La courbe en $U$ inversé : une représentation des processus de transformation rurale}

Pour expliquer ces observations, l'option retenue a été de représenter la relation diversification-revenu par une courbe en $U$ inversé. Lorsque les revenus sont très faibles (quand les ménages se consacrent entièrement à des stratégies de survie), les sources de revenus sont rarement diversifiées et l'agriculture accapare toute l'activité des ménages. Lorsque les revenus augmentent et que les ménages deviennent un peu plus riches, ils restent certes exposés à des risques (principalement liés aux aléas) mais ils disposent aussi d'une marge de manœuvre accrue pour se constituer un filet de sécurité. Avec l'augmentation progressive du revenu, les ménages commencent à diversifier leurs activités pour faire face aux risques et trouver des revenus additionnels. À ce stade, la diversification n'intervient qu'au niveau des ménages (diversification interne aux ménages), tandis que l'économie de la région demeure très agricole. Le processus de diversification se poursuit jusqu'à ce que les gains et le patrimoine des ménages soient suffisants pour que les revenus de la diversification leur permettent progressivement de satisfaire leurs besoins élémentaires et de gérer leurs risques. À ce stade, les ménages commencent à se spécialiser dans différentes activités - dans l'agriculture ou hors de l'agriculture - et il en résulte une économie régionale globalement plus diversifiée (diversification entre les ménages).

Un indicateur est proposé - le différentiel de diversification - pour représenter la progression d'une région sur ce continuum. La très forte corrélation observée entre le différentiel de diversification et le revenu des ménages permet de penser que les régions étudiées tendent à suivre la courbe en $U$ inversé au fil de leur développement. La relation diversification-revenu semble toutefois comprendre une composante exponentielle : dès lors qu'une région " passe le cap » et que les ménages commencent à se spécialiser économiquement, la croissance du revenu au niveau régional, jusque-là très lente, semble rapidement décoller et conduire la région sur une trajectoire qui l'éloigne de la pauvreté. 


\section{Les trappes à pauvreté et l'insaisissable économie rurale non agricole}

Un constat important de l'analyse proposée par RuralStruc est que la plupart des régions étudiées en Afrique subsaharienne progressent très lentement sur la courbe en U inversé. En fait, de nombreux ménages africains semblent se heurter à un mur invisible dans le processus de transition : le type de diversification des revenus qu'ils pratiquent ne leur permet pas de gagner assez d'argent pour sécuriser leurs moyens d'existence (du fait de la faible rémunération des activités de diversification) et de ce fait, ils ne franchissent jamais le cap pour commencer à se spécialiser. Ils semblent pris au piège d'une pauvreté structurelle, une observation qui confirme la difficulté de la transformation rurale et la troisième hypothèse du programme : le processus de mondialisation s'accompagne de risques d'impasse de transition.

Un autre phénomène, enfin, est mis en évidence par l'enquête : le processus de spécialisation au stade final de la courbe en $U$ inversé intervient principalement dans l'agriculture, tandis que la spécialisation dans d'autres activités économiques est plus rare. Ce constat paradoxal peut être expliqué par un biais méthodologique : l'enquête a été exclusivement réalisée en zone rurale et tend donc à informer principalement sur les processus de spécialisation dans le secteur agricole. De fait, les ménages qui se spécialisent dans des activités non agricoles le font souvent en zone urbaine, ce qui veut dire qu'ils migrent. Ainsi, et peut-être plus fondamentalement, ce résultat traduit la nature quelque peu éphémère de l'économie rurale non agricole, qui tend à simultanément croître et se dissoudre sous l'effet de l'urbanisation. Non seulement les ménages qui se spécialisent hors agriculture tendent à migrer en zone urbaine, mais les zones urbaines se développent parallèlement avec la transformation progressive des bourgs ruraux en villes. Ce phénomène du « déplacement des villes vers la campagne » est la conséquence de densités de population croissantes et de l'expansion territoriale des villes liée au processus endogène de croissance urbaine.

\section{Principales leçons pour les politiques publiques}

Les résultats tirés de l'enquête RuralStruc permettent d'offrir une représentation des processus de transformation rurale et de proposer un cadre explicatif des dynamiques de diversification et de spécialisation. Ils rappellent simultanément l'importance des caractéristiques nationales (dotation en facteurs, fonctionnalité des marchés, climat des affaires, cadre institutionnel, gouvernance globale, stabilité politique, etc.) qui déterminent la marge de manœuvre dont disposent les ménages qui tentent d'échapper à la pauvreté. Les résultats de RuralStruc apportent des éclairages importants sur la situation particulière des pays dont le développement s'est amorcé tardivement, illustrée par les régions étudiées en Afrique subsaharienne, où le stade très précoce de la transition économique limite les possibilités des ménages en matière de diversification des revenus et d'accès à des activités fortement rémunératrices. 
La courbe en U inversé n'est en aucun cas déterministe ; elle offre en réalité un cadre conceptuel pour essayer d'expliquer où en sont les régions dans le processus de diversification-spécialisation. Ce cadre permet de mener une réflexion systématique sur les changements intervenus et de repérer les causes possibles des impasses de transition observées. Il ne prétend pas prédire les trajectoires de développement futures, qui dépendront d'abord des particularités de chaque contexte local et de ses interactions avec le monde extérieur.

Pour les nombreuses régions rurales d'Afrique subsaharienne prises dans des trappes à pauvreté, les solutions devront venir d'interventions publiques adaptées au contexte national et d'initiatives capables de renforcer l'intégration régionale. Le meilleur moyen d'aborder le retard de l'Afrique subsaharienne dans le processus de transition est de promouvoir des politiques de croissance rurale à même d'accroître simultanément la demande rurale et d'y répondre. Une leçon importante des transitions passées montre que c'est l'augmentation des revenus agricoles qui stimule la demande rurale. Et pour pouvoir répondre à cette demande par une offre de produits et de services adéquate, les pouvoirs publics doivent favoriser l'investissement local par une fourniture adaptée en biens publics.

Si cette stratégie de développement rural est souhaitable pour tous les pays en développement, elle est essentielle pour les pays d'Afrique subsaharienne. Mais les travaux de RuralStruc ont également constaté l'existence d'une population rurale marginalisée dans les autres pays étudiés, avec de fortes inégalités entre zones urbaines et rurales - une situation qui n'est pas politiquement soutenable et nécessite des interventions ciblées.

\section{Principes généraux}

Il n'existe pas de solution aisée aux immenses défis que posent la diminution de la pauvreté, la croissance rurale et la transition économique. À défaut de remède miracle, les vingt dernières années de politiques de développement rural ont permis d'établir une longue liste d'interventions publiques utiles, dont les principales sont l'amélioration de marchés imparfaits (par la diminution des coûts de transaction), le développement des marchés incomplets (marché du crédit, du conseil et de l'assurance), la fourniture de biens publics (infrastructure, recherche, information et renforcement des capacités) et l'introduction de mécanismes de gestion des risques.

Il n'est pas facile de réunir tous les ingrédients d'une politique efficace, mais il est encore plus difficile de trouver le dosage précis pour réussir. Les politiques doivent d'abord être adaptées aux situations locales : la tâche la plus difficile consiste donc à identifier la combinaison de mesures qui sera efficace dans un contexte donné. Ce processus suppose de faire des choix importants en termes de priorités et d'objectifs. Car dans la plupart des pays (et pas seulement dans les pays en développement), un problème important pour les décideurs politiques est la nécessité de parer simultanément à une multitude de problèmes pressants, ce que les contraintes financières et de ressources humaines rendent généralement impossible. 
Compte tenu des résultats du programme, qui révèlent une très forte hétérogénéité des situations entre pays, régions et ménages, deux recommandations peuvent être formulées : (1) la relance des stratégies de développement au niveau national et régional et (2) la réalisation de diagnostics régionaux.

Relancer des stratégies de développement. L'élaboration d'une stratégie globale a été négligée ces dernières décennies du fait du retrait de l'État, d'une segmentation excessive des politiques sectorielles (qui conduit au cloisonnement) et de la détérioration des systèmes publics d'information et de statistiques - un handicap de taille pour les dirigeants politiques.

Dans ce contexte, il est urgent de réinvestir dans la création de connaissances. Comme l'ont montré les états des lieux par pays réalisés dans la première phase du programme RuralStruc, les informations socio-économiques générales sont insuffisantes et les données nécessaires pour appréhender la dynamique des économies rurales sont particulièrement rares. Les systèmes statistiques publics doivent être redynamisés et redéfinis et les organismes publics doivent pouvoir recueillir des informations et en rendre compte, mais aussi les analyser pour en tirer des conclusions utiles pour l'action publique. À défaut, les décideurs politiques seront incapables de concevoir des mesures pour accompagner l'évolution des économies rurales, la mobilité croissante des personnes et les nouveaux modes d'organisation des ménages (comme les systèmes en archipel) qui en résultent. La relance de stratégies de développement au niveau national et infranational suppose de réinvestir dans les processus. La consultation est indispensable à l'appropriation - facteur décisif d'une vision partagée nécessaire à l'engagement. Un tel processus prend du temps, implique une planification adéquate et un important effort de renforcement des capacités pour gérer les systèmes d'information, analyser les résultats et suivre les progrès obtenus.

Réaliser des diagnostics régionaux. Des diagnostics régionaux sont indispensables pour hiérarchiser les objectifs, cibler les interventions et planifier les actions. Il est nécessaire de cerner les contraintes qui pèsent sur la croissance agricole - première étape indispensable pour accroître la demande rurale et favoriser la diversification des économies locales - et de concevoir des politiques pour y répondre. Ces politiques doivent opérer des arbitrages, fixer des objectifs, planifier puis suivre l'exécution des interventions. Il faut cependant veiller à ne pas se trouver pris au piège d'une politique monosectorielle (par exemple, en se focalisant sur les problèmes agricoles) et à adopter des approches plus larges qui reconnectent l'agriculture au développement rural et le développement rural à un cadre de développement multisectoriel et régional intégré (c'est-à-dire de « développement territorial »).

Ces deux recommandations concernent la méthodologie d'élaboration des politiques et ne prescrivent pas de trains de mesures particuliers. Les interventions décidées à partir de ces recommandations doivent être adaptées aux circonstances et aux processus propres à chaque pays. Un patrimoine spécifique ou des avantages naturels prononcés (par exemple la richesse du sous-sol ou des atouts touristiques) peuvent offrir une marge de manœuvre supplémentaire pour soutenir de nouvelles activités et favoriser la transformation rurale. 


\section{Axes d'intervention}

Pour une grande majorité de situations rurales où l'activité agricole demeure centrale pour les ménages, il est toutefois possible de proposer de grandes orientations de politiques ou des axes d'intervention. Il est souhaitable que les décideurs politiques les gardent à l'esprit lorsqu'ils élaborent des stratégies de développement visant à sortir des trappes à pauvreté et à faciliter le processus général de transformation rurale. Trois axes d'intervention peuvent aider les gouvernements à éviter le piège des longues listes d'action où tout est prioritaire. Correspondant aux situations particulières des pays dont le développement s'est amorcé tardivement (en particulier ceux d'Afrique subsaharienne), ces axes reposent sur les principaux constats du programme RuralStruc et ciblent : (1) l'aide aux exploitations familiales, (2) la promotion des cultures vivrières de base et (3) le renforcement des liaisons villes-campagnes pour le développement territorial.

Aider les exploitations familiales. Les résultats de RuralStruc plaident pour une aide aux exploitations familiales et contribuent au débat sur la taille optimale des exploitations, relancé par la crise des prix alimentaires de 2008 et l'intensification du phénomène d'accaparement des terres qui lui est associée, en particulier en Afrique.

Un faux dualisme est au cœur de ce débat, qui oppose les petits exploitants et l'agriculture de subsistance d'un côté à l'agriculture commerciale à grande échelle de l'autre, alors que la réalité est un continuum dans lequel l'agriculture familiale est presque toujours le mode de production dominant. L'agriculture familiale - par opposition à l'agriculture managériale ou capitaliste à grande échelle - nourrit la majeure partie de la population mondiale. Les exploitations familiales peuvent être orientées vers l'agriculture vivrière ou l'agriculture commerciale ou associer les deux. Un vaste corpus de données empiriques montre que les exploitations de ce type peuvent avoir des coûts de production compétitifs par rapport aux grandes exploitations managériales. En Afrique subsaharienne, elles sont souvent compétitives sur le marché national mais désavantagées sur les marchés mondiaux du fait de facteurs sans lien avec leur taille (par exemple, l'environnement économique et le cadre institutionnel).

L'accent mis aujourd'hui sur la sécurité alimentaire a éclipsé la multifonctionnalité de l'agriculture (notamment son rôle écologique, économique, social et culturel); or, les exploitations familiales en sont les principaux vecteurs parce qu'elles sont ancrées dans leur contexte local. La priorité accordée à la sécurité alimentaire a également conduit de nombreux décideurs politiques à perdre de vue la contribution de l'agriculture à l'emploi et son rôle moteur dans la transformation structurelle à moyen terme. Parce qu'elles recourent abondamment à des systèmes techniques de production gourmands en main-d'œuvre, les exploitations familiales sont les plus aptes à absorber la poussée attendue de la population active (195 millions de jeunes ruraux dans les quinze prochaines années en Afrique subsaharienne). L'agriculture managériale, beaucoup plus intensive en capital, offre un moindre potentiel de création d'emplois. 
Les investissements dans l'agriculture commerciale à grande échelle (y compris les investissements étrangers) peuvent offrir d'importantes opportunités de croissance, de diversification des marchés et de développement des zones faiblement peuplées, mais ils doivent aussi être évalués du point de vue des emplois qu'ils peuvent créer. Ces investissements doivent être ciblés en priorité sur les segments de la chaîne de valeur qui manquent de capital (approvisionnement en intrants, commercialisation, transformation) afin de libérer le formidable potentiel de croissance de production des exploitations familiales.

Si l'aide aux exploitations familiales peut revêtir différentes formes, cet ouvrage se garde de présenter une longue liste de recommandations et préfère suggérer trois types d'actions pour régler les problèmes les plus aigus : (1) la sécurisation des droits fonciers, (2) la fourniture de biens publics et (3) le soutien aux organisations de producteurs.

Sécuriser les droits fonciers. Les ménages agricoles sont exposés à des risques importants. Pour sécuriser leur environnement, il faut d'abord faciliter l'accès aux terres agricoles et garantir les droits fonciers, deux conditions indispensables à l'investissement et l'innovation. L'accès des jeunes à la terre et la transmission du patrimoine agricole doivent être facilités.

Pallier le déficit en biens publics. La plupart des exploitations familiales sont fortement contraintes par une très faible capacité d'investissement, conséquence d'une pauvreté récurrente. Le ciblage sélectif d'une aide directe peut aider à surmonter cette contrainte, mais il est encore plus efficace de développer l'offre de biens publics, en particulier l'information, la formation et le renforcement des capacités des exploitants, ainsi que l'infrastructure rurale (irrigation à petite échelle, routes, production et distribution d'électricité). Ces infrastructures peuvent aussi, lorsque c'est justifié, faciliter l'accès à des zones peu peuplées et encourager les migrations internes.

Appuyer les organisations de producteurs. En raison de leur taille et de leur capacité productive souvent limitées, de nombreuses exploitations familiales sont incapables de réaliser des économies d'échelle dans le domaine de l'approvisionnement en intrants, de la commercialisation de la production et de la transformation des produits. L'action collective permet souvent de surmonter cette contrainte : un soutien aux organisations de producteurs peut améliorer l'intégration aux chaînes de valeur, faciliter la conclusion de contrats avec les opérateurs de l'aval des filières et renforcer le pouvoir de négociation des agriculteurs.

Promouvoir les cultures vivrières de base. Dans les pays à base agricole, quatre arguments fondés sur l'observation des réalités de terrain plaident pour une priorité accordée aux cultures vivrières de base. Le premier découle de la place prépondérante de ces cultures. Dans la plupart des pays en développement, l'écrasante majorité des ménages agricoles produisent des denrées alimentaires de base (90 \% en moyenne dans l'enquête RuralStruc). En conséquence, les politiques ciblées d'encouragement à la production et à la commercialisation de ces cultures peuvent avoir des effets importants sur l'ensemble de l'économie rurale, en termes de main-d'œuvre, de revenu et de croissance. Le ratio entre les producteurs 
cultivant ces produits de base et les producteurs d'autres cultures est aisément de 10 pour 1 et souvent bien plus élevé.

Le deuxième argument est lié au rôle spécifique des cultures vivrières de base dans la gestion des risques. Parce qu'il est fréquent que les marchés alimentaires en zone rurale ne fonctionnent pas correctement, de nombreux ménages ruraux sont exposés à des périodes de pénurie alimentaire et conservent souvent une part importante de leur production à des fins d'autoconsommation. En réduisant ce risque alimentaire, l'augmentation de la production vivrière peut libérer le potentiel d'innovation technique, accélérer la diversification agricole et encourager la participation aux chaînes de valeur modernes.

Le troisième argument tient au considérable potentiel du secteur des cultures vivrières. La demande d'aliments est appelée à croître régulièrement dans un avenir prévisible, stimulée par la croissance démographique et l'urbanisation. Même si l'augmentation des revenus modifie les habitudes de consommation, les produits de base - surtout les céréales - représenteront la majorité de la demande d'aliments pendant de nombreuses années. De plus, la hausse tendancielle des prix agricoles offre progressivement une meilleure rémunération aux producteurs tout en limitant la concurrence des importations qui deviennent moins attractives.

Le quatrième et dernier argument en faveur des cultures vivrières est la valeur ajoutée qu'elles peuvent créer à l'échelle locale en raison du considérable potentiel de transformation locale des produits. Leur développement pourrait ainsi fortement contribuer à renforcer les liens entre villes et campagnes et la diversification rurale.

Les mesures de politique publique visant à accroître la productivité des cultures vivrières et à améliorer le fonctionnement de leurs marchés sont très diverses mais, dans le cas de l'Afrique subsaharienne, deux actions prioritaires s'imposent : (1) la réduction des pertes postrécolte et (2) la libéralisation du commerce interrégional.

Réduire les pertes postrécolte. Ces pertes sont un problème récurrent face auquel les progrès ont été minimes. Leur coût économique est élevé (10 à $20 \%$ dans le cas des céréales et sans doute davantage pour les racines, tubercules et plantains) et ce sont principalement les agriculteurs qui l'assument. Il existe des solutions techniques, mais des efforts sont nécessaires pour adapter les dispositifs institutionnels et financiers afin de rendre rentable l'utilisation des systèmes de stockage (comme le warrantage).

Libéraliser le commerce interrégional. L'Afrique subsaharienne est potentiellement un énorme marché, mais son plein accès est actuellement contraint par la fragmentation politique du continent et les multiples obstacles récurrents au commerce. Bien que des progrès aient été faits pour encourager une meilleure intégration régionale, le commerce régional peine à se développer du fait des barrières non douanières, de l'inapplication des accords commerciaux régionaux et des coûts de transaction élevés associés au transport terrestre. Pour stimuler les échanges régionaux, les interventions les plus prometteuses résident dans l'amélioration des réseaux d'infrastructures et, surtout, dans la volonté politique des membres des communautés économiques régionales. 
Néanmoins, ce soutien aux cultures vivrières, s'il est essentiel, n'est pas suffisant. En raison de la valeur assez faible de ces cultures par rapport à d'autres productions - par exemple les produits horticoles ou l'élevage et les produits animaux - l'augmentation de leur productivité ne peut être la seule solution pour faire reculer la pauvreté. Il faut aussi saisir les autres opportunités, lorsqu'elles existent.

Renforcer les liaisons villes-campagnes pour le développement territorial. Le développement de liens forts entre les petites villes et les zones rurales qui les entourent est particulièrement important pour le développement. Historiquement, les liens entre ville et campagne se sont forgés sous l'effet de la croissance de la demande rurale de biens et de services, qui générait de nouvelles activités productives, lesquelles se concentraient naturellement dans les bourgs ruraux et les petites villes afin de bénéficier d'économies d'échelle. Ces dernières décennies, ce scénario a changé : partout dans le monde, l'urbanisation a été de plus en plus marquée par une "métropolisation » rapide structurée par les grandes villes et leurs zones périphériques, qui polarisent l'activité économique et concentrent les meilleures opportunités d'emploi. Conséquence de l'amélioration des transports et des réseaux d'information, la métropolisation a donné lieu à des migrations directes de grande ampleur des zones rurales vers les zones métropolitaines. Très souvent, les migrants esquivent entièrement les petites villes dans lesquelles des liens rural-urbain et agricole-non agricole pourraient être forgés. Et même lorsqu'ils demeurent dans des villes de petite à moyenne importance, les migrants renforcent une urbanisation informelle qui se produit sans biens et services publics adéquats. Ces dynamiques font obstacle à un développement urbain durable et empêchent le renforcement des liens entre villes et campagnes.

Consolider le niveau intermédiaire du développement territorial en stimulant la vitalité économique des villes de petite et moyenne importance - qui sont le " chaînon manquant » des territoires - semble une étape importante pour favoriser la transformation rurale dans le contexte d'une mondialisation qui tend à favoriser les réseaux longue distance par rapport aux réseaux locaux. Les interventions dans ce domaine peuvent offrir des solutions gagnant-gagnant qui non seulement créent de meilleurs débouchés commerciaux locaux, facilitent l'accès aux services, renforcent les communautés locales et contribuent à la fabrication du tissu économique et social d'une région, mais réduisent aussi les inconvénients de la méga-urbanisation. Ce type de dynamique territoriale est plus souple et ne crée pas de contraste aussi brutal entre les conditions de vie et d'activité des zones urbaines et rurales; il permet de travailler sur les deux fronts de la fracture rural-urbain et crée de solides bases pour une économie rurale non agricole plus durable. Cette perspective reconnaît la multifonctionnalité de l'agriculture et le rôle moteur qu'elle peut jouer dans le développement rural et régional. Deux types d'actions peuvent renforcer la dynamique villes-campagnes : (1) l'amélioration des services urbains et (2) le renforcement des capacités des institutions locales. 
Améliorer les services urbains dans les villes de petite et moyenne importance. L'infrastructure de transport est indispensable pour connecter les villes de petite et moyenne importance à leur entourage immédiat et renforcer leurs fonctions économiques. Cependant, comme le révèlent les enquêtes RuralStruc dans les zones rurales bien connectées du Kenya occidental et du bassin arachidier du Sénégal, l'infrastructure routière ne peut à elle seule favoriser la croissance et le développement territorial. Une offre adéquate de biens et de services publics est essentielle et doit être un objectif politique majeur. L'offre en matière de services de santé et d'éducation ainsi qu'un approvisionnement effectif en eau, en électricité et en télécommunications, sont de la plus haute importance. Le secteur privé peut difficilement fournir la plupart de ces biens et services dans les premiers stades du développement, mais des incitations fiscales peuvent être créées pour encourager les prestataires de services et les entrepreneurs privés à s'impliquer plus activement dans certains de ces secteurs. Ce type d'amélioration des services et des soutiens spécifiques (en particulier en termes de renforcement des capacités et d'accès au crédit) peuvent conforter les activités non agricoles, notamment pour les petites entreprises qui accompagnent la croissance du secteur agricole et sont la principale composante d'un développement territorial dynamique.

Renforcer les capacités des institutions locales. Parallèlement à l'amélioration des biens et des services publics, il importe de renforcer les institutions et les systèmes de gouvernance au niveau local et de faciliter la décentralisation qui, dans de nombreux pays, est plutôt restée très théorique. Le renforcement des capacités des organismes publics et des organisations de la société civile existantes est un premier pas important pour une stratégie de développement local intégrée efficace. Des institutions locales fonctionnelles dotées d'un pouvoir décisionnel décentralisé offrent la piste la plus prometteuse pour identifier les potentialités et ressources locales à même d'être employés dans la poursuite d'un développement territorial équilibré et durable. 


\section{Chapitre 1}

\section{Du contexte aux choix d'analyse}

Initié en 2005 dans le contexte d'un intense débat international autour de la libéralisation des marchés agricoles et de ses conséquences pour l'agriculture des pays en développement, le programme RuralStruc visait à porter un nouveau regard sur l'agriculture et son rôle dans le développement, et plus particulièrement à reconnecter les questions liées à la libéralisation du commerce à la discussion plus générale du changement structurel et de l'évolution des économies rurales dans le cadre de la mondialisation (voir encadré 1.1).

Cette initiative était motivée à la fois par le manque d'informations systématiques sur les processus à l'œuvre dans les économies rurales des pays en développement et par la question de la signification de ces processus pour le changement structurel et le développement économique. Des thèmes tels que l'intégration des agriculteurs dans les chaînes de valeur mondiales, les migrations et les fonds envoyés par des travailleurs à l'étranger, le développement de l'économie rurale non agricole ou les futurs possibles de l'agriculture sont de fréquents sujets d'étude et d'analyse des chercheurs spécialistes du développement et des questions agraires. La communauté internationale des donateurs, les États et les acteurs locaux évoquent aussi ces thèmes. Les nombreux travaux d'envergure publiés dans ces domaines donnent un large éventail d'informations sur les recompositions en milieu rural. Cependant, ces informations s'appuient souvent sur des études de cas ponctuelles dont il est difficile de tirer des conclusions générales et les analyses sont rarement connectées à la problématique du changement structurel. Cette situation crée un véritable " défi de connaissance » dans la mesure où une parfaite compréhension des dynamiques rurales est l'indispensable fondement des stratégies de développement et des politiques agricoles.

Ces objectifs initiaux ont déterminé les orientations du programme RuralStruc et son cadre général - à savoir, une démarche comparative portant sur sept pays à différents stades de changement structurel et d'intégration dans l'économie mondiale (d'ouest en est : le Mexique, le Nicaragua, le Sénégal, le Maroc, le Mali, le Kenya et Madagascar). Ces objectifs ont également sous-tendu l'approche collaborative du programme - une caractéristique déterminante de sa mise en œuvre. D'étroits partenariats ont été noués avec les équipes de recherche de chaque pays afin de renforcer une démarche basée sur la collecte directe de données locales et de nourrir le débat sur les politiques au niveau national ${ }^{1}$. 


\section{ENCADRÉ 1.1}

\section{RuralStruc : qu'y a-t-il derrière ce nom ?}

Le programme est officiellement intitulé Dimensions structurelles de la libéralisation pour l'agriculture et le développement rural. Le choix de l'acronyme RuralStruc illustre le souhait de réintroduire les questions structurelles dans un débat essentiellement centré sur le commerce.

RuralStruc renvoie à la fois aux structures rurales et aux implications des recompositions structurelles globales sur l'agriculture et les économies rurales. Le logo du programme s'inspire de l'image de l'iceberg : la libéralisation du commerce est la partie apparaissant à la surface, tandis que les transformations structurelles correspondent à la partie immergée, bien plus conséquente.

\section{Un contexte mondial déroutant, en rapide mutation}

Au cours des cinq dernières années, pendant qu'était mené le travail de terrain approfondi du programme, le paysage international ainsi que le champ et les thèmes du débat se sont radicalement transformés. Il est important de ne pas perdre de vue ces remaniements constants car ils constituent la réalité immédiate à laquelle se réfèrent les décideurs politiques.

\section{Le point de départ}

Lorsque le programme RuralStruc était en préparation (2005-2006), deux cadres de référence structuraient le débat international sur le développement : les Objectifs du Millénaire pour le développement des Nations unies (OMD) et le cycle de développement de l'Organisation mondiale du commerce (OMC), ou Programme de Doha pour le développement (PDD), fixé en 2001 lors de la conférence ministérielle de Doha. L'agriculture s'inscrivait évidemment dans ces deux cadres, mais bien qu'elle y ait parfois occupé une position déterminante (comme dans le cas du PDD), elle n'y a jamais tenu une place centrale.

Les OMD offraient un cadre général axé sur la réduction de la pauvreté. Le premier objectif - « Réduire de moitié la pauvreté et la faim avant 2015 »concerne indéniablement l'agriculture car d'une part, $70 \%$ des pauvres dans le monde (qui représentent $45 \%$ de la population mondiale) résident en zone rurale et vivent principalement de l'agriculture et, d'autre part, le recul de la faim exige une amélioration de l'offre alimentaire et de l'accès aux aliments. Les analyses réalisées par la Banque mondiale (2005) ont également réaffirmé le rôle crucial de l'agriculture dans une "croissance pro-pauvres ». Mais, alors que la pauvreté demeurait au cour des débats, le développement agricole n'était qu'un outil parmi d'autres évoqués pour lutter contre la pauvreté, à côté de nombreuses autres options thématiques et non sectorielles.

Parallèlement, les négociations au sein de l'OMC se sont logiquement concentrées sur la libéralisation du commerce, l'agriculture étant un des secteurs à libéraliser. 
Pourtant, l'agriculture a été la principale pierre d'achoppement dans le processus de négociation. Les pays en développement en ont fait leur argument central pour entreprendre les pays développés sur la question plus générale de la libéralisation des produits industriels et des services, qui a mené à l'échec de la conférence ministérielle de Cancún en 2003 et a amorcé un débat sur les coûts et les avantages de la libéralisation des échanges pour l'agriculture. Cette focalisation sur l'agriculture et le commerce et sa position dominante dans le débat international étaient une des justifications majeures du programme RuralStruc.

Depuis 2005 cependant, l'opinion internationale sur le développement s'est radicalement transformée. Les OMD ont été longtemps relégués au second plan et sont demeurés comme un rappel un peu lointain de l'engagement de la communauté internationale en faveur de la réduction de la pauvreté et du développement mondial. Ils ont néanmoins suscité un regain d'attention lors du Sommet des Nations unies de septembre 2010, qui évaluait les progrès accomplis et concluait que les objectifs ne seraient pas tous atteints (Nations unies 2010), et ils reviendront formellement sur le devant de la scène à l'approche de l'échéance de 2015.

Du côté de l'OMC, le débat a perdu de sa vitalité pour plusieurs raisons imbriquées. La première est bien sûr l'émergence de nouvelles problématiques au cœur des priorités internationales, notamment un nouveau débat sur l'agriculture (voir section suivante). La seconde est la profusion des recherches produisant des estimations en demi-teinte des gains attendus de la libéralisation du commerce. Ce corpus de travaux souligne les situations particulières de nombreux pays en développement, en particulier en Afrique, où la libéralisation du commerce pourrait produire, non pas des gains, mais des pertes nettes, ce qui accentue l'incertitude - si ce n'est la confusion - des débats ${ }^{2}$. Ces constats ont contribué à recentrer les négociations sur les subventions agricoles des pays membres de l'OCDE (Organisation de coopération et de développement économiques) et sur l'accès des pays en développement à leurs marchés. Ils ont aussi conforté l'opposition au Cycle de Doha et débouché sur une succession d'impasses, en particulier en matière agricole : l'échec de la réunion ministérielle de Hong Kong (2005) s'est soldé par une suspension des négociations (juillet 2006), suivie de vaines tentatives d'accord sur l'accès aux marchés agricoles et non agricoles (réunions de Genève en juillet 2008) et depuis par un report récurrent de la conclusion du Cycle de Doha. Une certaine « lassitude des négociations » explique aussi la perte de vigueur du débat au sein de l'OMC, de même que l'intérêt croissant porté aux accords de libre-échange (ALE) bilatéraux ou régionaux et la décision des principaux protagonistes de poursuivre à l'échelle bilatérale ce qui s'est avéré impossible de réaliser à l'échelle mondiale.

\section{Les nouvelles préoccupations}

Au cours des cinq dernières années, les circonstances ont pesé sur le débat international concernant l'agriculture avec la cristallisation ou l'apparition de trois problèmes majeurs : les inquiétudes croissantes suscitées par le réchauffement climatique, l'éruption de la crise des prix des produits alimentaires puis de la crise financière. 
Le réchauffement climatique est très présent dans le débat mondial, au moins depuis le Sommet de la Terre de Rio (1992) et la Conférence de Kyoto (1997), mais les préoccupations se sont accentuées ces dernières années à la suite de deux grands travaux de recherche : le Rapport Stern sur l'économie du changement climatique (Stern 2007) et Changements climatiques 2007, le rapport du Groupe d'experts intergouvernemental sur l'évolution du climat (GIEC) (Pachauri et Reisinger 2007). Ces analyses ont renforcé la prise de conscience de la communauté internationale et recentré les négociations en cours, qui ont conduit au Sommet de Copenhague de décembre 2009 (sans empêcher son échec). Elles soulignaient les conséquences négatives prévisibles des changements climatiques sur les ressources naturelles et l'agriculture, comme des phénomènes climatiques extrêmes (sécheresses, inondations, canicules), des changements de la température et de la pluviométrie et l'élévation du niveau de la mer. Toutes ces conséquences aggraveront probablement les difficultés qu'ont les agriculteurs et les travailleurs agricoles à pérenniser leurs moyens de subsistance. De toutes les régions du monde, l'Afrique subsaharienne est celle qui souffrira le plus des changements climatiques ; le GIEC prévoit en effet des pertes agricoles annuelles comprises entre 2 et $7 \%$ du PIB de la région en 2100. Le Rapport sur le développement dans le monde de 2010, Développement et changement climatique (RDM10) (Banque mondiale 2009a) a dressé un bilan exhaustif des difficultés auxquelles sont exposés les pays en développement, qui supporteront la majeure partie $(75 \%$ à $80 \%)$ des coûts des dommages dus au changement climatique. Parmi ces difficultés, citons la dépendance de leur production (principalement l'agriculture) à l'égard des écosystèmes et du capital naturel, la concentration de leur population dans des sites physiquement exposés et les faibles capacités financières et institutionnelles dont ils disposent pour s'adapter. Des mesures d'atténuation spécifiques seront nécessaires pour empêcher que 120 millions d'individus ne viennent grossir les rangs de ceux qui souffrent de la faim et l'agriculture jouera un rôle vital dans la gestion des ressources et le piégeage du carbone.

La crise des prix des produits alimentaires (2007-2008) a suscité un regain d'intérêt international pour les questions alimentaires et agricoles. Cet intérêt est renforcé par les projections démographiques qui estiment que la population mondiale atteindra 9 milliards d'individus en 2050. Les prix alimentaires, qui augmentaient progressivement depuis 2006, ont connu une brusque envolée début 2008 qui a mobilisé l'aide internationale. Bien que les prix aient ensuite reculé, les prévisions tablaient sur une plus forte volatilité et des niveaux relativement élevés à moyen terme et, de fait, ils ont rebondi fin 2010. Plusieurs facteurs sont à l'origine de la hausse des prix alimentaires et leur importance relative fait débat. Côté offre, les principales explications avancées sont les déficits de production dus aux conditions météorologiques conjugués à la hausse de prix des carburants et à une baisse tendancielle des niveaux de stock. Côté demande, il s'agirait de l'évolution à long terme de la structure de la consommation liée à la rapide transformation des habitudes alimentaires dans les pays émergents, du développement des biocarburants face au renchérissement du pétrole et de la spéculation (même limitée) sur les marchés financiers. Indépendamment de l'importance relative de ces facteurs, 
tout le monde s'accorde à penser qu'il n'y a pas de risque de pénurie alimentaire mondiale à moyen terme - le problème ne réside pas tant dans un manque d'aliments que dans le cô̂t de ces aliments. Le fonctionnement des marchés alimentaires et l'accès aux denrées pour les consommateurs à faible revenu sont donc des préoccupations centrales. La difficulté est de ne pas se focaliser sur des problèmes de court terme et, simultanément, d'aider les agriculteurs à recueillir les bénéfices des prix élevés actuels, d'atténuer les impacts des prix sur les consommateurs les plus pauvres, d'augmenter la production alimentaire disponible localement pour contrer la hausse des prix locaux et, enfin, d'améliorer le revenu des producteurs grâce à un pouvoir de négociation accru et des rendements plus élevés.

La brutale crise financière de septembre 2008 a eu des conséquences majeures sur l'économie mondiale et la reprise a été lente et reste fragile. La rapide contagion du retournement du secteur immobilier américain au secteur financier mondial a profondément touché tout à la fois les pays riches et les pays pauvres, entraînant une contraction et une récession dans plusieurs économies développées et un brutal ralentissement de la croissance de nombreux pays en développement. Ce ralentissement a été particulièrement problématique pour les pays qui, ayant peu de ressources financières, sont confrontés à une réduction radicale de leurs revenus (due à la forte diminution de l'investissement direct étranger, des recettes budgétaires, de l'aide étrangère et des fonds envoyés par les travailleurs à l'étranger).

La crise financière et celle des prix alimentaires ont suscité des débats différents (et sans lien direct) sur les remèdes mais, dans une certaine mesure, elles ont toutes deux déclenché des réactions protectionnistes temporaires comme l'augmentation des droits de douane, la mise en place de nouvelles barrières non tarifaires, le retour provisoire des quotas ou encore les interdictions d'exportation. Ce risque de "vague protectionniste » a directement battu en brèche les arguments en faveur de la libéralisation du commerce qui avaient marqué le débat international depuis le début des années 2000. Simultanément, la crise des prix alimentaires et les sources de rentabilité inattendues qu'elle a mises en évidence ont également débouché sur de nouvelles stratégies axées sur la sécurité alimentaire, qui privilégient de nouveaux modèles de production basés sur des investissements ou réinvestissements rapides en intrants (parfois avec des subventions), en infrastructures, en irrigation et en projets agricoles de grande ampleur. L'accaparement des terres par des investisseurs étrangers suscite des inquiétudes croissantes, en particulier en Afrique subsaharienne (Coalition foncière internationale - ILC 2009 ; Banque mondiale 2010b). Il est à l'origine de situations conflictuelles entre les acteurs locaux dans plusieurs pays et relance le vieux débat sur les mérites comparés de la petite agriculture et de l'agriculture à grande échelle (respectivement assimilées, à tort, aux petits exploitants et à l'agriculture commerciale).

\section{Quel rôle pour l'agriculture?}

Heureusement, et au-delà de la conjoncture des dernières années, deux contributions importantes ont amené la communauté internationale à s'intéresser 
davantage à l'agriculture " pour elle-même ». La première est l'Évaluation internationale des connaissances, des sciences et des technologies agricoles pour le développement (IAASTD), un ambitieux effort international de recensement des connaissances sur l'agriculture et d'évaluation de leur adéquation à la lumière des défis mondiaux qui se posent au développement durable. Ses résultats ont été examinés et ratifiés par 110 pays lors du sommet de Johannesburg (2008) et publiés (IAASTD 2009).

La seconde contribution est la publication du Rapport sur le développement dans le monde 2008, L'Agriculture au service du développement (RDM08) (Banque mondiale 2007). Établi en 2006-2007 et publié fin 2007, ce rapport a donné l'impulsion nécessaire pour susciter un regain d'intérêt à l'égard de l'agriculture et porter sur elle un nouveau regard. Il réaffirme vigoureusement que l'agriculture est un des principaux secteurs d'activité économique dans la plupart des pays en développement (source d'emplois, de croissance et d'avantages comparatifs), un secteur social important de par le fort pourcentage de population concerné et un important utilisateur de ressources naturelles. Après une marginalisation de près de trente ans de l'agriculture dans l'économie du développement - conséquence des réformes du Consensus de Washington (de Janvry 2009) - le RDM08 dresse un bilan précis et utile des connaissances sur les mécanismes du développement agricole et sur le rôle catalyseur de l'agriculture dans le développement économique. Il s'appuie sur une vision régionalisée de l'agriculture mondiale et examine les fonctions et défis particuliers de l'agriculture dans le processus de développement, en fonction de son importance dans l'économie de chaque région (voir encadré 1.2).

Bien que ce rapport traite assez peu des liens que l'agriculture entretient avec les autres secteurs ${ }^{3}$, son approche régionalisée et ciblée a été un important facteur de succès et sa large diffusion a facilité le retour de l'agriculture dans le débat international sur le développement. Cependant, comme pour le rapport de l'IAASTD, la dynamique qu'il avait amorcée s'est trouvée quelque peu ralentie par l'agenda international très chargé et les problèmes à court terme nés de la crise des prix alimentaires. En outre, quelques mois seulement après la publication du RDM08, la Banque mondiale et d'autres organismes internationaux ont diffusé des messages différents et, dans une certaine mesure, contradictoires. À titre d'exemple, le Rapport sur le développement industriel 2009 de l'Organisation des Nations unies pour le développement industriel (ONUDI 2008) insistait sur le rôle de l'industrie, considérée comme le principal moteur de changement, en particulier pour les pays « du Bottom Billion » (correspondant au milliard de personnes les plus pauvres) et pour les pays à revenu intermédiaire ${ }^{4}$. Adoptant une perspective différente, l'édition suivante du Rapport sur le développement dans le monde, Repenser la géographie économique (RDM09) (Banque mondiale 2008a), soulignait la nécessité d’augmenter les densités démographiques, de raccourcir les distances économiques et de réduire les divisions politiques (voir encadré 3.1). Le RDM09 démontrait qu'il est possible d'atteindre ces objectifs en intensifiant les processus d'agglomération et d'intégration, et soulignait ainsi le rôle de l'urbanisation ${ }^{5}$. 


\section{GNCADRÉ 1.2}

\section{Le Rapport sur le développement dans le monde 2008 (RDM08) et ses trois mondes}

Proposant une approche multiniveau de l'agriculture pour le développement, le RDM08 distingue trois mondes de l'agriculture en fonction de sa contribution à la croissance et de la part rurale dans la pauvreté totale d'un pays. Ces trois mondes sont : les pays à base agricole, les pays en mutation et les pays urbanisés. Chacun de ces mondes appelle un plan d'action particulier pour une agriculture au service du développement dans un objectif de croissance soutenable et de réduction de la pauvreté.

Dans les pays à base agricole, catégorie qui regroupe la majorité des pays d'Afrique subsaharienne, l'agriculture et les secteurs qui lui sont associés sont essentiels pour la croissance mais aussi pour la lutte contre la pauvreté de masse et l'insécurité alimentaire, car ils sont source d'emplois, d'activité et de revenus. Dans les pays en mutation, soit la plupart des pays d'Asie du Sud et de l'Est, le Moyen-Orient et l'Afrique du Nord, le creusement rapide des disparités entre les revenus ruraux et urbains et la persistance d'une extrême pauvreté rurale sont d'importantes sources de tensions sociales et politiques ; la diversification rurale et la croissance des revenus agricoles sont les réponses à ces difficultés. Enfin, dans les pays urbanisés - la majorité des pays d'Amérique latine et une grande partie de l'Europe et de l'Asie centrale - l'agriculture peut contribuer à réduire la pauvreté rurale qui perdure si les petits exploitants approvisionnent directement les marchés alimentaires modernes, si de bons emplois sont créés dans l'agriculture et l'agro-industrie et si des marchés de services à l'environnement se constituent.

Le RDM08 recense trois voies de sortie de la pauvreté : (1) l'entreprise agricole, (2) le marché du travail rural et (3) l'économie rurale non agricole associée à la migration dans

Figure d'encadré 1.2.1 Contribution de l'agriculture à la croissance, 1990-2005

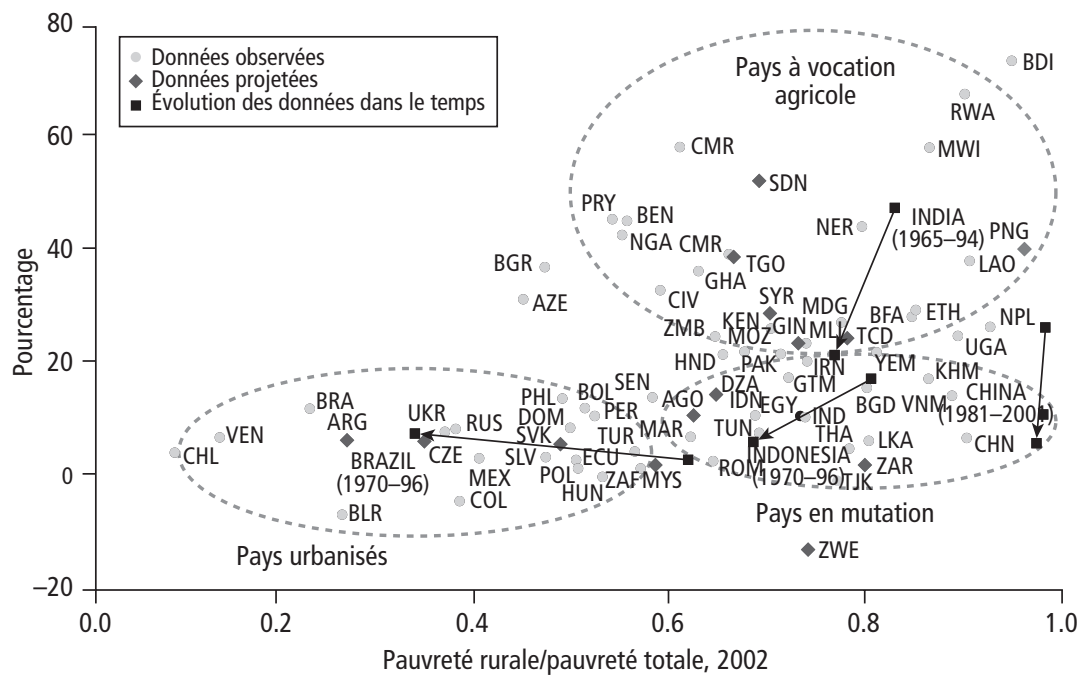

(Suite de l'encadré page suivante) 
les villes ou d'autres pays. II est fréquent que deux voies ou plus opèrent simultanément et les activités agricoles et non agricoles peuvent avoir des effets complémentaires non négligeables. Mais bien que les ménages ruraux pratiquent l'agriculture, le travail salarié et la migration, une de ces activités est habituellement la source de revenus dominante.

Tableau d'encadré 1.2.1 Caractéristiques des trois mondes agricoles du RDM08

\begin{tabular}{lccc}
\hline & $\begin{array}{c}\text { Pays à base } \\
\text { agricole }\end{array}$ & $\begin{array}{c}\text { Pays en } \\
\text { mutation }\end{array}$ & $\begin{array}{c}\text { Pays } \\
\text { urbanisés }\end{array}$ \\
\hline Population rurale (millions), 2005 & 417 & 2220 & 255 \\
Population rurale (\%), 2005 & 68 & 63 & 26 \\
PIB par habitant (2000 US\$), 2005 & 379,0 & 1068 & 3489 \\
Part de l'agriculture dans le PIB (\%), 2005 & 29 & 13 & 6 \\
Croissance annuelle du PIB agr. (\%), 1993-2005 & 9,0 & 2,9 & 2,2 \\
Croissance annuelle du PIB non agr. (\%), 1993-2005 & 3,5 & 7,0 & 2,7 \\
Taux de pauvreté rurale (\%), 2002 & 51 & 28 & 13 \\
\hline
\end{tabular}

Source: Banque mondiale 2007, p. 37-39.

Note : Le seuil de pauvreté est de 1,08 US\$ par jour en PPA 1993.

Au final, malgré cet environnement très instable et quelque peu déroutant, l'agriculture a cependant retrouvé sa place au cœur des préoccupations et les donateurs et les États réinvestissent la question de son développement. Une équipe spéciale de haut niveau (High Level Task Force) sur la crise mondiale de la sécurité alimentaire a été mise en place en 2008 auprès du Secrétaire général des Nations unies et coordonne les efforts internationaux. Au sommet de L'Aquila (juillet 2009), le Groupe des huit principaux pays industrialisés (G8) s'est engagé à mobiliser 20 milliards de dollars en cinq ans en faveur de la sécurité alimentaire, engagement qui a été confirmé au sommet du G20 de Pittsburgh en septembre 2009. Le Programme mondial pour l'agriculture et la sécurité alimentaire (GAFSP) a été officiellement lancé en avril 2010 avec une première contribution de près d'un milliard de dollars. Enfin, en novembre 2010, lors du sommet du G20 de Séoul, il a été décidé de lancer un « G20 agricole » pour favoriser la coopération internationale, en particulier dans la lutte contre la volatilité des prix alimentaires.

Bien que le concept de sécurité alimentaire soit plus étroit que le développement agricole et rural, ce nouveau contexte permet d'élargir le débat et de proposer un point de vue qui ne cantonne pas l'agriculture à l'offre alimentaire mais recouvre aussi ses autres fonctions (environnementales, économiques, sociales, culturelles). Comme l'indique l'IAASTD, cette multifonctionnalité ${ }^{6}$ est un trait distinctif de l'agriculture, puisqu'elle est la principale activité et le principal moyen d'existence des ruraux, ainsi qu'un vecteur majeur pour le recul de la pauvreté rurale. Elle est à la base de la diversification rurale et du développement des liens entre villes et campagnes et elle joue un rôle pivot dans la fourniture de services à l'environnement. Sa fonction de moteur de la transformation structurelle doit cependant être réaffirmée et il convient de s'interroger sur la viabilité 


\section{GNCADRÉ 1.3}

\section{Libéralisation ou mondialisation ?}

Dans la définition initiale du programme RuralStruc, la libéralisation était entendue comme un processus de changement global amorcé dans les années 1980, qui comprenait un train de réformes portant sur les échanges extérieurs, le marché intérieur, le désengagement de l'État et les privatisations et, dans de nombreux pays en développement, le fonctionnement des institutions et la décentralisation.

L'objectif était d'examiner toutes les dimensions structurelles de ce nouveau contexte, ce qui explique le choix de l'intitulé du programme. Cependant, malgré cette acception large de la libéralisation, cet intitulé s'est rapidement révélé inadapté. Tout d'abord, la compréhension des objectifs du programme était souvent limitée aux mesures standard des réformes et, de ce fait, il était souvent perçu comme ayant une position d'évaluation critique qui n'était pas recherchée. Ensuite, car cette erreur d'interprétation des objectifs limitait implicitement la compréhension globale du programme, à savoir une analyse des processus de recomposition en cours.

Des échanges avec la communauté des donateurs et avec les partenaires nationaux, il ressort qu'il aurait sans doute été plus clair de préférer le terme " mondialisation » au terme « libéralisation » dans l'intitulé du programme. Car si le terme « mondialisation » peut suggérer un champ d'étude trop large, le contexte auquel se réfère RuralStruc correspond bien au nouvel ordre international apparu au début des années 1980 et à ses conséquences pour l'agriculture, les économies rurales et le processus global de transition économique. Ce nouvel ordre se caractérise par de nouveaux rôles pour l'État et les acteurs privés et par un vaste mouvement d'intégration de l'économie mondiale.

Dans sa mise en œuvre et dans la présentation de ses résultats, le programme RuralStruc a clairement et progressivement adopté ce positionnement plus large. L'intitulé "Changements structurels des économies rurales dans la mondialisation » a d'ailleurs été employé pour les rapports nationaux et les policy briefs de la phase 2, et le titre de cet ouvrage fait clairement référence à la mondialisation.

effective des options de sortie de la pauvreté rurale à l'ère de la mondialisation ${ }^{7}$. Cette question critique doit être hissée aujourd'hui au premier plan du débat politique et devrait justifier de renouer avec l'élaboration de cadres d'action publique calés sur une indispensable vision à long terme.

\section{Principaux objectifs et hypothèses du programme : reconnecter les débats}

À l'origine, le programme RuralStruc avait trois objectifs : (1) enrichir le corpus de connaissances sur les transformations structurelles, les processus d'intégration et leurs effets sur l'agriculture et l'économie rurale des pays en développement (voir encadré 1.3) ; (2) alimenter et améliorer les débats nationaux et internationaux en promouvant et en réarticulant ces thématiques et (3) apporter des éclairages pour l'action publique. 
Alors que le troisième objectif du programme renvoyait directement à sa conception participative et à la mise en œuvre opérationnelle de ses résultats, les deux premiers étaient plus analytiques. Ils impliquaient un positionnement clair et le programme a choisi de situer ses travaux sur l'agriculture et les recompositions rurales dans le référentiel du changement structurel afin de réarticuler le débat sur le développement avec une perspective globale et d'éviter d'enfermer l'analyse dans des approches sectorielles.

La transformation structurelle des économies et des sociétés est un thème central des études sur le développement. Les données historiques et statistiques ${ }^{8}$ font apparaître une évolution progressive de l'agriculture (activité principale originelle de toutes les populations sédentaires) à l'industrie (activités secondaires), puis aux services (activités tertiaires). La dynamique bien connue sous-jacente à cette transformation structurelle - la transition économique d'une configuration à l'autre - réside dans les gains de productivité de l'agriculture liés à l'innovation qui favorise l'évolution des techniques et permet des transferts de main-d'œuvre et de capital vers d'autres activités économiques.

Ce processus s'accompagne d'une restructuration spatiale graduelle par laquelle des activités éparses (typiquement l'agriculture) cèdent la place à des activités plus concentrées (typiquement l'industrie), avec une migration du travail et de la population des zones rurales vers les villes. Parallèlement à cette urbanisation croissante, la transformation économique globale engendre une augmentation des revenus et des richesses qui améliore les conditions de vie. Ce phénomène, conjugué aux progrès de la médecine, amorce à son tour une transition démographique, à savoir la baisse progressive des taux de mortalité puis de natalité, dont le décalage dans le temps explique la dynamique de croissance de la population.

Ce processus de transformation structurelle s'accomplit à des rythmes différents selon les régions et peut suivre des voies multiples, mais il a été observé dans le monde entier. Il a commencé dès la fin du XVIII ${ }^{\mathrm{e}}$ siècle en Europe de l'Ouest avec le binôme révolution agricole-révolution industrielle pour se poursuivre dans les nouveaux pays d'origine européenne (principalement les États-Unis), puis d'autres régions d'Europe, et enfin dans la majeure partie de l'Amérique latine et de nombreux pays d'Asie.

C'est en référence à ce processus que s'est forgé le référentiel classique du développement à l'origine du courant économique du même nom ${ }^{9}$. Actuellement, un des principaux défis réside dans l'accélération des changements liés à la mondialisation et, par suite, dans les asymétries croissantes entre régions du monde situées à différents stades de ce processus de transformation structurelle.

Ce positionnement conceptuel a considérablement marqué le contour et influé sur les contenus du programme RuralStruc ainsi que sur ses hypothèses. Alors que le débat sur la libéralisation du commerce se centrait sur ses gains attendus et sur leurs conséquences pour la croissance et la pauvreté ${ }^{10}$, le programme se proposait de relancer le débat sur la transition économique dans le contexte de la mondialisation et d'en exposer précisément les possibles difficultés structurelles sans se borner aux seuls problèmes réputés transitoires ${ }^{11}$. RuralStruc entendait reconnecter la discussion sur l'agriculture avec des problématiques plus 
globales comme le creusement des écarts de productivité entre pays, les retards constatés dans la diversification économique ou les défis démographiques auxquels sont confrontées plusieurs régions du monde - autant de sujets qui sont des angles morts récurrents du débat international, dont la prise en compte est pourtant essentielle pour la transformation structurelle des pays en développement.

Trois hypothèses ont été retenues pour structurer le programme de recherche en référence aux débats sur l'agriculture, les marchés alimentaires et la diversification rurale (voir encadré 1.4 ). La première hypothèse $(\mathrm{H} 1)$ concerne la restructuration mondiale des marchés agroalimentaires et l'asymétrie grandissante entre régions dans la concurrence internationale. Le postulat est que ces phénomènes entraînent une différenciation croissante des structures agricoles, de commercialisation, de transformation et de distribution. Cette hypothèse soulève plusieurs interrogations : quelles sont les perspectives d'intégration des agriculteurs dans les chaînes de valeur émergentes et quels risques d'exclusion encourent-ils ? Quelle est l'ampleur de ces processus et quels en sont les rythmes et les caractéristiques ? Induisent-ils une segmentation accompagnée d'une concentration, d'une marginalisation, voire d'une exclusion à la fois à l'intérieur et en dehors du secteur agricole et, par suite, l'émergence ou la consolidation d'une agriculture à plusieurs vitesses?

La deuxième hypothèse $(\mathrm{H} 2)$ concerne les processus d'adaptation des ménages ruraux face à l'évolution de l'agriculture et son impact sur la viabilité des exploitations agricoles. Les ménages ruraux adoptent de nouvelles stratégies d'activités et de revenus caractérisées par une nouvelle place de l'agriculture et

\section{ENCADRÉ 1.4}

\section{Les trois hypothèses du programme RuralStruc}

H1 : La restructuration mondiale des marchés agroalimentaires renforce un processus de différenciation et de segmentation au sein des économies agricoles.

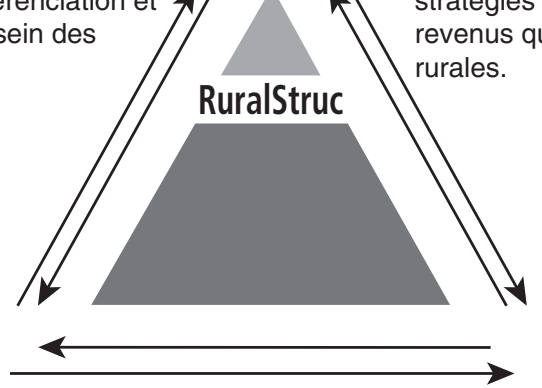

H2 : Les ménages agricoles s'adaptent au nouveau contexte en adoptant des stratégies composites d'activité et de revenus qui transforment les économies rurales.

H3 : Les tendances à la marginalisation introduites par ces processus engendrent des risques d'impasses de transition liées à la rareté des alternatives en termes d'activités et d'emploi. 
par l'importance croissante des autres activités rurales et des transferts (transferts privés relatifs à la migration et, dans certains cas, transferts publics liés à des systèmes d'aides spécifiques). Cette hypothèse suscite les questions suivantes : quelles sont les caractéristiques de ces nouvelles configurations d'activités ? En quoi diffèrent-elles d'un pays à l'autre? Ces dynamiques sont-elles nouvelles ou suivent-elles les trajectoires historiques de la transformation structurelle? Comment contribuent-elles à la recomposition des zones rurales et des liens entre villes et campagnes? Sont-elles des solutions efficaces pour pérenniser les moyens d'existence des ruraux?

Les dynamiques de différenciation à l'œuvre dans l'agriculture et les possibles difficultés d'adaptation des ménages ruraux (faute d'alternatives effectives en matière d'activités et de revenus) sont susceptibles de déboucher sur des impasses de transition dans le processus de transformation structurelle. C'est la troisième hypothèse du programme (H3), qui s'adresse principalement à la situation des pays qui restent aujourd'hui à «base agricole » (RDM08). Dans ces pays, le poids de l'agriculture dans la structure de l'emploi et des activités, une forte urbanisation sans industrialisation ou création d'emplois significative, la faible diversification de l'économie dans un contexte de concurrence internationale croissante et une poussée démographique sans précédent engendrent un défi sans égal pour le développement. Peut-on penser que par suite du manque d'alternatives locales (Kydd 2002) et d'opportunités de migration, une partie de ces pays se trouvera dans l'impasse sans possibilités d'échapper à la pauvreté ? Quelles pourraient être alors les conséquences sociales, économiques et politiques de ces impasses de transition?

\section{Structure générale du programme : études de cas nationales, enquêtes régionales et processus collaboratifs}

Pour évaluer la pertinence de ces hypothèses et répondre aux questions posées, le programme RuralStruc s'est attaché à mettre en évidence les principales similitudes et différences existant entre les pays étudiés, en référence aux processus d'adaptation au nouveau contexte et en tenant compte des modalités de leur transformation rurale et de leurs trajectoires de changement structurel. Le programme reposait sur un cadre collaboratif associant les partenaires locaux au processus d'analyse dans le double objectif d'une «meilleure compréhension pour de meilleures politiques » (la devise du programme).

\section{Une approche comparative}

La comparaison est un puissant outil d'analyse car elle met en lumière les convergences et les divergences et fait apparaître les facteurs explicatifs déterminants ; mais elle comporte aussi des risques et peut entraîner de profondes erreurs méthodologiques. Ainsi, le programme RuralStruc n'a pas établi de comparaisons entre pays (par exemple entre le Mexique et Madagascar), une option qui 
n'aurait pas fait sens et aurait surtout entraîné un biais classique de sélection ${ }^{12}$. De même, face aux difficultés bien connues de l'analyse ex post, l'objectif n'était pas d'évaluer les impacts, un choix qui aurait été confronté aux difficultés habituelles d'information (et en particulier à l'absence d'années de référence pour l'évaluation) et à la délicate question du sens des causalités. C'est pourquoi le terme « impact » a été volontairement évité dans l'intitulé officiel du programme pour lui préférer celui de « dimension».

L'objectif de l'approche comparative était d'illustrer les processus de changement dans l'agriculture et l'économie rurale dus à la libéralisation, à l'intégration économique et à la mondialisation, afin de dégager et de comprendre des traits communs et des différences utiles pour l'élaboration des politiques publiques. Dans sa mise en œuvre, le programme s'est efforcé d'adopter une perspective globale, pluridisciplinaire et historique sur les dynamiques de changement en

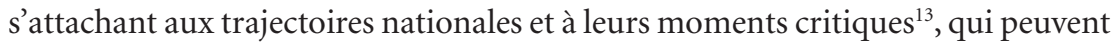
modifier la nature des relations entre l'agriculture, le secteur rural et l'économie dans son ensemble.

Sélection des pays. La sélection de cas nationaux aux fins de comparaison implique des arbitrages entre des critères objectifs liés aux finalités de la recherche et des aspects opérationnels liés aux modalités des partenariats locaux, aux conditions de mise en œuvre (surtout les délais et les ressources financières et humaines) et à l'adhésion et l'intérêt des donateurs.

Lors des discussions préliminaires, les bailleurs de fonds du programme ont fait le choix de privilégier l'Afrique subsaharienne dont la justification était la situation structurelle critique du sous-continent et les nombreux efforts entrepris pour revitaliser son secteur agricole. Ce réinvestissement progressif des donateurs dans l'agriculture faisait écho à l'engagement des gouvernements africains dans la mise en œuvre du Programme détaillé de développement de l'agriculture africaine (PDDAA) du Nouveau partenariat pour le développement de l'Afrique $(\mathrm{NEPAD})^{14}$, programme qui est devenu la référence africaine - et internationale pour l'action dans l'agriculture en Afrique et qui est en cours d'exécution.

Pour mettre en œuvre l'approche comparative, le choix fut de sélectionner un petit échantillon de pays. Ces pays n'étaient pas censés représenter la diversité des trajectoires de développement possibles, mais ils devaient correspondre néanmoins à un gradient de situations dans le processus de transformation structurelle, certains étant très avancés alors que, pour d'autres, la transition économique et le rythme d'intégration dans l'économie mondiale avaient été lents ou inégaux.

Parmi les sept cas nationaux retenus, le Mexique a été choisi pour illustrer une transition économique avancée, avec un fort taux d'urbanisation et un rôle limité de l'agriculture dans l'économie. L'économie rurale mexicaine connaît cependant des recompositions majeures liées aux importants flux migratoires vers les ÉtatsUnis et aux processus marqués d'intégration et de libéralisation, accélérés par la mise en œuvre de l'Accord de libre-échange nord-américain (ALENA) en 1992.

À l'opposé, l'Afrique subsaharienne (ASS) - représentée par le Kenya, Madagascar, le Mali et le Sénégal - illustre le stade initial de la transition économique, 
avec des processus d'intégration et de libéralisation partiels, amorcés par des réformes de l'État et des marchés, mais un rôle de l'agriculture et des autres activités primaires qui reste dominant.

Enfin, le Maroc et le Nicaragua représentent une phase intermédiaire de transformation structurelle, correspondant aux pays caractérisés par des processus d'intégration rapides, dus ici à la proximité de zones économiques puissantes avec lesquelles des accords de libre-échange ont été récemment mis en œuvre ${ }^{15}$. Dans ces pays, l'agriculture (caractérisée par des structures de production duales) conserve une importance primordiale dans le débat public alors que les migrations internationales jouent également un rôle important dans l'économie politique.

Outre les critères d'intégration graduelle et différenciée aux marchés mondiaux, trois critères macroéconomiques ont également été utilisés lors de la sélection des pays étudiés : le PIB par habitant, la part de l'agriculture dans le PIB et la part de la population active ayant une activité agricole. Ces critères sont des indicateurs élémentaires du stade de transition d'un pays. En dehors du Mexique, les pays sélectionnés sont des pays à faible revenu ou à revenu intermédiaire de tranche inférieure. Ils présentent des situations très diversifiées en termes de pauvreté, de développement humain, de gouvernance et de climat des affaires, avec de très nets contrastes entre les pays d'ASS et les autres et une gradation claire des indicateurs d'un pays à l'autre (voir tableau 1.1 ${ }^{16}$.

Ainsi, en référence à la typologie du RDM08, les pays sélectionnés représentent les trois mondes de l'agriculture : (1) pays à base agricole (Kenya, Madagascar, Mali) ; (2) pays en mutation (Sénégal, Maroc, Nicaragua) et (3) pays urbanisés (Mexique $)^{17}$. Dans ces pays, la contribution de l'agriculture à la croissance économique diminue progressivement, mais elle conserve un rôle important dans l'emploi national - entre $70 \%$ et $80 \%$ de la population active pour les pays d'ASS et moins de $30 \%$ au Mexique, au Maroc et au Nicaragua ${ }^{18}$. Ces sept pays correspondent également aux trois stades d'urbanisation du RDM09 : urbanisation naissante, intermédiaire et avancée ${ }^{19}$.

La taille de la population a également compté dans le processus de sélection. Afin d'éviter les situations extrêmes, les pays les plus peuplés (comme la Chine, l'Inde, l'Indonésie et le Brésil) mais aussi les pays démographiquement de petite taille ont été écartés ${ }^{20}$. Cette décision est contestable car il n'y a pas de corrélation directe entre la transition économique et la taille de la population, mais le RDM09 nous rappelle que « la taille est importante » et qu'elle peut être un solide atout pour la transformation structurelle, car les grands marchés intérieurs offrent des économies d'échelle et une demande facilement accessible, ce qui donne une marge de manœuvre appréciable aux entreprises nationales face à la concurrence croissante due à la mondialisation. C'est le cas notamment en matière d'industrialisation, de recherche et de renforcement des capacités ${ }^{21}$. Par conséquent, les pays sélectionnés sont de taille démographique modeste à moyenne : hormis le Mexique, ils comptent tous entre 5 et 35 millions d'habitants. Ces critères ont conduit à écarter les pays asiatiques ${ }^{22}$. 


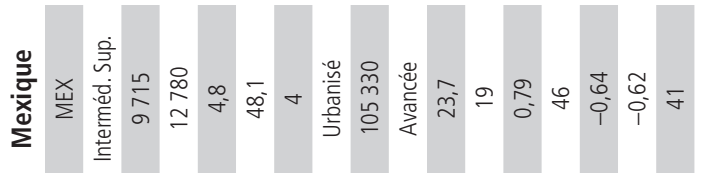

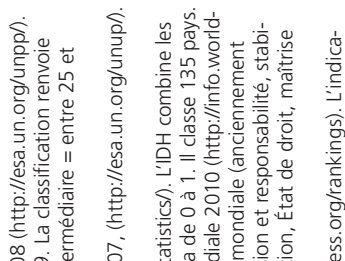

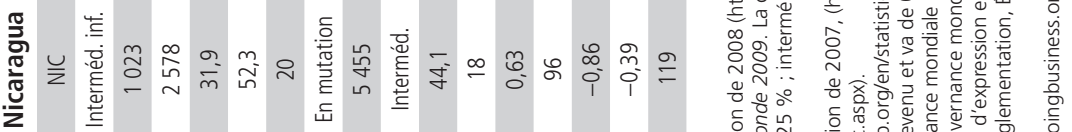

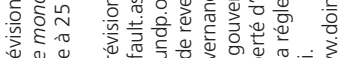

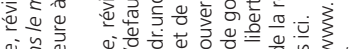

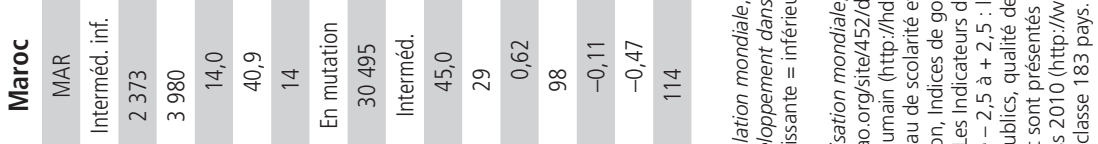

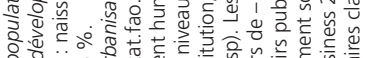

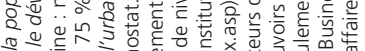

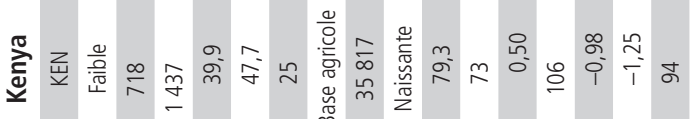

o

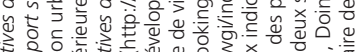

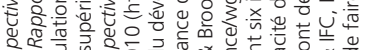
这过

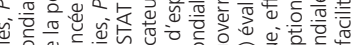

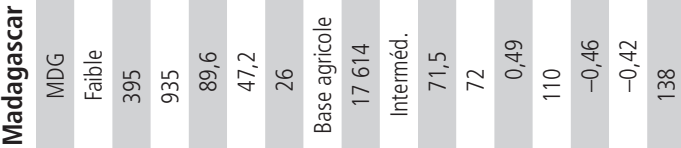

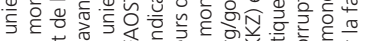

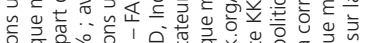

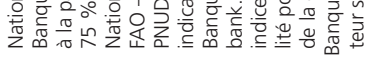

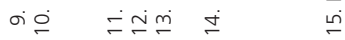

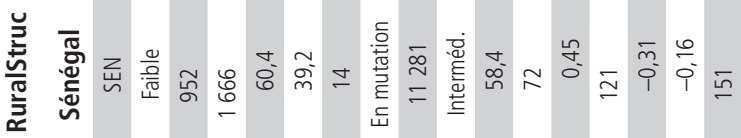

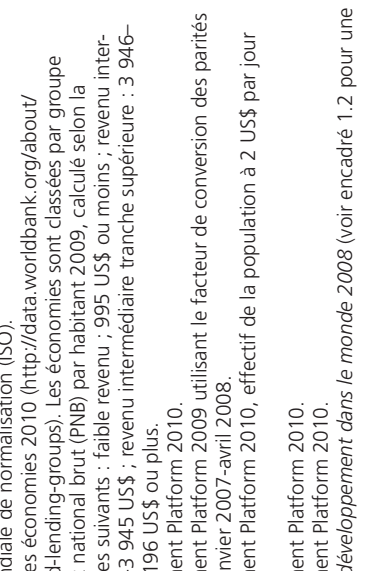

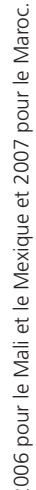

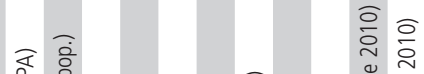

告

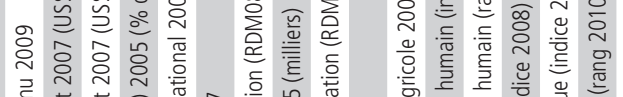

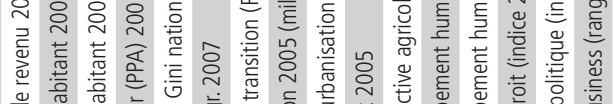

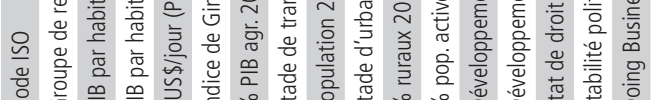

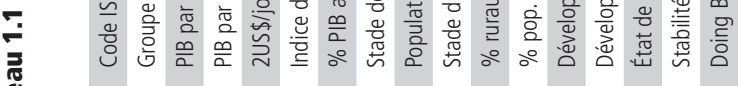

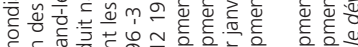

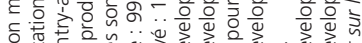

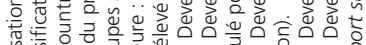

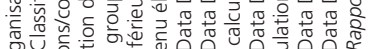

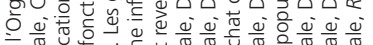

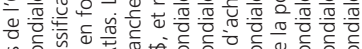

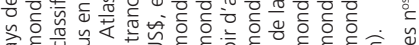

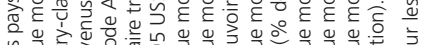

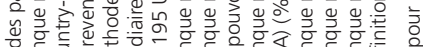

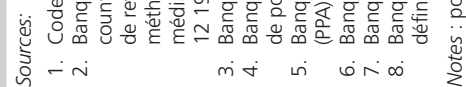


Les pays d'ASS sélectionnés illustrent la diversité des situations des pays à faible revenu (Madagascar, le Mali et le Sénégal font partie des pays les moins avancés). Ils se différencient par la géographie (Afrique australe, orientale et occidentale, y compris un pays sans accès à la mer, le Mali), leur histoire coloniale (anciennes colonies françaises ou britanniques), leur structure d'activité (y compris le rôle des migrations) et par l'état du débat national autour de l'agriculture et de la privatisation ${ }^{23}$.

Étant donné ses caractéristiques démographiques et économiques, le choix du Mexique était contraire à de nombreux critères de sélection énoncés : c'est un pays à revenu intermédiaire de la tranche supérieure, membre de l'OCDE, et une économie émergente. L'intérêt de sa sélection était de présenter une toile de fond illustrant la restructuration d'une économie rurale dans un contexte de forte libéralisation et d'intégration économique ${ }^{24}$.

Aucun échantillon n'est évidemment parfait. Cependant, la sélection proposée par le programme RuralStruc présente un large éventail de situations qui répondent à ses objectifs et permettent de dresser un tableau différencié des processus de transformation à l'œuvre. Les pays retenus apportent un éclairage sur les caractéristiques structurelles des économies à différents stades de diversification économique et d'urbanisation, illustrent différentes tendances migratoires et différents types de politiques publiques ainsi qu'une variété de configurations agricoles diversifiées. Les agricultures se différencient par leurs productions, la taille de leurs principales chaînes de valeur et l'orientation de leurs marchés (national ou international, produits de base ou à forte valeur ajoutée). Ces caractéristiques sont le produit des conditions naturelles et historiques qui ont modelé les systèmes agraires et les marchés locaux. Ainsi les agricultures étudiées par le programme sont généralement centrées sur les cultures annuelles (céréales principalement), parfois à base d'irrigation, mais elles comprennent aussi des produits de base d'exportation traditionnels comme la canne à sucre, le coton, l'arachide et le café. L'échantillon aurait pu bénéficier de l'inclusion d'une économie agricole de plantation, où les cultures pérennes (café, cacao, palmier) modèlent depuis longtemps les systèmes agraires (cas de certains pays du golfe de Guinée en Afrique de l'Ouest), mais ces situations avec cultures pérennes sont toutefois représentées ${ }^{25}$.

Mise en œuvre opérationnelle. Le programme RuralStruc a été structuré en deux phases. L'objectif de la première phase était de produire des synthèses générales par pays à partir d'études documentaires sur le rôle de l'agriculture dans l'économie. Ces études ont examiné la structuration des marchés des principaux facteurs de production et leur évolution, le développement et la différenciation des structures d'exploitation, ainsi que les risques de blocages et d'impasses de transition et les possibilités d'adaptation. Cette première phase a également permis de repérer les informations manquantes sur les processus de changement structurel dans l'agriculture et d'échanger avec les partenaires nationaux sur l'approche générale du programme.

La seconde phase était initialement destinée à produire des analyses à la fois régionales et sur les principales filières, à partir d'entretiens qualitatifs avec des 
agriculteurs, des intermédiaires et d'autres opérateurs économiques, et à aborder des problèmes plus spécifiques identifiés pendant la première phase. L'ampleur du déficit d'informations constaté au cours de cette première phase sur les processus de transformation rurale en général, et sur les activités des ménages ruraux, leurs revenus et leur intégration aux marchés en particulier, a toutefois conduit à modifier significativement la seconde phase pour y incorporer un recueil de données primaires au niveau des ménages ruraux ${ }^{26}$.

Dans chaque pays, l'objectif des enquêtes de ménages a consisté à produire des informations à la fois qualitatives et quantitatives originales sur les processus à l'œuvre, à mieux comprendre le rôle de l'agriculture dans les économies locales, ainsi que les moyens d'existence des ruraux, notamment les types de revenus, et les différentes combinaisons possibles d'activités et de sources de revenus ${ }^{27}$.

\section{Le travail de terrain}

La conception générale du travail de terrain et la sélection des régions ont été réalisées de la manière suivante :

Conception et limites. La décision de procéder à des enquêtes de ménages centrées sur les activités et les revenus a influencé la mise en œuvre du programme et ses résultats. La préférence pour les ménages ruraux et pas seulement agricoles était justifiée par la nécessité de déterminer plus précisément le rôle de l'agriculture par rapport aux autres activités et sources de revenus rurales. Ce choix a imposé de traiter avec des catégories d'analyse dont la définition est souvent incertaine, comme le terme « rural » dont la définition officielle diffère d'un pays à l'autre (voir encadré 1.5).

Cette priorité accordée aux revenus des ménages a conduit à s'engager dans le délicat exercice de leur repérage et recollement qui suppose, en zone rurale, d'identifier puis d'estimer les revenus agricoles. Le programme a résolu ces difficultés au moyen d'un cadre d'enquête conséquent, comportant des questionnaires détaillés ${ }^{28}$.

Le travail d'enquête s'est heurté à quelques difficultés au niveau local (comme des retards et des informations manquantes résultant des violences postélectorales au Kenya début 2008), mais la principale contrainte a porté sur les limites inhérentes aux enquêtes à un seul passage, conséquence de la durée du programme et de son financement. Cet unique passage d'enquêtes a posé une importante difficulté car les hypothèses du programme avaient été posées en termes dynamiques. Il a aussi été une source de biais du fait de la variation interannuelle des revenus agricoles due, par exemple, à l'impact de la sécheresse sur les rendements.

Pour atténuer les limites d'un passage unique, il aurait fallu étalonner les enquêtes sur des panels existants, mais cette option est apparue irréaliste car d'une part, il aurait été difficile, dans les délais impartis, de gérer des années de référence disparates et des conceptions et méthodologies d'enquêtes différentes et, d'autre part, car les études de panel spécifiquement ciblées sur les 


\section{ENCADRÉ 1.5}

\section{Rural et urbain : quelle définition pour chaque pays ?}

La définition du terme " rural » varie d'un pays à l'autre, mais il existe toutefois des points communs. Cette définition est rarement positive : le plus souvent, le qualificatif correspond à la population résiduelle après soustraction de la population urbaine à la population totale (définition de la FAO). Cependant, il n'existe pas de définition uniforme du terme " urbain » - cet attribut repose le plus souvent sur la taille des agglomérations, mais il peut aussi se fonder sur la densité de la population, les circonscriptions administratives et, parfois, sur l'offre de services. Les pays étudiés par le programme RuralStruc définissent le terme « rural » de la façon suivante :

Kenya : I'Office national de la statistique (Kenya National Bureau of Statistics) qualifie de « rurale » toute localité dont la population est inférieure à 2000 habitants.

Madagascar : les zones rurales sont les districts dans lesquels la population active agricole (telle que définie par le recensement agricole) est supérieure à 50 \% (RS 2 Madagascar, 26).

Mali : les ménages ruraux comprennent tous les ménages des zones rurales, lesquelles sont définies comme le contraire de la ville. Les ménages ruraux sont engagés dans des activités agricoles, définies au sens large, par l'intermédiaire d'au moins un de leurs membres (RS 2 Mali, 20).

Mexique : le système statistique national définit une localité rurale comme un lieu accueillant moins de 2500 habitants. Cependant, ce seuil est débattu et l'équipe nationale du programme a retenu la référence courante de 5000 habitants (RS 2 Mexique, 6).

Maroc : les zones rurales sont définies par défaut comme toute zone non comprise dans le périmètre d'une zone urbaine. Le périmètre des zones urbaines évolue sous l'effet de l'expansion des villes et de la reclassification des localités rurales en localités urbaines. II n'y a pas de définition statistique de la population rurale (RS 2 Maroc, 6).

Nicaragua : les zones rurales sont officiellement définies comme étant les districts de moins de 1000 habitants (RS 2 Nicaragua, 11).

Sénégal : le rural est défini par opposition à l'urbain, qui a une définition administrative : toutes les « communes » sont classées comme urbaines, même si elles ont tous les attributs d'une zone rurale, notamment une économie à base agricole (RS 2 Sénégal, 39).

revenus ruraux sont rares. Dans les pays en développement, les données de panel concernant les ménages (lorsqu'elles existent) ont généralement été développées pour estimer la pauvreté, notamment dans le contexte des programmes d'ajustement structurel entrepris dans les années 1980-1990. Elles concernent le plus souvent les dépenses des ménages et ciblent souvent les ménages urbains, qui forment généralement la part la plus importante des échantillons nationaux ${ }^{29}$.

Si les études de cas localisées sont nombreuses, rares sont les données disponibles sur les revenus ruraux. Cette situation résulte généralement de 
l'appauvrissement des systèmes statistiques nationaux, mais elle tient aussi souvent à des observations statistiques ciblées non pas sur l'économie rurale mais seulement sur l'agriculture. Une telle approche complique la conceptualisation des processus de transformation rurale à l'œuvre. L'une des rares exceptions est le projet Activités rurales génératrices de revenus (RIGA), développé par la FAO en collaboration avec la Banque mondiale, qui propose un cadre d'analyse cohérent pour une comparaison internationale des sources de revenus ruraux (voir encadré 1.6$)^{30}$.

Le RDM08 s'est beaucoup appuyé sur les résultats du projet RIGA, en particulier pour analyser la diversification des activités et des sources de revenus ruraux et leur rôle en matière de réduction de la pauvreté ${ }^{31}$. Ce recours à RIGA n'était toutefois pas possible pour les analyses conduites par RuralStruc en raison d'années de référence différentes et de l'absence de plusieurs pays inclus dans le programme.

Puisqu'il n'y avait pas de solution aisée pour étalonner les enquêtes de ménages, les inconvénients liés au passage unique ont été atténués en complétant les enquêtes par un travail de terrain supplémentaire et des études documentaires portant à la fois sur certaines filières de production et sur les caractéristiques des

\section{ENCADRÉ 1.6}

\section{Le projet Activités rurales génératrices de revenus (RIGA)}

Le projet RIGA (Rural Income Generating Activities) visait à combler des lacunes importantes dans la compréhension de l'économie rurale non agricole par la constitution d'une base de données à partir d'études de mesure des niveaux de vie (Living Standards Measurement Studies, LSMS) et d'autres enquêtes polyvalentes auprès des ménages effectuées par la Banque mondiale et la FAO. RIGA a analysé les sources de revenus des ménages ruraux à partir de 32 enquêtes réalisées dans 18 pays : Albanie 2002 et 2005 ; Bangladesh 2000 ; Bolivie 2005 ; Bulgarie 1995 et 2001 ; Équateur 1995 et 1998 ; Ghana 1992 et 1998 ; Guatemala 2000 et 2006 ; Indonésie 1992 et 2000 ; Kenya 2004-2005 ; Madagascar 1993-1994 et 2001 ; Malawi 2004 ; Népal 1995-1996 et 2003-2004; Nicaragua 1998, 2001 et 2005 ; Nigeria 2004 ; Pakistan 1991 et 2001 ; Panama 1997 et 2003 ; Tadjikistan 2003 ; et Vietnam 1992-1993, 1997-1998 et 2002.

La base de données RIGA est composée d'une série de variables relatives aux revenus ruraux construites à partir des sources de données originales. En dépit d'écarts de qualité et du niveau hétérogène des informations disponibles dans chaque enquête, l'échantillon de pays et le choix d'indicateurs offrent une couverture géographique, une qualité et une comparabilité qui permettent la comparaison internationale.

De nombreuses analyses fondées sur le projet RIGA ont été publiées. Cette base de données permet aux chercheurs (1) d'évaluer la participation aux activités rurales génératrices de revenus et les revenus qu'elles procurent ; (2) d'analyser le rôle du patrimoine des ménages dans la participation à chaque activité ; (3) d'analyser le rôle ce patrimoine dans les revenus tirés de chaque activité et (4) de désagréger les activités rurales non agricoles par secteur.

Source : Carletto et al. 2007 et http://www.fao.org/economic/riga/fr/. 
zones enquêtées. Ces travaux ont permis de procéder à une contextualisation grâce à une analyse fine des résultats des enquêtes auprès des ménages incorporant la dimension historique des processus de changement à l'œuvre, en particulier la restructuration des marchés agricoles. Les équipes de recherche nationales ont sélectionné les filières en fonction de leur importance dans l'économie nationale et régionale. Pour chaque filière, l'analyse a porté sur les caractéristiques de l'offre et de la demande, leur évolution dans le contexte de la libéralisation, ainsi que sur les processus d'intégration et de différenciation résultant de la restructuration mondiale des marchés agroalimentaires.

Les filières sélectionnées, qui comprenaient à la fois des denrées alimentaires de base et des matières premières, sont indiquées au tableau 1.2.

Tableau 1.2 Principales filières analysées dans les études nationales RuralStruc

\begin{tabular}{lc}
\hline \multicolumn{1}{c}{ Pays } & \multicolumn{1}{c}{ Filières } \\
\hline Mali & Viande et produits laitiers, céréales sèches, riz, oignons, coton \\
Sénégal & Arachide, manioc, riz, produits laitiers, maïs, tomates \\
Madagascar & Riz, maïs, pommes de terre, produits laitiers, haricots verts \\
Kenya & Maïs, lait, canne à sucre \\
Maroc & Céréales, viande rouge, huile d'olive, tomates, agrumes \\
Nicaragua & Céréales de base, légumes, produits laitiers, café, sésame \\
Mexique & Maïs, produits laitiers, fruits et légumes \\
\hline
\end{tabular}

Source : Rapports RuralStruc phase 2.

Sélection des régions étudiées. L'objectif des enquêtes auprès des ménages n'était pas d'obtenir un échantillon statistiquement représentatif mais de dresser un tableau détaillé des réalités rurales. C'est pourquoi, dans chaque pays, le programme a sélectionné des régions illustrant les principales dynamiques déjà identifiées.

Les régions ont été choisies par les équipes nationales sur la base des résultats de la phase 1 et de leurs connaissances du contexte afin d'illustrer les changements au niveau régional permettant de comprendre les processus de transformation à l'œuvre dans chaque pays. Plusieurs critères ont été retenus en fonction des spécificités nationales, mais tous avaient trait à l'accès au marché (infrastructures et proximité des villes), à la présence de filières intégrées, au niveau des investissements publics, à la dotation en biens publics et à la situation relative aux ressources naturelles.

Trois types de régions ont été définis :

- des régions "gagnantes », où les dynamiques d'intégration aux marchés (qu'elles aient trait à des filières spécifiques, à la proximité de centres urbains ou à de bonnes infrastructures) offrent des opportunités et sont d'importants vecteurs de changement ;

- des régions «perdantes ", caractérisées par des tendances à la marginalisation dues à des contraintes locales (faible dotation en facteurs et en biens 
publics), à des connexions médiocres aux marchés, ou à des taux de pauvreté élevés, et où les conditions d'existence des ménages apparaissent de plus en plus difficiles ;

- des régions intermédiaires, où les tendances sont imprécises et dépendront de l'évolution du contexte économique et du cadre institutionnel (apparition de nouvelles opportunités et réduction des contraintes existantes).

À partir de cette typologie générale, au moins trois régions pour chacun des sept pays (une par type) mais parfois plus ont été sélectionnées pour le recueil des données (voir tableau 1.3). Les équipes nationales ont ensuite choisi les localités étudiées dans chaque région sur la base de leurs connaissances locales en vue d'illustrer les dynamiques existantes. Enfin, dans chaque localité, la sélection des ménages enquêtés s'est faite par tirage au sort.

Tableau 1.3 Régions d'enquêtes sélectionnées dans les pays RuralStruc

\begin{tabular}{lccc} 
& & \multicolumn{3}{c}{ Classification ex ante } & \\
\cline { 2 - 4 } Mali & Régions gagnantes & Régions intermédiaires & Régions perdantes \\
\hline Sénégal & Delta du Sénégal & Diéma & Tominian \\
& & Bassin arachidier & Casamance \\
Madagascar & Antsirabe & Nord (Mekhé) & \\
\hline Kenya & Sud (Nioro) & Morondava \\
\hline Maroc & Nakuru North & Alaotra & Nyando \\
\hline Nicaragua & Souss & Itasy & Changouia \\
\hline Mexique & El Viejo & Saïss & Terrabona \\
\hline
\end{tabular}

Source : Rapports RuralStruc phase 2.

Pour certaines régions, l'analyse détaillée des premiers résultats des enquêtes a permis de repérer des différences marquées au niveau infrarégional. Ces constats ont conduit à scinder certaines régions pour la suite de l'analyse afin de mieux rendre compte des dynamiques existantes, en évitant notamment les effets réducteurs de moyennes calculées à partir de situations très contrastées. Ce choix s'est généralement fondé sur des différences statistiquement significatives observées quant au niveau de revenu des ménages des différentes localités étudiées dans la même région et, parfois, sur des conditions locales particulières, comme l'éloignement des centres urbains ou des conditions naturelles particulières. Plusieurs régions du Sénégal, de Madagascar et du Mexique ont été ainsi divisées en $\operatorname{deux}^{32}$, ce qui a permis de tenir compte des différences dans le niveau de patrimoine des ménages et les structures agraires qui s'expriment dans les structures de revenus ${ }^{33}$. 
Ainsi, du fait des conditions d'échantillonnage, les enquêtes RuralStruc ne sont statistiquement représentatives qu'à l'échelle locale (village ou communauté). Cependant, comme les équipes nationales ont sélectionné les régions en fonction de leur dotation en facteurs et de leur intégration aux marchés, les résultats donnent une estimation fiable des tendances régionales à l'œuvre au niveau national en termes de développement agricole, de revenus ruraux et de diversification rurale. Ils illustrent la diversité des situations rurales au sein de chaque pays, qui est confirmée par les rapports nationaux de la phase 2 (voir chapitre 3 ).

Près de 8000 enquêtes auprès de ménages ruraux couvrant 57000 personnes ont été réalisées dans 26 régions $^{34}$ des sept pays sélectionnés entre novembre 2007 et mai 2008 - avant que la crise des prix alimentaires ne prenne toute son ampleur. Les données recueillies reflètent essentiellement la campagne agricole 2007. 40 \% des ménages interrogés se trouvent dans les trois pays situés hors d'ASS.

La démarche et la méthodologie des enquêtes ainsi que les questionnaires ont été identiques dans toutes les régions. In fine, et en dépit des limitations mentionnées, ce dispositif a produit un ensemble d'observations comparables, portant sur la même période (une des principales différences avec RIGA), qui documente à la fois les tendances d'évolution générales et la grande diversité des situations existantes au sein des sociétés rurales ${ }^{35}$.

\section{Le partenariat en action}

Une caractéristique originale du programme RuralStruc, qui fait aussi sa force, est le choix d'un appui sur des partenariats locaux et d'une mise en œuvre fondée sur des équipes nationales. Ce choix a facilité le déroulement du programme, notamment la collecte des données, mais il a aussi fortement amélioré la qualité des observations et de l'analyse en apportant une garantie additionnelle de précision et de cohérence aux informations recueillies et à la compréhension générale des processus à l'œuvre. Au final, il a facilité aussi bien l'appropriation locale que le débat sur les politiques publiques.

Entre l'atelier de lancement d'avril 2006 et la publication de la première version de cette synthèse en juin 2009, les équipes nationales et de coordination ont été en contact continu ; leurs échanges se sont intensifiés lors du démarrage et de la conclusion de chaque phase du programme, mais aussi lors des missions conjointes sur le terrain et à l'occasion de plusieurs rencontres de l'ensemble des équipes.

Des termes de référence identiques, ajustés en collaboration avec les équipes, ont été utilisés pour chaque phase et un processus consultatif a été adopté pour la conception du questionnaire d'enquête. Le même cadre méthodologique, comprenant des définitions communes et les variables transversales clés à mobiliser pour l'analyse, a été appliqué dans les sept pays avec les adaptations locales nécessaires. Les principales difficultés concernant la définition des variables et l'analyse des données ont été discutées lors d'ateliers, tout comme la structure du rapport de phase 2. Par ailleurs, un effort commun a été entrepris pour constituer une base de données agrégée reprenant un cœur de variables, qui ont été extraites ou 
calculées par chaque équipe nationale à partir des données d'enquête. Enfin, les résultats ont fait l'objet d'une analyse approfondie croisant des données internationales, les données d'enquête et les rapports nationaux afin de consolider les conclusions du programme.

Toutes les équipes ont présenté les résultats obtenus dans chaque pays après la phase 1 et les résultats de la phase 2 ont déjà été diffusés dans une partie des pays participants. Le format et le rythme de ces restitutions (qui devraient se poursuivre après la clôture officielle du programme) dépendent des conditions du débat public dans chaque pays et de la disposition des partenaires locaux et des donateurs à s'y engager.

Jusqu'ici, des manifestations officielles de restitution de la phase 2 ont été organisées au Mali (en avril 2010) et au Sénégal (en juin 2010) en suivant la même démarche. L'équipe nationale a établi un ensemble de policy briefs sur les résultats et les recommandations du programme qui ont été discutés lors d'un atelier avec des représentants d'organisations professionnelles, de la société civile, de l'État, des collectivités locales, du secteur privé et des donateurs.

La diffusion internationale va se poursuivre en partenariat avec les équipes nationales. Outre les présentations du programme et ses premiers résultats réalisés depuis 2006, un atelier s'est tenu en amont de la $27^{\circledR}$ Conférence internationale des économistes agricoles à Pékin (août 2009) et les travaux ont été présentés au NEPAD (Addis-Abeba, mai 2011) ${ }^{36}$.

\section{Synopsis de l'ouvrage}

Cet ouvrage, qui présente les résultats définitifs du programme RuralStruc, propose une analyse comparative de la transformation rurale en lien avec le débat sur l'agriculture, le développement rural et le changement structurel. Il s'appuie sur un important travail de terrain et d'analyse réalisé par des équipes nationales à partir d'enquêtes auprès de ménages ruraux et d'études documentaires dont les résultats sont présentés dans 14 rapports nationaux ${ }^{37}$. Ces contributions nationales sont complétées par une revue de la littérature qui présente le contexte nécessaire pour interpréter les résultats du programme et souligner sa contribution aux recherches thématiques et au débat politique.

Le livre est organisé en six chapitres, dont les articulations sont présentées à la figure 1.1. Le lecteur peut lire les chapitres successivement ou consulter directement ceux qui l'intéressent. Les contraintes éditoriales n'ont pas permis d'incorporer les annexes techniques. Elles sont accessibles dans l'appendice publié sur le site de la Banque mondiale à la page Internet dédiée au programme RuralStruc et apportent des informations complémentaires sur la méthodologie, les partenariats locaux, la description des régions étudiées et des données chiffrées qui ne pouvaient être reproduites dans le document principal.

Ce premier chapitre a exposé les motivations à l'origine du programme en le resituant dans les débats internationaux passés et présents, ainsi que la méthodologie retenue. Il a décrit son organisation en deux phases impliquant des 
Figure 1.1 Comment naviguer dans le livre

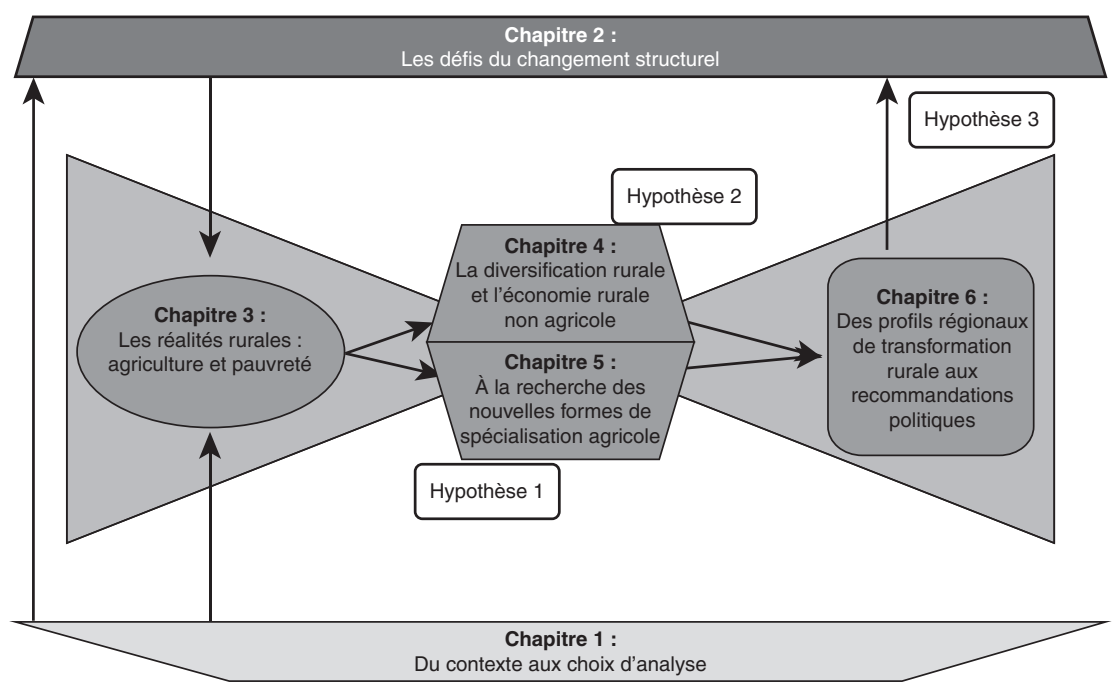

recherches qualitatives et quantitatives et a présenté les trois hypothèses du programme relatives : (1) à l'ampleur des processus d'intégration dans l'agriculture et à leurs conséquences pour les ménages et le secteur agricole ; (2) au développement de l'économie rurale non agricole et à son influence sur les réalités rurales et (3) aux risques d'impasses de transition, qui excluent certains ménages et régions du processus de transformation structurelle.

Le chapitre 2 sur les défis de la transformation structurelle résume les processus de transformation, en particulier les caractéristiques des transitions économiques et démographiques. Il apporte un cadre de référence à l'analyse développée dans l'ouvrage et pose la question de la viabilité des étapes historiques de la transformation structurelle dans le contexte de la mondialisation. Il évoque la situation des pays les moins avancés - principalement en Afrique subsaharienne - qui sont encore aux premiers stades de leurs transitions. Il présente également les caractéristiques de la structure démographique et économique des pays du programme RuralStruc et souligne leurs principaux défis et les diverses fonctions de l'agriculture.

Le chapitre 3 sur les réalités rurales axe l'analyse des régions étudiées sur le rôle de l'agriculture dans les activités et les revenus des ménages et sur l'ampleur de la pauvreté. Après avoir souligné le rôle pivot de l'agriculture dans des contextes régionaux très différenciés, ce chapitre s'appuie sur les données d'enquêtes microéconomiques pour présenter les caractéristiques des revenus des ménages. Deux thèmes dominants ressortent de cette analyse : l'ampleur et la persistance de la pauvreté absolue et l'importance renouvelée des activités agricoles des ménages, surtout en Afrique subsaharienne. Ce chapitre montre que cette pauvreté reste avérée quels que soient les modes de calcul : par habitant, par équivalent adulte et même en kilocalories. La dernière partie examine les stratégies d'activités observées 
lors de l'enquête en faisant référence à la typologie du RDM08, qui évoque trois voies de sortie de la pauvreté rurale : la spécialisation dans l'agriculture, le travail salarié en zone rurale et la migration. Deux grands groupes de ménages sont définis : ceux qui sont fortement spécialisés dans les activités de l'exploitation agricole et ceux qui sont plus diversifiés. Les ménages très spécialisés dans des activités hors exploitation sont peu nombreux.

Le chapitre 4 porte sur la diversification et l'économie rurale non agricole. Il examine l'ampleur de la diversification dans les régions étudiées, les différents types d'activités et de revenus hors de l'exploitation (travail salarié agricole, travail salarié non agricole, activité indépendante, transferts publics et privés et rentes) et dégage les caractéristiques des ménages qui exercent ces activités. Ce chapitre fait le point sur le débat actuel et clarifie les définitions de l'économie rurale non agricole et des activités « hors de l'exploitation» (ou « externes »), qui comprennent le travail salarié agricole, qui sont retenues par le programme. Il étudie la deuxième hypothèse et examine la contribution des types de diversification au changement et à la restructuration des économies rurales. Il différencie les stratégies d'adaptation déployées au premier stade de la diversification, des stratégies de diversification plus structurelles et effectives rencontrées aux stades ultérieurs.

Le chapitre 5, qui s'intéresse aux nouveaux profils de spécialisation agricole, est consacré à l'agriculture des régions étudiées. Après avoir examiné le mouvement de restructuration lié à la libéralisation et aux nouveaux marchés agroalimentaires, ce chapitre aborde les caractéristiques des activités des exploitations agricoles. Il explore la première hypothèse relative aux conséquences des transformations à l'œuvre dans le secteur. Plus que la « nouvelle agriculture » suggérée dans la littérature, les résultats mettent en lumière la persistance de pratiques agricoles anciennes, dont l'importance de l'autoconsommation et de la production de denrées alimentaires de base (liée aux stratégies de gestion des risques et à un environnement de marché peu porteur) qui se traduit par la faiblesse de la contractualisation. Ils rappellent cependant l'importance généralisée de la connexion des ménages agricoles aux marchés.

Le chapitre 6 enfin s'attache aux profils régionaux de transformation rurale aux recommandations en matière de politiques publiques. Il analyse les déterminants des niveaux de revenus dans chaque région étudiée et précise les caractéristiques de la relation diversification-spécialisation qui joue un rôle central dans le processus de transformation structurelle. À partir d'analyses statistiques, il identifie un profil en $U$ inversé qui explique le processus de transformation rurale. Il souligne la situation particulière de l'Afrique subsaharienne, où les obstacles sur la voie de la transformation semblent piéger la plupart des régions étudiées dans la pauvreté, une situation qui révèle le risque d'impasse de transition posé par la troisième hypothèse du programme. La seconde partie du chapitre s'appuie sur ces constats pour proposer des recommandations pour les politiques publiques. Elle suggère des orientations générales à caractère méthodologique et propose trois axes d'intervention ciblés spécifiquement sur les régions d'ASS susceptibles de les aider à surmonter les risques de leur transition. 


\section{Notes}

1. Des rapports nationaux ont été établis à chaque phase du programme. Les rapports de la phase 1 et ceux de la phase 2 sont respectivement appelés RS 1 [pays] et RS 2 [pays]. La liste des rapports est présentée au début de la bibliographie.

2. Parmi les travaux de recherche récents et souvent contradictoires, voir par exemple, Bouët et al. (2005), Boussard, Gérard et Piketty $(2005,2006)$, Polaski (2006) ou, plus récemment, Peréz, Schlesinger et Wise (2008) sur l'Amérique latine, Zepeda et al. (2009) sur le Kenya, et les travaux coordonnés par Anderson sur « Krueger/Schiff/ Valdés Revisited » (Anderson 2010).

3. Plus précisément, le rapport sous-estime les nouvelles conditions des rapports intersectoriels dans le contexte de la mondialisation. Ce point est développé au chapitre 2. Pour une critique du cadre d'analyse retenu par le RDM08, voir entre autres Akram-Lodhi (2008) et Oya (2009).

4. Le " milliard inférieur » fait référence au livre de Paul Collier, publié en 2007, qui examine les 50 pays dits « en faillite " (dont $70 \%$ en ASS), piégés durablement dans la pauvreté. Collier, qui est un des deux auteurs du rapport de l'ONUDI, a alimenté la controverse sur le rôle de l'agriculture, notant qu'elle ne sera pas capable de faire reculer la pauvreté et que la seule option possible est un vaste mouvement d'exode rural (Collier 2008, 2009). Pour plus de précisions sur ce débat, voir le chapitre 6.

5. Afin de conjuguer les perspectives des deux RDM, deux départements de la Banque mondiale - le Groupe Réduction de la pauvreté et équité (PREM) et le département Finances, économie et développement urbain (FEU) - ont engagé un programme de travail commun sur les conditions de réduction de la pauvreté au cours du processus d'urbanisation dans les pays en développement. Voir Simler et Dudwick (2010). Ce travail s'est achevé en 2011 (Dudwick et al. 2011).

6. La reconnaissance de la multifonctionnalité comme caractéristique particulière de l'agriculture fait l'objet d'un débat ancien et tendu, qui reste étroitement mêlé aux discussions en cours sur les politiques agricoles. De nombreux États européens ont adopté des politiques encourageant la multifonctionnalité dans les années 1990, malgré une forte opposition au sein de l'OMC de pays (principalement ceux du groupe Cairns et les États-Unis) qui voyaient dans ces mesures des distorsions de marché. Sur ces débats et sur la multifonctionnalité de l'agriculture en général, voir Barthélémy et al. (2003), Losch (2004), Caron et Le Cotty (2006) et Groupe Polanyi (2008). En outre, entre 2000 et 2006, la FAO a mis en œuvre un projet intitulé Rôles de l'agriculture, dans le cadre duquel a été développé un vaste ensemble d'études de cas et d'analyses sur les rôles de l'agriculture aux différentes phases du développement (FAO 2007).

7. Une longue tradition de recherches stimulantes dans le domaine des études agraires s'interroge sur les conséquences des processus de changement à l'œuvre et sur les risques d'impasses économiques et sociales qu'ils engendrent. Voir les analyses publiées par le Journal of Agrarian Change et Akram-Lodhi et Kay (2009a et b) pour un récent panorama des débats et de leur évolution.

8. Voir par exemple Johnston et Kilby (1975), Chenery et Syrquin (1975) et Timmer $(1988,2009)$.

9. L'économie du développement, apparue à la fin de la Seconde Guerre mondiale, est fondée sur l'idée de rattraper le processus de croissance des pays industrialisés avec pour référence le décollage économique des pays d'Europe occidentale au XIX ${ }^{\mathrm{e}}$ siècle. Elle est à l'origine d'une vision évolutionniste contestée selon laquelle les pays doivent 
passer par des "étapes nécessaires ». La question des transitions économiques et démographiques est étudiée plus amplement au chapitre 2.

10. La recherche économique se penche rarement sur les autres conséquences de la libéralisation. Pour ses effets potentiels sur l'emploi, voir par exemple Hoekman et Winters (2005). Pour ses dimensions environnementales, voir Cook et al. (2010).

11. La littérature économique considère généralement que les difficultés liées à la libéralisation sont temporaires. C'est le cas du chômage, qui est censé refléter le délai nécessaire à l'adaptation au nouveau contexte et à la réalisation d'un nouvel équilibre (Winters, McCulloch et McKay 2004).

12. Du fait du processus de sélection et de l'autosélection des études de cas nationales, toute conclusion tirée d'une comparaison directe pour expliquer les variables serait entachée d'une erreur systématique (Collier et Mahoney 1996).

13. Le concept de moment critique (critical juncture) est issu des travaux sur la dépendance de sentier et correspond aux points cruciaux auxquels une option précise est sélectionnée par des gouvernements, des coalitions ou des forces sociales et conduit à la formation de modèles institutionnels récurrents. Voir Mahoney (2001) et Pierson (2000).

14. Le PDDAA est un des programmes phares du Nouveau Partenariat pour le développement de l'Afrique (NEPAD). Lancé en 2003, il vise à développer l'investissement agricole pour le porter à $10 \%$ des budgets nationaux (Déclaration de Maputo) et à faciliter la préparation de programmes d'investissement. Il privilégie quatre piliers pour améliorer la productivité et la croissance : gestion des terres et des eaux, accès aux marchés, approvisionnement alimentaire et réduction de la faim, recherche agricole. Voir http://www.nepad-caadp.net.

15. L'Union européenne et les États-Unis dans le cas du Maroc, et les États-Unis dans le cas du Nicaragua (avec l'Accord de libre-échange Amérique centrale - République dominicaine [CAFTA-DR]).

16. Dans le tableau 1.1 et dans l'ensemble du document, les données nationales ont été classées de gauche à droite, d'abord par région (ASS, Afrique du Nord et Amérique latine), puis par niveau de revenus sur la base des résultats des enquêtes (voir chapitre 3).

17. Le fait que le Sénégal, un pays où $72 \%$ de la population active est agricole, soit classé parmi les pays « en mutation » illustre l'ambiguïté inhérente à l'utilisation de critères tels que « rural » ou «pauvreté rurale» au lieu de la population active agricole. La définition du terme " rural » diffère d'un pays à l'autre (voir encadré 1.5) et elle est restrictive au Sénégal. Le Nicaragua n'est pas mentionné dans l'analyse des trois mondes du RDM, qui exclut les pays de moins de 5 millions d'habitants (bien que le Nicaragua ait franchi ce seuil en 2000). Cependant, en utilisant le même critère, le Nicaragua ferait partie des pays en mutation.

18. La proportion de la population active agricole a été calculée à partir des données de la FAO fondées sur celles de l'Organisation internationale du travail (OIT). Dans le cas du Nicaragua, la part de la population active dans l'agriculture semble fortement sous-estimée. En effet, selon la Banque centrale du Nicaragua, ce secteur employait $29 \%$ de la population active (RS 1 Nicaragua, 28).

19. Les groupements de pays sont toujours contestables. Néanmoins, on peut s'étonner de voir l'urbanisation au Kenya qualifiée de «naissante » étant donné la formidable croissance urbaine observée dans le pays. Voir encadré 3.2 et Harre, Moriconi-Ebrard et Gazel (2010). 
20. «Petit» et « grand» sont des valeurs relatives. Seulement 11 des 192 pays membres des Nations unies comptent plus de 100 millions d'habitants ; 25 pays comptent plus de 50 millions d'habitants et 50 pays plus de 20 millions d'habitants - mais 80 pays ont moins de 5 millions d'habitants. La population nationale médiane est de 7 millions.

21. Si l'argument ayant conduit à exclure les pays les plus peuplés de la sélection se comprend aisément, l'argument contre les plus petits est plus délicat. Ces pays peuvent eux aussi rencontrer des difficultés de transition économique dans le monde d'aujourd'hui, mais en revanche les populations en jeu sont moins représentatives des difficultés de la transition et des risques d'impasse.

22. La Chine et l'Inde sont des exceptions mondiales, mais la plupart des pays d'Asie (par exemple la Thaïlande, le Viêt Nam, l'Indonésie et les Philippines) ont aussi des populations importantes. Lorsqu'on exclut les républiques de l'ex-Union soviétique et les pays en situation de conflit et de postconflit (Cambodge et Sri Lanka), les choix sont limités. Dans la fourchette démographique, le Népal ou la Malaisie auraient pu être des cas intéressants, cette dernière étant déjà profondément engagée dans sa transformation structurelle. À ce titre, la sélection du Nicaragua est contestable - c'est un pays en situation de postconflit, frappé par une guerre civile entre 1978 et 1989, et sa population est peu nombreuse. Le Guatemala et le Honduras avaient été envisagés pour illustrer les pays membres du CAFTA-DR, mais le Nicaragua a été sélectionné pour des raisons opérationnelles.

23. Du fait de son insularité, Madagascar est un cas spécifique en Afrique australe. Le Mozambique et la Zambie constituaient d'autres options mais, là encore, l'existence de partenaires locaux l'a emporté.

24. Le Mexique a lui aussi un statut particulier au sein des pays en développement en raison de sa politique agricole ancienne, fondée pendant la période révolutionnaire avec une réforme agraire en vigueur des années 1920 aux années 1970. Malgré la libéralisation du marché foncier en 1992, cette trajectoire a été déterminante pour la structure de l'agriculture mexicaine (RS 1 Mexique).

25. La sélection comprend des régions pratiquant des cultures tropicales pérennes. Les études de cas nationales en offrent des exemples au Kenya et au Nicaragua (café). Les cultures pérennes sont également très développées au Maroc (agrumes et olives principalement).

26. Décision de la première réunion du comité scientifique du programme en mars 2007. Le programme a bénéficié d'une structure de gouvernance comprenant un comité scientifique d'orientation de 10 membres et un comité de pilotage des donateurs.

27. La phase 1 s'est déroulée d'avril 2006 à mars 2007. La phase 2 devait être mise en œuvre entre juin 2007 et juin 2008 mais les nouveaux choix concernant la réalisation d'enquêtes de ménages ont imposé un nouveau calendrier. La phase 2 a été lancée en septembre 2007 et a duré jusqu'en juin 2009, avec des prolongations au Kenya, au Maroc et au Mexique jusqu'à janvier-mars 2010.

28. Voir l'annexe 1 de l'appendice en ligne sur le site http://www.worldbank.org/afr/ ruralstruc, qui présente la méthodologie détaillée des travaux de terrain (unités d'analyse, procédures d'échantillonnage, dates de collecte des données), les difficultés rencontrées et les solutions techniques adoptées.

29. C’est le cas en général des études de mesure des niveaux de vie (LSMS) réalisées par la Banque mondiale depuis les années 1980, qui couvrent 32 pays. En Afrique, la plupart des enquêtes sur la pauvreté réalisées auprès des ménages ont été conduites à l'échelle nationale, avec une approche très similaire. 
30. Dans les prochaines années, le nouveau programme d'enquêtes LSMS portant sur l'agriculture (LSMS-ISA), lancé en 2009 par la Banque mondiale et la Fondation Bill et Melinda Gates dans sept pays d'ASS, fournira des données de panel ciblées sur l'agriculture et les relations entre les activités agricoles et non agricoles.

31. Les résultats du projet RIGA sont aussi une référence majeure du Rapport sur la pauvreté rurale 2010 du Fonds international pour le développement agricole (FIDA), qui exploite également les données d'enquêtes du programme RuralStruc (FIDA 2010).

32. Au Sénégal, le delta du Sénégal et le nord du bassin arachidier ont été divisés en deux: Bas Delta et Haut Delta pour la première région et Mekhé 1 et 2 pour la seconde. De même à Madagascar, les régions d'Antsirabe et d'Alaotra ont elles aussi été divisées en deux : Antsirabe 1 et 2 et Alaotra 1 et 2 . Au Mexique, il a été décidé d'abandonner les résultats d'Ixmiquilpan, dans la région Otomi de l'État d'Hidalgo, qui figurait initialement parmi les régions dites perdantes car les incohérences présentes dans les résultats d'enquêtes étaient insurmontables. Le Sotavento (État de Veracruz) a été divisé en deux zones : plaines (Tierras Bajas) et montagnes (Sierra de Santa Marta). Cette dernière zone, caractérisée par l'éloignement, une faible dotation en biens publics et une population principalement indigène, offre les caractéristiques d'une région perdante et compense pour partie l'abandon d'Ixmiquilpan.

33. Des cartes des régions étudiées sont présentées à la fin de l'ouvrage. Leurs principales caractéristiques sont indiquées à l'annexe 3 de l'appendice en ligne : http://www.worldbank.org/afr/ruralstruc.

34. En raison des ajustements régionaux, les résultats sont présentés pour 30 régions et sous-régions ( 5 des 26 régions étudiées sont divisées en deux, et la région d'Ixmiquilpan au Mexique a été abandonnée). Voir le tableau à l'annexe 1 de l'appendice en ligne : http://www.worldbank.org/afr/ruralstruc.

35. Les bailleurs de fonds du programme ont accepté d'autoriser l'accès public aux bases de données de RuralStruc (bases de données nationales et base de données fusionnée centrale). Cette mise en ligne, comprenant une documentation adaptée respectant les normes internationales (métadonnées et description des variables), sera progressivement réalisée avec l'appui du Programme statistique accéléré (PSA-ADP) coordonné par la Banque mondiale et le Secrétariat de PARIS21. Les données de la base centrale sont déjà accessibles dans la bibliothèque Micro Data Library de la Banque mondiale à l'adresse : http://microdata.worldbank.org/index.php/catalog/670.

36. Les manifestations et événements lors desquels une diffusion des résultats a eu lieu sont indiqués à l'annexe 2 de l'appendice en ligne : http://www.worldbank.org/afr/ ruralstruc.

37. Les 14 rapports nationaux sont publiés sur la page du programme RuralStruc sur le site de la Banque mondiale à l'adresse : http://www.worldbank.org/afr/ruralstruc. 



\section{Chapitre 2}

\section{Les défis du changement structurel}

Le premier chapitre a mis en évidence plusieurs sujets de débat récents qui tiennent une place croissante dans l'agenda international et ont transformé le paysage politique. Parmi ceux-ci, la crise financière a contribué de manière inattendue à la résurgence d'un thème passé depuis longtemps à la trappe des débats sur les politiques économiques : celui du changement structurel. Bien que ce sujet n'ait rien perdu de sa pertinence dans les milieux académiques, il était devenu le grand absent des préoccupations des décideurs. Reconnecter ces questions structurelles au débat sur les politiques était une motivation centrale du programme RuralStruc.

Le retour politique des questions liées au changement structurel est encore très timide. Les problèmes liés à la crise économique et les plans de sauvetage à court terme mobilisent l'attention des gouvernements et des donateurs et limitent donc leur capacité à réinvestir les politiques structurelles à long terme. Néanmoins des indices de changement sont perceptibles. Deux contributions récentes méritent d'être mentionnées : le rapport de l'Institut de recherche des Nations unies pour le développement social (UNRISD) Combattre la pauvreté et l'inégalité : changement structurel, politique sociale et conditions politiques (UNRISD 2010) et le rapport économique sur l'Afrique 2011 de l'Union africaine et de la Commission économique pour l'Afrique intitulé Governing Development in Africa: The Role of the State in Economic Transformation (UNECA 2011). Des marques d'intérêt se manifestent également du côté des donateurs. Ainsi Justin Lin, l'économiste en chef de la Banque mondiale, a récemment appelé à plusieurs reprises à repenser le développement dans le cadre de ce qu'il nomme la «nouvelle économie structurelle» (Lin 2010).

Deux explications peuvent être avancées à ce regain d'intérêt pour les questions structurelles. Tout d'abord, la crise financière a soulevé la question de la soutenabilité du modèle de croissance et de ses déséquilibres au niveau mondial. De nouvelles solutions structurelles sont ainsi recherchées pour permettre un régime de développement plus soutenable et plus inclusif, un processus qu'illustrent les nombreuses tentatives d'amélioration de la gouvernance mondiale par des régulations internationales et le rôle croissant du G20. Ensuite, les mesures de sauvetage radicales qu'ont dû prendre les gouvernements des pays riches face aux conséquences de la crise financière rappellent clairement les limites du tout marché et indiquent que les États ont encore un rôle à jouer, en particulier lorsqu'il s'agit de questions réglementaires et structurelles. 
Dans ce contexte très évolutif, ce chapitre se propose de présenter les processus de transformation structurelle à l'œuvre et d'examiner les défis propres aux pays et régions du programme RuralStruc en matière de transition économique et démographique. Il s'attache en particulier à la situation de l'Afrique subsaharienne qui est la dernière région du monde à engager sa transformation structurelle.

\section{Différences régionales et positionnement des pays du programme}

Les pays du programme RuralStruc se différencient très nettement par leurs structures économiques et démographiques. Ces structures reflètent le stade auquel ils se trouvent dans le processus de transformation structurelle mais elles illustrent aussi, plus généralement, les grandes dynamiques régionales mondiales.

\section{Des transitions économiques inégales}

Les sept pays du programme RuralStruc ont été sélectionnés parce qu’ils représentent différents profils structurels. Une analyse approfondie des données macroéconomiques de chaque pays aurait pu être présentée dans cet ouvrage, mais il a été retenu de ne s'attacher qu'à quelques données synthétiques significatives et aux faits stylisés les plus importants afin de situer clairement les sept pays dans le processus de transformation ${ }^{1}$.

La figure 2.1 rappelle les fortes disparités de revenu entre les régions du monde et surtout l'ampleur des écarts de croissance. Elle compare l'ASS dans son ensemble aux trois pays du programme RuralStruc situés en dehors de l'Afrique subsaharienne et à d'autres pays de référence et souligne la longue stagnation du sous-continent par rapport aux autres régions (le Nicaragua, dont la trajectoire de croissance reflète les conséquences de sa guerre civile, fait exception).

Les figures 2.2 et 2.3 représentent la transition économique des pays du programme RuralStruc et celle d'autres pays de 1965 à 2005 sur la base de trois indicateurs - le PIB par habitant, la part de l'agriculture dans le PIB et la part de l'agriculture dans l'emploi - qui offrent un bon moyen de mesurer l'évolution structurelle d'une économie initialement centrée sur l'agriculture. Ces figures révèlent de nettes différences dans l'ampleur et le rythme de la transformation structurelle.

La figure 2.2 présente l'évolution de la part de l'agriculture dans l'emploi et le PIB. Les courbes représentent en quelque sorte les "signatures » des pays, d'une part parce qu'elles peuvent faire penser à une signature, mais aussi parce qu'elles résument la trajectoire de croissance propre à chacun. La figure illustre l'évolution du rôle de l'agriculture dans les différentes régions du monde au travers des pays choisis et montre sa part décroissante dans le PIB de l'ASS à l'Asie puis à l'Amérique latine. Elle montre aussi la diminution de l'emploi agricole dans la population active, un changement initialement lent qu'illustre 
Figure 2.1 Comparaison du PIB par habitant et de son évolution entre pays et régions, 1960-2009

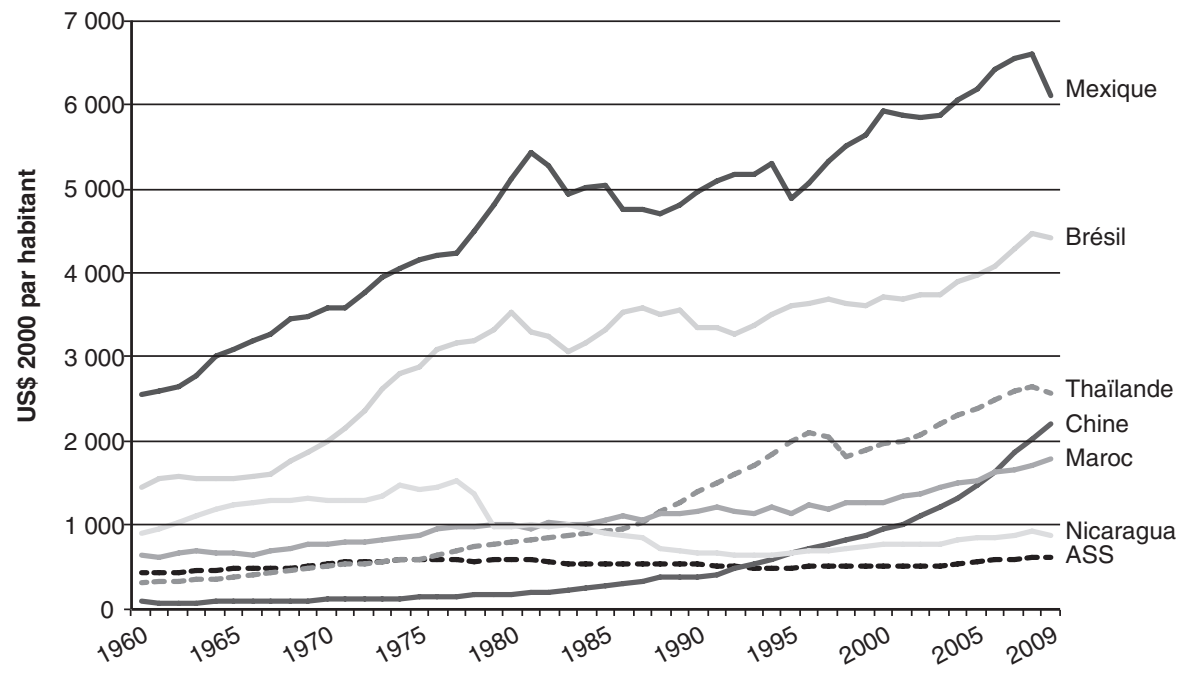

Source: Indicateurs du développement dans le monde (WDI).

Note: Pour alléger le graphique, l'Afrique subsaharienne est représentée au lieu des quatre pays du programme. À cette échelle, les écarts entre les pays d'ASS sont réduits.

la structure stagnante de l'emploi observée en Afrique subsaharienne mais aussi en Chine.

La différence entre la part de l'agriculture dans le PIB (PIB agricole) et sa part dans l'emploi (population active agricole) illustre une caractéristique bien connue de la transformation structurelle : une inégalité de revenus entre l'agriculture et les autres secteurs de l'économie qui reflète les écarts de productivité des facteurs (Timmer et Akkus 2008). La figure 2.3 présente cet écart structurel, qui est aussi un indicateur des disparités de revenus entre ruraux et urbains. L'écart structurel se creuse aux premières étapes du développement économique sous l'effet de l'expansion rapide de l'activité économique dans les villes et de l'accumulation de richesse qui en résulte. Il se réduit ensuite avec la diversification de l'économie et la poursuite de l'urbanisation, ce qui conduit à la convergence progressive des secteurs rural et urbain au sein d'une économie parfaitement intégrée, avec un écart proche ou égal à zéro.

S'agissant des pays du programme RuralStruc, la comparaison des valeurs de ces écarts confirme que le Mexique est pleinement engagé dans sa transformation structurelle; c'est une économie diversifiée dans laquelle l'agriculture ne joue plus un rôle prépondérant ( $5 \%$ du PIB). Cependant, malgré cette transition, des risques de marginalisation croissante de l'économie rurale mexicaine persistent : 25 millions de personnes vivent encore en zone rurale et l'agriculture concentre entre 15 et $20 \%$ de la population active. Les difficultés du Mexique dans ce processus de convergence sont révélées par la comparaison avec le Brésil, où l'écart se resserre beaucoup plus rapidement ${ }^{2}$. 
Figure 2.2 Part de l'agriculture dans le PIB et la population active, 1965-2005

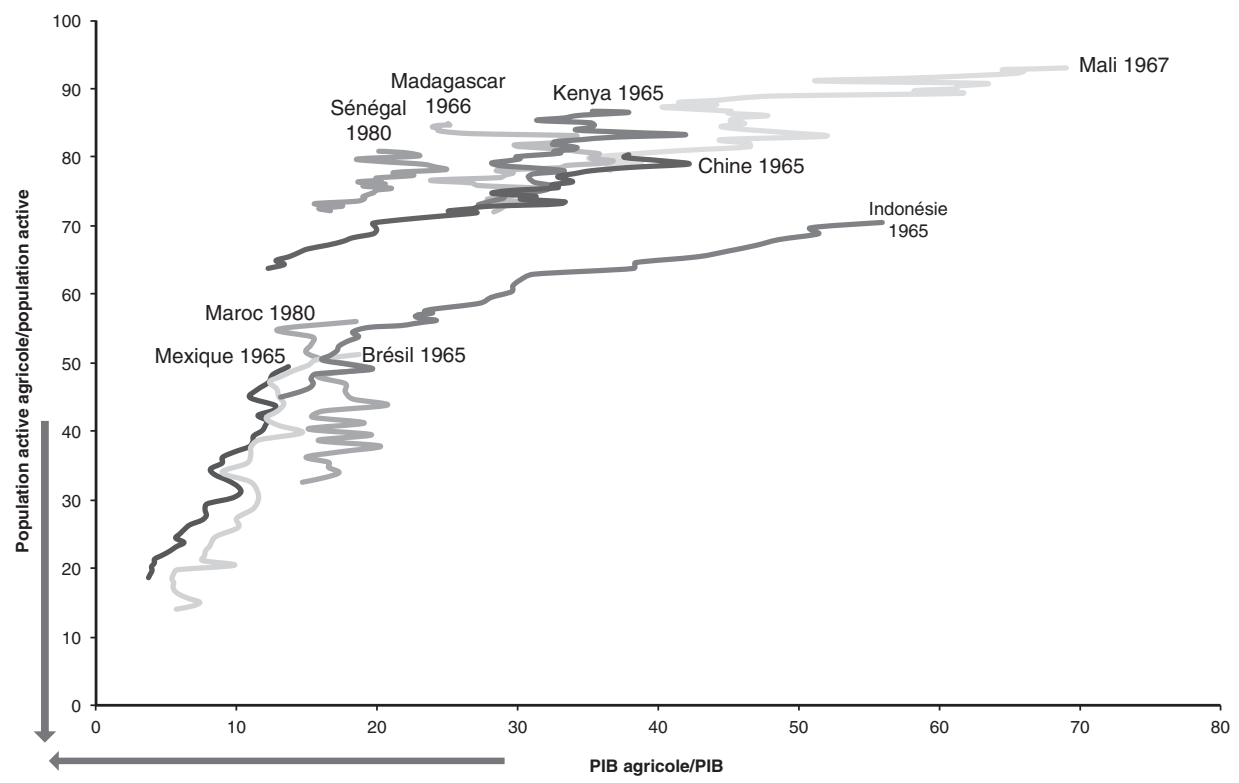

Sources : WDI, FAO.

Note : La dernière année est 2005 ; l'année de départ est indiquée à côté du nom de chaque pays.

Figure 2.3 Écart structurel et PIB par habitant, 1965-2005 (moyennes sur cinq ans)

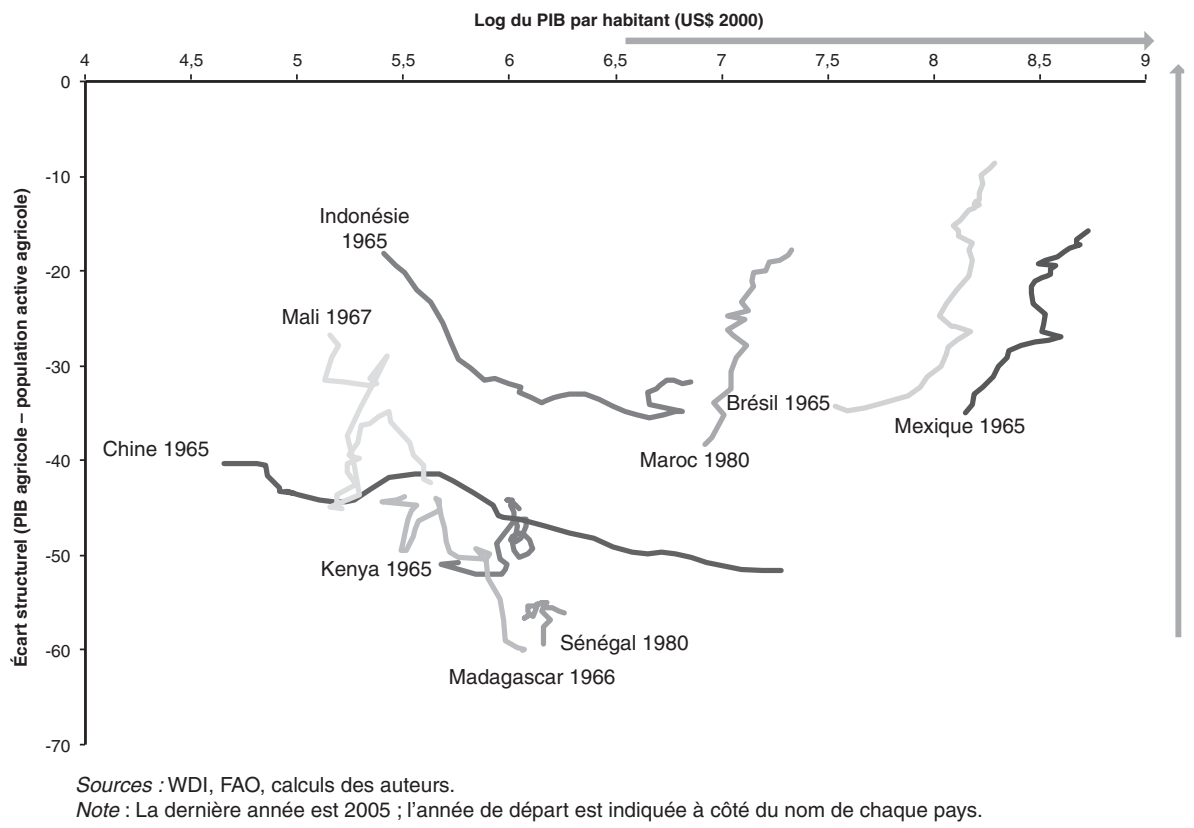


Les cas du Maroc et du Nicaragua ${ }^{3}$ sont plus incertains car l'agriculture y conserve une place importante dans la valeur ajoutée globale (15\% et $20 \%$ respectivement). La convergence entre le secteur rural et le secteur urbain est bien avancée et ce processus a été particulièrement rapide au Maroc. Cependant, la réussite de leur transition économique impliquera pour les deux pays de gérer au mieux l'intégration de leur économie intérieure sans marginaliser les régions reculées (régions montagneuses au Maroc et régions autonomes de la zone caraïbe au Nicaragua). Les politiques agricoles pourraient jouer un rôle important en limitant les processus d'exclusion. Le gouvernement marocain a pris des mesures en ce sens en 2008 avec le lancement du Plan Maroc vert. Cette nouvelle stratégie de développement rural repose sur deux piliers : le développement du secteur agro-industriel (avec un schéma associant des noyaux de petits exploitants approvisionnant des entreprises de transformation tournées vers l'exportation, appelées " agrégateurs ») et la promotion de l'agriculture familiale. L'attention qu'il convient d'accorder à ces deux piliers et les effets possibles de différentes approches sur la marginalisation des ménages ruraux suscitent cependant de vives controverses (RS 1 et RS 2 Maroc).

En Afrique subsaharienne en revanche, la transformation structurelle est à peine amorcée. Cinquante ans après leur indépendance, les pays d'ASS restent marqués par le poids du secteur agricole dans le PIB - il ressort en moyenne à $20 \%$, mais il dépasse $30 \%$ dans la plupart des pays, notamment au Kenya, au Mali et à Madagascar ${ }^{4}$. Les pays d'ASS se caractérisent également par le poids de l'agriculture dans la structure de l'emploi. Hormis en Afrique du Sud, l'agriculture concentre en moyenne $65 \%$ de la population active du sous-continent et ce chiffre se situe entre $70 \%$ et $85 \%$ dans de nombreux pays, au rang desquels figurent les quatre pays du programme RuralStruc. L'agriculture demeure ainsi la principale source d'activité économique et de revenu des ménages.

Le phénomène le plus frappant est la grande inertie des structures économiques de l'Afrique subsaharienne. Alors que les populations africaines révèlent une forte mobilité, dont témoigne l'urbanisation galopante, les trajectoires évoluent peu (voir figures 2.2 et 2.3). Avec un taux d'urbanisation (pourcentage de la population vivant dans les villes) frôlant $40 \%$, la population urbaine a été multipliée par 12 depuis 1960 (voir tableau 2.5). Pourtant, cette dynamique ne s'est accompagnée d'aucune industrialisation significative. Cette « urbanisation sans industrialisation » si souvent constatée en ASS se démarque des tendances observées dans les autres régions en développement, en particulier en Asie, où la transformation structurelle a été très rapide (voir figure 2.4). En conséquence, les services occupent une part écrasante dans l'économie des pays d'Afrique subsaharienne ; ils concernent principalement les activités urbaines informelles et souvent la commercialisation des produits agricoles ${ }^{5}$.

L'absence d'un secteur manufacturier dynamique en ASS explique que la population active ait surtout augmenté dans l'agriculture et le secteur urbain informel, qui ont joué un rôle d'amortisseur pour la population africaine. Le secteur urbain informel représente $30 \%$ à $45 \%$ du PIB non agricole et $70 \%$ à $90 \%$ de l'emploi non agricole total (Jütting et De Laiglesia 2009). 
Figure 2.4 Évolution de la structure du PIB : comparaison ASS - Asie, 1965-2005
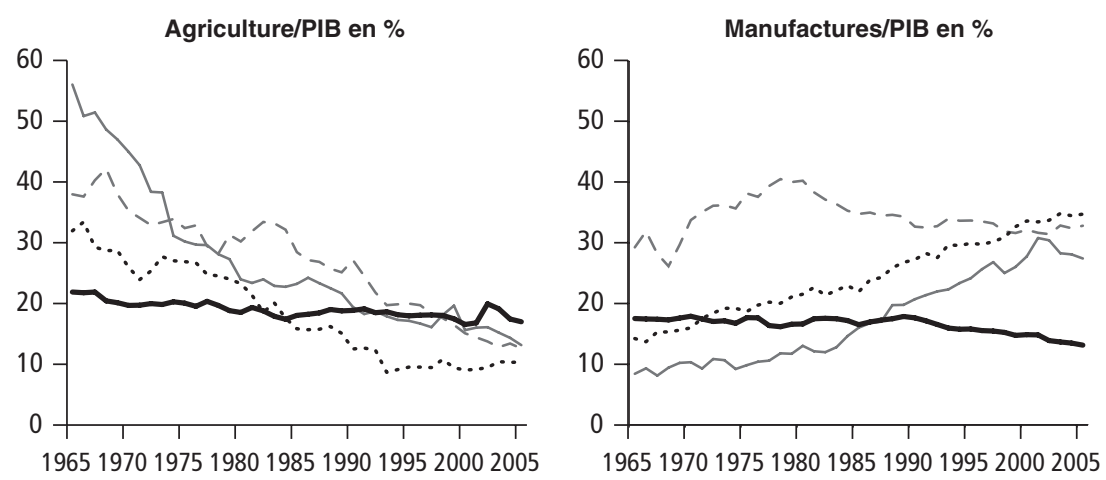

$$
\text { - - Chine } \quad \text { Indonésie } \cdots . . . \text { Thaïlande } \longrightarrow \text { ASS }
$$

Sources : WDI Banque mondiale 2009.

Note: Les pays d'Amérique latine ne sont pas représentés car leur diversification économique s'est essentiellement opérée avant 1965.

Avec un marché régional fragmenté formé de 42 États (ASS continentale uniquement), une intégration régionale en demi-teinte et des taux de pauvreté élevés qui limitent l'accumulation locale de capital, la croissance des économies d'ASS reste très tributaire de l'extérieur. Elle est en conséquence sujette aux chocs commerciaux liés à la volatilité des prix et dépendante d'investissements directs étrangers et d'une aide publique qui sont souvent procycliques. Ces caractéristiques, conjuguées à la faible productivité de la main-d'œuvre (due à la distribution sectorielle de la main-d'œuvre évoquée plus haut), à une fréquente instabilité politique et à la poussée démographique (voir section suivante), expliquent la très faible croissance et la forte volatilité de l'économie subsaharienne sur la longue durée par rapport au reste du monde (voir tableau 2.1). Cette trajectoire de croissance chaotique a fortement contribué à la vision à court terme de nombreux dirigeants politiques et investisseurs privés.

Depuis deux ans, la littérature consacrée au développement économique de l'Afrique multiplie les titres très optimistes avec: Les Lions en marche et Une Afrique émergente où (...) la pauvreté diminue... bien plus vite que ce que vous croyez $^{6}$ ! Ces déclarations de victoire doivent pourtant être mises en perspective à l'aune de la longue période.

En effet, si on peut se réjouir de la récente période de croissance et de l'apparente résilience de l'Afrique à la crise financière mondiale, il n'est pas certain qu'elles résultent de choix structurels qui soutiendront la croissance à long terme ${ }^{7}$. Sauf rares exceptions (situations de rattrapage ou d'amélioration de la gouvernance), la croissance a été initialement amorcée par un boom des matières premières ; elle a été plus lente en ASS que dans d'autres pays en développement (en particulier l'Asie de l'Est); et elle s'est surtout concentrée dans les services et le BTP - le taux d'investissement est resté le plus faible de toutes les régions en 
développement (Ali et Dadush 2010). Jusqu'ici, ces progrès récents n'ont pas mis fin à l'anémie structurelle du sous-continent et, d'ailleurs, les écarts entre l'ASS et le reste du monde ont continué de se creuser ${ }^{8}$.

Tableau 2.1 Dynamique comparée de la croissance régionale du PIB par habitant, 1960-2007

\begin{tabular}{lcc}
\hline \multicolumn{1}{c}{ Région } & $\%$ annuel & $\begin{array}{c}\text { Coefficient } \\
\text { de variation }\end{array}$ \\
\hline Afrique du Nord / Moyen-Orient & 2,06 & 1,68 \\
Afrique subsaharienne & 0,72 & 3,10 \\
Amérique latine / Caraïbes & 1,73 & 1,38 \\
Asie de l'Est et Pacifique & 5,44 & 0,76 \\
Asie du Sud & 2,72 & 0,99 \\
\hline
\end{tabular}

Source: Arbache et Page 2007.

\section{De nouvelles modalités de transition démographique}

Transition économique et transition démographique sont étroitement liées. La population mondiale continue d'enregistrer une vive croissance, mais avec des taux très différents d'une société à l'autre, qui traduisent différents stades de transition démographique - un processus qui se caractérise par une baisse successive des taux de mortalité et des taux de natalité9. En conséquence, les pays en développement concentrent une part grandissante de la population mondiale. Cette évolution va représenter un défi pour la croissance économique en exacerbant les asymétries régionales, et elle affectera, à terme, la structure économique des différentes régions du monde.

Selon les estimations de l'ONU, la population mondiale devrait atteindre 9,2 milliards en 2050 - soit 2 milliards de plus qu'aujourd'hui (voir tableau 2.2) ${ }^{10}$. Ces statistiques suscitent de nombreux débats ${ }^{11}$ mais la distribution régionale de cette croissance démographique recueille insuffisamment d'attention. Alors que l'Europe présente les caractéristiques du dernier stade de la transition démographique (population vieillissante et en recul), l'Afrique subsaharienne et l'Asie centrale et du Sud sont encore en plein essor. Elles croissent cependant à des rythmes différents : la population d'ASS devrait doubler d'ici 2050 pour atteindre 1,7 milliard d'habitants, tandis qu'en Asie centrale et du Sud, la population ne devrait augmenter «que » de $40 \%{ }^{12}$. Aussi, d'ici 2050, l'Afrique subsaharienne devrait être la deuxième région la plus peuplée du monde après l'Asie du Sud. Parallèlement, la croissance démographique de l'Asie de l'Est (Chine principalement) devrait s'interrompre sous l'effet d'une forte progression des revenus mais aussi probablement des politiques de natalité radicales mises en place dans les années 1970, une hypothèse qui fait débat. L'Asie de l'Est sera ainsi progressivement confrontée au problème que connaît l'Europe aujourd'hui - le poids d'une population vieillissante - qui pèsera lourdement sur sa croissance. Une situation à laquelle le Japon est déjà confronté. 
Tableau 2.2 Population mondiale par région en valeur absolue et en pourcentage, 1960-2050 (en millions)

\begin{tabular}{|c|c|c|c|c|c|c|c|c|c|c|}
\hline \multirow{2}{*}{$\frac{\text { Année }}{\text { Asie de l'Est }}$} & \multicolumn{2}{|c|}{1960} & \multicolumn{2}{|c|}{1990} & \multicolumn{2}{|c|}{2010} & \multicolumn{2}{|c|}{2050} & \multicolumn{2}{|c|}{$2010-2050$} \\
\hline & 779 & $26 \%$ & 1337 & $25 \%$ & 1564 & $23 \%$ & 1600 & $17 \%$ & 36 & $2 \%$ \\
\hline Asie centrale et du Sud & 627 & $21 \%$ & 1250 & $24 \%$ & 1780 & $26 \%$ & 2494 & $27 \%$ & 713 & $40 \%$ \\
\hline Afrique subsaharienne & 229 & $8 \%$ & 518 & $10 \%$ & 863 & $12 \%$ & 1753 & $19 \%$ & 890 & $103 \%$ \\
\hline Amérique latine / Caraïbes & 220 & $7 \%$ & 442 & $8 \%$ & 589 & $9 \%$ & 729 & $8 \%$ & 141 & $24 \%$ \\
\hline Amérique du Nord & 204 & $7 \%$ & 283 & $5 \%$ & 352 & $5 \%$ & 448 & $5 \%$ & 97 & $28 \%$ \\
\hline Europe & 604 & $20 \%$ & 721 & $14 \%$ & 733 & $11 \%$ & 691 & $8 \%$ & -42 & $-6 \%$ \\
\hline Autres régions & 359 & $12 \%$ & 739 & $14 \%$ & 1028 & $15 \%$ & 1434 & $16 \%$ & 406 & $40 \%$ \\
\hline Monde & 3023 & $100 \%$ & 5290 & $100 \%$ & 6909 & $100 \%$ & 9150 & $100 \%$ & 2241 & $32 \%$ \\
\hline
\end{tabular}

Source : Nations unies, Perspectives de la population mondiale, révision de 2008.

Note : Pour la définition des régions, voir http://esa.un.org./wpp/Excel-Data/definition-of-regions.htm.

Ces rythmes de croissance démographique différenciés (qui pourraient changer en cas de circonstances exceptionnelles) produiront une nouvelle cartographie du monde et modifieront probablement les équilibres de pouvoirs. Guengant (2007) rappelle qu'en 2050, la part de l'ASS dans la population mondiale devrait avoir retrouvé son niveau antérieur - environ $20 \%$ - et dépassé celle de la Chine (on notera que leurs populations respectives étaient très proches au XVI $I^{\mathrm{e}}$ siècle $-\mathrm{de}$ l'ordre de 100 à 120 millions). L'Europe et l'Amérique du Nord devraient représenter ensemble moins de $15 \%$ de la population mondiale totale (voir tableau 2.2).

Les différentes perspectives démographiques des pays du programme RuralStruc illustrent les variations autour de ces grandes tendances régionales. Trois pays d'ASS - le Kenya, Madagascar et le Mali - dépassent la moyenne régionale. La croissance démographique au Nicaragua est très proche de celle de l'Amérique centrale (38\%), tandis qu'on observe un net ralentissement au Mexique - qui fait pourtant partie de la même sous-région de l'ONU - correspondant à la fin de sa transition démographique (voir tableau 2.3).

La principale préoccupation économique relative à la transition démographique porte sur l'évolution de la structure d'activité de la population, qui est le reflet de sa structure par âge (Bloom, Canning et Sevilla 2001). Cette évolution apparaît dans les ratios de dépendance (ou ratios d'activité) qui indiquent la proportion d'actifs et d'inactifs dans l'économie ${ }^{13}$. Au premier stade de la transition démographique, la population est jeune et compte un important pourcentage de jeunes inactifs ; au deuxième stade, ces cohortes deviennent actives et - si les conditions de la croissance sont réunies (environnement économique, institutionnel et politique propice) - offrent une prime potentielle à l'économie appelée « dividende démographique ». Le troisième stade correspond au vieillissement de ces cohortes, qui augmente le ratio de dépendance (ou diminue le ratio d'activité).

La figure 2.5 illustre ces transitions démographiques échelonnées et différenciées. Du fait de la vive croissance démographique qu'elle enregistre depuis les années 1960 (supérieure à 2,5\% par an pendant quarante ans, avec un pic de $3 \%$ 
Tableau 2.3 Population des pays du programme RuralStruc, 1960-2050 (en millions)

\begin{tabular}{lcccccc}
\hline & Pays & $\mathbf{1 9 6 0}$ & $\mathbf{1 9 9 0}$ & $\mathbf{2 0 1 0}$ & $\mathbf{2 0 5 0}$ & \multicolumn{2}{c}{$\mathbf{2 0 1 0 - 2 0 5 0}$} \\
\hline Kenya & 8,1 & 23,4 & 40,8 & 85,4 & 44,5 & $109 \%$ \\
Madagascar & 5,1 & 11,2 & 20,1 & 42,7 & 22,5 & $112 \%$ \\
Mali & 5 & 8,6 & 13,3 & 28,3 & 14,9 & $112 \%$ \\
Sénégal & 3 & 7,5 & 12,8 & 26,1 & 13,2 & $103 \%$ \\
Maroc & 11,6 & 24,8 & 32,3 & 42,6 & 10,2 & $32 \%$ \\
Nicaragua & 1,8 & 4,1 & 5,8 & 8,1 & 2,3 & $40 \%$ \\
Mexique & 37,9 & 83,4 & 110,6 & 128,9 & 18,3 & $17 \%$ \\
\hline
\end{tabular}

Source : Nations unies, Perspectives de la population mondiale, révision de 2008.

dans les années 1980), l'Afrique subsaharienne a connu, dans les années 1980 et 1990, le ratio d'activité le plus faible jamais enregistré, avec à peu près un seul actif pour un inactif ${ }^{14}$. Comprendre cette énorme contrainte permet de remettre en perspective les deux décennies de crise économique et d'ajustement structurel qu'a connues l'ASS et sa situation actuelle. Sur la même période, l'Asie de l'Est a bénéficié d'un remarquable dividende démographique. Son ratio d'activité a dépassé deux actifs par inactif et a nourri la croissance économique régionale ${ }^{15}$. L'Asie du Sud, dont la transition est en retard d'une trentaine d'années sur celle de l'Asie de l'Est, devrait enregistrer ce dividende démographique vers 20352040, tandis que l'ASS devra attendre jusqu'en 2050 pour recueillir les bénéfices d'une structure démographique plus favorable.

Figure 2.5 Ratio d'activité par grandes régions, 1950-2050

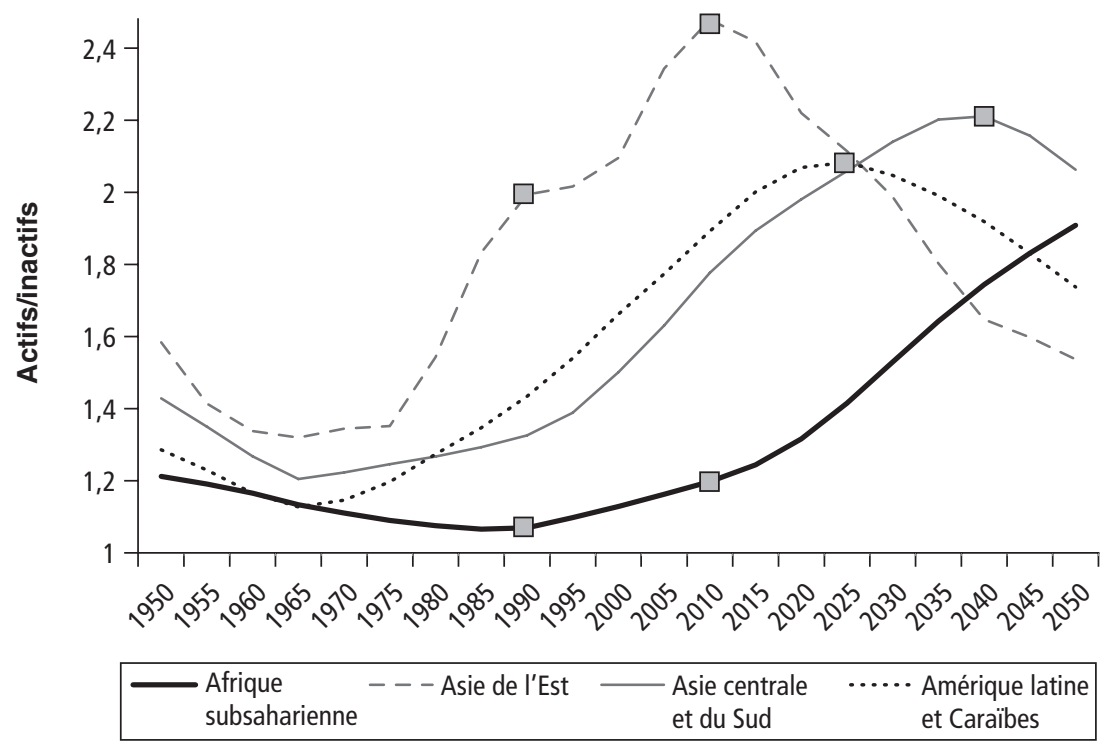

Sources : Nations unies, Perspectives de la population mondiale, révision de 2008. 
Examinées du point de vue des cohortes annuelles - en particulier des cohortes annuelles de jeunes qui entrent sur le marché du travail - ces tendances démographiques augurent d'une forte poussée de l'offre de main-d'œuvre dans les prochaines décennies en ASS et en Asie du Sud.

La figure 2.6 présente la taille des cohortes annuelles entrant sur le marché du travail et illustre les mêmes tendances dans les régions les plus peuplées du monde (Asie et ASS $)^{16}$. Elle donne une estimation des besoins d'absorption de maind'œuvre des diverses économies régionales. La cohorte annuelle de nouveaux actifs en Afrique subsaharienne est d'environ 17 millions aujourd'hui et devrait atteindre 25 millions dans quinze ans. Le pic se produira après 2050. Pour un pays d'ASS de taille moyenne (15 millions d'habitants), la cohorte annuelle était de 250000 personnes dans les années 2000 et devrait atteindre 400000 dans les années 2020. Cela signifie qu'en ASS, environ 330 millions de personnes entreront sur le marché du travail dans les quinze prochaines années - ce qui est à peu près équivalent à la population des États-Unis ${ }^{17}$. Ce chiffre n'est pas une projection : ces 330 millions de nouveaux entrants sur le marché du travail sont déjà nés.

Le tableau 2.4 indique ce que représentent ces tendances pour les pays du programme RuralStruc. En ASS, le pic d'offre de main-d'œuvre se produira après 2050 ; c'est la grande différence par rapport aux autres pays, où le pic s'est déjà produit ou sera prochainement dépassé. Dans les prochaines décennies, l'obtention d'une croissance économique suffisante pour créer de la demande de maind'œuvre sera un enjeu majeur pour les sociétés et les gouvernements d'Afrique subsaharienne (Banque mondiale 2009c ; UNRISD 2010).

Figure 2.6 Cohortes d'entrants sur le marché du travail par grandes régions, 1955-2050

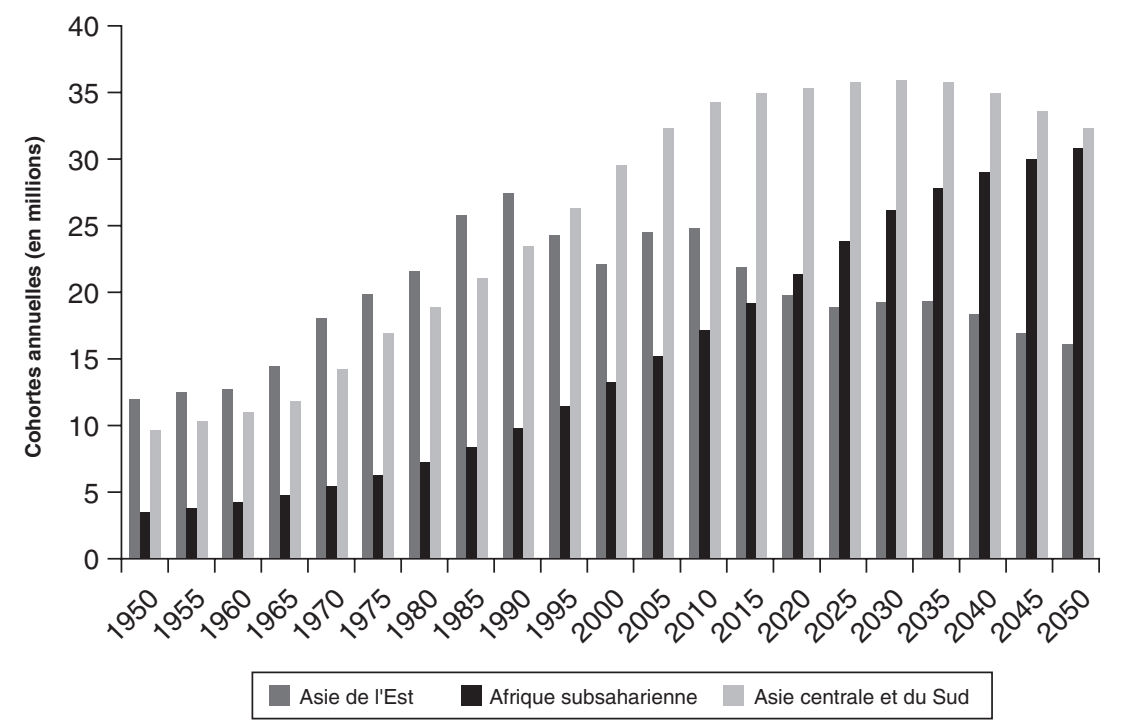

Sources : Nations unies, Perspectives de la population mondiale, révision de 2008. Note: Les cohortes annuelles correspondent à 1/10 de la tranche d'âge des 15-24 ans. 
Tableau 2.4 Entrants sur le marché du travail dans les pays du programme RuralStruc (en milliers)

\begin{tabular}{lccc}
\hline \multicolumn{1}{c}{ Pays } & $\begin{array}{c}\text { Nouveaux entrants sur } \\
\text { le marché du travail en 2010 }\end{array}$ & $\begin{array}{c}\text { Pic d'entrées } \\
\text { sur le marché du travail }\end{array}$ & Date du pic \\
\hline Kenya & 847 & 1545 & $>2050$ \\
Madagascar & 405 & 736 & $>2050$ \\
Mali & 278 & 524 & $>2050$ \\
Sénégal & 269 & 452 & $>2050$ \\
Maroc & 638 & 644 & 2005 \\
Nicaragua & 125 & 129 & 2015 \\
Mexique & 1984 & 2008 & 2015 \\
\hline
\end{tabular}

Source : Nations unies, Perspectives de la population mondiale, révision de 2008.

\section{Le changement structurel dans une économie mondiale ouverte}

À l'aune de la nouvelle configuration de l'économie mondiale, la vision évolutionniste qui sous-tend le modèle orthodoxe de transition économique n'est pas suffisamment remise en cause aujourd'hui. Le processus de mondialisation qui s'est amorcé dans les années 1970 est sans égal et trop souvent qualifié à tort de seconde mondialisation par référence à une première mondialisation survenue entre les années 1860 et la Première Guerre mondiale. Cette période n'a toutefois pas concerné l'ensemble du monde. Elle était caractérisée par un mouvement croissant de biens, de main-d'œuvre et de capital qui concernait avant tout l'Europe, sa périphérie immédiate (Russie et Empire ottoman) et les «nouveaux mondes » - les États-Unis principalement (Berger 2002). Il s'agissait plus exactement d'un processus de convergence au sein de l'économie nord-atlantique, nourri par les flux migratoires (O’Rourke et Williamson 1999) et gouverné par un ordre géopolitique couvrant les empires coloniaux européens et une sphère d'influence américaine en Amérique latine.

Les processus qui sont aujourd'hui à l'œuvre reflètent une intégration croissante de l'ensemble du monde. Cette mondialisation est facilitée par les progrès techniques continus dans le transfert des marchandises, des capitaux et des informations, renforcée par les politiques de libéralisation amorcées au début des années 1980. Elle est caractérisée par une plus forte concentration des actifs détenus par des entreprises de taille mondiale et par les investisseurs institutionnels, mais aussi par le développement des échanges à l'intérieur des grandes firmes et l'externalisation de la production. La mondialisation se traduit également par une convergence vers des préoccupations communes relatives au changement planétaire, surtout en ce qui concerne l'impact des activités humaines sur les ressources naturelles et le climat. 
Ces caractéristiques favorisent une profonde interconnexion des marchés et des sociétés humaines qui pèse de plus en plus fortement sur leurs structures. Elles tendent à affaiblir simultanément les liens locaux au profit de relations plus distantes (notamment en termes de production) et à creuser les asymétries entre les différentes régions du monde.

\section{La séquence historique du changement structurel est-elle viable aujourd'hui?}

Une des principales questions pour la période actuelle est de savoir si et dans quelle mesure la séquence historique de la transformation structurelle est viable pour les pays qui ont amorcé leur processus de changement tardivement. Ce débat est très largement piégé. Bien qu'elle présente de nombreuses variantes, la trajectoire de transformation structurelle est un phénomène historique stylisé, qui est certes confirmé par l'analyse statistique ${ }^{18}$. Il s'agit là d'un constat et non d'un jugement de valeur. Mais les notions de « développement » et d' " émergence » sont bien évidemment ambiguës car elles sont porteuses de biais inhérents à la vision européenne (occidentale) de l'histoire du monde ${ }^{19}$ selon laquelle la trajectoire structurelle des pays aujourd'hui développés constituerait la métrique de l'évolution des économies et des sociétés humaines (Gabas et Losch 2008). En dépit de cette difficulté fondamentale, cet ouvrage a néanmoins été contraint à repositionner les pays étudiés par rapport aux transitions historiques observées et à faire ainsi référence aux " précurseurs » et aux « retardataires » dans ce processus de changement.

Timmer et Akkus (2008) affirment que la lenteur de la transformation structurelle de certains pays tient principalement à des difficultés de croissance économique et n'implique pas une remise en cause du processus historique de transition. Même si cette affirmation est vraie dans l'absolu, elle sous-estime pourtant le poids des circonstances propres aux transitions passées et la difficulté d'une transformation structurelle à l'identique aujourd'hui.

L'importance $d u$ «moment». Quel que soit le pays ou la région, ses perspectives de changement ne dépendent pas seulement de ses conditions internes en termes de démographie, d'éducation, ou de ressources naturelles, mais aussi de ses liens avec le reste du monde (Gore 2003). Ainsi, par exemple, les possibilités d'industrialisation ne sont pas indépendantes des conditions de la concurrence internationale. L'adoption d'une perspective historique est donc essentielle dans l'analyse des processus de transformation structurelle parce que les opportunités, les contraintes et les rapports de force sont en constante évolution.

Aujourd'hui, le référentiel évolutionniste tend à omettre trois grandes caractéristiques des processus de transition passés. La première est l'équilibre mondial des pouvoirs à l'époque des transitions de l'Europe occidentale et de l'Amérique du Nord qui se sont déroulées au XIX ${ }^{e}$ siècle et sur la plus grande partie du $\mathrm{xx}^{\mathrm{e}}$ siècle. Ces transitions initiales de sociétés agraires vers des économies plus diversifiées ne peuvent être isolées de l'hégémonie politique et militaire européenne, qui s'est amorcée au $\mathrm{XVI}^{\mathrm{e}}$ siècle et s'est ouvertement exprimée par la colonisation et l'instauration de traités dits inégaux ${ }^{20}$. 
Cette hégémonie, qui est totalement enracinée dans l'histoire du capitalisme mondial (Braudel 1979 ; Wallerstein 1989), a réduit ou tout simplement éliminé les concurrences extérieures ${ }^{21}$ et a débouché sur des conditions d'offre et de demande particulièrement avantageuses fondées sur des marchés captifs. Combinée à la révolution agricole du XVIII siècle (Mazoyer et Roudart 1997), elle a ouvert la voie à la spécialisation et à l'industrialisation (notamment grâce à l'existence d'une offre alimentaire sécurisée et reposant sur des importations à bas coûts) et elle a facilité une forte rentabilité des entreprises propice à l'accumulation de capital et aux investissements. Les États-Unis ont reproduit et accentué les caractéristiques de cette transition européenne lors de leur propre transition opérée à partir du milieu XIX $\mathrm{X}^{\mathrm{e}}$ siècle.

La seconde omission concerne le vaste mouvement d'émigration enregistré en Europe, qui était étroitement lié à l'hégémonie du continent (Hatton et Williamson 2005). Entre 1850 et 1930, près de 60 millions d'Européens sont partis pour les « nouveaux mondes » : les Amériques, l'Australie, la Nouvelle-Zélande et l'Afrique (principalement l'Algérie et l'Afrique du Sud) ${ }^{22}$. Ces "migrations blanches» (Rygiel 2007) ont facilité l'ajustement des économies européennes et la gestion des excédents de main-d'œuvre résultant de leur transition démographique, plus précisément de l'exode rural et du rythme insuffisant des créations d'emplois dans les villes malgré un solide processus d'industrialisation (Losch 2008).

La troisième omission est relative aux conditions des transitions latino-américaines et asiatiques, qui sont fréquemment citées en renfort pour attester de l'intangibilité du processus de transformation structurelle. Les pays d'Amérique latine et d'Asie ont en effet amorcé leur transition dans une période très particulière de l'histoire mondiale qui est celle du « développement national autocentré » (Giraud 1996) qui a caractérisé le régime international entre la crise financière de 1929 et les années 1970 (c'est-à-dire le début de la mondialisation). Partout dans le monde, les États nations ont mis en œuvre leur propre «planification du développement» (McMichael 1996), caractérisée par la substitution des importations, le protectionnisme et une forte intervention de l'État (Chang 2002). Les politiques publiques ont été de toute première importance tant pour l'industrialisation (Evans 1995 ; Amsden 2001) que pour la modernisation de l'agriculture (Djurfeldt et al. 2005) et elles ont facilité l'apparition de l'État dit « développementiste ». Les pays indépendants d'Amérique latine ont engagé ce processus entre les deux guerres mondiales et ont maintenu ces politiques pendant les trente années d'après-guerre ${ }^{23}$. Ils ont été suivis par de nombreux pays d'Asie qui venaient d'accéder à l'indépendance dans les années 1950 et les financements liés à la guerre froide ont joué tout leur rôle dans les deux cas. Bien que ces stratégies de développement dirigées par l'État aient donné des résultats mitigés et aient été finalement abandonnées en invoquant leur inefficacité, elles ont contribué dans la plupart des pays à la création d'un solide tissu économique et institutionnel (en termes de compétences, de processus, d'expériences) qui a grandement facilité l'adaptation des pays à la mondialisation lors du retrait de l'État.

L'Afrique subsaharienne a clairement suivi une autre trajectoire. L'absence de transformation structurelle qui la caractérise depuis cinquante ans peut être en 
grande partie expliquée par une séquence historique qui a conduit à l'insertion tardive du sous-continent dans l'économie mondiale et par les conditions dans lesquelles cette insertion s'est finalement opérée. Un premier facteur explicatif concerne les conditions de la domination coloniale, avec ses marchés captifs et ses obstacles explicites à l'industrialisation et à l'éducation, qui ont débouché sur une spécialisation marquée dans les secteurs primaires. S’y ajoutent les conditions politiques dans lesquelles les États africains ont été créés, en particulier des frontières héritées de l'époque coloniale et l'adoption de systèmes administratifs extérieurs, qui ont débouché sur des États jeunes qui manquaient d' « épaisseur institutionnelle » (Amin et Thrift 1993), une situation rendant l'objectif d'intégration politique nationale notablement difficile et coûteux.

Enfin et peut-être surtout, les États africains ont été confrontés au début des années 1980 aux conséquences combinées de la mondialisation, des politiques d'ajustement structurel et d'une démographie pénalisante (en particulier le ratio d'activité le plus bas jamais enregistré à l'échelle mondiale), alors qu'ils avaient tout juste vingt ans. Cette jeunesse a fortement pesé sur leur capacité institutionnelle à répondre efficacement à ces défis - un constat majeur et trop souvent ignoré. Car les États africains n'ont pas disposé de la marge de manœuvre nécessaire pour entreprendre des politiques de modernisation volontaristes et pour élaborer et mettre en œuvre des stratégies de développement cohérentes, comme cela s'est fait en Asie et en Amérique latine.

Les difficultés de la reproduction. Les transitions passées se sont inscrites dans les spécificités de leur époque ; et les pays dont le développement s'est amorcé tardivement doivent gérer les caractéristiques d'une économie mondiale ouverte, une situation qui offre d'importantes opportunités, qui en écarte d'autres, mais qui accentue surtout les asymétries.

Un creusement des écarts et une réduction des distances. Chaque pays est à un stade de transition économique et démographique qui lui est propre, mais tous ont affaire au même monde. Du fait de leurs trajectoires de développement particulières et de la diversité des modalités et des moments de leur intégration dans l'économie mondiale, ils sont dotés d'avantages comparatifs différents. Ces avantages comparatifs ne sont toutefois pas figés et les pays dont la transition s'est amorcée tardivement peuvent bénéficier des progrès techniques et de l'expérience des précurseurs pour développer leurs propres bases de compétences et d'actifs. En parallèle, ils peuvent aussi saisir de nouvelles opportunités pour accéder à des marchés mondiaux en expansion.

Cependant, ces bénéfices tirés des expériences passées et du nouveau contexte sont limités par les importantes asymétries de productivité et de compétitivité, en croissance permanente, qu'ils rencontrent sur le marché international mais aussi sur leurs propres marchés intérieurs. Ils doivent se battre comme sur un « terrain découvert un jour d'orage » (Birdsall 2006), où leur productivité est concurrencée par des entreprises étrangères (surtout celles des grands pays émergents), alors que l'instabilité de l'environnement économique mondial et les impacts du changement 
climatique constituent des contraintes nouvelles. Il s'agit là de défis gigantesques pour la transformation structurelle aujourd'hui.

Globalement, l'ASS présente un écart de productivité de 1 à 5 avec les autres pays en développement et de 1 à 100 avec les pays de l'OCDE ${ }^{24}$. Un écart de cette ampleur est un obstacle colossal et persistant pour gagner en compétitivité au niveau mondial : même s'il existe des avantages comparatifs pour certains facteurs (le coût du travail par exemple), ils ne sont pas suffisants. Car la compétitivité ne dépend pas seulement des coûts de production mais aussi de la capacité d'une économie à répondre aux exigences des marchés à la fois en qualité et en volume. En effet, bien que les exigences de qualité soient la première barrière à l'entrée pour la production de biens à haute valeur ajoutée, le volume de l'offre détermine aussi la part de marché, qui constitue in fine l'indicateur clé de la compétitivité.

Cette observation vaut pour tous les secteurs d'activité, pour l'industrie manufacturière comme pour l'agriculture, et pour tous les pays. Le contexte actuel de croissance de la demande en produits alimentaires et leurs prix élevés est favorable à tous les producteurs agricoles du monde, mais ceux des pays ayant accusé un retard de développement sont à l'évidence moins bien armés pour exploiter cette nouvelle opportunité. Ils devront rapidement à la fois accroître la qualité de leurs produits et augmenter leur offre. À défaut, ce sont les pays concurrents qui répondront à cette demande nouvelle et leurs parts de marché en souffriront.

Ces asymétries de productivité et de compétitivité dans le contexte d'une économie ouverte affectent également la dimension locale de la transformation structurelle. Les échanges commerciaux, quelle que soit la distance en jeu, ont été grandement facilités par le processus de libéralisation et leur rapidité est démultipliée par les progrès des télécommunications et des transports. Une conséquence majeure de cette nouvelle donne est cependant l'affaiblissement les liens locaux entre l'agriculture, l'industrie et l'urbanisation - une articulation structurante qui a puissamment contribué aux transitions économiques anciennes - dans la mesure où ces liaisons locales sont largement battues en brèche par la propension aux importations (UNRISD 2010). Si les importations présentent souvent l'avantage non négligeable d'une disponibilité à coût réduit, elles ne renforcent toutefois pas la dimension locale du développement.

La dépendance à l'égard des importations a modifié les modes d'urbanisation dans de nombreux pays en développement, où il est fréquent que les villes (surtout les plus grandes) comptent plutôt sur les importations que sur leurs ressources propres ou celles des régions avoisinantes. Cette situation a contribué à la formidable expansion du secteur informel, qui sert de tampon pour absorber l'écart entre l'offre et la demande de main-d'œuvre. Ce processus d' « informalisation » est exacerbé en Afrique subsaharienne où, malgré une croissance économique très lente sur la longue période, l'urbanisation n'a pas été freinée (Fay et Opal 2000). Même sans promesses d'emploi, les villes conservent leurs attraits : services, opportunités accrues, mode de vie, etc.

Même s'il est très hétérogène ${ }^{25}$, le secteur informel peut être décrit comme un secteur à faible productivité, caractérisé par le sous-emploi, l'absence de 
sécurité et la faiblesse des revenus ${ }^{26}$. Ces facteurs contribuent à une croissance de bidonvilles qui prolifèrent autour des villes des pays en développement (ONU-Habitat 2003 ; Davis 2006).

Une marge de manouvre étroite. Outre les écarts de compétitivité et la géographie changeante des échanges internationaux, les perspectives de transition des pays dont le développement s'est amorcé tardivement sont compliquées par deux contraintes qui n'étaient pas présentes lors des transitions précédentes : la réduction du champ des interventions publiques et la limitation des possibilités de migration internationale.

L'agenda politique actuel prive les pays confrontés aux défis du changement structurel de plusieurs des interventions publiques qui ont marqué les transitions de nombreux pays d'Amérique latine et d'Asie. Aujourd'hui, le consensus économique mondial repose sur la libéralisation du marché et l'élimination des politiques jugées distorsives. Cette perspective interdit de nombreuses interventions qui ont servi autrefois à encourager la modernisation et à accroître la productivité de l'agriculture et de l'industrie manufacturière, en particulier en Asie (Chang 2002). C'est le cas par exemple des politiques qui ont permis la modernisation de la Corée du Sud et de Taïwan dans la seconde moitié du $\mathrm{xx}^{\mathrm{e}}$ siècle, qui n'auraient pas été possibles sous le régime OMC d'aujourd'hui (Birdsall, Rodrik et Subramanian 2005).

S'agissant de l'agriculture en Afrique subsaharienne, Bezemer et Headey (2008) rappellent que ces contraintes extérieures ont été accentuées par le biais urbain persistant des politiques menées par les États africain $s^{27}$. Ce biais s'est traduit par une lourde taxation des exportations agricoles et une faible protection contre les importations alimentaires, qui a bénéficié aux consommateurs urbains au détriment des agriculteurs ${ }^{28}$.

Il existe aujourd'hui cependant un assouplissement du champ des interventions politiques jugées acceptables par le consensus international, par exemple le cas des subventions dites « intelligentes » car ciblées sur des sujets considérés comme pertinents (smart subsidies). Cette nouvelle marge de manœuvre n'est toutefois pas adaptée à la situation de nombreux États qui n'ont pas les moyens d'apporter ce type de soutien en plus des impératifs de financement des investissements et services de base (biens publics). Dans un contexte marqué par l'ampleur des contraintes budgétaires, un environnement économique mondial instable et des niveaux d'aide internationale fluctuants, les marges de manœuvre devront être trouvées du côté des réformes de la fiscalité.

La seconde contrainte de la période actuelle concerne les migrations internationales, qui ne sont plus une option viable pour ceux qui quittent l'agriculture et ne trouvent pas d'autres alternatives d'emploi dans leur pays. Les migrations internationales sont devenues un objet de recherche privilégié des études sur le développement, même si elles sont surtout traitées du point de vue de l'impact des envois de fonds des travailleurs émigrés ${ }^{29}$ - des transferts qui représentent des flux de capitaux supérieurs à ceux de l'aide publique au développement. Mais la relative facilité qu'ont les migrants à envoyer leurs gains masque le fait que 
la migration elle-même est relativement restreinte. Même si le nombre total de migrants internationaux (individus vivant hors de leur pays d'origine) est estimé à quelque 200 millions, il apparaît difficilement imaginable de répéter dans l'ordre géopolitique actuel les migrations de masse qui se sont opérées au départ de l'Europe à partir du milieu du $\mathrm{XIX}^{\mathrm{e}}$ siècle. Il est certain que si les frontières internationales étaient ouvertes, " les gens viendraient sans hésiter » (Pritchett 2006), mais la migration est une question politiquement sensible et les frontières restent fermées aux personnes, alors qu'elles sont largement ouvertes aux biens, aux capitaux et à de nombreux services ${ }^{30}$.

Les routes migratoires les plus actives et les plus rémunératrices se concentrent à la périphérie de l'Union européenne et des États-Unis ${ }^{31}$ et, à l'avenir, les possibilités de migration dépendront sans doute de l'évolution démographique des pays à hauts revenus (et de la Chine) et de leur demande de main-d'œuvre étrangère. Il est probable que les pays situés dans leur périphérie continueront de satisfaire cette demande et, en conséquence, la plupart des autres pays en développement ne pourront pas reproduire les trajectoires migratoires suivies par les pays frontaliers du monde riche. Une bonne illustration est fournie par le Mexique et le Maroc dont environ $10 \%$ des ressortissants vivent aujourd'hui à l'étranger. L'opportunité de cette migration à grande échelle joue un rôle majeur dans l'économie politique de ces deux pays : elle fournit des transferts de liquidités massifs et sert de soupape de sécurité aux tensions internes associées à leur transformation structurelle. Mais la reproduction d'un tel modèle ne serait pas réaliste pour l'Afrique subsaharienne : si $10 \%$ de la population devaient migrer, ce sont 85 millions de personnes qui quitteraient le sous-continent aujourd'hui, principalement à destination de l'Europe. Un scénario politiquement inimaginable.

\section{Quelles sont les options de transition aujourd'hui ?}

Ce contexte général limite la capacité des régions en retard de développement à reproduire le processus de transition historique et les trajectoires possibles de changement font l'objet de débats au sein des bailleurs de fonds et des milieux universitaires. Les discussions portent plus particulièrement sur l'ASS, qui est souvent mise en perspective avec l'Asie, notamment quant aux alternatives d'emploi pour la main-d'œuvre qui quitte l'agriculture (Headey, Bezemer et Hazell 2010).

À cet égard, c'est la répartition de la population entre zones rurales et zones urbaines qui déterminera l'ampleur des défis géographiques et sectoriels liés à la croissance de l'offre de main-d'œuvre; et la base de données Perspectives de l'urbanisation mondiale des Nations unies (World Urbanization Prospects) offre d'utiles estimations, même si celles-ci sont discutables.

Le tableau 2.5 présente l'évolution historique et prévisionnelle du taux d'urbanisation des principales régions du monde. Bien que l'ASS connaisse une vive croissance urbaine, même comparée à l'Asie du Sud, elle devrait pourtant rester principalement rurale jusque vers 2030. Elle est par ailleurs la seule région du monde dont la population rurale augmentera encore en valeur absolue en 2050 (voir tableau 2.6) : un accroissement de 150 millions de personnes (près de 
$30 \%)$, quand la part des ruraux diminuera dans les autres régions (- $50 \%$ en Asie de l'Est, - $10 \%$ en Asie du Sud, - 45 \% en Europe).

Tableau 2.5 Taux d'urbanisation par grandes régions, 1960-2050 (en \%)

\begin{tabular}{lccccccc}
\hline \multicolumn{1}{c}{ Région } & $\mathbf{1 9 6 0}$ & $\mathbf{1 9 9 0}$ & $\mathbf{2 0 1 0}$ & $\mathbf{2 0 2 5}$ & $\mathbf{2 0 3 5}$ & $\mathbf{2 0 5 0}$ & Multiplicateur \\
\hline Asie de l'Est & 20,2 & 33,0 & 48,5 & 59,2 & 65,5 & 74,1 & 4,8 \\
Asie centrale et du Sud & 18,1 & 27,2 & 32,2 & 39,6 & 46,5 & 57,2 & 5,1 \\
Afrique subsaharienne & 14,8 & 28,2 & 37,3 & 45,2 & 51,2 & 60,5 & 9,5 \\
Amérique latine / Caraïbes & 48,9 & 70,6 & 79,4 & 83,5 & 85,7 & 88,7 & 4,4 \\
Amérique du Nord & 69,9 & 75,4 & 82,1 & 85,7 & 87,6 & 90,2 & 2,0 \\
Europe & 56,9 & 70,5 & 72,6 & 76,2 & 79,5 & 83,8 & 1,5 \\
Monde & 32,9 & 43,0 & 50,6 & 57,2 & 62,2 & 69,6 & 3,5 \\
\hline
\end{tabular}

Source : Nations unies, Perspectives de I'urbanisation mondiale, révision de 2007.

Tableau 2.6 Population rurale par grandes régions, 1960-2050 (en millions)

\begin{tabular}{lrrrrrrrrrr}
\hline \multicolumn{1}{c}{ Région } & \multicolumn{2}{c}{1960} & \multicolumn{2}{c}{1990} & \multicolumn{2}{c}{2010} & & $\mathbf{2 0 5 0}$ & $\mathbf{2 0 1 0 - 2 0 5 0}$ \\
\hline Asie de l'Est & 622 & $31 \%$ & 896 & $30 \%$ & 805 & $24 \%$ & 414 & $15 \%$ & -391 & $-49 \%$ \\
Asie centrale et du Sud & 513 & $25 \%$ & 910 & $30 \%$ & 1207 & $35 \%$ & 1067 & $38 \%$ & -140 & $-12 \%$ \\
Afrique subsaharienne & 195 & $10 \%$ & 372 & $12 \%$ & 541 & $16 \%$ & 693 & $25 \%$ & 151 & $28 \%$ \\
Amérique latine / Caraïbes & 112 & $6 \%$ & 130 & $4 \%$ & 121 & $4 \%$ & 82 & $3 \%$ & -39 & $-32 \%$ \\
Amérique du Nord & 61 & $3 \%$ & 70 & $2 \%$ & 63 & $2 \%$ & 44 & $2 \%$ & -19 & $-30 \%$ \\
Europe & 261 & $13 \%$ & 213 & $7 \%$ & 201 & $6 \%$ & 112 & $4 \%$ & -89 & $-44 \%$ \\
Autres régions & 264 & $13 \%$ & 425 & $14 \%$ & 474 & $14 \%$ & 369 & $13 \%$ & -105 & $-22 \%$ \\
Monde & 2029 & $100 \%$ & 3016 & $100 \%$ & 3413 & $100 \%$ & 2782 & $100 \%$ & -631 & $-18 \%$ \\
\hline
\end{tabular}

Sources : Nations unies, Perspectives de l'urbanisation mondiale, révision de 2007 et Perspectives de la population mondiale, révision de 2008 ; calculs des auteurs.

Comme rappelé précédemment, 330 millions d'individus déjà nés entreront sur le marché du travail subsaharien dans les quinze prochaines années. Selon les taux d'urbanisation prévisionnels (voir figure 2.7), 195 millions d'entre eux seront en zone rurale (59\%) et 137 millions en zone urbaine (41\%). Ces travailleurs seront une opportunité de croissance pour la région mais aussi un sérieux défi pour le marché du travail ${ }^{32}$.

Quelles sont dès lors les possibilités de créations d'emplois ? Plus généralement, quelles sont les mesures publiques prioritaires qui, d'un côté, faciliteront une croissance durable et la transition économique et, de l'autre, soutiendront la transition démographique et permettront d'en bénéficier?

Dans ce débat, les points de vue sont souvent très divisés entre les tenants de l'industrialisation et de l'agriculture (ou entre pro-urbains et ruralistes). Pour les premiers, l'industrie manufacturière est le seul véritable moteur possible du développement africain et donc de la transformation structurelle du sous-continent. La productivité agricole serait trop faible, les défis trop importants et les progrès atten- 
Figure 2.7 Cohortes annuelles entrant sur les marchés du travail rural et urbain et part de la population rurale en Afrique subsaharienne, 1955-2050

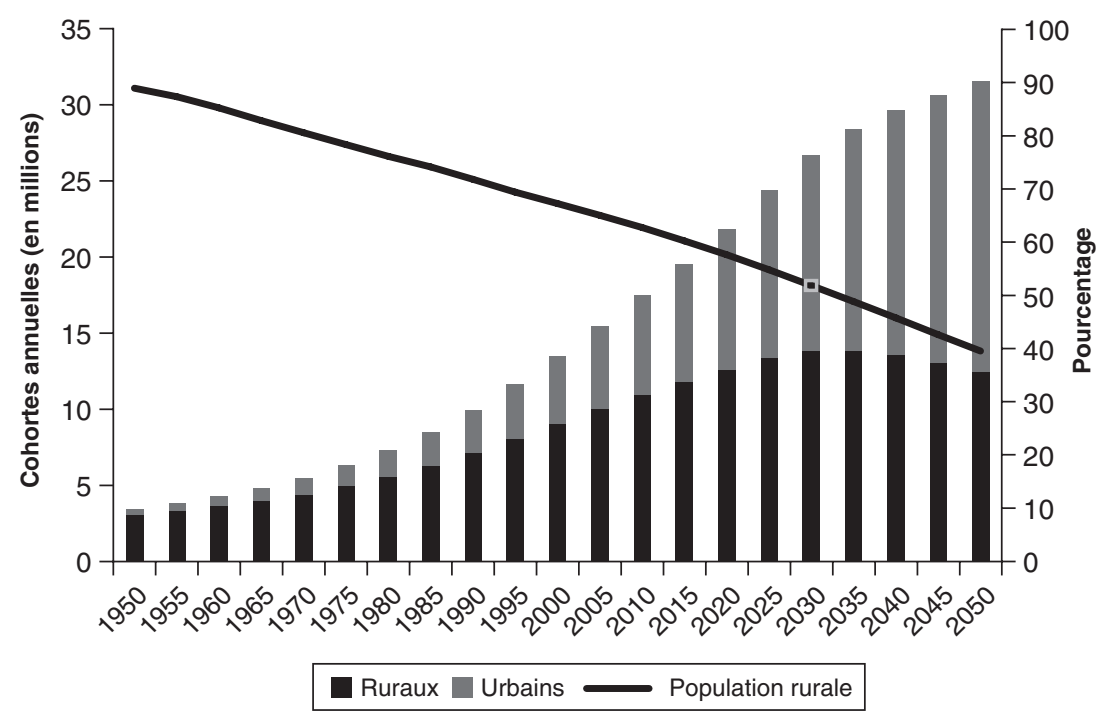

Sources : Nations unies, Perspectives de l'urbanisation mondiale, révision de 2007 et Perspectives de la population mondiale, révision de 2008 ; calculs des auteurs.

dus trop lents ; il est donc plus réaliste de développer l'industrie manufacturière et les services. Les tenants de l'agriculture soulignent pour leur part que la pauvreté est d'abord une question rurale et que les ruraux pauvres sont profondément engagés dans l'agriculture. Celle-ci peut donc être un outil primordial de réduction de la pauvreté, surtout dans le contexte très favorable de croissance de la demande alimentaire. De plus, le développement agricole crée des opportunités de diversification à travers la transformation des produits mais permet aussi une hausse de la demande rurale nourrie par l'augmentation des revenus agricoles.

Pour aider à la clarification de ce débat ancien, il est utile d'examiner de plus près l'économie des villes et des campagnes et leurs capacités respectives à absorber une population active croissante.

Côté urbain, des décennies de stagnation structurelle des économies subsahariennes sont un cuisant rappel de l'échec des modèles de transition traditionnels dans la région ${ }^{33}$. Comme noté précédemment, l'exode rural et la sortie de l'agriculture ont principalement nourri le secteur urbain informel. L'industrie manufacturière n'a jamais vraiment décollé et l'industrialisation intervenue plus tard a été en grande partie victime de ses propres limites ou des politiques de la période d'ajustement structurel.

Bien que l'industrie manufacturière soit très peu développée en ASS, beaucoup pensent cependant que les conditions actuelles offrent une nouvelle opportunité d'industrialisation. Les principaux arguments avancés portent sur l'amélioration du climat des affaires observée dans de nombreux pays, la hausse progressive des 
coûts industriels en Asie due à l'augmentation des salaires (en Chine particulièrement) et de nouvelles opportunités d'industrialisation fondée sur des segments de produits (light manufacturing) plutôt que sur la fabrication de produits finis (UNIDO 2008) - une conséquence du développement de l'externalisation et des échanges intragroupes qui caractérise la mondialisation. Cette option est avantageuse pour les pays les moins avancés parce qu'elle demande moins de capital et de compétences et qu'elle est possible dans un environnement économique et institutionnel plus fragile.

Certains économistes évoquent aussi de nouvelles possibilités de développement du secteur des services. Les multiples potentialités offertes par l'externalisation et les options liées au développement des technologies de l'information et de la communication (TIC) et notamment les ordinateurs en réseaux (informatique dématérialisée ou cloud computing) sont fréquemment citées, surtout lorsqu'on évoque la possibilité de sauter l'étape de l'industrialisation. Des opportunités existent ; mais il n'est pas certain qu'elles soient suffisantes pour que les pays concernés puissent éviter l'industrialisation, surtout dans un environnement où les services deviennent de plus en plus échangeables. La concurrence jouera à plein et il sera très difficile de gagner des parts de marché dans les services (UNRISD 2010). Les pays en développement ne doivent pas sous-estimer les exigences inhérentes à cette stratégie.

Comme noté plus haut, il n'y a pas eu d'industrialisation en ASS au cours des quarante dernières années en dépit d'un formidable processus d'urbanisation offrant tous les avantages économiques de la densité vantés par le RDM09 sur la géographie économique. Mais pour passer de l'environnement actuel à un secteur manufacturier dynamique, il ne suffira pas d'exploiter certains avantages comparatifs (par exemple les coûts de main-d'œuvre), il faudra aussi engager de lourds investissements. L'État doit jouer ici un rôle particulier en fournissant des infrastructures et en offrant des incitations à l'investissement privé, même si les types d'incitations qu'il doit offrir font l'objet de débats (Lin et Chang 2009). Toutefois, étant donné les défis de l'ASS à court et moyen terme (l'horizon de quinze ans souvent mentionné dans ce chapitre), il est difficile d'imaginer que l'industrie manufacturière puisse créer des centaines de milliers d'emplois par an. Il faut donc donner la priorité à la modernisation de l'outil productif existant, ce qui suppose d'offrir des incitations adaptées et de soutenir d'abord les pans du secteur informel les plus prometteurs - ceux qui ont un potentiel de modernisation.

Côté rural, en écho au titre du livre de Christiaensen et Demery paru en 2007 (Down to Earth), il faut du réalisme - un retour sur terre - ou, plus exactement, un retour à l'arithmétique de base (Headey, Bezemer et Hazell 2010). Les « grands nombres » présentés dans ce livre ne recèlent aucune ambiguïté : environ $65 \%$ de la population subsaharienne vit encore en zone rurale, $65 \%$ de la population active travaille dans l'agriculture et $60 \%$ des individus qui entreront sur le marché du travail d'ici à 2025 seront des ruraux. Il faut donc traiter les questions rurales pour régler la pauvreté et gérer aussi la transition économique et démographique. Les activités rurales compteront « pour beaucoup dans l'équation de 
l'emploi des jeunes " (Banque mondiale 2009c) et un échec du développement rural accélérera le dépeuplement des campagnes, renforçant encore les difficultés des villes.

Les « activités rurales » désignent à la fois l'agriculture et l'économie rurale non agricole, qui sont étroitement liées. Comme le décrit une abondante littérature, l'augmentation des revenus agricoles nourrit la demande rurale, qui à son tour favorise le développement d'activités nouvelles, la transformation rurale et le changement économique.

La demande croissante de produits alimentaires induite par le boom démographique et l'urbanisation garantira une croissance ferme de l'agriculture dans les prochaines décennies. Mais dans ce contexte favorable pour les pays à base agricole, la question centrale porte sur le modèle de croissance sectoriel qui sera encouragé ; car ce choix conditionnera la capacité de l'agriculture à absorber la main-d'œuvre et la soutenabilité globale de son développement. Une politique qui encourage les exploitations familiales et les techniques consommatrices en travail n'aura pas les mêmes effets sur l'absorption de la main-d'œuvre qu'une politique favorisant les grandes entreprises managériales et les techniques de production capitalistiques. De même, des politiques promouvant la multifonctionnalité de l'agriculture en ciblant plus particulièrement la gestion des ressources n'auront pas le même effet sur la soutenabilité qu'une politique encourageant une forte intensification fondée sur les facteurs de production industriels.

Des ressources naturelles limitées pourraient poser cependant un obstacle considérable à l'absorption durable de la main-d'œuvre par l'agriculture et au développement agricole en général (Alexandratos 2005), en particulier en ce qui concerne le stock de terres arables dont la superficie est souvent inconnue par manque de systèmes d'information fiables. D'autant plus que la disponibilité foncière est un concept relatif. Le potentiel de production et d'emploi des terres dépend en effet de l'utilisation qui en est faite et des possibilités d'accès (technologies, infrastructures et biens publics : accès à l'eau et irrigation, routes, éradication des maladies endémiques, etc.). Chaque situation connaît ses contraintes et ses opportunités propres, qui affectent directement les options de développement.

Cet état des lieux du débat souligne la nécessité d'appréhender les caractéristiques des situations rurales afin de mieux évaluer leurs contraintes et la marge de manœuvre, et de déterminer les options possibles pour favoriser la transformation rurale. Mais s'il est indispensable de mieux apprécier les ressources en facteurs et leur disponibilité, ce n'est pas suffisant : il faut aussi comprendre les réalités de l'environnement économique dans lequel les ménages ruraux gagnent leur vie et développent leurs activités, ainsi que la nature et l'ampleur de chaque activité et source de revenus et les effets que pourraient avoir sur elles les dynamiques de changement - notamment celles induites par la mondialisation. Les chapitres suivants examinent cette réalité à travers les résultats du travail de terrain réalisé par le programme RuralStruc. 


\section{Notes}

1. Ce travail a été entrepris dans la première phase du programme. Il s'est appuyé sur des sources internationales pour faciliter le positionnement global du programme et la contextualisation des études pays réalisées par les équipes nationales.

2. La situation de la Chine mérite d'être mentionnée : en dépit d'une vive croissance, d'une forte progression du PIB par habitant et d'une diminution considérable du poids économique de l'agriculture, l'écart structurel continue de se creuser. Cette situation très particulière est le résultat conjugué de villes en plein essor, surtout sur le littoral, et de zones rurales à la traîne, où vit encore une part importante de la population (760 millions de personnes, ou $56 \%$, en 2008). Cette population conserve principalement des activités agricoles (selon la $\mathrm{FAO}$ - qui surestime probablement le poids de l'agriculture - la population active agricole avoisine 500 millions de personnes) et l'écart entre les revenus ruraux et urbains est une source de tensions sociales et politiques croissantes.

3. Le Nicaragua n'est pas représenté dans la figure 2.3 car sa série temporelle est trop courte.

4. L'agriculture pèse très lourd dans le commerce extérieur. C'est la première source de devises des pays qui n'ont pas d'activité extractive et ne produisent pas de pétrole.

5. Les autres grands secteurs sont les industries extractives et le BTP, qui ont été séparés respectivement des secteurs primaire et secondaire pour dégager les tendances particulières de l'agriculture et de l'industrie manufacturière.

6. Il s'agit respectivement de Lions on the Move (McKinsey 2010), Emerging Africa (Radelet 2010) et African Poverty is Falling... Much Faster Than You Think (Sala-iMartin et Pinkovskiy 2010). Les lions africains de McKinsey semblent avoir été nommés pour compléter le "zoo de l'émergence » (Gabas et Losch 2008) qui compte déjà les dragons et tigres asiatiques et les jaguars d'Amérique latine. À noter que les « lions » comptés par McKinsey comprennent toutefois l'ensemble du continent africain, dont le PIB se partage entre l'Afrique du Nord (41 \%), l'Afrique du Sud (21\%) et le reste de l'ASS (38\%) - sur la base de la répartition du PIB pour 2008 en US\$ constants 2000 (WDI, Banque mondiale).

7. Le PIB par habitant a crû d'environ 3,5\% par an entre 2004 et 2008 et, après avoir baissé en 2009, il a rebondi à 2,3\% en 2010 .

8. S'appuyant sur les Indicateurs du développement dans le monde de la Banque mondiale, Arrighi et Zhang (à paraître) comparent le PNB par habitant de l'ASS (Afrique du Sud comprise) au PNB de ce qu'ils nomment le Premier Monde (Amérique du Nord, Europe de l'Ouest et du Sud y compris la Scandinavie, Israël, l'Australie, la Nouvelle-Zélande et le Japon). Ils montrent que la part de l'ASS (en pourcentage du PNB par habitant du Premier Monde) a été ramenée de 5,6\% en 1960 à 2,3 \% en 2005.

9. Au premier stade de la transition, la baisse du taux de mortalité intervenant avant tout déclin comparable du taux de natalité engendre une forte croissance démographique, qui ralentit ensuite progressivement lorsque le taux de natalité diminue. Aujourd'hui, les progrès de la médecine et des conditions de vie accentuent l'ampleur du processus et raccourcissent son cycle.

10. Les Perspectives de la population mondiale des Nations unies (World Population Prospects) sont une référence incontournable. Les projections s'appuient sur un ensemble d'hypothèses - en particulier le taux de fécondité - révisées tous les deux ans. Les résultats de la « variante moyenne » de la révision de 2008 ont été sélectionnés. 
11. De nombreux spécialistes pensent que les projections des Nations unies sont sous-estimées (Guengant et May 2009) car elles s'appuient sur un paradigme de convergence avec un objectif de taux de fécondité pour 2050 que contredisent de nombreux recensements nationaux. Au Mali par exemple, le recensement de 2009 (INSTAT 2009) conclut à une augmentation annuelle de 3,6 \% de la population malienne entre 1998 et 2009, un taux bien plus élevé que le taux de 2,4\% cité dans les Perspectives de la population mondiale.

12. Au-delà de ce portrait général d'une population en plein essor, on observe d'importantes différences entre les pays d'Afrique subsaharienne. Le taux de croissance de nombreux pays a été affecté par la pandémie du SIDA, surtout en Afrique australe, et la fécondité totale (nombre d'enfants par femme) enregistre un recul inégal. Elle demeure très élevée dans la plupart des pays du Sahel et d'Afrique centrale et dans la Corne de l'Afrique (six à sept enfants par femme), alors que certains pays (comme le Sénégal, le Nigeria et le Kenya) ont amorcé un ralentissement progressif et hésitant (quatre à cinq enfants par femme). Ces deux groupes représentent $85 \%$ de la population d'ASS. Les pays pleinement engagés dans leur transition (trois à quatre enfants par femme) sont le Ghana, la Côte d'Ivoire et l'Afrique australe (excepté le Mozambique). L'Afrique du Sud est bien plus avancée dans le processus (2,5 enfants par femme). Voir Nations unies, World Fertility Data 2008, Guengant (2007) et Gendreau (2010).

13. Le ratio couramment employé est le ratio de dépendance, qui rapporte la population active (de 15 à 64 ans) à la population inactive (moins de 15 ans et plus de 64 ans). Le ratio est calculé en divisant le nombre total d'inactifs par les actifs. Cependant, le programme a décidé d'employer le ratio d'activité (population active/population inactive) qui est plus parlant. Il faut souligner que ces ratios surestiment la population active : le groupe des individus « en âge de travailler » comprend de nombreux inactifs et chômeurs. Il faudrait donc utiliser les vrais ratios de dépendance ou d'activité, y compris le taux d'emploi. Dans de nombreux pays en développement et particulièrement en ASS, cette approche est compliquée par la taille du secteur informel et le manque d'informations sur le marché du travail. Pour plus d'informations sur ce point, voir entre autres Oudin (2003).

14. Le ratio était inférieur à 1 dans certains pays. Le ratio d'activité réel, comprenant le taux d'emploi effectif, serait encore plus défavorable.

15. L'écart de structure d'activité entre l'ASS et l'Asie de l'Est est plus élevé aujourd'hui : 1,2 contre 2,5 (voir les repères sur les courbes de la figure 2.5).

16. Les cohortes sont couramment calculées en prenant $1 / 10^{\mathrm{e}}$ du groupe des $15-24$ ans, ce qui produit une estimation des nouveaux entrants sur le marché du travail, c'està-dire des jeunes qui recherchent un emploi ou une activité procurant un revenu. Ce chiffre est différent de l'augmentation de la population active, que l'on peut estimer en utilisant l'augmentation nette $(n+1-n)$ de la population active (15-64 ans). Le second calcul produit une cohorte annuelle moins nombreuse que le premier : 12 millions en 2010 pour l'ASS contre 17 millions. Outre qu'elle est imprécise, la variation du niveau de la population active peut être trompeuse car les marchés du travail de la plupart des pays en développement comptent de nombreux individus qui continuent de travailler après 65 ans, notamment dans le secteur agricole et informel urbain, mais aussi des retraités du secteur formel qui tentent d'augmenter les revenus de leur pension. La cohorte annuelle des entrants sur le marché du travail donne donc une estimation plus fiable de la nouvelle offre de main-d'œuvre. 
17. Les chiffres pour l'Asie du Sud et centrale sont les suivants : une cohorte annuelle de 35 millions de personnes en 2010, 37 millions en 2025, et un montant cumulé de 575 millions de nouveaux entrants sur le marché du travail sur la période de quinze ans.

18. Timmer et Akkus (2008) ont testé l'évolution de la trajectoire structurelle dans 86 pays. Les résultats confirment la robustesse de ce processus historique. Les auteurs ont inclus les sept pays du programme RuralStruc dans leur échantillon ; ils ne s'écartent pas de la tendance générale.

19. Pour Rist (1996), le développement est une «croyance européenne » enracinée dans une vision unilatérale de l'histoire, ce que Goody (2006) appelle le « vol de l'histoire ». Le terme «nouveaux mondes » révèle la même perspective européenne, qui accordait alors peu de considération aux peuples indigènes.

20. Il est impossible de développer ici les conditions qui ont conduit à l'hégémonie européenne, mais la « découverte » et la domination des Amériques semblent avoir été déterminantes (Grataloup 2007). Pomeranz (2000) relève que l'hégémonie était également liée à la présence providentielle de charbon en Europe de l'Ouest, qui a profondément changé la relation du continent aux ressources naturelles par rapport à celle de la Chine.

21. Bairoch (1997) note qu'en 1750, l'Inde et la Chine concentraient un peu plus de la moitié de la production manufacturière mondiale.

22. Les estimations diffèrent selon la prise en compte ou non des migrants qui sont retournés dans leur pays d'origine. Les migrations européennes aux Amériques se sont principalement orientées vers les États-Unis, qui absorbaient 1,3 million d'immigrants par an au tournant du $\mathrm{xx}^{\mathrm{e}}$ siècle (Daniels 2003) sur un total d'environ 35 millions. Le Canada et la partie méridionale de l'Amérique du Sud (Argentine, sud du Brésil, Chili) étaient eux aussi des destinations privilégiées.

23. Voir les travaux considérables de la Commission économique des Nations unies pour l'Amérique latine (CEPAL).

24. La productivité globale est calculée en rapportant la valeur ajoutée à la population active totale. Les valeurs constantes moyennes par travailleur - sur la base des séries de 2000 à 2005 - avoisinent 500 US\$ pour l'ASS, 2500 US\$ pour les autres pays en développement et 50000 US\$ pour les pays de l'OCDE (CNUCED 2006).

25. Ranis et Stewart (1999) distinguent deux sous-secteurs informels : un sous-secteur traditionnel de type « éponge » issu de l'excédent de main-d'œuvre agricole avec des revenus parfois inférieurs aux revenus ruraux; et un sous-secteur informel satellite du secteur urbain formel, aujourd'hui en cours de modernisation.

26. Ces trappes à sous-emploi ont été pointées par Todaro (1971) il y a quarante ans.

27. Une abondante littérature a été consacrée à ce biais urbain et à ses effets sur le développement depuis les premiers travaux de Lipton (1977) et la contribution de Bates (1981), qui néglige certains facteurs liés aux particularismes de chaque pays. Ce biais s'explique avant tout par l'héritage de la colonisation et par un contexte politique défavorable, dans lequel les populations rurales africaines ont eu des difficultés à faire entendre leur voix, tandis que les électeurs urbains étaient plus directement à même de faire pression sur les gouvernements. Des régimes politiques autoritaires, une faible menace d'insurrection communiste en zone rurale (par rapport à l'Asie), la faible densité de population et les difficultés de communication sont les principales explications avancées à la faible mobilisation des populations rurales.

28. Anderson et Masters (2009) montrent une « déprotection» tendancielle sur quarante ans tandis que d'autres pays en développement protégeaient leur agriculture, mais aussi certains pays de l'OCDE.

29. Voir entre autres Maimbo et Ratha (2005) et Lucas $(2005,2008)$. 
30. Les discussions sur la libéralisation des migrations se réduisent souvent à la libéralisation des échanges de main-d'œuvre. La principale référence est ici le mode 4 de l'Accord général sur le commerce des services de l'OMC (AGCS) qui concerne le «mouvement des personnes physiques» (individus qui quittent leur pays d'origine pour fournir des services dans un autre, c'est-à-dire des travailleurs migrants). Sur cette perspective du commerce, voir Winters et al (2003).

31. Il ne faut pas sous-estimer les migrations entre des pays en développement. La moitié des migrants des pays en développement résident en effet dans d'autres pays en développement. Cependant, comme l'ont démontré Ratha et Shaw (2007), qui examinent ces « migrations sud-sud», $80 \%$ de ces migrations se produisent entre des pays frontaliers qui présentent des écarts de revenus relativement faibles. C'est pourquoi elles ne représentaient que 10 à $30 \%$ des gains des pays en développement provenant des travailleurs à l'étranger en 2005. Ces fortes différences de rendement entre les pays de destination sont entièrement confirmées par les enquêtes du programme RuralStruc (voir chapitre 4).

32. À titre de comparaison, l'augmentation de la population active en Asie centrale et du Sud (l'autre région en plein essor) produira 575 millions de nouveaux travailleurs sur la même période, $64 \%$ (370 millions) en zone rurale et $36 \%$ (205 millions) dans les villes.

33. Le modèle dualiste proposé par Lewis (1954), qui suggère des transferts de maind'œuvre d'un secteur traditionnel à base agricole (conjuguant faible productivité et excédent de main-d'œuvre) à un secteur urbain moderne en voie d'industrialisation, a été une contribution très importante à l'économie du développement. Voir une récente application du point de vue de Lewis sur le rôle de l'agriculture dans la transition dans Berthelier et Lipchitz (2005). 



\section{Chapitre 3}

\section{Les réalités rurales : agriculture et pauvreté}

Lorsque les équipes nationales du programme RuralStruc ont entrepris le travail d'enquête, la présomption était celle d'une forte différenciation régionale correspondant à la variabilité des types d'intégration aux marchés, des dynamismes locaux et des richesses produites (traduites en niveaux de revenus). Cette hypothèse était fondée sur l'appartenance des pays du programme aux trois mondes de l'agriculture du Rapport sur le développement dans le monde de 2008. Plus précisément, l'attente était de trouver des situations très contrastées entre des régions qui avaient été classées a priori dans des configurations distinctes - gagnantes, perdantes et intermédiaires - qui correspondaient à des opportunités différentes en termes de voies possibles de sortie de la pauvreté, et à des stades variés de transformation structurelle.

Pourtant, les résultats obtenus ont été étonnamment plus nuancés. Les différences entre les régions sont bien sûr importantes et l'écart le plus marqué est observé entre les régions d'Afrique subsaharienne et les autres régions, ce qui témoigne de niveaux très différents de richesse et de développement. Mais deux traits communs se dégagent néanmoins de cette diversité : l'importance systématique de l'agriculture dans les activités des populations rurales, et l'ampleur phénoménale de la pauvreté qui sévit presque partout, tant en valeur absolue que relative.

Ce chapitre donne un aperçu des réalités rurales dans les régions étudiées. Il examine la place de l'agriculture dans les activités et les revenus, compare les revenus ruraux estimés aux seuils de pauvreté internationaux et nationaux et présente leur distribution. Il affine ensuite les estimations de revenus et étudie la vulnérabilité alimentaire des ménages les plus pauvres. Enfin, il reprend la typologie établie par le RDM08 pour définir les principales catégories de ménages sur la base de la structure de leurs revenus, qui permet de discuter les tendances de la diversification rurale et les options possibles pour sortir de la pauvreté.

\section{Le rôle central de l'agriculture dans les différents contextes régionaux}

Les régions étudiées par le programme RuralStruc sont avant tout des régions agricoles, sans industrie extractive importante ${ }^{1}$. Elles pratiquent surtout des cultures annuelles dans lesquelles les cultures vivrières de base, principalement des céréales, 
tiennent une place essentielle. La plante cultivée est souvent le maïs au Mexique, au Nicaragua (avec le haricot) et au Kenya, le riz à Madagascar, le riz, le millet et le sorgho au Sénégal et au Mali, et le blé et le blé dur au Maroc. Les produits de base traditionnels destinés à l'exportation ou à l'agro-industrie locale sont présents dans toutes les régions, aux côtés des fruits et légumes et de l'élevage avec, dans certains cas, la fabrication de produits laitiers (voir chapitre 5).

Il n'était donc pas surprenant de constater que les ménages enquêtés sont très engagés dans l'agriculture au sens large - c'est-à-dire les cultures, l'élevage, la chasse, la pêche, la collecte de ressources naturelles, et la transformation de produits associés. La surprise est venue de la proportion d' " exploitants agricoles » - soit de ménages ruraux pratiquant des activités agricoles sur leur exploitation ${ }^{2}$ - alors qu'une plus forte proportion de ruraux pleinement engagés dans d'autres activités était anticipée. En effet, dans la majorité des régions, $95 \%$ à (plus souvent) $100 \%$ des ménages interrogés sont des exploitants agricoles (voir figure 3.1). Abstraction faite des familles d'Alaotra à Madagascar (un des principaux greniers à riz du pays), où certains ménages dépendent du seul travail salarié agricole, les deux grandes exceptions sont la région du Souss au Maroc et celle de Tequisquiapan au Mexique, qui ont toutes deux des liens étroits avec les villes et une économie locale plus diversifiée, ce qui correspond au stade de développement du pays.

Dans le Souss, 25 \% des ménages n'ont pas d'exploitation agricole. Cette diversification résulte de la proximité de plusieurs zones étudiées avec Agadir (une ville de près de 800000 habitants et la cinquième ville marocaine par la taille), de son secteur touristique et, surtout, du développement de l'emploi salarié agricole dans le secteur commercial des fruits et légumes de la plaine côtière. La région du Sotavento au Mexique présente elle aussi une proportion de ménages non agricoles un peu plus élevée que dans les autres régions étudiées (15\%), mais c'est Tequisquiapan qui offre l'exemple extrême, avec seulement $28 \%$ d'exploitants agricoles. La région enquêtée à Tequisquiapan correspond à six localités sélectionnées dans une vallée au nord de la ville de San Juan del Rio (approximativement 210000 habitants), située à $150 \mathrm{~km}$ au sud-est de la ville de Querétaro (dont la zone métropolitaine accueille près d'un million d'habitants). Dotée d'un solide réseau urbain, cette région enregistre depuis vingt ans une vive croissance ${ }^{3}$, marquée par le développement de l'agro-industrie (légumes et volailles exportés aux États-Unis) et de l'industrie manufacturière (maquiladoras et industries de haute technologie comme l'aéronautique), qui est à l'origine d'un marché du travail dynamique et a incité de nombreux ruraux à quitter l'agriculture (Rello et Morales 2002).

Les exemples du Souss et de Tequisquiapan soulignent l'importance du contexte régional et montrent son influence sur les activités des ménages ruraux. La densité de la population et le taux d'urbanisation (qui reflètent différents stades du processus de transition démographique et économique), les caractéristiques du réseau urbain (sa concentration et sa hiérarchie) et le développement des infrastructures de transport (qui détermine la fluidité des flux de personnes et de biens) exercent tous une influence sur le paysage régional. Ils contribuent à l'hétérogénéité observée des économies rurales en particulier, et à celles des différents pays et de leurs régions en général - le thème central du RDM09 sur la géographie économique (voir encadré 3.1). 


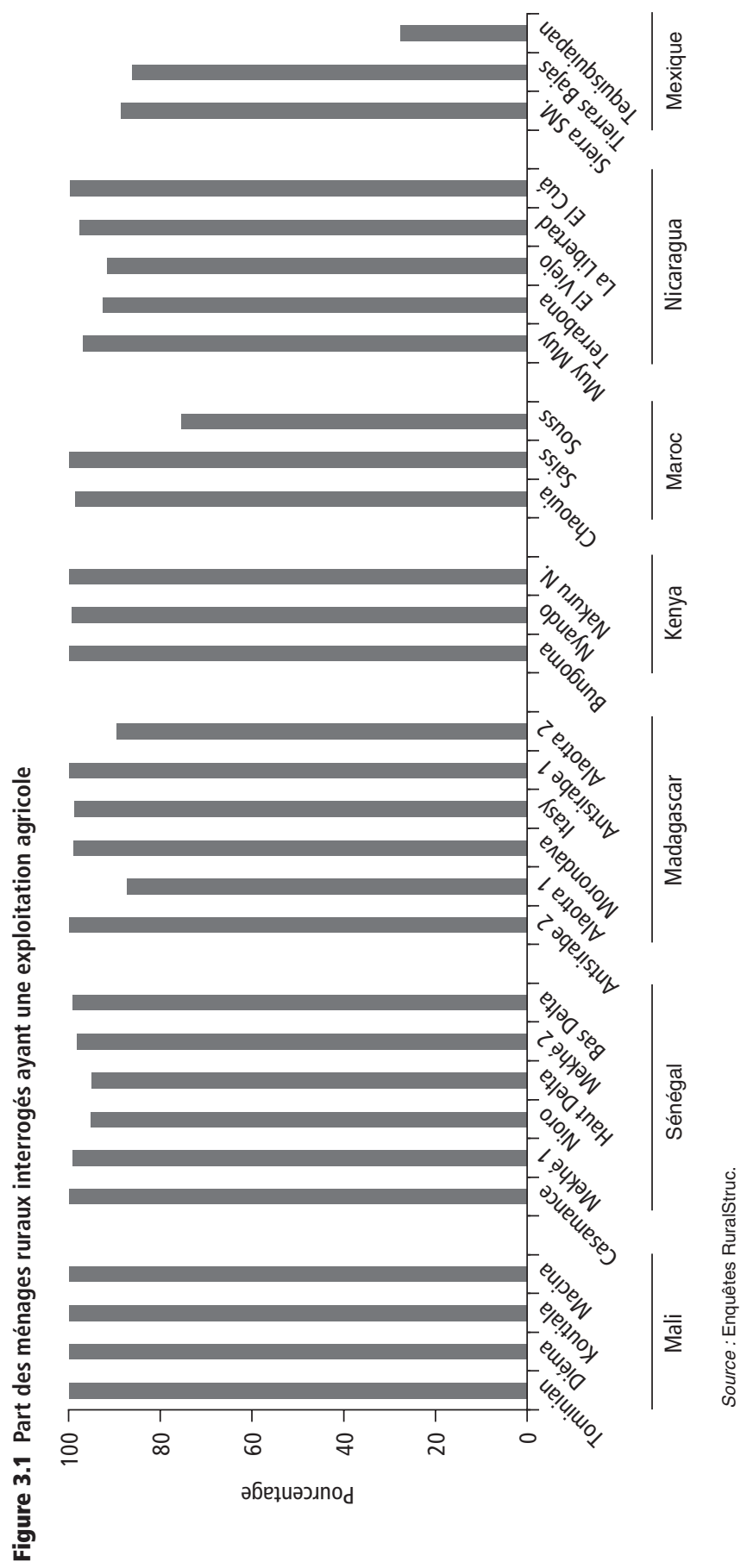




\section{GNCADRÉ 3.1}

\section{Densité, distance et division : les trois grandes caractéristiques géographiques du développement économique selon le RDM09}

Le Rapport sur le développement dans le monde de 2009 (RDM09), intitulé Repenser la géographie économique (World Bank 2008a), distingue trois dimensions géographiques du développement économique qui déterminent les forces du marché : (1) la densité (ou valeur produite au $\mathrm{km}^{2}$ ), (2) la distance (entre les régions en retard et les régions motrices où se concentre l'activité) et (3) la division (l'importance des obstacles liés aux frontières, aux monnaies, aux réglementations, à l'origine ethnique, etc.).

Les trois D - densité, distance et division - correspondent à trois échelles - locale, nationale et internationale. Ils engendrent des disparités de bien-être entre les régions et entre les pays qui peuvent déstabiliser les parties d'un pays, des nations tout entières et même certaines régions du monde. Pour réduire ces disparités, les pouvoirs publics disposent de nombreux instruments, que le RDM09 classe en trois catégories (les trois I) : (1) les institutions (réglementation foncière, du travail et du commerce), qui sont « spatialement neutres », (2) les infrastructures (qui facilitent la circulation des biens, des personnes, des services et des idées), qui sont « spatialement connectives » et (3) les interventions (comme les incitations fiscales et l'accès préférentiel au marché), qui sont « spatialement ciblées ».

Le RDM09 propose une règle empirique pour l'intégration économique : " un I pour un $\mathrm{D} »$ (un instrument par dimension) : pour un problème unidimensionnel (densité, distance ou division), des institutions spatialement neutres ; pour un défi à deux dimensions, des institutions et des infrastructures ; et pour un problème mêlant les trois dimensions, les trois instruments.

Bien que le rapport propose des outils pour analyser les asymétries, son approche standardisée et évolutionniste (voir chapitre 2) a suscité des critiques pour sa vision unilinéaire de la transition rural-urbain et pour ses nombreux angles morts, comme le rôle des marchés financiers dans la recomposition de la géographie mondiale (voir par exemple, Hart 2010).

L'accès aux marchés et aux biens publics (souvent disponibles en zone urbaine) et la facilité des contacts liée à la qualité des infrastructures de communication ont une forte influence sur la diversification des ménages ruraux. Les cartes de la figure 3.3 indiquent le temps de trajet en heures jusqu'à la ville de 50000 habitants la plus proche. Elles montrent à la fois la structure urbaine du pays et l'efficacité de son réseau de transport et révèlent une remarquable hétérogénéité entre les quatre pays d'ASS du programme RuralStruc ${ }^{4}$.

Néanmoins, et malgré de nettes différences entre ces contextes régionaux, la part moyenne du revenu des ménages provenant d'activités propres à l'exploitation agricole reste élevée dans l'échantillon RuralStruc et confirme le rôle important de l'agriculture dans les régions étudiées ${ }^{5}$. En effet, le revenu de l'exploitation représente plus de $50 \%$ du revenu global dans 22 des 30 régions et $70 \%$ ou plus dans 11 régions (figure 3.2 ). Une autre tendance, qui sera analysée plus avant, illustre l'importance de l'agriculture : la part du revenu des ménages provenant de l'exploitation agricole augmente avec la richesse régionale dans cinq des sept pays étudiés, les deux exceptions étant le Kenya et le Mexique ${ }^{6}$. 


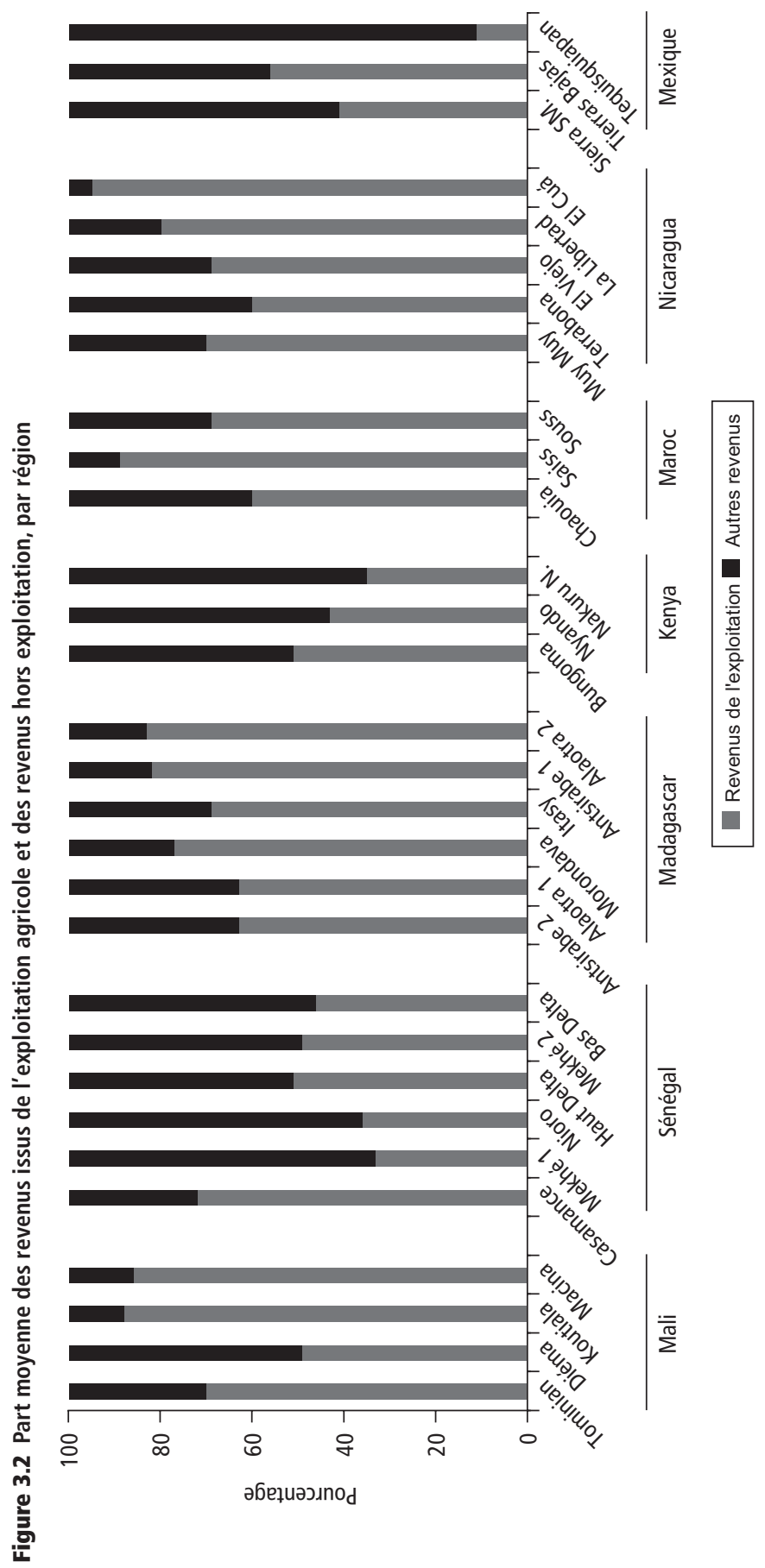


Figure 3.3 Temps de trajet jusqu'à la ville de 50000 habitants la plus proche dans les quatre pays d'ASS
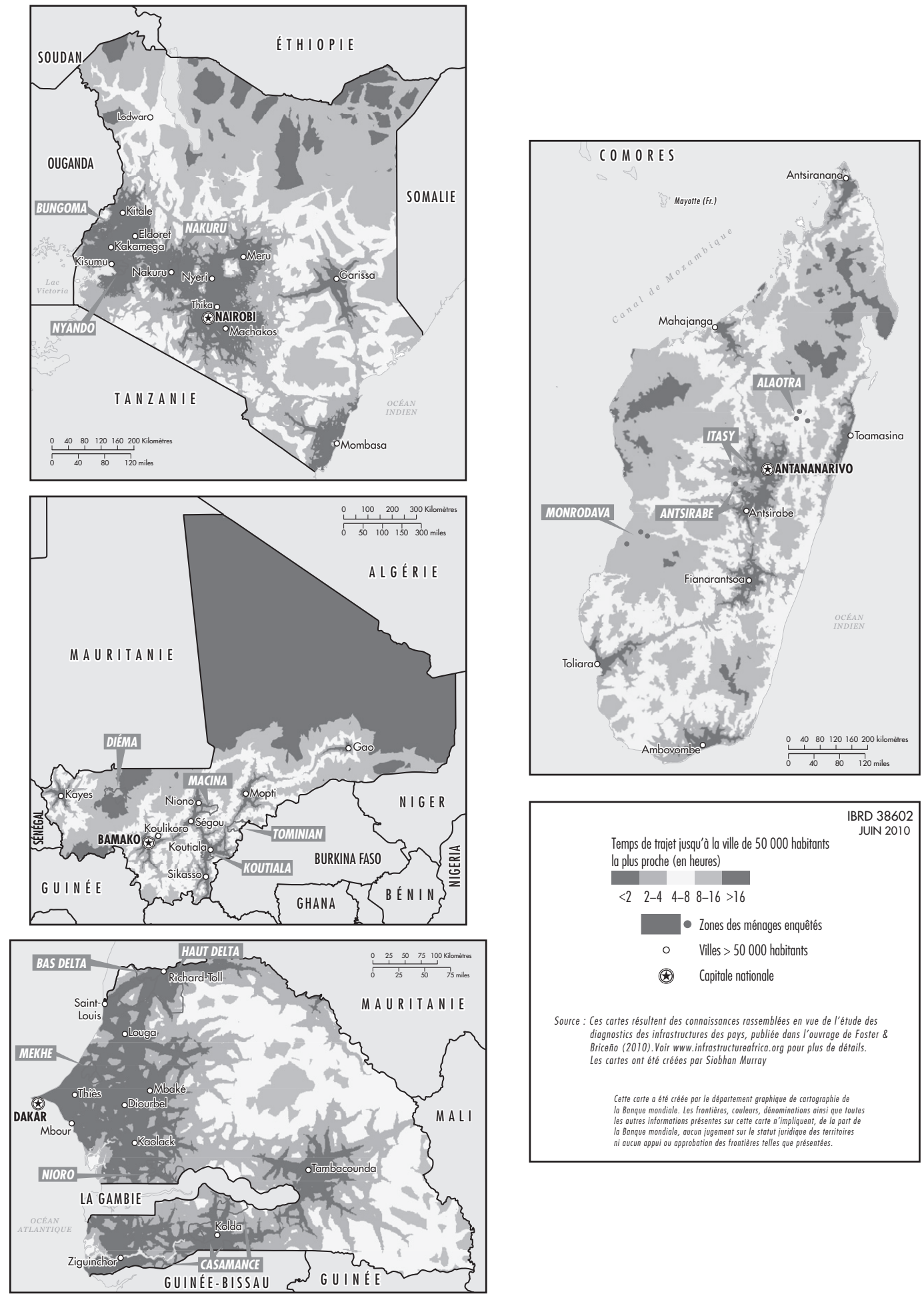


\section{ENCADRÉ 3.2}

\section{Urbanisation, réseaux de transports et moyens d'existence des ruraux}

Si dans l'ensemble, les pays d'Afrique subsaharienne font partie du premier monde à vocation agricole du RDM08 (le Sénégal, une des très rares exceptions, est classé comme un pays en mutation), les pays du programme RuralStruc présentent cependant des dynamiques économiques très différenciées. L'importance de la population et sa densité, le taux d'urbanisation et les types de réseaux urbains, ainsi que la qualité et la densité des infrastructures de communication ont tous une influence sur le degré de connexion au marché et sur les activités des ménages ruraux.

Les cartes de la figure 3.3 mettent en évidence l'importance des régions reculées et les déséquilibres régionaux du point de vue de l'accès au marché. Elles montrent aussi que les pays sont à différents stades d'urbanisation et ont des structures de peuplement divergentes. Le Mali et Madagascar présentent des situations très polarisées où le réseau de transport détermine la structure globale, tandis que le Kenya et le Sénégal illustrent des processus de densification très marqués.

Au Kenya, où l'urbanisation est très dynamique (la part de la population urbaine a bondi de $32 \%$ à $45 \%$ en dix ans), les ruraux qui vivent sur les hauts plateaux centraux, dans la partie centrale de la vallée du Rift et dans les régions à l'ouest du pays peuvent se rendre dans une ville d'au moins 50000 habitants en moins de deux heures. Nakuru North, une des régions étudiées, est très proche de Nakuru, quatrième ville du pays (population estimée à 544000 habitants en 2010) après Nairobi, Mombasa et Kisumu. Mais c'est la partie occidentale du pays qui enregistre depuis vingt ans un spectaculaire processus de densification progressive de ses zones rurales, marqué par l'émergence de deux conurbations. L'une, la conurbation occidentale, au nord de Kendu Bay sur les berges du lac Victoria, accueille près de 3,9 millions d'habitants et forme un réseau de 13 villes dont les plus grandes sont Kisumu et Bungoma. L'autre est la conurbation de Nyanza-Kisii, au sud de Kendu Bay, qui compte 2,1 millions de personnes et quatre grandes villes (Harre, Moriconi-Ebrard et Gazel 2010). La région enquêtée de Bungoma fait partie de la zone rurale de la conurbation occidentale, tandis que la région de Nyando se situe entre les deux conurbations (à l'est de Kendu Bay) et affiche, elle aussi, de fortes densités de population.

Au Sénégal, la tendance historique de peuplement de la partie occidentale du pays s'est accélérée depuis le milieu du xx siècle avec le développement du bassin arachidier et de ses grandes villes (Thiès, Kaolack, Diourbel) qui doivent leur prospérité à l'industrie de l'arachide. Toutefois, on observe depuis trente ans un déplacement progressif vers la région côtière. La majorité de la population sénégalaise se situe dans un rayon de 100 km de la côte atlantique et peut se rendre à Dakar, Mbour, Thiès ou Saint-Louis en moins de deux heures. Le contraste des densités avec le Mali voisin est remarquable.

Cependant, ces cartes ne disent rien des asymétries de distribution de la population urbaine entre les villes d'un même pays. Ainsi, l'indice de primauté (population de la plus grande ville/population de la deuxième ville) révèle le poids extrême des capitales sénégalaise et malienne (voir tableau 3.2.1) et, bien que l'urbanisation se densifie de manière générale en Afrique de l'Ouest, la situation qui persiste dans ces deux pays affaiblit leurs structures urbaines. Voir l'étude Africapolis (Denis et Moriconi-Ebrard 2009) et l'étude plus ancienne West Africa Long-Term Perspective Study (Club du Sahel-OCDE 1998). 
Tableau d'encadré 3.2.1 Niveau de concentration urbaine dans les pays du programme RuralStruc

\begin{tabular}{|c|c|c|c|c|}
\hline Pays & Première ville & Deuxième ville & $\begin{array}{c}\text { Indice } \\
\text { de primauté }\end{array}$ & $\begin{array}{c}\text { Année } \\
\text { de référence }\end{array}$ \\
\hline Kenya ${ }^{a}$ & Nairobi & Mombassa & 4,2 & $2010^{e}$ \\
\hline Madagascar ${ }^{b}$ & Antananarivo & Toamasina & 7,8 & 2005 \\
\hline Malia & Bamako & Sikasso & 11,3 & $2010^{e}$ \\
\hline Sénégala & Dakar & Thiès & 10,5 & $2010^{e}$ \\
\hline Maroc $^{\mathrm{a}}$ & Casablanca & Rabat & 1,9 & $2010^{e}$ \\
\hline Nicaragua ${ }^{b}$ & Managua & León & 6,5 & 2005 \\
\hline Mexique $^{b}$ & Mexico & Guadalajara & 4,9 & 2003 \\
\hline
\end{tabular}

Sources : a e-Geopolis/Menapolis \& Africapolis, ${ }^{\mathrm{b}}$ UnStats.

Note: ${ }^{\mathrm{e}}=$ estimations.

Les régions étudiées au Mali, à Madagascar, au Maroc et au Nicaragua sont fortement engagées dans l'agriculture alors que, sans surprise, la situation au Mexique est très différente (même si la zone de Tierras Bajas a aussi une certaine spécialisation agricole). Le Sénégal et le Kenya ont des profils différents : hormis la Casamance, qui présente de nombreuses caractéristiques voisines de celles du Mali, toutes les régions étudiées au Sénégal et au Kenya affichent un niveau élevé de revenus hors exploitation agricole - de l'ordre de $60 \%$. Ces chiffres tiennent en partie aux fortes connexions avec les villes dues aux densités plus élevées et à de meilleurs réseaux d'infrastructures (voir encadré 3.2), mais l'importance de ces activités n'implique pas nécessairement une déconnexion de l'agriculture car nombre d'entre elles ont en fait des liens avec le secteur agricole, en particulier le commerce des produits agricoles et le travail salarié dans l'agro-industrie.

\section{Une pauvreté rurale endémique}

Si l'agriculture conserve un rôle aussi important dans les régions étudiées, quelles sont leurs caractéristiques en termes de niveau et de distribution des revenus? Pour répondre à cette question, les résultats des ménages ont été agrégés au niveau régional afin de situer l'échantillon par rapport aux références existantes et les écarts de revenu entre ménages au sein chaque pays et entre les pays sont analysés ${ }^{7}$. Étant donné la méthodologie adoptée, ces comparaisons n'ont qu’une valeur indicative.

\section{Revenu moyen et niveau de pauvreté}

Les résultats des enquêtes font apparaître un large éventail de situations en termes de niveau et de distribution des revenus, qui permettent de mieux caractériser les régions, leurs similitudes et leurs différences.

Présentation générale. Le très faible niveau des revenus dans les régions étudiées est frappant, même lorsqu'on distingue les régions d'ASS, où la pauvreté est accablante, des autres régions (voir tableau 3.1 et figure 3.4$)^{8}$. Sans surprise, en raison 


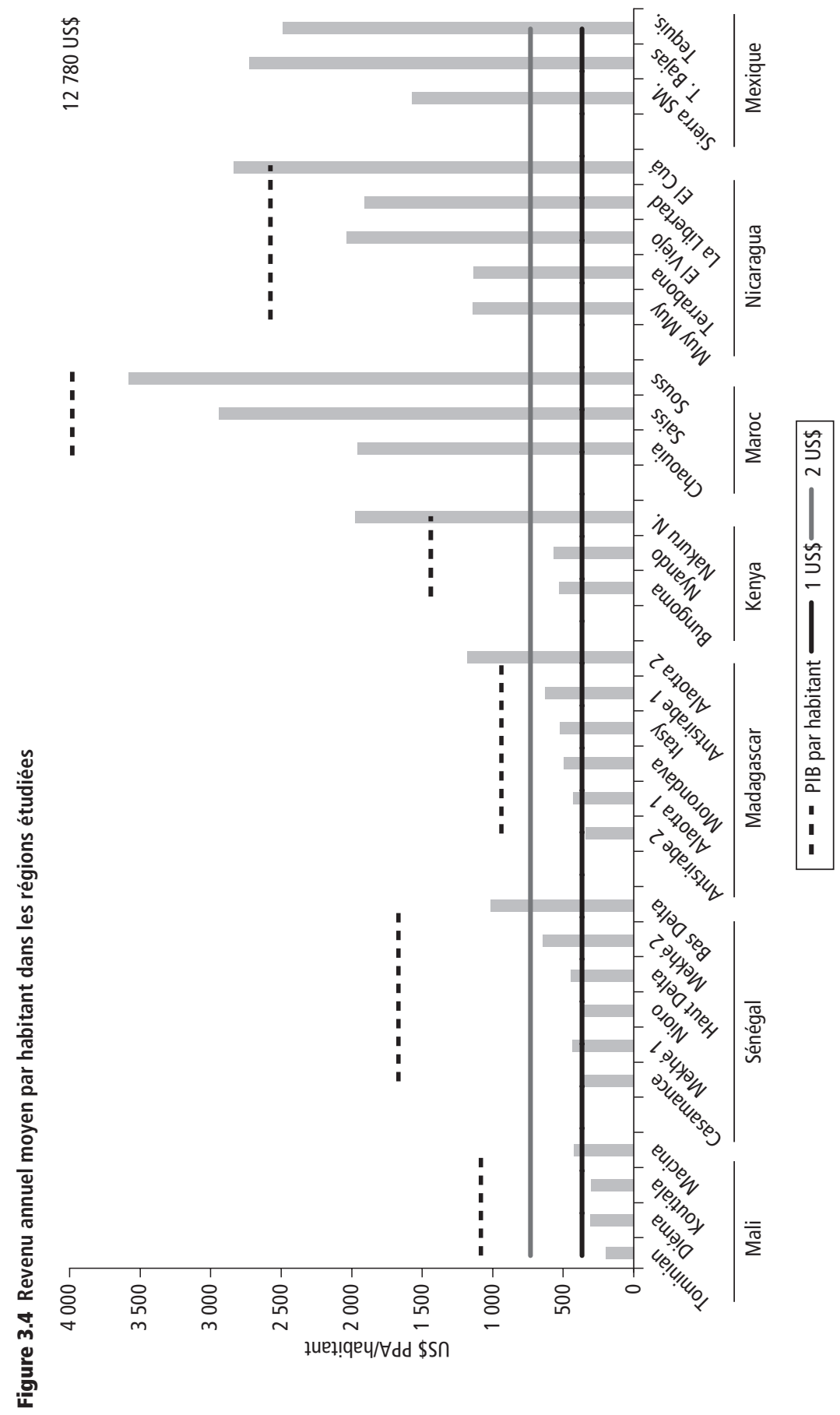




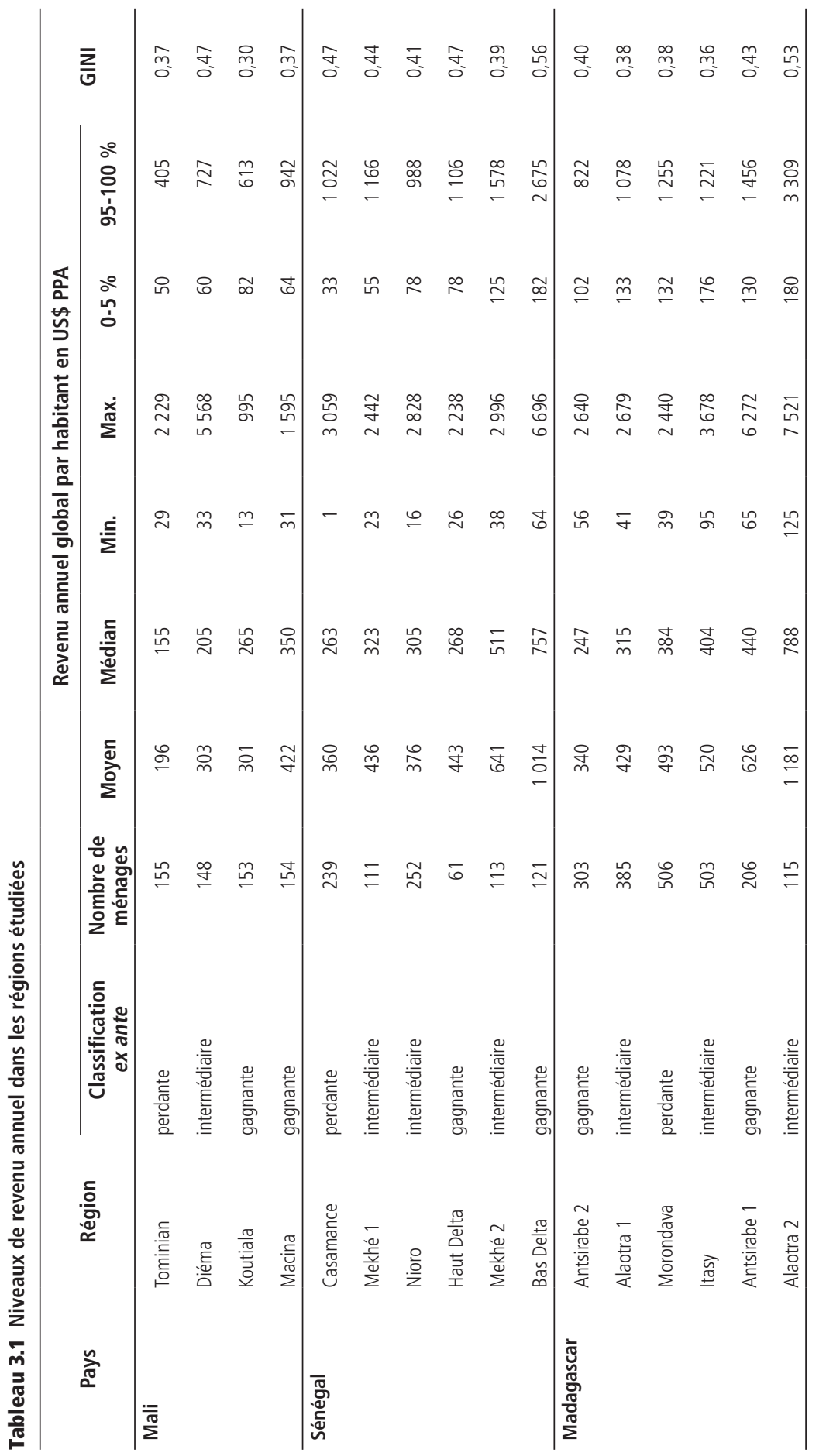




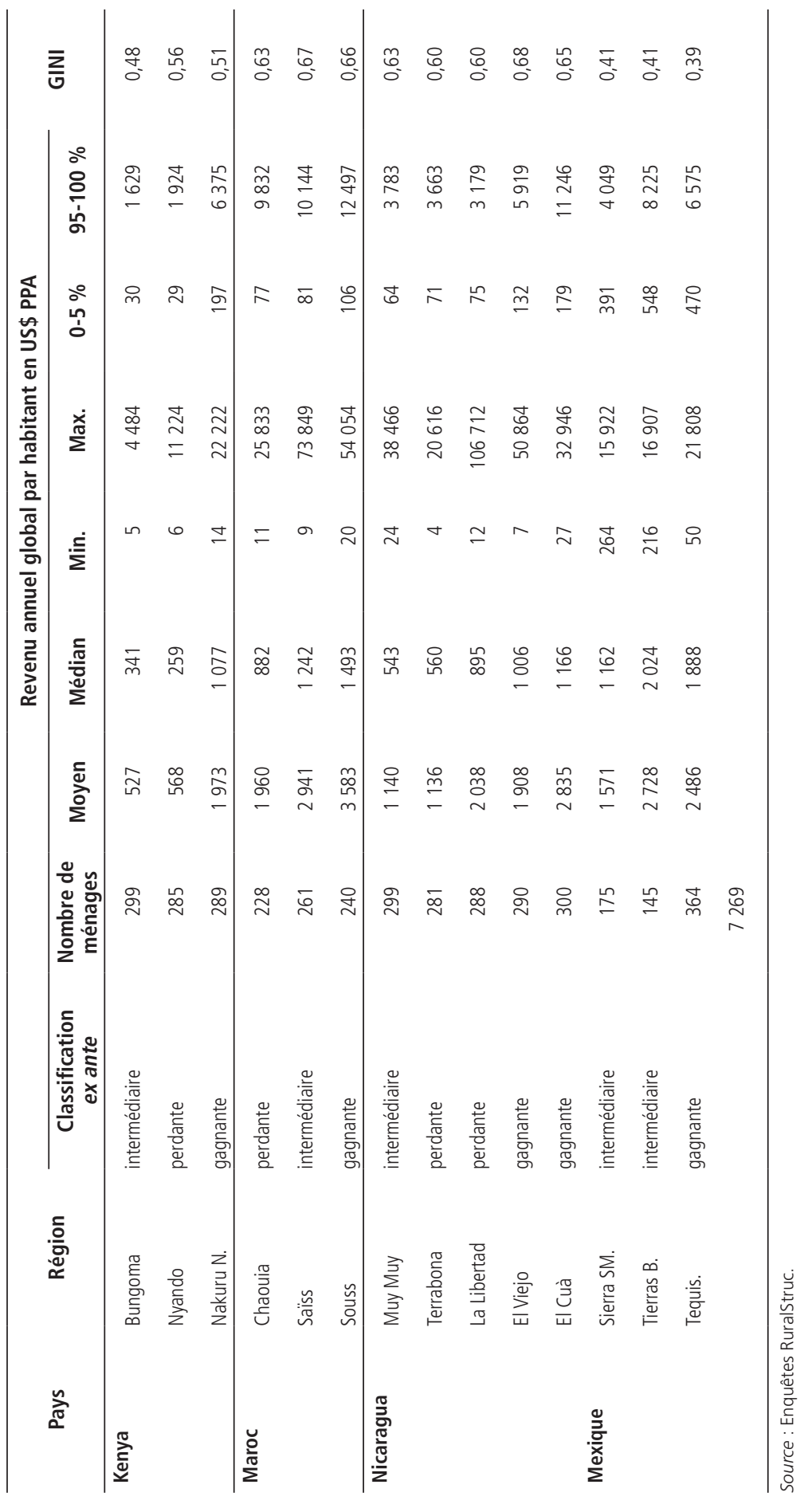


de la fracture marquée et bien connue de niveau de vie entre villes et campagnes, le revenu moyen des zones rurales enquêtées est inférieur au PIB national par habitant : seules les zones étudiées d'Alaotra 2 (Madagascar) et d'El Cuá (Nicaragua) dépassent ce seuil.

L'écart le plus élevé entre les revenus des ménages observés et le PIB par habitant publié est enregistré au Mexique, où le revenu moyen des régions étudiées est quatre à sept fois inférieur à la moyenne nationale (12 780 US\$ PPA). La situation mexicaine mérite d'être discutée car ce pays est de loin le plus avancé dans sa transformation structurelle et peut donc éclairer la discussion sur les caractéristiques du processus. L'écart observé confirme les fortes inégalités de revenus dans le pays et la distribution spatiale inégale de la pauvreté, très concentrée dans les zones rurales, ce que confirme l'indice de Gini (RS 1 Mexique) ${ }^{9}$. Cet écart tient à la difficulté à faire converger les revenus ruraux et urbains, un des problèmes structurels les plus sensibles du processus de transformation, qui pose d'ailleurs une difficulté particulière aux pays qui opèrent une transition rapide, comme la Chine (voir la discussion sur l'écart structurel au chapitre 2). Il révèle en outre une spécificité rurale qui est exacerbée par la méthodologie de l'enquête mais qui revêt aussi une dimension plus générique. En effet, l'enquête ayant porté sur des localités rurales (moins de 5000 habitants suivant la définition retenue), elle a exclu de nombreux ménages plus aisés (dont certains ménages agricoles) qui ont préféré migrer dans de gros bourgs ruraux ou de petites villes où ils ont accès à un meilleur niveau de services (RS 2 Mexique, 28). Comme on le verra aux chapitres 4 et 6 , il est ainsi plus difficile de saisir une réalité rurale en évolution dans laquelle le « rural » se fond progressivement dans l' « urbain » sous l'effet conjugué de l'exode rural et de l'urbanisation.

L'analyse des niveaux de pauvreté en référence aux seuils de pauvreté «absolue » et " relative » de 1 US\$ et 2 US\$ PPA par jour, révèle des différences radicales entre les pays d'ASS et les autres pays enquêtés ${ }^{10}$. En ASS, presque toutes les régions étudiées atteignent tout juste ou sont proches du seuil de 1 US\$, la région la plus pauvre du Mali (Tominian) étant même très en deçà. Seules les régions les plus riches du Sénégal, de Madagascar et du Kenya se situent au-dessus des 2 US\$ par jour. Si Nyando et Bungoma au Kenya sont aussi pauvres que les autres régions d'ASS, Nakuru North fait notablement exception : son revenu moyen estimé est comparable à celui des autres pays hors ASS (voir figure 3.4).

L'écart de revenu moyen par habitant entre les zones les plus pauvres et les plus riches est un bon indicateur de différenciation régionale au sein de chaque pays. L'écart le plus faible est observé au Maroc, avec un ratio de seulement 1,8, et le plus élevé à Madagascar et au Kenya $(3,5)^{11}$. Quant à la distribution des revenus, elle présente de fortes inégalités - une caractéristique courante de la plupart des situations agraires - confirmées par les revenus très élevés des $5 \%$ de ménages les plus riches, qui rappellent les limites des valeurs moyennes ${ }^{12}$. Ainsi, l'utilisation du revenu médian par habitant modifie l'importance des écarts entre régions étudiées. Bien que le classement ordinal des régions des plus pauvres aux plus riches reste identique, les profils sont plus compacts, en particulier au Maroc et au Nicaragua (voir figure 3.5). 


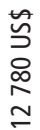
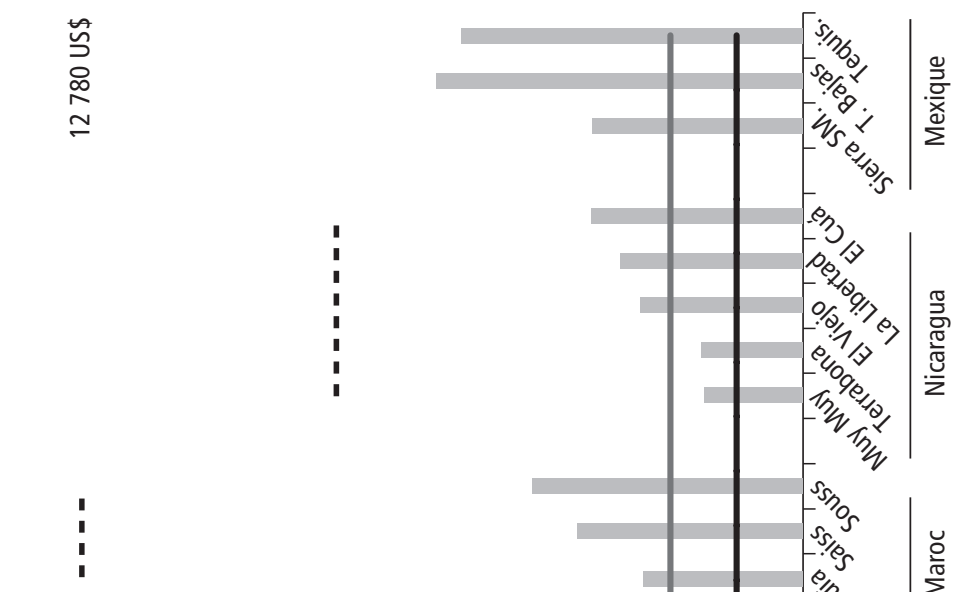

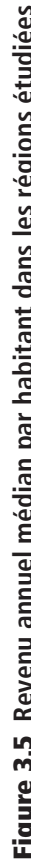
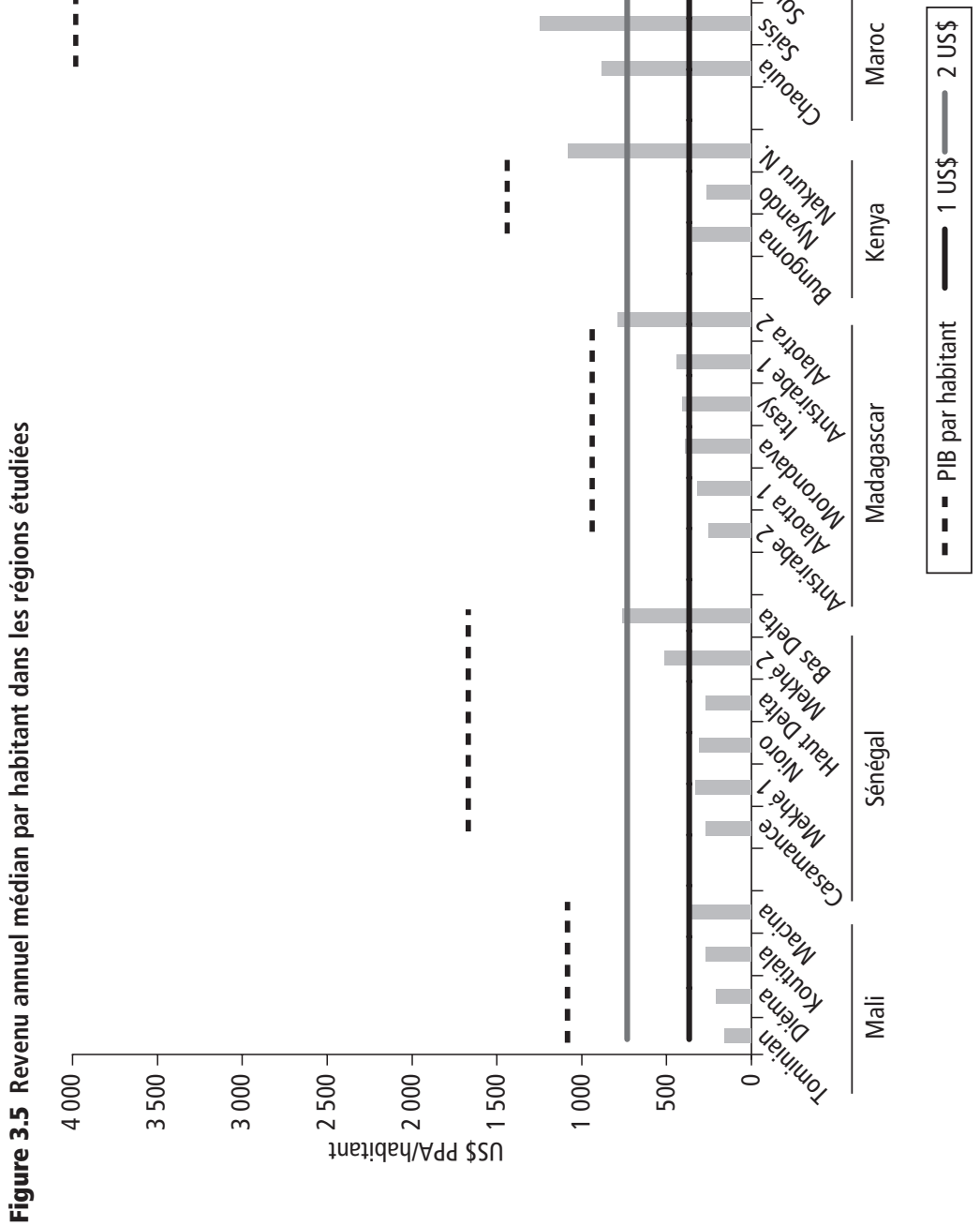
Malgré les limites de l'échantillon, les écarts de niveau et de distribution des revenus entre les zones rurales des sept pays donnent des informations sur le degré de leur transformation structurelle : en Afrique subsaharienne, qui est aux premiers stades de sa transition économique, l'écrasante majorité des ruraux est pauvre et les inégalités sont faibles (indices de Gini entre 0,35 et 0,45 ). Au Maroc et au Nicaragua, où s'opère une transition rapide, les revenus ruraux moyens sont notablement plus élevés mais avec de fortes inégalités (indices de Gini entre $0,6$ et 0,7$)$; il en résulte des différences très sensibles entre revenus moyens et revenus médians. Le Mexique, le pays dont la transformation structurelle est la plus avancée, présente les revenus ruraux médians les plus élevés dans deux des zones étudiées et des indices de Gini plus faibles $(0,4)^{13}$. Dans ce pays, la question des inégalités se pose aujourd'hui en termes de rapport villes-campagnes. La marginalisation des zones rurales - el campo - est d'ailleurs aujourd'hui une préoccupation politique majeure ${ }^{14}$.

Une apparente déconnexion entre le revenu des ménages (voir figure 3.4) et la distance au marché (voir figure 3.3) caractérise l'Afrique subsaharienne. Les revenus ne semblent pas avoir de liens avec la proximité au marché, mais ils semblent néanmoins corrélés avec la part des revenus d'exploitation par région, indiquée à la figure 3.2. D’un côté, le Mali et Madagascar se caractérisent par une forte hétérogénéité de l'accès au marché selon les régions et par l'importance de la part des revenus d'exploitation. De l'autre côté, le Sénégal et le Kenya présentent un accès plus aisé aux marchés et des activités hors exploitation plus développées (qui peuvent représenter jusqu'à $40 \%$ des revenus). Pourtant, la pauvreté des zones rurales est identique dans les quatre pays. Les trois exceptions (relatives) à cette pauvreté parmi les 19 régions africaines étudiées sont Nakuru North au Kenya, le Bas Delta au Sénégal et une sous-région d'Alaotra à Madagascar. Nakuru et le Bas Delta n'ont pas de meilleur accès aux villes que les autres régions étudiées (Bungoma et Nyando et le bassin arachidier respectivement). Cette observation rappelle une évidence : le temps de trajet aux marchés urbains n'est pas une panacée et les caractéristiques de l'urbanisation (diversification économique, biens publics, services et niveau de revenu urbain) jouent un rôle important.

Caractérisation et classification des régions étudiées. Que signifient ces résultats du point de vue de la classification ex ante des régions gagnantes, perdantes et intermédiaires utilisée par les équipes nationales pour sélectionner les régions à étudier ? En considérant le revenu moyen des ménages agrégé au niveau régional comme un bon indicateur de la richesse des ruraux et du dynamisme rural, les résultats de l'enquête sont étroitement alignés sur l'estimation ex ante (voir tableau 3.1). On note toutefois de légères différences de classement (réduites si on utilise les valeurs médianes) et quelques résultats divergents qui sont interprétés ci-après ${ }^{15}$.

Au Mali, Koutiala, au centre de la zone cotonnière, avait été choisie comme région gagnante. Elle était censée illustrer le succès de la " révolution blanche » du coton dans les zones de savane. Cependant les résultats décevants en termes de revenus révèlent une crise du secteur qui touche tous les aspects du dynamisme 
régional. La mauvaise tenue des prix internationaux du coton sur une longue période et les incertitudes découlant du retard des réformes du secteur cotonnier ont entraîné une diminution progressive des surfaces cultivées et une baisse des revenus agricoles. De plus, la grande taille des familles et les migrations vers la zone cotonnière de populations originaires d'autres régions du Mali (initialement motivées par la forte rentabilité du coton) ont été à l'origine d'une surexploitation croissante des ressources et d'une baisse des rendements. La situation de Koutiala illustre le célèbre paradoxe de Sikasso (du nom de l'autre grande zone de culture du coton au Mali), qui exprime la contradiction entre la réussite exemplaire du secteur cotonnier et le niveau relativement faible des revenus par personne dans la région de production (voir encadré 3.3).

Au Sénégal, le niveau de revenu des régions concorde généralement avec leur classification ex ante, mais les cas du bassin arachidier et du Delta méritent qu'on s'y arrête. L'un des principaux constats au Sénégal est l'absence globale de variation régionale. La Casamance est la plus pauvre des régions étudiées mais du fait d'une crise dans le secteur de l'arachide, le bassin arachidier (cheville ouvrière historique de l'économie sénégalaise) n'est désormais guère mieux loti. Mekhé 2 n'a pu obtenir des rendements économiques un peu supérieurs que par la diversification des cultures (manioc) et des activités (artisanat). Malgré sa production contractuelle de tomates, le Haut Delta est dans une situation comparable aux autres régions à la traîne, et bien plus pauvre que le Bas Delta.

Au Kenya, Nyando et Bungoma avaient été choisis pour illustrer des situations différentes. Bungoma, la région intermédiaire - dotée de meilleures ressources naturelles et dont les activités agricoles sont plus diversifiées - devait être dans une situation plus favorable. Pourtant, les revenus estimés des deux régions sont également préoccupants et ne sont guère différents. Ces régions ont toutes deux une activité très développée de production de canne à sucre, caractérisée par une faible rentabilité, et connaissent un niveau d'autoconsommation élevé. En revanche, Nakuru North, où les revenus sont 3,5 fois supérieurs, confirme son statut de région gagnante et illustre la success story kényane. Située dans la vallée du Rift, bénéficiant de bonnes conditions naturelles et d'un réseau urbain local dense et bien connecté, la région produit du maïs et des produits à forte valeur ajoutée dans le secteur du lait et de l'horticulture. Grâce à son positionnement stratégique sur le corridor Mombasa/ Nairobi/Ouganda, la ville de Nakuru offre de nombreuses opportunités d'activités non agricoles. Nakuru North se caractérise en outre par un faible ratio de dépendance (inférieur de moitié à celui des deux autres régions) qui révèle des capacités productives plus élevées par ménage et donc un potentiel de revenu supérieur.

La région d'Antsirabe à Madagascar est une région agricole très diversifiée (riz et céréales de climat tempéré, horticulture, produits laitiers), qui est bien connectée aux marchés et bénéficie d'une bonne infrastructure. Antsirabe, troisième ville du pays avec 200000 habitants environ, n'est qu'à $150 \mathrm{~km}$ de la capitale, Antananarivo, à laquelle elle est reliée par une route bitumée en bon état. La région d'Antsirabe avait été initialement sélectionnée pour illustrer une région gagnante mais des contraintes naturelles (mauvaises conditions météorologiques et problèmes phytosanitaires, notamment une maladie de la pomme de terre) 


\section{ENCADRÉ 3.3}

\section{Le paradoxe de Sikasso... et de Koutiala}

Au Mali, le coton est un secteur stratégique, souvent considéré comme le moteur du développement du sud du pays. Le secteur du coton concerne directement 275000 producteurs et près de 3 millions de personnes. Appelée " l'or blanc du Mali », la fibre de coton est la première exportation du pays depuis plusieurs décennies. Le marché se développe régulièrement depuis les années 1960, surtout depuis la dévaluation du franc CFA en 1994, avec de rares interruptions dues à des crises de gestion de la filière, dont la plus grave a été le " hold-up du coton » en 2001 lorsque la production a diminué de moitié par suite d'une grève des semis de la part des producteurs qui n'étaient pas satisfaits des nouveaux prix. Monopsone public, la Compagnie malienne de développement des textiles (CMDT), qui s'est engagée dans un difficile processus de privatisation (inachevé à ce jour), a profondément influencé le développement du secteur. La CMDT était chargée de la fourniture des intrants, de la vulgarisation agricole, de la collecte, de l'égrenage et de la commercialisation et, plus largement, du développement rural de la région cotonnière (routes, renforcement des capacités des organisations de producteurs, crédit rural, support technique, formation et programmes d'alphabétisation). Grâce à l'expansion du secteur, les agriculteurs ont pu investir en matériel et en animaux et accroître leur patrimoine - une évolution qui a contribué à la réputation du coton comme puissant moteur de réduction de la pauvreté et de développement régional. Cependant, l'Enquête malienne d'évaluation de la pauvreté (EMEP) (DNSI 2004) et d'autres études ont montré que, dans les zones de production cotonnière telles que Sikasso, la pauvreté était endémique avec des taux de malnutrition infantile parmi les plus élevés du pays. Les principales caractéristiques de ce paradoxe, selon Wodon et al. (2005) et Mesplé-Somps et al. (2008), sont les suivantes:

La pauvreté dans les régions de culture du coton est globalement moins marquée que dans d'autres régions.

La consommation des ménages est très sensible aux prix du coton et aux volumes produits et à d'autres conditions qui affectent l'agriculture locale, en particulier les précipitations. Le fait que l'enquête EMEP ait été réalisée en 2001 - année de la grève du coton - a eu une influence directe sur ses résultats.

Les producteurs de coton maliens sont clairement mieux équipés en biens durables (bicyclettes, motos, radios, téléviseurs) que les agriculteurs d'autres régions. Cet équipement prolonge les bénéfices de la production cotonnière sur le long terme, quelles que soient les circonstances d'une année particulière. Elle reflète aussi l'accès préférentiel au crédit dans le secteur cotonnier.

Le niveau d'instruction est généralement plus élevé dans les régions cotonnières, aussi bien au niveau de l'école primaire que de l'alphabétisation des adultes.

Le coton étant réputé pour sa rentabilité monétaire, Sikasso est la seule région après la capitale, Bamako, qui présente un flux migratoire net positif. Cependant, les migrations ont affecté le revenu par habitant et la région est en quelque sorte victime de son succès.

Si ces indicateurs peuvent donner une image positive de la zone cotonnière, il ne faut pas perdre de vue que le bien-être y est très dépendant des prix et assez fragile à long terme, compte tenu de la dégradation des ressources naturelles.

(Suite de l'encadré page suivante) 
Les résultats de la phase 2 du programme RuralStruc renforcent ces constats. Le ratio de dépendance à Koutiala est le plus élevé des quatre régions étudiées, ce qui réduit les effets positifs de la production cotonnière sur le revenu moyen. Bien que le prix du coton ait été bas sur la période de référence de l'enquête (campagne 2006-2007), le niveau de revenu dans la région cotonnière de Koutiala était comparable à celui de la région de Diéma, une zone reculée en culture sèche, caractérisée par un niveau élevé d'émigration internationale. Cependant, les producteurs de coton de Koutiala s'en sortent mieux que ceux de la zone de Tominian, la plus pauvre des zones étudiées. Ces résultats décevants au plan des revenus masquent toutefois un aspect important : dans les régions cotonnières, les agriculteurs sont généralement moins vulnérables parce qu'ils sont mieux équipés et ont un meilleur niveau de capitalisation, surtout en bétail, qui fait office de ressource-tampon.

Sources: RS 2 Mali ; communication avec l'équipe Mali du programme RuralStruc, 2009.

ont considérablement pesé sur les rendements, et en conséquence sur les revenus agricoles, lors de la campagne qui a fait l'objet de l'enquête. En outre, l'analyse a révélé que la région est très hétérogène, avec des zones reculées connaissant des difficultés de commercialisation qui se traduisent par une part d'autoconsommation plus forte et des niveaux de rémunération plus faibles. Ces facteurs ont conduit à distinguer deux sous-régions pour l'interprétation des résultats.

Au Nicaragua, la surprise est venue des deux régions dédiées à la production animale - Muy Muy et La Libertad - qui étaient censées illustrer des situations différentes. Muy Muy (située dans ce qui est appelé la « voie lactée » ou ceinture de produits laitiers) avait été choisie comme région intermédiaire en raison du développement de filières de produits laitiers intégrées, mais les estimations de revenus ont révélé une réalité plus âpre que prévu, qui tient principalement au fait que les agriculteurs ne bénéficient pas des hausses de prix du lait, captées en aval. La Libertad au contraire, qui avait été sélectionnée comme une région perdante en raison de nombreuses contraintes - comme sa localisation dans une région montagneuse reculée, une infrastructure de transport insuffisante et un manque d'investissements publics -, s'est révélée dans une situation de revenus nettement plus favorable, en raison notamment de tailles d'exploitation plus grandes et de l'existence de réseaux de vente de fromage affiné à la ferme.

Enfin, au Mexique, les résultats agrégés de la région du Sotavento se sont révélés comme prévu inférieurs à ceux de Tequisquiapan. Cependant et paradoxalement, le revenu moyen de la sous-région de Tierras Bajas est plus élevé que celui de Tequisquiapan, la région gagnante, ce qui prouve que la rentabilité de la production intensive de maïs peut être supérieure et plus lucrative à celle d'une très large spécialisation dans des activités non agricoles (voir plus loin et l'encadré 5.5).

Distribution des revenus ruraux. Un examen plus approfondi de la distribution des revenus confirme le net écart entre les pays d'ASS et les autres et l'importance des inégalités intrarégionales. L'agrégation des résultats des enquêtes au niveau national et leur répartition par classes de revenus (définies comme des intervalles de 1 US\$ PPA) mettent en évidence des distributions très différentes (voir figures 3.6 et 3.7). 
Figure 3.6 Distribution des ménages par classe de revenus dans les pays d'ASS

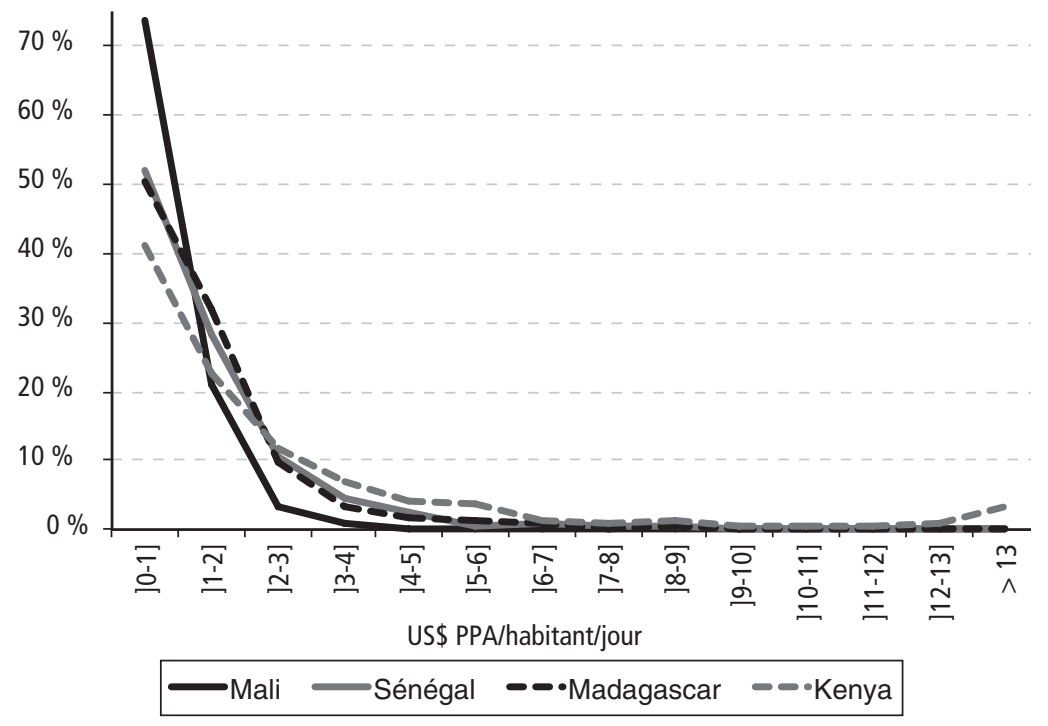

Source : Enquêtes RuralStruc.

Figure 3.7 Distribution des ménages par classe de revenus dans les pays étudiés hors ASS

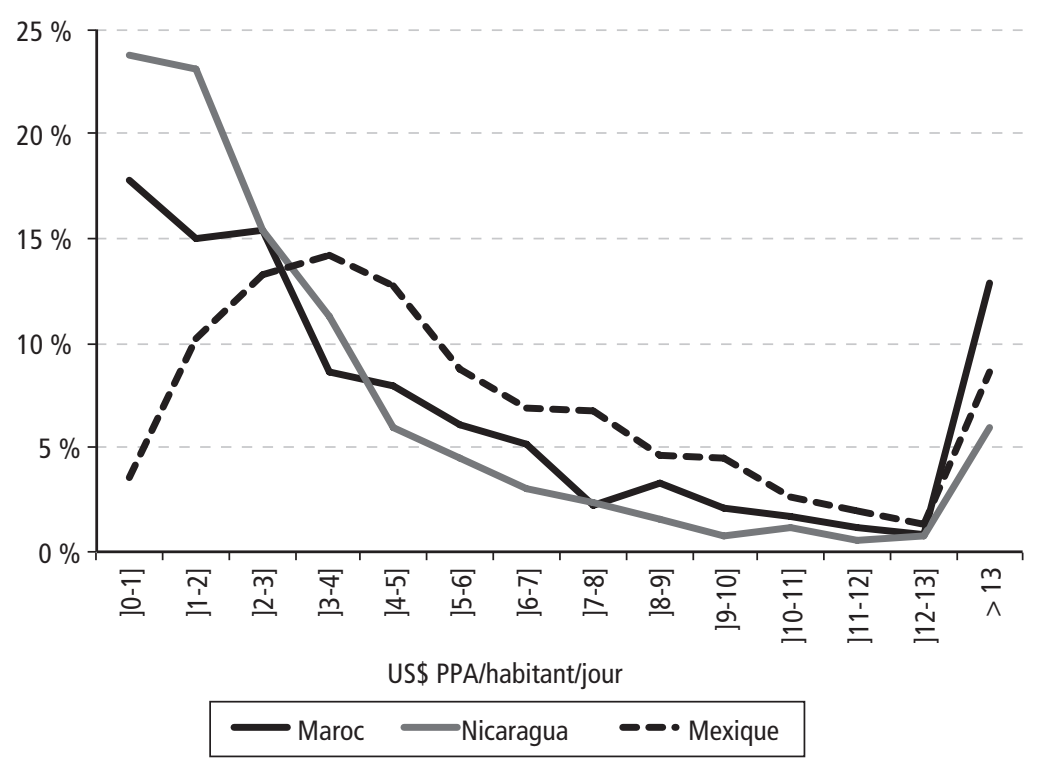

Source : Enquêtes RuralStruc. 
Dans l'échantillon, l'ampleur de la pauvreté absolue (1 US\$/jour/personne) va de $3 \%$ de la population dans les régions mexicaines à $74 \%$ dans les régions maliennes. Dans les régions d'ASS étudiées, $90 \%$ à $95 \%$ des ménages se situent dans les trois premières classes (la situation du Kenya étant un peu plus favorable et celle du Mali moins favorable). Au Mexique, au Nicaragua et au Maroc, la distribution est plus lisse et l'échantillon mexicain présente une structure très différente, qui culmine dans la classe de revenus 3-4 US\$. Dans les trois pays hors ASS, des revenus par personne et par jour supérieurs à 13 US\$ sont relativement courants (entre $5 \%$ et $15 \%$ de l'échantillon).

Afin de mieux caractériser les régions et la structure de leurs revenus, les résultats ont également été représentés par quintiles de ménages formant chacun $20 \%$ de l'échantillon (voir figures 3.8 et 3.9). Cette répartition apporte un nouvel éclairage sur la réalité rurale des régions étudiées.

L'ampleur de la pauvreté des premiers quintiles, qui est considérable, représente le problème le plus préoccupant. Les revenus par habitant les plus faibles sont observés dans les régions les plus pauvres du Mali, du Sénégal et du Kenya, avec une moyenne annuelle de 64 US\$ PPA à Tominian, de 54 US\$ PPA en Casamance et respectivement de 51 US\$ et 61 US\$ à Nyando et Bungoma. Ces valeurs ne représentent que $15 \%$ du seuil de pauvreté absolue de 1 US\$ par jour. À Madagascar, les ménages des premiers quintiles sont un peu mieux lotis, avec un revenu annuel avoisinant 150 US\$. Une surprise de taille vient des régions les plus pauvres du Maroc et du Nicaragua, qui sont aussi pauvres que les régions étudiées à Madagascar. Hormis le Mexique, le revenu du premier quintile est toujours inférieur à 1 US\$ par jour.

Les distributions par quintile présentent deux caractéristiques communes :

Le revenu global moyen par personne augmente de manière assez linéaire du quintile 1 au quintile 4 (le revenu du quintile $n$ étant de 1,3 à 2 fois le revenu du quintile $n-1$ ), tandis qu'un écart plus marqué est observé pour le quintile 5 (le revenu du quintile 5 représentant respectivement 2,7 et 5,4 fois le revenu du quintile 4 à Diéma, au Mali, et à El Viejo, au Nicaragua).

Le profil du quintile 5 diffère d'une région à l'autre mais la distribution des revenus à l'intérieur de ce quintile indique clairement le même phénomène dans de nombreuses régions : la moyenne du quintile 5 est tirée vers le haut par une poignée de ménages mieux lotis qui bénéficient de conditions sociales et économiques très particulières (par exemple l'envoi ponctuel de fonds importants par des travailleurs migrants, des loyers perçus de locations immobilières, ou une dotation exceptionnellement forte en terres et en capital qui se traduit par une production agricole plus élevée $)^{16}$.

\section{Analyse détaillée des classes de revenu}

Le recours à une approche par équivalent-adulte puis une analyse de la sécurité alimentaire par la conversion du revenu en kilocalories permettent d'étudier plus précisément la réalité économique des ménages enquêtés. Le patrimoine des exploitants agricoles et des ménages dont le chef de famille est une femme est ensuite comparé aux moyennes de l'échantillon. 


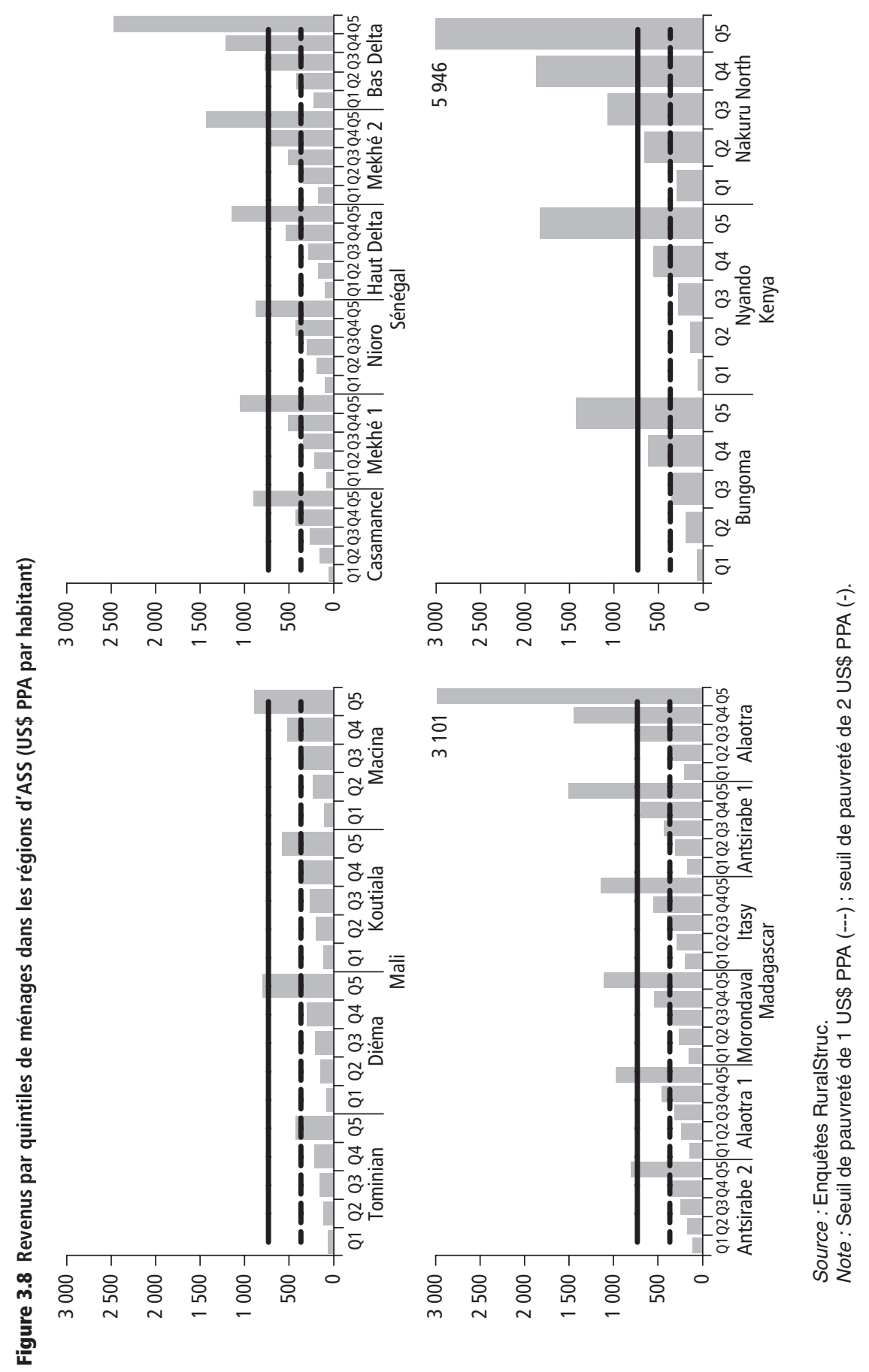




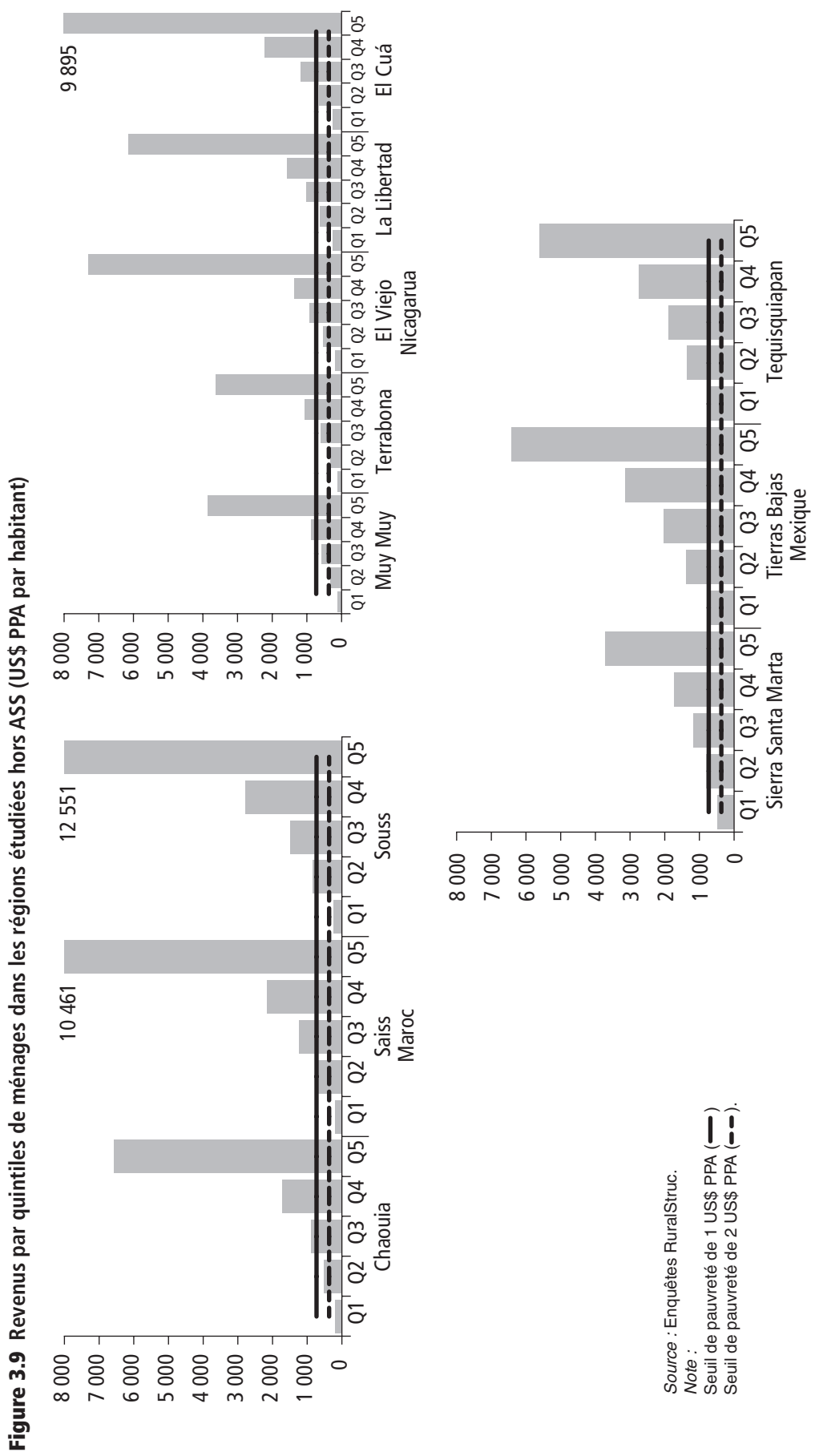


Utilisation de ratios par équivalent-adulte. Dans les sections précédentes, des ratios par habitant ont été utilisés pour comparer les résultats des enquêtes aux seuils de pauvreté et au PIB qui sont communément exprimés par tête. L'utilisation de ratios par équivalent-adulte (EqA) permet d'affiner l'analyse en tenant compte des différences très importantes constatées dans la structure et la composition des ménages et qui existent entre les ménages, selon les régions et les pays.

Une abondante littérature est consacrée aux échelles d'équivalence; le programme RuralStruc a adopté une conversion fondée sur les besoins nutritionnels par âge et par sexe. Dans la mesure où cette approche surestime la place de l'alimentation par rapport aux autres besoins, elle pénalise les ménages à revenus élevés, mais elle correspond à la réalité structurelle de la plupart des ménages enquêtés, au sein desquels les dépenses alimentaires et l'autoconsommation tiennent une place essentielle ${ }^{17}$.

Les différences de structure des ménages dépendent de la dynamique démographique et elles sont accentuées par les structures sociales et les habitudes culturelles propres à chaque pays. La figure 3.10 fait apparaître de fortes différences entre les pays d'Afrique de l'Ouest, caractérisés par des familles nombreuses, et les autres pays, qui ont généralement des familles nucléaires classiques. Les grandes structures familiales traditionnelles du Mali et du Sénégal - qui rassemblent plusieurs familles nucléaires sous l'autorité d'un ancien, le plus souvent le chef de lignée et détenteur des droits fonciers - jouent encore un rôle économique central.

Les différences de taille et de structure se traduisent par différents ratios de dépendance, qui ont une incidence directe sur la capacité de production (nombre de membres du ménage économiquement actifs/membres inactifs), les structures de consommation et le revenu disponible exprimé en EqA. Comme le montre le tableau 3.2, les ratios de dépendance élevés constatés dans les pays d'ASS confirment le poids des jeunes et témoignent de la transition démographique inachevée du sous-continent (voir chapitre 2).

En ASS, le ratio de dépendance des ménages enquêtés avoisine 1,1 à 1,2 au Mali et à Madagascar, 1,0 à 1,1 au Sénégal et atteint 1,3 au Kenya, excepté à Nakuru, dont la situation est particulièrement atypique ${ }^{18}$. Les autres pays de l'échantillon sont bien plus avancés dans le processus de transition démographique qui se traduit par des ratios inférieurs : c'est le cas au Maroc et dans deux des régions étudiées au Mexique ; mais le Nicaragua et la Sierra de Santa Marta du Sotavento mexicain s'écartent de ce schéma ${ }^{19}$. Ces différences structurelles sont importantes au plan de la capacité productive et en référence aux défis immédiats liés à l'augmentation de la population active. Cette conversion en équivalent-adulte améliore la comparabilité entre les revenus moyens et entraîne une augmentation de $14 \%$ à $28 \%$ des niveaux de revenu moyen selon les régions.

Les ménages dotés d'une exploitation agricole sont-ils mieux lotis ? Les ménages enquêtés sont principalement des exploitants agricoles. Quel est leur niveau de revenu estimé par rapport aux ménages sans exploitation ? La comparaison des deux catégories de ménages, présentée au tableau 3.3, produit des résultats inattendus. L' «agriculteur pauvre » est une figure courante dans les zones 


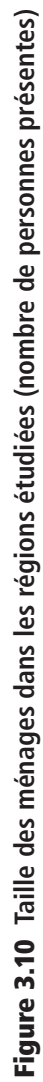

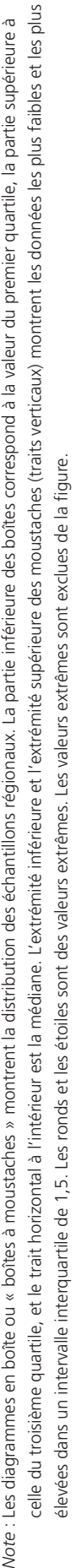


Tableau 3.2 Structure des ménages et revenu par équivalent adulte

\begin{tabular}{|c|c|c|c|c|c|c|c|}
\hline \multirow{2}{*}{$\mathrm{Pay}$} & & \multicolumn{2}{|c|}{ Taille du ménage } & \multirow{2}{*}{$\begin{array}{l}\text { Ratio de } \\
\text { dépendance }\end{array}$} & \multicolumn{2}{|c|}{$\begin{array}{l}\text { Revenu total } \\
\text { en US\$ PPA }\end{array}$} & \multirow{2}{*}{$\begin{array}{c}\text { Différence } \\
\%\end{array}$} \\
\hline & & $\begin{array}{l}\text { Nombre de } \\
\text { personnes }\end{array}$ & $\begin{array}{c}\text { Nombre } \\
d^{\prime} E q A\end{array}$ & & $\begin{array}{l}\text { par } \\
\text { tête }\end{array}$ & $\begin{array}{l}\text { par } \\
\text { EqA }\end{array}$ & \\
\hline \multirow[t]{4}{*}{ Mali } & Tominian & 11,1 & 9,3 & 1,09 & 196 & 234 & 19 \\
\hline & Diéma & 18,8 & 15,3 & 1,19 & 303 & 368 & 21 \\
\hline & Koutiala & 14,8 & 12,1 & 1,25 & 301 & 368 & 22 \\
\hline & Macina & 12,9 & 10,5 & 1,15 & 422 & 516 & 22 \\
\hline \multirow[t]{6}{*}{ Sénégal } & Casamance & 14,3 & 11,8 & 1,15 & 360 & 439 & 22 \\
\hline & Mekhé 1 & 14,7 & 12,2 & 0,99 & 436 & 527 & 21 \\
\hline & Nioro & 11,8 & 9,5 & 1,15 & 376 & 484 & 29 \\
\hline & Haut Delta & 12,1 & 10,1 & 0,85 & 443 & 524 & 18 \\
\hline & Mekhé 2 & 15,0 & 12,4 & 1,04 & 641 & 769 & 20 \\
\hline & Bas Delta & 10,7 & 9,0 & 1,00 & 1014 & 1205 & 19 \\
\hline \multirow[t]{6}{*}{ Madagascar } & Antsirabe 2 & 5,8 & 4,8 & 1,19 & 340 & 409 & 20 \\
\hline & Alaotra 1 & 5,2 & 4,4 & 1,01 & 429 & 506 & 18 \\
\hline & Morondava & 5,5 & 4,5 & 1,23 & 493 & 597 & 21 \\
\hline & Itasy & 5,5 & 4,5 & 1,21 & 520 & 622 & 20 \\
\hline & Antsirabe 1 & 5,7 & 4,8 & 1,21 & 626 & 744 & 19 \\
\hline & Alaotra 2 & 6,0 & 5,1 & 0,90 & 1181 & 1346 & 14 \\
\hline \multirow[t]{3}{*}{ Kenya } & Bungoma & 6,7 & 5,6 & 1,30 & 527 & 641 & 22 \\
\hline & Nyando & 6,3 & 5,4 & 1,35 & 568 & 660 & 16 \\
\hline & Nakuru N. & 6,5 & 5,7 & 0,61 & 1973 & 2258 & 14 \\
\hline \multirow[t]{3}{*}{ Maroc } & Chaouia & 7,1 & 6,1 & 0,68 & 1960 & 2280 & 16 \\
\hline & Saïss & 6,6 & 5,8 & 0,59 & 2941 & 3419 & 16 \\
\hline & Souss & 5,8 & 5,1 & 0,57 & 3583 & 4131 & 15 \\
\hline \multirow[t]{5}{*}{ Nicaragua } & Muy Muy & 5,8 & 4,7 & 1,02 & 1140 & 1417 & 24 \\
\hline & Terrabona & 5,5 & 4,5 & 0,84 & 1136 & 1458 & 28 \\
\hline & El Viejo & 5,6 & 4,5 & 0,94 & 2038 & 2575 & 26 \\
\hline & La Libertad & 5,8 & 4,8 & 0,89 & 1908 & 2329 & 22 \\
\hline & El Cuá & 6,0 & 4,9 & 1,00 & 2835 & 3610 & 27 \\
\hline \multirow[t]{3}{*}{ Mexique } & Sierra SM. & 4,6 & 4,0 & 0,85 & 1571 & 1824 & 16 \\
\hline & Tierras Bajas & 4,3 & 3,7 & 0,63 & 2728 & 3144 & 15 \\
\hline & Tequis. & 4,6 & 3,9 & 0,61 & 2486 & 2879 & 16 \\
\hline
\end{tabular}

Source : Enquêtes RuralStruc. 
Tableau 3.3 Revenus des ménages avec et sans exploitation agricole

\begin{tabular}{|c|c|c|c|c|c|c|c|c|c|}
\hline \multirow{3}{*}{ Pays } & & \multicolumn{4}{|c|}{ Ménages avec exploitation } & \multicolumn{4}{|c|}{ Ménages sans exploitation } \\
\hline & & \multicolumn{2}{|c|}{ Observations } & \multicolumn{2}{|c|}{ US\$ PPA par EqA } & \multicolumn{2}{|c|}{ Observations } & \multicolumn{2}{|c|}{ US\$ PPA par EqA } \\
\hline & & Nombre & $\%$ & $\begin{array}{l}\text { Revenu } \\
\text { moyen }\end{array}$ & $\begin{array}{l}\text { Revenu } \\
\text { médian }\end{array}$ & Nombre & $\%$ & $\begin{array}{l}\text { Revenu } \\
\text { moyen }\end{array}$ & $\begin{array}{l}\text { Revenu } \\
\text { médian }\end{array}$ \\
\hline \multirow[t]{4}{*}{ Mali } & Tominian & 155 & 100 & 234 & 187 & 0 & 0 & - & - \\
\hline & Diéma & 148 & 100 & 368 & 252 & 0 & 0 & - & - \\
\hline & Koutiala & 153 & 100 & 368 & 318 & 0 & 0 & - & - \\
\hline & Macina & 154 & 100 & 516 & 418 & 0 & 0 & - & - \\
\hline \multirow[t]{6}{*}{ Sénégal } & Casamance & 239 & 100 & 439 & 316 & 0 & 0 & - & - \\
\hline & Mekhe 1 & 110 & 99 & 531 & 394 & 1 & 1 & 120 & 120 \\
\hline & Nioro & 240 & 95 & 460 & 358 & 12 & 5 & 972 & 585 \\
\hline & Haut Delta & 58 & 95 & 525 & 307 & 3 & 5 & 489 & 527 \\
\hline & Mekhe 2 & 111 & 98 & 775 & 609 & 2 & 2 & 448 & 448 \\
\hline & Bas Delta & 120 & 99 & 1212 & 889 & 1 & 1 & 421 & 421 \\
\hline \multirow[t]{6}{*}{ Madagascar } & Antsirabe 2 & 303 & 100 & 409 & 296 & 0 & 0 & - & - \\
\hline & Alaotra 1 & 336 & 87 & 526 & 388 & 49 & 13 & 373 & 321 \\
\hline & Morondava & 501 & 99 & 597 & 469 & 5 & 1 & 591 & 676 \\
\hline & Itasy & 497 & 99 & 625 & 490 & 6 & 1 & 373 & 250 \\
\hline & Antsirabe 1 & 206 & 100 & 744 & 525 & 0 & 0 & - & - \\
\hline & Alaotra 2 & 103 & 90 & 1455 & 1052 & 12 & 10 & 405 & 369 \\
\hline \multirow[t]{3}{*}{ Kenya } & Bungoma & 299 & 100 & 641 & 429 & 0 & 0 & - & - \\
\hline & Nyando & 283 & 99 & 661 & 306 & 2 & 1 & 495 & 495 \\
\hline & Nakuru N. & 289 & 100 & 2258 & 1213 & 0 & 0 & - & - \\
\hline \multirow[t]{3}{*}{ Maroc } & Chaouia & 225 & 99 & 2280 & 1002 & 3 & 1 & 2309 & 1890 \\
\hline & Saïss & 261 & 100 & 3419 & 1503 & 0 & 0 & - & - \\
\hline & Souss & 181 & 75 & 4758 & 2122 & 59 & 25 & 2208 & 1157 \\
\hline \multirow[t]{5}{*}{ Nicaragua } & Muy Muy & 290 & 97 & 1436 & 670 & 9 & 3 & 803 & 734 \\
\hline & Terrabona & 260 & 93 & 1457 & 690 & 21 & 7 & 1470 & 1081 \\
\hline & El Viejo & 264 & 92 & 2678 & 1176 & 24 & 8 & 1440 & 1279 \\
\hline & La Libertad & 283 & 98 & 2353 & 1251 & 7 & 2 & 1350 & 1269 \\
\hline & El Cuá & 299 & 100 & 3619 & 1428 & 1 & 0 & 995 & 995 \\
\hline \multirow[t]{4}{*}{ Mexique } & Sierra SM. & 155 & 89 & 1937 & 1444 & 20 & 11 & 947 & 645 \\
\hline & T. Bajas & 125 & 86 & 3383 & 2506 & 20 & 14 & 1651 & 1158 \\
\hline & Tequis. & 101 & 28 & 3697 & 2873 & 263 & 72 & 2565 & 2055 \\
\hline & & 6749 & & & & 520 & & & \\
\hline
\end{tabular}

Source : Enquêtes RuralStruc. 
rurales des pays en développement et on pourrait penser que les ménages qui n'ont que des activités rurales non agricoles ont un avantage en termes de revenus. Pourtant il n'en est rien. Dans les six régions où plus de $10 \%$ des ménages enquêtés n'ont pas d'exploitation agricole, les exploitants ont un revenu deux fois supérieur en moyenne à celui des ménages qui n’ont pas d'exploitation (excepté Tequisquiapan, où l'écart est moins élevé).

La situation se comprend très bien à Madagascar, où les familles qui n'ont pas d'accès foncier comme à Alaotra sont plus pauvres et vivent principalement de salaires agricoles peu élevés. Le cas de la Sierra de Santa Marta dans la région mexicaine du Sotavento est comparable : les ménages sans exploitation ont très peu de sources de revenus dans cette région assez reculée. En revanche, les situations du Souss (Maroc) et de Tierras Bajas et Tequisquiapan (Mexique) sont paradoxales car on aurait pu anticiper des revenus plus élevés des activités non agricoles. Ce résultat surprenant de l'enquête tempère l'idée courante d'activités rurales non agricoles dynamiques. Nous y reviendrons au chapitre $4^{20}$.

Situation économique des ménages dont le chef de famille est une femme. À l'exception des deux pays d'Afrique de l'Ouest et du Maroc, les ménages dont le chef de famille est une femme représentent environ $10 \%$ de l'échantillon. Ce pourcentage est nettement plus élevé à Alaotra 1 et El Viejo (20\%) et à Nyando $(30 \%)^{21}$.

Ces écarts trouvent de multiples explications liées à des différences d'approche et de gestion des accidents de la vie (décès, divorce), qui varient fortement selon le contexte culturel, mais aussi à des contextes migratoires très diversifiés. Dans les familles nucléaires, comme au Nicaragua et au Mexique (et dans une moindre mesure à Madagascar et au Kenya), c'est souvent le mari qui quitte le foyer pour une migration de longue durée, alors qu'en Afrique de l'Ouest ce sont surtout les jeunes dépendants. La guerre civile au Nicaragua a elle aussi laissé son empreinte dans les structures familiales.

Lorsque le chef de famille est une femme, la taille du ménage est souvent moins importante, ce qui est logique dans un contexte de famille nucléaire. L'écart de revenu moyen entre les ménages selon le genre du chef de famille est moins important qu'anticipé (voir tableau 3.4) : dans les ménages dont le chef de famille est une femme, les revenus sont généralement inférieurs de $10 \%$ avec quelques exceptions (Macina et, encore une fois, El Viejo et Nyando). À Tequisquiapan et dans la Sierra de Santa Marta au Mexique, en revanche, le revenu moyen de ces ménages est notablement plus élevé. Bien que l'enquête ait eu des difficultés à saisir la réalité des migrations (voir chapitre 4), ces résultats parlent d'eux-mêmes. Le niveau des revenus reflète le rôle des envois de fonds de travailleurs à l'étranger et des tailles de ménages plus petites - une conséquence de migrations à long terme (ces ménages sont généralement aux premiers stades du cycle familial et les migrants sont d'abord des hommes de moins de quarante ans qui ont laissé la responsabilité du ménage à leur femme). 
Tableau 3.4 Proportion, taille et revenu annuel des ménages dont le chef de famille est une femme

\begin{tabular}{|c|c|c|c|c|c|c|c|}
\hline \multirow{3}{*}{ Pays } & \multirow{3}{*}{ Région } & \multirow{2}{*}{\multicolumn{2}{|c|}{$\begin{array}{l}\text { Ménages dont } \\
\text { le chef de } \\
\text { famille est une } \\
\text { femme }\end{array}$}} & \multirow{2}{*}{\multicolumn{2}{|c|}{$\begin{array}{c}\text { Taille du ménage en } \\
\text { EqA }\end{array}$}} & \multirow{2}{*}{\multicolumn{2}{|c|}{$\begin{array}{c}\text { US\$ PPA par EqA } \\
\begin{array}{c}\text { Ménage dont le chef } \\
\text { de famille est }\end{array} \\
\end{array}$}} \\
\hline & & & & & & & \\
\hline & & Nombre & $\%$ & un homme & une femme & un homme & une femme \\
\hline \multirow[t]{4}{*}{ Mali } & Tominian & 0 & 0,0 & 9,3 & - & 235 & - \\
\hline & Diéma & 0 & 0,0 & 15,3 & - & 368 & - \\
\hline & Koutiala & 1 & 0,7 & 12,1 & 4,4 & 367 & 495 \\
\hline & Macina & 2 & 1,3 & 10,6 & 6,2 & 520 & 203 \\
\hline \multirow[t]{6}{*}{ Sénégal } & Casamance & 7 & 2,9 & 11,9 & 9,2 & 441 & 365 \\
\hline & Mekhé 1 & 3 & 2,7 & 12,4 & 6,3 & 519 & 799 \\
\hline & Nioro & 13 & 5,2 & 9,6 & 7,7 & 473 & 698 \\
\hline & Haut Delta & 5 & 8,2 & 10,5 & 5,8 & 499 & 794 \\
\hline & Mekhé 2 & 1 & 0,9 & 12,5 & 4,4 & 772 & 399 \\
\hline & Bas Delta & 6 & 5,0 & 9,1 & 7,0 & 1207 & 1163 \\
\hline \multirow[t]{6}{*}{ Madagascar } & Antsirabe 2 & 25 & 8,3 & 4,9 & 3,6 & 409 & 406 \\
\hline & Alaotra 1 & 82 & 21,3 & 4,6 & 3,6 & 516 & 471 \\
\hline & Morondava & 82 & 16,2 & 4,8 & 3,2 & 601 & 574 \\
\hline & Itasy & 54 & 10,7 & 4,7 & 3,1 & 616 & 670 \\
\hline & Antsirabe 1 & 14 & 6,8 & 4,9 & 2,3 & 736 & 852 \\
\hline & Alaotra 2 & 11 & 9,6 & 5,3 & 3,6 & 1362 & 1188 \\
\hline \multirow[t]{3}{*}{ Kenya } & Bungoma & 33 & 11,0 & 5,7 & 4,7 & 628 & 745 \\
\hline & Nyando & 87 & 30,5 & 5,8 & 4,6 & 818 & 300 \\
\hline & Nakuru N. & 48 & 16,6 & 5,9 & 4,4 & 2255 & 2272 \\
\hline \multirow[t]{3}{*}{ Maroc } & Chaouia & 11 & 4,8 & 6,2 & 4,1 & 2299 & 1922 \\
\hline & Saïss & 1 & 0,4 & 5,8 & 3,0 & 3426 & 1587 \\
\hline & Souss & 4 & 1,7 & 5,1 & 3,8 & 4175 & 1521 \\
\hline \multirow[t]{5}{*}{ Nicaragua } & Muy Muy & 35 & 11,7 & 4,8 & 4,3 & 1472 & 1000 \\
\hline & Terrabona & 41 & 14,6 & 4,5 & 4,3 & 1467 & 1406 \\
\hline & El Viejo & 65 & 22,6 & 4,4 & 4,9 & 2891 & 1491 \\
\hline & La Libertad & 30 & 10,3 & 4,8 & 4,7 & 2342 & 2216 \\
\hline & El Cuá & 42 & 14,0 & 4,9 & 4,7 & 3670 & 3241 \\
\hline \multirow[t]{3}{*}{ Mexique } & Sierra SM. & 24 & 13,7 & 4,1 & 2,8 & 1776 & 2621 \\
\hline & T. Bajas & 13 & 9,0 & 3,8 & 2,7 & 3187 & 2712 \\
\hline & Tequis. & 50 & 13,7 & 4,1 & 2,8 & 2820 & 3247 \\
\hline
\end{tabular}

Source : Enquêtes RuralStruc. 
Viabilité des ménages à faibles revenus et insécurité alimentaire. La distribution des revenus en quintiles de ménages opérée plus haut a mis en évidence la situation intenable des ménages du premier quintile dans toutes les régions étudiées, excepté le Mexique. Leur situation s'améliore légèrement en utilisant des ratios par EqA (avec une augmentation de $15 \%$ à $30 \%$ au niveau de l'échantillon régional - voir tableau 3.2) mais demeure dramatique. Comment les ménages les plus pauvres survivent-ils et comment pourvoient-ils à leurs besoins avec des revenus aussi bas?

Afin de mieux appréhender la situation des ménages du premier quintile, leur revenu estimé en valeur monétaire a été converti en kilocalories (kcal) pour déterminer s'ils leur permettent de couvrir leurs besoins alimentaires minimaux. Cette approche est une approximation - on ne peut réduire les besoins des ménages aux seuls besoins alimentaires - mais elle donne une estimation et permet d'affiner la comparaison entre les régions étudiées. Les revenus des ménages en EqA ont été convertis en kilocalories sur la base du coût local de la principale denrée alimentaire de base. Les revenus exprimés en kcal par EqA et par jour ont ensuite été comparés aux besoins alimentaires journaliers moyens, estimés par l'Organisation mondiale de la santé (OMS) à $2450 \mathrm{kcal}$ par personne adulte et par jour ${ }^{22}$.

Le coût d'une kilocalorie varie d'un pays à l'autre et entre les régions d'un même pays (voir tableau 3.5) : il va de 0,10 US\$ PPA pour $1000 \mathrm{kcal}$ de maïs au Mexique à 0,49 US\$ PPA à Nyando, Kenya. Le coût d'une kilocalorie dépend de la céréale cultivée localement et de l'environnement global de la filière. Ainsi les céréales sèches du Mali (millet, sorgho, maïs), principalement consommées dans les régions non irriguées, sont nettement moins chères que le riz (0,11 US\$ PPA contre 0,19 US\$ PPA pour $1000 \mathrm{kcal})$; mais le riz coûte moins cher au Sénégal que dans d'autres pays (0,15 US\$PPA), avec de faibles écarts régionaux. Ce coût réduit résulte d'une forte concurrence sur le marché entre les brisures de riz d'importation et le riz local. De même au Mexique, la faiblesse des coûts par kilocalorie résulte à la fois de la pression permanente d'importations à bas coûts en provenance des États-Unis et des soutiens publics aux grandes exploitations agricoles commerciales qui bénéficient de mécanismes de crédit et d'une assistance technique à l'acquisition et à l'utilisation de paquets techniques. Ces soutiens leur permettent de rester compétitifs face au maïs importé et d'atteindre une productivité relativement satisfaisante.

Le tableau 3.5 donne une nouvelle vision de la réalité des ménages du premier quintile et permet de comprendre comment les ménages ruraux les plus pauvres tentent de s'adapter à des niveaux de revenus monétaires aussi bas ${ }^{23}$.

Quatorze des 27 zones étudiées où le revenu journalier du premier quintile est inférieur au seuil de pauvreté de 1 US\$ PPA (c'est-à-dire tous les pays sauf le Mexique) pourraient théoriquement couvrir leurs besoins alimentaires les plus élémentaires. Dans les 13 autres zones, la situation des plus pauvres est extrêmement critique et confirme une forte insécurité alimentaire. Dans tous les pays d'ASS étudiés, les régions les plus pauvres se caractérisent toutes par 
Tableau 3.5 Revenu total du premier quintile en US\$ PPA et en kilocalories

\begin{tabular}{|c|c|c|c|c|c|c|c|c|}
\hline \multirow{3}{*}{ Pays } & \multirow{3}{*}{ Région } & \multirow{3}{*}{$\begin{array}{c}\text { Prix de } \\
1000 \text { kcal } \\
\text { en US\$ PPA }\end{array}$} & \multicolumn{4}{|c|}{$\begin{array}{c}\text { Revenu total du Q1 } \\
\text { par EqA par jour }\end{array}$} & \multirow{3}{*}{$\begin{array}{c}\text { kcal } \\
\text { disponibles } \\
\text { / besoins } \\
\text { journaliers }\end{array}$} & \multirow{3}{*}{$\begin{array}{c}\text { Principales } \\
\text { denrées } \\
\text { de base }\end{array}$} \\
\hline & & & \multicolumn{2}{|c|}{ en US\$ PPA } & \multicolumn{2}{|c|}{ en kcal } & & \\
\hline & & & Moyen & Indice & Moyen & Indice & & \\
\hline \multirow[t]{4}{*}{ Mali } & Tominian & 0,12 & 0,21 & 100 & 1730 & 100 & 0,7 & Millet, sorgho \\
\hline & Diéma & 0,12 & 0,26 & 123 & 2132 & 123 & 0,9 & Millet, sorgho \\
\hline & Koutiala & 0,11 & 0,39 & 188 & 3557 & 206 & 1,5 & $\begin{array}{c}\text { Millet, sorgho, } \\
\text { maïs }\end{array}$ \\
\hline & Macina & 0,19 & 0,36 & 171 & 1870 & 108 & 0,8 & Riz \\
\hline \multirow[t]{6}{*}{ Sénégal } & Casamance & 0,15 & 0,18 & 86 & 1197 & 69 & 0,5 & Riz \\
\hline & Mekhé 1 & 0,16 & 0,25 & 120 & 1556 & 90 & 0,6 & Riz \\
\hline & Nioro & 0,15 & 0,34 & 164 & 2268 & 131 & 0,9 & Riz \\
\hline & Haut Delta & 0,16 & 0,30 & 144 & 1863 & 108 & 0,8 & Riz \\
\hline & Mekhé 2 & 0,15 & 0,56 & 271 & 3755 & 217 & 1,5 & Riz \\
\hline & Bas Delta & 0,15 & 0,72 & 349 & 4825 & 279 & 2,0 & Riz \\
\hline \multirow[t]{6}{*}{ Madagascar } & Antsirabe 2 & 0,23 & 0,38 & 183 & 1647 & 95 & 0,7 & Riz \\
\hline & Alaotra 1 & 0,21 & 0,49 & 235 & 2319 & 134 & 0,9 & Riz \\
\hline & Morondava & 0,20 & 0,53 & 253 & 2626 & 152 & 1,1 & Riz \\
\hline & Itasy & 0,25 & 0,64 & 309 & 2564 & 148 & 1,0 & Riz \\
\hline & Antsirabe 1 & 0,23 & 0,57 & 274 & 2471 & 143 & 1,0 & Riz \\
\hline & Alaotra 2 & 0,21 & 0,64 & 308 & 3041 & 176 & 1,2 & Riz \\
\hline \multirow[t]{3}{*}{ Kenya } & Bungoma & 0,44 & 0,20 & 98 & 462 & 27 & 0,2 & Maïs \\
\hline & Nyando & 0,49 & 0,16 & 78 & 329 & 19 & 0,1 & Maïs \\
\hline & Nakuru N. & 0,34 & 0,92 & 441 & 2693 & 156 & 1,1 & Maïs \\
\hline \multirow[t]{3}{*}{ Maroc } & Chaouia & 0,18 & 0,58 & 281 & 3241 & 187 & 1,3 & Blé \\
\hline & Saïss & 0,16 & 0,61 & 296 & 3841 & 222 & 1,6 & Blé \\
\hline & Souss & 0,21 & 0,77 & 372 & 3679 & 213 & 1,5 & Blé \\
\hline \multirow[t]{5}{*}{ Nicaragua } & Muy Muy & 0,18 & 0,36 & 176 & 2026 & 117 & 0,8 & Maïs \\
\hline & Terrabona & 0,20 & 0,40 & 194 & 2013 & 116 & 0,8 & Maïs \\
\hline & El Viejo & 0,20 & 0,64 & 311 & 3222 & 186 & 1,3 & Maïs \\
\hline & La Libertad & 0,19 & 0,79 & 382 & 4174 & 241 & 1,7 & Maïs \\
\hline & El Cuá & 0,18 & 0,88 & 426 & 4912 & 284 & 2,0 & Maïs \\
\hline \multirow[t]{3}{*}{ Mexique } & Sierra SM. & 0,10 & 1,49 & 720 & 14942 & 864 & 6,1 & Maïs \\
\hline & T. Bajas & 0,10 & 2,25 & 1086 & 22549 & 1304 & 9,2 & Maïs \\
\hline & Tequis. & 0,10 & 2,18 & 1050 & 21795 & 1260 & 8,9 & Maïs \\
\hline
\end{tabular}

Source : Enquêtes RuralStruc. 
une très forte vulnérabilité des ménages. Le cas de Bungoma et de Nyando au Kenya est particulièrement grave et exacerbé par le coût élevé du maïs dans l'ouest du pays. Muy Muy et Terrabona au Nicaragua présentent également une forte vulnérabilité. Globalement, dans les pays d'ASS (voir tableau 3.6), $10 \%$ ou plus des ménages sont incapables d'atteindre le seuil de $2450 \mathrm{kcal}$ dans 11 des 19 régions étudiées. Cette proportion atteint plus de $20 \%$ des ménages dans trois zones: Casamance, Antsirabe 2 et Nyando.

En conclusion, l'approche par les kilocalories offre un complément utile aux comparaisons monétaires. Elle permet de mieux comprendre l'apparente absence de viabilité des ménages à faibles revenus et confirme l'extrême dénuement des plus pauvres. L'insécurité alimentaire persiste et demeure très présente dans plusieurs régions, un constat confirmé par l'appréciation des chefs de famille sur leur sécurité alimentaire : $23 \%$ à $40 \%$ des ménages au Mali, $15 \%$ à $43 \%$ au Sénégal et plus de $40 \%$ dans certaines régions de Madagascar (Antsirabe) et du Nicaragua (El Viejo) déclarent que leur sécurité alimentaire s'est dégradée au cours des cinq dernières années, tant en qualité qu'en quantité (figure 3.11). Il est possible que cette impression ait été accentuée par l'amorce de la crise des prix alimentaires au cours de l'année de l'enquête (fin 2007-début 2008) et qu'elle se soit détériorée dans les mois qui ont suivi. Elle confirme l'âpre réalité de nombreux ménages ruraux dans de nombreuses régions étudiées.

\section{Les stratégies d'activités des ménages}

Face à des situations aussi difficiles dans de nombreuses régions étudiées, quelles sont les stratégies d'activités adoptées par les ménages ruraux pour subvenir à leurs besoins et bâtir un avenir pour leurs enfants?

Le RDM08, qui examine les voies de sortie de la pauvreté rurale, offre un cadre utile pour l'analyse des résultats du programme RuralStruc. Adoptant l'approche développée par le projet RIGA (voir chapitre 1), il recense quatre stratégies d'activités pour les ménages ruraux (World Bank 2007, 75) : (1) les ménages axés sur l'agriculture, qui tirent la majeure partie de leurs revenus des activités conduites sur leurs exploitations agricoles ${ }^{24}$; (2) les ménages axés sur la main-d'œuvre, qui vivent du travail salarié, agricole ou non agricole, ou d'activités d'auto-emploi (self-employment) en dehors de l'agriculture ; (3) les ménages axés sur la migration, qui choisissent de quitter complètement le secteur rural, ou de s'appuyer sur les transferts de membres de la famille qui ont migré, ou encore sur les transferts publics ; enfin, (4) les ménages diversifiés, qui tirent leurs revenus d'une combinaison des trois stratégies précédentes.

\section{Mise en pratique de la typologie du RDM08}

Partant de ces définitions ${ }^{25}$, le tableau 3.7 et la figure qui lui est associée (figure 3.12) présentent les résultats des enquêtes en fonction des catégories du 
Tableau 3.6 Distribution des ménages par classes de revenus exprimés en kilocalories (en \%)

\begin{tabular}{|c|c|c|c|c|}
\hline \multirow{2}{*}{ Pays } & \multirow{2}{*}{ Région } & \multicolumn{3}{|c|}{ Classes de revenu total en kcal par EqA par jour } \\
\hline & & $<=2450$ & $2451-4900$ & $>=4900$ \\
\hline \multirow[t]{4}{*}{ Mali } & Tominian & 19 & 41 & 40 \\
\hline & Diéma & 12 & 24 & 64 \\
\hline & Koutiala & 5 & 10 & 86 \\
\hline & Macina & 14 & 25 & 60 \\
\hline \multirow[t]{6}{*}{ Sénégal } & Casamance & 22 & 22 & 56 \\
\hline & Mekhé 1 & 17 & 15 & 68 \\
\hline & Nioro & 12 & 25 & 63 \\
\hline & Haut Delta & 13 & 31 & 56 \\
\hline & Mekhé 2 & 4 & 9 & 87 \\
\hline & Bas Delta & 4 & 4 & 92 \\
\hline \multirow[t]{6}{*}{ Madagascar } & Antsirabe 2 & 29 & 41 & 31 \\
\hline & Alaotra 1 & 11 & 38 & 51 \\
\hline & Morondava & 6 & 28 & 65 \\
\hline & Itasy & 6 & 38 & 56 \\
\hline & Antsirabe 1 & 8 & 27 & 65 \\
\hline & Alaotra 2 & 3 & 19 & 77 \\
\hline \multirow[t]{3}{*}{ Kenya } & Bungoma & 15 & 14 & 71 \\
\hline & Nyando & 22 & 21 & 57 \\
\hline & Nakuru N. & 1 & 1 & 97 \\
\hline \multirow[t]{3}{*}{ Maroc } & Chaouia & 7 & 7 & 86 \\
\hline & Saïss & 8 & 4 & 87 \\
\hline & Souss & 8 & 5 & 87 \\
\hline \multirow[t]{5}{*}{ Nicaragua } & Muy Muy & 12 & 13 & 75 \\
\hline & Terrabona & 13 & 13 & 74 \\
\hline & El Viejo & 8 & 6 & 85 \\
\hline & La Libertad & 5 & 8 & 88 \\
\hline & El Cuá & 3 & 8 & 89 \\
\hline \multirow[t]{3}{*}{ Mexique } & Sierra SM & 0 & 0 & 100 \\
\hline & T. Bajas & 0 & 0 & 100 \\
\hline & Tequis. & 1 & 1 & 98 \\
\hline
\end{tabular}

Source : Enquêtes RuralStruc. 


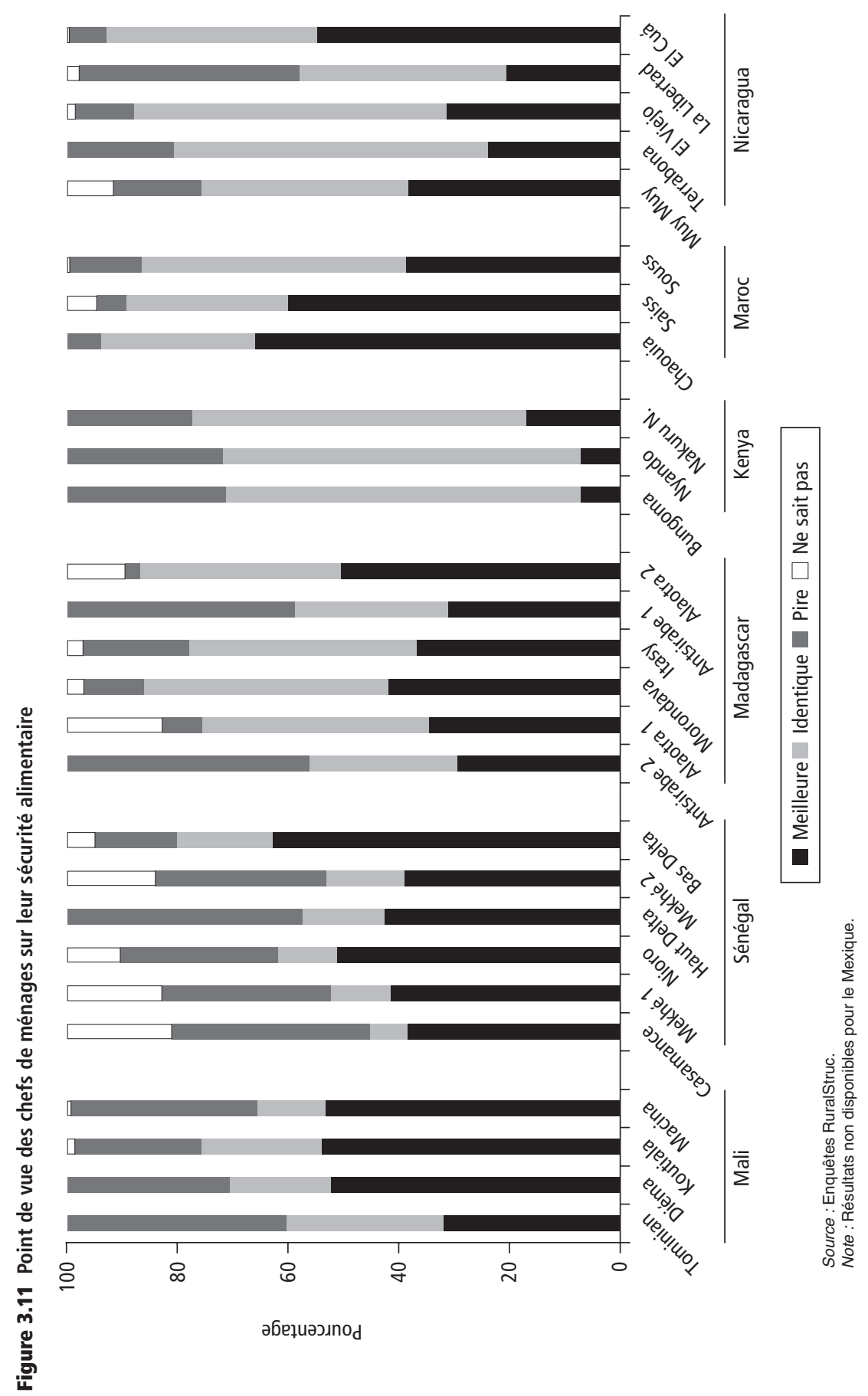




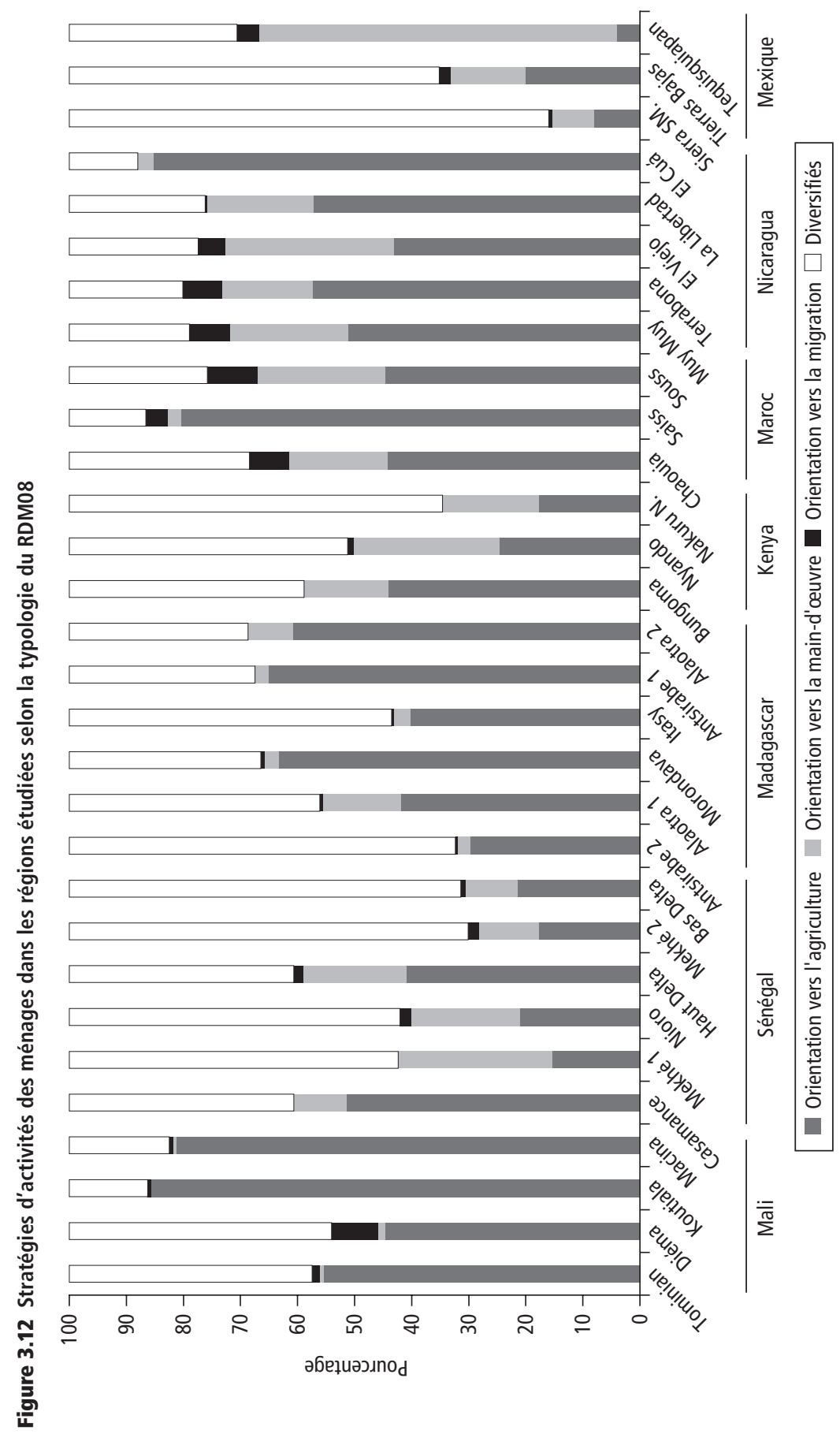

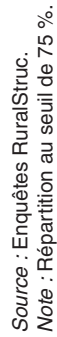


RDM08 et donnent un aperçu de la répartition des ménages entre les quatre stratégies d'activités. La part la plus importante revient à la catégorie axée sur l'agriculture et confirme le rôle de l'agriculture et des revenus tirés des exploitations agricoles dans les régions étudiées. Dans 18 régions sur 30, les exploitations agricoles sont la principale source de revenus; et dans 12 régions l'agriculture est la principale stratégie pour plus de $50 \%$ des ménages interrogés. Dans quatre régions (Koutiala et Macina au Mali, Saïss au Maroc et El Cuá au Nicaragua), elle fait même vivre 80 \% des ménages. Au Kenya et au Sénégal, l'orientation agricole ne semble pas aussi généralisée, tandis que le Mexique confirme son exception.

Comme indiqué plus haut, une seule région n'est pas tournée vers l'agriculture : à Tequisquiapan, le salariat et l'auto-emploi concernent une écrasante majorité de ménages $(80 \%)$, ce qui corrobore le faible nombre d'exploitations agricoles parmi les ménages enquêtés. Dans les autres régions, cette orientation excède à peine $30 \%$, excepté à Mekhé 1 au Sénégal, Nyando au Kenya et El Viejo au Nicaragua, où un tiers des ménages sont orientés vers le salariat et l'auto-emploi. Les stratégies migratoires ne ressortent jamais comme une tendance lourde, même dans les pays comme le Maroc, le Nicaragua et le Mexique, où de nombreux ménages ont une tradition de migration ${ }^{26}$. Diéma au Mali, la Chaouia et le Souss au Maroc, et Muy Muy et Terrabona au Nicaragua sont les seules régions qui présentent qui une proportion de ménages axés sur la migration et les transferts de $7 \%$ à $8 \%$.

Ainsi, lorsque les ménages se spécialisent, cette spécialisation s'opère principalement dans l'agriculture. À l'autre extrême, la catégorie diversifiée est bien représentée dans toutes les zones étudiées et elle domine dans 12 régions, avec un maximum de $84 \%$ dans la Sierra du Sotavento (Mexique). La diversification peut être néanmoins surestimée ; elle est très sensible au seuil de $75 \%$ du revenu retenu par le RDM08, qui tend à polariser les résultats. D'ailleurs, Davis et al. (2007) estiment que ce seuil correspond plus à un niveau de spécialisation qu'à une orientation. Afin de tester la sensibilité du seuil, l'échantillon a également été ventilé sur la base d'un seuil de $60 \%$ (voir tableau 3.7). Cette différence de $15 \%$ modifie fortement le profil d'ensemble : la part du groupe diversifié est divisée par deux, excepté dans le Sotavento ce qui témoigne de la solidité de son orientation diversifiée. Dans quelques régions (Antsirabe 1, Alaotra 2, Chaouia, Muy Muy, Tequisquiapan), l'importance de cette catégorie est divisée par trois et plus. La catégorie diversifiée ne demeure dominante que dans les deux zones du Sotavento. La nouvelle répartition des ménages bénéficie principalement au groupe tourné vers l'agriculture (au Mali et à Madagascar) et au groupe orienté vers le salariat et l'auto-emploi (Kenya et Sénégal). Dans le cas de Tequisquiapan, le groupe des ménages axés sur ces activités de main-d'œuvre passe à $86 \%$.

Si on compare les résultats de RuralStruc avec ceux du projet RIGA pour le Nicaragua et Madagascar - les deux seuls pays communs aux deux projets (mais avec des années de référence différentes, respectivement 2001 et 1993) - de nettes différences apparaissent, en particulier au Nicaragua (voir tableau 3.7), 
où le pourcentage de ménages orientés vers les activités de main-d'œuvre est de $48 \%$ selon le projet RIGA, alors qu'il est au maximum de $30 \%$ dans l'étude RuralStruc. En revanche, le pourcentage de ménages tournés vers l'agriculture est bien plus faible : $19 \%$ pour le projet RIGA contre $43 \%$ à $85 \%$ pour les enquêtes RuralStruc. La différence n'est pas aussi marquée à Madagascar bien que la période de référence couvre quinze ans. L'explication probable de ces écarts est que les résultats du projet RIGA s'appuient sur des observations agrégées au niveau national, alors que les données du programme RuralStruc illustrent des situations régionales spécifiques. Bien que les méthodologies d'enquête, le niveau d'analyse et les années de référence diffèrent, ces écarts illustrent la difficulté à établir des mesures de revenus internationalement comparables - un constat qu'indiquait clairement le RDM08 (World Bank 2007, voir encadré 3.2).

\section{Les besoins d'analyses complémentaires}

Cette typologie des stratégies d'activités des ménages permet d'expliquer la configuration des économies régionales étudiées. À ce stade de l'analyse, elle confirme la domination des activités conduites dans le cadre d'exploitations agricoles et le rôle moindre des autres stratégies reposant sur des activités hors exploitations ou sur la migration. Elle rappelle aussi que les alternatives à l'agriculture sont très limitées et pointe les limites des opportunités locales, qui n’apparaissent pas nécessairement lorsque les données sont agrégées au niveau national.

Comme l'a montré le RDM08, il est difficile d'évaluer l'efficacité de ces stratégies d'activités pour sortir de la pauvreté. L'absence de données dynamiques, la forte hétérogénéité des ménages et le faible nombre de ménages par stratégie à l'échelle régionale empêchent toute analyse des niveaux de revenus par types de stratégie ${ }^{27}$.

In fine, l'application de la typologie du RDM au large éventail de situations observées dans le programme RuralStruc débouche sur deux grands groupes de ménages, les uns très spécialisés dans les activités liées à leur exploitation agricole, les autres plus diversifiés, sans spécialisation notable dans une autre forme d'activité. Mais, au-delà de cette première approche, les caractéristiques des différentes activités pratiquées par les ménages restent méconnues. Quelles sont-elles dans les régions étudiées ? C'est à cette caractérisation que s'attachent les deux prochains chapitres. 


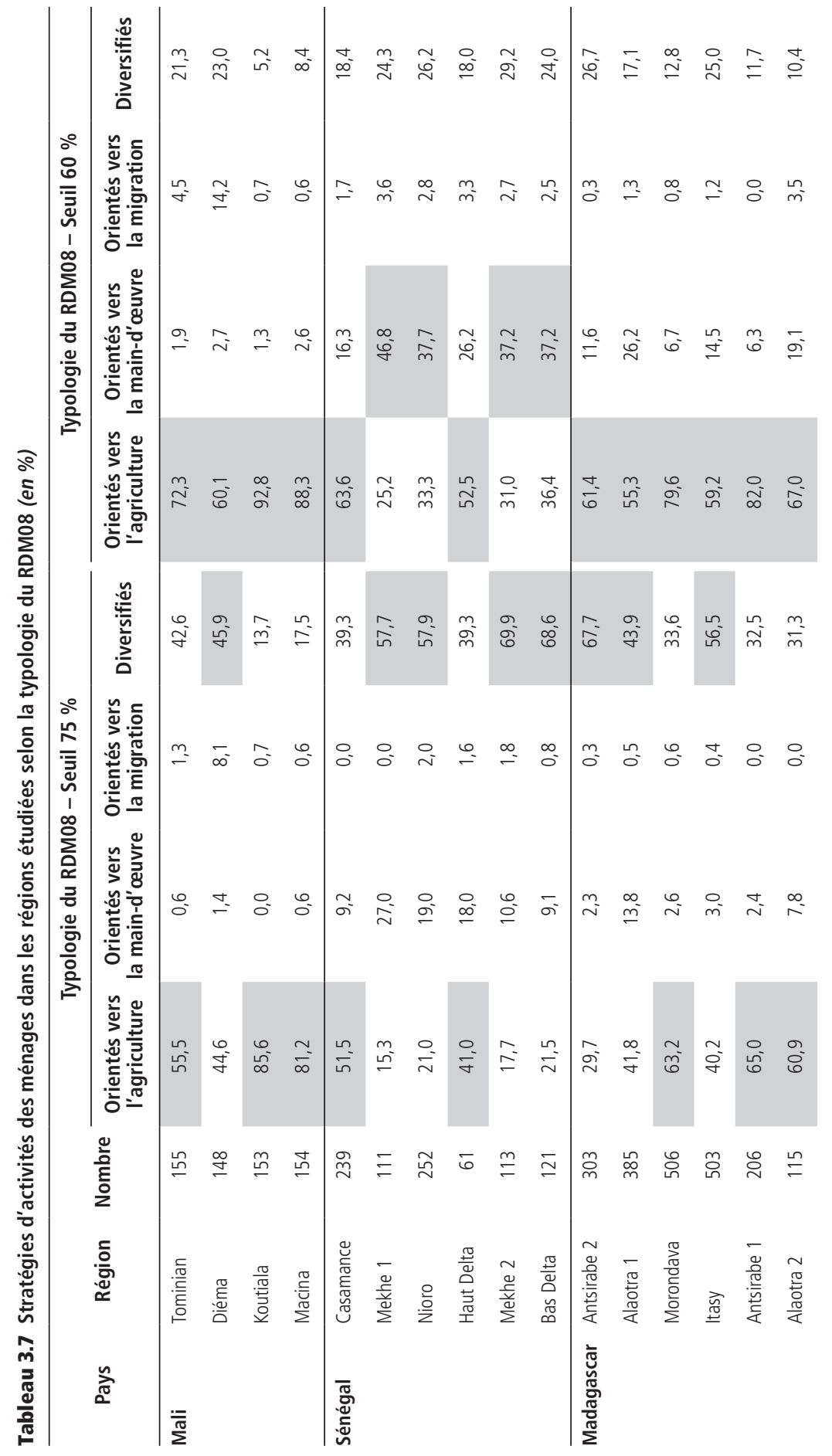




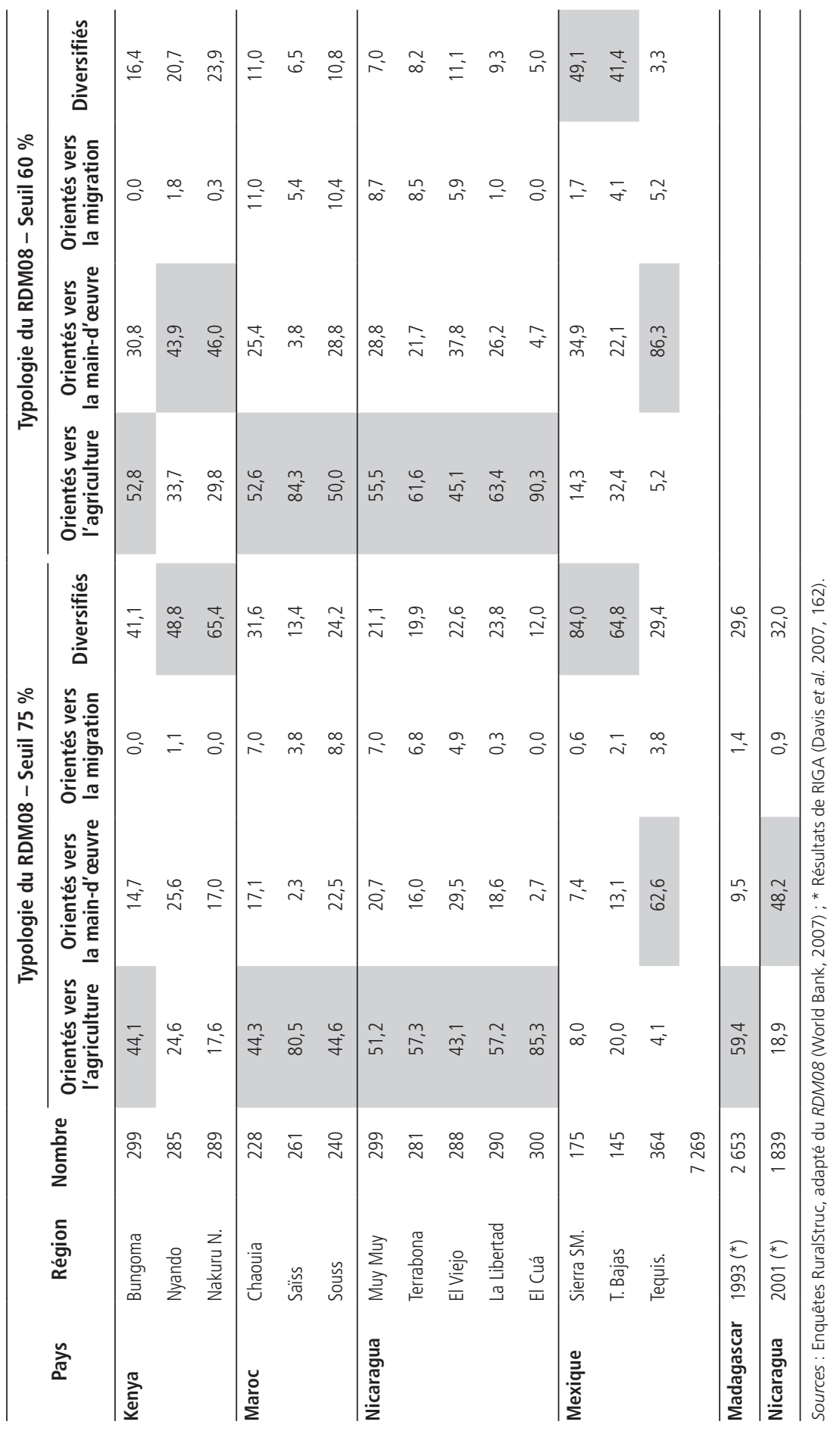




\section{Notes}

1. L'annexe 3 de l'appendice en ligne : http://www.worldbank.org/afr/ruralstruc donne un bref aperçu des caractéristiques de ces régions.

2. Le programme définit un ménage exploitant agricole comme un ménage ayant des activités directes d'agriculture au sens large et qui tire des revenus, en espèces ou en nature, de ces activités, indépendamment du niveau des actifs productifs et des droits de propriété (par exemple, parcelles dont l'exploitant est propriétaire, ou louées ou prêtées). Voir chapitre 4 pour la définition des activités des ménages.

3. La vallée de San Juan compte quatre villes de 25000 à 55000 habitants, dont Tequisquiapan (27 000).

4. Le seuil de 50000 habitants, qui vient de l'indice d'agglomération du RDM09, est une des variables utilisées dans les analyses de régression réalisées sur l'échantillon d'enquêtes (voir l'annexe 5 de l'appendice en ligne : http://www.worldbank.org/afr/ ruralstruc). Il n'a pas été possible d'établir des cartes équivalentes pour les trois pays hors ASS, mais leurs taux d'urbanisation et leurs réseaux d'infrastructures auraient produit une couleur gris foncé-noir pour la plupart des régions étudiées.

5. Ce calcul est effectué sur la totalité de l'échantillon régional, qui comprend tous les ménages (avec et sans exploitation agricole) et s'appuie sur la part des moyennes régionales correspondant à la structure régionale des revenus (voir chapitre 6 pour une analyse du calcul des moyennes). Les activités hors exploitation agricole sont décrites au chapitre 4 : elles comprennent le salariat agricole et non agricole, les activités d'auto-emploi, les transferts publics et privés et les rentes.

6. Dans chaque pays, les régions ont été classées de gauche à droite, des plus pauvres aux plus riches en termes relatifs. Cette configuration apparaît clairement à la figure 3.4.

7. Pour permettre des comparaisons, les revenus des ménages par habitant agrégés au niveau régional et exprimés en monnaie locale ont été convertis en dollars à parité de pouvoir d'achat (US\$ PPA) pour l'année 2007, qui est l'année de référence des informations recueillies (voir annexe 1 de l'appendice en ligne : http://www.worldbank. org/afr/ruralstruc). La même conversion en dollars internationaux a été appliquée au PIB par habitant et aux seuils de pauvreté nationaux initialement exprimés en monnaie locale.

8. Le revenu total estimatif par ménage est un agrégat des revenus monétaires et en nature (autoconsommation) évalués aux prix du marché (voir annexe 1 de l'appendice en ligne : http://www.worldbank.org/afr/ruralstruc

9. Cet écart de revenu est renforcé par la sélection des régions étudiées, car la partie méridionale du pays est plus touchée par la pauvreté rurale et caractérisée par des exploitations de plus petite taille.

10. L'annexe 4 de l'appendice en ligne : http://www.worldbank.org/afr/ruralstruc présente les seuils de pauvreté pour chaque pays. Cependant, les définitions nationales de la pauvreté obéissent souvent à des considérations politiques et la grande diversité des seuils ne facilite pas l'analyse générale. Onze des 19 régions et sous-régions étudiées en Afrique subsaharienne se situent en dessous des seuils de pauvreté nationaux (les exceptions étant le Kenya et Madagascar, où les seuils de pauvreté sont très bas).

11. Le chiffre enregistré au Maroc est remarquable car la relative homogénéité entre les régions contraste nettement avec l'hétérogénéité marquée à l'intérieur des régions (parmi les plus fortes des sept pays) exprimée par les indices de Gini. La présence de ménages à revenus élevés, dont les revenus proviennent principalement de rentes 
(revenus locatifs), a évidemment une incidence sur la moyenne de l'échantillon et explique l'apparente homogénéité, qui est compromise par la distribution des revenus (RS 2 Maroc, 151). L'hétérogénéité intrarégionale résulte aussi de la définition des régions étudiées - en particulier dans le Saïss et le Souss, et de la décision prise par l'équipe nationale de grouper les localités de plaines et de montagnes. Dans le Souss, il aurait été possible d'identifier une sous-région pour Taliouine, dans la zone montagneuse.

12. Plus généralement et ce n'est peut-être pas surprenant, les indices de Gini tendent à être plus élevés dans les régions les plus riches dans tous les pays, hormis le Mali où la région la plus riche - Macina - a un indice identique aux autres régions. Cette particularité résulte de l'homogénéité des exploitations (dotations foncières et techniques de production) au sein des périmètres d'irrigation de l'Office du Niger, où les enquêtes ont été réalisées.

13. Cette évolution présente des similitudes avec la courbe de Kuznets, qui a été invalidée par de nouvelles données (voir Bourguignon et Morrisson 1998 ; Deininger et Squire 1998). Cependant l'analyse ne porte pas ici sur les résultats d'ensemble des pays mais sur les seules zones rurales, et l'évolution des inégalités apporte un éclairage sur le processus de la transformation rurale (voir chapitre 6).

14. Les inégalités, la pauvreté rurale et le mécontentement croissant suscité par les conséquences de l'ALENA ont été à l'origine d'un puissant mouvement social amorcé en 2002 par les organisations de producteurs ruraux et appelé ¡El campo no aguanta mas! (Le pays n’en peut plus !). Voir Sánchez Albarrán (2007) et Puricelli (2010).

15. Le Maroc est le seul pays où la classification ex ante est parfaitement respectée.

16. Cette caractéristique est illustrée par les statistiques descriptives du quintile 5 présentées à l'annexe 4 de l'appendice en ligne : http://www.worldbank.org/afr/ruralstruc.

17. Voir l'annexe 1 de l'appendice en ligne : http://www.worldbank.org/afr/ruralstruc.

18. Le ratio de dépendance moyen observé à Nakuru North correspond aux autres données disponibles, qui indiquent aussi des ratios voisins de 0,60 (RS 2 Kenya, 79), et il est conforme aux statistiques nationales. Une explication possible réside dans les caractéristiques démographiques de la ville de Nakuru, qui est exceptionnellement jeune : $55 \%$ de la population a moins de 20 ans et $75 \%$ a moins de 30 ans (Republic of Kenya 2005). En outre, seulement 55 \% des ménages enquêtés de Nakuru North ont des enfants présents, ce qui pourrait s'expliquer par la migration permanente des jeunes dans des familles d'accueil en zone urbaine.

19. La croissance démographique du Nicaragua décélère depuis le milieu des années 1990. Cependant, les zones rurales présentent un profil particulier lié aux conséquences de la guerre civile (moins d'hommes adultes) et des migrations à long terme (les migrants à long terme ne sont pas comptés dans le nombre de personnes présentes sur lequel le ratio est calculé). Cette situation migratoire concerne aussi la Sierra de Santa Marta, qui présente également des taux de natalité plus élevés, caractéristiques des populations indiennes.

20. Au Nicaragua, bien que le pourcentage de ménages sans exploitation soit plus faible que dans le Souss et au Mexique, les résultats d'une comparaison entre ménages avec ou sans exploitation agricole varient en fonction du type de ratio (moyen ou médian). Le revenu médian des ménages sans exploitation est plus élevé que celui des ménages avec exploitation, mais le résultat est inverse pour le revenu moyen. Ces résultats contraires signifient qu'il existe un petit nombre d'agriculteurs spécialisés et mieux dotés en ressources. 
21. Le cas de Nyando semble faire exception. Les données de panel du Tegemeo Institute confirment une rapide augmentation du nombre de ménages dont le chef de famille est une femme ( $80 \%$ sont veuves). Le SIDA est l'explication la plus probable.

22. La méthodologie adoptée et le tableau de conversion sont présentés à l'annexe 1 de l'appendice en ligne : http://www.worldbank.org/afr/ruralstruc.

23. Le tableau montre également de nouveaux écarts de revenus entre les régions. En prenant Tominian - la région la plus pauvre de l'échantillon du programme RuralStruc-comme référence (indice 100), la conversion du revenu en kilocalories modifie l'échelle entre les régions les plus riches et les plus pauvres : si on exclut les régions mexicaines, où le coût d'une kcal est très particulier, l'écart le plus marqué entre les plus riches et les plus pauvres est pratiquement divisé par deux (2,8 à 1,0 contre 4,4 à 1,0$)$.

24. Le RDM08 recense en fait cinq stratégies. Il distingue deux sous-catégories dans la catégorie des ménages tournés vers l'agriculture : l'agriculture de subsistance et l'agriculture tournée vers le marché. Ce dernier groupe est analysé au chapitre 5 avec les résultats du programme concernant sur l'insertion au marché.

25. Le seuil pour chaque groupe est fixé à $75 \%$ du revenu total. Les ménages orientés vers l'agriculture dépendent principalement des produits (toutes catégories) tirés de leur exploitation agricole ; les ménages axés sur la main-d'œuvre dépendent des salaires (toutes catégories) et des activités d'auto-emploi ; enfin, les ménages orientés vers la migration tirent leurs revenus de transferts (publics et privés) et d'autres sources comme les rentes. Dans les ménages diversifiés, aucune de ces sources de revenu ne représente plus de $75 \%$ du revenu total.

26. Pour une analyse de la difficulté à rendre compte des fonds envoyés par les travailleurs à l'étranger, voir le chapitre 4 .

27. «La structure des revenus d'un ménage ne dit pas si celui-ci a adopté une stratégie de revenu adéquate. Chaque stratégie peut constituer une voie de sortie de la pauvreté mais de nombreux ménages ne parviennent pas à améliorer leur situation à long terme, ce qui reflète l'hétérogénéité marquée existant dans chacune des activités et le fait que les revenus varient largement pour chacune des stratégies. » (World Bank 2007, 77). 


\section{Chapitre 4}

\section{Les multiples facettes de la diversification rurale et l'économie rurale non agricole}

Sans surprise, les activités agricoles sont généralisées dans les régions étudiées par le programme RuralStruc, mais la diversification des activités rurales y est aussi extrêmement fréquente. Cette observation soulève deux questions : quelles sont les caractéristiques de ces activités rurales développées en dehors des exploitations agricoles? Et quels sont les déterminants de leur expansion ? Ces questions servent de point de départ à une discussion de la deuxième hypothèse du programme $(\mathrm{H} 2)$, qui concerne l'adaptation des ménages ruraux aux mutations de leur environnement. Ces processus d'adaptation sont-ils nouveaux et ont-ils reconfiguré les zones rurales? Ou sont-ils semblables aux trajectoires historiques de la transformation structurelle ? Surtout, contribuent-ils à l'amélioration des moyens de subsistance des ruraux? Autrement dit, l'économie rurale non agricole, si souvent mise en avant dans la littérature, est-elle la meilleure réponse à une pauvreté rurale récurrente?

Les résultats des enquêtes dressent un tableau assez nuancé des transformations en cours. Ils montrent des types de diversification très différenciés, étroitement liés aux opportunités propres à chaque région. Après un examen de la littérature consacrée à la diversification, ce chapitre aborde les différentes catégories d'activités et de revenus ruraux observées chez les ménages enquêtés : le travail salarié (agricole et non agricole), les activités indépendantes d'auto-emploi (self-employment), les transferts et les rentes.

\section{La question de la diversification rurale}

Avant de proposer une classification des activités et des revenus ruraux, il convient de revenir sur le débat relatif à la diversification. Une définition précise des catégories d'activités est en effet indispensable pour éviter les sources d'incompréhension ou les biais d'analyse. 


\section{Aperçu du débat sur la diversification rurale}

Un important courant de recherche a montré que les ménages ruraux des pays en développement tirent de plus en plus leurs revenus d'activités non agricoles et de transferts. Une récente revue de cette littérature (Haggblade, Hazell et Reardon 2007) a décrit les multiples facettes de l'économie rurale non agricole. Haggblade (2007) souligne que le débat ancien qu'elle suscite met en jeu quatre angles d'approche, tous ancrés dans le champ de l'économie du développement. Ainsi, l'économie rurale non agricole peut être considérée du point de vue de ses liens avec la croissance agricole, de sa contribution à l'emploi, de son rôle dans le développement régional ou de sa contribution directe aux stratégies de revenu des ménages. C'est cette dernière perspective qui a été retenue par le programme RuralStruc.

La diversification croissante des ménages ruraux résulte d'une double série de facteurs : des facteurs d'attraction plutôt considérés comme positifs (pull factors) et des facteurs d'évasion plutôt négatifs (push factors). Du côté pull, il faut souligner l'importance des nouvelles opportunités d'emploi dues à l'amélioration des connexions entre les zones rurales, les marchés et les villes, conséquence du développement économique et de l'amélioration générale des infrastructures de transport et de télécommunications (par exemple, les téléphones mobiles et les nouveaux services qui y sont associés, comme les systèmes de transfert d'espèces). Mais la diversification découle aussi, côté push, des conditions d'activité plus difficiles des exploitations agricoles. Il s'agit notamment des conséquences de la croissance démographique, qui peut engendrer des pressions croissantes sur les ressources naturelles et une baisse des revenus agricoles (parcelles plus petites, terres surexploitées et dégradées), mais aussi des nombreux changements de l'environnement institutionnel et économique résultant des politiques de libéralisation et des effets de la mondialisation intervenus depuis les années 1980. Ces transformations de l'environnement ont certes créé de nouvelles opportunités de marché, mais la fin de la réglementation des prix, la suppression des subventions (en particulier pour les intrants) et le retrait des appuis techniques financés par des fonds publics sont aussi à l'origine d'un environnement plus instable et souvent plus difficile pour les ménages agricoles. Et ces difficultés sont aggravées dans les zones reculées, où les imperfections du marché sont plus nombreuses (marchés inexistants, coûts de transaction élevés) et la fourniture de biens publics insuffisante.

Face à toutes ces mutations et aux coûts d'accès croissants à de nombreux services (en particulier l'éducation et la santé en Afrique subsaharienne), nombreux sont les ménages ruraux qui ont besoin de revenus plus élevés et plus stables. Confrontés à des difficultés financières croissantes, ils sont contraints de déployer des stratégies de gestion des risques ou d'adaptation pour trouver un complément de revenu non agricole ${ }^{1}$. Comme le résument Barrett et Reardon, « la diversification est la norme. Très peu d'individus tirent la totalité de leurs revenus d'une seule source, détiennent tout leur patrimoine sous forme d'un actif unique ou affectent tous leurs actifs à une seule activité » (2000,1-2).

L'ampleur de la diversification rurale suscite un débat intense. Les résultats très divergents présentés dans la littérature découlent de différences importantes qui 
sont liées à la définition des activités, à l'objectif des études (estimation du revenu ou estimation de l'emploi par exemple) et au type de données utilisées (données primaires ou données secondaires et méthodes de collecte). Les résultats traduisent aussi la forte hétérogénéité des structures de revenus entre les pays, entre les régions d'un même pays et entre les ménages d'une même région, ainsi que la rareté des données sur les revenus ruraux. La compilation d'informations provenant de sources très diverses en ensembles de données agrégées est une caractéristique courante de la littérature consacrée à l'économie rurale non agricole (voir chapitre 1).

S’appuyant sur de nombreuses sources existantes, Haggblade, Hazell et Reardon (2010) estiment que les activités non agricoles représentent environ $30 \%$ de l'emploi rural à temps plein en Asie et en Amérique latine, $20 \%$ en Asie de l'Ouest et en Afrique du Nord, et seulement $10 \%$ en Afrique subsaharienne. Toutefois, lorsqu'ils prennent en compte les données relatives au revenu, qui comprennent les revenus des activités saisonnières et à temps partiel, les estimations sont nettement plus élevées : 50 \% pour l'Asie et l'Amérique latine et $35 \%$ pour l'Afrique. Toutes les données disponibles suggèrent que la vieille vision d'économies rurales exclusivement tournées vers l'agriculture ne correspond plus vraiment à la réalité ${ }^{2}$.

\section{Comment classifier les activités et les revenus ruraux}

Toute discussion sur la diversification des moyens d'existence des ruraux est difficile car l'économie rurale non agricole suscite plusieurs débats parallèles qui ne se situent pas tous du point de vue des ménages (voir les quatre angles d'approche d'Haggblade). Néanmoins, même en prenant simultanément en compte toutes ces perspectives, l'étude de la diversification rurale est compliquée par l'absence de consensus sur la définition des catégories d'activités et de revenus.

Barrett et Reardon (2000) classifient les activités économiques d'un ménage rural en fonction de trois paramètres : le secteur (primaire, secondaire ou tertiaire), la fonction (activité indépendante ou salariée) et la localisation (sur place ou ailleurs). Selon cette classification, l'économie rurale non agricole comprend toutes les activités qui ne sont pas des activités agricoles, c'est-à-dire toutes les activités secondaires, tertiaires et primaires non agricoles, quels que soient le lieu et la fonction des activités.

Afin de préciser le débat, Davis et al. (2007) ont divisé les activités rurales en six catégories: (1) l'agriculture, (2) l'élevage, (3) l'emploi salarié agricole, (4) l'emploi salarié non agricole, (5) l'auto-emploi non agricole et (6) les transferts (privés et publics). Les trois premières catégories sont considérées comme des activités " agricoles », tandis que les trois dernières sont " non agricoles ». De plus, les deux premières catégories sont des activités propres à l'exploitation agricole ou « internes » à l'exploitation ( «on-farm»), tandis que les catégories 4 et 5 sont des activités hors exploitation ou « externes» ( off-farm»). Le travail salarié agricole est toujours considéré comme une activité hors exploitation, mais cette catégorie du off-farm peut être trompeuse, car tantôt elle est appliquée exclusivement au travail salarié agricole, tantôt à l'ensemble des activités qui ne sont pas exercées sur une exploitation agricole (activités 3 à 6). 
Les transferts forment une catégorie à part car ce ne sont pas des activités génératrices de revenus, mais une source de revenus. Il s'agit de fonds transférés par des membres du ménage qui vivent ailleurs (c'est le cas des fonds envoyés de l'étranger), par d'autres ménages (dons) ou par des organismes, publics ou non (subventions ou allocations sociales). Le programme a également étudié le cas particulier des rentes, qui sont généralement constituées de revenus locatifs (d'actifs corporels) ou de titres.

Le programme RuralStruc a choisi de classifier les revenus du point de vue des ménages et non des activités parce son objectif est d'identifier des configurations exprimant la complexité des stratégies adoptées par les ménages ruraux. Par conséquent, le groupe des activités hors exploitation couvre l'ensemble des activités exercées et des revenus générés hors de l'exploitation familiale, indépendamment du secteur ou de la fonction. Il comprend l'emploi salarié agricole et tous les autres revenus et activités non agricoles; il correspond à l'économie rurale non agricole plus le travail salarié agricole ${ }^{3}$.

En conséquence, les revenus de l'exploitation (on-farm) proviennent des productions agricoles et de l'élevage, de la transformation des produits à la ferme ${ }^{4}$ et de la chasse, de la pêche et de la collecte de ressources naturelles ${ }^{5}$. Les revenus hors exploitation (off-farm) comprennent l'emploi salarié (agricole et non agricole), les activités d'auto-emploi, les transferts publics et privés et les rentes (voir figure 4.1).

Il existe un large éventail de stratégies des ménages qui correspond à de multiples combinaisons de ces activités et de ces revenus; et la diversification en dehors de l'exploitation n'implique pas l'abandon total de la production agricole et de l'élevage. La combinaison d'activités propres à un ménage dépend de la rentabilité de ces activités, des actifs dont il dispose, ainsi que des opportunités offertes par l'environnement économique en termes d'options d'investissement et des risques. Le travail et le capital peuvent être réaffectés localement quand des activités plus rentables existent ou réaffectés ailleurs lorsque le déplacement des facteurs est la seule option.

Figure 4.1 Classification des activités et des revenus des ménages ruraux

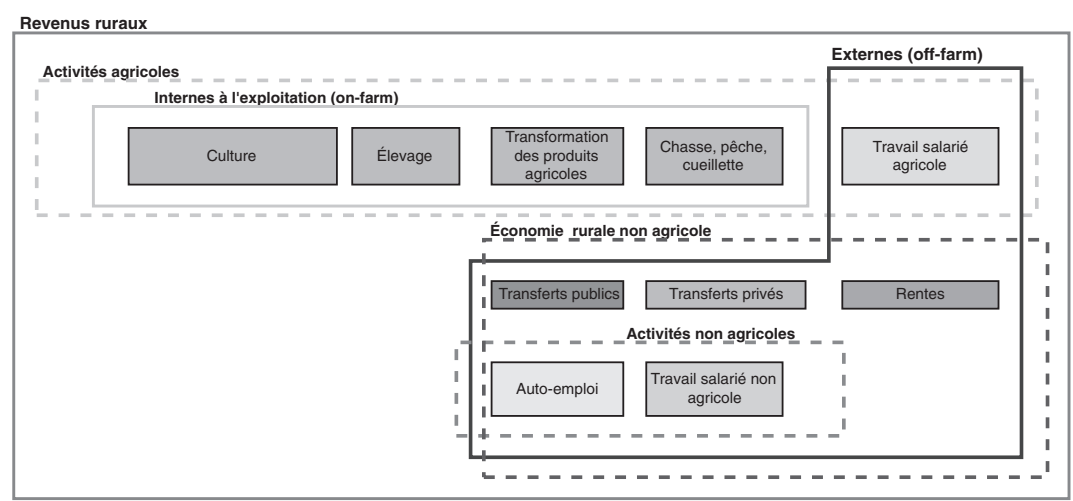

Source : Auteurs. 


\section{Les réalités des activités off-farm dans les régions étudiées}

L'échantillon du programme RuralStruc présente une grande diversité de stratégies d'activités et de revenus. L'importance des revenus hors exploitation sera d'abord considérée au niveau agrégé, puis chaque type d'activité sera analysé successivement.

\section{Importance et nature des activités et revenus hors exploitation}

Dans les régions étudiées, les activités et revenus hors exploitation sont très répandus. Ils se caractérisent par leur faible rentabilité et par l'absence de configuration spécifique, tant du point de vue du type que de la distribution.

Un développement généralisé mais une faible rentabilité. Bien que l'agriculture demeure l'épine dorsale des activités en milieu rural dans la plupart des régions étudiées (comme l'a montré le chapitre 3), les autres activités sont partout présentes et peuvent fournir un complément de revenu significatif allant jusqu'à remplacer progressivement les revenus d'exploitation dans certains cas. La figure 4.2 indique les taux de participation des ménages ruraux enquêtés aux activités hors

Figure 4.2 Participation aux activités off-farm dans les régions étudiées (pourcentage de ménages)

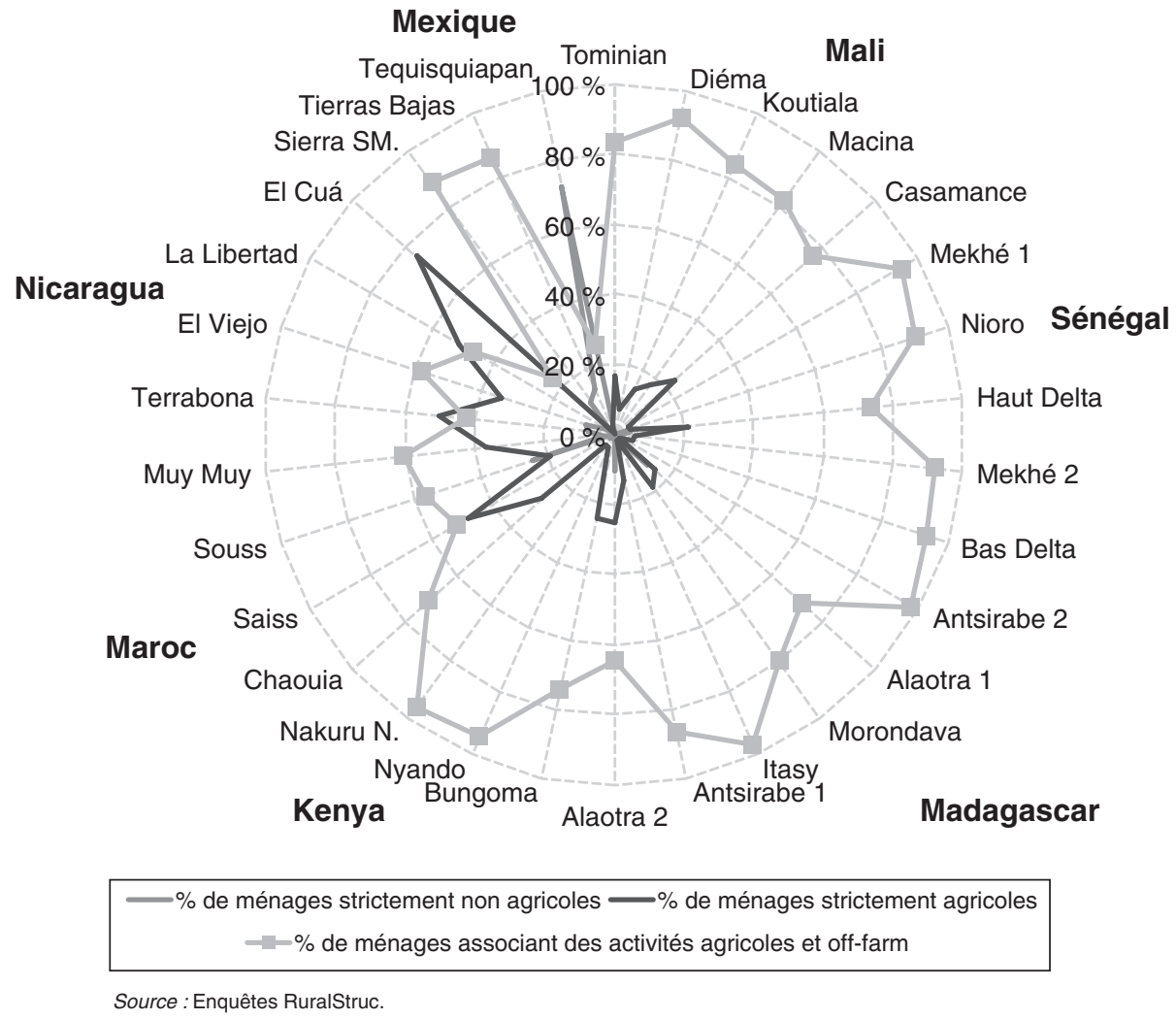


exploitation. Elle distingue les ménages qui sont uniquement exploitants agricoles (sans activités ou revenus hors exploitation), les ménages combinant à la fois revenus d'une exploitation agricole et d'autres types de revenus, et enfin les ménages sans exploitation agricole (qui n'ont donc pas de revenus d'exploitation).

En ASS, le taux de participation des ménages ruraux (qui sont presque tous des exploitants agricoles) à des activités externes est extrêmement élevé (entre $80 \%$ et $95 \%$ ), plus élevé que dans les autres régions, où les ménages tendent à se spécialiser sur leur exploitation. Cette forme de spécialisation est particulièrement visible au Maroc et au Nicaragua, où elle concerne une forte proportion des ménages sans activités en dehors de l'exploitation (en particulier $50 \%$ dans le Saïss et à Terrabona et $75 \%$ à El Cuá.). Les trois régions mexicaines sont particulières : de nombreux ménages de Tequisquiapan n'ont pas d'exploitation agricole tandis que les zones du Sotavento demeurent très diversifiées.

Traduite en revenus, la contribution des activités off-farm au revenu global des ménages est très variable d'une région à l'autre, comme le montre la figure 4.3. Contrairement aux résultats présentés par Reardon et al. (2007), un travail qui a compilé 40 études en Afrique et en Amérique latine, les écarts entre les régions d'ASS et les autres sont moins importants que les écarts observés à l'intérieur de chaque groupe de pays, et les régions d'ASS ne semblent pas moins diversifiées que les autres.

Le paradoxe révélé par ce graphique est l'écart entre la valeur des revenus externes et leur part dans le revenu global des ménages. Hors d'Afrique subsaharienne, la valeur et la part suivent la même tendance, alors qu'en ASS - hormis à Nakuru North, Kenya - des revenus d'activités off-farm très faibles représentent une part importante du revenu total des ménages. Cette configuration, particulièrement marquée au Sénégal, renvoie à l'importance de la pauvreté (étudiée au chapitre 3) et au manque de possibilités de diversification, un point sur lequel nous reviendrons plus loin.

La comparaison entre pays montre que les activités externes génèrent de faibles revenus en ASS, où elles rapportent moins de 400 US\$ PPA par EqA par an au ménage moyen (moins de 200 US\$ PPA au Mali, à Madagascar et en Casamance, au Sénégal). Les exceptions sont le Bas Delta (Sénégal) et Nakuru North (Kenya), où la valeur de ces revenus est plus élevée et reflète des économies régionales plus dynamiques. Ce dynamisme ne peut cependant être réduit à la proximité d'une ville. En effet, dans d'autres régions du Kenya et du Sénégal proches d'une ville, la diversification ne procure pas un niveau de revenus significatif. C'est le cas par exemple de Nyando au Kenya, malgré sa proximité de Kisumu, qui est pourtant une agglomération plus grande que Nakuru (voir figure 3.2 au chapitre 3). Hors ASS au contraire, excepté dans les régions très agricoles, la valeur des revenus off-farm est plus élevée (de 600 US\$ PPA à 1600 US\$ PPA par EqA). Tequisquiapan (2 600 US\$ PPA), où $70 \%$ des ménages n'ont pas d'exploitation agricole, est un cas unique : il illustre la situation de régions plus riches, où le rôle de l'agriculture a sensiblement diminué mais où le niveau de vie des ménages ruraux n'est pas nécessairement plus élevé que dans les régions plus tournées vers l'agriculture (voir chapitre 3). 


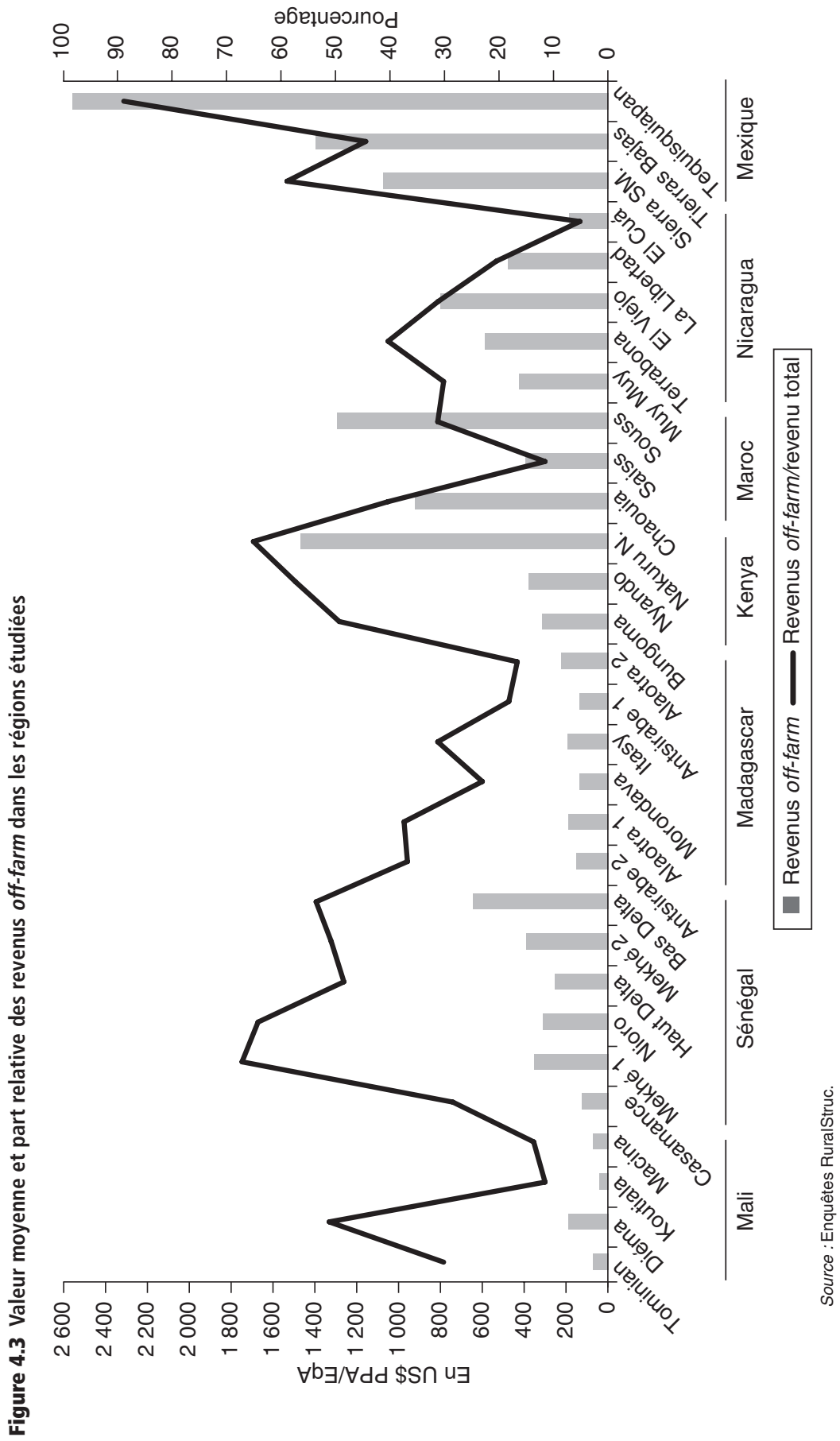


Hétérogénéité des sources de revenus hors exploitation. La ventilation des revenus par source fait apparaître d'importantes différences entre les régions étudiées. Elle révèle des situations et des stratégies diversifiées et souligne les opportunités et les contraintes de l'environnement local qui déterminent les options économiques. Le tableau 4.1 présente les principales activités externes des ménages selon leur ordre d'importance et la contribution de chaque type de revenu au revenu offfarm global. Au niveau régional, deux grandes tendances se dégagent en termes de types et de combinaisons d'activités.

La première tendance est liée à la richesse des régions : les revenus externes sont plus diversifiés dans les régions les plus riches. Hors ASS, les ménages ont un plus large éventail d'activités, leurs principales sources de revenus externes sont plus équilibrées et les trois sources les plus importantes représentent généralement $75 \%$ à $80 \%$ du revenu externe total (avec quelques exceptions). En ASS en revanche, la première activité procure le plus souvent la majeure partie du revenu off-farm tandis que les trois activités principales représentent fréquemment $90 \%$ à $95 \%$ du total.

L'autre grande tendance est l'importance des activités d'auto-emploi en ASS et son importance décroissante dans les régions plus riches. L'auto-emploi est en effet la première activité externe dans 15 des 19 régions d'ASS mais dans une seulement des 11 régions hors ASS (deux, si on exclut le cas particulier des rentes au Maroc - voir plus loin la section sur les autres revenus off-farm). Dans la plupart des régions d'Afrique, une combinaison d'auto-emploi et de migration domine les stratégies externes, tandis qu'au Kenya et à Madagascar, l'auto-emploi est associé respectivement à l'emploi salarié non agricole et au salariat agricole.

\section{Caractéristiques des activités off-farm}

Afin d'étudier les choix de diversification dont disposent les ménages, cette section et la suivante examineront les contours et l'importance de chaque catégorie de revenu. Bien que les données ne soient pas suffisantes pour tirer des conclusions sur l'efficacité absolue de chaque type d'activité pour permettre une sortie de la pauvreté, il est possible d'effectuer des comparaisons entre les profils de diversification observés dans différentes régions et de tirer des conclusions intermédiaires quant à l'ampleur des opportunités de diversification non agricole.

L'analyse portera sur le niveau de développement de chaque activité (le pourcentage de ménages concernés) et les revenus qu'en tirent les ménages (présentés par personne active $)^{6}$. L'enquête n'était pas assez détaillée pour déterminer l'activité économique particulière de chaque membre actif du ménage ; c'est pourquoi, pour un ménage donné, le total des revenus de chaque activité a été divisé par le nombre total d'actifs. Cette approche sous-estime probablement la rentabilité de chaque activité et, en conséquence, l'indicateur de revenu par actif doit être considéré comme une approximation ${ }^{7}$.

L'emploi salarié agricole : une activité courante mais peu rémunératrice. Il est nécessaire d'analyser tout d'abord les liens existants entre la structure des exploitations agricoles et l'importance du travail salarié avant d'examiner ensuite ses caractéristiques dans les régions étudiées. 
Tableau 4.1 Principaux revenus et activités off-farm (\% du revenu hors exploitation moyen)

\begin{tabular}{|c|c|c|c|c|}
\hline Pays & Région & $1^{\text {re }}$ activité externe & $2^{\mathrm{e}}$ activité externe & $3^{e}$ activité externe \\
\hline \multirow[t]{4}{*}{ Mali } & Tominian & Transferts privés (48 \%) & Auto-emploi (37 \%) & Salaire non agr. (7 \%) \\
\hline & Diéma & Transferts privés (86 \%) & Auto-emploi (11\%) & Salaire agr. (3 \%) \\
\hline & Koutiala & Auto-emploi (63 \%) & Transferts privés (20\%) & Salaire non agr. (7 \%) \\
\hline & Macina & Auto-emploi (43 \%) & Transferts privés (22 \%) & Salaire agr. (17 \%) \\
\hline \multirow[t]{6}{*}{ Sénégal } & Casamance & Auto-emploi (69 \%) & Transferts privés (20 \%) & Salaire non agr. (10 \%) \\
\hline & Mekhé 1 & Auto-emploi (69 \%) & Transferts privés (19 \%) & Salaire non agr. (12\%) \\
\hline & Nioro & Auto-emploi (77 \%) & Transferts privés (13\%) & Salaire non agr. (8 \%) \\
\hline & Haut Delta & Auto-emploi (76 \%) & Salaire non agr. (15\%) & Transferts privés (9 \%) \\
\hline & Mekhé 2 & Auto-emploi (68 \%) & Salaire non agr. (19 \%) & Transferts privés (13\%) \\
\hline & Bas Delta & Auto-emploi (58 \%) & Salaire non agr. (22 \%) & Rentes (13\%) \\
\hline \multirow[t]{6}{*}{ Madagascar } & Antsirabe 2 & Auto-emploi (67 \%) & Salaire agr. (21 \%) & Transferts privés (7 \%) \\
\hline & Alaotra 1 & Auto-emploi (52 \%) & Rentes (19 \%) & Salaire agr. (18 \%) \\
\hline & Morondava & Auto-emploi (50 \%) & Salaire agr. (24 \%) & Salaire non agr. (16 \%) \\
\hline & Itasy & Auto-emploi (53 \%) & Salaire agr. (25 \%) & Salaire non agr. (12\%) \\
\hline & Antsirabe 1 & Auto-emploi (62 \%) & Salaire agr. (26 \%) & Salaire non agr. (6 \%) \\
\hline & Alaotra 2 & Auto-emploi (57 \%) & Rentes (23 \%) & Salaire agr. (16 \%) \\
\hline \multirow[t]{3}{*}{ Kenya } & Bungoma & Salaire non agr. (54 \%) & Auto-emploi (38 \%) & Salaire agr. (5 \%) \\
\hline & Nyando & Salaire non agr. (56 \%) & Auto-emploi (31%) & Salaire agr. (8 \%) \\
\hline & Nakuru N. & Auto-emploi (72 \%) & Salaire non agr. (24 \%) & Rentes (2 \%) \\
\hline \multirow[t]{3}{*}{ Maroc } & Chaouia & Rentes (30 \%) & Transferts privés (23 \%) & Auto-emploi (22 \%) \\
\hline & Saïss & Rentes (47 \%) & Transferts privés (15 \%) & Auto-emploi (15 \%) \\
\hline & Souss & Rentes (40 \%) & Auto-emploi (24 \%) & Salaire non agr. (14 \%) \\
\hline \multirow[t]{5}{*}{ Nicaragua } & Muy Muy & Salaire agr. (37 \%) & Transferts privés (30 \%) & Salaire non agr. (17 \%) \\
\hline & Terrabona & Transferts privés (32 \%) & Salaire non agr. (31\%) & Auto-emploi (27 \%) \\
\hline & El Viejo & Salaire agr. (58 \%) & Transferts privés (19 \%) & Salaire non agr. (17 \%) \\
\hline & La Libertad & Salaire agr. (67 \%) & Auto-emploi (20 \%) & Salaire non agr. (7 \%) \\
\hline & El Cuá & Salaire non agr. (28 \%) & Salaire agr. (26 \%) & Auto-emploi (23 \%) \\
\hline \multirow[t]{3}{*}{ Mexique } & Sierra SM & Auto-emploi (38 \%) & Transferts publics (32 \%) & Salaire agr. (15 \%) \\
\hline & T. Bajas & Transferts publics (32\%) & Auto-emploi (30 \%) & Salaire agr. (15\%) \\
\hline & Tequis. & Salaire non agr. (47 \%) & Salaire agr. (24\%) & Auto-emploi (21\%) \\
\hline
\end{tabular}

Source : Enquêtes RuralStruc.

Travail salarié agricole et structure des exploitations. En raison du rôle important de l'agriculture dans l'emploi des pays en développement, le travail salarié agricole est une activité courante et une option fréquemment utilisée par les ménages ruraux à la recherche d'un complément de revenus. Cependant, le développement 
de l'emploi salarié dans l'agriculture est fortement tributaire de la demande locale en main-d'œuvre, laquelle dépend du degré de différenciation des structures d'exploitation. En effet, l'existence de grandes exploitations incapables de couvrir leurs besoins en main-d'œuvre est généralement une condition préalable au développement du salariat agricole. Celui-ci peut également être favorisé par la présence de certaines cultures qui nécessitent une main-d'œuvre abondante et ne sont pas totalement mécanisables (comme l'horticulture et les cultures arbustives) 8 .

Les exploitations familiales dominent dans les régions étudiées par le programme RuralStruc, particulièrement en ASS mais aussi au Maroc, au Nicaragua et au Mexique. Le programme définit l'agriculture familiale comme « une forme de production qui se caractérise par le lien structurel particulier existant entre les activités économiques et la structure familiale. Cette relation influe sur le processus de décision, c'est-à-dire sur le choix des activités, l'organisation du travail familial, la gestion des facteurs de production et la transmission du patrimoine » (Bélières et al. 2002, p. 3). Il ressort clairement de cette définition qu'au sein de ces structures familiales, les membres du ménage accomplissent la majeure partie du travail agricole et ne perçoivent pas de rémunération directe de leur travail. Cependant, les exploitations familiales peuvent aussi recourir à de la main-d'œuvre extérieure lorsqu'elles ne parviennent pas à couvrir leurs besoins, par exemple en période de pointe d'activité. Cette main-d'œuvre extérieure peut consister en des groupes d'entraide locaux (parents et autres membres de la communauté qui travaillent sans compensation monétaire sur une base de réciprocité) et en travailleurs salariés - travailleurs temporaires ou employés agricoles permanents9.

La demande de main-d'œuvre s'accroît avec l'augmentation de la taille des exploitations familiales ou le développement d'exploitations managériales ou entrepreneuriales à grande échelle qui ont structurellement besoin de maind'œuvre extérieure. Rare en Afrique subsaharienne, où la majorité des exploitations sont des unités de petite taille avec un patrimoine limité, cette demande est plus fréquente dans les autres régions.

L'échantillon du programme RuralStruc présente différents niveaux de différenciation des exploitations. Les exploitations ${ }^{10}$ sont plus grandes dans les deux pays d'Amérique latine, surtout au Nicaragua où elles couvrent en moyenne 15 à 20 hectares (ha) ${ }^{11}$, tandis qu'elles sont plus modestes en ASS, surtout au Kenya et à Madagascar où la moyenne tourne autour de 1 ha. Certaines régions étudiées dans les Hautes Terres de Madagascar comptent des exploitations encore plus petites, une conséquence du terrain accidenté mais, surtout, de la croissance de la population dans un contexte où les terres arables sont limitées ${ }^{12}$. Le cas des deux pays d'Afrique de l'Ouest est plus spécifique. Les grandes exploitations y sont développées mais en raison de structures familiales qui sont elles aussi plus importantes (voir chapitre 3) et regroupent souvent plusieurs ménages sur la même exploitation ${ }^{13}$. C'est pourquoi la figure 4.4 présente la distribution des tailles d'exploitation en hectare par actif familial, car une représentation de la taille des exploitations en moyenne régionale serait trompeuse. Les extrêmes sont particulièrement marqués au Nicaragua, où les inégalités foncières sont fortes. À La Libertad par exemple, une région d'élevage reculée de la frontière agricole, 


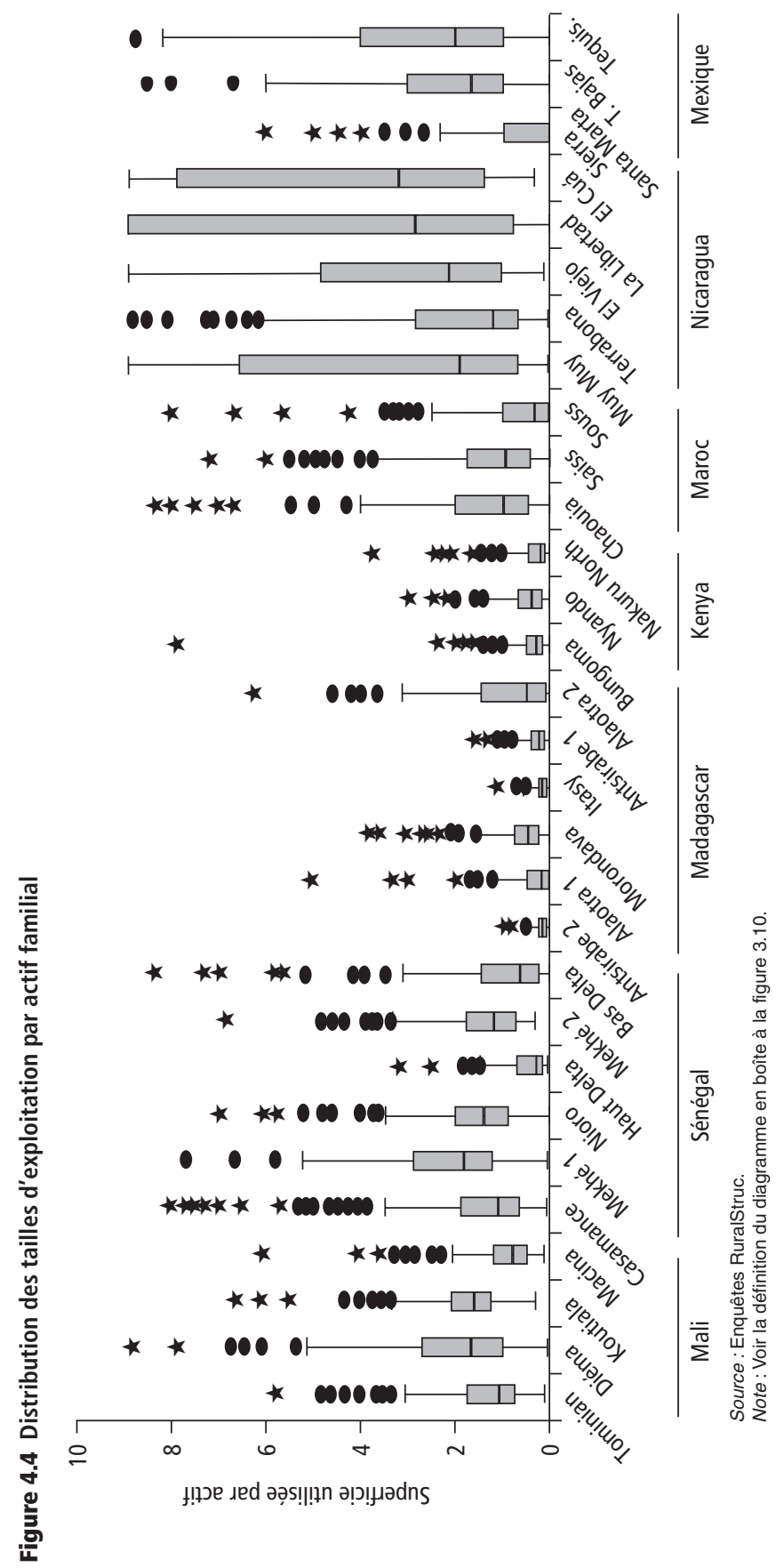


$20 \%$ des ménages les plus riches détiennent de grands domaines (latifundia) qui couvrent 53 \% de la superficie totale des ménages enquêtés. À El Viejo, une région de plaines de la côte Pacifique connue pour ses inégalités foncières et pour les conflits qui s'y rapportent et caractérisée par la production intensive de cultures d'exportation, comme le sésame ou la canne à sucre, $6 \%$ des enquêtés sont des ménages sans terre. Une situation comparable existe à l'Alaotra, à Madagascar, où $10 \%$ des ménages n'ont pas d'accès à la terre ${ }^{14}$ (ce sont principalement des familles qui ont migré dans la région en réponse à l'importante demande en travail agricole). Au Maroc également, l'accès à la terre peut être difficile, surtout dans les régions où de vastes superficies ont été accaparées par des projets publics de développement agricole. Ces situations très différenciées sont rares en ASS, où l'accès à la terre repose principalement sur des droits fonciers coutumiers.

Néanmoins, quelles que soient les structures d'exploitation, il existe toujours une demande en main-d'œuvre agricole, au moins pendant la haute saison (la récolte en général, mais aussi le repiquage du riz irrigué $)^{15}$. Or, dans un contexte de faible différenciation des exploitations, la principale contrainte est que l'offre en maind'œuvre augmente pour tous pendant la saison sèche, lorsque la demande de travail est faible, alors que les pénuries de main-d'œuvre sont fréquentes au moment de la récolte. Cette situation explique le développement de groupes d'entraide et favorise les migrations de courte durée venant d'autres régions où la saison des récoltes est différente ou dont les disponibilités en main-d'œuvre sont plus grandes. C’est le cas des migrations au départ du bassin arachidier vers le Delta du fleuve Sénégal, ou de migrations comparables au Maroc pour la récolte du blé, ou encore à El Cuá, au Nicaragua, pour la récolte du café. Plus généralement, les déséquilibres cycliques entre l'offre et la demande en main-d'œuvre reflètent le sous-emploi structurel caractéristique de nombreuses zones rurales des pays en développement.

Ampleur et caractéristiques du travail salarié agricole. L'emploi salarié agricole est assez courant dans les régions étudiées : un quart des ménages interrogés perçoivent des salaires agricoles et ils sont presque $40 \%$ dans les régions structurellement plus contrastées du Mexique et du Nicaragua. Les ménages malgaches, dont un grand nombre sont des pauvres sans terre, sont eux aussi fortement engagés dans le salariat agricole ( $46 \%$ des ménages).

Il faut noter cependant que la méthodologie de l'enquête engendre un biais. L'entrée par les stratégies des ménages ne permet pas de rendre pleinement compte de l'importance du travail salarié agricole : d'une part car les salaires agricoles perçus par des membres du ménage lors de migrations courtes vers d'autres régions sont comptabilisés dans la catégorie des transferts ; d'autre part car les salaires payés localement aux migrants venant d'autres régions sont comptés du côté du ménage du migrant. Ces limites sous-estiment certainement le poids local véritable des salaires agricoles.

Les données recueillies sur les salaires agricoles appellent deux observations. La première, qui n'est peut-être pas surprenante, est que le travail salarié agricole occupe principalement les ménages les plus pauvres de chaque région et que sa fréquence décroît lorsque le revenu global augmente ${ }^{16}$. La relation inverse entre 
l'emploi salarié agricole et la richesse du ménage est clairement illustrée par la part des salaires agricoles dans le revenu de chaque quintile ${ }^{17}:$ les salaires agricoles représentent entre $20 \%$ et $30 \%$ du revenu global des ménages des deux quintiles inférieurs, puis son poids diminue fortement. L'exception est le Nicaragua, où la part reste élevée jusqu'au quintile 4 (à Muy Muy, El Viejo et La Libertad) ${ }^{18}$.

Le travail salarié agricole est une importante source de revenus pour les ménages les plus pauvres des premiers quintiles, surtout dans les régions qui comptent des ménages sans terre ${ }^{19}$. Dans certains cas extrêmes, les ménages louent leur terre à des agriculteurs plus importants et plus aisés, ou à des agro-industries, car ils n'ont pas les moyens de mettre en valeur leurs parcelles : ils peuvent ainsi se retrouver travailleurs agricoles sur leurs propres terres. Cette situation a été observée en particulier dans le Souss (RS 2 Maroc).

La seconde observation concerne la faiblesse des revenus procurés par les emplois agricoles. La figure 4.5 illustre particulièrement les différences de prix de la main-d'œuvre entre les régions étudiées d'ASS et les régions hors Afrique subsaharienne. Le salaire minimum officiel va de 3 à 6 US\$ PPA par jour en ASS et de 8 à 10 US\$ PPA dans les autres pays ${ }^{20}$. Mais les salaires agricoles observés lors des enquêtes, qui sont en majorité payés de manière informelle, sont plus faibles, à l'exception des situations où le marché du travail connaît de fortes tensions (temporaires ou non). C'est le cas des régions qui enregistrent des pics de demande élevés - comme les régions productrices de riz au Mali, à Madagascar et dans le Delta au Sénégal - et de celles où les grandes exploitations ou les agro-industries sont nombreuses, notamment dans l'horticulture (Souss, El Viejo, Tequisquiapan) ou dans l'élevage (La Libertad et Tequisquiapan).

La principale difficulté est liée au fait que souvent, notamment dans les régions les plus pauvres, les prix locaux du travail correspondent surtout à la haute saison, lorsque les exploitations familiales sont en manque de main-d'œuvre, alors qu'il n'y a pas de demande en basse saison lorsqu'elles ont du travail à offrir. Les salaires reportés sont des prix journaliers pour la main-d'œuvre ponctuelle et ils ne peuvent pas être convertis en salaires mensuels ou annuels ${ }^{21}$. Paradoxalement, lorsqu'il existe des migrations interrégionales - comme dans la région du Delta au Sénégal et à El Cuá au Nicaragua - ce sont les travailleurs migrants venus d'autres régions qui bénéficient de cette demande temporaire de main-d'œuvre. De ce fait, le revenu moyen tiré de l'emploi agricole est très faible dans les régions d'ASS (entre 200 US\$ et 300 US\$ PPA annuels par actif) et encore plus insignifiant au Mali. Des revenus supérieurs à 1000 US\$ PPA (équivalents à 2,7 US\$ PPA par jour) ne sont observés que dans certaines des régions précitées du Maroc, du Nicaragua et du Mexique, qui offrent des possibilités de travail salarié plus permanent.

Ces données d'enquête, et le nombre relativement limité de ménages qui ont une activité salariée agricole, confirment la modeste contribution des salaires agricoles à la diversification des revenus et au recul de la pauvreté (Reardon et al. 2007). Même à Tequisquiapan, où le salaire agricole journalier moyen indiqué est de 18 US\$ PPA (grâce au développement conséquent de l'agro-industrie), le revenu moyen ramené à l'année est faible, autour de 6 US\$ PPA par actif et par jour. Un ouvrier agricole ne peut en fait gagner 18 US\$ par jour que pendant quelques jours. 


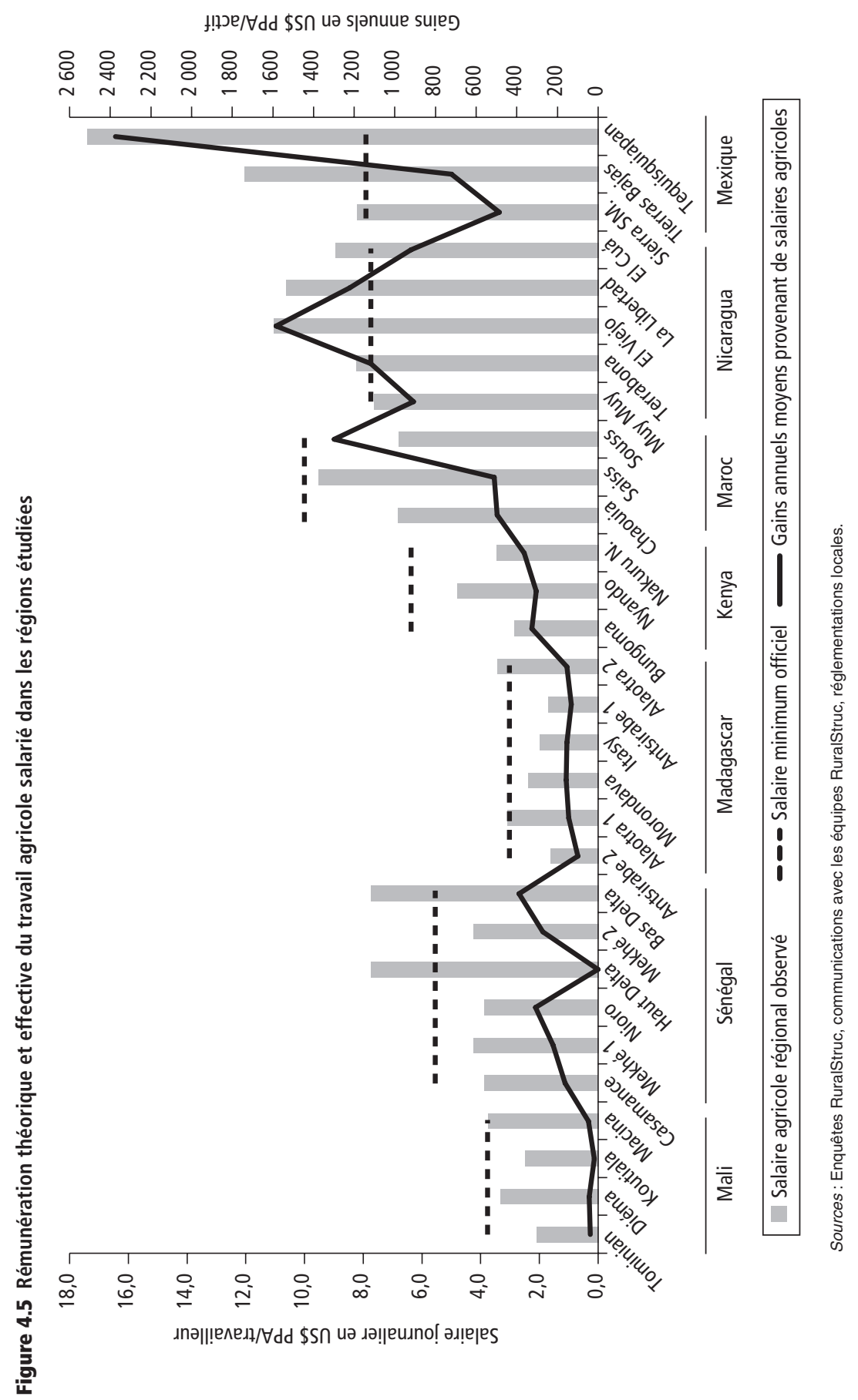


La principale conclusion en matière d'emploi salarié agricole est le manque d'opportunités réellement rémunératrices. Les emplois agricoles sont dans leur écrasante majorité temporaires et, surtout, ils offrent des revenus très limités lorsqu'ils sont rapportés à l'année. Ils représentent un complément de revenu modeste pour de nombreux ménages ruraux (un quart de l'échantillon), même s'ils s'avèrent impératifs pour les plus pauvres, qui ont peu d'autres alternatives. Seuls les emplois véritablement permanents peuvent faire une différence et permettre d'échapper à la pauvreté ; mais ils sont souvent trop mal payés et surtout trop rares pour offrir une solution viable au plus grand nombre.

L'emploi salarié non agricole : circonscrit à des situations régionales spécifiques. Le développement du travail salarié non agricole est une étape cruciale dans le modèle standard de la transformation structurelle; et pour de nombreux pays en développement engagés dans leur transition économique, les activités salariées tiennent d'ores et déjà une place importante dans la production de biens et services non agricoles. L'amplitude de l'emploi salarié non agricole dépend toutefois et avant tout des caractéristiques économiques nationales et régionales et du degré de diversification de chaque économie locale.

Ici encore, il convient de mentionner les problèmes méthodologiques dus au statut de l'emploi salarié non agricole en tant que catégorie. Sa définition est très vague dans la mesure où il recouvre toutes les activités salariées qui ne sont pas liées à la production agricole stricto sensu. Cette définition très large comprend ainsi les activités extractives (mines, carrières), les activités en aval de la production agricole notamment agro-industrielles (nettoyage, calibrage, transformation et conditionnement), l'industrie manufacturière (produits intermédiaires ou finis), le BTP et tous les types de services, publics ou privés, dans l'éducation, la santé, l'information, les transports, la garde des enfants, la sécurité, etc. Toutes ces activités comprennent des emplois qualifiés et non qualifiés et peuvent être développées par des entreprises très diverses au plan de la taille, du capital et du type de gestion.

Le périmètre de cette catégorie et l'importance du secteur informel et des petites entreprises dans la plupart des pays en développement impliquent qu'il peut être parfois difficile de distinguer l'activité indépendante de type auto-emploi du travail salarié non agricole. Cela soulève des questions quant au statut effectif de certains types d'emploi qui, bien que théoriquement salariés, sont majoritairement exécutés dans de petits ateliers ou de petites entreprises à petite échelle - par exemple chauffeur de taxi ou apprenti - et sont donc difficiles à analyser lorsqu'ils entrent dans le même groupe que le travail de bureau formel ou le travail dans une usine. Cette catégorie est hétérogène et il faut garder cette caractéristique à l'esprit lors de la comparaison de contextes économiques et institutionnels différents.

Dans les régions du programme RuralStruc, environ $25 \%$ des ménages enquêtés exercent une activité salariée agricole, mais seulement $15 \%$ des ménages ont un emploi salarié non agricole avec de fortes différences observées entre régions (voir figure 4.6). 


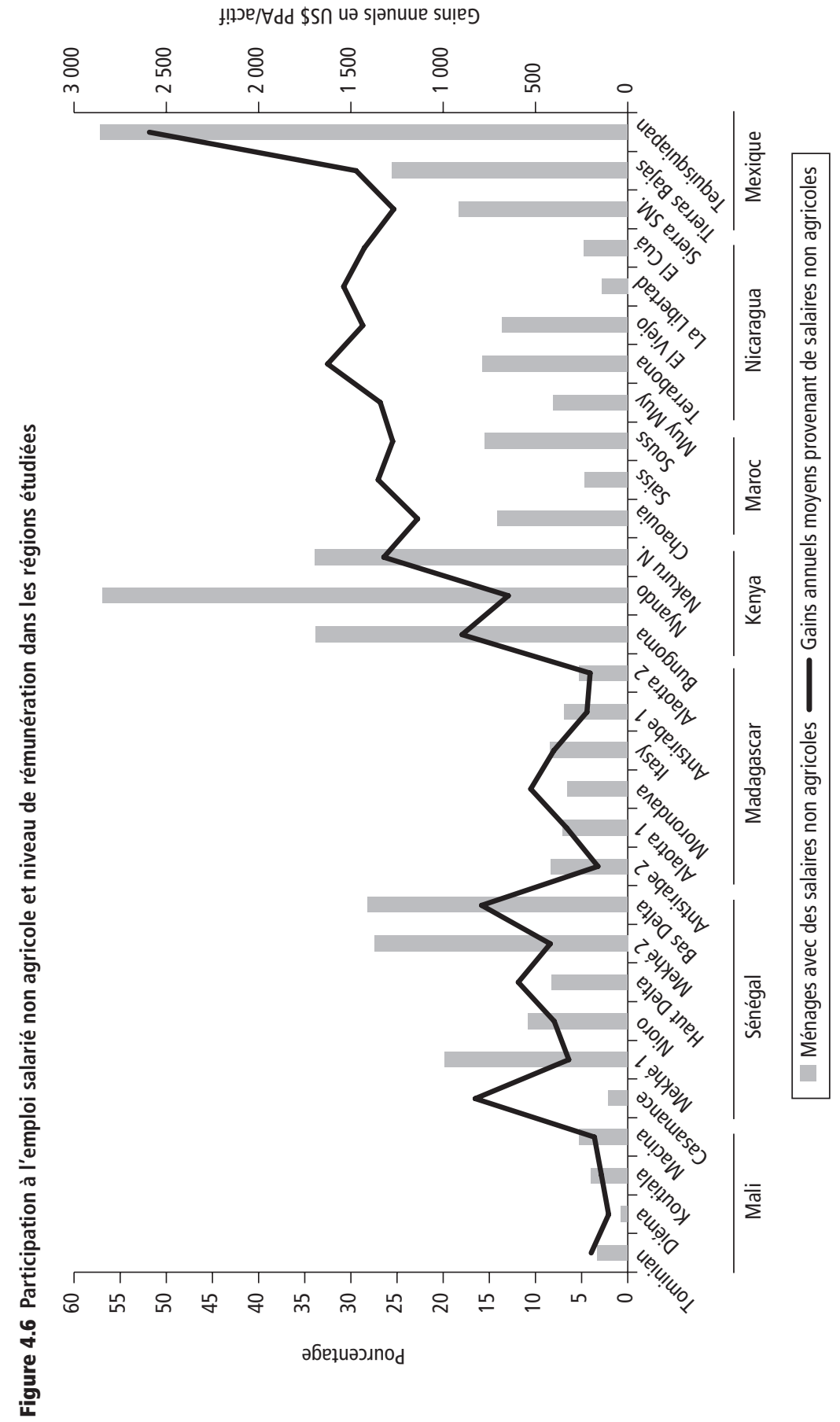


Le Kenya et le Mexique se distinguent car $40 \%$ des ménages ont un travail salarié non agricole, tandis que Madagascar et surtout le Mali sont très loin derrière. Même au Mexique et au Kenya, les disparités sont fortes : 57 \% des ménages ont des activités salariées non agricoles à Tequisquiapan et Nyando, $34 \%$ à Bungoma et Nakuru, et seulement $21 \%$ dans les deux sous-zones du Sotavento. Au Sénégal, dans le Bas Delta et le nord du bassin arachidier (Mekhé), les chiffres ressortent respectivement à $20 \%$ et $25 \%$. Le Souss, Terrabona et El Viejo, au Maroc et au Nicaragua, atteignent $15 \%$, soit la moyenne de l'échantillon.

Ces fortes différences posent la question des déterminants de l'emploi salarié non agricole. Les écarts observés entre les ménages trouvent comme souvent des explications à la fois au niveau microéconomique et au niveau méso/macroéconomique. Au niveau microéconomique, comme l'atteste la littérature ${ }^{22}$, la capacité des ménages à saisir les opportunités d'emploi locales dépend avant tout de leurs compétences, ce qu'atteste la forte corrélation positive entre le niveau d'instruction et la participation au travail salarié non agricole ${ }^{23}$. Le tableau 4.2 illustre la grande hétérogénéité des situations entre pays du programme RuralStruc en matière d'éducation. En revanche, le niveau de revenu des ménages ne semble avoir aucun effet sur leur taux d'activité salariée non agricole. Les ménages participent à l'emploi salarié non agricole quel que soit leur groupe de revenus, et la corrélation entre activité salariée non agricole et niveau de revenu total peut même être négative dans certains cas.

Au niveau méso- et macroéconomique, hormis les rares emplois dans les secteurs de l'éducation, de la santé et de l'administration locale (instituteurs, assistants médicaux, fonctionnaires), les débouchés en termes de travail salarié

Tableau 4.2 Niveau d'instruction des ménages enquêtés (moyenne nationale en \%)

\begin{tabular}{|c|c|c|c|c|c|c|}
\hline & & $\begin{array}{c}\text { Pas } \\
\text { d'instruction }\end{array}$ & $\begin{array}{l}\text { Primaire } \\
\text { commencé }\end{array}$ & $\begin{array}{l}\text { Primaire } \\
\text { terminé }\end{array}$ & $\begin{array}{l}\text { Secondaire } \\
\text { commencé }\end{array}$ & $\begin{array}{l}\text { Secondaire } \\
\text { terminé ou } \\
\text { université }\end{array}$ \\
\hline \multirow{7}{*}{ 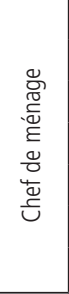 } & Mali & 84 & 10 & 2 & 4 & 0 \\
\hline & Sénégal & 79 & 16 & 3 & 2 & 0 \\
\hline & Madagascar & 18 & 56 & 7 & 18 & 1 \\
\hline & Kenya & 0 & 68 & 14 & 8 & 9 \\
\hline & Maroc & 50 & 15 & 22 & 10 & 3 \\
\hline & Nicaragua & 39 & 4 & 52 & 5 & 1 \\
\hline & Mexique & 24 & 38 & 22 & 1 & 15 \\
\hline \multirow{7}{*}{ 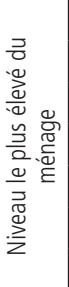 } & Mali & 40 & 20 & 33 & 7 & 0 \\
\hline & Sénégal & 17 & 40 & 25 & 14 & 4 \\
\hline & Madagascar & 6 & 34 & 8 & 28 & 24 \\
\hline & Kenya & 0 & 26 & 28 & 26 & 20 \\
\hline & Maroc & 7 & 4 & 42 & 39 & 9 \\
\hline & Nicaragua & 0 & 0 & 8 & 56 & 36 \\
\hline & Mexique & n.d. & n.d. & n.d. & n.d. & n.d. \\
\hline
\end{tabular}

Source : Enquêtes Ruralstruc. 
non agricole dépendent des caractéristiques régionales : niveau de ressources naturelles, peuplement et croissance démographique, qualité des infrastructures et dotation en biens publics, densité et accès au réseau urbain (illustrés au chapitre 3 par les cartes de la figure 3.3) et présence de secteurs d'activités dynamiques, porteurs de croissance économique et générant une demande de main-d'œuvre. Mais les effets de localité peuvent être considérables et peser sur les résultats régionaux si les ménages ruraux enquêtés sont dans le voisinage d'une usine qui offre des centaines d'emplois.

Dans les régions étudiées, c'est le cas à Terrabona au Nicaragua, où une industrie textile liée au développement des zones franches offre des emplois industriels (voir encadré 4.1), mais surtout à Tequisquiapan, Mexique, où une longue tradition de maquiladoras stimulée par l'ALENA est à l'origine de la dissémination de petites unités de production en zone rurale (voir encadré 4.2). Les emplois y sont relativement bien rémunérés et ont un impact important sur les revenus locaux.

Dans le Souss marocain, le développement des services touristiques dans la ville voisine d'Agadir et dans les stations balnéaires offre quelques opportunités. Dans les deux autres régions du Maroc, ce sont la transformation et la commercialisation des produits agricoles, ainsi que le BTP et les services qui génèrent les principales sources d'emploi non agricole.

Au Sénégal, le Bas Delta bénéficie d'une connexion à la ville de Saint-Louis, tandis que la situation de Mekhé, sur le grand axe routier reliant Saint-Louis à Dakar, explique une activité salariée non agricole relativement plus importante des ménages enquêtés. Ce n'est pas le cas à Nioro et surtout en Casamance, qui sont plus éloignés de la zone de forte densité économique du littoral - et la Casamance est en outre de l'autre côté de la Gambie. Les ménages enquêtés y sont pourtant proches d'agglomération mais, comme on l'a vu au chapitre 3 , les différences d'opportunités et d'accès au marché n'ont pas d'incidence significative sur le revenu global des ménages.

La situation la plus paradoxale est sans doute celle du Kenya, un des pays les plus urbanisés d'Afrique subsaharienne, où l'urbanisation demeure extrêmement dynamique (voir encadré 3.2). Cependant, l'expansion urbaine s'est effectuée sans industrialisation (cette absence d'industrialisation étant une caractéristique majeure de l'urbanisation en ASS), de sorte que l'emploi non agricole consiste principalement en emplois peu qualifiés et souvent temporaires dans l'industrie agroalimentaire (canne à sucre, conserverie), dans le BTP et l'artisanat, et dans les services peu rémunérés (commerce, transport, restauration).

Étant donné la diversité des profils des régions et des pays, la participation des ménages à l'emploi non agricole et les revenus qu'ils en tirent sont très hétérogènes. Ces revenus sont faibles dans les régions étudiées, avec une différence très marquée entre les régions d'ASS et les autres. En ASS, le revenu annuel moyen par actif est de l'ordre de 500 US\$ PPA, tandis qu'au Maroc, au Nicaragua et au Mexique, il est proche de 1500 US\$ PPA. Nakuru et Tequisquiapan appellent des remarques particulières. À Nakuru, le revenu moyen est de 1500 US\$ PPA, bien plus proche des niveaux observés au Nicaragua et au Maroc que du reste du Kenya, tandis qu’à Tequisquiapan, la rémunération annuelle du travail salarié 


\section{GNCADRÉ 4.1}

\section{Zones franches industrielles et travail salarié non agricole au Nicaragua}

Au Nicaragua, le nombre d'usines situées dans des zones franches industrielles (ZFI) est en forte augmentation depuis les années 1990. Las Mercedes, le premier parc industriel ouvert en 1976 comptait 11 usines. Aujourd'hui, le système des ZFI comprend une douzaine de parcs industriels et une cinquantaine d'entreprises, principalement originaires de Taïwan et des États-Unis, qui produisent en grande majorité des vêtements pour l'exportation, essentiellement aux États-Unis. Le secteur est particulièrement générateur d'emplois : le nombre de postes de travail est passé de 1003 en 1992 à 38792 en 2001 et avoisine les 70000 aujourd'hui. Quinze mille créations d'emplois supplémentaires sont attendues dans les trois à cinq prochaines années. On estime que $55 \%$ des travailleurs sont des femmes jeunes avec un niveau de formation réduit.

L'emploi industriel est très concentré dans les zones de Matagalpa et Managua et bénéficie aux zones rurales voisines (Corral et Reardon 2001). Dans les enquêtes RuralStruc, il concerne principalement Terrabona, où les emplois des ZFI génèrent des salaires annuels allant de 2500 à 4500 US\$ PPA par habitant. Ces chiffres sont conformes aux estimations d'autres études, qui évaluent le salaire mensuel maximum à 500 US\$ en 2009. En janvier 2010, le gouvernement du Nicaragua, les syndicats et le secteur privé ont signé un accord qui fixe les ajustements de salaires dans les ZFI pour les trois prochaines années. L'objectif est de protéger les emplois et d'offrir une certaine prévisibilité afin que les investisseurs puissent établir des projections financières pour leurs entreprises. Cet accord, dit « Accord de consensus social/emploi » porté de la Commission tripartite du travail de la zone franche, prévoit des augmentations de salaire minimum sur les trois prochaines années de $8 \%, 9 \%$ et $10 \%$.

Sources : Enquêtes RuralStruc ; RS 2 Nicaragua.

\section{ENCADRÉ 4.2}

\section{Les maquilas du secteur textile à La Fuente, Tequisquiapan, Mexique}

Le village de La Fuente dans le municipio de Tequisquiapan offre une bonne illustration du processus de « densification » et de diversification de l'économie rurale dans le sud de l'État de Querétaro. La Fuente, qui compte 3884 habitants (recensement de 2005), est situé à $18 \mathrm{~km}$ de Tequisquiapan (26 858 habitants) et à $24 \mathrm{~km}$ de San Juan del Rio, une ville de 210000 habitants reliée par une autoroute inter-États à Querétaro et Mexico. Malgré son environnement urbanisé, La Fuente conserve une forte activité agricole, I'agriculture représentant $24,3 \%$ de la valeur ajoutée locale en 2000 , contre 3,5\% à Tequisquiapan. Le village accueille trois maquilas, qui emploient respectivement 150, 100 et 80 personnes. Tous les travailleurs, des femmes en grande majorité, résident à La Fuente ou dans des villages voisins.

(Suite de l'encadré page suivante) 
Les deux plus grandes usines se spécialisent dans la ropa barata (confection bon marché) - vêtements de base pour l'exportation - et subissent de plein fouet l'intense concurrence de la Chine depuis plusieurs années. La troisième usine, Lecuria La Fuente, est spécialisée dans la lingerie fine pour le segment supérieur du marché intérieur et vend ses produits sous la marque Vanity à des boutiques haut de gamme comme Liverpool et Palacio de Hierro. Ce positionnement et les tâches plus qualifiées ont jusqu'ici protégé l'entreprise de la concurrence étrangère. La société a été créée par deux personnes, dont le directeur, un ingénieur textile né dans le village. Le terrain de l'usine a été acheté à un ejidatario - un résident local qui a bénéficié de droits de propriété associés au système ejido (terrain collectif distribué lors de la réforme agraire).

Les tissus sont directement importés de Corée du Sud et du Japon et coupés au laser avant d'être assemblés en pièces de lingerie. Ce travail étant très spécialisé (il faut 10 mois pour former une ouvrière), il est bien rémunéré. Les contrats de travail sont établis sur la base d'un prix par minute et d'un nombre de minutes par pièce et les ouvrières sont payées en fonction de leur productivité au-dessus ou au-dessous du temps moyen nécessaire pour coudre une pièce. Le contrat-type d'un travailleur permanent procure un salaire mensuel de 2400 pesos (330 US\$ PPA), auquel s'ajoutent la Sécurité sociale et les avantages sociaux. Une bonne ouvrière peut gagner $30 \%$ de plus : 3120 pesos ou 427 US\$ PPA, soit 5130 US\$PPA par an. Des contrats à court terme (hebdomadaires) sont proposés dans les périodes de pointe à une main-d'œuvre de réserve formée ; ces contrats sont mieux payés (+ $30 \%)$, à 800 pesos par semaine, mais ne comportent pas d'avantages sociaux. À titre indicatif, un ouvrier agricole est payé 500 pesos par semaine et un travailleur occasionnel (le cas le plus fréquent) 120 à 140 pesos par jour (16 à 19 US\$ PPA).

Le programme RuralStruc a enquêté 49 ménages à La Fuente. Seulement 14 d'entre eux avaient une exploitation (voir tableau ci-dessous). Bien qu'il existe des emplois non agricoles bien rémunérés, les ménages agricoles gagnent plus en moyenne que les ménages non agricoles (13 645 US\$ PPA contre 8286 US\$PPA). Cet écart tient en grande partie au fait que les ménages agricoles ont de multiples activités, principalement un emploi salarié agricole et non agricole (généralement pratiqué par les femmes). La moitié des ménages de l'échantillon perçoit des salaires agricoles et l'autre moitié des salaires non agricoles; $15 \%$ perçoivent les deux. $55 \%$ des ménages de La Fuente ont un revenu annuel moyen par EqA supérieur à 2000 US\$ PPA et $10 \%$ un revenu supérieur à 5000 US\$ PPA.

Tableau d'encadré 4.2.1 Niveau et structure des revenus à La Fuente

\begin{tabular}{|c|c|c|c|c|c|c|c|c|c|c|}
\hline \multirow[b]{2}{*}{ Ménages } & \multirow[b]{2}{*}{ Nombre } & \multicolumn{2}{|c|}{$\begin{array}{l}\text { Revenu total } \\
\text { moyen } \\
\text { (en US\$ PPA) }\end{array}$} & \multicolumn{7}{|c|}{$\begin{array}{l}\text { Moyenne de la part de chaque source } \\
\text { (\% du revenu total) }\end{array}$} \\
\hline & & 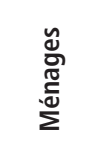 & 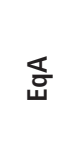 & 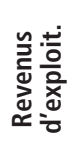 & 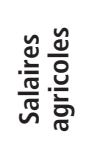 & 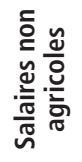 & 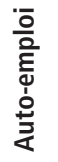 & 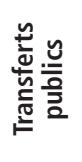 & 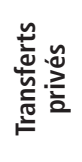 & 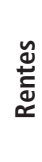 \\
\hline Sans exploitation & 35 & 8286 & 2542 & 0 & 34 & 39 & 20 & 2 & 4 & 1 \\
\hline Avec exploitation & 14 & 13645 & 3800 & 28 & 22 & 25 & 6 & 7 & 0 & 13 \\
\hline
\end{tabular}

Sources : Entretiens RuralStruc, janvier 2008 ; enquêtes RuralStruc ; RS 2 Mexique. 
non agricole peut atteindre 2500 US\$ PPA par actif, un niveau très au-dessus de toutes les autres situations observées dans les enquêtes RuralStruc.

Le cas de Nakuru et celui de Tequisquiapan - et plus généralement du Kenya et du Mexique - illustrent la discussion sur l'importance de l'environnement économique pour les possibilités de travail salarié non agricole. À Nakuru North, où $34 \%$ des ménages environ ont un travail salarié non agricole, la proximité de la ville et le patrimoine touristique voisin (parc national de Nakuru) permettent à certains membres des ménages d'obtenir un emploi dans l'administration locale, l'éducation, le commerce, le transport (la ville est située sur le principal couloir de transport du Kenya) et le tourisme. À Nyando en revanche, plus de la moitié des ménages ont un emploi salarié non agricole (20\% de plus qu'à Nakuru) mais leurs revenus sont plus faibles. Les travailleurs de Nyando occupent des emplois peu rémunérés dans les sucreries ou dans les petites activités de services. Ainsi, les différences en termes de types d'emploi salarié sont décisives. Au Mexique, les revenus plus élevés observés à Tequisquiapan résultent directement des emplois bien rémunérés et parfois très spécialisés des maquilas (voir encadré 4.2).

La distribution des revenus annuels des ménages par classes confirme les différences en termes de types d'emplois et de revenus qu'ils procurent (voir figure 4.7). Au Kenya, plus de $50 \%$ des ménages gagnent moins de 2000 US\$ PPA et seulement $5 \%$ gagnent plus de 10000 US\$, car l'emploi salarié non agricole est très peu rémunéré. Les revenus des ménages de Tequisquiapan sont nettement différents :

Figure 4.7 Distribution des ménages par classes de revenu non agricole, Kenya et Mexique

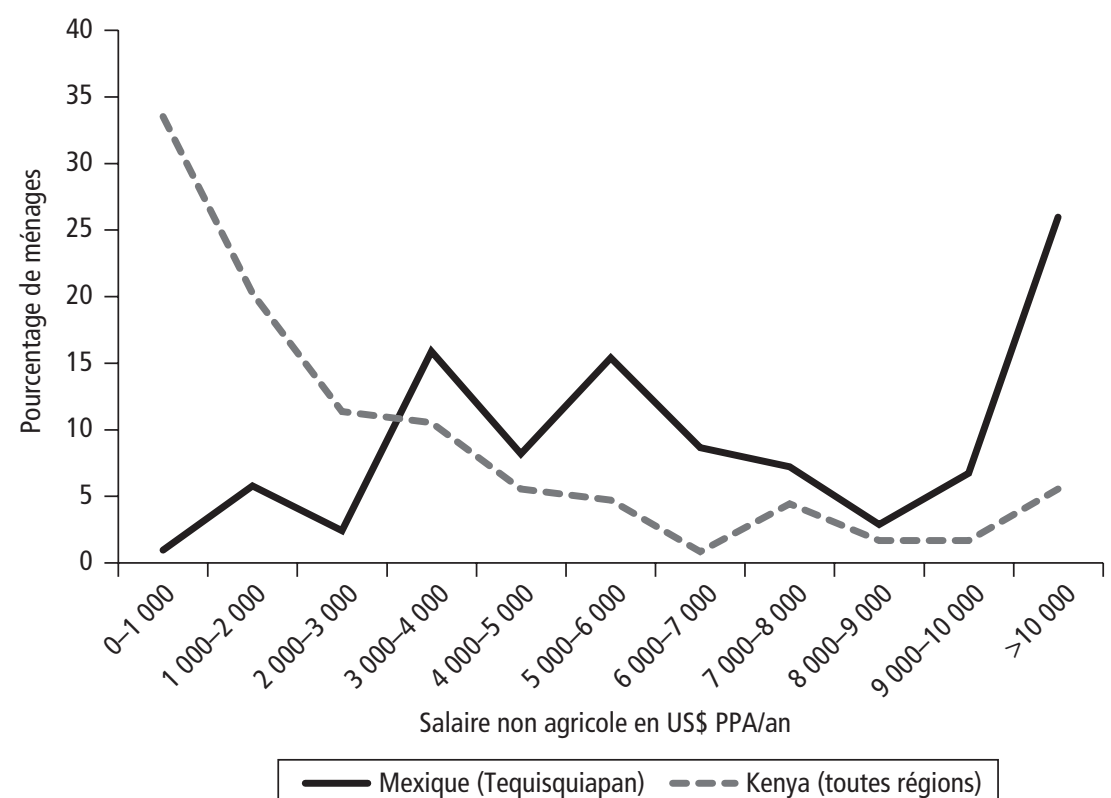

Source : Enquêtes RuralStruc. 
$25 \%$ de ceux qui ont un emploi salarié non agricole gagnent plus de 10000 US\$ PPA, ce qui est possible lorsque deux membres du ménage travaillent dans une maquiladora (voir encadré 4.2) ou lorsque l'emploi occupé est plus qualifié.

Dans l'ensemble, les possibilités de travail salarié non agricole sont assez réduites et très dépendantes de l'économie régionale. Les possibilités, lorsqu'elles existent, prennent le plus souvent la forme d'emplois peu qualifiés et peu rémunérés, excepté dans l'industrie manufacturière. Sans renforcement significatif du niveau de diversification économique (qui n'est pas nécessairement liée à l'urbanisation, comme l'illustre le Kenya), la majorité des ménages n'auront pas cette possibilité à court ou moyen terme.

L'auto-emploi: une stratégie "fourre-tout " généralisée. Comme précisé en début de chapitre, l'auto-emploi est la source de revenu externe la plus courante dans la plupart des régions étudiées et la principale possibilité de diversification pour les plus pauvres. Le travailleur salarié « prend » un travail, tandis que le travailleur indépendant " crée » son travail en saisissant les opportunités de développer ses activités en fonction de ses compétences et de son capital.

L'auto-emploi couvre ainsi un large éventail d'activités commerciales et artisanales, comprenant notamment des activités reposant sur la transformation, le transport, la distribution et la vente de produits agricoles et naturels locaux (comme le bois, les produits forestiers et le charbon de bois), le transport et le commerce de produits manufacturés pour le marché rural local (petites quincailleries), l'artisanat (poterie, vannerie, bijouterie, confection, fabrication de chaussures) et les services (coiffure, restauration, écrivain public ou réparation de matériels agricoles, de véhicules, de téléviseurs et d'autres appareils électriques).

Cette diversité est illustrée à la figure 4.8, qui indique les activités des ménages enquêtés au Sénégal. Elle montre le nombre d'actifs dans les grandes catégories d'auto-emploi par sexe.

Les revenus de ces activités dépendent étroitement du pouvoir d'achat des clients, lequel est directement lié à la richesse de la région. Les revenus des activités d'auto-emploi sont aussi diversifiés que les activités elles-mêmes. Lorsque l'auto-emploi ne requiert pas de matériel spécialisé ni de compétence particulière, les revenus sont proches du prix local du travail.

La proportion de ménages ayant une activité d'auto-emploi est très variable d'une région à l'autre. Les enquêtes ont également relevé un écart important dans les revenus de ces activités entre les ménages. Comme le montre la figure 4.9, ces caractéristiques évoluent en sens opposé et illustrent deux types de situations.

La première caractérise les régions où le taux d'auto-emploi est moyen à élevé (35 \%-80 \%) mais où les revenus de ces activités sont faibles - à savoir la plupart des régions d'ASS et celles du Sotavento au Mexique, où le taux d'auto-emploi est le plus élevé de l'enquête. Dans ces régions, le revenu annuel moyen de ces activités indépendantes avoisine 500 US\$ PPA/actif, tandis qu'il atteint 750 US\$ PPA/actif dans le bassin arachidier et dans le Delta au Sénégal et dans le Sotavento au Mexique.

La seconde situation est rencontrée dans les régions où l'auto-emploi est peu développé (5 \% à $15 \%$ ). C’est le cas au Maroc, au Nicaragua et dans une certaine 
Figure 4.8 Types d'activités d'auto-emploi au Sénégal

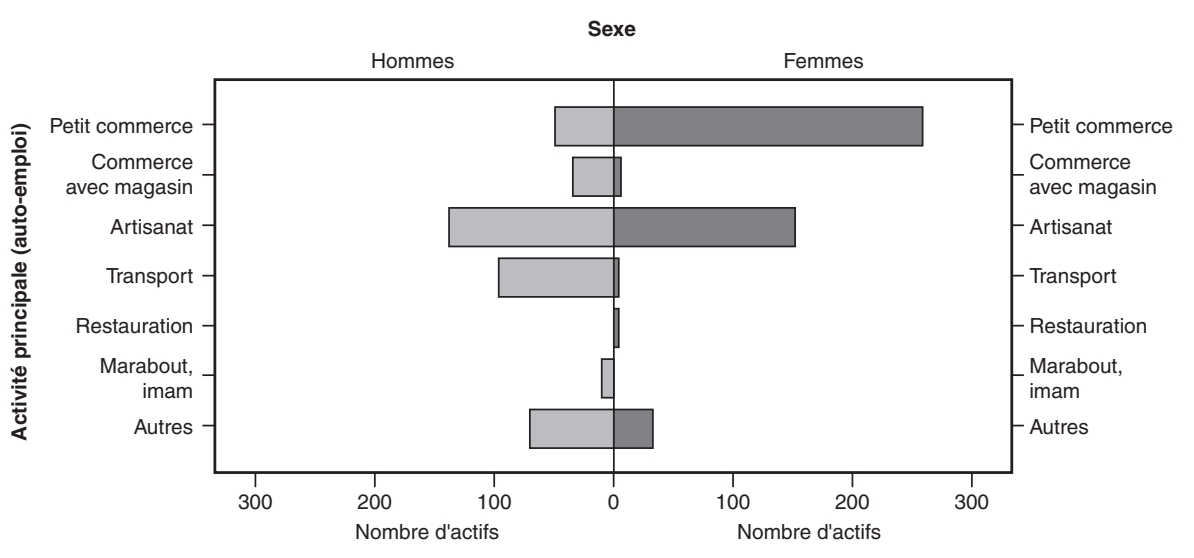

Source : Enquêtes RuralStruc.

mesure à Tequisquiapan au Mexique, où les ménages ruraux ont peu d'activités indépendantes parce qu'ils ont d'autres possibilités, comme le travail salarié et la migration. Les revenus du travail indépendant sont bien plus élevés dans ces régions - de l'ordre de 1500 US\$ PPA/actif/an en moyenne - et plus encore dans le Souss et à Tequisquiapan (2 500-3 000 US\$ PPA/actif/an), une situation qui reflète surtout des contextes régionaux plus riches. Nakuru au Kenya est un cas à part, car les niveaux de revenus de l'auto-emploi rivalisent avec ceux observés au Maroc et au Nicaragua. Cependant, ces revenus y sont très inégalement distribués et, bien que de nombreux ménages aient ce type d'activité (77\%), quelques-uns ont des revenus très élevés qui tirent la moyenne régionale vers le haut ${ }^{24}$.

Deux tendances se dégagent de cette diversité. La première correspond à ce qu'on pourrait qualifier de "diversification positive », où l'auto-emploi tient une place importante dans le revenu des ménages. C'est en général une activité à plein-temps - une micro-entreprise dotée d'équipements - qui explique que les ménages disposant d'un plus gros patrimoine ou de meilleurs actifs - ou de la capacité à réaliser un investissement initial important grâce à leur capital financier, social ou humain - aient plus de chance de profiter des opportunités du marché local. Sur l'échantillon total des enquêtes, $41 \%$ des ménages ont une activité d'auto-emploi mais seulement $13 \%$ en retirent plus de 5000 US\$ PPA par an ${ }^{25}$. Ces ménages ne sont pas nécessairement installés dans des régions où l'auto-emploi est généralisé : $58 \%$ des ménages marocains et $27 \%$ des ménages nicaraguayens qui ont une activité indépendante sont dans ce groupe, contre $22 \%$ au Mexique et au Kenya et seulement $13 \%$ au Sénégal.

La seconde tendance correspond à une " diversification neutre », dans laquelle les ménages les plus pauvres et les plus marginalisés déploient des stratégies d'adaptation et de survie en exerçant des activités indépendantes mineures à très faible rentabilité. Ces activités complètent le plus souvent le revenu de leur exploitation agricole mais elles sont bien trop peu rémunératrices pour être une option 


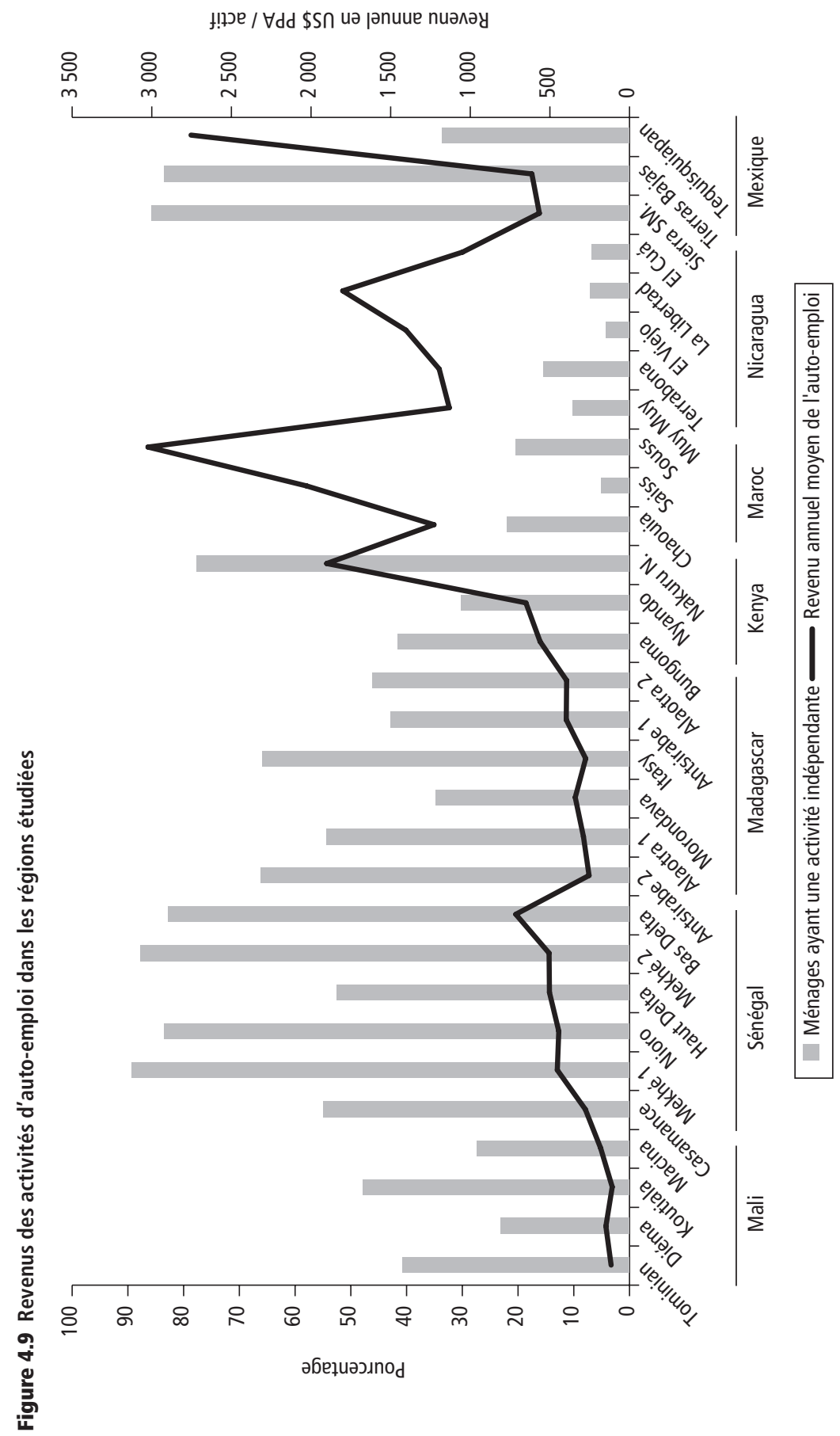


viable de sortie de la pauvreté : $51 \%$ des ménages interrogés ayant une activité d'auto-emploi gagnent moins de 1000 US\$ PPA par an, soit 2,7 US\$ PPA par jour.

Le dernier constat est que ces activités indépendantes ne se limitent pas aux lieux qui n'offrent aucune activité salariée permanente hors de l'agriculture (une idée communément admise). La diversité des situations régionales montre qu'une stratégie d'adaptation dans les zones rurales pauvres offrant peu d'activités alternatives et une stratégie de diversification positive dans les régions plus riches et diversifiées sont toutes deux possibles. En outre, les revenus de l'auto-emploi bénéficient pleinement des environnements économiques les plus dynamiques.

\section{Les autres revenus ruraux : des compléments significatifs}

Les revenus off-farm ne proviennent pas seulement des activités pratiquées localement par les membres du ménage, mais aussi des activités exercées dans des lieux distants par des travailleurs migrants qui envoient une partie de leurs gains. Ces envois de fonds jouent un rôle très important dans plusieurs régions étudiées ; ils forment la majeure partie de la catégorie des " transferts privés », qui peut également comprendre, quoique bien plus rarement, des cadeaux ou des dons provenant d'autres ménages.

Les transferts publics constituent eux aussi des revenus externes - ce sont les subventions versées par l'État ou les collectivités locales (soutien aux activités économiques ou à des groupes sociaux) mais aussi par des ONG ou d'autres communautés locales. Dans les enquêtes RuralStruc, les transferts publics sont principalement observés au Mexique et ne sont significatifs que dans ce pays ${ }^{26}$.

Enfin, la dernière catégorie de revenus off-farm est constituée par les rentes, qui comprennent les revenus de location d'actifs corporels (terrains, matériels et logements) et auraient compris les revenus de valeurs mobilières si celles-ci avaient été recensées. Entre $5 \%$ et $10 \%$ des ménages interrogés ont indiqué percevoir des revenus locatifs, hormis au Kenya où ce pourcentage est bien plus élevé (40\%). Cependant, ces revenus sont très faibles : pour $45 \%$ des ménages qui en perçoivent, ils représentent moins de 100 US\$ PPA par $\mathrm{an}^{27}$.

Les migrations : des configurations régionales très différenciées. Les ménages ont toujours déployé des stratégies de subsistance combinant des actifs fixes et mobiles, constitués de capital physique et de capital humain (Augustins 1989). Aujourd'hui, des millions de personnes se déplacent chaque année dans une autre région ou une autre ville ou franchissent des frontières et des océans afin de réduire l'écart entre leur situation et celle des habitants d'endroits plus riches (Black, Natali et Skinner 2005). D’un point de vue plus structurel et historique, la migration consiste souvent en un déplacement intersectoriel de main-d'œuvre et résulte des écarts de revenu du travail salarié entre les secteurs économiques, notamment entre l'agriculture et le reste de l'économie (Larson et Mundlak 1997). Ces migrations de main-d'œuvre sont un des plus puissants moteurs du changement structurel (voir chapitre 2).

Il est fréquent que les migrations ne soient pas permanentes ou qu'elles ne concernent pas l'ensemble du ménage. Ce type de mouvement intermédiaire 
engendre de fréquents transferts de biens et d'argent entre différents contextes géographiques et entre différents membres du ménage. Le développement des migrations temporaires (qui peuvent être de longue durée), facilité par l'amélioration des conditions de transport, a ainsi entraîné une augmentation significative des transferts privés, en particulier des envois de fonds internationaux (appelés aussi « remises ») dont le rôle dans le développement économique a été abondamment analysé ces dix dernières années.

Cependant, l'analyse globale peut être trompeuse. Non seulement les caractéristiques de la mobilité varient fortement d'une région à l'autre, ce que montrent les pays du programme RuralStruc (voir encadré 4.3), mais l'impact des envois de fonds differe aussi en fonction du niveau d'analyse. Les transferts privés provenant de l'étranger peuvent fortement peser dans les comptes nationaux mais avoir un impact très différent au niveau des régions ou des ménages. C'est particulièrement le cas dans les zones rurales où le rôle des migrations a souvent été exagéré. Comme le notent Reardon et al. (2007), aussi bien la littérature que l'opinion courante des responsables politiques tendent à souligner l'importance des remises des travailleurs migrants, alors que de nombreuses études de terrain indiquent que le pourcentage de ménages effectivement concernés par la migration est en réalité assez faible.

\section{ENCADRÉ 4.3}

\section{Caractéristiques des migrations internationales dans les pays du programme RuralStruc}

Les caractéristiques des migrations internationales dépendent de la géographie et des trajectoires nationales de développement. Les Mexicains, les Marocains et les Nicaraguayens ont profité du positionnement géographique de leurs pays, frontaliers avec des pays à revenus plus élevés, et environ $10 \%$ de la population de ces pays vit à l'étranger. Mais I'option migratoire est moins aisée en Afrique subsaharienne, hormis au Mali dont environ $11 \%$ de la population vit à l'étranger. Le Kenya et Madagascar ont des taux d'émigration extrêmement bas.

Tableau d'encadré 4.3.1 Importance des migrations internationales dans les pays étudiés

\begin{tabular}{lccccccc}
\hline & Mali & Sénégal & Madagascar & Kenya & Maroc & Nicaragua & Mexique \\
\hline $\begin{array}{l}\text { Stocks d'émigrants en 2005 (en } \\
\text { millions) }\end{array}$ & 1,2 & 0,5 & 0,2 & 0,4 & 2,7 & 0,7 & 11,5 \\
Population en 2005 (en millions) & 11,4 & 11,7 & 17 & 33,4 & 29,9 & 5,6 & 104,3 \\
Émigrants/population (\%) & 10,6 & 4,0 & 0,9 & 1,3 & 9,1 & 12,2 & 11,0 \\
$\begin{array}{l}\text { Envois de fonds en 2005 } \\
\text { (en millions d'US\$) }\end{array}$ & 175 & 511 & 16 & 494 & 4724 & 600 & 21802 \\
Envois de fonds (\% PIB) & 3,9 & 6,7 & 0,4 & 3,4 & 9,4 & 13,3 & 3,5 \\
Envois de fonds (US\$/migrant) & 144 & 1103 & 106 & 1156 & 1738 & 878 & 1895 \\
\hline
\end{tabular}

Sources : Ratha et Shaw 2007 ; World Development Indicators. 
La destination des migrants exerce une forte influence sur les revenus procurés par la migration. Au Mexique et au Maroc, où une écrasante majorité de migrants travaillent dans des pays de l'OCDE, le revenu moyen par migrant est élevé (près de 2000 US\$). Le Mali se situe à l'autre extrême : la région de Kayes dans l'ouest du pays, près de la frontière sénégalaise, a une longue tradition d'émigration en France, mais $90 \%$ des migrants maliens restent en Afrique de l'Ouest, principalement en Côte d'Ivoire, et leurs revenus représentent moins de $10 \%$ de ceux des migrants mexicains et marocains. Le Nicaragua et le Sénégal sont dans une situation intermédiaire : à peu près la moitié de leurs migrants travaille dans des pays riches, tandis que l'autre moitié travaille dans des pays voisins (Costa Rica pour le Nicaragua, Gambie et Mauritanie pour le Sénégal), avec un impact proportionnel sur le niveau des fonds envoyés. Au Sénégal, les transferts augmentent régulièrement depuis 2005.

Tableau d'encadré 4.3.2 Destination des flux migratoires dans les pays étudiés

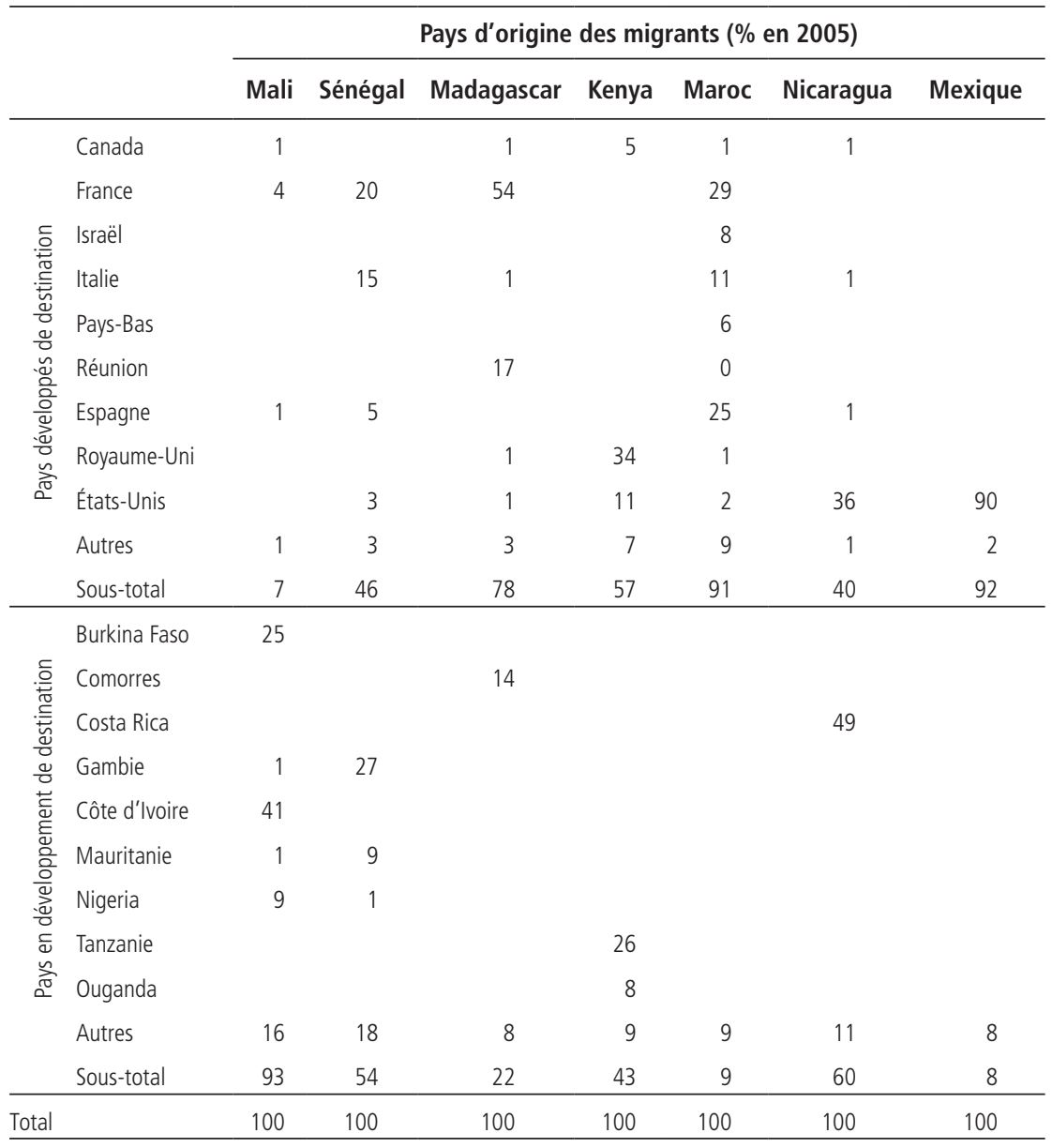

Sources : Ratha et Shaw 2007 ; World Development Indicators. 
L'analyse de l'impact des migrations est compliquée par la difficulté à saisir les diverses caractéristiques de la mobilité - selon la destination ou selon la durée. Les différents types de migration n'ont pas la même importance d'un pays à l'autre, ce qui se traduit par des caractéristiques migratoires différenciées. En outre, il est difficile d'estimer le montant des fonds envoyés de l'étranger, parce qu'ils sont irréguliers et empruntent de nombreux circuits ${ }^{28}$ et que certaines personnes interrogées hésitent à donner des informations à leur sujet ${ }^{29}$.

Dans les régions du programme RuralStruc, tous les types de migration sont représentés : nationale ou internationale, de courte ou de longue durée ${ }^{30} .24 \%$ des ménages interrogés ont une expérience migratoire, la fourchette centrale allant de $15 \%$ à $40 \%$. Les exceptions sont l'Alaotra à Madagascar, La Libertad et El Cuá au Nicaragua, et le Sotavento au Mexique, où moins de $10 \%$ des ménages sont concernés par les migrations. À l'opposé, Tominian et Diéma au Mali et Nioro au Sénégal dépassent 60 \% (voir figure 4.10).

Les déterminants de la migration sont multiples et sont liés aux facteurs d'attraction/évasion (pull et push) propres aux différentes régions. La situation économique du ménage, l'absence de débouchés au niveau local ou l'espérance d'un Eldorado distant d'une part, et les obstacles exogènes qui limitent les routes migratoires possibles d'autre part se combinent pour modeler les décisions individuelles et collectives. La migration est plus souvent un choix du ménage qu'un choix individuel et parfois, pour les migrations internationales, ce peut être une décision de la communauté tout entière. C'est pourquoi la migration repose souvent sur la capacité de certains groupes à créer et maintenir des liens de solidarité avec les membres de la diaspora.

En ce qui concerne les migrations internationales, la décision de migrer est rarement suffisante car le voyage peut être long et coûteux, surtout lorsque la destination est un pays riche, où la réglementation est de plus en plus défavorable. Compte tenu des coûts de voyage et des autres coûts connexes, les ménages dont certains membres émigrent sont généralement plus aisés que la moyenne, car non seulement la migration est coûteuse, mais la famille doit le plus souvent attendre des mois ou des années avant que le migrant puisse effectuer ses premiers envois de fonds.

Les ménages qui comptent des migrants à l'étranger sont en général plus qualifiés et ont de meilleurs réseaux sociaux, l'un et l'autre facilitant la réussite de la migration. Le revenu et le capital humain et social - principaux déterminants de la migration internationale - se conjuguent pour offrir des opportunités particulières aux ménages concernés. Un déterminant à lui seul ne présente pas de relation claire avec une migration réussie - ils sont tous les trois importants.

Les fonds envoyés par les travailleurs à l'étranger sont la première source de revenus hors exploitation agricole dans seulement trois des 30 régions étudiées (Tominian et Diéma au Mali, Terrabona au Nicaragua). Ils sont la deuxième source de revenus externes dans neuf autres régions, notamment au Sénégal, au Maroc et au Nicaragua (voir tableau 4.1). Cependant, même dans les régions où la migration est importante, les revenus procurés par les migrants sont très hétérogènes (voir figure 4.10). 


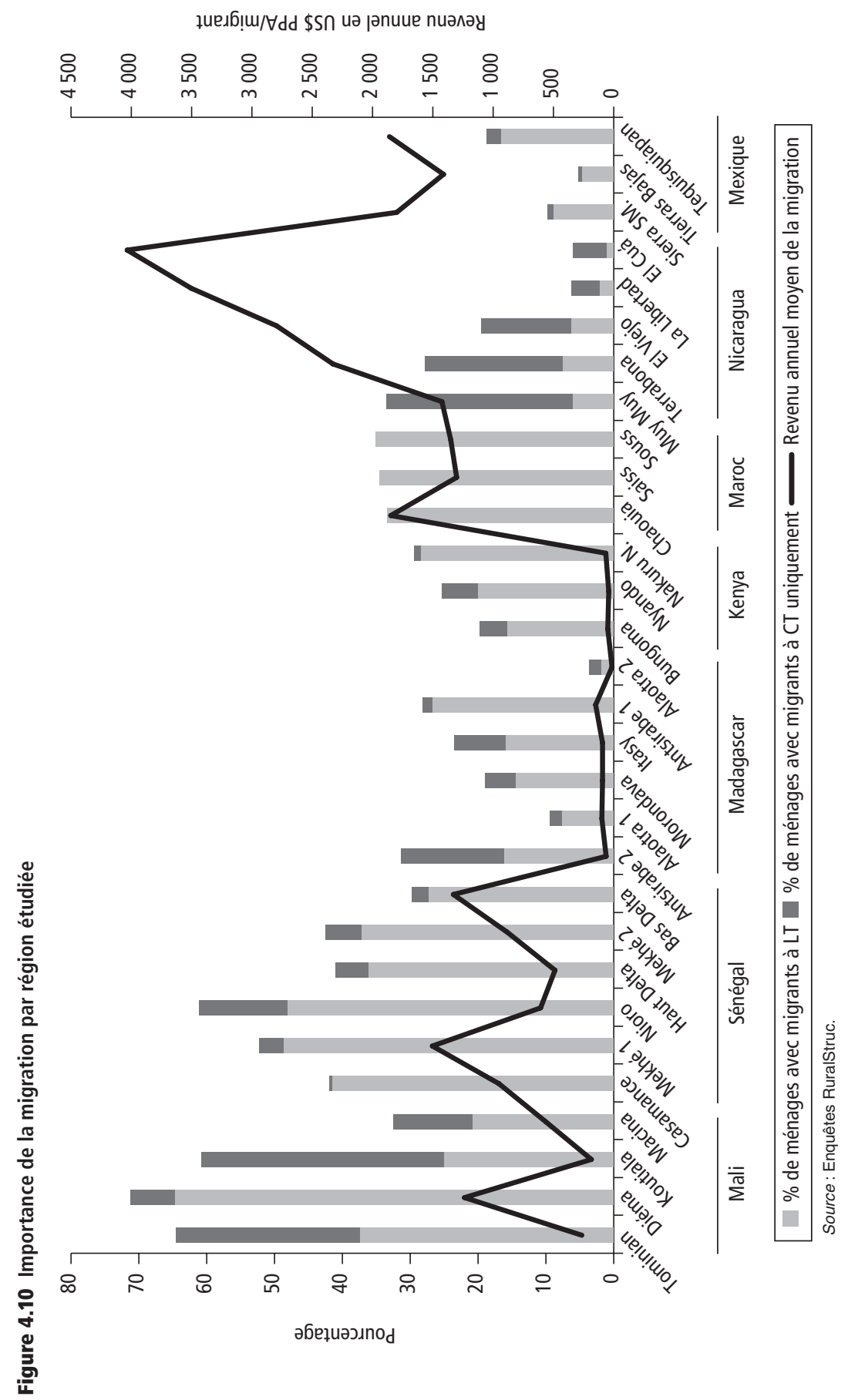


Un noyau de régions affiche des revenus compris entre 1000 US\$ PPA et 2000 US\$ PPA (Diéma, Mekhé 1 et Bas Delta, toutes les zones étudiées au Maroc et au Mexique), tandis que les données relatives au Kenya et à Madagascar font apparaître des transferts très faibles. Les cinq régions étudiées au Nicaragua font exception, avec un revenu moyen supérieur à 2500 US\$ PPA ${ }^{31}$. Les revenus des migrants mexicains semblent étonnamment modestes en comparaison, surtout en regard du développement avéré des migrations dans ce pays ${ }^{32}$.

Ce tableau très diversifié permet de dégager trois grandes configurations migratoires fondées sur l'importance régionale et la durée des migrations et sur les principales destinations des migrants (voir figure 4.11).

Elles correspondent à 23 des 30 régions étudiées et leurs principales caractéristiques sont présentées au tableau 4.3 .

La première configuration correspond aux migrations internationales de longue durée, à destination des pays de l'OCDE principalement (Diéma, le Souss et le Saïss, Tequisquiapan). La deuxième représente les migrations vers des pays voisins ; elle est illustrée par le cas très particulier du Nicaragua (Terrabona, La Libertad, Muy Muy), avec des migrations de courte durée au Costa Rica et au Salvador, où les migrants ont des activités salariées, principalement dans l'agriculture (cultures d'exportation) et les services (surtout le BTP, les services de ménage à domicile et la sécurité). Les revenus plus élevés observés s'expliquent par le fait que les migrants conservent des liens plus étroits avec leur foyer du fait de la proximité de leurs destinations. Ils retournent souvent dans leur famille, au bout de quelques mois, et transportent la plus grande partie de leur revenu sur eux en espèces, ce qui n'est pas le cas des migrants à long terme, qui organisent des transferts de fonds à un rythme plus irrégulier. La troisième configuration correspond aux migrations internes - à destination de la capitale, de grandes villes ou d'autres zones rurales. Ces migrations sont fortement orientées vers du travail de courte durée - ce que montrent Tominian et Koutiala au Mali et El Viejo au Nicaragua - mais elles

Tableau 4.3 Principales configurations migratoires dans les régions étudiées

\begin{tabular}{|c|c|c|c|c|c|}
\hline & & \multicolumn{4}{|c|}{ Pourcentage de ménages avec migrants } \\
\hline & & $>50 \%$ & $30-50 \%$ & $10-30 \%$ & $<10 \%$ \\
\hline \multirow{8}{*}{ 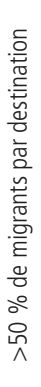 } & \multirow[t]{3}{*}{ Étranger } & \multirow[t]{3}{*}{ Diema } & Saïss & Terrabona & La Libertad \\
\hline & & & Souss & \multirow[t]{2}{*}{ Tequisquiapan } & \\
\hline & & & Muy Muy & & \\
\hline & Capitale & Tominian & & & \\
\hline & \multirow[t]{4}{*}{ Autres régions } & Koutiala & Chaouia & El Viejo & El Cuá \\
\hline & & & Antsirabe 2 & Kenya (tous) & Tierras Bajas \\
\hline & & & & Madagascar (autres) & Sierra SM \\
\hline & & & & & Alaotra $1 \& 2$ \\
\hline
\end{tabular}

Source : Enquêtes RuralStruc.

Notes : $=>40 \%$ des migrations sont de courte durée. 
peuvent être aussi de longue durée. Dans les régions et pour les ménages les plus pauvres (par exemple, au Kenya, à Madagascar et au Mali), ces migrations ne visent pas seulement à générer des revenus, elles permettent aussi de réduire le nombre de bouches à nourrir entre deux campagnes agricoles, lorsque l'exploitation ne requiert pas de main-d'œuvre familiale qui se trouve alors en excédent massif (RS 2 Mali). Lorsqu'elles sont plus durables, elles sont un moyen radical de réduire les charges des ménages ${ }^{33}$. Ces migrants, qui peuvent travailler comme domestiques ou accomplir de menus travaux, gagnent souvent très peu d'argent et trouvent simplement un moyen de couvrir leurs besoins les plus élémentaires.

Les sept autres régions étudiées (les six régions sénégalaises et Macina) présentent un tableau très contrasté du point de vue de la destination des migrants (voir figure 4.11) et illustrent des combinaisons de toutes les options migratoires possibles. C'est le cas par exemple du bassin arachidier au Sénégal, où la configuration migratoire illustre une stratégie régionale tous azimuts de renforcement des activités et des revenus. De bonnes connexions avec Dakar, Thiès et Saint-Louis offrent de multiples opportunités qui aident les ménages à faire

Figure 4.11 Destination des migrants par région étudiée (en \% du total des migrants)

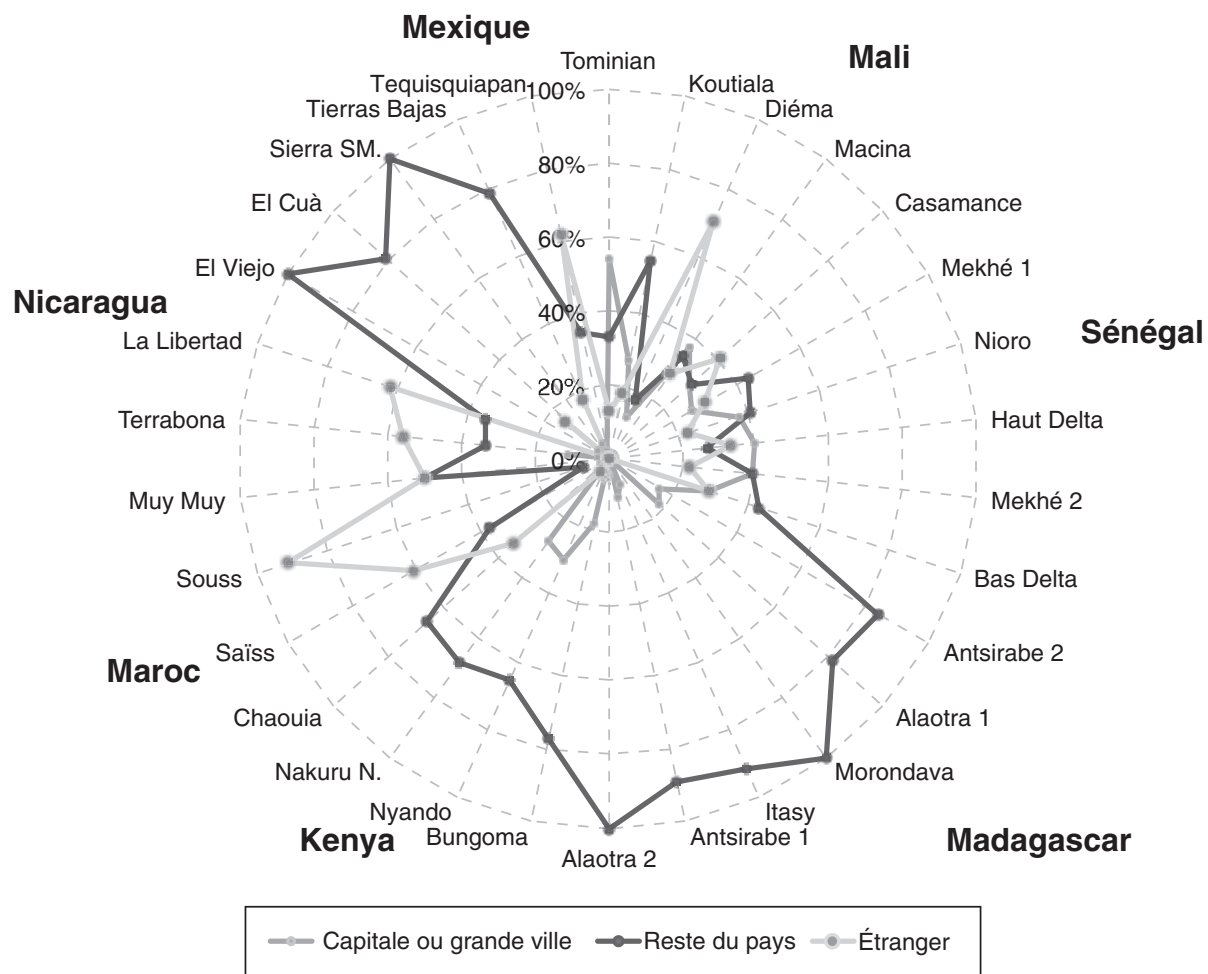


face à la profonde crise du secteur de l'arachide. Néanmoins, cette stratégie d'activités multiples - caractérisée par l'accumulation de « petits boulots » dans le village, dans la petite ville voisine ou dans la capitale, ou pour certains ménages, à l'étranger - n'apporte qu'une réponse partielle et n'est pas une véritable option de sortie de la pauvreté, comme l'atteste la faiblesse des revenus de la région (voir encadré 4.4).

Cette analyse de l'activité de migration des ménages et des revenus qu'elle procure appelle deux conclusions. La première est l'importance écrasante de la géographie. Les lieux proches de pays à revenus élevés (Mexique, Maroc) ou qui ont un accès aisé à des pays dynamiques à revenus intermédiaires (Nicaragua) ont un net avantage car le coût et la difficulté d'accès sont de moindres contraintes et les travailleurs peuvent trouver des emplois mieux rémunérés. Sans être un obstacle absolu (comme le montrent le Sénégal et le Mali), la distance complique le tableau surtout lorsqu'elle est importante et qu'il n'y a pas de transport terrestre (les cas du Kenya mais surtout de Madagascar sont révélateurs).

La seconde conclusion concerne une configuration émergente qui remodèle progressivement de nombreuses économies rurales. L'amélioration des transports et des télécommunications a accru les opportunités de connexion à un large ensemble de lieux - les villes proches, les capitales régionales et nationale, des localisations hors du pays d'origine - qui débouchent sur un fonctionnement en réseau connectant les membres d'un même ménage travaillant dans

\section{ENCADRÉ 4.4}

\section{Migrer à Dakar pour y vendre des cartes téléphoniques: une voie illusoire de sortie de la pauvreté}

La vente de cartes de téléphone, une activité qui se multiplie dans toutes les villes d'Afrique, est un bon exemple des « petits boulots » adoptés par de nombreux ménages sénégalais. Beaucoup de jeunes originaires de zones rurales tentent leur chance aux feux rouges de Dakar pendant deux ou trois mois.

Les chiffres expliquent la réalité de ce type de travail. Ces colporteurs gagnent 75 FCFA (0,28 US\$ PPA) par carte de 1000 FCFA vendue (marge de 7,5 \%) ou 900 FCFA pour une carte de 10000 FCFA (9\%). Ils peuvent gagner 525 FCFA par jour (2 US\$) en vendant sept cartes de 1000 FCFA, sachant qu'il est rare de vendre une carte de 10000 FCFA. Sur ce maigre revenu, ils doivent payer leurs repas et un endroit où dormir. Le prix d'un abri rudimentaire pour passer la nuit est d'environ 30000 FCFA par mois, de sorte qu'un travailleur doit atteindre un objectif de vente de 400 cartes téléphoniques par mois pour se loger - et la concurrence est rude. Ces coûts peuvent être réduits jusqu'à un certain point - en partageant une chambre avec de nombreuses personnes ou même en dormant dans la rue - mais avec ce type de travail, il est difficile de couvrir ses frais et on a peu de chance de rapporter de l'argent au village. 
des lieux multiples et à des périodes différentes. Ces réseaux correspondent à de nouveaux systèmes composites multilocalisés, qui redéfinissent les liens entre villes et campagnes. Dans ces « modèles en archipel », le ménage demeure solidement ancré son territoire rural et intégré dans son environnement (économiquement, socialement et intellectuellement), avec un centre de décision (le chef de famille) qui gère les flux de revenus provenant de membres du ménage qui vivent et travaillent en différents lieux (comme des îles articulées avec leur capitale $)^{34}$. Bien que ces réseaux familiaux puissent faciliter les migrations permanentes et le départ définitif des campagnes, ils sont le plus souvent un moyen de " partir pour rester $»^{35}$. En associant des stratégies d'activités et de revenus multiples, les ménages s'adaptent à leur environnement en mutation et maintiennent leur affiliation avec leur communauté locale, même si certains membres doivent partir pour y parvenir.

Les transferts publics : spécifiques au Mexique. Dans les régions étudiées, les transferts publics ne sont significatifs qu'au Mexique. Économie à revenu intermédiaire de la tranche supérieure avec une forte base fiscale, l'État mexicain a mis en œuvre un système d'aides publiques développé ciblé sur les zones rurales (Léonard et Losch 2009). Les programmes de transfert visent avant tout la diminution de la pauvreté, avec des filets de sécurité à caractère social ciblant des groupes spécifiques (par exemple, Oportunidades pour les plus démunis) et des subventions au secteur agricole à travers plusieurs programmes (Procampo pour la production, Aserca pour la commercialisation et Alianza pour l'investissement). Alors que Procampo concerne toutes les exploitations agricoles quel que soit leur niveau de richesse - l'aide reçue dépend de la superficie cultivée - les deux autres programmes sont destinés aux grandes exploitations ou à l'agro-industrie. Ces transferts ont joué un rôle important et des travaux de recherche ont confirmé leur effet multiplicateur sur les revenus (Sadoulet, de Janvry et Davis 2001). Ils sont complétés depuis quelques années par de nouveaux programmes liés à la décentralisation (par exemple Ramo 033 ou les programmes pour les «zones enclavées ») et à la protection de l'environnement, qui bénéficient principalement aux collectivités locales et aux communautés.

Plus de 15 programmes publics ont été observés dans la structure de revenu des ménages enquêtés. La plupart des exploitants agricoles participaient au programme Procampo, ce qui explique que $80 \%$ des ménages reçoivent des transferts publics dans les deux zones du Sotavento alors qu'ils ne sont que $32 \%$ à Tequisquiapan, où très peu de ménages ont une exploitation. Cependant, ces transferts ne sont pas toujours correctement ciblés. Dans le Sotavento, ils représentent entre $12 \%$ et $20 \%$ du revenu des ménages dans tous les quintiles, ce qui indique une déconnexion entre le niveau de revenu et les allocations perçues (voir encadré 4.5). 


\section{GNCADRÉ 4.5}

\section{Inégalités d'accès aux transferts publics dans la région du Sotavento, Mexique}

Dans les zones étudiées des Tierras Bajas et de la Sierra de Santa Marta au Mexique, les transferts publics sont respectivement la première et la deuxième source de revenus externes. Ils représentent $32 \%$ du revenu off-farm et, en moyenne, $15 \%$ du revenu total (RS 2 Mexique-Sotavento, 25).

Comme le montre la figure ci-après, un des résultats les plus frappants des enquêtes dans ces deux régions concerne les inégalités de distribution des subventions publiques, tant du point de vue des groupes sociaux que des zones géographiques. Les ménages les plus riches bénéficient de transferts publics sept fois plus élevés que les ménages les plus pauvres et $50 \%$ plus élevés que la somme des subventions perçues par les ménages des trois quintiles inférieurs. Ces inégalités ont une forte dimension spatiale : dans les Tierras Bajas, où résident les trois quarts des ménages les plus riches enquêtés dans le Sotavento, le montant moyen des transferts publics est plus élevé (1 797 US\$ PPA) que dans la zone montagneuse (1 329 US\$ PPA) mais, surtout, le niveau des subventions est fortement corrélé aux revenus.

En réalité, il semble qu'au lieu de lisser les inégalités et de compenser les écarts de dotation en facteurs, les transferts publics contribuent à une différenciation accélérée avec une " transition » vers une spécialisation agricole des plus grandes exploitations et des mesures d'accompagnement (filets de sécurité) pour les autres ménages.

Figure d'encadré 4.5.1 Transferts publics par zone étudiée et niveau de revenu des ménages

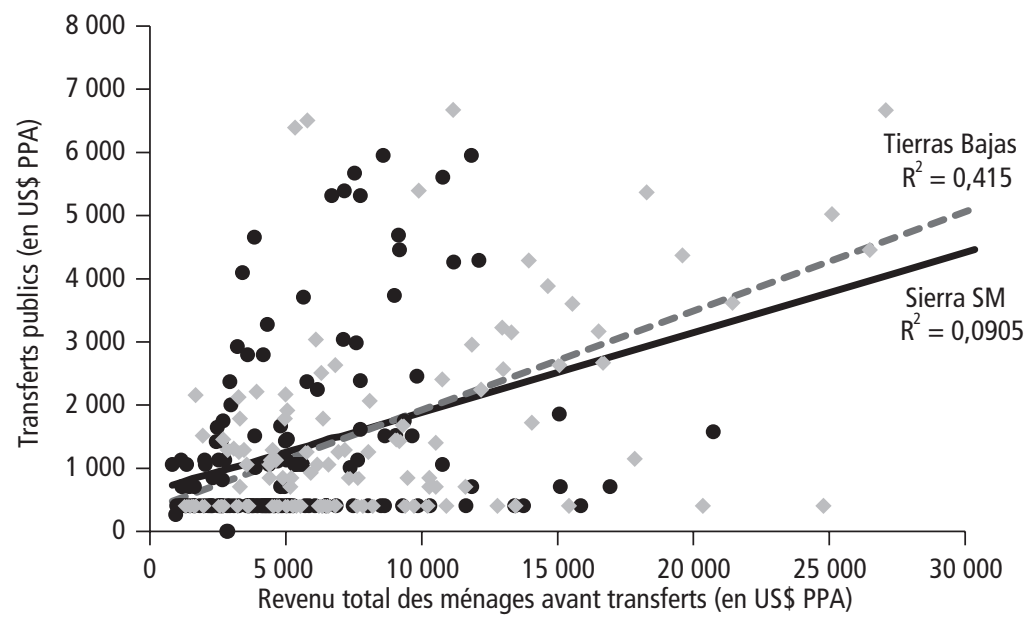

Sierra Santa Marta Tierras Bajas 


\section{Diversification off-farm et transformation rurale}

De manière générale, la littérature suggère que la diversification est la norme au sein des économies rurales des pays en développement et qu'elle conduit à l'émergence d'économies rurales non agricoles dynamiques. Les résultats du programme RuralStruc dressent pourtant un tableau plus nuancé. Les enquêtes montrent en effet que, si la diversification est partout présente, ses caractéristiques sont très différentes d'une région à l'autre.

Les différences inter- et intrarégionales illustrent la forte hétérogénéité des situations étudiées. Le niveau général de développement économique est une des distinctions essentielles entre les pays d'ASS et les autres pays. Cette distinction ne porte pas sur les taux de participation des ménages à des activités hors exploitation, qui sont très proches, mais sur les caractéristiques de la diversification - les types d'activités et leur rentabilité économique.

Deux types de diversification sont observés dans l'échantillon du programme RuralStruc. Dans les pays d'Afrique subsaharienne, la diversification représente surtout des stratégies d'adaptation - une réponse à une pauvreté forte et persistante. Les activités liées à l'exploitation agricole dominent et s'accompagnent d'un sous-emploi structurel qui reflète la saisonnalité de l'agriculture, le manque de diversification de l'économie et le manque d'alternatives d'emploi. Les activités hors exploitation sont surtout des activités d'auto-emploi, à faible rentabilité, tandis que les emplois salariés (dans l'agriculture et plus encore dans les autres secteurs) sont rares, peu rémunérateurs (ce qui illustre la situation du marché du travail) et pour la plupart temporaires. De ce fait, les revenus off-farm sont très faibles et n'apportent qu'une réponse partielle à la pauvreté, même s'ils peuvent tenir une place importante dans le revenu global des ménages dans les régions confrontées à des situations agricoles difficiles, comme le bassin arachidier au Sénégal. Dans chaque région, une fraction des ménages obtient de meilleurs revenus : ceux qui peuvent trouver un emploi non agricole permanent (pour lequel un bagage éducatif est un solide atout) ou développer certains types d'activités indépendantes (pour lesquelles les compétences mais aussi le niveau des revenus de départ peuvent faire la différence). Mais ces exceptions ne modifient pas le tableau d'ensemble.

Dans les autres pays, plus riches et à l'économie plus diversifiée que celle des pays d'ASS, les activités externes sont plus lucratives. Elles reflètent une diversification plus positive et représentent souvent une activité à temps complet. Cela signifie que certains ménages (ou membres du ménage) se spécialisent dans ces activités externes tandis que d'autres ménages (ou membres de ces ménages) se spécialisent dans l'agriculture. Cette situation est clairement illustrée dans les régions étudiées au Nicaragua et au Maroc, et à Tequisquiapan au Mexique, où les ménages conjuguant une exploitation agricole avec des activités hors exploitation sont nettement moins nombreux qu'en ASS.

Ces observations du programme RuralStruc suggèrent que, dans une certaine mesure, les caractéristiques de la diversification rurale reflètent le processus de 
transition économique d'ensemble : une diversification naissante à faible rendement aux premiers stades de la transformation structurelle, et une diversification plus mature aux stades ultérieurs, qui consolide le processus de changement.

Enfin, trois derniers résultats transversaux intéressants méritent d'être mentionnés. Le premier contredit en partie l'idée communément admise que l'urbanisation et la densité démographique peuvent en elles-mêmes nourrir la croissance économique. Les médiocres résultats économiques des ménages enquêtés dans la zone dense de la bande littorale du Sénégal et dans la partie occidentale du Kenya montrent que, si l'urbanisation et la densité peuvent faciliter et accélérer le processus de transformation rurale, leurs caractéristiques (la qualité de cette densification par exemple) sont importantes, au même titre que les caractéristiques de l'économie dans son ensemble, notamment son degré de diversification et sa productivité.

Le deuxième résultat a trait à la difficulté de saisir l'intégralité du processus de diversification économique. Puisqu'elles ciblaient les ménages ruraux (ménages dans des zones définies comme rurales), les enquêtes n’ont pas concerné les ménages qui ont migré en zone urbaine au cours du processus de transformation. Ces nouveaux ménages urbains ont pu accéder à de meilleurs services et à de meilleures conditions de vie, avoir logiquement davantage d'activités en dehors de l'agriculture et même, dans certains cas, garder leur exploitation d'origine - une situation observée dans l'enquête mexicaine. Ce biais méthodologique implique que la distinction entre bourgs ruraux et petites villes est quelque peu théorique et que saisir la réalité du continuum rural-urbain, qui évolue parallèlement au processus de densification, est un défi considérable pour les systèmes d'information. Cette question souligne également la nature relativement éphémère de l'économie rurale non agricole, qui tend à se développer avec et à se dissoudre concomitamment dans le processus d'urbanisation - ceux qui se spécialisent dans des activités non agricoles migrant en zone urbaine et les villes "se déplaçant vers la campagne », en conséquence de densités démographiques plus fortes et de l'expansion territoriale des villes liée à la croissance urbaine.

Le troisième résultat concerne les leçons tirées des migrations. S’il est difficile de saisir les informations sur le montant des transferts, les enquêtes montrent que la migration concerne $24 \%$ des ménages interrogés, un pourcentage assez faible mais cohérent avec de nombreuses études rurales. Les enquêtes montrent également que la rentabilité économique de la migration dépend de la destination des migrants, qui est elle-même influencée par la géographie, en particulier par la proximité de pays à revenus élevés. Outre l'avantage direct des fonds envoyés par les travailleurs migrants, la migration peut aussi procurer une rentabilité indirecte sous forme d' « effet réseau ». Les résultats des enquêtes pointent le développement de systèmes en archipel, dans lesquels les revenus d'un ménage proviennent de membres situés dans des lieux différents. Ces travailleurs peuvent se répartir le long d'un continuum géographique allant du rural au périurbain et à l'urbain et peuvent être situés dans d'autres régions du pays ou même à l'étranger, mais ils restent tous membres du même ménage. Ce type d'organisation facilitée par l'amélioration des infrastructures de transport et de communications permet une 
plus grande diversification, une meilleure gestion des risques et améliore les perspectives économiques des ménages.

Si les caractéristiques des trajectoires historiques du changement structurel sont présentes, le développement de ces liaisons d'un nouveau type pourrait modifier les modalités de la transformation rurale en créant de nouvelles opportunités. Mais elles requièrent un accès aux services et une bonne dotation en biens publics - en plus des infrastructures - permettant de renforcer les liens entre le rural et l'urbain et de créer une densité efficace.

\section{Notes}

1. Ellis (1998) explique la confusion courante entre les stratégies de gestion des risques et les comportements d'adaptation : la gestion des risques est une stratégie ex ante pour anticiper un choc ou un échec, tandis que l'adaptation est une réponse ex post à une crise. Il note toutefois que l'adaptation peut également correspondre à l'émergence de nouveaux modes de vie et configurations d'activités en réponse aux difficultés financières et à la situation de crise structurelle.

2. Pour une approche générale, voir Barrett et Swallow (2005), Ellis (2000, 2004) et Wiggins et Davis (2003) ; pour les questions régionales, voir Reardon, Berdegue et Escobar (2001) pour l'Amérique latine, et Barrett, Reardon et Webb (2001) et Bryceson (1999, 2002) pour l'Afrique.

3. C'est la définition adoptée notamment par Barrett et Reardon (2000), Davis et al. (2007) et Haggblade, Hazell et Reardon (2010).

4. De nombreux auteurs incluent la transformation des produits dans les activités rurales non agricoles (voir Haggblade, Hazell et Reardon, 2010). Le programme considère que la transformation des matières premières à la ferme doit entrer dans les activités de l'exploitation car, dans la plupart des cas, elle contribue directement à la valeur ajoutée apportée aux productions. C'est vrai en particulier en ASS, où la transformation concerne souvent les produits de l'exploitation familiale elle-même. Lorsque les produits sont transformés par des agro-industries ou de petites entreprises indépendantes, les revenus du travail sont évidemment hors exploitation et considérés comme de l'emploi salarié non agricole ou une activité d'auto-emploi.

5. La pratique occasionnelle de la chasse, de la pêche et de la cueillette n'est pas une activité agricole en soi, mais comme il s'agit de pratiques rurales courantes d'exploitation des ressources naturelles, il est possible de les inclure dans les revenus de l'exploitation.

6. L'annexe 4 de l'appendice en ligne : http://www.worldbank.org/afr/ruralstruc présente ces résultats pour chaque région étudiée.

7. Au lieu de confiner l'analyse des activités hors exploitation au niveau des ménages, le programme a choisi d'utiliser cet indicateur parce qu'il permet de prendre en compte les différences dans le nombre d'actifs par ménage.

8. Sur le travail salarié agricole lié à l'horticulture, voir McCulloch et Ota (2002) pour le Kenya, et Maertens et Swinnen (2007) pour le Sénégal.

9. Les salaires agricoles peuvent être entièrement payés en numéraire ou entièrement ou partiellement payés en nature, par exemple une partie de la production, les repas ou le logement sur l'exploitation pour les employés permanents. Les travailleurs agricoles sont souvent des travailleurs occasionnels, ce qui complique l'estimation de la valeur annuelle des salaires agricoles. 
10. La variable est ici la «surface utilisée », c'est-à-dire la surface de l'exploitation utilisée par le ménage, qu'il en soit propriétaire ou non, pour la culture et l'élevage, y compris les jachères (voir annexe 4 de l'appendice en ligne : http://www.worldbank.org/afr/ ruralstruc).

11. La structure des exploitations mexicaines reflète la réforme agraire, même si de fortes disparités persistent à l'échelle nationale. Les régions étudiées se caractérisent néanmoins par des exploitations de taille petite à moyenne. La taille moyenne des exploitations étudiées dans la région du Sotavento avoisine 10 ha dans les plaines et 6 ha dans les montagnes; elles sont plus petites à Tequisquiapan ( 2 ha $)$ et coexistent avec plusieurs agro-industries qui emploient des jornaleros (journaliers).

12. À Madagascar, la taille moyenne des exploitations est passée de 1,2 ha à 0,86 ha entre les deux derniers recensements (1985 et 2005) (RS 1 Madagascar).

13. La population active par ménage compte six à neuf personnes dans les régions étudiées du Mali et du Sénégal, soit deux à trois fois plus que dans d'autres régions étudiées (voir annexe 4 de l'appendice en ligne : http://www.worldbank.org/afr/ ruralstruc).

14. Outre l'Alaotra et El Viejo, les autres régions étudiées marquées par la présence de nombreux ménages sans terre sont Tequisquiapan (19\%), le Souss $(10 \%)$ et le Sotavento $(7 \%)$. Dans l'enquête, les ménages sans terre ont été définis comme des ménages qui pratiquent l'agriculture par le biais de l'emploi salarié agricole mais qui n'ont aucun accès à des terres agricoles, quel que soit le mode d'accès (type de tenure foncière).

15. Les pointes de travail existent pour toutes les grandes productions régionales : le riz à Madagascar (Alaotra, Itasy), au Mali (Macina) et au Sénégal (Bas Delta) ; l'horticulture à Madagascar (Itasy et Antsirabe) et au Maroc (Souss et Saïss); l'ananas dans les Tierras Bajas du Sotavento au Mexique (alors que le maïs est au contraire fortement mécanisé) ; et la canne à sucre à El Viejo (Nicaragua) et à Nyando et Bungoma (Kenya).

16. La part des salaires agricoles dans le revenu global des ménages est négativement corrélée au niveau du revenu total. Le résultat est légèrement négatif pour l'ensemble de l'échantillon (Pearson $=-0,068$ ) et plus élevé hors ASS (Pearson $=-0,24)$. De même, le niveau des salaires agricoles diminue avec la taille de l'exploitation, ce qui indique une meilleure dotation en facteurs et peut-être des ménages plus aisés, mais aussi un meilleur taux d'emploi de la main-d'œuvre familiale. Les corrélations entre la taille totale de l'exploitation (superficie utilisée) et la valeur des salaires agricoles sont importantes : $-0,114^{* *}$ à Madagascar $;-0,096^{* *}$ au Kenya $;-0,112^{\star *}$ au Maroc ; $-0,059^{\star}$ au Nicaragua ; et $0,059^{\star}$ au Mexique ( ${ }^{\star}$ significatif à 0,$05 ;{ }^{\star *}$ significatif à 0,01$)$.

17. La part du travail salarié agricole dans le revenu total est ventilée par quintile et par région étudiée au chapitre 6 , figure 6.1.

18. Le Mali et le Sénégal sont là encore des cas particuliers. Même si les membres des ménages ont parfois une activité salariée agricole, elle est très occasionnelle et les montants qu'ils en retirent sont faibles (quelques points de pourcentage du revenu total pour tous les quintiles). Ce faible développement du travail salarié s'explique par l'importance de la main-d'œuvre familiale, qui limite la demande de main-d'œuvre extérieure.

19. Les salaires agricoles représentent environ $50 \%$ du revenu total des ménages sans terre à l'Alaotra, $65 \%$ dans le Souss et à Tequisquiapan, $75 \%$ dans le Sotavento et plus de $90 \%$ au Nicaragua. 
20. Les chiffres correspondent au salaire minimum national au Mali et au Mexique et au salaire minimum rural dans les autres pays.

21. Dans la région du Delta au Sénégal, Les Grands Moulins du Sénégal, filiale de La Compagnie Fruitière, sont une des très rares agro-industries actives dans la production horticole d'exportation (principalement des tomates-cerises cultivées sous serre). La société emploie 1200 travailleurs temporaires pendant quatre mois et 80 travailleurs permanents. Le salaire des travailleurs temporaires est de 50000 FCFA par mois (193 US\$ PPA), ce qui est très proche du salaire agricole journalier de 8 US\$ PPA observé dans la région du Bas Delta (pour un mois standard de 22 jours travaillés). Mais quand les résultats de ces rares élus, qui gagnent la totalité de leur salaire en quatre mois, sont rapportés sur un an, le taux journalier passe à 2,1 US\$ PPA (entretiens du programme RuralStruc, mars 2008).

22. Voir par exemple Reardon, Berdegué et Escobar (2001) et de Janvry et Sadoulet (2001).

23. Les corrélations de Pearson entre le niveau d'instruction du membre le plus instruit de chaque ménage et le niveau des salaires non agricoles sont positivement significatives dans tous les pays mais surtout en ASS : Mali $\left(0,286^{* *}\right)$, Sénégal $\left(0,225^{* *}\right)$, Madagascar $\left(0,220^{\star *}\right)$, Kenya $\left(0,286^{\star \star}\right)$, Maroc $\left(0,083^{\star}\right)$, Nicaragua $\left(0,194^{\star *}\right)$ et Mexique $\left(0,194^{* *}\right){ }^{*}$ significatif à 0,$05 ;{ }^{* *}$ significatif à 0,01$)$.

24. $40 \%$ des ménages gagnent moins de 500 US\$ PPA/actif/an, un chiffre plus proche des autres moyennes d'ASS (61 \% gagnent moins de 1000 US\$ PPA/actif/an).

25. Ce seuil est quelque peu arbitraire. Il correspond à 14 US\$PPA par jour. $22 \%$ des ménages gagnent plus de 3000 US\$ et $4 \%$ plus de 10000 US\$.

26. D'autres transferts ont été observés, principalement à Tominian au Mali (subsides financiers distribués par une organisation religieuse d'aide aux familles pauvres) et dans certains villages de Madagascar. Ils sont marginaux.

27. Le Maroc fait exception : 19 ménages de l'enquête ont des revenus locatifs supérieurs à 10000 US\$ PPA par an, qui correspondent essentiellement à des loyers urbains dans des villes de la région. Ces valeurs extrêmes affectent les moyennes régionales et expliquent que les rentes soient la première source de revenus externes au Maroc, ce qui est une distorsion majeure. L'exclusion de ces ménages de l'échantillon a été envisagée mais il a été décidé de les conserver parce qu'ils font partie de la réalité rurale (RS 2 Maroc, 40).

28. Les circuits bancaires et de transfert d'argent officiels sont d'importants vecteurs, mais des flux conséquents sont transférés depuis l'étranger via des réseaux informels.

29. C'est le cas en particulier au Mexique, où il est de plus en plus difficile d'obtenir des informations sur les fonds envoyés par les travailleurs à l'étranger car de nombreuses personnes enquêtées refusent de répondre. Ces réticences tiennent principalement à la migration illégale qui (bien qu'elle soit extrêmement développée) mobilise l'attention des autorités, et à des craintes relatives à la place croissante des organisations criminelles dans les transferts monétaires. Les enquêteurs du programme RuralStruc se sont heurtés à ce problème.

30. Pour les besoins de l'enquête, les migrants à long terme ont été définis comme des personnes géographiquement éloignées du ménage pendant plus de six mois par an et qui envoient (ou n'envoient pas) des fonds, quel qu'en soit le montant.

31. Les revenus élevés à El Cuá et La Libertad (plus de 4000 US\$ PPA par migrant) doivent être replacés dans leur contexte très particulier lié au très faible nombre de ménages concernés $(5 \%)$ et aux pays d'accueil des migrants : principalement les États-Unis et l'Espagne. 
32. Bien que le Sotavento ne soit pas une zone d'émigration traditionnelle, les migrations à court et long terme vers les périmètres irrigués de la côte Pacifique se sont développées. Les revenus déclarés lors de l'enquête concernaient surtout les migrations à long terme. Leur faible montant est entièrement lié aux difficultés présentées par les conditions de l'enquête.

33. Les corrélations de Pearson entre le nombre de membres du ménage présents et le nombre total de migrants sont positivement significatives au Mali $\left(0,390^{\star *}\right)$, au Sénégal $\left(0,144^{* *}\right)$ et à Madagascar $\left(0,168^{* *}\right){ }^{* *}$ la corrélation est significative à 0,01 .)

34. Le modèle de l'archipel a été initialement développé par les études andines dans les années 1970 pour décrire les multiples habitats des ménages dans des situations écologiques variées liées à l'altitude (voir Van Buren 1996). Il a été appliqué aux nouvelles configurations des économies rurales caractérisées par l'importance des migrations à court et long terme, notamment au Mexique. Voir Quesnel et Del Rey (2005), Léonard, Quesnel et Del Rey (2004), Del Rey (2008) et Gastellu et Marchal (1997). Le concept a été également utilisé pour qualifier les dynamiques spatiales propres à la mondialisation (Veltz 1996; Viard 1998).

35. C'est le titre évocateur de l'ouvrage de Cortes (2000) consacré aux communautés paysannes de Bolivie. 


\section{Chapitre 5}

\section{À la recherche des nouvelles formes de la spécialisation agricole}

L'importance persistante de l'exploitation agricole dans les activités et revenus des ruraux est un important constat des enquêtes RuralStruc (voir chapitre 3). Dans la quasi-totalité des régions étudiées, $90 \%$ à $100 \%$ des ménages ruraux ont une exploitation, la principale exception étant Tequisquiapan au Mexique. Les revenus de l'exploitation tiennent partout une place conséquente quoique variable en fonction de l'importance des processus de diversification rurale décrits au chapitre précédent.

Un des objectifs centraux du programme, énoncé dans sa première hypothèse $(\mathrm{H} 1)$, était d'étudier dans quelle mesure la restructuration des marchés agroalimentaires liée à la libéralisation et à la mondialisation avait entraîné l'émergence de chaînes de valeur modernes et d'évaluer la persistance de productions et de configurations de marché plus traditionnelles. Une question connexe concernait le développement de la spécialisation en agriculture - une des voies de sortie de la pauvreté énoncées par le RDM08 - par le biais d'une intégration verticale plus poussée.

La fameuse « révolution des supermarchés » et les nouveaux processus d'intégration à des chaînes de valeur mondialisées ont très diversement touché les pays en développement. Les différences de situation sont directement liées à l'organisation des marchés nationaux et à leurs connexions avec l'économie mondiale et, plus généralement, au stade dans le processus de transition économique. Pour cette raison, le bouleversement des marchés agroalimentaires et son impact (un sujet de prédilection dans la littérature) relèvent quelque peu de l'épouvantail. Il tend à ignorer le fait que de larges pans du monde rural demeurent mal connectés et il surestime des dynamiques d'intégration qui ne sont que très progressives. Étant donné la diversité des situations représentées dans les pays du programme RuralStruc, des écarts significatifs étaient anticipés d'un pays à l'autre. Cependant, les résultats obtenus révèlent que les nouveaux modes d'intégration demeurent assez limités et l'analyse des activités conduites par les exploitations agricoles s'est quelque peu apparentée à la quête d'une nouvelle agriculture insaisissable. Bien entendu, le choix des pays influe sur ces résultats et, malgré les changements majeurs observés dans les régions étudiées au Mexique, le tableau d'ensemble reste assez peu contrasté - marqué en tout point par l'ampleur et les conséquences de la pauvreté rurale. 
Après un bref aperçu des caractéristiques générales et des conséquences de la restructuration des marchés agroalimentaires mondiaux, ce chapitre analyse les principaux traits des activités conduites par les exploitations agricoles dans les régions étudiées, en particulier l'importance respective de l'autoconsommation et de la commercialisation. Il examine les différents profils de spécialisation ou de diversification des cultures et conclut par un état des lieux des processus d'intégration aux marchés actuellement à l'œuvre.

\section{Le grand chambardement ${ }^{1}$}

Le processus de libéralisation de l'agriculture initié depuis plusieurs décennies n'est pas encore achevé. L'État s'est désengagé de l'agriculture comme il l'a fait des autres secteurs économiques à partir des années 1980, mais à un rythme plus lent - car l'agriculture reste considérée comme un secteur stratégique ${ }^{2}$. Ce processus se poursuit toujours au sein de l'OMC, dans le cadre d'un cycle de Doha qui peine à arriver à son terme (voir chapitre 1). Cependant, si la libéralisation des marchés internationaux est particulièrement difficile lorsqu'il s'agit des soutiens et de l'accès au marché, celle des marchés nationaux a enregistré des transformations plus beaucoup plus radicales. En effet, le démantèlement des instances de régulation et des entreprises publiques et la vague de privatisations qui a suivi ont permis l'entrée sur le marché de nouveaux acteurs (ayant souvent de fortes connexions internationales), la diffusion progressive de nouvelles règles, l'apparition de nouveaux rapports de force et l'émergence d'un nouvel ordre alimentaire international ${ }^{3}$.

\section{Les recompositions à l'œuvre}

La situation des marchés connaît une double transformation, au niveau national et au niveau international, qui débouche sur de nouvelles règles du jeu qui modifient la marge de manœuvre des opérateurs locaux.

La libéralisation des marchés intérieurs. Au niveau national, les principaux changements résident dans le désengagement de l'État et le processus de privatisation, qui ont été accompagnés par la mise en œuvre de nouvelles réglementations.

Avant la libéralisation. Dans les pays du programme RuralStruc comme dans de nombreux pays en développement, les marchés agricoles se caractérisaient avant la libéralisation par un système dual avec différents niveaux d'intervention de l'État. D’un côté, la plupart des marchés domestiques de denrées alimentaires de base et les marchés traditionnels d'exportation étaient contrôlés et strictement réglementés par des offices de commercialisation, avec des industries gérées par l'État, des prix administrés pour les produits d'exportation mais aussi pour les prix de gros et de détail de nombreux produits alimentaires. La plupart de ces organismes publics étaient des monopsones, surtout pour les principaux produits d'exportation, mais aussi parfois pour les denrées alimentaires de base (avec occasionnelle- 
ment quelques cas de monopoles associés). Ces structures avaient été créées dans un quadruple objectif : (1) promouvoir la croissance du secteur agricole car, selon le paradigme " développementiste ", l'accumulation de capital dans l'agriculture était le premier stade du processus de développement ; (2) stabiliser les prix des producteurs (et leurs revenus) sur la durée d'une campagne agricole et réduire les fluctuations entre les campagnes afin de diminuer les risques ; (3) accroître les prix à la production agricole et renforcer les incitations à l'investissement des agriculteurs en diminuant le nombre d'intermédiaires au sein des filières agricoles ; et (4) faciliter les exportations en gérant l'ensemble de l'offre agricole nationale.

De l'autre côté, quelques marchés traditionnels de produits alimentaires non essentiels (principalement des produits frais comme les fruits, les légumes et les produits laitiers) étaient pratiquement libres, avec une intervention de l'État très modeste voire nulle, et sans réglementation des prix. Ces marchés se caractérisaient par des transactions au comptant, impliquant de nombreux acheteurs et vendeurs de petite taille, non spécialisés et non organisés. Les classifications et les normes de produits étaient rares, voire inexistantes ; les systèmes d'information de marché étaient dans l'ensemble médiocres ; et les contrats informels, dont le respect était principalement assuré par les rapports sociaux, étaient la règle (Fafchamps 2004).

En raison de la faiblesse du secteur privé, les États intervenaient également dans la transformation, principalement par le biais d'entreprises parapubliques. Cette situation était fréquente dans des industries clés du secteur d'exportation traditionnel, comme l'arachide, l'huile de palme, le thé, le café, le cacao et le sucre. De nombreuses productions agro-industrielles étaient aussi développées par des sociétés d'État sur le principe d'une intégration verticale justifiée par les économies d'échelle. Ce modèle était également promu pour répondre aux exigences techniques d'une transformation rapide due au caractère périssable des produits, ainsi qu'aux impératifs de qualité des produits d'exportation comme l'huile de palme et le thé.

Le désengagement de l'État. Dans les années 1980 et 1990, la mise en œuvre de politiques agricoles pilotées par les marchés a été la clé de voûte de la libéralisation dans les pays en développement. Souvent mises en œuvre dans le contexte de programmes d'ajustement structurel - conçus pour rétablir les équilibres budgétaires et commerciaux, réduire ou éliminer les distorsions, faciliter une meilleure transmission des prix, et stimuler l'investissement et la production (Akiyama et al. 2003 ; Barrett et Mutambatsere 2005) - ces réformes se justifiaient par les inefficiences des structures gérées par l'État. Les offices de commercialisation, les agences de développement et les entreprises publiques n'atteignaient plus leurs objectifs initiaux et étaient perçues comme des symboles de l'inefficacité publique. Plus généralement, le désengagement de l'État était une condition préalable à la complète libéralisation des marchés et c'est la raison pour laquelle les réformes ont commencé par le démantèlement et la privatisation de ces structures, accompagnés par la réduction des tarifs douaniers, des taxes à l'exportation, des subventions aux consommateurs et la suppression des prix administrés au producteur. 
Les tableaux 5.1 et 5.2 donnent quelques exemples d'organismes publics qui ont été démantelés dans les pays du programme RuralStruc ${ }^{4}$. Ces restructurations se sont déroulées sur une longue période - de la fin des années 1970 à la fin des années 1990. Le point de départ, l'ampleur et le rythme de la libéralisation étaient propres à chaque pays, ce qui explique les fortes variations observées.

Les nouvelles régulations de marchés. Le désengagement de l'État des marchés agricoles et le démantèlement des organismes parapublics et des systèmes de réglementation ont donné naissance à de nouveaux cadres économiques et institutionnels nationaux. Ces changements ont eu plusieurs conséquences qui peuvent se résumer en deux caractéristiques liées. Tout d'abord, les filières ont été rapidement soumises aux forces du marché et tributaires des fluctuations de l'offre et de la demande. De nouveaux acteurs privés sont apparus, mais un grand nombre d'entre eux ont été rapidement éliminés du fait de la concurrence intense qui a suivi le retrait de l'État. Pour survivre, les alliances extérieures étaient nécessaires

Tableau 5.1 Les restructurations dans le secteur agricole et agroalimentaire des pays du programme RuralStruc (hors ASS)

\begin{tabular}{|c|c|c|}
\hline & Avant la libéralisation & Après la libéralisation \\
\hline \multicolumn{3}{|l|}{ Maroc } \\
\hline \multirow{3}{*}{$\begin{array}{l}\text { ONICL } \\
\text { (Office national interprofessionnel } \\
\text { des céréales et légumineuses) }\end{array}$} & \multirow{3}{*}{$\begin{array}{l}\text { Office public de commercialisation: } \\
\text { contrôle total de la commercialisa- } \\
\text { tion des céréales avec fixation des } \\
\text { prix (surtout pour le blé) et un strict } \\
\text { contrôle des importations }\end{array}$} & \multirow{2}{*}{$\begin{array}{l}\text { 1988-1996 : libéralisation progressive } \\
\text { du marché des céréales }\end{array}$} \\
\hline & & \\
\hline & & $\begin{array}{l}\text { Subsistance de quotas pour la « farine } \\
\text { nationale » }\end{array}$ \\
\hline OCE & \multirow{2}{*}{$\begin{array}{l}\text { Office public de commercialisation: } \\
\text { monopole sur les exportations } \\
\text { d'agrumes, de produits horticoles, de } \\
\text { produits en conserve, etc. }\end{array}$} & \multirow{2}{*}{$\begin{array}{l}1985 \text { : fin du monopole et libéralisa- } \\
\text { tion des exportations }\end{array}$} \\
\hline $\begin{array}{l}\text { (Office de commercialisation et } \\
\text { d'exportation) }\end{array}$ & & \\
\hline
\end{tabular}

\begin{tabular}{|c|c|c|}
\hline Nicaragua & & \\
\hline ENABAS & $\begin{array}{l}\text { Agence publique de commercialisa- } \\
\text { tion : monopole sur la commerciali- }\end{array}$ & $\begin{array}{l}1984 \text { : élimination des différentiels de } \\
\text { prix pour les céréales de base }\end{array}$ \\
\hline Básicos) & $\begin{array}{l}\text { sation des denrées alimentaires de } \\
\text { base et des cultures d'exportation } \\
\text { comme l'arachide, le sésame et } \\
\text { le soja }\end{array}$ & $\begin{array}{l}1990 \text { : libéralisation totale de la } \\
\text { commercialisation des denrées alimen- } \\
\text { taires de base }\end{array}$ \\
\hline
\end{tabular}

\begin{tabular}{|c|c|c|}
\hline \multicolumn{3}{|l|}{ Mexique } \\
\hline \multirow{3}{*}{$\begin{array}{l}\text { CONASUPO } \\
\text { (Compañía Nacional de Subsisten- } \\
\text { cias Populares) }\end{array}$} & \multirow{3}{*}{$\begin{array}{l}\text { Entreprise gérée par l'État: mono- } \\
\text { pole sur les importations, supervi- } \\
\text { sion des exportations et approvision- } \\
\text { nement du marché intérieur pour les } \\
\text { produits alimentaires de base avec } \\
\text { encadrement des prix }\end{array}$} & \multirow{2}{*}{$\begin{array}{l}1989 \text { : fin du monopole de commer- } \\
\text { cialisation sur les importations et sur } \\
\text { le marché intérieur pour tous les pro- } \\
\text { duits alimentaires de base sauf le maïs } \\
\text { et les haricots }\end{array}$} \\
\hline & & \\
\hline & & $\begin{array}{l}1999 \text { : fin des interventions sur le mar- } \\
\text { ché du maïs et des haricots }\end{array}$ \\
\hline INMECAFE & \multirow{2}{*}{$\begin{array}{l}\text { Office public de commercialisation: } \\
\text { soutien à la production, à la transfor- } \\
\text { mation et à la commercialisation et } \\
\text { monopole sur les exportations de café }\end{array}$} & \multirow{2}{*}{$\begin{array}{l}1993 \text { : démantèlement de l'office et } \\
\text { libéralisation }\end{array}$} \\
\hline & & \\
\hline
\end{tabular}

Source : Rapports nationaux du programme RuralStruc, phases 1 et 2. 
(pour accéder notamment aux capitaux) et ce phénomène a accentué les asymétries qui caractérisaient la plupart des filières, marquées par l'atomisation des producteurs et le faible nombre des opérateurs de l'aval (exportateurs ou industriels). Il en a résulté un processus de concentration, allant jusqu'à déboucher parfois sur une prise de contrôle de certains secteurs, et l'émergence d'acteurs de grande taille qui ont profondément transformé la dynamique de marché.

Ensuite, la suppression des réglementations et de l'administration des prix a augmenté les incertitudes et les coûts de transaction des acteurs privés dans un environnement de plus en plus concurrentiel. Les entreprises de négoce et les industriels ont réagi en sécurisant leurs approvisionnements par des contrats avec des producteurs, des organisations de producteurs et des opérateurs commerciaux. Certaines firmes se sont engagées dans des processus d'intégration renforcés en achetant des opérateurs locaux ou en organisant leurs propres réseaux d'approvisionnement, en apportant parfois un encadrement technique aux producteurs. Ces évolutions ont profondément modifié les règles du jeu.

Tableau 5.2 Les restructurations dans le secteur agricole et agroalimentaire des pays du programme RuralStruc (en ASS)

\begin{tabular}{|c|c|c|}
\hline & Avant la libéralisation & Après la libéralisation \\
\hline \multicolumn{3}{|l|}{ Mali } \\
\hline OPAM & \multirow{2}{*}{$\begin{array}{l}\text { Office public de commercialisation: } \\
\text { monopole sur la commercialisation } \\
\text { des céréales }\end{array}$} & 1986 : fin du monopole \\
\hline $\begin{array}{l}\text { (Office des produits agricoles du } \\
\text { Mali) }\end{array}$ & & $\begin{array}{l}1989 \text { : libéralisation des importations } \\
\text { et de la commercialisation domestique }\end{array}$ \\
\hline Office du Niger & $\begin{array}{l}\text { Organisme parapublic: gestion de } \\
\text { l'eau, des terres et des infrastruc- } \\
\text { tures d'irrigation dans le périmètre } \\
\text { de l'Office ; monopole de la distribu- } \\
\text { tion et de la transformation du riz }\end{array}$ & $\begin{array}{l}1994 \text { : fin des interventions sur le riz } \\
\text { (hormis pour la vulgarisation agricole) }\end{array}$ \\
\hline CMDT & \multirow[b]{2}{*}{$\begin{array}{l}\text { Entreprise semi-publique (40 \% au } \\
\text { groupe français Dagris, aujourd'hui } \\
\text { Geocoton) : fourniture d'intrants, } \\
\text { vulgarisation agricole, commer- } \\
\text { cialisation et transformation des } \\
\text { semences de coton, fourniture de } \\
\text { la fibre de coton à l'industrie textile } \\
\text { publique nationale (Comatex) et } \\
\text { exportations }\end{array}$} & \multirow[t]{2}{*}{ Libéralisation en cours depuis 2004} \\
\hline $\begin{array}{l}\text { (Compagnie malienne de développe- } \\
\text { ment des textiles) }\end{array}$ & & \\
\hline \multicolumn{3}{|l|}{ Sénégal } \\
\hline ONCAD & \multirow{2}{*}{$\begin{array}{l}\text { Office public de commercialisation: } \\
\text { monopole sur la commercialisation } \\
\text { des produits agricoles domestiques } \\
\text { (arachides, céréales) et les importa- } \\
\text { tions; supervision des coopératives } \\
\text { de producteurs }\end{array}$} & 1979 : liquidation \\
\hline $\begin{array}{l}\text { (Office national de commer- } \\
\text { cialisation et d'assistance au } \\
\text { développement) }\end{array}$ & & $\begin{array}{l}1991 \text { : libéralisation du marché local } \\
\text { et des importations de riz }\end{array}$ \\
\hline SONACOS & \multirow{2}{*}{$\begin{array}{l}\text { Entreprise gérée par l'État : produc- } \\
\text { tion d'huile d'arachide }\end{array}$} & \multirow[t]{2}{*}{2006 : privatisation } \\
\hline $\begin{array}{l}\text { (Société nationale de commercialisa- } \\
\text { tion des oléagineux du Sénégal) }\end{array}$ & & \\
\hline
\end{tabular}




\begin{tabular}{|c|c|c|}
\hline \multicolumn{3}{|l|}{ Madagascar } \\
\hline \multirow{3}{*}{$\begin{array}{l}\text { BCSR } \\
\text { (Bureau de commercialisation et de } \\
\text { stabilisation du riz) }\end{array}$} & \multirow[t]{3}{*}{$\begin{array}{l}\text { Office public de commercialisation: } \\
\text { monopole total sur le riz }\end{array}$} & $\begin{array}{l}\text { 1983-1986 : fin du monopole sur la } \\
\text { commercialisation domestique du riz }\end{array}$ \\
\hline & & 1990 : privatisation des importations \\
\hline & & 1991 : fin du stock tampon \\
\hline HASYMA & Entreprise semi-publique (36 \% au & 2004 : privatisation (90 \% du capita \\
\hline (Hasy Malagasy) & $\begin{array}{l}\text { groupe français Dagris, aujourd'hui } \\
\text { Geocoton) : fourniture d'intrants, } \\
\text { vulgarisation agricole, commer- } \\
\text { cialisation et transformation de la } \\
\text { semence de coton, fourniture de } \\
\text { fibre de coton à l'industrie textile } \\
\text { publique nationale et exportations }\end{array}$ & détenu par Dagris) \\
\hline
\end{tabular}

\begin{tabular}{|c|c|c|}
\hline \multicolumn{3}{|l|}{ Kenya } \\
\hline $\begin{array}{l}\text { NCPB } \\
\text { (National Cereals and Produce } \\
\text { Board) }\end{array}$ & $\begin{array}{l}\text { Office public de commercialisation: } \\
\text { monopole sur la commercialisation } \\
\text { des céréales (marché intérieur et } \\
\text { exportations) }\end{array}$ & $\begin{array}{l}\text { 1991-1995 : privatisation et libéralisa- } \\
\text { tion de la commercialisation }\end{array}$ \\
\hline \multirow{4}{*}{$\begin{array}{l}\text { KCC } \\
\text { (Kenya Cooperative Creameries) }\end{array}$} & \multirow{4}{*}{$\begin{array}{l}\text { Société coopérative : monopole sur } \\
\text { la transformation et la vente de pro- } \\
\text { duits laitiers dans toutes les zones } \\
\text { urbaines }\end{array}$} & 1992 : fin du monopole \\
\hline & & $\begin{array}{l}\text { 1999: effondrement résultant de la } \\
\text { nouvelle concurrence }\end{array}$ \\
\hline & & $\begin{array}{l}2000 \text { : rachat et création de KCC } \\
\text { Holdings }\end{array}$ \\
\hline & & $\begin{array}{l}2003 \text { : reprise par l'État et revitalisa- } \\
\text { tion ; création de New KCC }\end{array}$ \\
\hline $\begin{array}{l}\text { CBK } \\
\text { (The Coffee Board of Kenya) }\end{array}$ & $\begin{array}{l}\text { Office public de commercialisation: } \\
\text { monopole sur la collecte, la transfor- } \\
\text { mation et l'exportation du café }\end{array}$ & $\begin{array}{l}2001 \text { : fin du monopole. Rôle consul- } \\
\text { tatif uniquement }\end{array}$ \\
\hline $\begin{array}{l}\text { TBK } \\
\text { (The Tea Board of Kenya) }\end{array}$ & $\begin{array}{l}\text { Office public de commercialisation: } \\
\text { réglementation du secteur du thé } \\
\text { (production, recherche, transfor- } \\
\text { mation, commerce et promotion } \\
\text { sur les marchés domestiques et } \\
\text { internationaux) }\end{array}$ & Pas de changement \\
\hline $\begin{array}{l}\text { KTDA } \\
\text { (Kenya Tea Development Authority) }\end{array}$ & $\begin{array}{l}\text { Agence publique de développe- } \\
\text { ment : gestion de production par la } \\
\text { fourniture d'intrants, les services de } \\
\text { vulgarisation agricole, la collecte, la } \\
\text { transformation et la commercialisa- } \\
\text { tion du thé }\end{array}$ & $\begin{array}{l}2000 \text { : privatisation. Rebaptisée Kenya } \\
\text { Tea Development Agency, apporte un } \\
\text { soutien au secteur }\end{array}$ \\
\hline
\end{tabular}

Source : Rapports nationaux du programme RuralStruc, phases 1 et 2.

Les nouveaux marchés agroalimentaires. Les profondes mutations intervenues sur les marchés intérieurs se sont inscrites dans un contexte de restructuration de grande ampleur des marchés agroalimentaires internationaux. Ces processus résultent de la dynamique de libéralisation décrite plus haut et de nouvelles tendances de la demande alimentaire liées à la mondialisation (voir figure 5.1).

La principale conséquence de cette évolution, amorcée dans les années 1980, est une intégration tendancielle croissante qui alimente et consolide la restructuration des marchés intérieurs. Ces processus d'intégration se caractérisent essentiellement par l'élaboration de normes et des relations plus étroites entre 


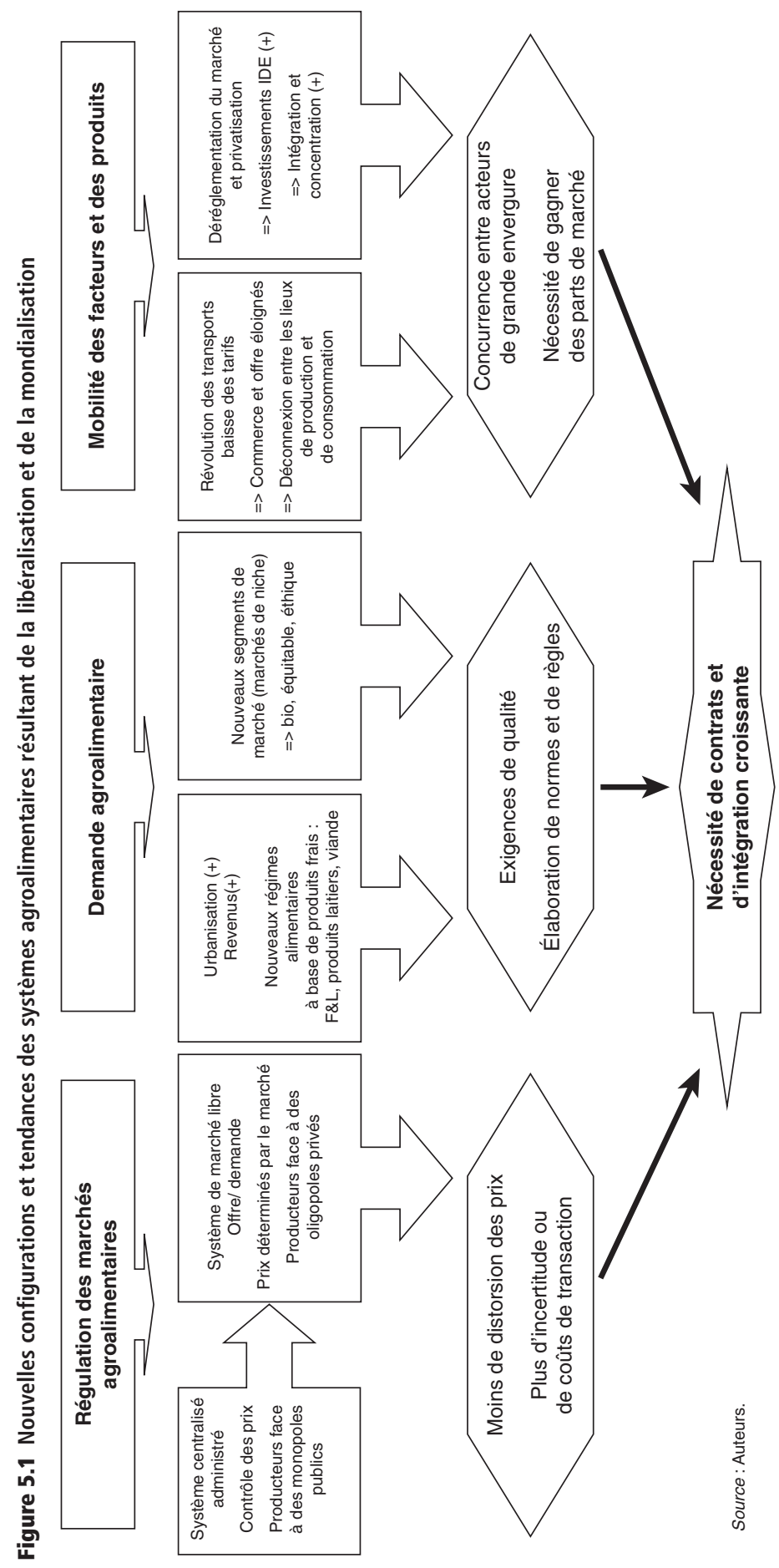


producteurs et acheteurs. Évidemment, ces transformations s'accomplissent à des rythmes très différents d'un pays à l'autre. L'objectif de la section qui suit est de donner un cadre de référence pour comprendre les changements à l'œuvre.

Les nouvelles caractéristiques de la demande agroalimentaire. Les grandes tendances de la demande peuvent se résumer comme suit : (1) la population mondiale est de plus en plus urbaine ; (2) l'augmentation des revenus entraîne une rapide évolution des habitudes alimentaires : les denrées alimentaires de base sont remplacées par des protéines et des produits à forte valeur ajoutée (viande et produits laitiers, fruits et légumes) ; (3) jusqu'à la période actuelle d'augmentation des prix alimentaires, la baisse structurelle des prix a stimulé la dynamique des marchés agroalimentaires; et (4) l'intégration croissante du commerce mondial et l'amélioration des systèmes de transport ont accéléré la convergence des régimes et des préférences alimentaires (FAO 2004).

Conséquence de ces évolutions simultanées, les filières orientées vers le consommateur final (fruits, légumes, viande, produits laitiers, produits de la mer) ont connu un développement rapide. Les télécommunications ont facilité le commerce à distance, et les progrès des techniques de transport et de stockage dans la seconde moitié des années 1980 ont permis aux producteurs de l'hémisphère sud d'expédier des produits frais (pommes, fraises et asperges par exemple) aux consommateurs de l'hémisphère nord. Cette expansion de la demande et du commerce de produits périssables et de produits à forte valeur ajoutée a imposé de renforcer les normes sanitaires ${ }^{5}$. Cette évolution transparaît clairement dans l'attention croissante apportée aux risques associés aux intrants agricoles (résidus de pesticides, produits vétérinaires, etc.) et aux contaminations microbiologiques. L'instauration de normes plus strictes de sécurité alimentaire et de standards de qualité dans les pays à hauts revenus a eu un fort impact sur l'évolution des filières. Les exportateurs et les distributeurs en particulier ont désormais des incitations pour recourir à de nouveaux types de contrats de production et de commercialisation et une assistance technique et financière peut être apportée pour conforter ces nouveaux liens avec les producteurs.

Ce passage de marchés organisés par l'offre à des marchés tirés par la demande dans un contexte de croissance des revenus (au niveau global) a également transformé les relations entre les opérateurs des filières de produits d'exportation traditionnels (les commodités). Aujourd'hui, les consommateurs des pays riches sont de plus en plus demandeurs d'informations sanitaires, sur les conditions de culture et de commercialisation des produits dans une préoccupation de pratiques agricoles socialement équitables et durables. Cette sensibilisation croissante des consommateurs a progressivement soutenu un ensemble d'initiatives alternatives au sein des systèmes agroalimentaires internationaux, nationaux et locaux et elle a favorisé des changements dans les pratiques de distribution avec la généralisation du commerce équitable, des produits biologiques et d'autres aliments alternatifs. L'émergence de ces marchés de niche a conduit à l'instauration de nouvelles normes et de nouveaux contrôles parallèlement à la mise en place de structures de certification plus génériques. Des efforts sont faits par exemple pour 
garantir la fiabilité des normes biologiques afin de mieux différencier les produits «bio » et pour promouvoir différentes formes de filières courtes permettant de favoriser le développement des communautés locales ${ }^{6}$.

Les contrats, dans leurs différentes formes et avec leurs différents degrés d'obligation, réduisent habituellement les risques encourus par l'acheteur et par le vendeur. Suite à la suppression des systèmes de commercialisation publics, leur développement est apparu comme un moyen de garantir les standards de qualité et les exigences des acheteurs. Pour le producteur, la vente sous contrat permet aussi une réduction du risque, surtout quand les caractéristiques du produit demandé sont complexes et les exigences de qualité élevées - des conditionnalités fréquentes pour accéder à certains marchés. C'est pourquoi les arrangements contractuels se sont progressivement étendus aux nouvelles chaînes de produits frais et aux marchés de niche, où les spécifications des produits sont clairement définies en termes de normes et où la valeur marchande finale des produits permet de couvrir les coûts spécifiques des contrats (sélection, négociation, suivi et exécution).

Nouveaux acteurs, mobilité des facteurs et nouvelles modalités du commerce. Depuis les années 1980, l'expansion du commerce à longue distance et le développement de l'investissement direct étranger (IDE), facilités par les politiques de libéralisation et la conclusion d'accords de libre-échange (ALE), ont modifié le périmètre de production et de commercialisation des produits agricoles. Il s'agit des conséquences d'une économie internationale plus ouverte résultant de la libéralisation économique mais aussi de progrès techniques avec, du côté logistique de gestion, l'impact des communications Internet sur la circulation des capitaux et de l'information et, du côté logistique des produits, les améliorations du transport maritime, du stockage et des procédés de transformation. Tous ces facteurs ont nettement renforcé l'efficience du commerce - international et national - et ont partout ouvert la voie depuis les années 1990 à la réalisation d'investissements de grande ampleur par de nouveaux acteurs, particulièrement dans la transformation et la distribution (Barrett et Mutambatsere 2005). Une poignée de multinationales verticalement intégrées et d'alliances stratégiques entre de grandes entreprises ont renforcé leur contrôle à la fois sur certains marchés nationaux (voir encadré 5.1) et sur le commerce, la transformation et la distribution des produits alimentaires à l'échelle mondiale (Vorley 2003). La « révolution des supermarchés» (voir encadré 5.2) est un exemple du formidable essor de ces processus.

Les différences de développement de la grande distribution entre pays peuvent s'expliquer par les conditions de la demande et de l'offre qui sont influencées par des facteurs socio-économiques et des choix d'investissement. Du côté de la demande, le niveau de revenu et l'urbanisation - qui déterminent le coût d'opportunité du temps (en particulier celui des femmes) -, la baisse des coûts de transaction résultant de l'amélioration des routes et des transports et la généralisation des réfrigérateurs façonnent les attentes des consommateurs en termes d'accessibilité, de diversité et de qualité des produits et renforcent l'attractivité de la distribution en supermarché. Mais ces facteurs ne suffisent pas à expliquer leur très vive expansion dans de nombreux pays en développement dans les années 1990 et 2000, alors que la plupart 
avaient un secteur de supermarchés très modeste avant 1990. En effet, les conditions du côté de l'offre ont aussi joué un rôle avec en particulier l'afflux d'investissements étrangers dans la distribution, qui a été favorisée par la libéralisation des règles en matière d'IDE, et les améliorations des systèmes d'approvisionnement, qui se sont conjugués avec les objectifs de modernisation des systèmes de distribution de nombreux gouvernements. Les pays du programme RuralStruc offrent un bon panorama de ces évolutions extrêmement contrastées (voir encadré 5.6).

\section{ENCADRÉ 5.1}

\section{La restructuration du secteur du maïs mexicain}

Le Mexique est un bon exemple de pays où le secteur agricole a connu d'importantes restructurations. Celles-ci se sont amorcées à la fin des années 1980 avec la fin du monopole sur la commercialisation des produits agricoles détenu par une entreprise d'État (la Conasupo - voir tableau 5.1) (Yunes Naude 2003), la déréglementation des prix, et la mise en œuvre de l'ALENA en 1993 (Appendini 2001). Dans ce processus de réformes, le secteur du maïs a bénéficié d'un traitement préférentiel en raison de sa place centrale dans le régime alimentaire mexicain, de son poids dans le secteur agricole et de son statut social, culturel et politique. Dans un premier temps, des aides transitoires ciblées, non génératrices de distorsions, ont été apportées aux producteurs par le biais d'une subvention fondée sur la taille des parcelles (Procampo) ; cette subvention devait expirer en 2008. Ensuite, un système transitoire de quotas a protégé la filière nationale d'une augmentation des importations liée à l'ALENA. Les importations américaines devaient être limitées à un quota annuel de 2,5 millions de tonnes en franchise douanière, avec une augmentation annuelle de $3 \%$. Au-delà du quota, elles devaient être taxées à $215 \%$. Il était également prévu de démanteler progressivement ce système de quotas à l'horizon 2008 (Lederman, Maloney et Servén 2005).

Ces nouvelles politiques ont été à l'origine d'un puissant processus de concentration. Les grandes exploitations commerciales mexicaines productrices de maïs ont bénéficié de multiples aides publiques, dont la subvention Procampo, qui était versée en fonction de la superficie cultivée et bénéficiait donc aux grandes exploitations. Bien que ce programme ait prévu un plafonnement des montants des subventions, il s'est avéré que les grandes exploitations commerciales (seulement $10 \%$ du total) captaient $53 \%$ de ses ressources en 2003. Les grandes exploitations ont également bénéficié de programmes conçus pour soutenir leur modernisation et leurs connexions avec les marchés. Les programmes Aserca et Alianza ciblaient les exploitations offrant les meilleures perspectives de gains de productivité et versaient d'importantes subventions pour la commercialisation et l'investissement. Avec le temps, le budget du ministère de l'Agriculture a favorisé ces derniers programmes. Alors que Procampo représentait $70 \%$ de son budget sous le président Zedillo (1995-2000), il a été ramené à 50 \% sous le président Fox (2001-2006), la différence allant au financement d'Alianza et d'Aserca (Zahniser et Coyle 2004).

La concentration de la production a remodelé la géographie du secteur (RS 2 Mexique, 36). Auparavant, les deux millions de petits exploitants du Mexique - concentrés au centre et au sud du pays - dominaient le marché national. Au cours des vingt dernières 
années, 300000 grandes exploitations commerciales se sont développées jusqu'à couvrir $23 \%$ des surfaces cultivées et approvisionner $35 \%$ du marché. La plupart sont situées dans le nord-ouest (surtout Sinaloa), où l'irrigation est très fréquente et la productivité peut atteindre 9,8 tonnes/ha (contre 1,4 tonne/ha pour les petits exploitants du reste du pays). Du côté de la transformation, le désengagement de l'État, la privatisation, la fin des prix imposés pour les galettes de maïs (tortillas) et les soutiens à l'industrie de la farine ont érodé le secteur artisanal de la tortilla. Un puissant oligopole de minotiers industriels en est venu à contrôler $52 \%$ de l'offre de farine (SAGARPA 2007) et s'est servi de ce contrôle pour intégrer les producteurs de tortillas par des systèmes de licence (Léonard 2010). Aujourd'hui, deux grands groupes approvisionnent le marché de la farine industrielle : Maseca (75\%) et Minsa (15\%).

La position dominante des producteurs industriels a été renforcée par leur intervention dans l'importation de maïs jaune en provenance des États-Unis et ils ont grandement bénéficié de la décision du gouvernement de n'appliquer que sporadiquement les quotas et les taxes sur les importations décrits plus haut (Wise 2009). Les importations de maïs, qui s'élevaient à 1,3 million de tonnes en 1992, atteignaient 8,8 millions de tonnes en 2008, un chiffre très supérieur aux quantités autorisées par le système des quotas. Sept sociétés contrôlent plus de la moitié de ces importations (à $95 \%$-98 \% du maïs jaune) (De Ita 2008) - les deux grandes minoteries - Maseca (dont ADM détient $25 \%$ du capital) et Minsa (associée à Corn Products International, qui a pris le contrôle de la société de raffinage du maïs Arancia en 1998), aux côtés de Cargill-Continental, de trois grandes sociétés actives dans la production de volailles et d'aliments pour animaux (Bachoco, Pilgrim's Pride et Purina) et de Diconsa, une entreprise publique issue de la scission de I'ancienne Conasupo, qui assure encore la fourniture de denrées alimentaires essentielles à des communautés rurales marginalisées.

L'incorporation du maïs jaune (traditionnellement destiné à I'alimentation animale) à la production de farine est un changement radical qui modifie la structure du marché mexicain du maïs et les habitudes de consommation (les tortillas sont traditionnellement fabriquées avec du maïs blanc). Elle est directement à l'origine de prix aux producteurs nationaux très inférieurs au prix de référence international, tandis que les prix des tortillas vendues aux consommateurs finaux ont continué d'augmenter (Appendini 2008).

\section{ENCADRÉ $\mathbf{5 . 2}$}

\section{La révolution des supermarchés}

La pénétration des circuits modernes de distribution de produits alimentaires n'est pas identique dans tous les pays en développement. Reardon et Timmer (2007) décrivent le processus de la façon suivante :

Le « décollage » du secteur des supermarchés intervenu dans les pays en développement au début et au milieu des années 1990 s'est opéré en trois vagues. La première a touché une grande partie de l'Amérique du Sud, I'Asie de l'Est - excepté la Chine - et I'Afrique du Sud, régions où la part de marché moyenne des supermarchés dans la distribution alimentaire est passée approximativement de 10-20\% seulement vers 1990 à 
50-60 \% au début des années 2000. La deuxième vague a touché certaines parties de I'Asie du Sud-Est, I'Amérique centrale et le Mexique, où la part des supermarchés est passée d'environ 5-10 \% en 1990 à 30-50 \% au début des années 2000, le décollage intervenant entre le milieu et la fin des années 1990. La troisième vague comprend les pays où la révolution des supermarchés ne s'est amorcée qu'à la fin des années 1990 ou au début des années 2000, couvrant environ 10-20\% de la distribution alimentaire nationale vers 2003. II s'agit d'une partie de l'Afrique et de quelques pays d'Amérique centrale et du Sud (comme le Nicaragua, le Pérou et la Bolivie), du Sud-Est asiatique et de la Chine, de I'Inde et de la Russie. L'Afrique subsaharienne présente une situation très diversifiée, l'Afrique du Sud étant le seul pays véritablement engagé dans la première vague de pénétration des supermarchés, et les autres pays étant soit dans la première phase de la troisième vague, soit dans ce qui peut être un décollage prochain - mais non encore amorcé - du développement des supermarchés (p. 284).

\section{Les conséquences attendues des recompositions des marchés sur l'agriculture}

Toutes ces transformations des marchés agroalimentaires ont des conséquences en amont au niveau des producteurs. Cependant, la force, la profondeur et la progression des impacts de ce grand chambardement sur l'agriculture demeurent méconnues.

Théoriquement, l'émergence de marchés mondiaux représente une opportunité pour les producteurs puisqu'ils peuvent bénéficier de nouveaux consommateurs et d'une demande permanente tout au long de l'année ; il faut toutefois qu'ils puissent s'y connecter. La contractualisation est souvent considérée comme un outil facilitant l'intégration des exploitants agricoles à ces nouveaux marchés, dans la mesure où elle peut permettre d'accroître et de stabiliser leurs revenus. Adoptant ce point de vue, le RDM08 affirme que la contractualisation et le développement de l'entrepreneuriat sont un moyen d'échapper à la pauvreté pour les exploitants agricoles des pays en développement. Il est vrai que ceux-ci ont souvent des contraintes de capital et de trésorerie et manquent fréquemment de facilités pour adopter les innovations techniques disponibles. Les contrats - avec des supermarchés, des industriels ou des exportateurs - peuvent ainsi aider à surmonter ces contraintes et cette perspective a suscité un regain d'intérêt des bailleurs de fonds pour les approches par filières ou chaînes de valeur - un mouvement qui a été à l'origine d'une abondante littérature et de nouveaux programmes et projets .

Cependant, comme indiqué plus haut et comme le rappellent notamment Reardon et Timmer (2007), la contractualisation implique des exigences croissantes en termes de normes et de standards, comprenant parfois des cahiers des charges concernant les pratiques de culture et de récolte, les conditions de transport, de transformation et de stockage des produits. Dès lors, les contrats et les nouveaux marchés avec lesquels ils mettent les producteurs en relation ne sont une opportunité que pour les producteurs en mesure de satisfaire à ces exigences. Pour les autres, le développement de la contractualisation des filières engendre un important risque de marginalisation, surtout lorsque le contexte économique et 
institutionnel n'est pas favorable à la grande majorité des producteurs - ce qui est le cas dans de nombreux pays et particulièrement en Afrique (Gibbon et Ponte 2005). Cette contractualisation croissante et la marginalisation qui l'accompagne sont ainsi susceptibles d'avoir un impact direct sur les structures agricoles. Et il s'avère essentiel de comprendre le développement de ces processus de différenciation afin de pouvoir anticiper leurs effets positifs et négatifs.

Le programme de recherche intitulé Regoverning Markets (voir encadré 5.3) a investi ces questions. Il a notamment constaté une croissance initiale de la participation des exploitants agricoles dans les chaînes de valeur modernes, y compris les petits producteurs, fréquemment suivie de leur marginalisation progressive lorsque des producteurs de plus grande taille pénètrent le marché et sont en mesure de livrer de plus grandes quantités de produits selon la qualité requise (Vorley, Fearne et Ray 2007 ; Reardon et Huang 2008). Cette différenciation progressive des producteurs est accentuée par les pratiques des grands distributeurs et des centrales d'achat des supermarchés qui, pour faciliter l'adoption de leurs cahiers des charges et réduire leurs coûts de transaction, choisissent souvent de travailler avec un petit nombre de fournisseurs capables de produire des volumes importants avec une qualité régulière.

\section{ENCADRÉ $\mathbf{5 . 3}$}

\section{Regoverning Markets}

Regoverning Markets est un programme de recherche en partenariat (2005-2007) dont l'objectif était l'analyse de la concentration croissante observée dans les secteurs de la transformation et de la distribution de systèmes agroalimentaires - au niveau national et sous-régional - et l'appréciation de ses effets sur les conditions de vie des populations rurales et des communautés des pays à faible revenu et à revenu intermédiaire. Le programme visait plus particulièrement à donner des conseils stratégiques au secteur public, aux acteurs des filières agroalimentaires, aux organisations de la société civile et aux agences de développement, afin de les aider à anticiper et gérer les effets des changements sur les marchés locaux et régionaux.

Le programme a concentré ses efforts sur les effets de la restructuration des marchés agroalimentaires, à la fois sur les différents segments en amont des filières - distribution (surtout les supermarchés), transformation, commerce de gros - et sur la production agricole. II a comparé des paires de pays et de produits à différents stades de restructuration, au moyen d'enquêtes auprès de ménages agricoles et d'analyses de filières. Les enquêtes ont été ciblées sur une sélection de produits à haute valeur ajoutée, principalement des produits frais comme les fruits, les légumes et les produits laitiers.

Source : http://www.regoverningmarkets.org.

Grâce au programme Regoverning Markets, les caractéristiques et les modalités de l'intégration dans les filières et celles du développement de la contractualisation sont désormais mieux connues; mais les informations sur l'ampleur de ces processus restent très incomplètes. Jusqu'à quel point ces nouvelles formes 
d'intégration des marchés ont-elles touché les pays en développement et, en particulier, quelles sont les différences d'impact selon le rythme des changements qui varient selon les pays? Quels sont les effectifs en jeu ? Et combien d'agriculteurs participent à ces nouvelles filières intégrées?

Les statistiques agricoles et douanières fournissent des données sur les produits et les exportations à haute valeur ajoutée ; mais elles ne disent rien du nombre de producteurs concernés par ces nouvelles filières - une situation qui représente un obstacle récurrent à l'évaluation des processus de changement. Les équipes du programme RuralStruc ont eu de grandes difficultés à recueillir des données exactes sur le degré de participation des producteurs à ces filières lors des revues du secteur agricole de la phase 1. Les quelques données obtenues indiquent cependant que, dans chaque pays, des milliers d'agriculteurs sont engagés dans ces nouvelles chaînes de valeur quand ce sont des centaines de milliers (voire des millions) de producteurs qui pratiquent toujours une agriculture plus traditionnelle. La réussite désormais célèbre de l'horticulture au Kenya offre une bonne illustration du potentiel - et des limites - de ces exportations à forte valeur ajoutée (voir encadré 5.4).

\section{ENCADRÉ $\mathbf{5 . 4}$}

\section{La success story des exportations horticoles kényanes : qui est concerné ?}

En une vingtaine d'années, les produits horticoles (fruits, légumes et fleurs coupées) se sont hissés au premier rang du commerce mondial agricole et représentent aujourd'hui $20 \%$ des exportations agricoles mondiales. En ASS, les exportations horticoles ont connu un développement rapide, mais la production demeure concentrée dans quelques régions. Le Kenya est un exemple bien connu ; c'est le deuxième exportateur horticole du sous-continent après l'Afrique du Sud, le deuxième pays en développement exportateur de fleurs après la Colombie et le deuxième fournisseur de légumes de l'Union européenne après le Maroc. L'horticulture est aujourd'hui le deuxième secteur exportateur de produits agricoles au Kenya après le thé (English, Jaffee et Okello 2006).

Cette expérience est une indéniable réussite en termes de parts de marché, de revenus d'exportation et de croissance, même si son impact sur les ressources en eau suscite des préoccupations. II est intéressant d'examiner le développement de ces exportations horticoles dans le contexte de la structure générale du secteur agricole kényan. Bien que les données disponibles soient peu nombreuses en raison de l'absence de statistiques, des enquêtes et des entretiens ciblés avec les principaux opérateurs, notamment les exportateurs, permettent de dresser un tableau général.

Plusieurs auteurs rapportent qu'au début des années 1990, la majorité des exportations horticoles étaient produites par de petits exploitants (Harris 1992 et Jaffee 1994, entre autres). Cependant, Dolan et Humphrey (2000) ont estimé qu'à la fin des années 1990, alors que les exportations horticoles s'étaient considérablement développées, $40 \%$ provenaient de terres louées par des exportateurs ou leur appartenant, $42 \%$ de grandes exploitations commerciales et seulement $18 \%$ de petits exploitants, 
qui avaient du mal à satisfaire aux exigences sanitaires et de qualité des acheteurs internationaux. Jaffee (2003) proposait des chiffres plus optimistes et évaluait la part du marché d'exportation des petits exploitants à $27 \%$ pour les légumes frais et à $85 \%$ pour les fruits frais, pour une part globale du secteur de $47 \%$. Ces estimations montrent que la croissance des exportations s'est majoritairement produite hors de la petite agriculture.

Il est tout aussi difficile d'estimer le nombre de petits exploitants dans le secteur. Selon la dernière enquête nationale - Welfare Monitoring Survey, 1994 -, le nombre d'exploitations dans le pays était estimé à 3,4 millions et moins de 20000 petits agriculteurs intervenaient dans les exportations horticoles (Jaffee 1995 ; Asfaw, Mithofer et Waibel 2007). English, Jaffee et Okello (2006) ont estimé que le secteur des exportations horticoles générait entre 120000 et 150000 emplois en 2003, dont un tiers dans le secteur des fleurs coupées, dont les petits exploitants sont absents. Les deux autres tiers sont les fruits et légumes, où l'emploi se partage entre petites exploitations (40 000 emplois), unités de transformation (10 000) et grands domaines et entrepôts (50 000).

Cette analyse indique que, bien que le secteur offre d'importants résultats au niveau macroéconomique et des opportunités à des dizaines de milliers de ménages (dont McCulloch et Ota ont montré qu'ils sont plus riches que le ménage moyen dans leur région), il faut garder à l'esprit la taille du secteur agricole mais aussi la dynamique du marché du travail. En 2010, 840000 personnes sont entrées sur le marché du travail - dont 650000 dans le seul secteur rural (voir chapitre 2). Muendo et Tschirley (2004) ont montré qu'au Kenya, plus de $90 \%$ des petits agriculteurs des régions non arides produisent des produits horticoles, principalement pour la consommation intérieure, et que les fruits et légumes destinés au marché national représentent plus de $90 \%$ de la production horticole totale en volume. Cette vue d'ensemble rappelle l'ampleur des défis qui subsistent malgré l'impact des exportations agricoles sur l'économie kényane, et le potentiel de croissance dans les autres secteurs de production agricole, qui mériteraient également de bénéficier de l'attention et de l'aide des décideurs politiques.

\section{Une nouvelle agriculture insaisissable}

Les transformations à l'œuvre dans les systèmes agroalimentaires et leurs conséquences du point de vue de l'intégration croissante de l'agriculture aux chaînes de valeur mondiales s'opèrent évidemment à des rythmes différents en fonction des caractéristiques locales et nationales. Les pays du programme RuralStruc ne font pas exception et les régions étudiées illustrent des situations très diverses.

Les résultats les plus frappants de la recherche de terrain menée par le programme résident dans le maintien d'une part importante de la production agricole consacrée aux cultures vivrières de base (staples) - même dans des régions d'agriculture d'exportation - et dans la forte proportion de l'autoconsommation. Cette dernière n'est pas surprenante en soi car une grande partie de la production vivrière des pays en développement est autoconsommée. Cependant, dans les régions qui avaient été identifiées ex ante comme " gagnantes ", une diversification des cultures et des liens avec les marchés plus étroits avaient été anticipés. Or, même dans les régions les mieux connectées de l'échantillon, la production agricole demeure relativement «traditionnelle » et tournée vers le marché national ${ }^{8}$. 
La section qui suit examine les caractéristiques de la production agricole dans les régions étudiées puis analyse le degré de diversification agricole et les conditions d'intégration au marché.

\section{Caractéristiques des revenus des exploitations agricoles}

Les revenus de l'exploitation (on-farm) proviennent d'un large éventail d'activités menées sur l'exploitation dont les caractéristiques sont liées aux conditions agroécologiques, aux profils spécifiques des systèmes agraires et à l'environnement de marché local.

Vue d'ensemble. Les revenus de l'exploitation peuvent être classés en quatre grandes catégories (voir figure 4.1) : l'agriculture (les produits des cultures) ; l'élevage ; les produits de la chasse, de la pêche et de la cueillette; les opérations de transformation à la ferme, comme la transformation du lait en fromage. La figure 5.2 montre que l'agriculture est la principale source de revenus et qu'elle domine les autres productions dans toutes les régions. Sa prédominance n'est menacée qu'à La Libertad au Nicaragua, dans la Chaouia au Maroc et à Nakuru North au Kenya, où l'élevage génère à peu près $50 \%$ des revenus internes.

La figure montre également que les ménages agricoles enquêtés ne tirent pas de revenus significatifs de l'exploitation des ressources naturelles ${ }^{9}$. Les principales activités de cette catégorie sont la pêche dans la zone de l'Office du Niger au Mali (Macina), dans la région du lac Victoria au Kenya (Nyando) et sur le littoral pacifique au Nicaragua (El Viejo), et la cueillette de fruits à Tominian et Koutiala (Mali). Quant à la transformation des produits, elle demeure étonnamment limitée ; lorsqu'il y a transformation, elle concerne les produits de l'élevage (principalement la production de fromages) au Nicaragua et au Maroc (Saïss), mais aussi le traitement initial du café (déparchage) au Nicaragua, la production d'huile d'olive au Maroc et de pâte d'arachide au Sénégal.

Pour poursuivre plus spécifiquement l'analyse de la production, la trentaine de produits identifiés lors des enquêtes, a été ventilée en cinq grandes catégories (voir tableau 5.3). Ce type de classification est toujours délicat, surtout lorsqu'il comprend différentes régions ayant leurs propres habitudes de consommation, car l'usage des produits peut être variable ${ }^{10}$.

Tableau 5.3 Classification des produits utilisée pour l'analyse des données

\begin{tabular}{ll}
\hline Cultures vivrières de base & $\begin{array}{l}\text { Riz, maïs, blé et blé dur, autres céréales (millet, sorgho, fonio, orge), manioc, } \\
\text { pommes de terre, autres denrées alimentaires de base (pois et haricots - } \\
\text { niébé, voandzou -, pois chiches, lentilles, etc.), soja }\end{array}$ \\
$\begin{array}{l}\text { Produits d'exportation traditionnels } \\
\text { Fruits et légumes }\end{array}$ & $\begin{array}{l}\text { Coton, arachide, sésame, café, canne à sucre } \\
\text { Produits de l'élevage }\end{array}$ \\
Outres & Lait, autres produits de l'élevage (beurre, viande, etc.), animaux vivants \\
\hline
\end{tabular}

Source : Enquêtes RuralStruc. 


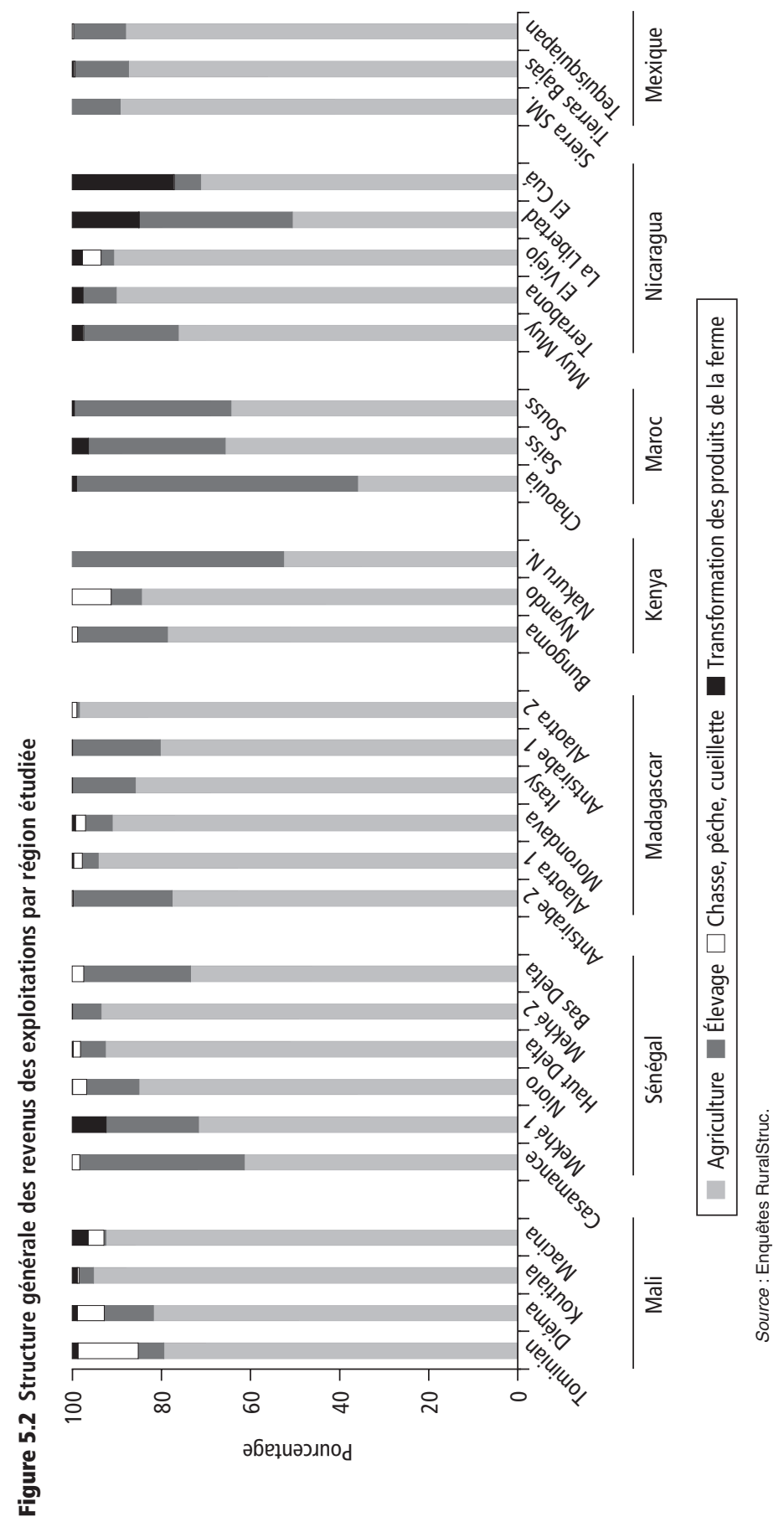


La figure 5.3 présente la structure générale du produit brut agricole des ménages dans les différentes régions ${ }^{11}$. La part des cultures vivrières de base est remarquable : elles sont cultivées par $90 \%$ des exploitations de l'échantillon (98\% en ASS et $76 \%$ ailleurs). Dans 18 des 30 régions étudiées, les cultures vivrières représentent plus de $50 \%$ du produit brut agricole, parfois $80 \%$. Les principales exceptions sont le Maroc et, en partie, le Kenya et le Sénégal. Ces cultures concernent en général un produit principal, habituellement des céréales : le riz dans toute l'île de Madagascar, à Macina au Mali, dans le Delta et en Casamance au Sénégal ; le millet et le sorgho dans les trois autres régions du Mali et dans le bassin arachidier au Sénégal ; le blé au Maroc; et le maïs au Kenya, au Mexique et au Nicaragua. La majeure partie de la production céréalière n'est pas irriguée, mais dans certains cas, les agriculteurs pratiquent la culture irriguée du riz (Madagascar, Sénégal et Mali) et du maïs (Mexique) ${ }^{12}$.

Les haricots sont la seconde culture vivrière de base au Nicaragua. À Antsirabe (Madagascar), la pomme de terre occupe une place importante ; bien que la filière de la pomme de terre se soit initialement développée pour répondre à la demande urbaine, le produit a progressivement transformé les habitudes de consommation locales; il est aujourd'hui très autoconsommé et vendu. Les racines, tubercules et plantains sont cultivés presque partout sauf au Maroc. Au Sénégal, le manioc s'est développé dans le bassin arachidier et est devenu une des principales options de diversification face à la dégradation du secteur arachidier.

L'élevage est présent dans toutes les régions étudiées et la commercialisation des animaux vivants est la règle, en particulier au Mali, qui est l'un des principaux fournisseurs de bétail des pays du golfe de Guinée. Une certaine spécialisation régionale apparaît dans le domaine des produits de l'élevage, notamment les produits laitiers. La « voie lactée » (via lactea) du Nicaragua (Muy Muy) produit du lait frais et des produits laitiers et les exploitations de La Libertad ont une activité traditionnelle de fabrication à la ferme d'un fromage de qualité médiocre (car il n'y a pas d'unités de transformation dans la région du fait de la mauvaise qualité des infrastructures - transport, électricité). Nakuru North au Kenya, Antsirabe à Madagascar et la région du Saïss au Maroc ont des ceintures laitières en périphérie des villes qui sont à l'origine du développement d'industries agroalimentaires. La Casamance au Sénégal a elle aussi une activité de transformation laitière et écoule ces produits sur le marché local. Les modes de commercialisation des produits de l'élevage et le développement de l'agro-industrie s'expliquent largement par la qualité des infrastructures de chaque région. Celle-ci détermine ce qui peut être vendu (par exemple, du lait frais réfrigéré pour l'industrie ou les supermarchés qui approvisionnent les villes, ou au contraire du fromage fait maison de faible qualité pour les marchés ruraux locaux - comme illustré par le Nicaragua) mais aussi l'ampleur de la demande locale accessible (proximité et accès aux centres urbains).

$\mathrm{Du}$ fait du caractère patrimonial de l'élevage, ses revenus (en valeur absolue et relative) peuvent être affectés par des conditions particulières. C'est ce qui s'est produit dans la Chaouia, au Maroc, où une très mauvaise campagne agricole a profondément affecté les rendements céréaliers et contraint de nombreux agriculteurs à vendre leur bétail sur pied et leurs petits ruminants ${ }^{13}$. De même, l'importance des revenus de l'élevage dans la zone cotonnière du Mali (Koutiala) reflète les bas prix 


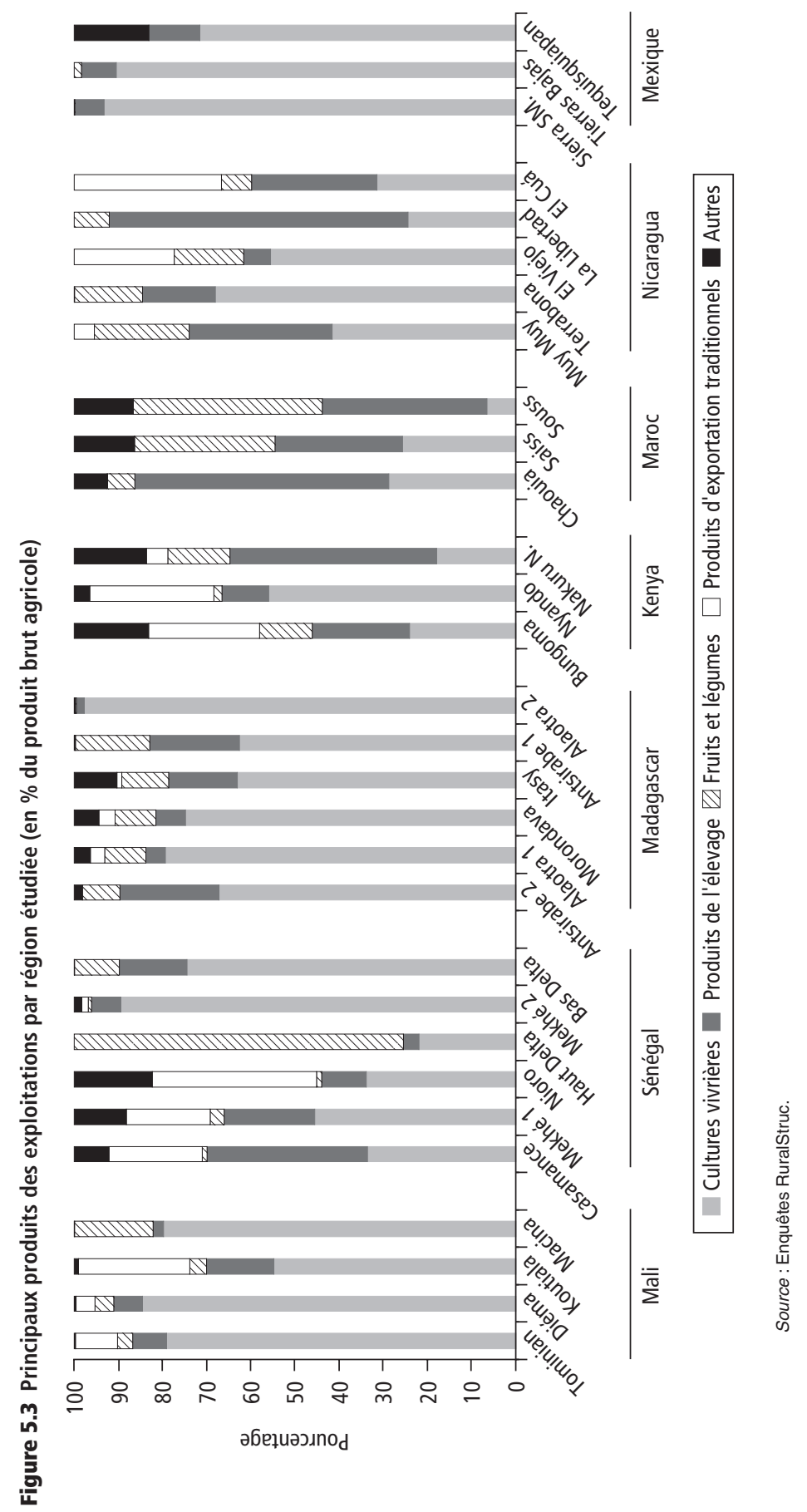


du coton dont les cultivateurs ont été victimes en 2007. De nombreux agriculteurs ont décapitalisé et vendu leurs bêtes pour conserver leur pouvoir d'achat. La bonne campagne à Macina a eu l'effet inverse avec des investissements accrus en bétail. Au Mali et, plus généralement dans toute l'Afrique subsaharienne, le statut patrimonial du bétail est patent : il fournit une force de traction et du fumier pour les cultures mais il constitue aussi une épargne sur pied conservée pour les moments difficiles.

L'horticulture est une activité courante - les légumes sont cultivés partout pour la consommation domestique. Cependant, dans de nombreuses régions étudiées, une spécialisation s'est produite, encouragée par les conditions naturelles favorables et stimulée par le développement urbain qui a entraîné des investissements privés spécifiques. C'est le cas en particulier dans le Saïss et le Souss au Maroc, où la production de fruits et légumes destinés à l'exportation ou à l'industrie agroalimentaire (fruits et tomates principalement) est devenue en vingt ans une activité de toute première importance, et où les transformateurs et les exportateurs encouragent le développement par des dispositifs contractuels. Le même phénomène s'est produit à Nakuru North ; bien que la zone étudiée ne soit pas située dans la célèbre zone productrice de fleurs de la région, les ménages produisent des tomates et les vendent à une conserverie. Dans le Haut Delta au Sénégal, la production de tomates s'est développée en raison de la présence d'une unité de transformation qui alimente le marché local en concentré de tomate. Le fait que de nombreux ménages enquêtés soient situés dans la zone de collecte de l'usine explique la forte part de l'horticulture dans leur produit brut agricole. La production de produits frais pour les villes s'est aussi développée à Antsirabe et Itasy (Madagascar), où il est possible de cultiver des fruits et légumes de climats tempérés (pêches, pommes et carottes par exemple $)^{14}$. La production d'oignons s'est fortement développée dans le Saïss (Maroc) et dans l'Office du Niger (Macina, Mali); elle approvisionne les marchés nationaux et régionaux et représente près de $20 \%$ du produit brut agricole des exploitations de la région. À Terrabona (Nicaragua), les ménages les plus riches pratiquent l'horticulture irriguée ; leurs produits sont principalement vendus au niveau national, sur les marchés traditionnels mais aussi dans le cadre de filières plus intégrées (comme les centrales d'achat des supermarchés).

Quant aux produits d'exportation traditionnels, leur importance s'explique par les circonstances propres à chaque région. Leur développement est généralement associé à l'histoire régionale et résulte d'avantages naturels et d'interventions spécifiques de l'État ou du secteur privé - les investissements destinés à l'approvisionnement des métropoles à l'époque coloniale ont souvent joué un rôle initial. De manière générale, les produits d'exportation ont profondément influencé le profil agricole des régions concernées du fait de leur poids économique et parfois politique ancien, même si celui-ci a pu s'estomper avec le temps. C'est le cas du coton à Koutiala et en Casamance, de l'arachide dans le bassin arachidier, du café à El Cuá et Bungoma, et de la canne à sucre à El Viejo, Nyando et Bungoma.

Le rapport autoconsommation-vente. Malgré les conditions agroécologiques, agraires, historiques et institutionnelles très différenciées propres à chaque contexte régional, l'importance de l'autoconsommation est la principale caractéristique du revenu des 
exploitations agricoles dans l'échantillon du programme RuralStruc ${ }^{15}$. Elle représente une part significative du produit brut agricole et les écarts entre les régions sont une illustration des différences de connexion aux marchés. Cette relation aux marchés est cependant plus complexe et ambivalente car, même lorsque l'autoconsommation est importante, elle n'implique pas nécessairement une déconnexion des marchés. En effet, les ménages ont différents modes de participation aux marchés : ils peuvent vendre une partie limitée de leur production, tout en vendant leur travail (voir chapitre 4) ; mais ils sont aussi des consommateurs qui achètent des biens (notamment de la nourriture) et des services. Dans le cas du Mali (voir tableau 5.4), même dans les régions où l'autoconsommation est importante, un fort pourcentage de ménages participe aux marchés alimentaires en qualité de consommateur ${ }^{16}$.

Néanmoins, comme le montre la figure 5.4, les niveaux d'autoconsommation demeurent très élevés; et les proportions constatées dépassent les anticipations compte tenu de la méthodologie de sélection des régions. Il existe cependant des différences sensibles entre les pays et régions - le Mexique constituant une exception notable (voir encadré 5.5) - mais aussi entre les niveaux de revenus.

Dans les pays et régions les plus riches, les ménages consomment apparemment une moindre part de leur production, tandis que les ménages les plus pauvres sont plutôt tournés vers l'autoconsommation. Les différences entre les quintiles de revenu sont notables partout mais elles semblent plus marquées à Madagascar, au Kenya et au Nicaragua ${ }^{17}$.

Les niveaux de la demande et du risque sont les deux principaux déterminants de l'autoconsommation. La demande dépend surtout de la distance aux marchés et des possibilités d'intégration par le biais de filières spécifiques. À Koutiala par exemple, l'autoconsommation est plus faible car une forte demande de coton donne des opportunités de sortir de l'agriculture de subsistance ; il en est de même des tomates et du manioc dans le Haut Delta et à Mekhé 2, respectivement.

Cependant, les ménages qui consomment une grande partie de leur production le font souvent parce que leurs produits sont peu demandés, généralement parce leur connexion aux marchés est mauvaise ${ }^{18}$. L'autoconsommation décroît dès lors qu'existe une demande effective, qu'elle soit due à la proximité avec un grand marché urbain ou à la présence d'un acheteur spécifique. Ces effets de demande tendent à apparaître principalement entre régions, car l'accès au marché varie peu à l'intérieur d'une même région, même si des différences sont possibles : c'est le cas notamment dans les zones reculées de la Chaouia ou du Souss au Maroc; et c'est ce constat qui a motivé la distinction entre deux zones à Antsirabe.

Tableau 5.4 Participation des ménages maliens aux marchés alimentaires

\begin{tabular}{lcc}
\hline & Ménages achetant des aliments & Ménages vendant des produits vivriers \\
\hline Tominian & $60 \%$ & $8 \%$ \\
Diéma & $64 \%$ & $53 \%$ \\
Koutiala & $58 \%$ & $77 \%$ \\
Macina & $71 \%$ & $89 \%$ \\
\hline
\end{tabular}

Source : Enquêtes RuralStruc. 
$\bar{\sigma}$

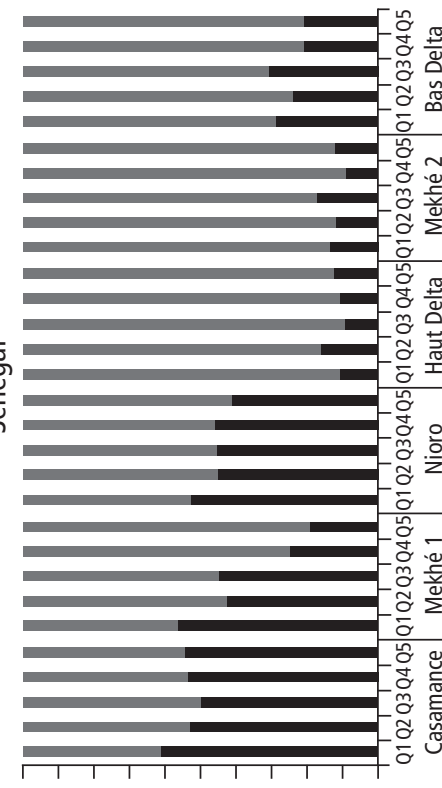

응ㅇㅇ옹ㅇㅇㅇㅇㅇㅇㅇㅇㅇㅇ

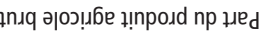
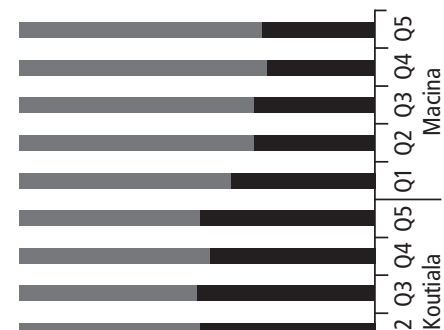

$\stackrel{-\frac{1}{\Sigma}}{\Sigma}$
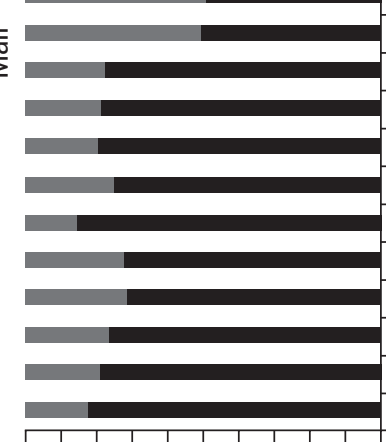

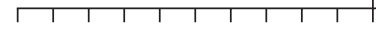

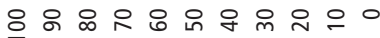

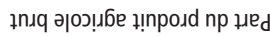

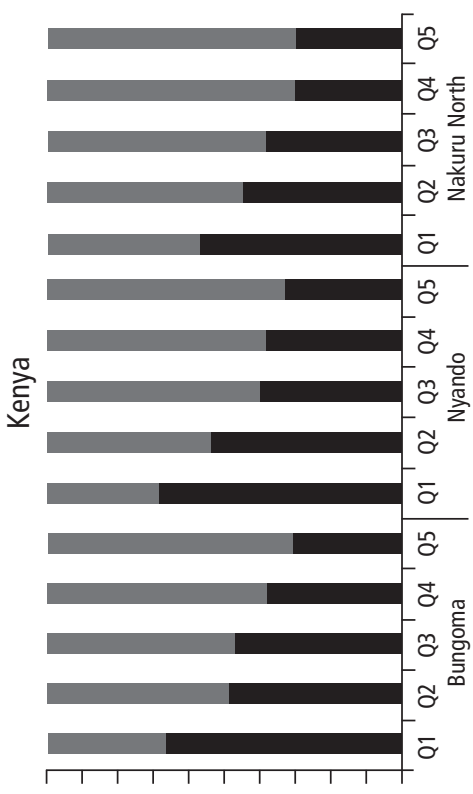

으요용요 웅유응

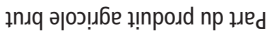

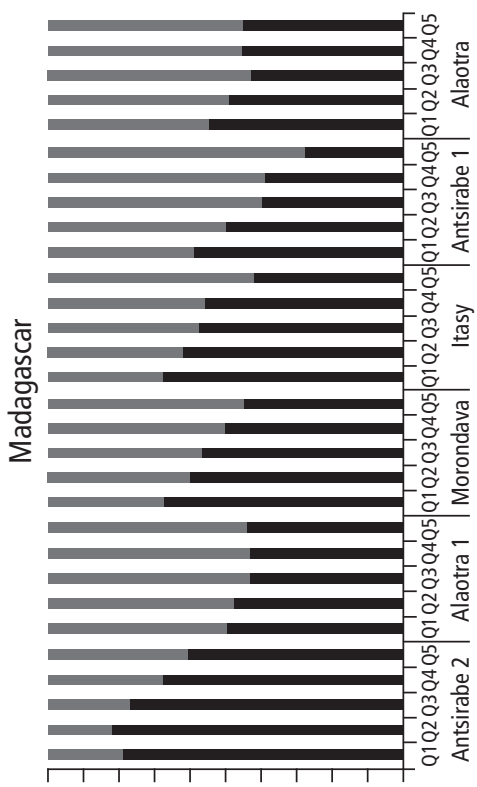

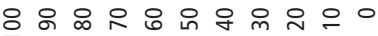

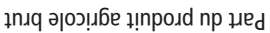



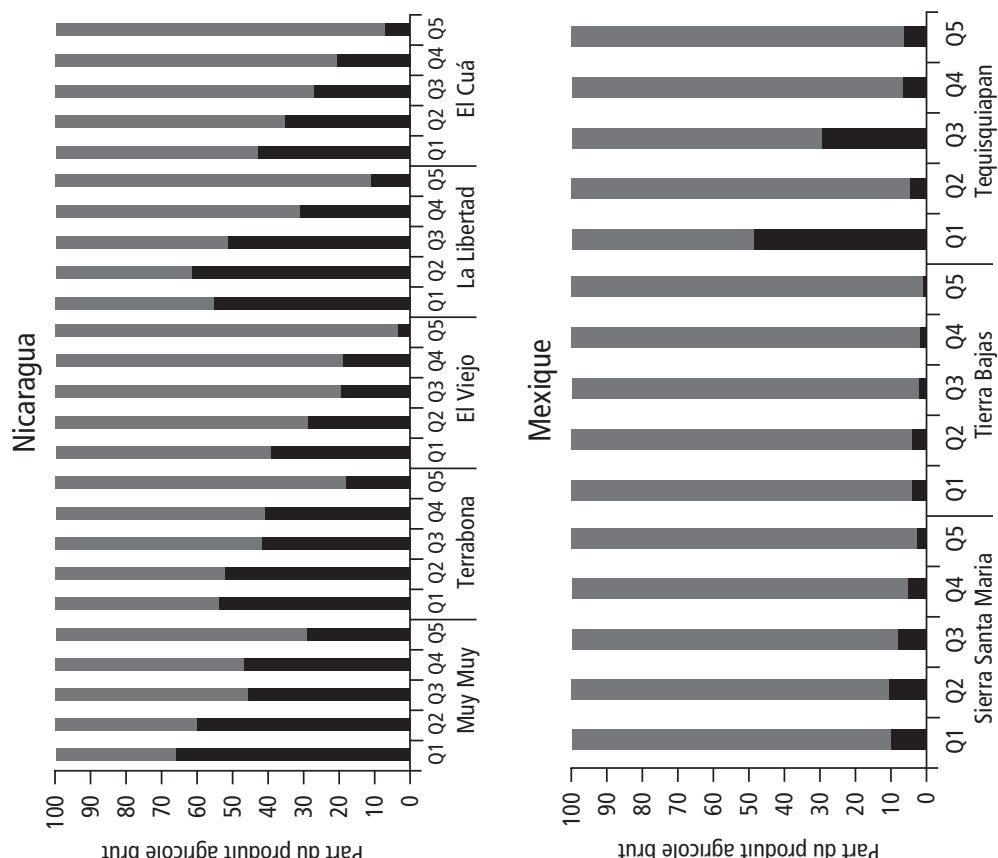

으 응응ㅇํ 웅유응

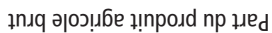
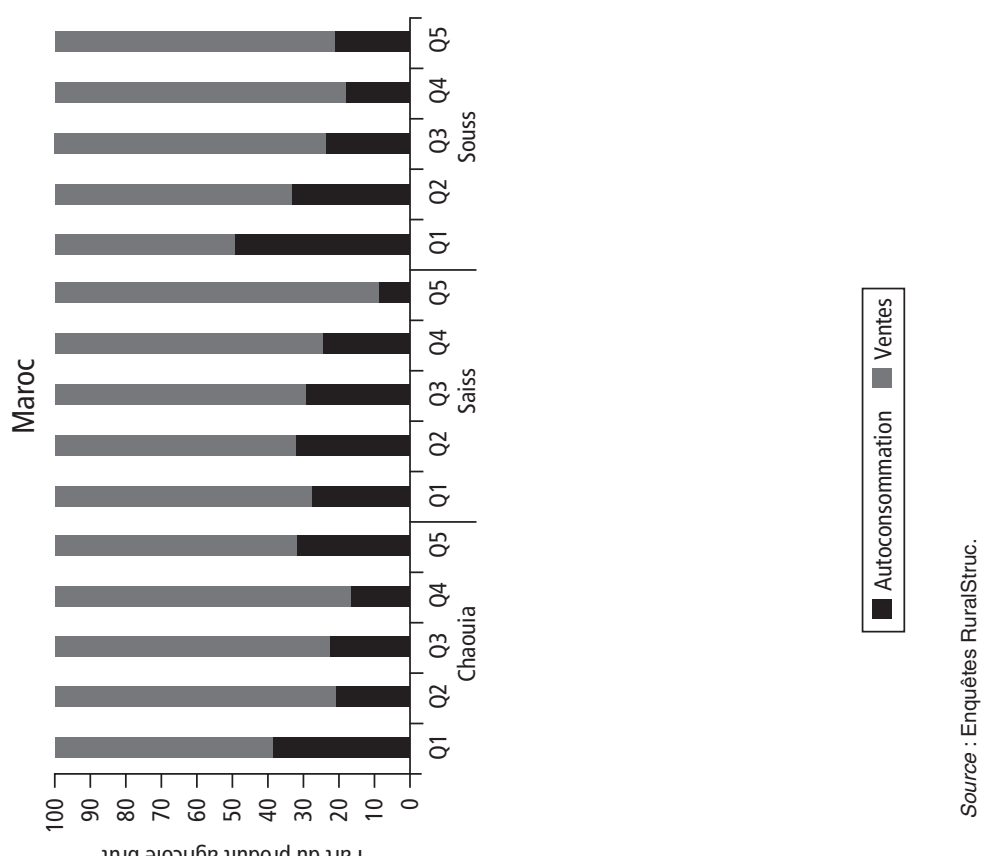

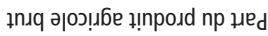


Le second déterminant a trait au niveau du risque qui est fortement corrélé au niveau de revenu. En effet, les ménages qui ont de très faibles revenus sont exposés à l'insécurité alimentaire (voir chapitre 3) et ils adoptent en conséquence des pratiques de gestion des risques qui les conduisent à s'assurer de leur approvisionnement alimentaire en produisant eux-mêmes. C'est ce qu'on peut appeler un effet d'offre : face à un risque plus élevé, les ménages ne veulent pas vendre leur production sur le marché et en consomment une part importante. Les effets d'offre tendent à se manifester davantage entre les quintiles de revenu d'une même région.

Le Nicaragua est un bon exemple de pays où les différences entre quintiles de revenu dominent les profils d'autoconsommation : dans les quintiles inférieurs, les taux d'autoconsommation atteignent ou avoisinent $60 \%$ mais, à l'intérieur de ces quintiles, une partie des ménages (20\% à $40 \%)$ n'ont aucun lien avec les marchés agricoles (c'est-à-dire que leur taux d'autoconsommation est de $100 \%$ ). Une seule région, La Libertad, est caractérisée par l'isolement physique et connaît donc des problèmes de transport, mais le déficit d'infrastructure n'y gomme pas un effet quintile prononcé.

Les stratégies de gestion des risques sont manifestement un déterminant important de la faiblesse des liens avec les marchés agricoles. Mais il faut aussi prendre en compte le rôle des réseaux commerciaux. En effet, les intermédiaires n'ont parfois guère intérêt à collecter de petites quantités produites par un grand nombre de très petits agriculteurs lorsqu'ils peuvent accéder à de plus grandes quantités produites par des agriculteurs plus importants - ce qui plaide clairement pour une action collective des petits producteurs. Des conditions locales particulières peuvent aussi influencer les stratégies des ménages, comme à Terrabona où les possibilités de travail salarié dans l'agriculture et dans les maquiladoras permettent une double stratégie : d'autoconsommation des produits de l'exploitation et d'insertion sur le marché du travail. Les ménages préfèrent pratiquer les cultures vivrières à des fins de consommation familiale et gagner de l'argent grâce à un emploi salarié pour couvrir d'autres besoins comme la scolarité, la santé ou les biens de consommation.

\section{Profils régionaux de diversification agricole}

La section précédente a examiné les types de productions pratiquées par les exploitations et l'importance de l'autoconsommation dans les régions étudiées, mais quel est le degré de spécialisation ou de diversification des exploitations?

La figure 5.5 présente la structure globale du produit brut agricole des exploitations des différentes régions en mettant en évidence la part de la production agricole autoconsommée et celle des deux premières productions commercialisées.

L'analyse des produits commercialisés confirme la forte persistance des produits vivriers de base que les ménages vendent dans toutes les régions et quel que soit leur niveau de revenu. Les produits vivriers représentent au minimum $25 \%$ de la production agricole dans toutes les régions, à l'exception principalement du Maroc où l'importance du blé a été masquée par une mauvaise campagne et par la vente de bétail qui a suivi. L'enquête montre que ces produits vivriers sont 


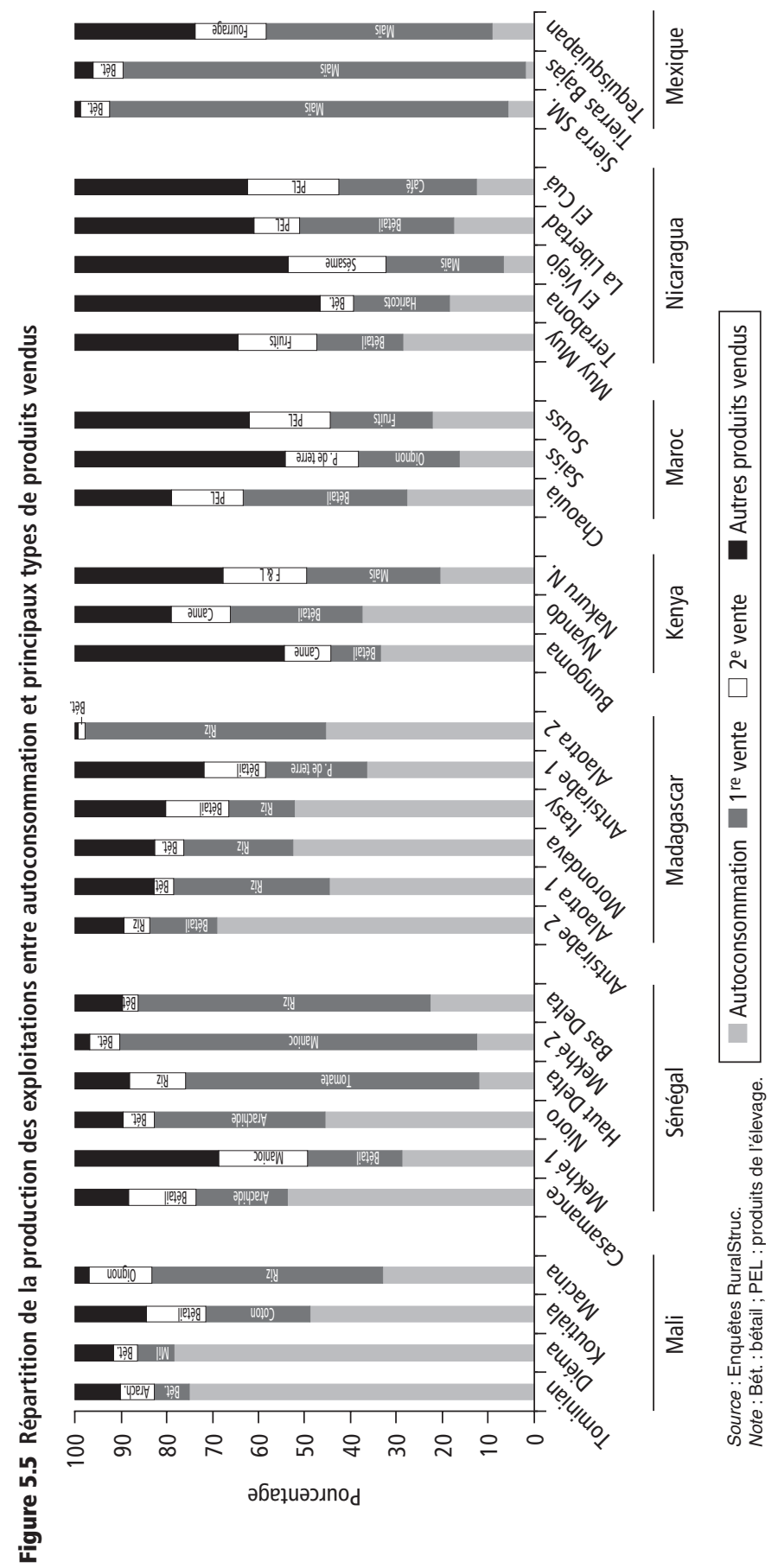


souvent une des meilleures options de commercialisation offertes aux ménages agricoles, même les plus aisés, et suggère des possibilités globalement faibles de spécialisation dans des cultures à plus forte valeur ajoutée.

L'analyse confirme l'importance de l'autoconsommation au niveau régional agrégé (et non plus par quintiles), surtout dans les régions les plus pauvres; mais elle fait également apparaitre des tendances et profils de diversification agricole différents. En Afrique subsaharienne, l'autoconsommation ou l'autoconsommation combinée au premier produit commercialisé représentent en moyenne plus de $70 \%$ du produit brut d'exploitation dans 13 des 19 régions étudiées. Alors que dans les régions hors Afrique subsaharienne, ce n'est le cas que dans 2 des 11 régions étudiées : les deux zones du Sotavento au Mexique, où est apparu au cours des dernières années un processus particulier de spécialisation poussée dans le maïs (voir encadré 5.5). Un constat général est que lorsque la richesse régionale augmente (des régions les plus pauvres à gauche de la figure aux régions les plus riches à droite), la part de l'autoconsommation recule alors que celle des " autres produits vendus » augmente.

La diversification des produits de l'exploitation differe non seulement entre régions pauvres et riches, mais aussi entre ménages pauvres et riches au sein de chaque région. Dans 20 des 30 régions étudiées, les ménages du cinquième quintile sont plus diversifiés que ceux du premier : les ventes de leurs trois produits principaux représentent une moindre proportion de leur revenu d'exploitation (environ $8 \%$ de moins en moyenne, mais l'écart peut atteindre $16 \%$ ). Ainsi, une diversification croissante des quintiles les plus pauvres aux plus riches est observée dans 10 des 11 régions hors ASS, mais dans seulement 10 des 19 zones d'ASS, ce qui concorde avec l'observation précédente d'une diversification moindre en Afrique subsaharienne.

Au niveau intrarégional, la diversification des exploitations se caractérise plus souvent par l'ajout de nouvelles cultures commerciales que par l'abandon d'un type de production en faveur d'un autre. En fait, dans 20 des 30 régions étudiées, la première production commercialisée des ménages du cinquième quintile et de ceux du premier quintile est identique. Il ne semble pas que les ménages pauvres soient contraints à ne vendre que des produits vivriers de base alors que les ménages plus riches pourraient vendre des produits d'exportation ou à forte

\section{ENCADRÉ $\mathbf{5 . 5}$}

\section{L'exception du Sotavento : spécialisation des exploitations et effondrement de l'autoconsommation}

Dans l'enquête RuralStruc, le Sotavento, dans l'État de Veracruz, est un cas à part - caractérisé par la spécialisation dans la culture du maïs, une intégration accrue aux circuits de commercialisation et un recul considérable de l'autoconsommation. 
L'essor du maïs dans les années 2000 s'est produit en dépit d'un environnement économique défavorable. En effet, entre 1994 et 2007, une vaste restructuration du secteur a fait chuter les prix réels du maïs de $60 \%$ tandis que les prix des intrants enregistraient une augmentation à peu près équivalente (Léonard et Palma 2002 ; Zahniser et Coyle 2004 ; RS 2 Mexique). Pourtant, la superficie cultivée en maïs a augmenté dans les deux zones étudiées du Sotavento (Tierras Bajas et Sierra de Santa Marta). La production a surtout augmenté dans les plaines - $30 \%$ - et n'a progressé que de $6 \%$ dans la zone montagneuse. À l'échelle de l'État de Veracruz, la surface cultivée en maïs a reculé de $18 \%$ sur la même période.

Cette tendance trouve deux explications (RS 2 Mexique - Sotavento). Dans les plaines, les vastes surfaces inondables sont très fertiles et la mécanisation permet de réaliser des économies d'échelle. De grandes exploitations se sont développées, mais les petits producteurs ont malgré tout pu participer au marché en dépit du nouveau contexte à travers des organisations de producteurs qui leur ont donné accès à des services de mécanisation, à des contrats (la plupart informels) avec des acheteurs ou avec de grandes exploitations et, enfin, aux incitations offertes au secteur commercial par les programmes Aserca et Alianza (Brun 2008). Dans la Sierra, l'éloignement a limité les possibilités de diversification des activités - aussi bien sur qu'en dehors de l'exploitation - et in fine, grâce au programme Procampo, le maïs est resté la meilleure option de consolidation des revenus. Ainsi, dans les deux zones du Sotavento, les exploitations ont renforcé leur spécialisation dans le maïs et les autres productions agricoles ont été progressivement abandonnées, comme le riz dans les plaines et même les haricots dans la Sierra, où ils étaient traditionnellement cultivés en alternance avec le maïs.

Cette spécialisation dans le maïs s'est accompagnée d'un effondrement de l'autoconsommation, en particulier dans les Tierras Bajas, où elle est aujourd'hui inexistante. Cette situation est très éloignée du système alimentaire traditionnel, fondé sur le maïs cultivé avec le haricot sur de petites parcelles destinées à la consommation familiale (la milpa). Quatre facteurs expliquent ce repli de I'autoconsommation. Premièrement, pour accéder au crédit public et au soutien technique des grandes entreprises, les producteurs ont dû leur vendre toute leur production. Deuxièmement, cette possibilité de vente de l'ensemble de la production a résolu un problème récurrent de stockage qui a renforcé l'adhésion des producteurs car les nouvelles variétés de maïs hybrides étaient très vulnérables aux rongeurs après la récolte. Troisièmement, dans les plaines du Sotavento, la récolte du maïs a été entièrement mécanisée par le biais de services fournis par les entreprises. Enfin, la possibilité pour les femmes d'accéder dans les Tierras Bajas à des activités à l'extérieur de l'exploitation s'est faite au détriment du temps disponible à consacrer à la fabrication de tortillas à partir du maïs cultivé sur l'exploitation. Ainsi, dans ce contexte de rapide restructuration du " complexe maïs-tortilla », les agriculteurs vendent leur maïs hybride et achètent de la farine de maïs ou des tortillas toutes prêtes sur les marchés locaux (Appendini et Gómez, à paraître).

Cette exception du Sotavento parmi les régions étudiées est une référence importante, car elle montre le puissant effet que peuvent avoir les nouveaux circuits de commercialisation soutenus par un ensemble de facteurs de changement. Elle montre aussi que des habitudes de production-consommation peuvent être radicalement transformées dans un laps de temps très court. Enfin, elle souligne l'impact des programmes d'aides publiques sur les processus d'adaptation des ménages. 
valeur ajoutée. Parmi les 20 régions où les ménages les plus riches et les plus pauvres ont la même production commerciale principale, cette production est une culture vivrière dans 11 cas. Il n'est pas rare de voir les ménages les plus aisés vendre principalement des produits vivriers, mais aussi de voir les ménages du premier quintile vendre des animaux ou des produits d'exportation (le café à El Cuá, l'arachide au Sénégal, le coton à Koutiala).

$\mathrm{Au}$ niveau interrégional, contrairement aux attentes, les exploitations de la région la plus riche d'un pays ne vendent pas nécessairement un produit à forte valeur ajoutée tandis que les régions les plus pauvres vendraient principalement des produits vivriers à faible valeur ajoutée. À cet égard, les régions enquêtées du Maroc et du Nicaragua sont de possibles exceptions, puisque les régions les plus riches sont aussi celles qui sont respectivement spécialisées dans les fruits et le café.

Le type des produits vendus dépend de la situation particulière de chaque région au plan des ressources naturelles, des biens publics, de l'investissement privé et de la présence ou de l'absence d'acheteurs. Lorsque de vastes mouvements d'adoption de cultures commerciales se produisent, ils semblent concerner tous les ménages d'une région. Les différences entre les ménages aisés et les ménages pauvres tendent à résider dans la diversification des sources de revenus de l'exploitation : les ménages plus aisés ont généralement des revenus plus diversifiés, chaque source représentant une part plus modeste du revenu total. L'hétérogénéité apparaît comme une caractéristique définitoire de ces profils de diversification. Les agriculteurs se servent des actifs dont ils disposent pour tirer parti des opportunités de l'environnement naturel et économique régional. Les productions vivrières de base et certains produits d'exportation semblent à la portée de tous les agriculteurs d'une même région; mais les ménages les plus aisés, qui ont davantage de ressources, peuvent mieux profiter des opportunités locales pour se diversifier davantage.

Ces mécanismes sont également à l'œuvre dans les zones de spécialisation agricole, comme au Mexique, où les ménages les plus aisés, qui ont davantage de ressources, peuvent se saisir des possibilités offertes par le contexte régional. Dans le cas du Sotavento, les conditions très favorables auxquelles ont accès les producteurs de maïs les incitent à la spécialisation dans le maïs plutôt qu'à la diversification dans d'autres productions (voir encadré 5.5). Cette spécialisation observée au Mexique est cependant une exception : dans les régions étudiées par le programme RuralStruc, les exploitations les plus riches sont incitées à la diversification.

\section{Profils régionaux d'intégration au marché}

Les profils de production et de diversification agricole décrits précédemment indiquent de fait une nette prépondérance de formes traditionnelles de commercialisation et d'intégration au marché dans les régions étudiées. En effet, les fameuses exportations à forte valeur ajoutée, qui étaient censées introduire de nouveaux modes de commercialisation connectés à des acheteurs sur les marchés très compétitifs des pays à hauts revenus, restent extrêmement modestes. 
Parallèlement, et au-delà de la pénétration très variable des systèmes modernes de distribution alimentaire parmi les pays du programme RuralStruc (voir encadré 5.6), la "révolution des supermarchés » apparaît comme un horizon assez éloigné (hormis peut-être au Mexique). Et même lorsqu'une pénétration significative des supermarchés est observée, les effets sur l'exploitant agricole moyen demeurent limités. Le projet de recherche Regoverning Markets a en effet montré : (1) qu'il existe un écart important entre le niveau général de pénétration des supermarchés et le taux plus spécifique de pénétration sur les segments à forte valeur ajoutée de la filière alimentaire (estimés à seulement $25 \%$ au Mexique) ; et (2) que les supermarchés tendent à acheter la majorité de leurs produits à des grossistes et parfois à de grandes entreprises sous contrat.

\section{ENCADRÉ 5.6}

\section{La grande distribution alimentaire : un développement contrasté dans les pays du programme RuralStruc}

Selon le programme Regoverning Markets (Reardon et Huang 2008), les pays du programme RuralStruc peuvent être classés en trois catégories en fonction du développement de l'agro-industrie alimentaire moderne : (1) les pays avancés, où plus de $40 \%$ des ventes de produits alimentaires sont réalisées en supermarché (Mexique) ; (2) les pays à un stade intermédiaire, où les supermarchés concentrent entre $10 \%$ et $40 \%$ des ventes alimentaires (Nicaragua, Kenya et Maroc) et (3) les pays à un stade initial, où les supermarchés réalisent moins de $10 \%$ des ventes (Madagascar, Mali et Sénégal).

Mexique : la grande distribution s'est développée en trois temps. Avant 1980, les supermarchés se concentraient dans les grandes villes du nord et du centre du pays et leur développement reposait surtout sur des capitaux domestiques, bien que certaines chaînes aient été créées avec des capitaux américains. Dans les années 1980, les supermarchés ont commencé à se développer hors de leurs bases régionales et ont entrepris de se consolider par des alliances avec des capitaux domestiques et étrangers dans un contexte de vive concurrence. À compter de 1990, on a assisté à une expansion très rapide, stimulée par l'entrée de chaînes géantes venues des États-Unis (Walmart) et de France (Carrefour) (Schwentesius et Gomez 2002). Aujourd'hui, les supermarchés concentrent $55 \%$ de la distribution alimentaire ; cependant, cette moyenne nationale masque de fortes disparités régionales et une nette fracture entre zones urbaines et zones rurales.

Nicaragua : les supermarchés ont commencé à se développer dans les années 1990. Dans un premier temps, les entreprises nicaraguayennes étaient les seules en lice, puis des entreprises costaricaines ont créé une chaîne de supermarchés concurrente et des entreprises régionales comme Hortifruti se sont lancées dans le commerce de gros. Dans les années 2000, Walmart a racheté des supermarchés régionaux et des sociétés intermédiaires comme Pali, La Union, Paiz et Hortifruti. Les supermarchés couvrent 
aujourd'hui à peu près $20 \%$ de la demande de produits à haute valeur ajoutée (RS 1 Nicaragua, 43-45).

Maroc : après des débuts modestes à Casablanca et Rabat dès le début des années 1960 avec Monoprix (France), les supermarchés ont commencé à se développer dans les années 1990, emmenés par plusieurs chaînes à capitaux marocains, en particulier Marjane, Label'Vie et Aswak Assalam. Le premier investissement étranger d'envergure a été réalisé en 2001 lorsque Auchan (France) a formé une coentreprise avec ONA (Omnium Nord Africain), le plus grand consortium marocain à capitaux privés, et a pris le contrôle de Marjane, puis d'Acima en 2002 (Codron et al. 2004). ONA détient $51 \%$ de la coentreprise et Auchan $49 \%$. Le nombre d'hypermarchés est passé de 6 magasins en 1993 à 19 en 2007 (RS 1 Maroc, 104).

Kenya : alors qu'ils n'occupaient qu'une petite niche il y a quinze ans, les supermarchés concentrent aujourd'hui $20 \%$ à $30 \%$ de la distribution alimentaire et poursuivent leur vive expansion. Le premier magasin en dehors de Nairobi a été construit par Uchumi à Nakuru en 1993, ce qui a déclenché une compétition au niveau national. La rivalité entre les deux grandes chaînes - Uchumi et Nakumatt - est devenue un important moteur de croissance car chaque nouvelle stratégie déployée par une des deux chaînes obligeait l'autre à l'imiter ou à déployer une contre-stratégie (Neven et Reardon 2004). En 2003, on comptait 225 magasins de grande distribution au Kenya : 209 supermarchés et 16 hypermarchés.

Madagascar : la part des supermarchés demeure modeste, mais ils se sont développés dans les grandes villes du pays à travers trois sociétés étrangères. Avant la récente crise politique du début 2009, qui a particulièrement touché le secteur de la grande distribution (pillages), la situation était la suivante : la chaîne sud-africaine Shoprite, présente à Madagascar depuis 1992 où elle a racheté les actifs locaux du français Champion, avait sept magasins (cinq à Antananarivo, un à Antsirabe et un à Toamasina), Leaderprice (France) comptait trois magasins à Antananarivo et Score (racheté par le groupe Vindemia, aujourd'hui filiale du français Casino) possédait trois hypermarchés à Antananarivo et deux supermarchés dans les autres provinces (RS 1 Madagascar, 63).

Sénégal : la distribution alimentaire moderne est très peu développée, avec seulement trois supermarchés à Dakar. Initialement ouverts par SCOA (France) à l'enseigne Score, ils sont franchisés par Casino (France) depuis 2007.

Mali : il n'y a pas de distribution alimentaire moderne notable au Mali.

Sources : Rapports nationaux du programme RuralStruc et autres références citées.

En dehors du Mexique, les régions étudiées présentent un panorama plus classique, modelé par des systèmes commerciaux anciens, principalement fondés sur des arrangements informels - quels que soient les types de produits et d'opérateurs. Plusieurs filières ont néanmoins des structures de marché particulières qui donnent lieu à une organisation spécifique.

La commercialisation traditionnelle domine. La commercialisation « traditionnelle » correspond à l'ensemble des intermédiaires ruraux qui font le lien entre les campagnes et les marchés nationaux, régionaux et internationaux (c'est-à- 
dire, les systèmes de distribution et les exportateurs). Il s'agit des grossistes et des agents ou courtiers ${ }^{19}$ qui travaillent pour eux, ainsi que des acheteurs indépendants. Ce type de commercialisation donne aux agriculteurs deux options au contour souvent imprécis. La première correspond à la vente au comptant, soit directement bord champ, soit au marché du village à un courtier ou un grossiste ; la seconde est la vente régulière à un grossiste, bien que cette option n'implique pas nécessairement d'arrangement formel ni ne garantisse forcément une quantité vendue ou un prix supérieur à ce qui aurait été obtenu au comptant. Cette seconde option représente néanmoins une certaine formalisation de la transaction commerciale dans la durée.

La commercialisation traditionnelle domine dans les régions étudiées. La figure 5.6 classifie les types de commercialisation en quatre grandes catégories de vente : au comptant, à des grossistes (les deux formes de commercialisation traditionnelle), à des coopératives, et à des agro-industries ${ }^{20}$. Sauf très rares exceptions, les deux premières catégories représentent la majeure partie de la valeur totale des ventes. Les ventes au comptant à la ferme ou au marché du village représentent $100 \%$ des ventes à Tominian, au Mali, et $95 \%$ dans le bassin arachidier du Sénégal et dans la Chaouia (Maroc). La commercialisation par des grossistes s'impose en particulier à Madagascar pour le riz (Alaotra, où sont basés les grossistes) et les produits horticoles (Antsirabe 1, à proximité de la ville d'Antsirabe), et au Nicaragua.

Étonnamment, en dehors du Mexique, la part vendue à des coopératives (un thème de prédilection de nombreuses politiques agricoles antérieures) est inexistante dans la grande majorité des localités enquêtées et au mieux anecdotique dans les autres. Dans le Sotavento, de nouvelles organisations de producteurs ont été formées pour soutenir le développement de la production de maïs, mais elles visaient surtout à accéder aux subventions publiques (voir encadré 5.5). À Tequisquiapan, les agriculteurs vendent du maïs ou des plantes fourragères à des organisations de producteurs, lesquelles ont souvent conclu des contrats avec de grandes entreprises d'élevage.

Les ventes aux agro-industries sont plus développées bien que leur importance varie nettement selon les régions. Logiquement, cette variabilité est liée à la présence ou à l'absence d'une unité de transformation (et en conséquence à la présence de productions impliquant une transformation locale). Ainsi, des niveaux de ventes significatifs à l'agro-industrie sont observés : à Koutiala, au Mali, où la totalité du coton est vendue aux usines d'égrenage de la CMDT (Compagnie malienne pour le développement des textiles); dans le Haut Delta, au Sénégal, où les tomates sont transformées par la SOCAS (Société de conserves alimentaires du Sénégal); et au Kenya, où la canne à sucre est vendue à plusieurs sucreries locales $^{21}$. Dans les autres régions, la commercialisation par les opérateurs agro-industriels est moins développée et représente généralement moins de $20 \%$ des ventes. C'est le cas des tomates, des agrumes et des olives dans le Saïss et le Souss (Maroc); des tomates et des produits laitiers à Nakuru North (Kenya); du café à El Cuá (Nicaragua); du riz aux rizeries en Alaotra 2 et des haricots verts destinés à l'exportation à Itasy (Madagascar). 


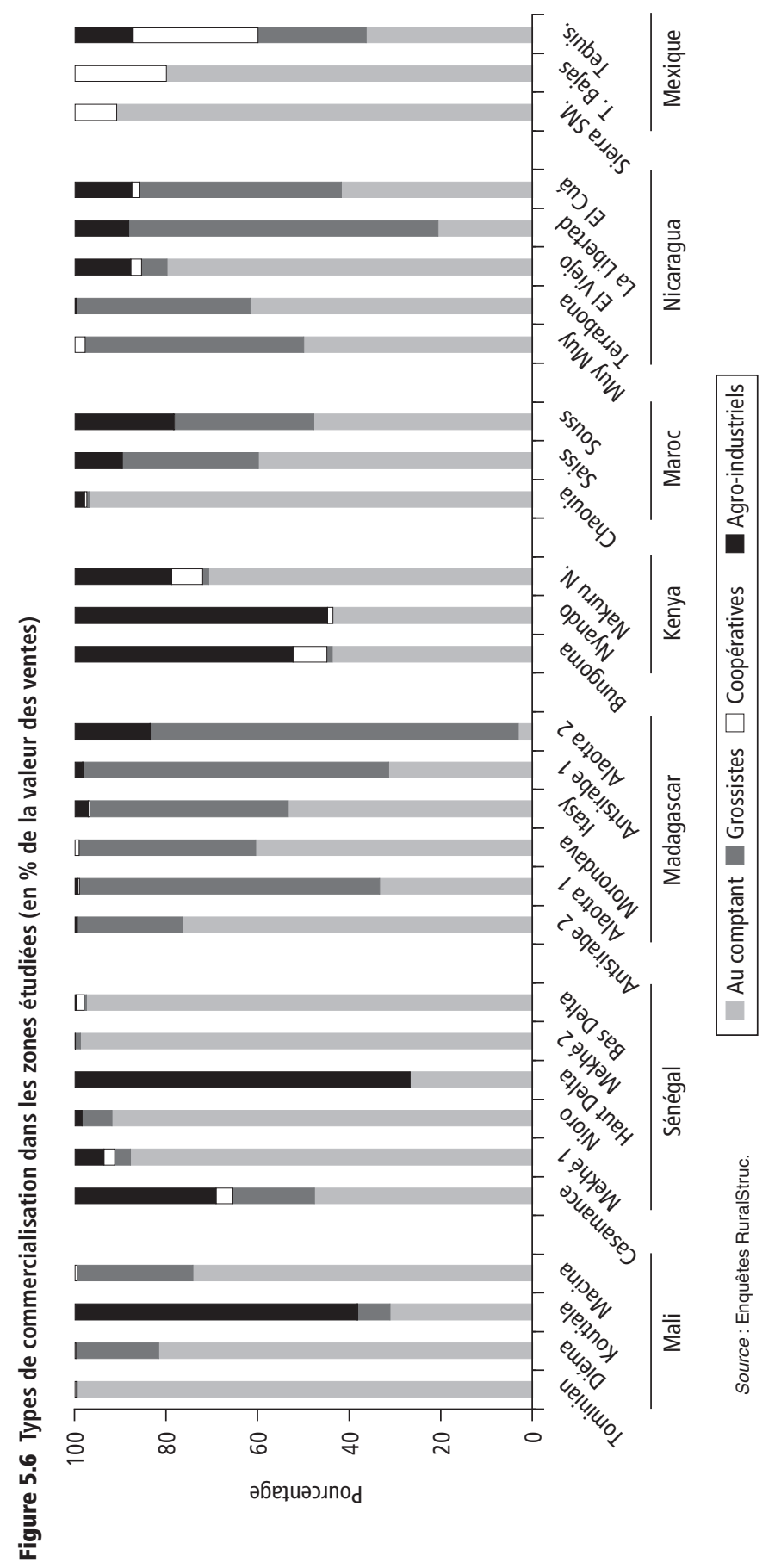


Une contractualisation sous-développée. Le développement des contrats est généralement considéré comme l'indicateur de l'intégration croissante entre les différents agents économiques d'une filière. En conséquence, les enquêtes RuralStruc se sont attachées à recenser ces dispositifs contractuels. L'interprétation des résultats appelle cependant trois mises en garde. En premier lieu, les équipes du programme ont sélectionné des régions et des localités qui illustreraient différentes situations et des stades d'intégration diversifiés. On ne peut donc tirer aucune conclusion des différences observées dans le nombre de contrats entre les régions ou sous-régions étudiées. Ensuite, l'analyse de la contractualisation impose la collecte d'informations extrêmement précises : la définition des types de contrats est essentielle et, si les contrats formels sont habituellement des contrats écrits, les contrats informels correspondent à une multiplicité de configurations dans lesquelles la confiance entre l'acheteur et le vendeur est l'élément primordial. Les conditions d'enquête empêchaient une telle investigation. Enfin, pour se prononcer sur l'amplitude de la contractualisation, il faut analyser son degré tout au long de la filière, ce que la méthodologie du travail de terrain ne permettait pas.

Néanmoins, même avec ces limites, il est évident que la contractualisation au niveau des producteurs est faible dans toutes les régions sélectionnées. Seulement 539 des ménages enquêtés (7,4\% de l'échantillon) ont déclaré avoir conclu au moins un contrat (voir tableau 5.5) ${ }^{22}$.

Ce niveau de contractualisation modeste - surtout la quasi-absence de contrats formels - est une caractéristique importante, qui illustre la faible intensité des processus d'intégration dans les régions étudiées et le faible développement des filières de produits à haute valeur ajoutée, au sein desquelles les exigences relatives à la qualité des produits justifient des contrats. Cette situation n'est pas totalement surprenante, même si plusieurs régions « gagnantes » avaient été sélectionnées pour identifier des dynamiques de marché liées à ces produits à haute valeur ajoutée ou aux dynamiques agro-industrielles.

$\mathrm{Y}$ compris dans certaines régions où ces filières sont présentes, les contrats avec les agro-industriels sont quasiment inexistants, en particulier dans deux régions du Nicaragua (Terrabona et Muy Muy), où une poignée d'agriculteurs seulement est directement connectée aux filières intégrées des fruits et légumes (supermarchés nationaux comme Walmart/La Union-Pali ou La Colonia) et aux filières du lait (supermarchés et industriels tels que Parmalat ou Eskimo). Ces observations illustrent un constat important : il est fréquent que la contractualisation n'intervienne pas au niveau des producteurs, mais plutôt en aval, entre le grossiste ou la coopérative et l'entreprise de transformation ou la centrale d'achat.

De manière générale, l'enquête révèle trois types de dispositifs et de liens contractuels entre les différents agents économiques : (1) des contrats informels résultant de relations anciennes, principalement avec des grossistes ; (2) des contrats d'approvisionnement avec un industriel et (3) une intégration directe aux filières à forte valeur ajoutée.

Les contrats informels. L'expansion de la demande urbaine de produits frais est à l'origine du développement de filières structurées par des grossistes et alimentées 
Tableau 5.5 Importance des arrangements contractuels formels et informels dans les régions étudiées

\begin{tabular}{|c|c|c|c|c|c|}
\hline \multirow{2}{*}{ Pays } & \multirow{2}{*}{ Région } & \multicolumn{2}{|c|}{$\begin{array}{c}\text { Ménages } \\
\text { avec contrat }\end{array}$} & \multirow{2}{*}{ Type } & \multirow{2}{*}{ Type de filière et opérateurs } \\
\hline & & Nombre & $\%$ & & \\
\hline \multirow[t]{4}{*}{ Mali } & Tominian & 1 & 0,6 & & \\
\hline & Diéma & 0 & 0,0 & & \\
\hline & Koutiala & 0 & 0,0 & $d f$ & Société cotonnière (CMDT) \\
\hline & Macina & 16 & 10,4 & I & Rizeries \\
\hline \multirow[t]{6}{*}{ Sénégal } & Casamance & 11 & 4,6 & & \\
\hline & Mekhé 1 & 26 & 23,4 & I & Grossistes en manioc \\
\hline & Nioro & 1 & 0,4 & & \\
\hline & Haut Delta & 54 & 88,5 & $\mathrm{~F}$ & Usine de concentré de tomates (SOCAS) \\
\hline & Mekhé 2 & 33 & 29,2 & 1 & Grossistes en manioc \\
\hline & Bas Detla & 12 & 9,9 & 1 & Rizeries \\
\hline \multirow[t]{6}{*}{ Madagascar } & Antsirabe 2 & 16 & 5,3 & । & Collecteurs de légumes \\
\hline & Alaotra 1 & 2 & 0,5 & & \\
\hline & Morondava & 15 & 3,0 & 1 & \\
\hline & Itasy & 50 & 9,9 & $\mathrm{~F}$ & $\begin{array}{l}\text { Usine de conditionnement de haricots verts } \\
\text { (Lecofruit) et tabac }\end{array}$ \\
\hline & Antsirabe 1 & 46 & 22,3 & $\mathrm{~F} / \mathrm{l}$ & Laiteries (Tiko) et collecteurs de légumes \\
\hline & Alaotra 2 & 8 & 7,0 & 1 & Rizeries \\
\hline \multirow[t]{3}{*}{ Kenya } & Bungoma & 75 & 25,1 & $\mathrm{~F}$ & Sucreries \\
\hline & Nyando & 7 & 2,5 & $\mathrm{~F}$ & Sucreries \\
\hline & Nakuru North & 16 & 5,5 & $\mathrm{~F} / \mathrm{l}$ & Laiteries et conserveries de tomate \\
\hline \multirow[t]{3}{*}{ Maroc } & Chaouia & 1 & 0,4 & & \\
\hline & Saïss & 20 & 7,7 & $\mathrm{~F}$ & Laiteries \\
\hline & Souss & 1 & 0,4 & & \\
\hline \multirow[t]{5}{*}{ Nicaragua } & Muy Muy & 9 & 3,0 & I & Laiteries (Parmalat et Eskimo) \\
\hline & Terrabona & 4 & 1,4 & & \\
\hline & El Viejo & 13 & 4,5 & $\mathrm{~F} / \mathrm{l}$ & Transformateurs de sésame et sorgho \\
\hline & La Libertad & 20 & 6,9 & 1 & Collecteurs de lait \\
\hline & El Cua & 47 & 15,7 & $\mathrm{~F} / \mathrm{l}$ & Coopératives de caféiculteurs \\
\hline \multirow[t]{4}{*}{ Mexique } & Sierra S. M. & 0 & 0,0 & $d f$ & Organisations de producteurs de maïs \\
\hline & Tierras Bajas & 6 & 4,0 & F/df & Minoteries et organisations de producteurs de maïs \\
\hline & Tequis. & 29 & 8,0 & $\mathrm{~F}$ & Organisations de producteurs de maïs \\
\hline & & 539 & 7,4 & & \\
\hline
\end{tabular}


par les producteurs dans le cadre de contrats informels. Cette situation est particulièrement fréquente si la concurrence entre intermédiaires est vive et le produit périssable.

À titre d'exemple, les secteurs des fruits et légumes à Antsirabe sont intégrés sur la base d'accords informels entre les organisations de producteurs et les courtiers qui approvisionnent les grossistes urbains. Les producteurs qui bénéficient de ces accords sont généralement les plus gros et les mieux dotés en facteurs (les corrélations sont statistiquement significatives), ce qui leur permet de dégager des marges supplémentaires. Dans le bassin arachidier au Sénégal (principalement Mekhé 2), les producteurs de manioc ont conclu des accords informels avec des intermédiaires sur la base de transactions répétées et de la réputation des acheteurs. Ces contrats garantissent le flux des approvisionnements aux zones urbaines malgré la dispersion de la production.

De même, au Nicaragua, face à la demande urbaine croissante et au développement des supermarchés, des grossistes ont récemment élargi leur périmètre de collecte. Dans certains villages de La Libertad, les accords verbaux se sont multipliés pour répondre à cette demande. Ces accords apportent de nombreux avantages aux agriculteurs : ils sont en particulier assurés de vendre leur lait tous les jours au lieu de devoir écouler leur production sous forme de fromage fabriqué sur l'exploitation une fois par semaine; ils ont également des coûts de commercialisation plus faibles. En général, les ménages qui peuvent conclure ces accords informels sont ceux qui ont des surfaces importantes et donc de plus grands troupeaux qui leur permettent de produire plus de lait. En moyenne, ils disposent de 2,3 fois plus de surface et de 3 fois plus de têtes de bétail (les corrélations sont statistiquement significatives).

Dans la région du Sotavento, des contrats informels ont été passés entre des organisations de producteurs et des groupements d'entreprises afin de permettre aux premiers d'accéder aux transferts publics et de leur offrir une aide technique et des intrants en contrepartie de la commercialisation de leurs productions. Bien que les producteurs n'aient pas indiqué avoir conclu de contrats, leur simple affiliation à une organisation de producteurs signifie le plus souvent des contrats de fait.

Contrats d'approvisionnement avec des entreprises agroalimentaires. Ces contrats sont une pratique très ancienne, qui s'est initialement développée pour garantir les approvisionnements (et donc la rentabilité) des investissements industriels. Les régions étudiées en offrent plusieurs exemples, surtout dans le secteur du lait. À Madagascar, la privatisation du monopole parapublic n'a pas beaucoup modifié la configuration de la filière, qui est en grande partie contrôlée par Tiko, une entreprise à capitaux privés qui joue (ou qui jouait jusqu'à la récente crise politique) un rôle pivot dans l'industrie laitière malgache. Tiko collectait plus de $90 \%$ du lait commercialisé dans la principale région de production (Antsirabe) et transformait la majorité des produits laitiers du pays. Avec Tiko, les producteurs sous contrat livraient le lait à des centres de collecte, où ils devaient remplir des critères de qualité stipulés dans un contrat formel. En retour, l'entreprise agroalimentaire fournissait des intrants et faisait parfois des avances de trésorerie. 
Ce sont les producteurs avec les plus grands troupeaux qui sont les plus engagés dans ces stratégies d'intégration. Des systèmes similaires existent dans le Saïss et le Souss, au Maroc, et à Nakuru North au Kenya.

Des contrats formels d'approvisionnement de même type existent aussi avec les sucreries au Kenya (Bungoma) et avec l'industrie de la tomate dans le Haut Delta (Sénégal) et à Nakuru. Cette configuration - avec une entreprise agroalimentaire et de nombreux fournisseurs qui sont de petits producteurs - peut correspondre à des situations de monopsone. Dans ce cas, il n'y a pas de contrat mais une contractualisation tacite résultant de l'unique option offerte aux producteurs : vendre à l'entreprise. Le coton est un bon exemple : $75 \%$ des exploitations familiales de la région de Koutiala cultivent du coton ; même si rien n'est écrit, elles ont un contrat de fait avec la CMDT. Le secteur est verticalement intégré, avec la fourniture d'intrants par l'intermédiaire des organisations de producteurs, un système de crédit garanti par les ventes de coton, des services de vulgarisation agricole et de soutien technique, et des prix fixés en début de campagne, qui sont en partie négociés ${ }^{23}$.

Les contrats liés à des exportations à haute valeur ajoutée. Les contrats conclus avec des entreprises d'exportation de produits à haute valeur ajoutée sont ceux qui sont généralement évoqués dans la littérature. Cependant, l'enquête RuralStruc n'en a trouvé que deux exemples. Le premier est Lecofruit à Ifanja, Itasy (Madagascar), un exemple souvent cité, qui concerne la culture de haricots verts destinés à l'exportation (voir encadré 5.7). L'autre concerne la région du café d'El Cuá, au Nicaragua. Dans cette région, une filière de café biologique a été développée, principalement promue par COMANUR-RL (Cooperativa Multisectorial Alfonso Núñez Rodriguez), qui vend à la fois des cafés conventionnels et biologiques. Les agriculteurs produisent du café biologique conformément à un strict cahier des charges pour la coopérative, à un prix prédéterminé, et celle-ci apporte en retour une assistance technique à ses membres, comprenant l'accès à du conseil de gestion à la parcelle, à du matériel végétal (nouvelles variétés de café), des intrants agricoles (engrais et autres produits phytosanitaires) et à des matériels ou des infrastructures.

Compte tenu du manque d'informations et des rares cas recueillis par les enquêtes, il est difficile de tirer des conclusions sur les conséquences de la contractualisation sur les revenus des ménages. Se pose en outre un problème de causalité inverse : en général, le faible niveau de production des ménages est un des principaux obstacles à la conclusion de contrats, car les systèmes d'approvisionnement et les entreprises agroalimentaires préfèrent travailler avec de gros producteurs (fournisseurs) pour réduire leurs coûts de transaction. De ce fait - à l'exception des producteurs de haricots verts à Madagascar, dont la taille des parcelles est limitée par la société avec lesquels ils passent contrat - ce sont généralement les ménages les mieux dotés en facteurs qui concluent des contrats ${ }^{24}$.

Néanmoins, les études de cas du programme RuralStruc permettent de penser que, sauf exception, les avantages tirés de la contractualisation restent limités. Les enquêtes montrent en effet que les écarts de revenu entre les ménages 


\section{GNCADRÉ 5.7}

\section{Lecofruit : les petits producteurs malgaches vendent sur les marchés européens}

Lecofruit (Légumes Condiments et Fruits de Madagascar SA) a été fondée en 1989 à Madagascar, au moment où l'État malgache a créé des zones franches et les a promues par des exonérations d'impôts et d'autres avantages fiscaux. Initialement, Lecofruit produisait des légumes au vinaigre en partenariat avec une centaine d'agriculteurs. Pour développer ses marchés à l'exportation, la société s'est associée à la société française Segma Maille, qui lui assurait des débouchés réguliers pour ses produits en Europe. Lecofruit a commencé à diversifier sa production avec les haricots verts, les pois mangetout, les concombres, les asperges et les légumes primeurs pour les exporter sur le marché européen. Aujourd'hui, elle concentre sa production sur les haricots verts extra-fins : la société a exporté 3000 tonnes de produits lors de la campagne 2004-2005, dont $70 \%$ de haricots verts. Environ $90 \%$ ont été transformés et mis en conserve dans son usine à Antananarivo, puis transportés en Europe par bateau. Les $10 \%$ restants étaient des haricots verts et des pois mangetout frais envoyés par avion.

En 2007-2008, la société a étendu ses activités pour travailler avec 10000 agriculteurs sous contrat dans la production de haricots verts. Les producteurs, situés sur les hauts plateaux de Madagascar, ont une longue tradition de production de légumes et de fruits. Pour optimiser les coûts de transport jusqu'à l'unité de transformation d'Antananarivo, la société cible les zones de cultures connectées aux grands axes routiers.

Les agriculteurs cultivent leur propre parcelle, ce qui permet d'éviter les problèmes fonciers récurrents de la zone des hauts plateaux. Les contrats de production sont standardisés et individuels, même si les producteurs doivent être affiliés à une organisation de producteurs. Un contrat est limité à une superficie d'environ $1000 \mathrm{~m}^{2}$ afin que les producteurs puissent respecter toutes les étapes de la production jusqu'à la récolte, car la production est très gourmande en main-d'œuvre. Les autres engagements concernent des recommandations techniques particulières (préparation du compost, labour, semis, etc.) et la nécessité d'une récolte quotidienne pour répondre à la catégorie « extra-fins ».

Les producteurs reçoivent des avances en liquide et des semences gratuites; le coût des engrais et des pesticides est déduit du paiement final, qui intervient à la livraison des haricots. Lecofruit fournit un " paquet » groupé de semences, d'engrais minéraux et de pesticides pour garantir le respect des seuils limites de résidus dans les produits agricoles exportés vers I'Union européenne. Certaines conditions sanitaires sont également stipulées dans les contrats, comme l'obligation de se laver les mains avec du savon non parfumé avant de cueillir les haricots. Enfin, les producteurs doivent vendre exclusivement à Lecofruit. Le paiement est périodique. Le prix est fixé à l'avance par la société et demeure inchangé tout au long de la saison - 630 ariary $/ \mathrm{kg}$ pour les haricots verts en 2007-2008 (0,83 US\$ PPA).

Bien que le rapport de force soit favorable à l'agro-industriel, le nombre d'agriculteurs sous contrat avec Lecofruit n'a jamais diminué, ce qui indique l'attrait des conditions offertes. Les agriculteurs peuvent notamment générer des revenus et des liquidités pour financer leurs autres activités agricoles ou couvrir leurs besoins.

Source : RS 2 Madagascar, 84-85. 
avec et sans contrat sont le plus souvent minimes. Le montant moyen maximum tiré de la production de haricots verts sous contrat à Itasy, Madagascar, est très faible : 43 US\$ par ménage et par an. De même, les producteurs de tomates sous contrat avec la SOCAS dans le Haut Delta au Sénégal ne sont pas beaucoup plus riches que les autres ménages enquêtés au Sénégal. Dans de nombreuses régions étudiées, les principaux avantages de la contractualisation concernent d'abord l'accès aux paquets techniques, au crédit et à une garantie de commercialisation.

\section{Spécialisation agricole et transformation rurale}

La spécialisation dans l'agriculture est l'une des trois voies de sortie de la pauvreté rurale. Les ménages enquêtés par le programme RuralStruc y sont très largement engagés - les revenus de l'exploitation agricole représentent une part importante du revenu total - mais ils demeurent globalement pauvres. Ce constat contradictoire, mis en évidence au chapitre 3 , justifiait l'examen attentif des revenus ruraux en général et des caractéristiques des revenus des exploitations agricoles en particulier.

La quasi-totalité des ménages d'ASS pratiquent l'agriculture mais la plupart sont aussi diversifiés et, à ce titre, ils ont également d'autres activités qui s'inscrivent dans le cadre de leurs stratégies d'adaptation - une situation qui explique la rareté d'une spécialisation totale dans les seules activités de l'exploitation agricole. En revanche, cette spécialisation peut être observée dans plusieurs régions du Nicaragua et du Maroc, où des filières plus robustes peuvent offrir des garanties de rentabilité suffisantes.

Dans l'ensemble, les revenus d'exploitation se caractérisent par des niveaux élevés d'autoconsommation, l'importance des cultures vivrières et des profils hétérogènes de diversification des produits qui découlent des opportunités propres à chaque région. Ce tableau est très éloigné de la «nouvelle agriculture » tant évoquée dans la littérature et ne reflète pas les processus d'intégration croissante, les nouveaux acteurs et les nouvelles règles qu'elle met en avant.

Dans les régions étudiées, l'importance persistante de l'autoconsommation tient à la fois à des effets d'offre et de demande. Les effets d'offre correspondent aux stratégies de gestion des risques déployées par les ménages pour garder le contrôle de leur approvisionnement alimentaire - une réaction directe à des marchés incomplets et imparfaits. Les effets de demande expriment le manque d'accès et d'intégration aux marchés qui se traduit par une faible demande de produits de la part des opérateurs de l'aval. Des infrastructures insuffisantes peuvent être un obstacle considérable qui peut être renforcé par des systèmes de commercialisation peu performants dans lesquels les intermédiaires n'ont pas d'incitation à aller collecter de faibles quantités de produits - une conséquence de la productivité modeste des cultures vivrières de base - et à faible valeur marchande, surtout lorsqu'il s'agit de zones peu denses où les coûts de ramassage sont élevés.

La plupart des agents de collecte qui travaillent dans les zones étudiées par le programme RuralStruc s'appuient sur des relations contractuelles informelles 
pour accéder à la production des exploitants agricoles, tandis que les entreprises agroalimentaires recourent aux contrats classiques. Cette contractualisation est cependant très rare, même parmi les exploitations solidement intégrées aux marchés par des relations continues avec les grossistes et d'autres acheteurs. En outre, elle intervient rarement au niveau des producteurs mais souvent en aval, entre le grossiste ou le groupement de collecte et l'entreprise de transformation ou le service des achats des distributeurs.

La part de l'autoconsommation diminue lorsque la richesse des régions et des ménages augmente, et les régions étudiées en Afrique subsaharienne sont moins avancées dans cette progression. Les ménages les plus riches ont des productions peu diversifiées, cette situation tenant principalement à l'environnement de marché qui offre moins d'opportunités de participation à de nouvelles filières. Il explique aussi en conséquence l'importance persistante des cultures vivrières dans la production, même lorsque les ménages s'éloignent de l'autoconsommation et même lorsque leur revenu s'améliore. Les cultures vivrières ne sont pas l'apanage des agriculteurs pauvres; la diversification de l'exploitation (son ampleur et le type de produits concernés) dépend souvent de dynamiques qui concernent une région dans son ensemble. Il en résulte que généralement tous les ménages peuvent participer à de nouvelles filières, leur niveau de participation étant déterminé par leurs actifs (facteurs de production, capital humain et social). Les fameuses filières à haute valeur ajoutée tournées vers l'exportation sont rares. Elles ne concernent qu'un petit nombre des agriculteurs enquêtés et leur expansion dépend des opérateurs en place (industriels, exportateurs) et de leur capacité de développement, notamment par des contrats avec des acheteurs étrangers.

Malgré les multiples mutations intervenues dans les secteurs agricoles de nombreux pays en développement au cours des dernières décennies, les anciennes configurations agricoles persistent. La spécialisation totale des exploitations est rare et la tendance d'ensemble est à la diversification des productions - un moyen de saisir les opportunités et de partager les risques dans des environnements économiques incertains.

\section{Notes}

1. L'objectif n'est pas ici de présenter une revue complète de l'abondante littérature sur la restructuration agroalimentaire mondiale mais de donner un bref aperçu des principaux développements des trente dernières années.

2. Quel que soit le régime politique, l'agriculture a toujours été une « affaire d'État» (Coulomb et al. 1990). En dernier ressort, c'est bien le secteur qui répond aux besoins élémentaires de la population... et des électeurs.

3. Pour une "généalogie » des food regimes, voir Friedmann et McMichael (1989) et McMichael (2009). McMichael suggère la consolidation progressive d'un nouvel « ordre alimentaire des grandes firmes mondiales » ("corporate food regime»).

4. Ces tableaux ne donnent que quelques exemples alors que la plupart des pays comptaient des dizaines d'organismes parapublics. Voir les rapports nationaux du programme RuralStruc pour plus d'informations. 
5. Le commerce des produits agricoles (en particulier les exportations de produits carnés) s'est également développé entre les pays de l'OCDE et de nombreux pays à revenu faible et intermédiaire; il est souvent favorisé par des exigences sanitaires et de sécurité alimentaire moins strictes.

6. Un exemple de cette tendance est l'émergence de la Fédération internationale des mouvements d'agriculture biologique (International Federation of Organic Agriculture Movements, IFOAM), qui fonde la certification sur des critères de santé, d'écologie, d'équité et le principe de précaution.

7. Voir World Bank (2007). Webber et Labaste (2010) donnent une bonne description des applications possibles de ces nouvelles approches.

8. Comme indiqué au chapitre 1 , les pays et régions sélectionnés ne comprennent pas de foyers anciens de cultures tropicales d'exportation dans lesquels les liens anciens avec les marchés ont profondément modifié les économies rurales. Cependant, plusieurs situations étudiées sont concernées par ces produits d'exportation et par des produits à haute valeur ajoutée. Le terme " traditionnel » renvoie ici aux cultures qui ne sont pas concernées par les nouvelles filières intégrées.

9. Il est difficile d'estimer les revenus de la cueillette car elle porte sur de faibles quantités de produits cueillis tout au long de l'année et souvent autoconsommés. Cependant, les fruits, les animaux et poissons sauvages tiennent souvent une place essentielle dans la sécurité alimentaire des ménages ruraux.

10. C'est le cas de la pomme de terre, un produit horticole autoconsommé qui peut être considéré comme une denrée alimentaire de base sur les hautes terres de Madagascar - l'une des deux régions étudiées, avec le Saïss au Maroc, où sa culture est développée. C'est aussi le cas de l'arachide, l'exportation traditionnelle du Sénégal, considérée comme une exportation bien qu'elle soit de plus en plus consommée localement par suite de l'évolution défavorable de la filière. La canne à sucre est un produit d'exportation traditionnel, mais au Kenya la production est principalement vendue sur le marché national et ne suffit d'ailleurs pas à répondre à la demande locale.

11. Dans ce chapitre consacré à la production et à la commercialisation des exploitations agricoles, les résultats des enquêtes sont présentés en utilisant le produit brut agricole (absolu et relatif) par ménage (c'est-à-dire la valeur totale des ventes et de l'autoconsommation des cultures et des produits d'élevage) plutôt que le revenu. Ce choix résulte des contraintes liées à la méthodologie des enquêtes qui ne permettait pas la répartition des coûts par type de production - les coûts totaux ont été appliqués respectivement au produit brut de la culture et au produit brut de l'élevage pour calculer les revenus de la culture et de l'élevage, puis le revenu total de l'exploitation.

12. Bien qu'il existe des systèmes traditionnels de culture irriguée à Madagascar, le développement de l'irrigation a surtout bénéficié d'infrastructures publiques à travers des projets d'irrigation, notamment au Sénégal et au Mali. La culture irriguée du maïs au Mexique est principalement pratiquée par les grandes exploitations commerciales. La situation de la zone des Tierras Bajas dans le Sotavento, avec ses plaines naturellement inondables, est unique en son genre.

13. Après une grave sécheresse, la production céréalière marocaine a chuté de $73 \%$ en 2007 ; la plupart des régions de production ont été touchées, en particulier la Chaouia (RS 2 Maroc, 145) et les ventes d'animaux ont nettement augmenté en conséquence, tant en valeur absolue que relative.

14. Un petit (et désormais célèbre, car fréquemment cité dans la littérature) marché de production de haricots verts destinés à l'exportation s'est développé à Itasy, étroitement 
lié à la présence de Lecofruit, une entreprise agroalimentaire tournée vers l'exportation (voir encadré 5.7).

15. L'autoconsommation comprend les cadeaux aux familles et aux réseaux sociaux et religieux. Elle comprend aussi les réserves alimentaires (voir annexe 1 de l'appendice en ligne : http://www.worldbank.org/afr/ruralstruc).

16. Le cas de Tominian est remarquable : $30 \%$ à $40 \%$ des ménages n'ont aucun lien avec les marchés alimentaires, ni comme vendeurs ni comme acheteurs.

17. Au Mali, les différences entre les quintiles sont très faibles. Cela peut s'expliquer par le faible niveau des revenus, même pour les ménages les plus riches, et par l'importance accordée aux réserves de céréales, car un stock important est un signe d'aisance. Par ailleurs, la campagne 2004-2005 a été très mauvaise et de nombreux ménages reconstituaient leurs stocks lorsque l'enquête a été réalisée en 2007.

18. Les liens des producteurs ruraux avec les marchés agricoles sont souvent étudiés; pourtant, les liens des consommateurs ruraux avec les marchés de biens et de services peuvent être eux aussi un obstacle. Lorsqu'il n'y a pas grand-chose ou rien à acheter, il n'y a pas d'incitation à vendre ou à développer la production. Ce «côté pile » des marchés est généralement absent du débat politique.

19. Contrairement au courtier, le grossiste achète le produit.

20. Cette catégorie comprend les entreprises agroalimentaires qui transforment les produits agricoles bruts en produits semi-transformés (par exemple le coton graine en fibre de coton) ou transformés (par exemple les tomates fraîches en concentré de tomates ou en tomates en conserve). Elle inclut aussi les entreprises qui procèdent au nettoyage, calibrage et conditionnement des produits comme les fruits et légumes, notamment pour l'exportation.

21. Les ventes aux entreprises agroalimentaires sont parfois effectuées par l'intermédiaire d'organisations de producteurs ou de « coopératives » qui sont en fait des créations de l'agro-industrie, qui est leur seul acheteur. C'est le cas avec la SOCAS au Sénégal et avec la CMDT au Mali.

22. Les dispositifs contractuels indiqués au tableau 5.5 comprennent les contrats écrits formels et les contrats informels que le producteur juge effectifs.

23. Pendant longtemps, les monopsones publics et semi-publics tels que la CMDT ont été tenus d'acheter toute la production des producteurs de coton. La CMDT a été privatisée en 2010 après des années de négociation ; mais la nouvelle configuration de la filière fondée sur des monopsones régionaux issus de la vente par lots de la CMDT ne changera pas fondamentalement les relations producteurs-acheteurs.

24. Cela semble être le cas en particulier pour les surfaces cultivables disponibles. Cependant, le petit nombre de ménages ayant des contrats formels ne permet de tirer aucune conclusion. À Antsirabe 1, où le nombre de contrats de l'échantillon est suffisant, le test de Student est significatif. 



\section{Chapitre 6}

\section{Des profils régionaux de transformation rurale aux recommandations politiques}

Les trois chapitres précédents ont présenté une analyse détaillée du niveau des revenus et des caractéristiques des activités rurales dans les régions étudiées : celles des exploitations agricoles et l'ensemble des autres activités en dehors de l'agriculture. L'objectif était de répondre aux questions sous-jacentes des hypothèses du programme. Comment les exploitations agricoles s'adaptent-elles à un environnement en mutation ? Se spécialisent-elles dans l'agriculture lorsqu'elles s'intègrent davantage aux marchés (H1) ? Comment les ménages ruraux combinent-ils les différentes activités pour diversifier leurs sources de revenus (H2) ? Une question importante demeure néanmoins à explorer : la relation entre les différents profils de spécialisation et de diversification qui ont été observés et le niveau du revenu total. Que dit cette relation sur la viabilité de différentes voies de sortie de la pauvreté, sur le processus global de transformation rurale et sur les risques d'impasses de transition $(\mathrm{H} 3)$ ?

Le chapitre 6 est consacré à l'examen de cette relation diversification-spécialisation. Après un retour sur le revenu total, et plus particulièrement sur ses déterminants qui n'avaient pas été analysés au chapitre 3, il détaille les profils régionaux de diversification des revenus et la relation entre le niveau du revenu et sa structure. Cette étape conduit à investir les dynamiques de spécialisation et de diversification régionales. Le chapitre propose ensuite une typologie des ménages fondée sur leur marge de manœuvre en fonction de leur niveau de revenu et en dégage des conclusions quant à l'importance des réalités rurales observées pour l'élaboration des politiques publiques. Il conclut par des recommandations susceptibles de faciliter la transformation rurale dans les contextes étudiés.

\section{Profils régionaux de diversification - spécialisation des revenus}

L'analyse quantitative des résultats d'enquêtes à partir de techniques usuelles de régression a tout d'abord permis de mieux comprendre les déterminants des revenus ruraux et de mettre en lumière leur forte hétérogénéité du point de vue des combinaisons d'actifs et des conditions du contexte régional. En revanche, 
l'analyse plus poussée du profil structurel des revenus au niveau de chaque région a ensuite permis de déceler d'importantes régularités : il existe en particulier une relation étroite entre le revenu, la spécialisation et la diversification, qui a trait aux dynamiques propres au changement structurel. Une meilleure compréhension de ces dynamiques permet de mettre en évidence des trappes à pauvreté dans la plupart des régions d'Afrique subsaharienne.

\section{Comprendre le niveau des revenus dans chaque région}

Le niveau de revenu des membres d'un ménage dépend d'un ensemble de facteurs comprenant le type d'activités économiques pratiquées et leur rentabilité, les facteurs dont il dispose, sa taille, sa structure démographique et son environnement économique. Les chapitres 4 et 5 ont examiné les activités des ménages et constaté une forte hétérogénéité entre ménages et régions, sans jamais dégager d'indication sur l'existence d'un type d'activité qui s'imposerait comme la meilleure option universelle. En conséquence, l'analyse du niveau des revenus porte non pas sur l'utilité respective des différentes activités mais plutôt sur les caractéristiques qui permettent à un ménage de tirer parti des opportunités régionales.

L'analyse s'articule autour de quatre types de déterminants principaux : les caractéristiques du ménage et son capital humain ; les facteurs explicatifs de la productivité de l'exploitation ; les conditions d'environnement et d'accès au marché; ; et les activités rurales non agricoles. Les analyses de régression conduites par le programme, la justification des variables utilisées et un résumé des principaux résultats sont présentés ci-après ${ }^{1}$.

Les analyses statistiques ont été principalement effectuées au niveau régional et portent sur les 30 régions étudiées. Elles ne portent que sur les ménages ayant une exploitation agricole ( $93 \%$ de l'échantillon), car la prise en compte des autres ménages aurait diminué la puissance explicative des variables relatives aux caractéristiques de l'exploitation agricole. Dans chaque régression, la variable dépendante est le logarithme du revenu du ménage par équivalent adulte (EqA) ${ }^{2}$.

Des calculs de régression ont également été réalisés au niveau agrégé, en regroupant tous les ménages enquêtés dans chaque région d'un même pays. Les régressions ont alors été conduites globalement sur l'ensemble des ménages sans tenir compte de leur appartenance régionale.

Cette approche a permis de capter l'impact sur le niveau de revenu de dotations en facteurs ou de conditions d'environnement qui varient significativement selon les régions (par exemple, l'accès à l'irrigation, comme au Mali, où Macina bénéficie d'aménagements que les autres régions n'ont pas), mais pas à l'intérieur d'une même région (par exemple, dans une région donnée, tous les ménages sont confrontés aux mêmes difficultés de transport).

Les tableaux 6.1 et 6.2 donnent un aperçu des résultats obtenus au niveau régional et au niveau agrégé et présentent les variables les plus significatives. Cependant, il convient de noter que de nombreux résultats du tableau 6.1 peuvent donner lieu à des interprétations multiples. En effet, les régressions ont une grande puissance explicative dans certaines régions, alors que dans d'autres 
elles informent peu sur les déterminants des différences de revenus. De manière générale, les tests donnent de meilleurs résultats dans les régions où le revenu de l'exploitation agricole représente une part importante du revenu total. Ainsi, par exemple, l'importance de l'auto-emploi au Sénégal explique probablement les résultats peu significatifs de la régression qui comprend de nombreuses variables relatives à l'exploitation agricole. Les principaux résultats par catégorie de variable sont commentés ci-après et l'indice de diversification est analysé dans la section suivante.

Démographie et capital humain. Le nombre total de membres du ménage (personnes présentes) est significatif dans 18 des 30 régions étudiées : c'est donc une des variables les plus robustes de la régression. Dans presque tous les cas, cette variable a un coefficient négatif, ce qui veut dire que dans la plupart des ménages, un membre supplémentaire « coûte » plus cher à entretenir que ce qu'il rapporte en revenus additionnels. Cette situation est rencontrée partout sauf à Koutiala, au Mali, où la relation entre personnes présentes et revenu est positive. Ce résultat peut indiquer que les familles de Koutiala manquent de main-d'œuvre, une hypothèse raisonnable eu égard aux exigences en travail de la culture du coton.

Étant donné la fréquence de l'excédent de main-d'œuvre au sein des ménages (analysé au chapitre 4 et illustré par les résultats des régressions), il est surprenant que les migrations ne soient des déterminants significatifs du revenu que dans cinq régions. Ce constat a deux causes possibles : tout d'abord, comme l'a montré le chapitre 4 , la rentabilité de la migration dépend étroitement de la destination du migrant, laquelle varie fortement d'un pays à l'autre mais moins à l'intérieur d'un pays, ce qui signifie que les régressions au niveau régional ou au niveau agrégé peuvent difficilement capter un effet. Ensuite, les migrations observées sont moins courantes que prévu ${ }^{3}$, les migrants à long terme n'étant présents que dans $20 \%$ de l'échantillon des ménages agricoles. Quant aux migrants à court terme, ils ne concernent que $10 \%$ de l'échantillon. Ces résultats de l'analyse statistique illustrent la discussion du chapitre 4 sur les importants obstacles à la migration qui privent de nombreux ménages de cette option.

Les conclusions tirées des variables relatives à l'éducation sont moins tranchées. Le niveau d'instruction du chef de famille est moins fréquemment associé au revenu au Sénégal, au Mali, au Maroc et au Mexique. En Afrique du Nord et de l'Ouest, cette situation s'explique par la faiblesse générale du niveau d'instruction (voir chapitre 4). Au Mali par exemple, $84 \%$ des chefs de famille interrogés n'ont pas suivi d'instruction formelle, bien que cette situation évolue. Les enquêtes montrent que la personne la plus instruite du ménage est rarement le chef de famille; les enfants sont plus instruits que leurs parents, ce qui implique un bénéfice de l'éducation à moyen terme ${ }^{4}$.

Facteurs relatifs à la productivité. Les résultats obtenus quant à l'importance de la dotation en facteurs des ménages appellent trois constats importants. Le premier est la persistance de l'importance incontestée de la terre, plus précisément 
Tableau 6.1 Résultats des régressions au niveau régional

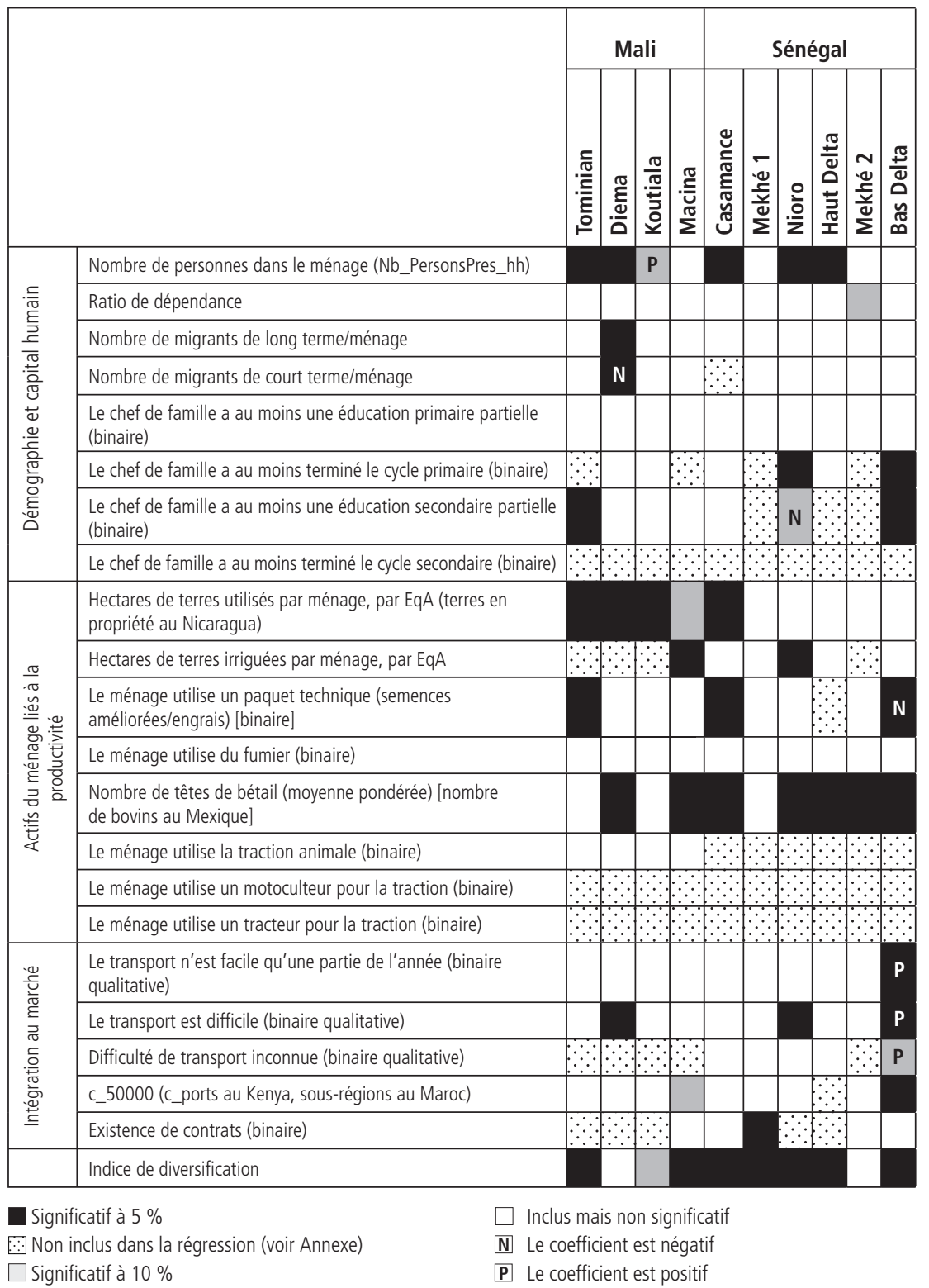

de la superficie dont dispose l'agriculteur. Elle est significative dans 22 régions sur 30, ce qui en fait la variable la plus forte de l'enquête. Elle a le coefficient le plus élevé dans sept régions et arrive en deuxième position dans cinq autres régions. Une conclusion partielle liée à ce résultat est que, malgré tous les 


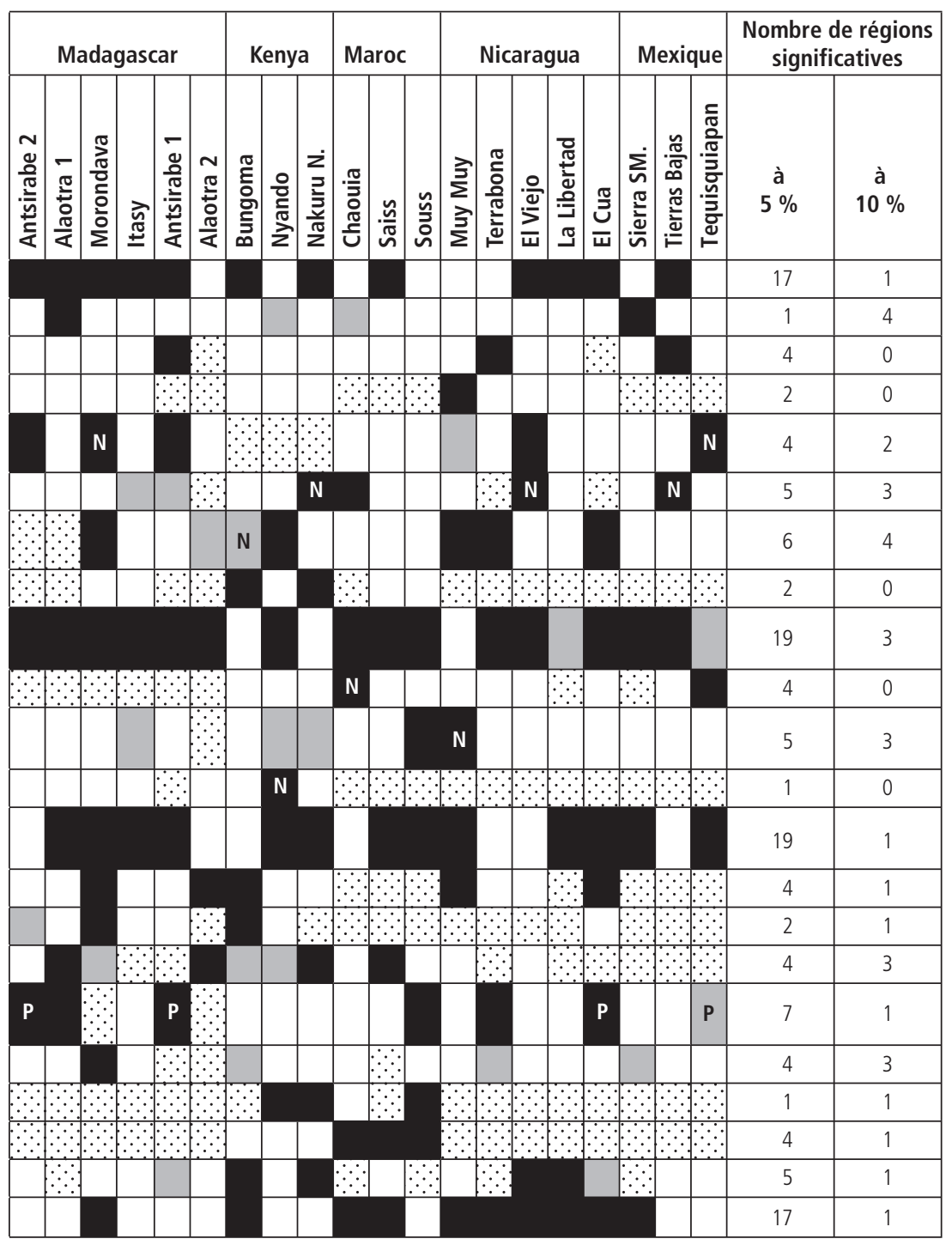

efforts consentis ces dernières décennies par la communauté du développement pour améliorer le niveau de production à la parcelle, le meilleur moyen pour un agriculteur d'améliorer son revenu reste encore d'augmenter ses surfaces. Ce résultat confirme une importante leçon du chapitre 5 , à savoir que les processus 


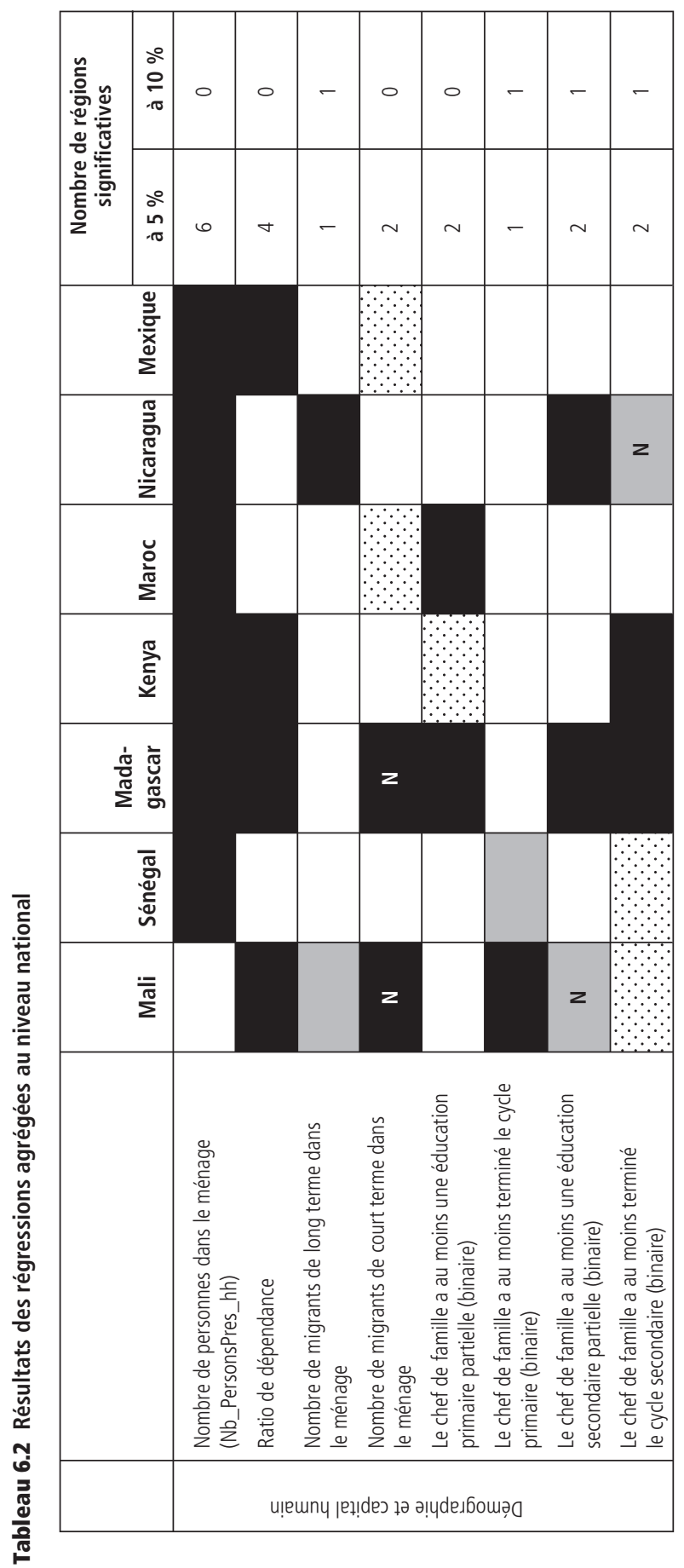




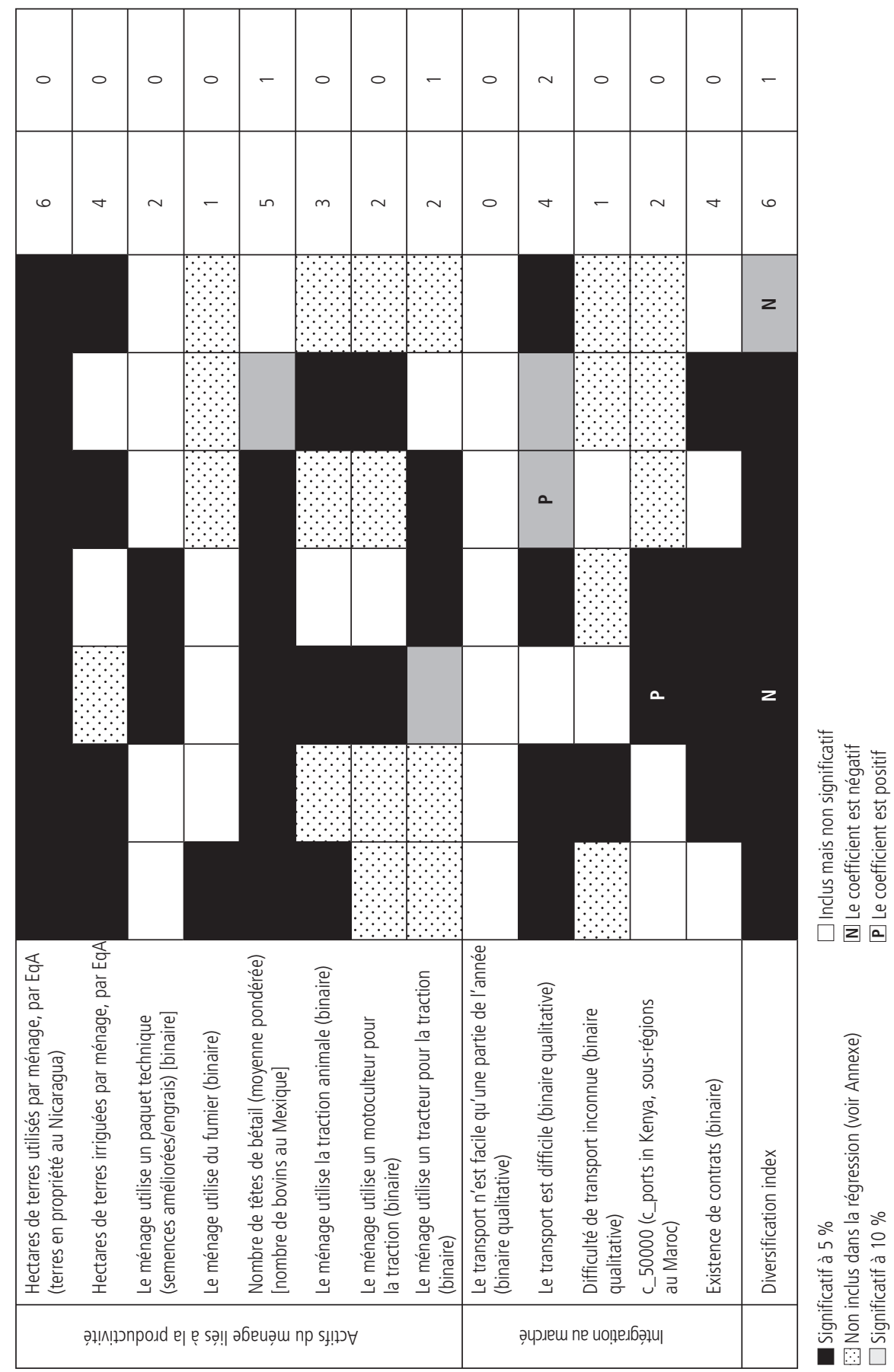


de différenciation agricole qui étaient anticipés d'une intégration économique accrue ne se sont pas encore matérialisés. Le deuxième constat conforte le premier : l'effet de la variable " paquet technique » est globalement négligeable 5 . Celle-ci n'est significative que dans huit régions, et dans deux d'entre elles a un effet négatif (ce qui veut dire que les agriculteurs qui ont le paquet technique s'en sortiraient moins bien que les autres). Peut-être plus surprenant encore, elle n'est significative au niveau agrégé que dans deux des sept pays du programme (Madagascar et Kenya). Le troisième constat enfin est que la taille du cheptel dont l'agriculteur est propriétaire est généralement et significativement associée au revenu. Cependant, comme le bétail peut être tout à la fois un produit, un actif productif, une épargne ou un attribut social, l'interprétation de cette variable cheptel est délicate.

Variables d'accès au marché. Même si l'insertion et l'intégration au marché sont difficiles à mesurer, un important constat des calculs de régression est que l'intégration au marché ne se traduit pas nécessairement par des gains de revenu : cela dépend du contexte. La régression suggère cette conclusion en examinant les liens avec les marchés selon deux axes : (1) la distance aux marchés (en prenant en compte la qualité des transports) et (2) le niveau d'intégration aux filières à travers le nombre de ménages qui ont des contrats (ce qui constitue un contrat est examiné au chapitre 5).

La variable temps de transport jusqu'aux marchés (c_50 000, voir tableau 6.1) n'a pas réellement permis de dégager des tendances claires. Tout comme la variable portant sur la qualité du transport qui est significative dans plusieurs régions mais avec des causalités inverses : dans six régions, réparties dans les différents pays du programme, la relation s'effectue dans le sens anticipé (à savoir une qualité de transport médiocre associée à des revenus plus faibles), mais dans cinq autres la relation est inverse ${ }^{6}$.

En ce qui concerne les contrats, la régression montre qu'ils ne sont significativement corrélés au revenu que dans quelques cas, notamment au Kenya et au Nicaragua. Mais cela n'a aucun lien avec la fréquence des contrats puisque des agriculteurs travaillent sous contrat dans tous les pays, et le Kenya et le Nicaragua ne sont pas particulièrement bien dotés. La différence réside plutôt dans la classe de revenu dans laquelle se concentrent les contrats. Les effets sont parfois inverses. Dans la région sénégalaise du Haut Delta par exemple, où plus de $90 \%$ des agriculteurs travaillent sous contrat avec la SOCAS, le transformateur de tomates local, les rares ménages qui n'ont pas de contrat sont en fait plus riches. Car ceux qui ont des contrats sont fortement tributaires de l'industriel : le contrat leur offre un accès préférentiel aux intrants agricoles qu'ils peuvent aussi employer pour d'autres cultures, mais en général cela ne suffit pas à réduire la pauvreté et les rend plus dépendants de l'usine. C'est le contexte régional qui détermine si un contrat permet aux agriculteurs d'accroître leurs revenus ou les empêche de saisir des opportunités plus lucratives.

Principales conclusions. Les résultats obtenus à ce stade des analyses de régression peuvent se résumer en deux points. Le premier est la persistance de 
tendances anciennes en matière de d'accumulation. Dans les régions où l'agriculture joue un rôle prépondérant, le niveau de revenu reste largement déterminé comme par le passé dans les différentes régions du monde : l'accès à la terre et l'expansion des terres cultivées sont les meilleurs moyens d'améliorer les revenus agricoles. De plus, en fonction des opportunités économiques en termes de diversification et des contraintes locales (niveau de dotation en ressources naturelles et d'accès aux ressources), les dynamiques démographiques peuvent jouer un rôle décisif. Souvent, lorsque la taille du ménage augmente, le revenu par tête diminue.

Le second résultat indique cependant que des changements sont à l'œuvre, mais de façon sporadique et sans suivre de schéma régulier. Les ménages réagissent à leur environnement du mieux qu'ils peuvent, en fonction de leur dotation en facteurs, pour accroître leur revenu. Les conditions d'environnement et les dotations en actifs étant très différentes d'une région à l'autre, les stratégies des ménages sont elles aussi très diverses. L'efficacité de stratégies spécifiques en termes de génération de revenus est tout aussi variable d'une région à l'autre, comme l'attestent clairement les résultats de l'analyse statistique. L'éducation est significativement corrélée aux revenus dans certaines régions et pas dans d'autres, sans sembler suivre une configuration particulière. Il en va de même de la force de traction employée et de la qualité des transports.

Cette hétérogénéité est bien illustrée au tableau 6.3, qui présente les trois variables les plus étroitement associées au revenu dans chaque région. La variable de premier rang est celle dont le coefficient est le plus élevé (en valeur absolue) et significative à au moins $5 \%$. Si moins de trois variables sont significatives à $5 \%$, celle dont le coefficient est le plus élevé à $10 \%$ est retenue.

Cette représentation synthétique est sans équivoque : la variable « hectares de terres utilisés » est le principal déterminant du revenu des ménages dans la moitié des régions étudiées par le programme. Ce résultat est flagrant pour les régions les plus pauvres (le cas de Madagascar est clair) mais également significatif au Maroc et au Mexique. À part cette variable de surface disponible, aucune autre ne s'impose comme déterminant majeur du revenu dans plus de six régions, et aucune configuration tangible ne s'impose.

\section{Une analyse plus détaillée des caractéristiques régionales}

Une première analyse de la structure consolidée du revenu des ménages par quintiles et par région permet d'améliorer la compréhension des profils régionaux de diversification. Un indice de diversification, utilisé pour l'analyse statistique, est ensuite proposé pour explorer les liens entre revenu et diversification.

Vue d'ensemble. La figure 6.1 présente un tableau d'ensemble des sources de revenu des ménages. Elle s'appuie sur les résultats discutés aux chapitres 4 et 5 et expose les structures de revenus par quintile et région ${ }^{7}$. Il s'agit de la première étape pour comprendre les tendances de diversification. Les graphiques indiquent les structures régionales du revenu par quintile en utilisant le revenu de l'exploitation (on-farm) d'une part, et les six autres catégories de revenus 
Tableau 6.3 Variables les plus étroitement associées au revenu, par région

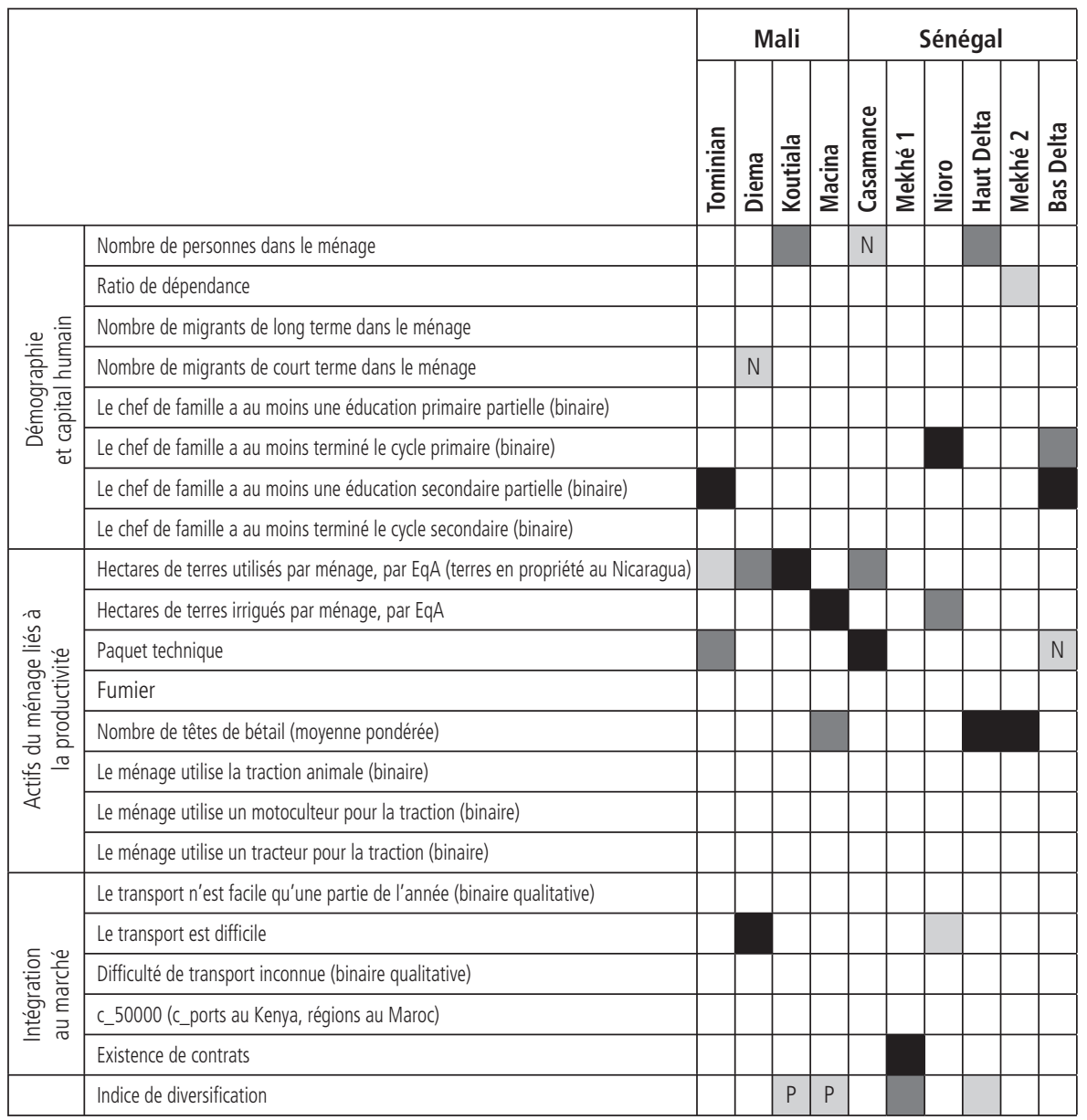

Variable la plus significative

$2^{\mathrm{e}}$ variable la plus significative

$\square 3^{e}$ variable la plus significative
$\mathrm{N}$ Le coefficient est négatif

P Le coefficient est positif

(off-farm) analysées au chapitre 4 d'autre part : les salaires agricoles, les salaires non agricoles, les activités indépendantes (auto-emploi), les transferts publics, les transferts privés et les rentes.

Ce tableau d'ensemble confirme la place privilégiée des revenus de l'exploitation agricole dans la structure des revenus, mais il fait également apparaître des différences entre les régions. La part du revenu de l'exploitation est élevée pour la plupart des quintiles au Mali, à Madagascar, au Nicaragua, en Casamance au Sénégal, et dans le Saïss au Maroc. Dans plusieurs régions, l'exploitation agricole est la principale source de revenu des ménages les plus riches. Les autres revenus 


\begin{tabular}{|c|c|c|c|c|c|c|c|c|c|c|c|c|c|c|c|c|c|c|c|c|c|c|c|}
\hline \multicolumn{6}{|c|}{ Madagascar } & \multicolumn{3}{|c|}{ Kenya } & \multicolumn{3}{|c|}{ Maroc } & \multicolumn{5}{|c|}{ Nicaragua } & \multicolumn{3}{|c|}{ Mexique } & \multicolumn{4}{|c|}{ Scores } \\
\hline \multirow[t]{17}{*}{ 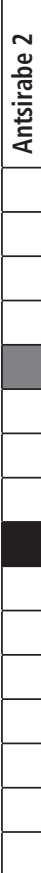 } & $\frac{\sigma}{\frac{\pi}{ \pm}}$ & 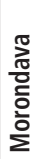 & $\underset{ \pm}{\stackrel{\leftrightarrows}{ \pm}}$ & 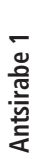 & 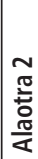 & 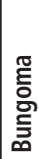 & $\begin{array}{l}\frac{0}{0} \\
\frac{0}{5} \\
\text { 을 }\end{array}$ & $\begin{array}{l}z \\
\text { 롤 } \\
\frac{\sqrt{\pi}}{2}\end{array}$ & 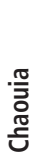 & $\frac{\tilde{n}}{\sqrt{n}}$ & 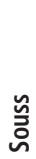 & $\underset{\sum}{\stackrel{\vec{j}}{\Sigma}}$ & 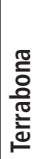 & 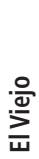 & 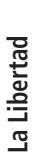 & 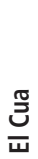 & $\frac{\sum_{\pi}^{\infty}}{i \frac{\pi}{4}}$ & 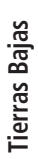 & 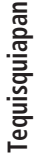 & 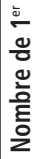 & 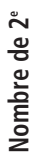 & 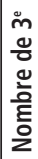 & $\begin{array}{l}\overline{\widetilde{\pi}} \\
\text { गे }\end{array}$ \\
\hline & & & & & & & & & & & & & & & & & & N & & 0 & 4 & 2 & 6 \\
\hline & & & & & & & & & & & & & & & & & & & & 0 & 0 & 1 & 1 \\
\hline & & & & & & & & & & & & & & & & & & & & 1 & 0 & 1 & 2 \\
\hline & & & & & & & & & & & & $\mathrm{N}$ & & & & & & & & 0 & 0 & 2 & 2 \\
\hline & & & & & & & & N & & & & & & & & & & & N & 1 & 1 & 3 & 5 \\
\hline & & & & & & & & & & & & & & & & & & & & 1 & 3 & 0 & 4 \\
\hline & & & & & & $\mathrm{N}$ & & & & & & & & & & & & & & 4 & 1 & 1 & 6 \\
\hline & & & & & & & & & & & & & & & & & & & & 1 & 1 & 0 & 2 \\
\hline & & & & & & & & & & & & & & & & & & & & 7 & 5 & 3 & 15 \\
\hline & & & & & & & & & & & & & & & & & & & & 2 & 1 & 0 & 3 \\
\hline & & & & & & & & & & & & $\mathbf{N}$ & & & & & & & & 2 & 1 & 1 & 4 \\
\hline & & & & & & & $\mathrm{N}$ & & & & & & & & & & & & & 0 & 0 & 1 & 1 \\
\hline & & & & & & & & & & & & & & & & & & & & 2 & 3 & 1 & 6 \\
\hline & & & & & & & & & & & & & & & & & & & & 1 & 0 & 2 & 3 \\
\hline & & & & & & & & & & & & & & & & & & & & 1 & 1 & 0 & 2 \\
\hline & & & & & & & & & & & & & & & & & & & & 2 & 1 & 0 & 3 \\
\hline \multirow[t]{6}{*}{$P$} & & & & & & & & & & & & & & & & & & & & 0 & 3 & 3 & 6 \\
\hline & & & & & & & & & & & & & & & & & & & & 1 & 0 & 1 & 2 \\
\hline & & & & & & & & & & & & & & & & & & & & 0 & 1 & 0 & 1 \\
\hline & & & & & & & & & N & & $\mathbf{N}$ & & & & & & & & & 2 & 1 & 0 & 3 \\
\hline & & & & & & & & & & & & & & & & & & & & 2 & 0 & 2 & 4 \\
\hline & & & & & & & & & & & & & & & P & & $P$ & & & 0 & 1 & 5 & 6 \\
\hline
\end{tabular}

sont très importants au Sénégal, au Kenya, au Mexique et dans la Chaouia et le Souss au Maroc. En outre, leur composition est très variable : l'auto-emploi est une activité essentielle au Sénégal ; les salaires non agricoles et l'auto-emploi sont importants au Kenya ; les salaires agricoles jouent un rôle important au Nicaragua ; et le Mexique est plus diversifié.

L'analyse révèle un éventail de situations et illustre le caractère hétérogène des profils régionaux de diversification des ménages. Même s'il est possible de proposer de grandes lignes d'explication au fait que certaines régions se diversifient et pas d'autres (par exemple, les avantages comparatifs, l'accès aux marchés, l'ur- 

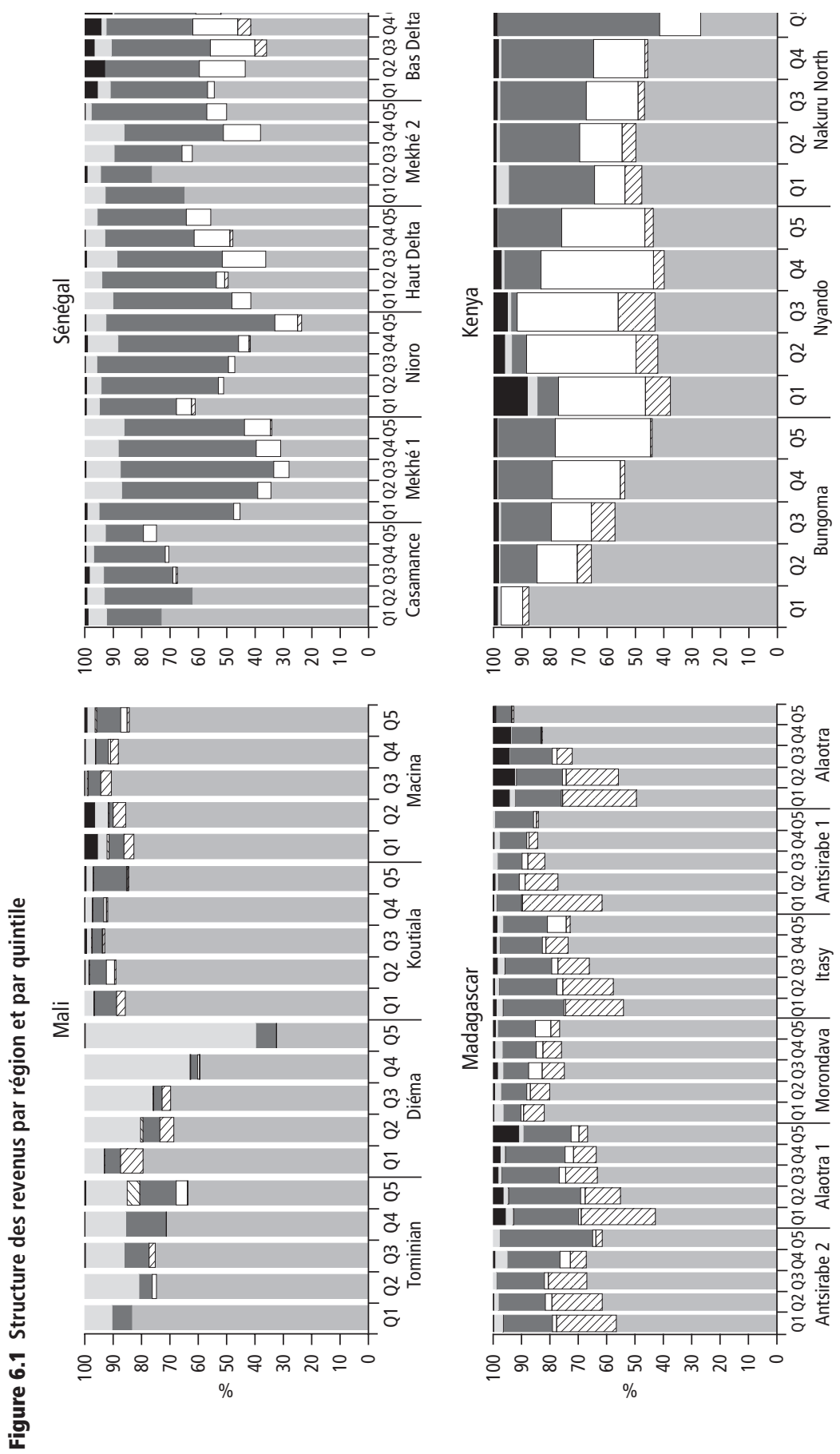


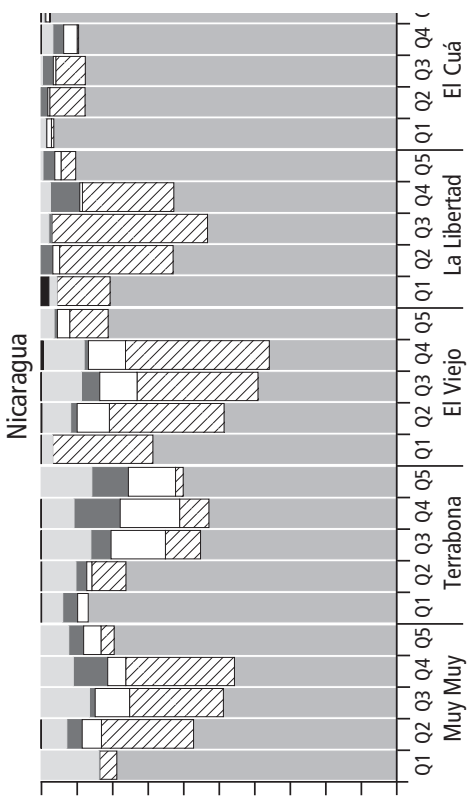

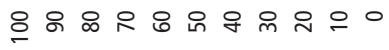

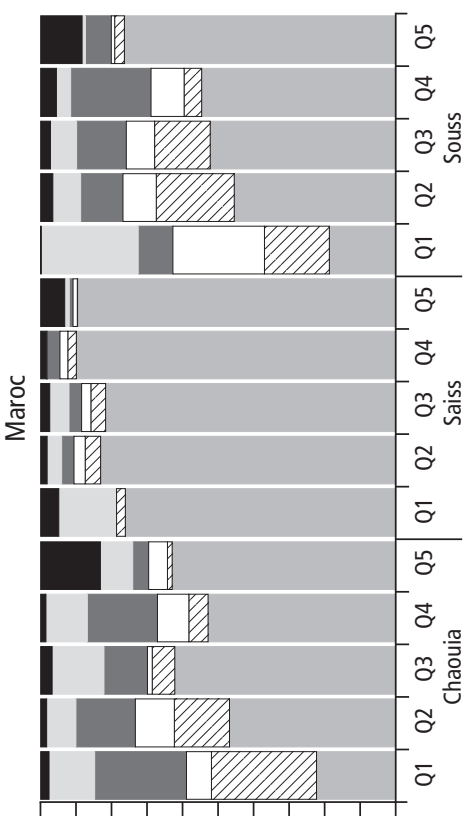

응ㅇㅇㅇㅇㅇㅇㅇㅇㅇㅇㅇㅇㅇㅇ
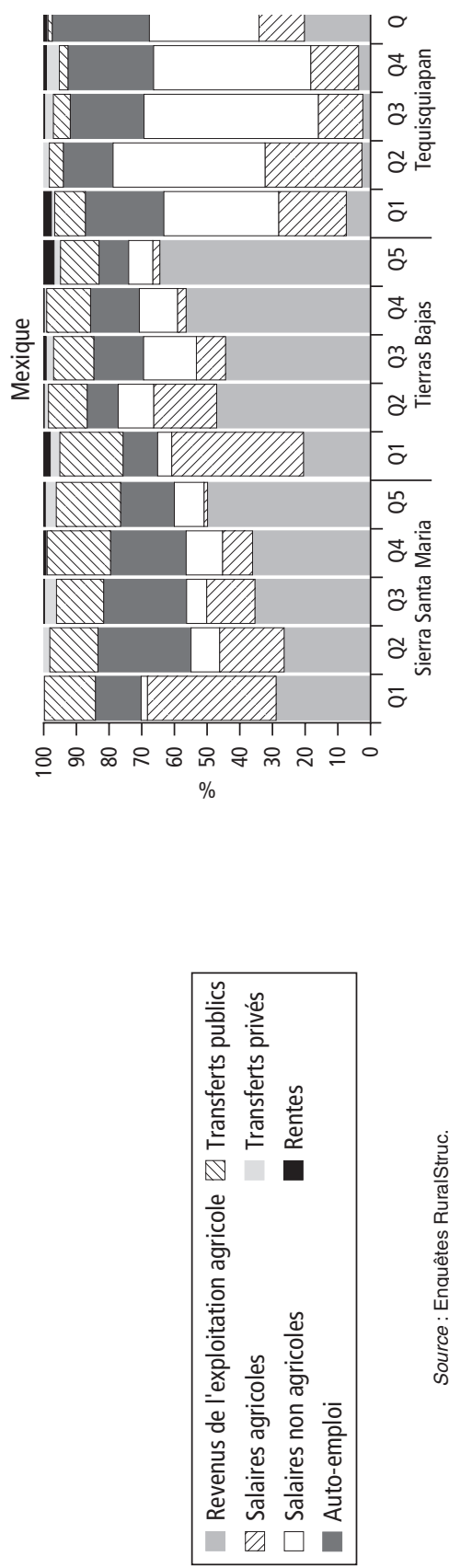
banisation, les institutions) et que, dans chaque région, certains ménages sont diversifiés et pas d'autres (les dotations en facteurs), les mécanismes qui contribuent effectivement aux multiples combinaisons de sources de revenus demeurent difficiles à cerner.

Caractérisation des tendances de diversification. Afin d'apporter un éclairage complémentaire, un indice de diversification des ménages, fondé sur l'indice de Herfindahl-Hirschman (iHH) a été établi ${ }^{8}$. La valeur de cet indice, qui va de 0 à 1 , augmente en fonction du nombre de sources de revenus des ménages. Une valeur élevée indique une diversification plus forte, tandis qu'une valeur faible indique une spécialisation plus poussée.

La figure 6.2, qui a été établie sur l'ensemble de l'échantillon, présente le niveau moyen de l'indice de diversification par région et par quintile de ménages ; elle fait apparaître des tendances à trois niveaux : pays, régions d'un même pays (effets régionaux) et quintiles de revenu d'une même région (effets de quintile).

Au niveau national. À l'échelle de la comparaison entre les pays du programme, l'indice de diversification diminue sensiblement des régions d'ASS vers les autres régions. Au Maroc, au Nicaragua et à Tequisquiapan au Mexique, la valeur moyenne de l'indice va de 0,15 à 0,2 , tandis que dans la plupart des autres régions étudiées (y compris 16 des 19 régions d'ASS), l'indice de diversification avoisine 0,3 .

Les exceptions sont rares et méritent d'être mentionnées. La diversification la plus faible est observée en Afrique subsaharienne, à Koutiala et Macina (Mali) et à Morondava (Madagascar) ; cette situation résulte de conditions régionales particulières (voir infra). Le niveau de diversification le plus élevé est observé dans les deux zones du Sotavento mexicain (Tierras Bajas et Sierra Santa Marta) et résulte principalement du mode de construction de l'indice'. Même en présence de ces exceptions, la diversification tend à diminuer lorsque le revenu national augmente.

Au niveau régional. Des profils régionaux apparaissent distinctement à l'intérieur d'un même pays, même si ces effets régionaux ne suivent aucune tendance spécifique. Certaines régions tendent à se diversifier lorsque le revenu régional augmente : au Sénégal, le Bas Delta est nettement plus diversifié que la Casamance ; et au Kenya, Nakuru North est plus diversifié que Nyando. Mais on observe également une tendance inverse, comme au Mali et au Mexique.

Les caractéristiques locales jouent pleinement. Ainsi, la plus forte spécialisation des ménages dans les deux régions les plus riches du Mali (Koutiala et Macina) résulte de l'attention portée de longue date par les pouvoirs publics au secteur cotonnier dans le cas de Koutiala et au secteur rizicole dans le cas des périmètres d'irrigation de l'Office du Niger à Macina. Au Mexique au contraire, les ménages de la Sierra Santa Marta ont un score de diversification nettement plus élevé que ceux des Tierras Bajas et de Tequisquiapan. Dans la Sierra, les ménages ne peuvent pas se spécialiser dans le maïs (comme leurs voisins des plaines) ou dans d'autres activités rurales (tels les ménages de Tequisquiapan) (voir figure 6.1). Cette situation tient à l'isolement, aux caractéristiques agroécologiques (montagnes contre 
plaines inondables) et au manque d'accès aux paquets techniques et aux gros acheteurs de maïs, mais aussi à des parcelles plus petites et à une moindre productivité des terres en zone de montagne.

Au niveau des ménages. Cependant, les effets régionaux s'avèrent moins prononcés que les effets de quintile au niveau intrarégional. Bien que les quintiles les plus riches soient plus diversifiés dans certaines régions et plus spécialisés dans d'autres, l'écart d'un quintile à l'autre est important dans la plupart des régions. Il existe manifestement un lien étroit entre le niveau du revenu et la diversification.

Premièrement, le sens de l'effet de quintile (à savoir si les ménages plus riches tendent à être plus spécialisés ou plus diversifiés) semble être identique dans les régions d'un même pays (avec quelques exceptions notables comme Morondava à Madagascar, la Casamance au Sénégal et El Cuá au Nicaragua). Ensuite, une première tentative de classification des régions selon la nature de la relation entre les niveaux de diversification et les quintiles de ménages apporte des résultats complémentaires. Bien que ces relations soient diverses, 11 régions présentent un profil qu'il est possible de décrire comme un U inversé (cas le plus courant). Dans ces régions, les ménages se diversifient progressivement des quintiles 1 à 3 ; mais ils recommencent à se spécialiser au-dessus des quintiles 3 ou $4^{10}$.

La relation diversification-revenu. Un examen plus minutieux de la relation entre diversification et revenu nécessite d'examiner la distribution complète des ménages, masquée dans l'analyse précédente par les moyennes des quintiles, sachant que ces moyennes sont particulièrement faussées par l'important différentiel de revenu entre les quintiles 4 et 5 dans toutes les régions (voir chapitre 3 ).

La projection de tous les ménages d'une région sur un graphique dont les axes représentent le revenu et la diversification et l'exécution de régressions polynomiales de second ordre confirment et renforcent la classification esquissée précédemment : 22 des 30 régions étudiées présentent un profil en $U$ inversé $^{11}$; 7 des 8 régions qui échappent à cette configuration affichent une courbe en $U$, tandis que la dernière présente une tendance baissière (voir encadré 6.1).

La persistance de la configuration en U inversé suggère que les ménages préfèrent se spécialiser. C'est en fait le choix de ceux qui ont les revenus les plus élevés. S’ils préfèrent se spécialiser mais ne le font pas aux niveaux les plus pauvres, il faut en conclure qu'ils n'en ont pas les moyens. Ainsi, l'implication d'une configuration en $U$ inversé est que les ménages pauvres se diversifient afin d'optimiser leurs revenus pour couvrir leurs besoins élémentaires et atténuer un niveau de risques très élevé mais, au-delà d'un certain seuil de revenu, ils commencent à se spécialiser (voir figure 6.3).

Les calculs de régression présentés aux tableaux 6.1 et 6.2 donnent d'autres indications sur cette relation étroite entre diversification et revenu. La variable « diversification » est significativement associée au revenu dans tous les pays (c'est-à-dire au niveau national agrégé) ; elle est également très significative au niveau des régions (17 régions sur 30 ), ce qui en fait la troisième variable la plus significative de la régression. 


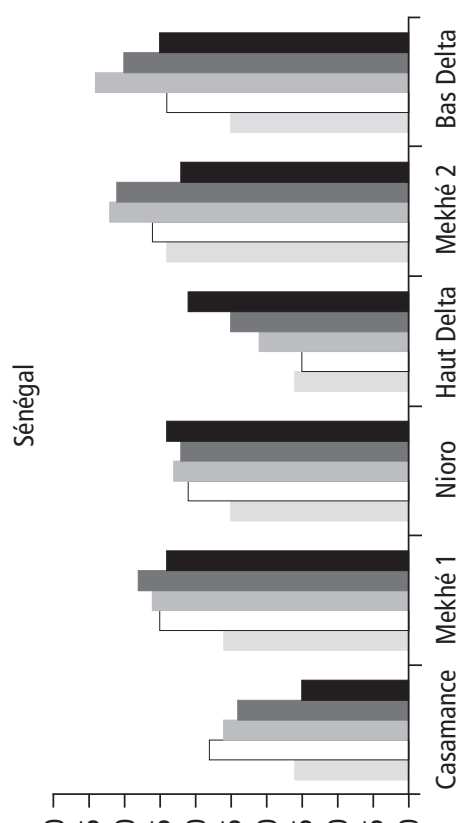

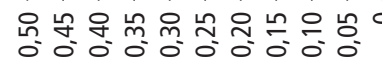

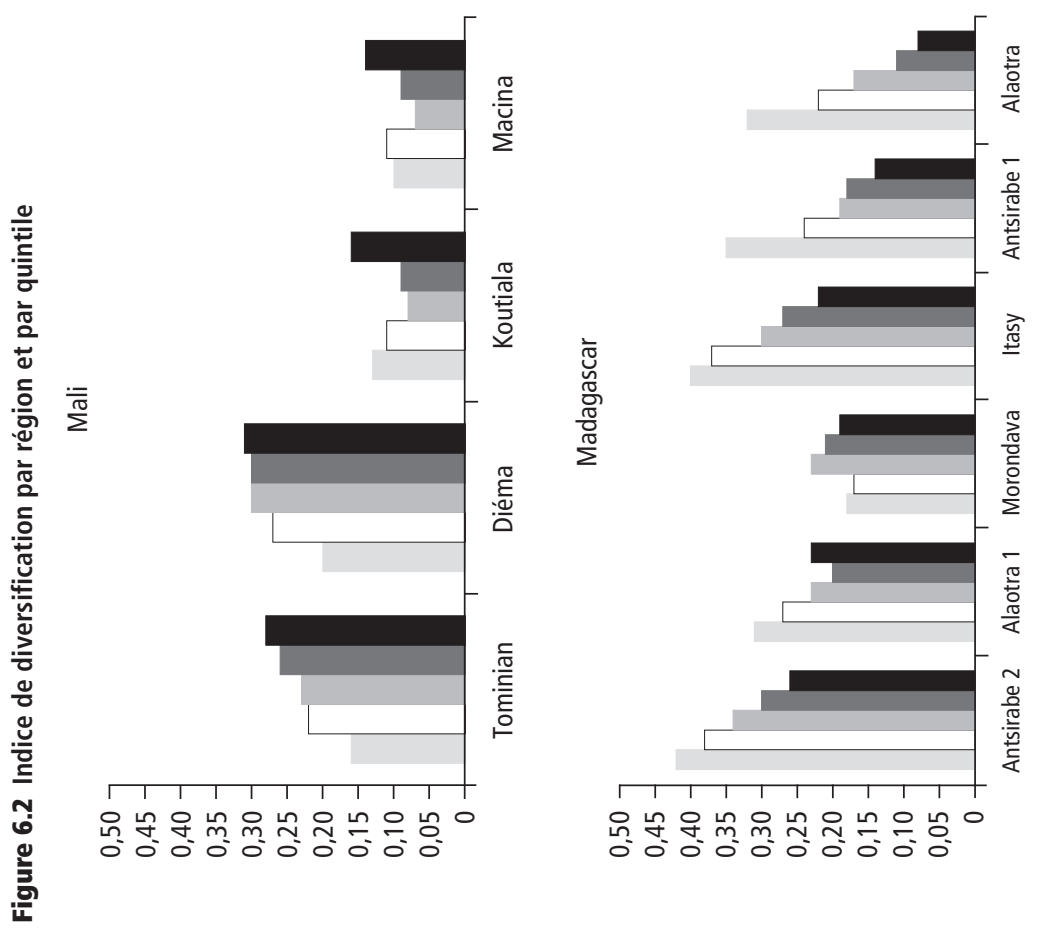




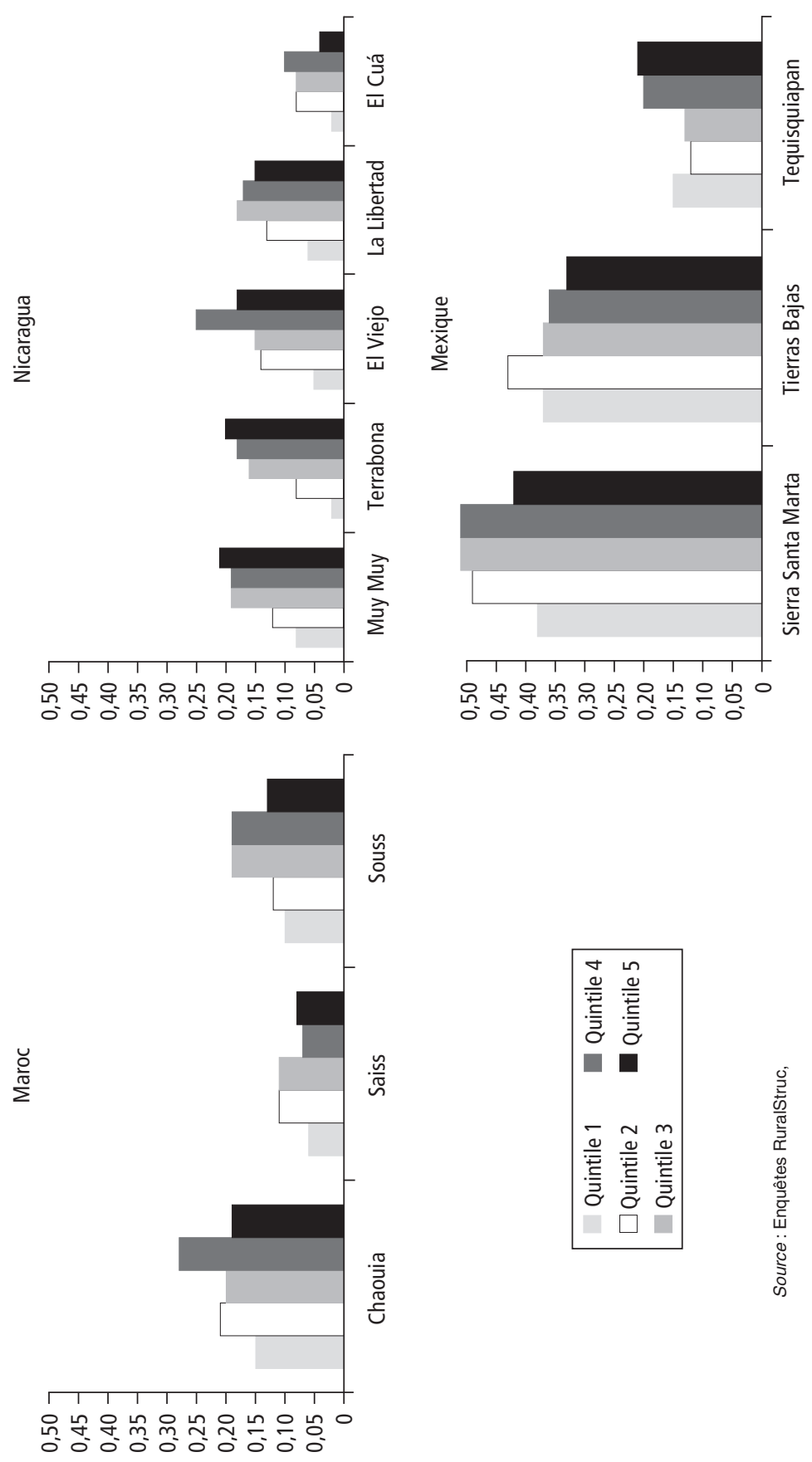


Figure 6.3 Représentation stylisée de la configuration en U inversé

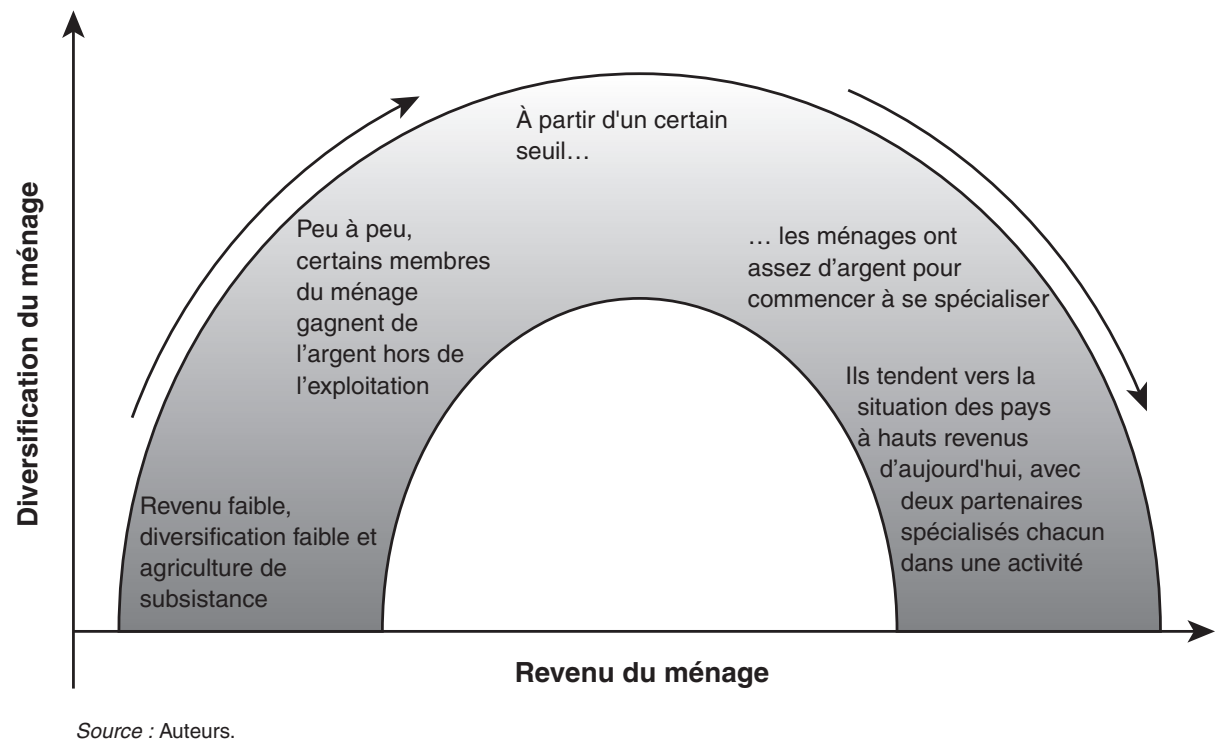

En outre, au niveau régional, le sens de l'effet de la variable diversification (le signe du coefficient de régression) tend à correspondre à celui du niveau national agrégé $^{12}$. Les deux pays dans lesquels la diversification est négativement corrélée au revenu au niveau agrégé sont Madagascar et le Mexique, qui figurent respectivement parmi les plus pauvres et les plus riches de l'échantillon. À Madagascar, cette relation découle de la structure déjà très diversifiée des revenus des ménages pauvres (voir encadré 6.1); au Mexique, elle tient à la spécialisation des ménages agricoles dans le maïs.

En conclusion, les données résultant de l'étude des structures de revenu et des analyses statistiques indiquent que la relation diversification-revenu est principalement gouvernée par un profil en U inversé, dans lequel les ménages les plus pauvres se diversifient pour atténuer les risques, tandis que les ménages plus aisés tendent à se spécialiser. La section suivante introduit un point de vue additionnel qui développe plus avant l'hypothèse du profil en U inversé et relie cette observation aux aspects plus généraux du changement structurel.

\section{Spécialisation des ménages, diversification régionale et changement structurel}

La littérature consacrée à la diversification rurale tend à se concentrer sur son propre développement et sur ses impacts sur l'économie rurale. L'érosion progressive des activités agricoles et le développement de nouvelles activités nourrissent le processus de transformation structurelle (Hazell, Haggblade et Reardon 2007). Cependant, il existe peu d'information sur les profils de diversification et 


\section{GNCADRÉ 6.1}

\section{Les clés des exceptions au profil en $\mathbf{U}$ inversé}

Les régions étudiées à Madagascar remettent en cause le profil en $U$ inversé : elles correspondent à cinq des huit régions dont les distributions ne suivent pas ce profil. Excepté Morondava, elles suivent une courbe en $U$, c'est-à-dire que les ménages sont initialement diversifiés, puis se spécialisent, et tendent finalement à se diversifier à nouveau.

Les ménages des quintiles les plus pauvres de Madagascar sont plus diversifiés que dans d'autres régions étudiées car certains d'entre eux ne parviennent pas à vivre de la seule agriculture de subsistance. En raison de la forte densité de la population, les exploitations sont de très petite taille (moins d'un demi-hectare) et le nombre de paysans sans terre n'est pas négligeable. Les plus pauvres doivent donc chercher du travail et trouvent des emplois salariés agricoles dans la riziculture (voir figure 6.1), une des activités les plus mal rémunérées de toute l'enquête. Les ménages tentent évidemment d'échapper à cette situation et parviennent parfois à atteindre un point auquel les revenus de leur exploitation leur permettent à eux seuls de subvenir à leurs besoins, ce qui équivaut à une spécialisation. La situation de Morondava, qui est l'exception parmi les régions malgaches étudiées et suit la courbe en $U$ inversé, s'explique par la moindre densité démographique et la taille plus grande des exploitations. Les ménages les plus pauvres peuvent y vivre de l'agriculture de subsistance. Par ailleurs, la région est moins spécialisée dans le riz et offre à ce titre moins d'opportunités de travail salarié. Pour améliorer son revenu dans cette région, il est nécessaire d'accéder à d'autres sources de revenus et de se diversifier avant de se spécialiser éventuellement ensuite dans quelques activités spécifiques.

Les deux autres régions étudiées qui ne suivent pas le profil en $U$ inversé sont Koutiala au Mali et El Cuá au Nicaragua. Koutiala, comme les régions de Madagascar, présente une forme en $U$. La cause en est ici la présence d'une culture commerciale avec une commercialisation garantie. Pour les ménages pauvres, développer leur activité dans le coton est la meilleure solution car il offre un environnement relativement sécurisé. Cependant, les limites paradoxales du développement des zones cotonnières (réduction des disponibilités foncières et baisse de la fertilité - voir encadré 3.3) impliquent que le coton ne peut procurer qu'un revenu limité aux ménages. Les ménages les plus riches sont ceux qui se diversifient.

El Cuá est la seule région dont la courbe est totalement baissière, ce qui illustre une tendance à la spécialisation pour tous les niveaux de revenu. Ici aussi, l'explication est la présence d'une culture commerciale ayant une grande facilité d'accès aux marchés. Cependant, la culture du café offre des rendements très supérieurs au coton, de sorte que la nécessité de compléter les revenus n'est pas un impératif.

de spécialisation, aussi bien au niveau des ménages qu'au niveau régional, alors que leur diversité met pourtant en lumière l'importance des dynamiques de transformation à l'œuvre.

Pour illustrer la diversité de ces profils et leurs liens avec la courbe en U inversé, considérons un pays imaginaire dans lequel le changement structurel 
se serait produit selon les étapes stylisées suivantes. Au début du processus de transformation, tous les habitants de ce pays pratiquent une agriculture de subsistance et personne n'a d'autre activité. Les prémices du changement voient apparaître quelques personnes qui s'essaient à d'autres activités que l'agriculture, mais il est peu probable qu'à ce stade ces " diversificateurs précoces » prennent le risque de négliger leur approvisionnement alimentaire et d'abandonner leurs parcelles de culture. En conséquence, la diversification observée à cette première étape de la transformation s'opère en grande partie au sein des ménages. Ensuite, le pays poursuivant sa transformation progressive et les marchés gagnant en fiabilité, les diversificateurs précoces peuvent atteindre un stade où ils sont suffisamment bien installés dans des activités non agricoles pour pouvoir compter entièrement sur d'autres sources de revenu pour assurer leur alimentation. Ils peuvent alors se désengager complètement de l'agriculture et consacrer la plus grande partie de leur temps à leurs nouvelles activités (petite entreprise ou travail salarié). Lorsque s'amorcent ce changement et cette spécialisation progressive dans des activités non agricoles, la diversification au sein des ménages commence à diminuer dans tout le pays ; mais la diversification entre les ménages, qui sont désormais spécialisés dans des activités différentes, s'accentue au niveau régional et national. Il en résulte un pays dans lequel de nombreux ménages se spécialisent et tirent des revenus d'un petit nombre d'activités (ou même d'une seule) qui sont différentes, tandis que les régions et le pays se sont globalement diversifiés.

Ces étapes stylisées mettent en évidence le besoin d'une approche nuancée de la relation entre niveaux de revenu et profils de diversification-spécialisation. $\mathrm{Au}$ lieu de faire référence à des stades de diversification ou de spécialisation absolue, il faut considérer la progression et les tendances, à la fois au sein et entre les ménages.

Au début du processus de transformation, la diversification « au sein des ménages » est la tendance principale, car les ménages tentent de se diversifier par rapport à leur activité principale sur l'exploitation agricole, mais la région dans son ensemble reste caractérisée par le poids de l'agriculture (spécialisation agricole globale). Avec la poursuite de la transformation, des tendances de spécialisation apparaissent, puis dominent progressivement au niveau de chaque ménage. Il en résulte une augmentation de la diversification « entre les ménages », et la région développe une économie plus diversifiée (voir figure 6.4).

Les données des enquêtes RuralStruc illustrent cette évolution des profils de diversification-spécialisation à la fois au niveau des ménages et à celui des régions. En outre, en raison des caractéristiques de l'échantillon de pays étudiés, les données permettent aussi de comparer entre eux les différents stades de transition à l'intérieur du processus de transformation structurelle.

Pour affiner l'analyse des processus de changement, il est possible d'utiliser, au lieu de l'indice de diversification moyen par région (iHH) présenté à la section précédente, un indicateur simple de la diversification qui est la part du revenu des ménages qui ne provient pas de l'exploitation agricole (le off-farm). $\mathrm{Au}$ premier stade de la transformation, cette part est faible et les ménages et 
Figure 6.4 La diversification au sein et entre les ménages et la courbe en U inversé

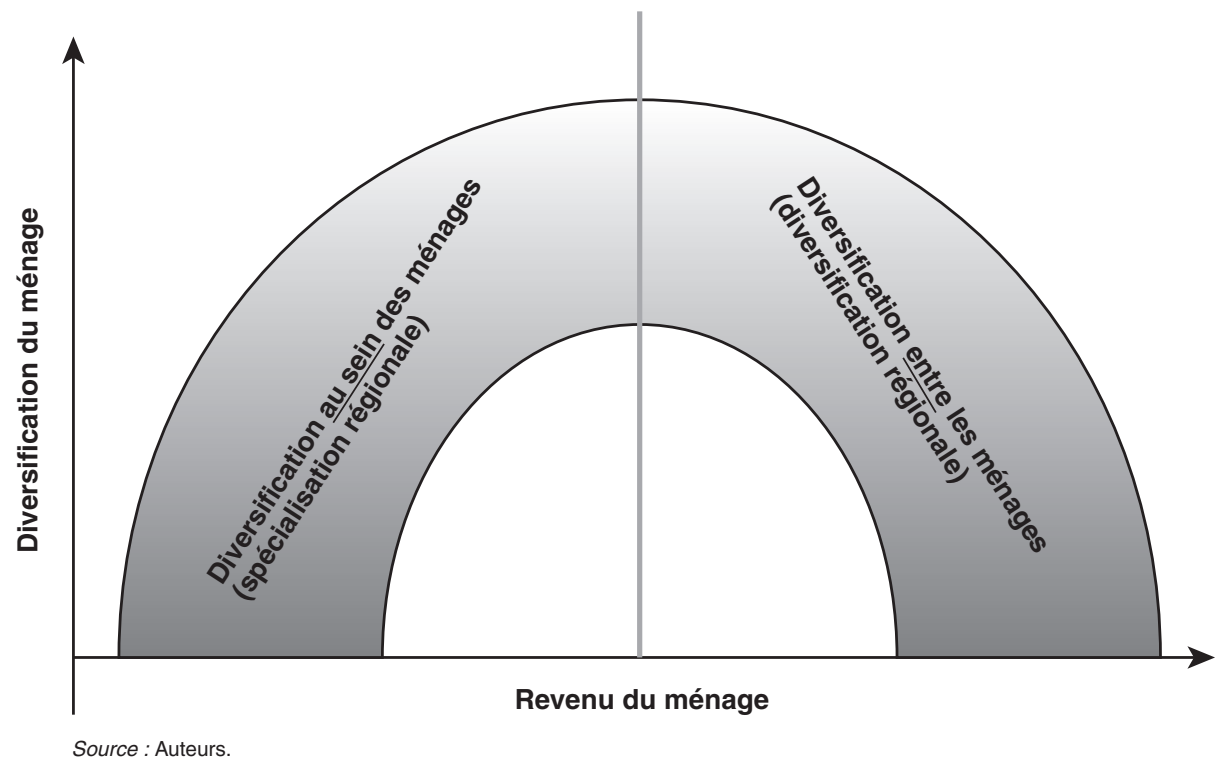

les régions sont spécialisés dans l'agriculture. Elle augmente ensuite lorsque la transformation structurelle s'amorce. Cependant, étant donné que les revenus non agricoles constituent un agrégat d'activités différentes, l'augmentation de leur part relative dans le revenu total signifie aussi que les ménages se spécialisent dans différentes activités non agricoles, une évolution qui résulte en une diversification accrue au niveau régional.

Pour examiner cette diversification régionale, il est possible d'utiliser la part moyenne des revenus hors exploitation dans le revenu total sachant que cette moyenne peut être calculée de deux façons : sous forme de moyenne des parts de chaque ménage, et sous forme de part des moyennes régionales (voir encadré 6.2).

Le tableau 6.4 montre qu'il existe des écarts importants entre la moyenne des parts des ménages (MdPM) et la part des moyennes régionales (PdMR). Ces deux moyennes renvoient à deux configurations différentes. Comme la MdPM représente la part du revenu hors exploitation des ménages, elle exprime les tendances au sein des ménages. Alors que la PdMR, en tant qu'agrégat régional, incorpore bien sûr les tendances des ménages, mais cette fois entre les ménages, et elle exprime aussi la tendance moyenne de diversification régionale.

L'écart entre ces deux moyennes donne des indications sur le profil de diversification-spécialisation dans chaque région étudiée. Ce profil peut être synthétisé en calculant un écart de diversification, défini comme la différence entre MdPM et PdMR (voir encadré 6.2). 
Tableau 6.4 L'écart de diversification dans les régions étudiées

\begin{tabular}{|c|c|c|c|c|c|}
\hline & & \multicolumn{2}{|c|}{ Profil de spécialisation/diversification } & \multirow[b]{2}{*}{$\begin{array}{c}\text { Écart de } \\
\text { diversification } \\
\text { (MdPM - PdMR) }\end{array}$} & \multirow[b]{2}{*}{$\begin{array}{c}\text { Revenu moyen } \\
\text { en US\$ PPA } \\
\text { par EqA }\end{array}$} \\
\hline & & $\begin{array}{l}\text { Moyenne des parts } \\
\text { de revenu off-farm } \\
\text { des ménages } \\
\text { (MdPM) }\end{array}$ & $\begin{array}{l}\text { Part des moyennes } \\
\text { régionales de } \\
\text { revenu off-farm } \\
\text { (PdMR) }\end{array}$ & & \\
\hline \multirow{4}{*}{$\frac{\overline{\bar{\pi}}}{\sum}$} & Tominian & 0,26 & 0,30 & $-0,04$ & 235 \\
\hline & Diéma & 0,35 & 0,51 & $-0,16$ & 368 \\
\hline & Koutiala & 0,12 & 0,12 & 0,00 & 368 \\
\hline & Macina & 0,15 & 0,14 & 0,01 & 516 \\
\hline \multirow{6}{*}{ 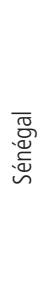 } & Casamance & 0,30 & 0,28 & 0,02 & 439 \\
\hline & Mekhé 1 & 0,64 & 0,67 & $-0,03$ & 527 \\
\hline & Nioro & 0,54 & 0,64 & $-0,10$ & 484 \\
\hline & Haut Delta & 0,41 & 0,49 & $-0,08$ & 524 \\
\hline & Mekhé 2 & 0,54 & 0,51 & 0,03 & 769 \\
\hline & Bas Delta & 0,55 & 0,54 & 0,01 & 1205 \\
\hline \multirow{6}{*}{ 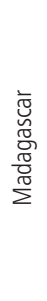 } & Antsirabe 2 & 0,36 & 0,37 & $-0,01$ & 409 \\
\hline & Alaotra 1 & 0,42 & 0,37 & 0,05 & 506 \\
\hline & Morondava & 0,22 & 0,23 & $-0,01$ & 597 \\
\hline & Itasy & 0,35 & 0,31 & 0,04 & 622 \\
\hline & Antsirabe 1 & 0,22 & 0,18 & 0,04 & 744 \\
\hline & Alaotra 2 & 0,31 & 0,17 & 0,14 & 1346 \\
\hline \multirow{3}{*}{ 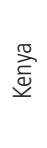 } & Bungoma & 0,37 & 0,49 & $-0,12$ & 641 \\
\hline & Nyando & 0,58 & 0,57 & 0,01 & 660 \\
\hline & Nakuru N. & 0,55 & 0,65 & $-0,10$ & 2258 \\
\hline \multirow{3}{*}{$\frac{\breve{O}}{\frac{.}{N}}$} & Chaouia & 0,55 & 0,40 & 0,15 & 2280 \\
\hline & Saïss & 0,16 & 0,11 & 0,05 & 3419 \\
\hline & Souss & 0,58 & 0,31 & 0,27 & 4131 \\
\hline \multirow{5}{*}{$\begin{array}{l}\frac{\pi}{5} \\
\frac{0}{0} \\
\frac{\pi}{0} \\
\frac{\pi}{Z}\end{array}$} & Muy Muy & 0,41 & 0,30 & 0,11 & 1417 \\
\hline & Terrabona & 0,35 & 0,40 & $-0,05$ & 1458 \\
\hline & El Viejo & 0,50 & 0,31 & 0,19 & 2575 \\
\hline & La Libertad & 0,30 & 0,20 & 0,10 & 2329 \\
\hline & El Cuá & 0,09 & 0,05 & 0,04 & 3610 \\
\hline \multirow{3}{*}{$\frac{\stackrel{0}{ }}{\frac{D}{x}}$} & Sierra SM. & 0,65 & 0,59 & 0,06 & 1824 \\
\hline & T. Bajas & 0,55 & 0,44 & 0,11 & 3144 \\
\hline & Tequis. & 0,93 & 0,89 & 0,04 & 2879 \\
\hline
\end{tabular}

Source : Enquêtes RuralStruc. 


\section{GNCADRÉ 6.2}

\section{Des moyennes de diversification du revenu à l'écart de diversification}

Le calcul de parts moyennes est une opération simple qui peut produire des résultats différents car deux modes de calcul sont possibles.

Dans le premier, on calcule la part du revenu hors exploitation dans le revenu total de chaque ménage, puis les parts moyennes sont calculées au niveau régional : c'est la moyenne des parts des ménages (MdPM). Selon le second mode, on calcule la valeur moyenne du revenu hors exploitation pour une région tout entière, puis on la divise par la valeur moyenne du revenu total des ménages de la région : c'est la part des moyennes régionales (PdMR). La distinction entre ces deux variables est que la MdPM lisse l'effet des valeurs extrêmes, ce qui n'est pas le cas de PdMR.

Les deux moyennes correspondent aux formules ci-dessous :

$$
\begin{aligned}
& \text { Moyenne des parts Part des moyennes } \\
& \frac{\sum_{l}^{n}\left(\frac{O_{i}}{y_{i}}\right)}{n} \\
& \left(\frac{\sum_{1}^{n} o_{i}}{n}\right) \\
& \left(\frac{\sum_{1}^{n} y_{i}}{n}\right)
\end{aligned}
$$

Dans les enquêtes RuralStruc, la différence entre ces deux moyennes est étroitement corrélée au revenu (la corrélation de Pearson est relativement élevée : 0,60). La valeur de cette différence est directement influencée par la distribution des ménages opérant une diversification sur le gradient des revenus. Un signe négatif signifie que les ménages les plus riches se diversifient alors que la majorité ne le fait pas. Un signe positif indique l'inverse : les ménages les plus pauvres se diversifient (ou restent diversifiés).

$$
\frac{\sum^{n}\left(\frac{o_{i}}{y_{i}}\right)}{n}-\frac{\sum_{i}^{n} o_{i}}{\sum_{i}^{n} y_{i}}
$$

La différence entre les deux moyennes s'appelle l'écart de diversification.

L'écart de diversification illustre le stade d'avancement d'une région dans le processus de transformation structurelle. Lorsqu'il est négatif, les ménages sont encore très impliqués dans l'exploitation agricole; ils s'essaient individuellement à la diversification sans abandonner leurs parcelles et la part des revenus hors exploitation dans leur revenu total demeure limitée. Même s'ils ont de nombreuses activités externes, ce sont généralement des activités d'adaptation ou de survie à faible 
rentabilité (voir chapitre 4). La région conserve globalement sa spécialisation agricole, mais un nombre limité de ménages (les plus riches) se sont déjà diversifiés ${ }^{13}$, ce qui tire la moyenne régionale vers le haut : c'est ce qui explique que MdPM soit inférieur à PdMR et que la valeur de l'écart puisse être fortement négative.

À l'inverse, un écart de diversification positif suggère que la part du revenu hors exploitation dans le revenu des ménages augmente. Avec l'élévation du revenu moyen, de nombreux ménages développent des activités en dehors de la ferme et l'effet des valeurs extrêmes est réduit (la valeur de PdMR diminue). Ce processus est renforcé par la spécialisation dans différentes activités qui est mise en évidence par le U inversé : une spécialisation en dehors de l'agriculture pour la plupart des ménages et une spécialisation agricole pour quelques-uns. Comme l'échantillon RuralStruc compte une grande majorité de ménages agricoles, la PdMR rend compte de façon privilégiée de ces ménages qui se spécialisent dans l'agriculture sur leur exploitation (ce sont les nouveaux extrêmes de ce côté-ci du U inversé), ce qui conduit à abaisser la valeur moyenne de la part des revenus hors exploitation et relève la valeur positive de l'écart.

Ainsi, l'écart de diversification, indicateur unique et composite, reflète les complexités de la diversification des revenus ruraux et illustre le processus de transformation rurale. Il explique la distribution en $U$ inversé dans laquelle les profils de diversification des ménages observés aux premiers stades de la transition économique cèdent la place à une spécialisation progressive et à de nouveaux profils de diversification régionale.

La figure 6.5 illustre cette relation entre l'écart de diversification et les revenus : elle représente chaque région sous forme d'un point de données dans l'espace à deux dimensions défini par l'axe des revenus et l'axe reportant l'écart de diversification.

L'écart de diversification peut présenter toutes sortes de valeurs négatives lorsque les revenus sont faibles mais, en général, il faut attendre qu'il soit positif pour qu'une nette augmentation des revenus se produise. La ligne de tendance de la figure 6.5 confirme une forte relation positive entre le revenu et l'écart de diversification. Mais il apparaît aussi que cette relation comporte une composante exponentielle : tant que l'écart n'a pas atteint un certain niveau, les revenus n'augmentent pas de manière générale mais, au-delà d'un certain seuil, ils augmentent rapidement. Ainsi, chaque région qui présente un écart de diversification supérieur à 0,05 a un revenu moyen supérieur à 1000 US\$ PPA/EqA/ an. En revanche, toutes les régions dont l'écart de diversification est négatif, à l'exception de deux régions, ont des revenus inférieurs à 1000 US\$ PPA.

Au-delà de la forme générale de la distribution, la figure 6.6 indique la position des pays d'ASS. L'écart de diversification est inférieur à 0,05 dans toutes les régions d'ASS sauf Alaotra 2, et toutes les régions hormis le Bas Delta et Nakuru North ont un revenu moyen inférieur à 800 US\$ PPA/EqA - très proche du seuil de 2 US\$ PPA/jour. Cette distribution signifie que 16 des 19 régions d'ASS se caractérisent par des revenus très bas et une faible diversification régionale. Dans ces 16 régions, la puissance explicative de l'écart de diversification sur le revenu est très faible, ce qui indique que les ménages se heurtent probablement à un obstacle : il est possible qu'ils ne parviennent pas à passer d'une situation 
Figure 6.5 La relation entre revenu et écart de diversification

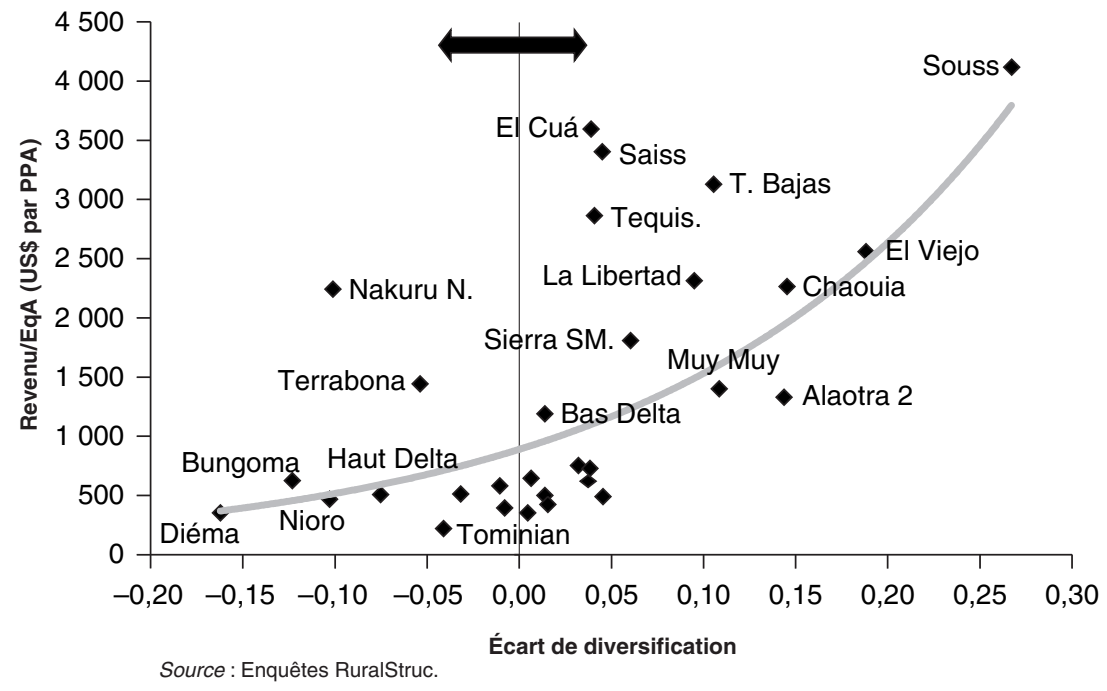

Figure 6.6 La relation revenu/écart de diversification selon les régions étudiées (ASS et hors ASS)

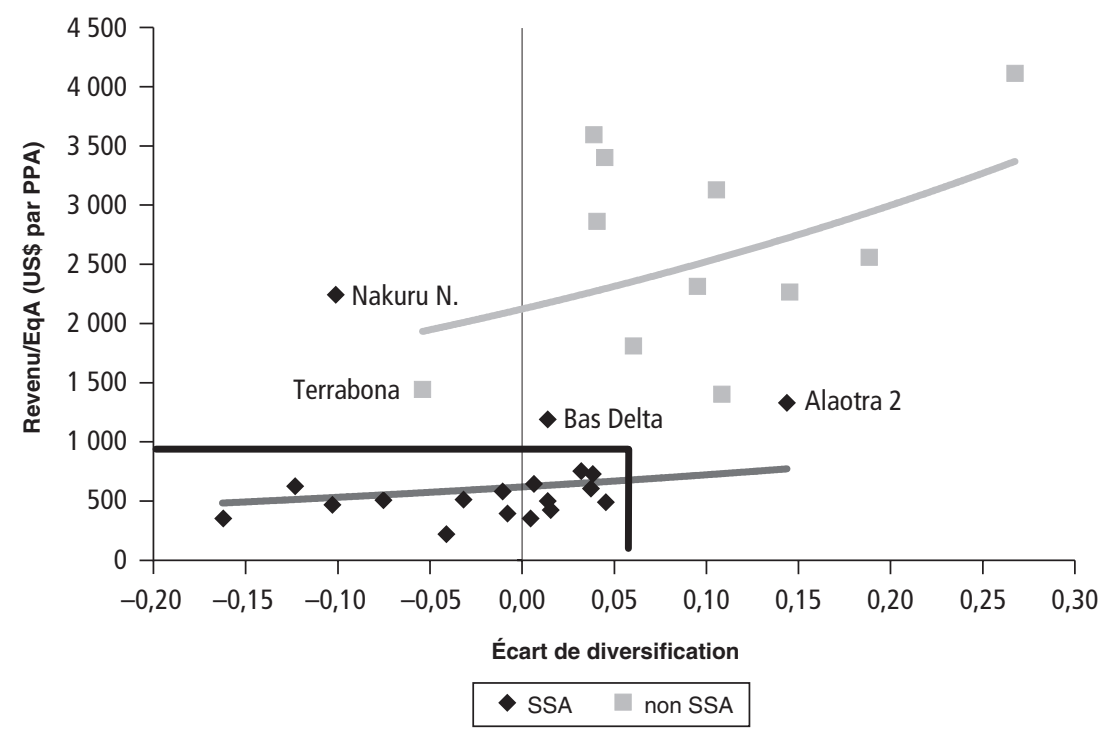

Source : Enquêtes RuralStruc.

où la diversification reste interne aux ménages à une situation où la diversification s'effectue entre les ménages, ce qui correspond pratiquement à leur spécialisation - à la fois parce que leurs revenus sont trop faibles et leurs options de diversification trop limitées. Un tel constat indique la présence possible de trappes à pauvreté (voir encadré 6.3) et renvoie aux impasses de transition mises en avant par la troisième hypothèse du programme ${ }^{14}$. 


\section{ENCADRÉ 6.3}

\section{Les trappes à pauvreté}

La trappe à pauvreté décrit une situation dans laquelle les ménages ne parviennent pas à accumuler des actifs et restent prisonniers de la pauvreté. " Ils ne peuvent pas avancer car ils retombent toujours vers l'arrière. » (Barrett et Carter 2001) Une abondante littérature décrit les causes, les symptômes et les mécanismes de cet engrenage, dont l'existence dépend de "rendements d'échelle croissants et de mécanismes d'exclusion qui empêchent localement certaines personnes de bénéficier de moyens d'existence ou de technologies à rendement plus élevé » (Barrett et Carter 2004, 15). Ce type de situation apparaît souvent lorsque les ménages pauvres prennent des décisions d'allocation sous-optimales de leurs ressources parce qu'ils sont confrontés à des risques auxquels ils ne peuvent pas faire face. Le phénomène de trappe à pauvreté est souvent accentué par l'existence de situations discriminantes, comme le manque d'accès au crédit ou de compétences financières, qui empêchent les ménages de trouver la moindre marge de manœuvre.

On peut donner pour exemple la situation d'un ménage qui dépense toutes ses ressources en semences et alloue toute sa main-d'œuvre familiale à la production vivrière. Si son insécurité alimentaire n'était pas aussi forte, il pourrait investir dans des engrais, qui augmenteraient considérablement la rentabilité de ses dépenses globales. Sans engrais, la dégradation du sol peut être rapide et la fertilité décroissante peut diminuer les rendements année après année, alors même que la famille peut continuer à s'accroître. Pour tenter de compenser la perte de productivité de l'exploitation, le ménage peut alors décider d'envoyer certains de ses membres travailler dans d'autres secteurs d'activité ou dans d'autres régions, mais sans que cette stratégie soit toujours pour autant couronnée de succès.

Cette stratégie d'adaptation est fréquemment observée dans les données collectées par le programme RuralStruc. Les régions où les ménages ruraux se débattent dans des trappes à pauvreté sont souvent caractérisées par une diversification dans des activités à faible rentabilité. Cette situation est illustrée à la figure 6.6 : certaines des régions étudiées apparaissent comme bloquées et dans l'impossibilité de se spécialiser et d'accroître leurs revenus.

Hors d'Afrique subsaharienne, les régions étudiées ne semblent pas rencontrer de telles trappes ou de tels obstacles. Sur la figure 6.6, hormis Terrabona, elles sont généralement réparties très au-dessus et à droite des régions d'ASS ${ }^{15}$ et elles ne sont pas regroupées autour d'une valeur spécifique de l'écart de diversification. Leur ligne de tendance est presque deux fois plus pentue que pour les régions d'ASS. Elles sont donc plus riches et plus diversifiées à l'échelle régionale, mais elles tendent aussi à mieux tirer parti d'une diversification régionale croissante.

L'explication de ces fortes différences régionales réside principalement dans la rentabilité des types d'activités économiques accessibles localement. Comme indiqué au chapitre 4 , dans les régions très pauvres d'ASS, un ménage peut être très diversifié dans de nombreuses activités à faible rentabilité - tous les membres actifs du ménage et souvent les enfants ont au moins une activité et parfois plusieurs. Dans ces contextes, il est probable que les gains du ménage ne 
sont pas suffisamment sûrs pour lui permettre de s'engager dans la spécialisation. Le ménage est alors pris au piège : il ne peut pas gagner plus et consomme tout ce qu'il gagne - et parfois il est même contraint de consommer une partie de son patrimoine. C'est la définition de la trappe à pauvreté.

Hors d'Afrique subsaharienne toutefois, les activités des ménages les plus pauvres de chaque région leur procurent un gain bien plus élevé (voir chapitres 4 et 5). Les possibilités de diversification leur apportent un niveau de sécurité suffisant pour leur permettre de commencer à se spécialiser. Ils deviennent alors plus productifs - ce qui implique des résultats plus élevés - et leur revenu augmente plus rapidement.

Les données microéconomiques du programme RuralStruc sur les profils de diversification et de spécialisation sont une illustration du stade de transformation d'un pays dans le processus de changement structurel. La plupart des régions d'un même pays suivent le même schéma, ce qui suggère l'importance de certaines caractéristiques nationales quant aux options de diversification ou de spécialisation possibles - dotations en facteurs, fonctionnalité des marchés, climat des affaires, dispositifs institutionnels, gouvernance globale et stabilité politique. Les options concrètes offertes par chaque contexte reflètent son stade dans le processus de transition économique.

Les tendances et caractéristiques présentées dans cette synthèse reposent sur des données d'enquêtes et correspondent aux situations des ménages enquêtés dans leurs régions respectives. Ces tendances ne sont en rien déterministes : elles indiquent uniquement la position de chaque région étudiée dans le processus de diversification-spécialisation. Elles reflètent les changements intervenus et suggèrent des explications aux impasses de transition observées. Mais elles ne prédisent pas des trajectoires futures, qui dépendront des spécificités du contexte local et de la nature de ses interactions avec le monde extérieur.

\section{Quelles recommandations pour l'élaboration des politiques publiques?}

Il ressort des analyses qui précèdent que si les déterminants du revenu et de la diversification des ménages ruraux sont avant tout microéconomiques (dotation en facteurs du ménage, caractéristiques du portefeuille d'activités, compétences managériales), les déterminants de la rentabilité d'une activité touchent aux conditions méso- et macroéconomiques. Les marchés sont décisifs, mais le cadre institutionnel est tout aussi important. La faible rentabilité des activités non agricoles et les difficultés de la diversification agricole observées en Afrique subsaharienne rappellent clairement les limites du contexte général.

L'élaboration de politiques publiques de soutien au processus de changement est un exercice difficile car il n'existe pas de solution miracle. L'hétérogénéité des situations locales mise en lumière par les résultats du programme RuralStruc illustre la nécessité de mettre en œuvre des approches régionalisées : il n'y existe pas de " prêtà-porter » des politiques permettant de répondre à toutes les situations et le «sur 
mesure » doit être la règle. Il est néanmoins possible de suggérer un certain nombre d'orientations pour l'action qui concernent d'une part les méthodes d'élaboration des politiques et, d'autre part, quelques axes prometteurs d'intervention possibles.

\section{À propos de la méthode}

La standardisation des réponses politiques obéissant à des « recettes » importées a longtemps été un obstacle considérable à l'élaboration de politiques adéquates. En conséquence, une étape nécessaire consiste à réengager des démarches d'élaboration de stratégies de développement nécessaires à l'identification et à la prise en compte des principaux défis et à la définition des priorités et des objectifs de l'action publique et collective.

Le réengagement dans la définition de stratégies de développement. L'examen des politiques de développement des vingt dernières années débouche rapidement sur une longue liste de mesures bien connues qui sont reproduites dans toute bonne publication consacrée au développement économique en général et au développement rural en particulier. Les principaux ingrédients recommandés comme nécessaires au succès sont la fourniture de biens publics (infrastructure, recherche, information et renforcement des capacités), l'amélioration de marchés imparfaits (approvisionnement en intrants, commercialisation des produits et diminution des coûts de transaction), les incitations au développement des marchés incomplets (crédit, assistance technique, assurances) et les mécanismes d'atténuation des risques. La difficulté consiste bien sûr dans l'assemblage et le dosage adéquats de ces différents ingrédients afin d'obtenir des politiques adaptées avec une mise en œuvre adéquate, en fonction des priorités retenues et des cibles d'intervention.

Pour identifier les priorités d'action, les résultats du programme rappellent l'importance d'un réengagement dans des stratégies de développement afin de faire face aux défis spécifiques propres aux pays en développement. C'est plus particulièrement le cas de l'Afrique subsaharienne, qui doit tout à la fois gérer sa transition démographique et sa transition économique, dans le contexte de la mondialisation, avec les contraintes nouvelles du changement climatique (voir chapitre 2).

De nombreux pays ont négligé l'élaboration de stratégies globales, le plus souvent depuis la fin des années 1970 - une conséquence des politiques de libéralisation et de désengagement de l'État, qui ont débouché sur la segmentation des politiques et le désinvestissement des systèmes d'information, dont la faiblesse constitue aujourd'hui un obstacle considérable à l'élaboration de politiques appropriées.

Une stratégie de développement est beaucoup plus que l'articulation de politiques sectorielles. C'est le résultat d'un processus débouchant sur une vision du futur partagée, résultant d'un accord entre les acteurs concernés ou les citoyens, qui permet à un pays de faire des choix et de définir des priorités. Comme le rappelle Stiglitz (1998), une stratégie de développement est un bien public et mérite à ce titre un soutien large des pouvoirs publics à sa conception. 
Le réengagement dans la conception de stratégies de développement implique d'abord et avant tout de réinvestir dans la création de connaissances. Comme l'ont bien illustré les études-pays conduites lors de la phase $1 \mathrm{du}$ programme, il existe un manque critique d'informations en général, et d'informations sur l'évolution des économies rurales en particulier. Les résultats des enquêtes ont révélé combien l'hétérogénéité des situations produit des configurations rurales complexes qu'il n'est pas possible d'appréhender sans système d'information efficace. Il est en conséquence nécessaire de rétablir et de redéfinir des systèmes statistiques permettant à l'action publique de prendre en compte l'évolution des économies rurales, la mobilité croissante des personnes et les nouveaux réseaux familiaux structurés sur des modèles en archipel (voir chapitre 4). Ce réengagement implique aussi de réinvestir dans les processus. L'étape de la consultation est ici déterminante, car l'appropriation est un facteur décisif. Une telle démarche prend du temps et doit être soigneusement planifiée. Enfin, la relance des stratégies de développement suppose d'investir et de réinvestir dans le renforcement des capacités. De nombreux pays n’ont pas (plus) les compétences pour gérer les systèmes d'information, analyser les résultats, suivre les évolutions et bâtir des scénarios. La situation est particulièrement critique en Afrique subsaharienne, où de nombreux États ont perdu ces compétences techniques dans le sillage de l'ajustement structurel et du désengagement de l'État, et où les nouvelles collectivités locales nées de la décentralisation sont dans l'ensemble mal préparées pour ce type de démarche, d'autant plus que les organisations de la société civile et les groupes de réflexion effectifs sont peu nombreux.

Hiérarchisation des priorités et cibles d'intervention. La multiplicité des besoins est un problème récurrent majeur pour les dirigeants politiques car les ressources financières et humaines ne permettent pas d'agir simultanément sur tous les fronts. Il faut faire des choix et ces choix sont difficiles dans les situations qui sont celles de nombreux pays en développement, où les moyens d'action sont limités.

Dans ce contexte, la hiérarchisation et la planification sont impératives. Elles doivent s'appuyer sur des analyses appropriées pour lesquelles il faut établir des diagnostics généraux, sectoriels et régionaux afin de repérer les « contraintes critiques ». Dans la perspective du programme RuralStruc centré sur la transformation rurale, la première étape d'une telle approche serait de déterminer les principales contraintes régionales à la croissance agricole - passage nécessaire pour accroître la demande rurale, clé de la diversification ${ }^{16}$.

Mais il faut ensuite analyser les priorités en termes d'objectifs, qui peuvent être fixés pour des groupes d'agents économiques, des secteurs (types de produits) et des régions. Le but du programme RuralStruc n'est évidemment pas de proposer des priorités et des objectifs pour les pays et régions étudiés, mais il est possible d'illustrer ce que pourrait être cette étape de ciblage en identifiant des priorités d'action qui pourraient contribuer à la définition des instruments d'intervention.

À cette fin, le programme a procédé à une définition sommaire de groupes cibles en fonction des niveaux de revenus observés. L'approche comporte évidemment des limites dans la mesure où une classification fondée sur une typologie 
plus détaillée, intégrant notamment les actifs des ménages et les opportunités et contraintes locales, serait nécessaire. L'exercice s'est cependant avéré utile car il permet de raisonner les options d'intervention possibles en fonction de la situation économique générale des ménages enquêtés.

Quatre groupes de ménages ont été définis en fonction de leur revenu total et de leur revenu d'exploitation afin d'évaluer leur capacité d'investissement - un indicateur central de la marge de manœuvre des ménages, qu'il faut estimer pour déterminer les incitations et les aides appropriées. Ces groupes sont: les ménages mieux lotis (> 4 US\$ PPA/EqA/jour); les ménages en capacité (> 2 US\$/jour); les ménages pauvres et les ménages extrêmement pauvres ( $<2$ US\$/jour). Les ménages pauvres sont ceux qui pourraient sortir de la pauvreté s'ils pouvaient doubler le revenu qu'ils tirent actuellement de leur exploitation. Les ménages extrêmement pauvres sont ceux qui ne le pourraient pas : même en doublant le revenu de leur exploitation, ils n'atteindraient pas 2 US\$/jour.

Bien que l'exercice soit très théorique, il est possible de considérer que pour les ménages qui disposent de plus de 2 US\$ par jour, les besoins élémentaires sont couverts, les revenus ne sont plus accaparés par la consommation et ils peuvent être affectés pour partie à l'investissement et à l'épargne. Au-dessus de 4 US\$/ jour, les possibilités d'allocation des revenus sont évidemment supérieures. En dessous de 2 US\$/jour, la situation est critique : tous les revenus sont consacrés à la couverture des besoins élémentaires et ils n'y suffisent pas.

Le tableau 6.5 présente le pourcentage de ménages enquêtés qui entrent dans chaque groupe de revenus et la figure 6.7 indique la répartition par groupe au niveau régional. Les résultats de cette classification révèlent les situations très difficiles rencontrées par les ménages dans les différentes régions d'ASS, en particulier au Mali et au Sénégal ; et ils illustrent clairement la référence discutée précédemment aux trappes à pauvreté. Ils rappellent la réalité extrêmement

Tableau 6.5 Distribution des ménages de l'échantillon RuralStruc par groupe cible

\begin{tabular}{lcccc}
\hline \multirow{2}{*}{ Pays } & \multicolumn{4}{c}{ Groupes cibles } \\
\cline { 2 - 5 } & Extrêmement pauvres & Pauvres & En capacité & Mieux lotis \\
\hline Mali & 69,8 & 19,7 & 7,7 & 2,8 \\
Sénégal & 58,5 & 15,6 & 11,8 & 14,0 \\
Madagascar & 49,9 & 25,8 & 13,6 & 10,8 \\
Kenya & 45,7 & 12,5 & 12,8 & 29,0 \\
Maroc & 21,9 & 8,0 & 13,6 & 56,5 \\
Nicaragua & 24,6 & 13,8 & 14,4 & 47,3 \\
Mexique & 7,3 & 2,6 & 10,2 & 79,8 \\
TOTAL & 40,2 & 16,0 & 12,6 & 31,1 \\
ASS & 53,6 & 20,2 & 12,3 & 13,9 \\
Non-ASS & 19,8 & 9,6 & 13,2 & 57,4 \\
\hline
\end{tabular}

Source : Enquêtes RuralStruc. 


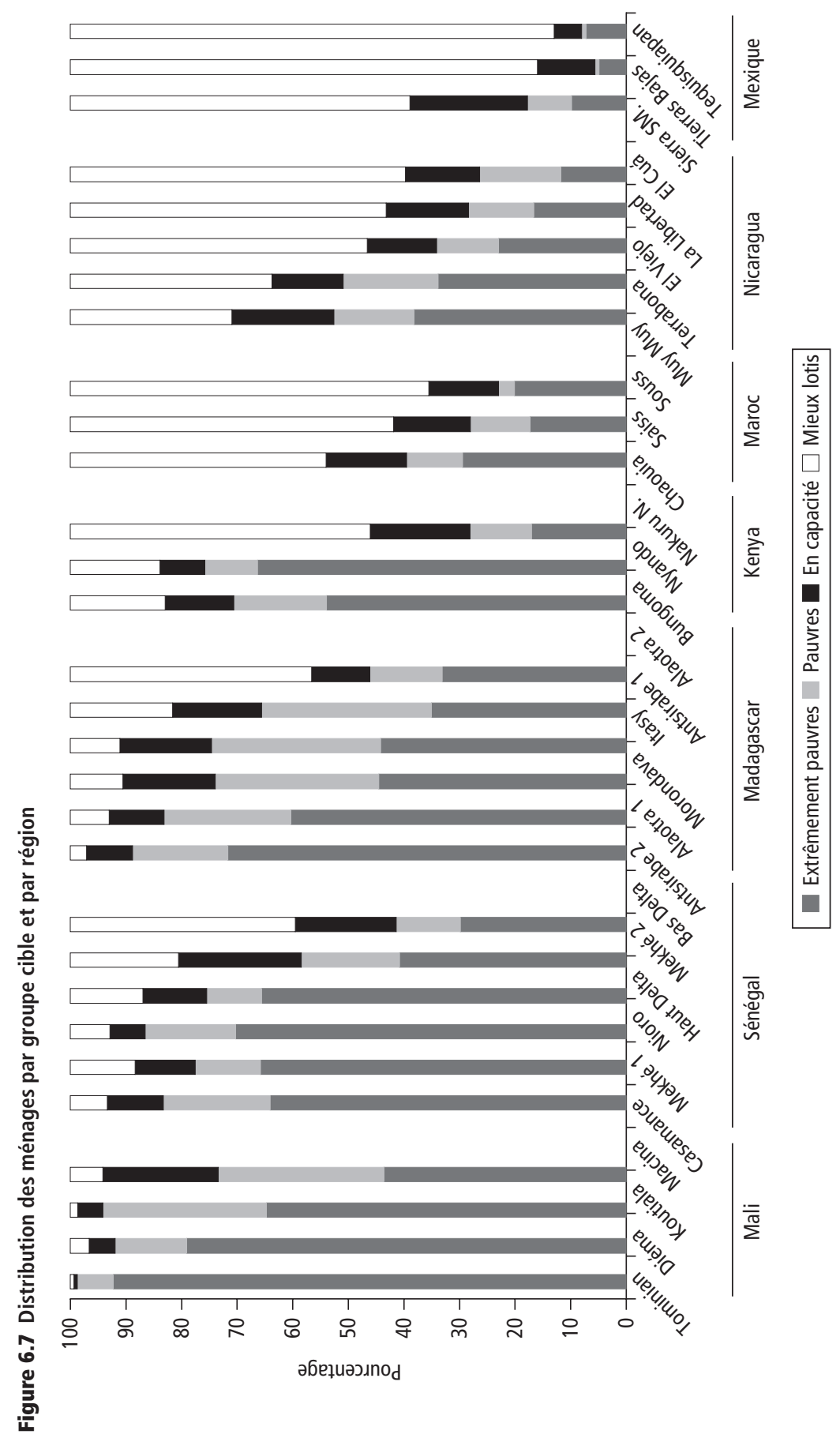


difficile de la plupart des ménages ruraux. Les deux groupes " pauvres ", qui comprennent la majorité des ménages enquêtés en Afrique subsaharienne, font face à des difficultés colossales. Dans le cas particulier du groupe extrêmement pauvre, les ménages seraient toujours pauvres même si leurs revenus d'exploitation étaient multipliés par deux. Or, dans la plupart des situations régionales étudiées, un tel saut apparaît impossible à court et moyen terme.

Ces résultats, bien que fondés sur une approche de ciblage sommaire, permettent néanmoins de réfléchir à des orientations d'intervention possibles. Il n'est pas réaliste d'attendre de ménages dont les revenus sont inférieurs à 2 US\$ $\mathrm{PPA} / \mathrm{EqA} /$ jour, et qui ne parviennent pas à couvrir leurs besoins élémentaires, d'entreprendre seuls un quelconque investissement. Ils auront besoin de biens publics au niveau local (transports, eau et électricité), de droits fonciers et de recherche agricole adaptée.

Il leur faudra également davantage d'aides directes sous forme de subventions intelligentes ${ }^{17}$, surtout pour faciliter l'accès aux intrants et aux services de vulgarisation agricole, ainsi qu'à des prêts à très faible taux d'intérêt, qui sont le seul moyen d'améliorer les systèmes agricoles existants, de promouvoir l'innovation et d'accroître la productivité ${ }^{18}$. Les risques de prix sont un autre obstacle majeur. La volatilité croissante des marchés mondiaux et la volatilité saisonnière bien connue des marchés alimentaires nationaux requièrent des mesures que le secteur privé a peu d'incitations à mettre en œuvre. Une aide publique est nécessaire pour mettre en place des systèmes d'information - une mesure préliminaire et indispensable - et les instruments de stabilisation doivent être adaptés aux situations locales, en fonction du type d'instabilité (endogène ou importée) ${ }^{19}$.

Tout soutien relatif à la fourniture de biens publics et à l'amélioration des marchés bénéficierait à tous les ménages agricoles, les pauvres comme ceux qui sont en capacité et/ou ceux qui sont mieux lotis. Ce type d'interventions n'opposerait pas les groupes de revenus les uns aux autres; elles pourraient être utiles à tous les ménages ruraux tout en permettant un rattrapage plus rapide des pauvres.

Cependant il est clair que, pour les ménages extrêmement pauvres, les perspectives de sortie de la pauvreté en restant totalement engagés dans l'agriculture sont très limitées, et que d'autres solutions seront nécessaires en termes d'activités et de revenus. Les alternatives sont réduites à court et moyen terme, mais l'amélioration des compétences et des capacités facilitera la diversification. En conséquence, en matière de biens publics, l'éducation doit être une priorité. Les enquêtes révèlent une situation hétérogène d'un pays à l'autre, mais qui progresse : la prochaine génération a atteint - au moins au plan formel - des niveaux d'instruction plus poussés, même si un effort considérable demeure indispensable. Car un meilleur niveau d'instruction facilite la mobilité sur le marché du travail et l'accès à des activités en dehors de l'agriculture.

Là encore, tous les ménages ruraux bénéficieront de l'amélioration du niveau d'instruction. Mais celle-ci sera également utile pour la conduite des exploitations agricoles, de meilleures compétences donnant accès à de nouveaux paquets techniques et à de nouvelles pratiques culturales (un atout pour l'amélioration de la productivité et l'adaptation aux nouvelles exigences des marchés). 


\section{Des axes d'intervention pour la transformation rurale}

Cette section s'est attachée jusqu'ici à l'hétérogénéité des contextes et à la nécessité d'un réengagement dans des stratégies de développement conçues à l'échelle nationale (ou même infranationale). Des résultats du programme RuralStruc se dégagent cependant des orientations pour l'action et des thèmes communs qui ont été regroupés en axes d'intervention, en gardant l'impératif de sélectivité à l'esprit. Ces axes d'intervention sont principalement ciblés sur l'Afrique subsaharienne, qui connait de formidables difficultés de transition. Ce ne sont pas des recommandations stricto sensu, mais plutôt des cadres de référence à garder à l'esprit pour la définition de politiques de développement ciblées.

L'agriculture doit demeurer une priorité. Dans les pays d'Afrique subsaharienne à base agricole, la principale impulsion pour le changement structurel et pour la sortie progressive des trappes à pauvreté doit venir du secteur agricole. Même si des politiques publiques d'appui au développement d'autres secteurs d'activités sont nécessaires, la transformation reposera d'abord sur l'augmentation des revenus agricoles et l'instauration d'un environnement économique plus sûr - des conditions propices à l'accroissement de la demande rurale qui facilitera la diversification.

Les constats recueillis au cours des deux phases du programme RuralStruc et ses résultats permettent de cibler trois grands axes d'intervention : (1) le soutien à l'agriculture familiale; (2) la promotion des cultures vivrières et l'amélioration de leurs marchés; et (3) le renforcement des liaisons villes-campagnes.

Appuyer les exploitations familiales. Les résultats du programme permettent de contribuer au débat (souvent trompeur) sur la taille optimale des exploitations, débat relancé récemment par la crise des prix alimentaires de 2008 et par l'accentuation du phénomène d'accaparement des terres qui l'a accompagnée (voir chapitre 1). Le débat a jusqu'à présent surtout été posé en termes de sécurité alimentaire ; et la confusion vient en partie de la publication de deux essais de Paul Collier $(2008,2009)$ consacrés à l'offre alimentaire, dont certains arguments provocateurs ont été utilisés pour nourrir la polémique opposant les tenants des petites aux partisans des grandes exploitations.

Ce débat postule un faux dualisme entre la petite agriculture de subsistance d'un côté et l'agriculture commerciale de l'autre, alors que la réalité est un continuum de situations façonnées par les dotations en facteurs locaux et l'environnement économique et institutionnel. Partout dans le monde, l'agriculture familiale demeure la forme d'agriculture dominante. Elle couvre un large spectre de situations, allant des micro-exploitations à des exploitations plus vastes (parfois des centaines d'hectares) qui recourent à la mécanisation et au travail salarié, et elles sont les principaux fournisseurs des marchés alimentaires mondiaux ${ }^{20}$. L'agriculture familiale peut être de subsistance, commerciale ou combiner les deux objectifs. Tout au long de l'histoire et dans toutes les régions du monde, elle a démontré sa remarquable capacité d'adaptation et son aptitude à répondre à une demande croissante. C'est indéniablement le cas aussi en Afrique subsaharienne, malgré des conditions extrêmement défavorables (Mortimer 2003; Toulmin et Guèye 2003). 
Les partisans de l'agriculture à grande échelle évoquent l'importance et la croissance des écarts de rendement à l'hectare entre les larges surfaces travaillées mécaniquement et les terres travaillées par de plus petits exploitants. Ils avancent qu'en favorisant la production à grande échelle et en produisant ainsi plus d'aliments localement, les pays en développement (en particulier l'ASS) pourraient maîtriser leurs déficits alimentaires croissants et réduire leur vulnérabilité aux fluctuations des prix alimentaires internationaux.

Les tenants de l'agriculture familiale mettent aussi en avant la sécurité alimentaire. Ils pointent le manque bien connu d'économies d'échelle dans l'agriculture et les nombreux échecs des projets agricoles à grande échelle ${ }^{21}$. Ils rappellent les nombreux avantages de la main-d'œuvre familiale, comme l'absence de coûts de supervision du personnel, le niveau d'implication des travailleurs (qui sont directement intéressés aux résultats de l'exploitation), la flexibilité et l'adaptation à des besoins en main-d'œuvre fluctuant au fil des mois et à des résultats économiques variables d'une campagne à l'autre, et enfin la connaissance fine du milieu local qui contribue à rendre le modèle familial plus efficient ${ }^{22}$. La récente étude de la Banque mondiale Réveiller le géant africain endormi (Awakening Africa’s Sleeping Giant - 2009b) a montré que la petite agriculture africaine avait des coûts de production compétitifs face à ceux des grandes exploitations (dans le cas étudié, celles de la région du Cerrado dans le centre-sud du Brésil), qu'elle était compétitive sur ses marchés nationaux, mais qu'elle était en revanche désavantagée sur les marchés mondiaux en raison de coûts logistiques d'exportation élevés (qui renvoient aux contextes économiques et institutionnels locaux et non à la taille des exploitations $)^{23}$.

Ainsi, ce débat opposant les tailles d'exploitations illustre les biais évoqués au chapitre 1 : une discussion sur des politiques qui auront des effets à long terme est influencée par des considérations de court terme (ici, les conséquences de la crise des prix alimentaires). En se focalisant sur la production, le débat omet de considérer le rôle plus large que joue l'agriculture dans le développement économique et il oublie sa contribution au changement structurel.

En Afrique subsaharienne, du fait d'une transition économique embryonnaire et de la transition démographique en cours, l'agriculture aura un rôle éminent à jouer à moyen terme, en particulier quant à l'absorption d'une main-d'œuvre en forte augmentation. Les 195 millions de jeunes ruraux qui devront trouver un emploi entre aujourd'hui et 2025 (voir chapitre 2) auront à travailler dans l'agriculture ou dans l'économie rurale non agricole.

Étant donné que la majorité des ruraux relèvent de l'agriculture familiale et que les activités non agricoles sont directement et indirectement soutenues par les revenus des agriculteurs (et se développeront avec leur amélioration), la question du développement rural est celle du modèle de développement agricole qui aura les meilleurs résultats sur le revenu global, l'emploi et le recul de la pauvreté.

Si les gouvernements devaient privilégier les grandes exploitations managériales - ce qui impliquerait sans aucun doute l'adoption à plus grande échelle de nouveaux systèmes techniques et la mécanisation - ils prendraient le risque de nuire au développement de l'emploi lié à l'agriculture familiale, plus intensive en main-d'œuvre. À cet égard, les récentes acquisitions de terres à grande échelle 
en Afrique par des opérateurs étrangers sont à la fois troublantes et paradoxales. Comme le rappelle Karen Brooks $(2010,9)$, « de nombreux jeunes africains ayant une expérience agricole intègrent la population active [alors que], du fait de contraintes en capital et en droits d'accès, la terre qui pourrait assurer leur avenir risque de passer sous le contrôle durable d'étrangers ».

Les choix politiques doivent éviter toute radicalisation. En fonction du contexte local et du type de production, les investissements dans l'agriculture à grande échelle, y compris les investissements étrangers, peuvent offrir des opportunités de croissance et d'emploi. Ils peuvent aider le développement de nouvelles filières en facilitant l'obtention de seuils de production minimaux et promouvoir le développement agricole dans des régions faiblement peuplées. Cependant, comme l'a justement rappelé l'étude Africa's Sleeping Giant, il serait préférable que ces investissements soient orientés vers des segments de filière où le capital fait défaut - comme la fourniture d'intrants, la commercialisation, ou la transformation des produits - ce qui permettrait de libérer l'énorme potentiel de production des exploitations familiales.

Ces arguments ont des conséquences importantes en termes de définition des politiques. Parmi les nombreux ingrédients de l'action publique relatifs à la fourniture de biens publics et à l'amélioration des marchés, la priorité doit être donnée à l'accès à la terre et aux droits fonciers. Ce choix est validé par les résultats de l'enquête RuralStruc, qui montrent que l'accès à la terre est le déterminant majeur des revenus des exploitations (voir le début de ce chapitre).

Dans les pays où le stock de terres disponibles représente une contrainte forte, l'amélioration de la productivité de la terre par des techniques intensives en main-d'œuvre est la seule solution pour accroître à la fois le revenu agricole et l'emploi agricole. Si les problèmes d'accès et de coût des intrants sont un obstacle important, l'adoption d'innovations techniques au niveau des exploitations offre un vaste éventail de réponses adaptées et soutenables ${ }^{24}$. Ces innovations doivent être couplées avec la sécurisation des droits fonciers, sans laquelle le niveau de risque économique est intenable.

Dans les pays où il est possible d'augmenter la superficie en terres cultivées, faciliter l'accès à ces ressources en terres par des infrastructures, une planification régionale adéquate et des droits fonciers respectés et sécurisés peut être un puissant moyen d'augmenter le revenu et l'emploi agricoles. C'est le cas dans de nombreuses régions d'Afrique, en particulier dans les zones de savane guinéenne, où seulement $10 \%$ des 400 millions d'hectares de terres arables potentielles sont actuellement cultivés (World Bank 2009b) ${ }^{25}$. Dans ces contextes particuliers, la première étape est de recenser le potentiel existant, une information essentielle dont la plupart des pays d'ASS sont démunis.

Par ailleurs, la question difficile et rarement abordée de l'accès des jeunes à la terre doit être posée. De nombreux jeunes sont bloqués dans des situations agraires où les anciens contrôlent l'accès au foncier et la gestion des exploitations. Les jeunes chefs de famille restent souvent dépendants de leur père ou de leur grand-père jusqu'à ce que ceux-ci décèdent, une situation qui bloque les initiatives et les innovations techniques que les jeunes pourraient plus aisément 
adopter. L'accès des jeunes ruraux aux terres agricoles, la transmission des actifs de l'exploitation aux jeunes travailleurs familiaux et l'arrêt d'activité des anciens sont des questions majeures qui doivent être prises en compte par les politiques publiques. C'est le passage nécessaire pour l'insertion économique des jeunes et le développement agricole.

La dernière recommandation porte sur la question des économies d'échelle des exploitations familiales, auxquelles leur production par nature limitée fait obstacle. Ce handicap peut être surmonté par des organisations de producteurs efficaces, sachant toutefois que des incitations et des aides sont nécessaires à leur développement. Les organisations de producteurs peuvent faciliter la commercialisation des produits par la collecte primaire et jouer un rôle important dans l'investissement en installations de stockage, en matériel de transformation des produits et dans l'organisation de systèmes d'approvisionnement en intrants. Le regroupement des productions peut faciliter la contractualisation avec les opérateurs aval (grossistes, entreprises agroalimentaires, exportateurs) et les organisations de producteurs peuvent user de leur pouvoir de négociation renforcé dans les négociations contractuelles.

Promouvoir les cultures vivrières. Dans l'échantillon du programme RuralStruc, la production vivrière représente plus de $50 \%$ du produit brut agricole dans 18 des 30 zones étudiées et atteint parfois $80 \%$. Ce résultat reflète une double réalité. Tout d'abord, il souligne l'importance de l'autoconsommation. La part de l'autoconsommation dans le produit brut agricole d'un ménage reflète les stratégies de gestion des risques (effet d'offre) que les ménages déploient dans un environnement qui demeure incertain (marchés incomplets et imparfaits et parfois conditions naturelles difficiles qui peuvent affecter la campagne agricole). Ce résultat reflète ensuite une demande concrète qui peut être limitée par le manque d'accès et d'intégration au marché (effet de demande). L'importance des produits vivriers de base est souvent liée au manque d'opportunités de marché dans les zones étudiées et à la rareté des filières alternatives aux productions traditionnelles. Bien que les exploitations soient plus diversifiées hors d'ASS (hormis dans les régions mexicaines pour des raisons spécifiques), l'importance des productions vivrières est une caractéristique générale qui concerne tous les ménages à tous les niveaux de revenus, et les ménages des quintiles supérieurs peuvent être fortement engagés dans leur production et leur commercialisation.

Ces résultats d'enquête justifient la priorité à donner aux politiques d'appui à la production vivrière et à l'amélioration de ses marchés. Cette priorité a été la pierre angulaire du changement structurel des économies asiatiques, et de leur révolution verte (Delgado, Hopkins et Kelly 1998). Les objectifs étaient tout à la fois de faire reculer la pauvreté, de réduire les coûts de l'alimentation mais - aussi - de gérer et de ralentir la sortie de l'agriculture - une condition d'ajustement nécessaire pour s'adapter au rythme de leurs transitions économiques.

Quatre arguments justifient de donner la priorité aux cultures vivrières. Le premier a trait à leur caractère inclusif - une conséquence de leur développement généralisé : la quasi-totalité des ménages agricoles produisent des denrées 
vivrières de base (98\% des ménages enquêtés en ASS et $76 \%$ dans les autres pays du programme), tandis que les autres productions concernent une population plus réduite. Bien qu'elles soient souvent appelées dans le débat, les exportations à haute valeur ajoutée ne touchent souvent que quelques dizaines de milliers de producteurs, voire moins, sur les centaines de milliers, voire les millions que compte chaque pays. Les politiques visant à encourager et soutenir les cultures vivrières peuvent ainsi toucher une écrasante majorité de ménages ruraux.

De manière générale, les produits vivriers ont une valeur marchande très inférieure à celle des produits de l'horticulture ou de l'élevage. Ils offrent des rendements plus faibles et il est clair qu'augmenter leur production ne peut suffire pour faire reculer la pauvreté. Cependant, la hausse des prix alimentaires entraîne une augmentation progressive de la rentabilité de l'agriculture vivrière et la contrainte de gains unitaires relativement faibles est compensée par l'ampleur de la production, qui permet d'accroître la demande en main-d'œuvre, d'augmenter le revenu total et de bénéficier d'effets de liaison en termes de croissance. En contribuant fortement aux revenus agricoles au niveau agrégé (et donc aux revenus ruraux), les productions vivrières peuvent stimuler la demande rurale et faciliter l'émergence d'autres activités. Par ailleurs, il est plus facile pour les producteurs d'accéder aux marchés des produits vivriers, dont les exigences sont moins rigoureuses que celles des produits à plus forte valeur ajoutée, surtout lorsqu'il s'agit de marchés à l'exportation.

Le deuxième argument concerne le rôle primordial des cultures vivrières dans la gestion des risques. Respectivement $75 \%$ et $30 \%$ des ménages interrogés en ASS et dans les autres régions se situent dans les deux groupes " pauvres ", dont le revenu total par équivalent adulte est inférieur à 2 US\$ PPA par jour. Ces ménages sont en situation de risques et l'insécurité alimentaire est une réalité pour un grand nombre d'entre $e^{26}{ }^{26}$. Dans une telle situation, l'autoconsommation et le stockage (lorsqu'il est possible) sont la règle et tout risque associé à de nouvelles cultures, de nouvelles techniques de production, de nouveaux circuits de commercialisation ou à la diversification des activités est soigneusement évité. En conséquence, l'augmentation de la production vivrière peut jouer un rôle de catalyseur : elle contribue à réduire les risques et peut ainsi libérer le potentiel d'innovation et de diversification, sur l'exploitation et en dehors de l'exploitation.

Le troisième argument en faveur des cultures vivrières est le formidable potentiel d'expansion du secteur. En effet, dans les prochaines décennies, la croissance démographique - à l'échelle nationale, régionale et mondiale - et l'urbanisation grandissante accroîtront continuellement la demande pour tous les types de denrées vivrières. L'évolution des régimes alimentaires liée à l'augmentation des revenus entraînera un développement rapide des produits carnés, laitiers et horticoles (Collomb 1999), mais les produits de première nécessité - surtout les céréales - représenteront pour longtemps l'essentiel de la demande.

En Afrique subsaharienne, la production vivrière a parfaitement réussi à se développer pour répondre à une demande croissante (Bricas, Zoungrana et Thirion 2009) et qui continuera à croître du fait des perspectives démographiques de la région. Le secteur représente déjà les trois quarts de la production agricole 
totale ${ }^{27}$. En outre, l'augmentation des prix internationaux réduira la concurrence des importations à bas prix et encouragera l'expansion de la production régionale. Les producteurs nationaux seront en meilleure position pour capter une partie des importations alimentaires, qui se chiffrent aujourd'hui en Afrique subsaharienne à 23 milliards d'US $\${ }^{28}$.

Le dernier argument en faveur d'une politique de soutien aux cultures vivrières a trait au colossal potentiel des activités de transformation en aval. La transformation initiale (égrenage et mouture habituellement) a généralement lieu sur l'exploitation pour l'autoconsommation, ou au village pour la consommation locale. Mais la plupart des ventes de denrées vivrières, surtout celles qui sont destinées aux consommateurs urbains des grandes villes, consistent en produits bruts et ce sont les agents économiques urbains qui captent la valeur ajoutée de leur transformation.

Le développement de la production vivrière pourrait aisément générer une plus forte valeur ajoutée locale, renforcer les liens entre les zones rurales et les petites villes voisines et contribuer à la diversification rurale. Cette évolution nécessiterait un climat plus favorable à l'investissement, mais ceux-ci ne sont pas nécessairement très élevés. La transformation locale peut être réalisée avec de petits matériels et des unités de transformation à forte consommation de maind'œuvre, qui peuvent se charger de la transformation initiale et secondaire et du conditionnement des produits pour les consommateurs urbains.

Si les arguments tirés du programme permettent d'affirmer la priorité à accorder au secteur vivrier, les recommandations en termes de politiques destinées à développer leur production sont plus difficiles à énoncer, car le risque est grand de répéter la longue liste des mesures d'amélioration de la productivitée ${ }^{29}$. En Afrique subsaharienne, il faut cependant résoudre deux grands problèmes : le premier concerne les pertes postrécolte, un thème ancien promu après les crises alimentaires des années 1970, mais qui reste d'actualité en raison du manque d'équipements de stockage dans la plupart des zones rurales. Les estimations sont difficiles, surtout pour les racines et tubercules, mais les pertes postrécolte de céréales sont généralement estimées entre $10 \%$ et $20 \%$ de la production totale ${ }^{30}$. De nombreuses mesures portant sur les étapes postrécolte peuvent être promues (tri, séchage, contrôle des nuisibles, traitement précoce), mais un bon stockage semble particulièrement important et peut être soutenu par des arrangements institutionnels adaptés comme les systèmes de warrantage, qui peuvent simultanément améliorer la trésorerie des producteurs et réduire leurs risques économiques (World Bank 2010a).

Le second problème a trait au commerce régional. L'Afrique subsaharienne est un marché colossal en pleine expansion, mais elle subit les contraintes du morcellement politique du sous-continent et de nombreuses frontières internationales, une situation soulignée dans le RDM09. En moyenne, dans les années 2000, $20 \%$ seulement des exportations agricoles de l'ASS étaient destinées à d'autres pays du sous-continent (Lipchitz, Torre et Chedanne 2010). Des progrès considérables ont été accomplis depuis une vingtaine d'années grâce à l'intégration régionale progressive et à la constitution des communautés économiques régionales (CER). 
De manière générale, les tarifs douaniers ont été éliminés à l'intérieur des CER, mais ces mesures n'ont pas développé pour autant le commerce régional (Faivre Dupaigre 2007). Les difficultés sont liées au non-respect des règles des CER, à la persistance de barrières non tarifaires relatives aux normes (sur les produits et les intrants) et à des pratiques irrégulières, principalement liées au franchissement des frontières (où les tracasseries bureaucratiques sont souvent la règle $)^{31}$. Une véritable volonté politique d'harmonisation et de facilitation des échanges constitue la réponse principale à apporter, sans ignorer les besoins d'investissements en infrastructures de transport (Ndulu 2006 ; Foster et Briceño-Garmendia 2010), qui sont l'objectif d'un des deux grands programmes du NEPAD.

Un appui privilégié aux cultures vivrières ne doit pas pour autant être la seule priorité et, lorsque d'autres opportunités de production existent ou apparaissent, elles doivent être soutenues. Les produits d'exportation traditionnels ou les produits à plus forte valeur ajoutée peuvent offrir d'importantes alternatives locales. C'est le cas des produits de l'élevage, qui sont développés dans un grand nombre des régions étudiées ${ }^{32}$. Dans la situation des pays étudiés hors ASS, l'existence d'une production agricole plus diversifiée et d'environnements économiques et institutionnels plus favorables offre une marge de manœuvre plus grande pour renforcer la diversification existante, en particulier en ce qui concerne les produits à haute valeur ajoutée - une conclusion conforme aux recommandations du RDM08 pour les pays déjà fortement urbanisés.

Resserrer les liens villes-campagnes pour le développement territorial. Le processus de transformation rurale est entièrement enchâssé dans la relation diversification-spécialisation dont les modalités déterminent le passage des économies régionales de la spécialisation agricole à la diversification rurale. Ce processus est rendu possible par la réduction des risques et l'augmentation de la rentabilité des activités agricoles (résultant d'une meilleure productivité agricole et de la baisse des coûts de transaction) qui accroissent la demande rurale de produits et services non agricoles. Celle-ci engendre de nouvelles activités (transformation des produits, achat et revente de biens marchands, services) qui se concentrent dans les bourgs ruraux et petites villes afin de bénéficier d'économies d'échelle, alors que l'agriculture est par nature exercée par de multiples unités de production dispersées sur l'ensemble du territoire rural.

Le renforcement des liens entre les petites villes et leur environnement joue un rôle essentiel dans le développement et il a favorisé la transition économique dans toutes les régions du monde. Ces liens créent de meilleurs débouchés locaux, facilitent l'accès aux services, développent les communautés et contribuent au renforcement du tissu économique et social d'une région. Ils s'intensifient progressivement avec l'augmentation de la production et des revenus agricoles, mais les changements sont lents et se produisent généralement sur plusieurs générations.

La question porte donc sur les moyens de renforcer ces liens villes-campagnes et la dimension territoriale (ou régionale) du développement, dans un contexte caractérisé dans de nombreux pays en transition par l'émergence de nouveaux modes d'urbanisation, avec une métropolisation rapide - une évolution qui sape 
la densification des liens locaux. Le chapitre 2 a rappelé combien l'amélioration des réseaux de transport facilitait l'accès aux grandes villes, qui offrent plus de services et de meilleures perspectives d'emploi, et cet accès entraîne souvent des migrations directes des zones rurales vers les zones métropolitaines (UNSRID 2010).

Ce mode d'urbanisation peut contrarier le développement des villes secondaires, là où les liens entre monde rural et monde urbain, entre activités agricoles et non agricoles, peuvent se former et offrir des effets multiplicateurs pour le développement. En outre, il complique la gestion urbaine des grandes villes, qui subissent un afflux de migrants ruraux pauvres et sans qualification venant grossir le secteur urbain informel. Cette population croissante dans les zones métropolitaines pose des difficultés aux aménageurs urbains en termes d'infrastructures, d'équipements et de services, dans la mesure où les consommateurs urbains sont trop démunis pour contribuer eux-mêmes à leur entretien (Paulais 2010 et 2012). Ce phénomène explique l'ampleur de la croissance des bidonvilles dans les zones métropolitaines des pays en développement - un autre problème d'envergure pour les pouvoirs publics (UN-Habitat 2003).

Les résultats de travaux récents sur l'importance des dynamiques rural-urbain au niveau régional confirment le rôle majeur des centres urbains de petite et moyenne importance et la nécessité de cibler les efforts de développement au niveau local. Christiaensen et Todo (2009) montrent que les sorties de l'agriculture vers ce qu'ils appellent le " chaînon manquant " (les villes secondaires et l'économie rurale non agricole qui leur est associée) ont de puissants effets de réduction de la pauvreté, ce qui n'est pas le cas lorsque les ruraux migrent directement vers les grandes métropoles - un résultat qui contredit les bénéfices de la concentration mis en avant par le RDM09. À partir de données sur la pauvreté de 49 pays, Christiaensen et Todo montrent que si la concentration de la population dans les mégalopoles accélère la croissance globale, elle engendre aussi des inégalités plus fortes, alors qu'une urbanisation mieux répartie et s'appuyant sur le chaînon manquant lisse le processus et permet un développement plus inclusif.

La question des moyens de soutenir les liens entre les petites villes et leur environnement immédiat est cruciale et a beaucoup mobilisé les universitaires et les praticiens du développement au cours des dernières décennies, sans qu'aucune solution définitive n’ait émergé ${ }^{33}$. Comme pour le développement économique global et la transition économique, il n'y a pas de solution miracle pour le développement territorial. Cependant, il existe des méthodes et des leçons en matière d'institutions locales et de renforcement des fonctions économiques des petites villes qui peuvent être utiles aux décideurs politiques - parmi celles-ci le développement inclusif fondé sur les exploitations familiales, la consolidation des marchés vivriers et la transformation des produits locaux sont essentiels.

Une démarche territoriale qui s'efforce de comprendre les atouts et faiblesses existant au niveau régional, et notamment les contraintes les plus critiques, est une étape nécessaire du développement rural et local ${ }^{34}$. Cela nécessite un diagnostic précis, établi conjointement par et avec les acteurs locaux, qui permet une hiérarchisation, un séquençage et un ciblage efficaces des priorités. Ce processus accompagne le développement des institutions et de la gouvernance 
locale. La décentralisation et le renforcement des organisations de la société civile permettent d'opérer des choix en matière de politiques locales; cependant, les institutions locales sont souvent faibles car la décentralisation a fréquemment précédé le développement des systèmes d'information et des capacités d'analyse nécessaires à une gouvernance efficace - des domaines qui requièrent d'évidents appuis externes.

Le renforcement des fonctions économiques des petites villes concerne leurs liens aux marchés et le type et le niveau des services qu'elles fournissent. Les infrastructures de transport peuvent être déterminantes (une opinion courante dans les débats sur le développement), mais elles ne sont pas suffisantes. Un important résultat des enquêtes RuralStruc est que des zones rurales bien connectées, bénéficiant d'un accès aisé aux grands centres urbains, ne sont pas nécessairement mieux loties que des zones plus reculées - une situation illustrée par la situation difficile des ménages ruraux dans la partie occidentale du Kenya ou dans le bassin arachidier au Sénégal (voir chapitre 3). Cette discussion renforce le débat sur le chaînon manquant, qui insiste sur la qualité de l'urbanisation et pas seulement sur la lutte contre une métropolisation excessive.

Les caractéristiques de l'urbanisation en Afrique de l'Ouest rappellent clairement que la métropolisation n'est pas incompatible avec le développement de villes de petite et moyenne importance mais que ces villes ne sont pas pour autant des vecteurs de la croissance régionale. En effet, comme le montre l'étude Africapolis (Denis et Moriconi-Ebrard 2009), le nombre de centres urbains de plus de 10000 habitants a fortement augmenté en Afrique de l'Ouest ; il a raccourci considérablement les distances entre les villes et reconfiguré la géographie régionale (Bossard 2009). Cependant, la question ici est le niveau de fourniture de biens publics et la qualité des infrastructures et des services. Ce sont des conditions nécessaires pour qu'une ville puisse assumer son rôle économique car, sans elles, l'urbanisation se réduit au développement d'agglomérations de pauvres. Dans de nombreux pays d'Afrique subsaharienne, la capitale domine et accapare les financements, empêchant ainsi la répartition des infrastructures et des services et le développement d'une urbanisation positive (voir encadré 3.2).

Ainsi, à l'échelle régionale, l'action publique doit se focaliser sur la fourniture adéquate de biens publics (liés à l'administration locale, à la santé, à l'éducation et aux infrastructures de communication - (et pas seulement aux routes) mais de services de base, comme l'eau et l'électricité - des services que le secteur privé ne peut aisément fournir dans les premières phases de développement. Ces biens publics sont indispensables pour faciliter l'investissement privé et améliorer les conditions de vie des résidents des petites villes et des bourgs ruraux - une condition nécessaire pour minimiser l'exode rural vers les zones métropolitaines. Plutôt que des approches spatialement neutres, les interventions publiques doivent être ciblées et cette démarche doit être la règle plutôt que l'exception réservée à des situations particulièrement défavorisée ${ }^{35}$. La fourniture de biens publics peut utilement s'accompagner de mesures fiscales visant à aider les prestataires de services et les entrepreneurs locaux. 
Les investissements et soutiens publics de ce type ont des effets positifs avérés sur la croissance régionale, le développement de l'emploi non agricole et le renforcement des liens villes-campagnes (Fan 2008). Ils peuvent directement promouvoir un développement territorial qui valorise les actifs spécifiques locaux, facilite l'amélioration du contenu en valeur ajoutée des produits locaux et renforce la fourniture de services à l'environnement ${ }^{36}$. Les clusters agroalimentaires qui mettent à profit les connaissances locales, les réseaux locaux et les appellations géographiques des produits peuvent être de puissants moteurs de développement ${ }^{37}$.

Une telle perspective reconnaît la multifonctionnalité de l'agriculture et son potentiel d'entraînement du développement rural et régional. Elle peut servir de fondation à un «nouveau contrat rural-urbain » (Gutman 2007), fondé sur un nouveau type de gouvernance territoriale, qui réconcilierait les " urbanistes » et les « ruralistes » et permettrait d'améliorer la dynamique du changement structurel en reconnectant les villes à leur environnement régional.

\section{Notes}

1. Pour une explication complète des analyses statistiques, en particulier les travaux de régression et une description des variables, voir l'annexe 5 de l'appendice en ligne : http://www.worldbank.org/afr/ruralstruc.

2. Tout a été mis en œuvre pour exécuter la même régression dans les 30 régions étudiées. Cela n'a pas toujours été possible, car certains éléments d'information n'étaient disponibles que dans certaines régions, mais aussi parce que certaines variables n'étaient pas localement pertinentes (par exemple l'irrigation, qui n'est pas présente partout). Cependant, en général, la spécification des régressions pour chaque région est similaire.

3. Les difficultés posées par la détermination des revenus des migrants sont évoquées au chapitre 4.

4. Il semble également y avoir d'importants effets de niveau, mais pas nécessairement d'« effets de diplôme ». La différence la plus significative en matière de revenus est associée à une scolarité menée jusqu'à la fin du primaire et à une éducation secondaire partielle.

5. L'enquête n'a pas effectué d'analyse détaillée des pratiques d'intensification. La variable « paquet technique » ne représente que l'accès aux engrais et à des semences améliorées.

6. Ces cinq régions sont le Bas Delta (Sénégal), les deux zones d'Antsirabe (Madagascar), El Cuá (Nicaragua) et Tequisquiapan (Mexique). Les résultats sont difficiles à interpréter mais ils indiquent néanmoins l'importance relative de la distance physique à la ville et de la qualité des routes. Lorsque la variable qualité des transports est négativement significative, la proximité physique d'une ville est très importante ; lorsqu'elle est positivement significative, un bon réseau routier est préférable à la proximité d'une ville (voir l'annexe 5 de l'appendice en ligne : http://www.worldbank.org/afr/ ruralstruc).

7. La figure représente les parts des moyennes régionales par source de revenus et quintile régional. Les différents modes de calcul des moyennes sont étudiés infra dans ce chapitre (voir encadré 6.2). 
8. L'indice de diversification (1-iHH) est défini comme l'opposé de l'indice Herfindahl-Hirschman (iHH).

Il se définit comme suit :

$$
1-I H H=1-\frac{\sqrt{\sum_{i=1}^{n} P_{i}^{2}}-\sqrt{\frac{1}{n}}}{1-\sqrt{\frac{1}{n}}}
$$

Où $i$ représente les différentes sources de revenu (revenus de l'exploitation, salaires agricoles, salaires non agricoles, auto-emploi, transferts publics, transferts privés, rentes), $n$ le nombre de sources de revenu, et $P$ la part de chaque source de revenu dans le revenu total. Étant donné que iHH élève les parts au carré, il renforce et révèle les principales caractéristiques du ménage.

9. L'indice de diversification étant fondé sur sept catégories de revenus (les revenus de l'exploitation et les six sources de revenus hors exploitation), la présence ou l'absence d'une de ces catégories peut avoir un effet notable sur le score global du ménage. Une des sept catégories correspond aux transferts publics, qui sont présents dans tous les quintiles dans chaque région au Mexique et nulle part ailleurs dans les régions enquêtées (à quelques infimes exceptions près). Cette situation augmente considérablement l'indice de diversification au Mexique par rapport aux autres pays mais dans une moindre mesure à Tequisquiapan où seulement $27 \%$ des ménages ont des revenus d'exploitation, de sorte que les subventions agricoles (Procampo) y sont plus faibles.

10. Ce profil est observé dans les régions suivantes: Casamance, Mekhé 1 et 2 et Bas Delta au Sénégal, Chaouia et Souss au Maroc, El Viejo, La Libertad et El Cuá au Nicaragua, et Tierras Bajas et Sierra Santa Marta au Mexique.

11. Cette forme en $U$ inversé est présente même lorsqu'on élimine les ménages qui ont les revenus les plus élevés, qui exercent une forte influence sur les moyennes du quintile 5. La seule exception est le Saïss, au Maroc, où l'exclusion des cinq ménages les plus riches produit un résultat de régression différent et une forme en $\mathrm{U}$ au lieu d'un U inversé (voir chapitre 4 pour une analyse des revenus élevés dus aux rentes au Maroc).

12. À titre d'exemple, la diversification est positivement corrélée au revenu au Sénégal et positivement significative dans cinq des six régions dans la régression au niveau régional. La variable de diversification n'est pas significative dans le Haut Delta en raison de la forte spécialisation dans la production de tomates (voir chapitre 5).

13. Une partie de ces ménages sont engagés dans des activités de services (santé, éducation, administration locale, ou commerce et transport), qui offrent de meilleurs revenus.

14. Les trois valeurs régionales extrêmes observées en ASS - Nakuru North, Alaotra 2 et Bas Delta - ont les revenus moyens régionaux les plus élevés de l'échantillon d'ASS et sont les régions enquêtées les plus riches de chaque pays. À Nakuru North, la valeur négative de l'écart suggère que le revenu régional est tiré par quelques ménages plus aisés qui ont des activités non agricoles lucratives. En Alaotra 2, la valeur positive de l'écart illustre une situation dans laquelle les ménages les plus riches sont très spécialisés dans les activités de leur exploitation agricole (la riziculture), ce qui abaisse la moyenne régionale et augmente la valeur positive de l'écart de diversification. La position plus neutre du Bas Delta, qui est proche des seuils, reflète la meilleure rentabilité des activités des ménages les plus aisés. 
15. À Terrabona, une des deux régions les plus pauvres du Nicaragua, les ménages ont principalement des activités agricoles sur l'exploitation avec une diversification hors exploitation dans des activités faiblement rémunératrices. Les ménages les plus riches sont ceux qui ont accès à des emplois mieux payés dans les maquiladoras.

16. La méthode du diagnostic de croissance (growth diagnostic) développée par Hausmann, Rodrik et Velasco (2005) et son concept de « contraintes critiques » (binding constraints) est une référence majeure qui pourrait être adaptée à une approche régionale.

17. La question des subventions est très sensible, mais les positions ont évolué récemment (World Bank 2007). Il n'est plus contesté aujourd'hui que des subventions intelligentes (smart subsidies) peuvent faciliter l'accès des producteurs aux marchés des intrants et aussi procurer des incitations aux fournisseurs. Les systèmes de bons d'achat sont une bonne option qui a été assez largement adoptée car ils facilitent le ciblage des groupes d'agriculteurs et des régions. La question cruciale est la bonne gestion de ce type de système et la capacité à s'en désengager.

18. Sur la base d'une revue à long terme et d'une modélisation, Fuglie (2009) a démontré que les niveaux de productivité en Afrique subsaharienne ont très peu augmenté en quarante-cinq ans, contrairement aux autres pays en développement.

19. Les systèmes de stabilisation des prix qui ont été développés partout dans le monde entre les deux guerres mondiales (et principalement déployés par les puissances coloniales dans leurs anciennes colonies) ont tous été démantelés lors de la vague de libéralisation des années 1980, sauf rares exceptions comme le Ghana Cocoa Marketing Board. De nombreuses tentatives ont été faites pour mettre en place des instruments de marché (options, contrats à terme) et des filets de sécurité pour les ménages les plus vulnérables ; mais les résultats ont été modestes et inégaux. Aujourd'hui, la nécessité de l'intervention de l'État dans la gestion des marchés est mieux acceptée, surtout depuis la crise des prix alimentaires de 2008. Cependant, les options structurelles pour réduire et gérer l'instabilité sont encore largement débattues. Sont mis en avant la nécessité de maintenir une forte croissance de la productivité par des investissements et de disposer d'instruments de gestion de crise (par exemple, des réserves nationales ad hoc ou des prix d'intervention). Voir Byerlee, Jayne et Myers (2005), Poulton et al. (2006), World Bank (2007), Galtier (2009) et Timmer (2010). Le rôle dominant des entreprises globales sur les marchés agricoles depuis le désengagement des États de la gestion de l'offre reste cependant un problème majeur. De nombreuses solutions proposées comptent fortement sur la coopération des entreprises - dont la disposition à coopérer n'est pas assurée (Losch 2007).

20. Comme rappelé au chapitre 4, l'agriculture familiale est définie par le lien étroit entre la structure des ménages et l'activité agricole en termes de patrimoine et de gestion. Elle s'oppose à l'agriculture managériale ou capitaliste entièrement fondée sur le salariat et l'actionnariat. Ce type d'agriculture cible la rentabilité de l'investissement en capital, tandis que l'agriculture familiale vise avant tout la rentabilité du travail (Lamarche 1991 ; Losch et Fréguin-Gresh à paraître).

21. Dans le cas de l'Afrique, voir Poulton et al. (2008), qui montrent également que les quelques succès apparents en Afrique orientale et australe ont été nourris par d'importantes aides publiques.

22. Voir Hazell et al. (2007b), Wiggins (2009) et Binswanger-Mkhize, McCalla et Patel (2009). L'absence d'économies d'échelle en agriculture connaît quelques exceptions, essentiellement liées à la transformation et au conditionnement des produits périssables. Byerlee et Deninger (2010) montrent également que les nouvelles technologies 
informatiques de gestion des exploitations et de conduite des opérations culturales pourraient remettre en cause l'avantage historique des petites exploitations.

23. L'étude a confirmé des économies d'échelle pour certains produits précis (palmier à huile, horticulture) et relevé les avantages d'échelle lorsqu'il faut satisfaire à des critères de qualité rigoureux.

24. La plupart de ces innovations techniques sont des pratiques agroécologiques qui ont été entièrement validées par l'Évaluation internationale des connaissances, des sciences et des technologies agricoles pour le développement (IAASTD 2009). Elles comprennent des pratiques culturales améliorées et une meilleure gestion des parcelles, comme le contrôle de l'érosion par la création de terrasses, la couverture des sols, l'agroforesterie et les systèmes d'agriculture-élevage intégrés.

25. Dans les pays du programme RuralStruc, c'est le cas au Mali (la large zone de savane proche de la frontière guinéenne, dans l'ouest du pays, ainsi que le delta intérieur du Niger), à Madagascar (les régions occidentale et septentrionale) et au Nicaragua (la côte caraïbe). Voir les rapports nationaux RS 1.

26. Dans 11 des 19 régions étudiées en ASS et dans 2 régions au Nicaragua, plus de $10 \%$ des ménages vivent dans l'insécurité alimentaire (voir chapitre 3).

27. Voir World Bank (2008a). La valeur globale des produits vivriers pèse lourdement lorsqu'elle est comparée à celle des exportations agricoles totales : selon Diao et al. (2007), la valeur de marché estimée en 2003 était de 50 milliards d'US\$, contre 16,6 milliards d'US\$ pour les exportations.

28. WITS/Comtrade (CTCI Révision 3), année 2008, groupes de produits 0 (produits alimentaires et animaux vivants) et 4 (huiles, graisses et cires d'origine animale ou végétale). Les importations de céréales (groupe de produits 04 ) représentent $39 \%$ des importations totales ( 9 milliards d'US\$).

29. L'irrigation, les semences et les engrais ont été les principaux ingrédients de la révolution verte asiatique. Ils ont été complétés par des investissements publics massifs en infrastructures, en recherche et en vulgarisation agricoles et par de forts soutiens et protections des prix des intrants et des produits.

30. Rien qu'en Afrique australe et orientale, les pertes estimées se chiffrent à presque 2 milliards d'US\$ (World Bank 2008b). À comparer aux importations de céréales qui se chiffrent pour toute l'ASS à 9 milliards d'US\$.

31. Pour une analyse détaillée des politiques et pratiques commerciales existantes en Afrique de l'Ouest (Communauté économique des États de l'Afrique de l'Ouest et Union économique et monétaire ouest-africaine), voir Rolland et Alpha 2010.

32. Dans 6 des 19 régions d'ASS, la part des produits de l'élevage dépasse $20 \%$ du produit brut agricole. Toutes les régions du Maroc et toutes celles du Nicaragua sauf deux se situent au-dessus de ce seuil.

33. Voir, entre autres, Davis et al. (2002), Satterthwaite et Tacoli (2003) et De Ferrandi et al. (2005).

34. Concernant le développement territorial, une importante référence internationale est la politique de développement rural de l'Union européenne et son programme phare, LEADER, qui répartit les financements structurels régionaux. Plusieurs initiatives de développement territorial prometteuses et fructueuses ont été déployées ou sont en cours en Amérique latine. Voir, par exemple, les débats liés à la nueva ruralidad (nouvelle ruralité) et le programme Dynamiques territoriales rurales géré par le Centre latino-américain pour le développement rural (RIMISP). Sur la nueva ruralidad, voir entre autres Pérez et al. (2008). Pour une comparaison de la nueva ruralidad à l'approche européenne de la multifonctionnalité, voir Bonnal et al. (2004). 
35. Ces approches spatialement neutres limitant le ciblage des interventions sont une recommandation majeure du RDM09 (voir encadré 3.1).

36. Gutman (2007) affirme que les services environnementaux pourraient stimuler le développement rural et inaugurer un nouveau type de relation rural-urbain, qu'il appelle un "nouveau contrat rural-urbain " (new rural-urban compact). Le financement de ces services est une question cruciale qui nécessitera une évolution du débat politique.

37. L'approche en termes de clusters agroalimentaires a donné de bons résultats dans plusieurs pays d'Amérique latine et a facilité le développement de systèmes agroalimentaires localisés fondés sur la promotion des actifs spécifiques locaux. Les « indications géographiques ", qui renvoient aux origines géographiques d'un produit (et ses facteurs naturels ou humains spécifiques), sont de plus en plus mises en avant dans le débat sur le développement, même si elles sont aujourd'hui fortement débattues dans le cadre de la libéralisation du commerce. C'est un des grands sujets des discussions sur les ADPIC (aspects des droits de propriété intellectuelle qui touchent au commerce) au sein de l'OMC. Sur les systèmes agroalimentaires localisés, voir Muchnik et al. (2007). Sur les indications géographiques, voir Giovannucci et al. (2009). 


\section{Annexe}

\section{Cartes des pays étudiés}

Pour plus d'informations sur les régions étudiées, voir l'annexe 3 de l'appendice en ligne : http://www.worldbank.org/afr/ruralstruc. 
Mali
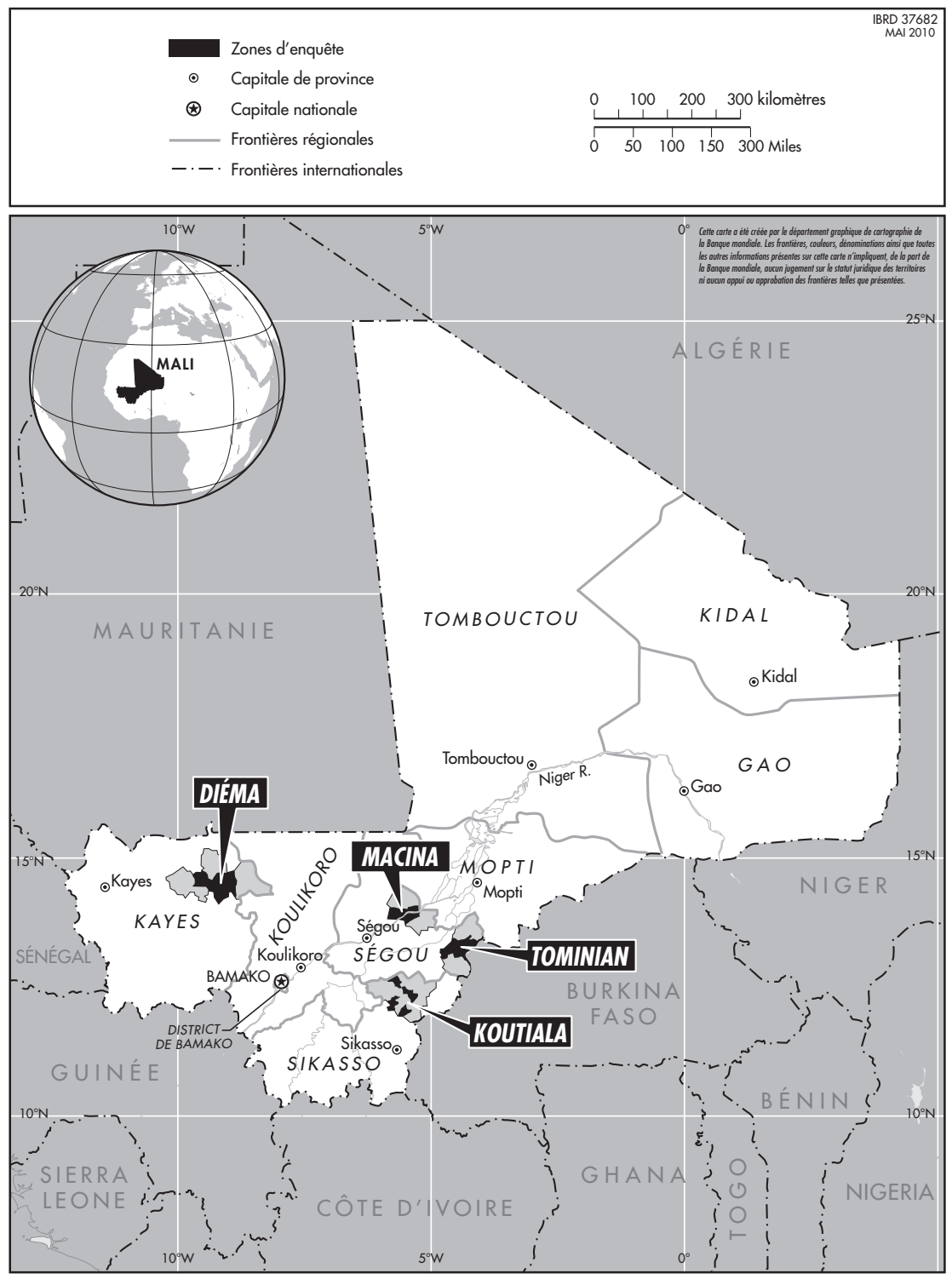


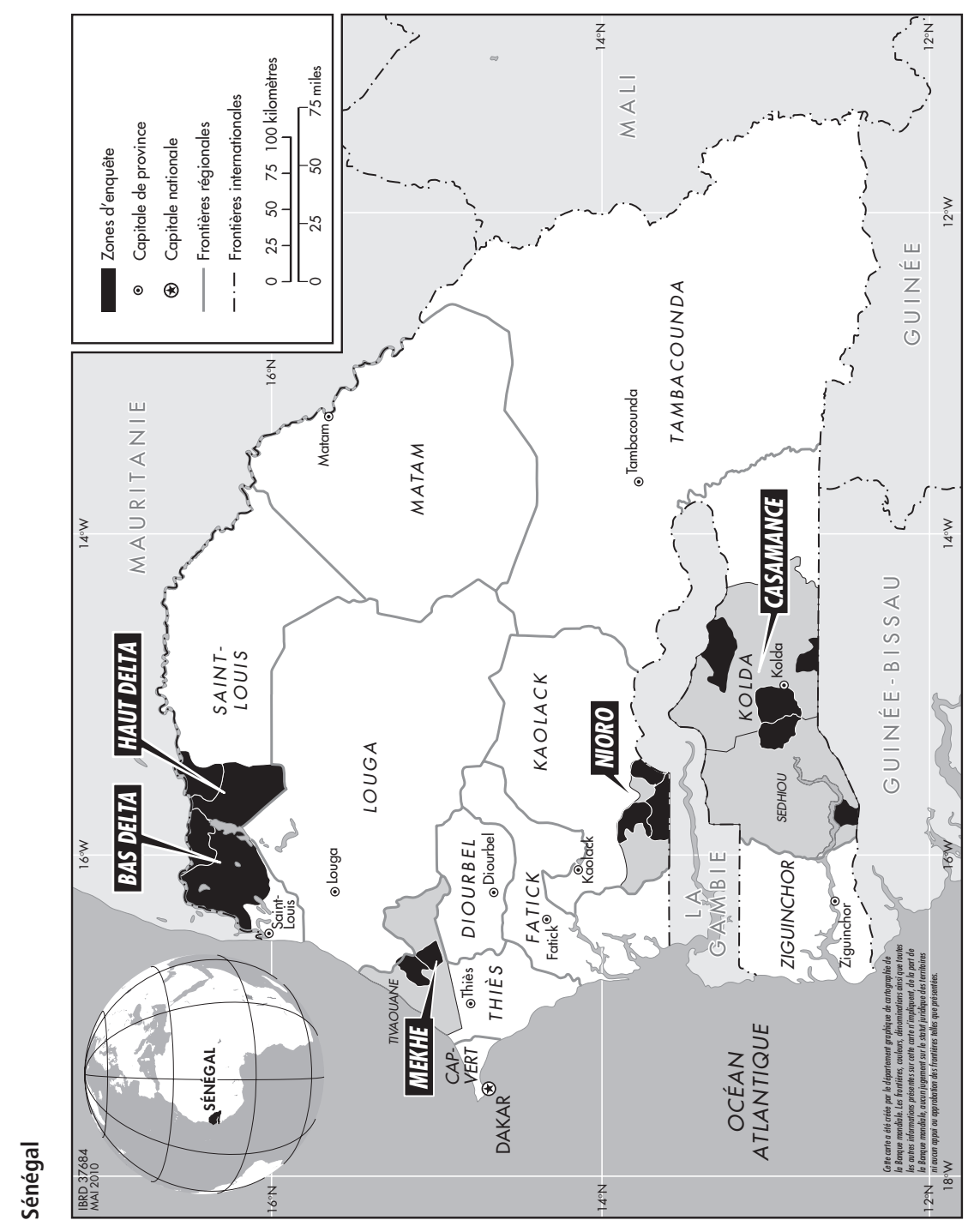




\section{Madagascar}

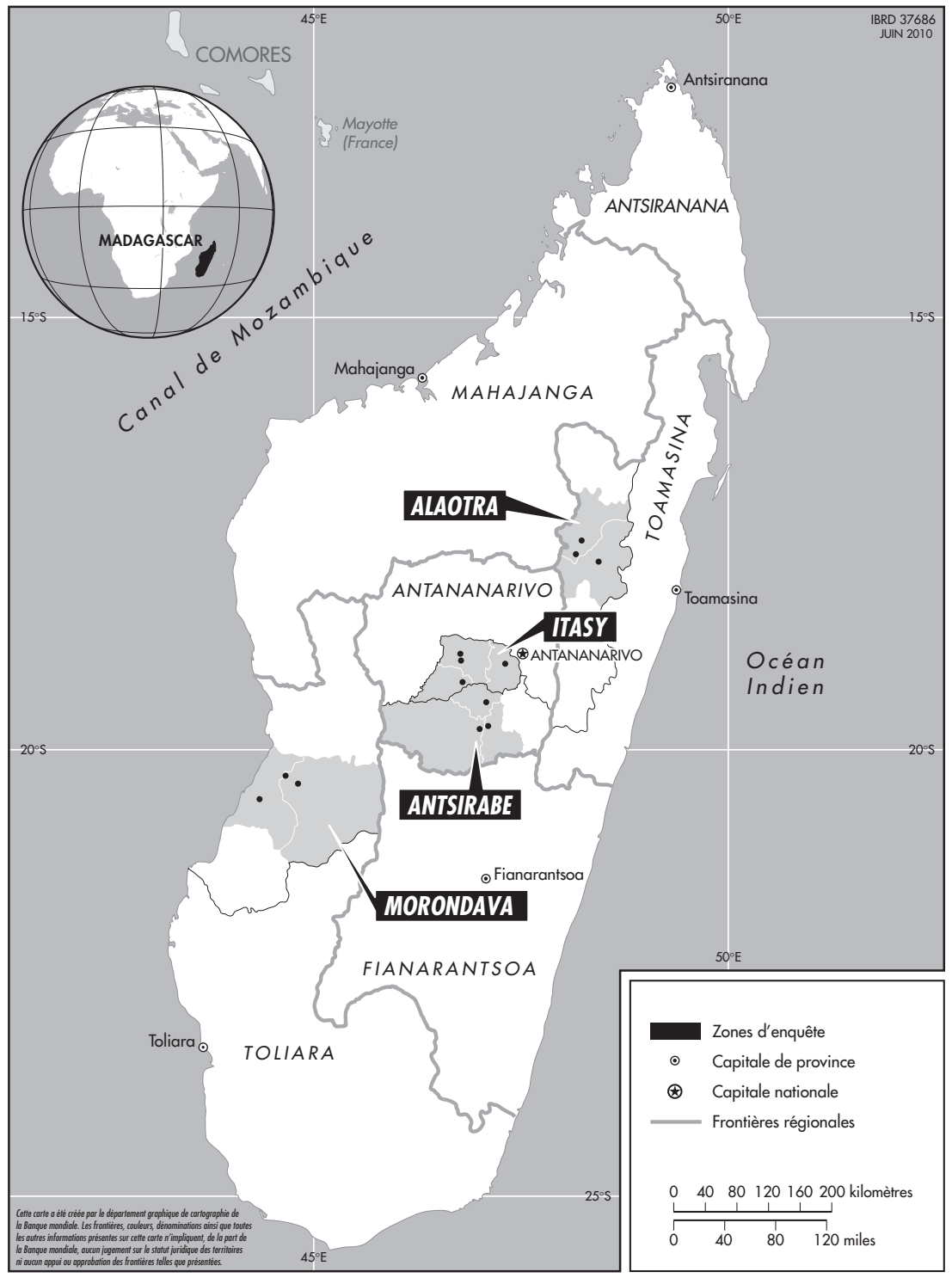




\section{Kenya}

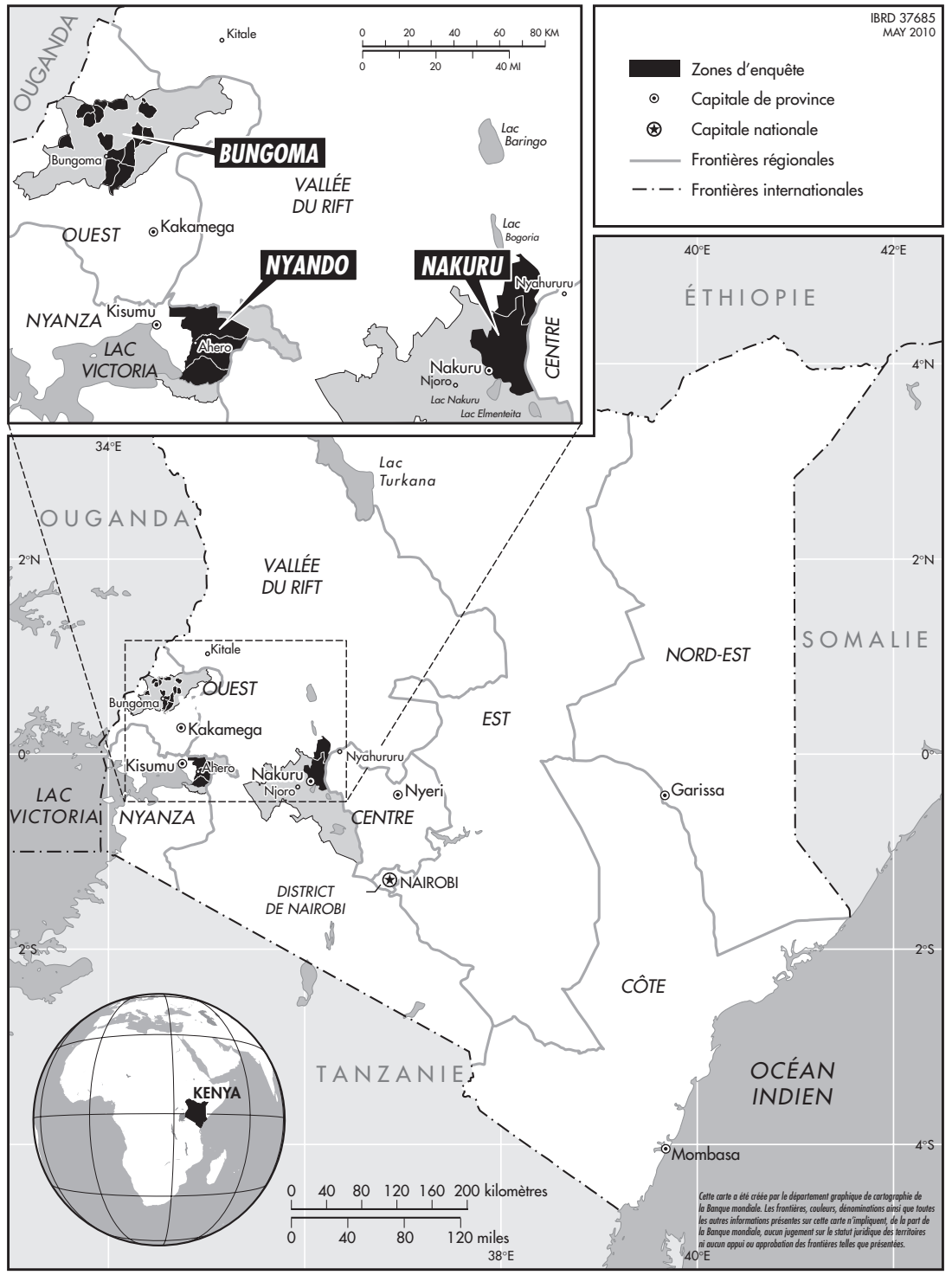




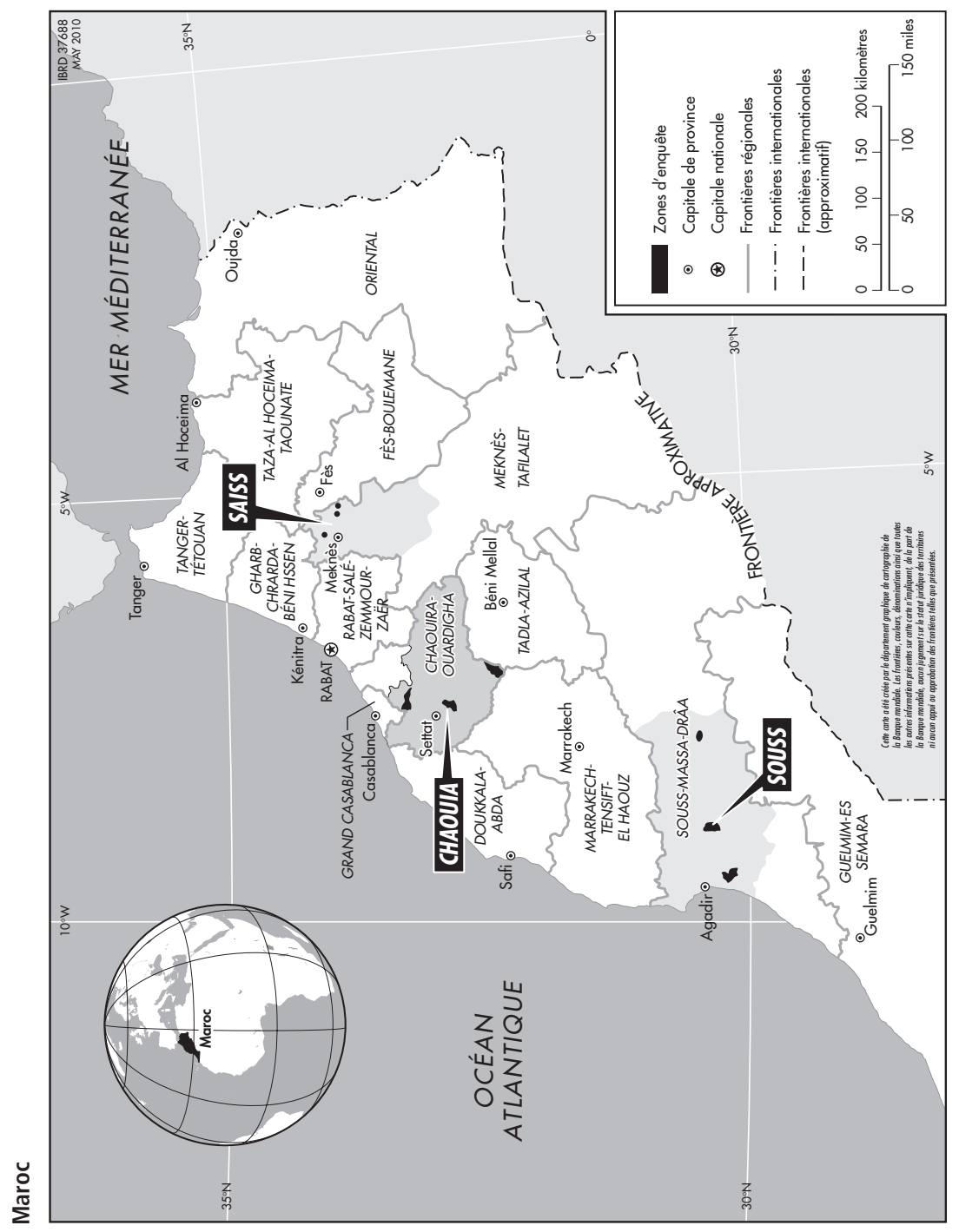




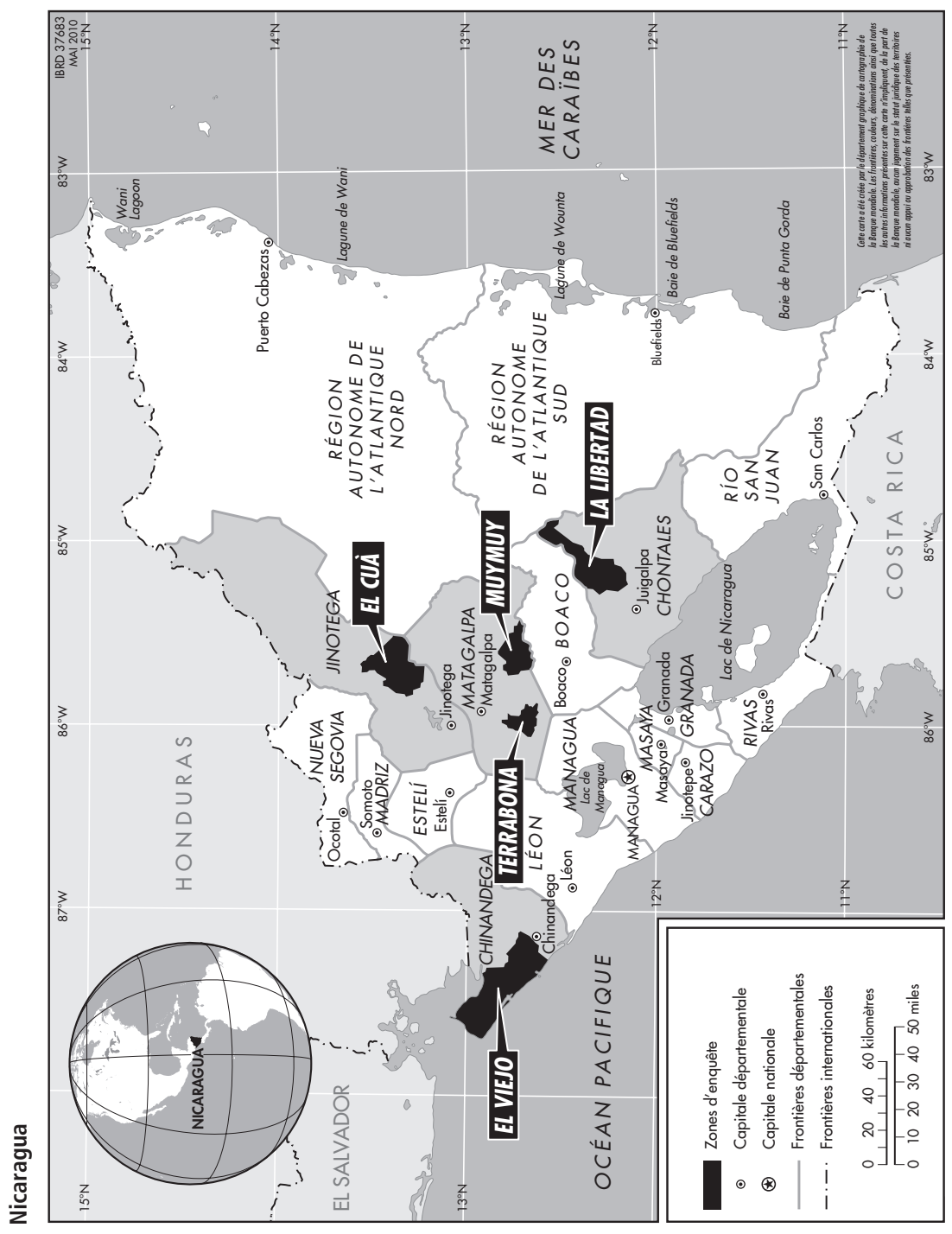




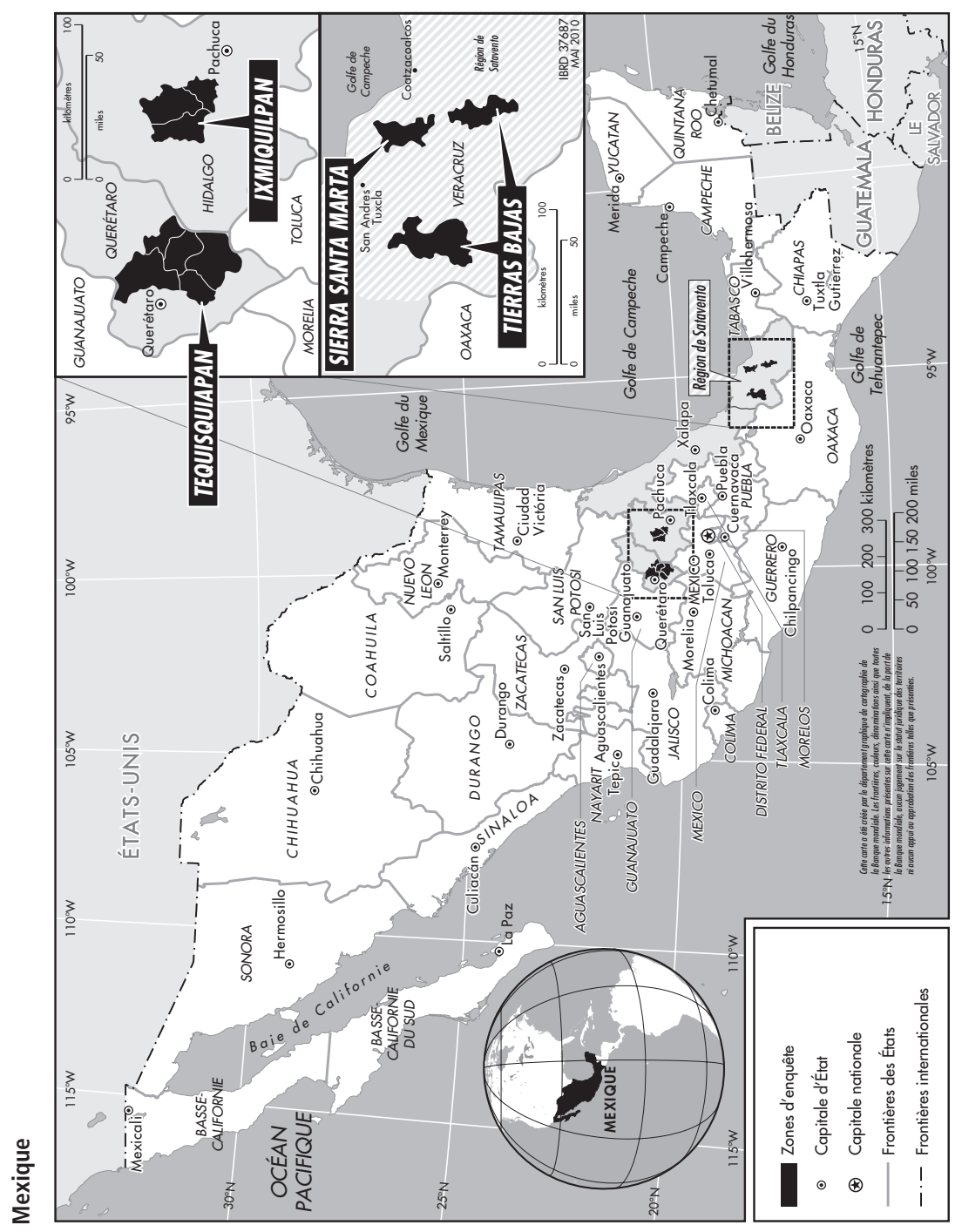




\section{Bibliographie}

\section{Rapports nationaux du programme RuralStruc}

\section{Kenya}

Gamba P. and B. Kibaara, 2007, Structural Implications of Economic Liberalization on Agriculture and Rural Development. RuralStruc Program Kenya - Phase I. Nairobi: Tegemeo Institute, Egerton University, 145 p.

Kirimi L., Mathengue M., Olwande J., Gamba P., Onyuma S. and J. Lagat, 2010, Globalization and Structural Change in Rural Economies. RuralStruc Program Kenya - Phase II. Nairobi: Tegemeo Institute, Egerton University, 153 p.

\section{Madagascar}

Pierre-Bernard A., Ramboarison R., Randrianarison L. et L. Rondro-Harisoa, 2007, Dimensions structurelles de la libéralisation pour l'agriculture et le développement rural. Programme RuralStruc Madagascar - Phase 1. Antananarivo : APB Consulting, EPP PADR, 267 p.

Randrianarison L., Andrianirina N., et R. Ramboarison, 2009, Changements structurels des économies rurales dans la mondialisation. Programme RuralStruc Madagascar - Phase 2. Antananarivo : APB Consulting, EPP - PADR, 285 p.

Rakotonarivo A., Ramialison Z. L., Martignac C. et B. Gastineau, 2008, Migrations internes et villes secondaires. Programme RuralStruc Madagascar - Phase 2. Antananarivo : ICM - CIRAD - IRD, $25 \mathrm{p}$.

\section{Mali}

Samake A., Bélières J.-F., Bosc P.-M. et O. Sanogo, 2007, Dimensions structurelles de la libéralisation pour l'agriculture et le développement rural. Programme RuralStruc MaliPhase 1. Bamako : CEPIA, 278 p.

Samake A., Bélières J.-F., Corniaux C., Dembele N., Kelly V., Marzin J., Sanogo O. et J. Staatz, 2008, Changements structurels des économies rurales dans la mondialisation. Programme RuralStruc Mali - Phase 2. Bamako : IER - CIRAD - MSU, 464 p. 


\section{Mexique}

Rello F. y F. Saavedra, 2007, Dimensiones estructurales de la liberalización para la agricultura y desarrollo rural. Programa RuralStruc Mexico - Fase I. Mexico: FLACSO, 219 p.

Rello F., Saavedra F., Brun V., Léonard E., Palma Grayeb R., Robles H., Muñoz C. y C. Gonzales, 2010, Cambios Estructurales de las Economías Rurales en la Globalización. Programa RuralStruc Mexico - Fase II. Mexico: FLACSO, 340 p.

Brun V., Léonard E. y R. Palma Grayeb, 2009, Inserción competitiva y trayectorias de las economías domésticas en el Sotavento de México. Entre integración agroindustrial y asistencia social. Programa RuralStruc Mexico - Fase II. Jalapa: CEMCA - IRD - Universidad Veracruzana - FLACSO, 33 p.

\section{Maroc}

Akesbi N., Benatya D. et N. El Aoufi, 2007, Dimensions structurelles de la libéralisation pour l'agriculture et le développement rural. Programme RuralStruc Maroc- Phase 1. Rabat: Institut agronomique et vétérinaire Hassan II, $181 \mathrm{p}$.

Icon2e, 2009, Changements structurels des économies rurales dans la mondialisation. Programme RuralStruc Maroc - Phase 2. Rabat : Icon2e, 234 p.

\section{Nicaragua}

Grigsby Vado A.H. and F.J. Pérez, 2007, Structural Implications of Economic Liberalization on Agriculture and Rural Development. RuralStruc Program Nicaragua - Phase I. Managua: Nitlapán, Universidad Centroamericana, 128 p.

Grigsby Vado A.H., Pérez F.J., Gómez L.I., García E.S., Alemán M.A. y Y.L. Marín, 2009, Cambios Estructurales de las Economías Rurales en la Globalización. Programa RuralStruc Nicaragua - Fase II. Managua: Nitlapán, Universidad Centroamericana, 191 p.

\section{Sénégal}

Faye J., Ba C.O., Dièye P.N. et M. Dansoko, 2007, Dimensions structurelles de la libéralisation pour l'agriculture et le développement rural. Programme RuralStruc Sénégal Phase 1. Dakar : IPAR - ASPRODEB, 229 p.

Ba C.O., Diagana B., Dièye P.N., Hathie I. et M. Niang, 2009, Changements structurels des économies rurales dans la mondialisation. Programme RuralStruc Sénégal - Phase 2. Dakar : IPAR - ASPRODEB, 168 p. 


\section{Autres références}

Akyiama T., Baffes J., Larson D.F. and P. Varangis, 2003, Commodity Markets Reform in Africa. Some Recent Experience. Washington DC: World Bank, Policy Research Working Paper 2995.

Akram-Lodhi A., 2008, “(Re)imagining Agrarian Relations? The World Development Report 2008: Agriculture for Development”. Development and Change, 39(6): 1145-1161.

Akram-Lodhi A. and C. Kay, 2009a, "Surveying the agrarian question (part 1): unearthing foundations, exploring diversity". The Journal of Peasant Studies, 37(1): 177-202.

Akram-Lodhi A. and C. Kay, 2009b, "Surveying the agrarian question (part 2): current debates and beyond". The Journal of Peasant Studies, 37(2): 255-284

Akram-Lodhi A. and C. Kay (eds.), 2009c, Peasants and Globalization. Political economy, rural transformation and the agrarian question. Oxon - New York: Routledge.

Alexandratos N., 2005, "Countries with Rapid Population Growth and Resource Constraints: Issues of Food, Agriculture, and Development". Population and Development Review, 31(2): 237-258.

Ali S. and U Dadush, 2010, "Is the African Renaissance For Real?" Carnegie Endowment for International Peace, International Economic Bulletin, September 30, 2010.

Amin A. and N. Thrift, 1993, "Globalization, institutional thickness and local prospects". Revue d'économie régionale et urbaine, 3: 405-427.

Amsden A., 2001, The Rise of "The Rest": Challenges to the West From Late-Industrializing Economies. Oxford University Press.

Anderson K., 2010, Krueger/Schiff/Valdés Revisited. Agricultural Price and Trade Policy Reform in Developing Countries since 1960. Washington DC: World Bank, Policy Research Working Paper 5165, 46 p.

Anderson K. and W. Masters (eds.), 2009, Distortions to Agricultural Incentives in Africa. Washington DC: World Bank, 618 p.

Anderson K. and A. Valdés (eds.), 2008, Distortions to Agricultural Incentives in Latin America. Washington DC: World Bank, 618 p.

Anderson K. and W. Martin, 2005, Agricultural Trade Reform and the Doha Development Agenda. Washington DC: World Bank.

Anseeuw W., Fréguin-Gresh S. et P. Gamba, 2008, "Une nouvelle politique agricole au Kenya: nécessaire mais suffisante?". In Devèze J.-C. (dir.), Défis agricoles africains. Paris : Karthala-AFD, p. 209-229.

Appendini K., 2001, De la milpa a los tortibonos. La restructuración de la política alimentaria en México. México: El Colegio de México - UNRISD, 290 p.

Appendini K., 2008, "Tracing the maize-tortilla chain”. UN Chronicle.

Appendini K. y G. R. Gómez (coord.), en prensa, La Paradoja de la Calidad. Los alimentos mexicanos en la región de América del Norte. Mexico: Pieran - El Colegio de México.

Arbache J. and J. Page, 2007, More Growth or Fewer Collapses? A New Look at Long Run Growth in Sub-Saharan Africa. Washington DC: World Bank, Policy Research Working Paper 4384.

Arrighi G. and L. Zhang, forthcoming, "Beyond the Washington consensus: A new Bandung?" In Shefner J. and P. Fernandez-Kelly (eds.), Globalization and beyond: New examinations of global power and its alternatives. University Park: Pennsylvania State University Press. 
Asfaw S., Mithöfer D. and H. Waibel, 2008, What Impact Are EU Supermarket Standards Having on Developing Countries Export of High-Value Horticultural Products? Evidence from Kenya. Contributed paper at the $105^{\text {th }}$ EAAE Seminar "International Marketing and International Trade of Quality Food Products", Bologna, Italy, March 8-10, 2007.

Augustins G., 1989, Comment se perpétuer? Devenir des lignées et destins des patrimoines dans les paysanneries européennes. Paris: Société d'ethnologie, $434 \mathrm{p}$.

Bairoch P., 1997, Victoires et déboires. Histoire économique et sociale du monde du xvi ie siècle à nos jours. Paris : Gallimard-Folio.

Barrett C.B. and M.R. Carter, 2001, “Can't get ahead for falling behind: new directions for development policy to escape poverty and relieve traps". Choices, 16:35-38.

Barrett C.B and M.R. Carter, 2004, The Economics of poverty traps and persistent poverty: an asset-based approach. Madison: BASIS - CRSP, University of Wiscosin.

Barrett C. B. and E. Mutambatsere, 2005, "Agricultural markets in Developing Countries". In Blume L.E. and S. N. Durlauf (eds.), The New Palgrave Dictionary of Economics, $2^{\text {nd }}$ Edition. London: Palgrave Macmillan.

Barrett C. B. and T. Reardon, 2000, Asset, Activity, and Income diversification Among African Agriculturalists: Some practical Issues. Ithaca - New York: Cornell University.

Barrett C. B., Reardon T. and P. Webb, 2001, "Nonfarm Income diversification and household livelihood strategies in rural Africa: concepts, dynamics, and policy implications". Food Policy, 26: 315-331.

Barrett C.B. and B.M. Swallow, 2005, "Dynamic Poverty Traps and Rural Livelihoods". In Ellis F. and H.A. Freeman (eds.), Rural Livelihoods and Poverty Reduction Policies. London: Routledge, p. 16-28.

Barthélémy D., Delorme H., Losch B., Moreddu C. et M. Nieddu (eds.), 2003, La Multifonctionnalité de l'activité agricole et sa reconnaissance par les politiques publiques. Actes du colloque international de la Société française d'économie rurale, 21-22 mars 2002. Dijon : Éducagri, 922 p.

Bates R.H., 1981, Markets and States in Tropical Africa. The Political Basis of Agricultural Policies. Berkeley: University of California Press.

Bélières J.-F., Bosc P.-M., Faure G., Fournier S. and B. Losch, 2002, What future for West Africa's family farms in a world market economy? London: IIED, Issue paper No. 113, $42 \mathrm{p}$.

Berger S., 2002, Our First Globalization: Lessons from the French. Cambridge: MIT.

Berthelier P. et A. Lipchitz, 2005, “Quel rôle joue l'agriculture dans la croissance et le développement ?” Revue Tiers Monde, XLVI (183) : 603-620.

Bezemer D. and D. Headey, 2008, "Agriculture, Development, and Urban Bias". World Development, 36(8): 1342-1364.

Binswanger-Mkhize H. P., McCalla A. F. and P. Patel, 2009, Structural Transformation and African Agriculture. Africa Emerging Markets Forum, Western Cape, South Africa, September 13-15, 2009.

Birdsall N., 2006, Stormy Days on an Open Field: Asymmetries in the Global Economy. Washington DC: Center for Global Development, Working Paper 81.

Birdsall N., Rodrik D. and A. Subramanian, 2005, “How to Help Poor Countries”. Foreign Affairs, 84(4): 136-152. 
Black R., Natali C. and J. Skinner, 2005, Migration and inequality. World Development Report 2006, Background Papers. Development Research Centre on Migration, Globalisation and Poverty, University of Sussex.

Bloom D., Canning D. and J. Sevilla, 2001, Economic Growth and the Demographic Transition. Cambridge: National Bureau of Economic Research, Working paper No. 8665.

Bonnal P., Bosc P.-M., Diaz J.M. y B. Losch, 2004, "Multifuncionalidad de la agricultura y Nueva Ruralidad ¿Reestructuración de las políticas públicas a la hora de la globalización?” In Pérez C. E. y M.A. Farah Q. (comp.), Desarollo Rural y Nueva Ruralidad en América Latina y la Unión Europea, p. 19-41.

Bossard L. (dir.), 2009, Atlas régional de l'Afrique de l'Ouest. Cahiers de l'Afrique de l'Ouest. Paris : OCDE - Club du Sahel et de l'Afrique de l'Ouest, 291 p.

Bouët A., Bureau J.-C., Decreux Y. and S. Jean, 2005. "Multilateral Agricultural Trade Liberalisation: The Contrasting Fortunes of Developing Countries in the Doha Round". World Economy, 28(9): 1329-1354.

Bourguignon F. and C. Morisson, 1998, "Inequality and development: the role of dualism". Journal of Development Economics, 57(2): 233-257.

Boussard J.-M., Gérard F. et M.-G. Piketty, 2005, Libéraliser l'agriculture mondiale? Théorie, modèles et réalités. Montpellier: CIRAD.

Boussard J.-M., Gérard F., Piketty M.-G., Ayouz M. and T. Voituriez, 2006, “Endegeneous risks and long run effects of liberalization on a global analysis framework". Economic Modelling, 23(3): 457-475.

Braudel F., 1979, Civilisation matérielle, économie et capitalisme. $X V^{e}-X V I H I^{e}$ siècle. Paris : Armand Colin, 3 tomes.

Bricas N., Zoungrana B. et M.-C. Thirion, 2009, Bassins de production et de consommation des cultures vivrières en Afrique de l'Ouest et du Centre. Montpellier : CIRAD - CILSS - AFD.

Brooks K., 2010, African Agriculture-What Do We Not Know? What Do We Need to Know? AAEA Annual Meeting, Denver, July 26, 2010.

Brun V., 2008, “Secteur privé et céréaliculture familiale dans le Mexique du libre-échange. Une étude dans les terres basses du sud-Veracruz". Économie rurale (303-304-305) : 90-107.

Bryceson D. F., 1999, African Rural Labour, Income Diversification and Livelihood Approaches: A long-term Development Perspective. Leiden: Afrika-Studiecentrum.

Bryceson D. F., 2002, “The Scramble in Africa: Reorienting Rural Livelihoods”. World Development, 30(5): 725-739.

Bureau J.-C., Jean S. and A. Matthews, 2006, “The consequences of agricultural trade liberalization for developing countries: distinguishing between genuine benefits and false hopes", World Trade Review, 5: 225-249.

Byerlee D., Jayne T.S. and R. Myers, 2005, Managing Food Price Risks and Instability in an Environment of Market Liberalization. Washington DC: World Bank.

Byerlee D. and K. Deininger, 2010, “The Rise of Large Farms: Drivers and Development Outcomes”. WIDER Angle Newsletter.

Carletto G., Covarrubias K., Davis B., Krausova M., Stamoulis K., Winters P. and A. Zezza, 2007, "Rural income generating activities in developing countries: re-assessing the evidence". Journal of Agricultural and Development Economics, 4(1): 146-193. 
Caron P. and T. Le Cotty, 2006, A review of the different concepts of multifunctionality and their evolution. Multagri Project, European Series on Multifunctionality, No. 10, 179 p.

Carton de Grammont H., 2009, "La desagrarización del campo mexicano". Convergencia, 50: 13-55.

Chang H-J., 2002, Kicking Away the Ladder: Development Strategy in Historical Perspective. London: Anthem Press.

Chenery H. and M. Syrquin, 1975, Patterns of Development: 1950-70. New York: Oxford University Press.

Christiaensen L. and L. Demery, 2007, Down to Earth. Agriculture and Poverty Reduction in Africa. Washington DC: World Bank, $105 \mathrm{p}$.

Christiaensen L. and Y. Todo, 2009, Poverty Reduction during the Rural-Urban Transformation - The Role of the Missing Middle. Washington DC: World Bank.

Cline W. R., 2007. Global Warming and Agriculture: Impact Estimates by Country. Washington DC: Center for Global Development - Peterson Institute for International Economics.

Club du Sahel-OECD, 1998, Preparing for the Future - A Vision of West Africa in the Year 2020. West Africa Long-Term Perspective Study (WALTPS), Paris: Club du Sahel OECD, $157 \mathrm{p}$.

Codron J.-M., Bouhsina Z., Fort F., Coudel E. and A. Puech, 2004, "Supermarkets in Low-income Mediterranean Countries: Impacts on Horticulture Systems". Development Policy Review, 22(5): 587-602.

Collier D. and J. Mahoney, 1996, "Insights and Pittfalls: Selection Bias in Qualitative Research?” World Politics, 49(1): 56-91.

Collier P., 2007, The Bottom Billion: Why the Poorest Countries are Failing and What Can Be Done About It. Oxford: Oxford University Press.

Collier P., 2008, “The Politics of Hunger: How Illusion and Greed Fan the Food Crisis". Foreign Affairs, 87(6).

Collier P., 2009, “Africa’s Organic Peasantry. Beyond Romanticism.” Harvard International Review, 32(2), 62-65.

Collomb P., 1999, Une voie étroite pour la sécurité alimentaire d'ici à 2050. Rome : FAO Paris : Économica.

Cook J., Cylke O., Larson D., Nash J. and P. Stedman-Edwards, 2010, Vulnerable Places, Vulnerable People. Trade Liberalization, Rural Poverty and the Environment. WWF - World Bank, Cheltenham - Northampton: Elgar.

Corral L. and T. Reardon, 2001, "Rural nonfarm incomes in Nicaragua”. World Development, 29(3): 427-442.

Cortes G., 2000, Partir pour rester. Survie et mutation de sociétés paysannes andines (Bolivie). Paris, IRD Éditions, $413 \mathrm{p}$.

Cotula L., Vermeulen S., Leonard R. and J. Keeley, 2009, Land Grab of Development opportunity? Agricultural investment and international land deals in Africa. London -Rome: IIED - FAO - IFAD.

Coulomb P., Delorme H., Hervieu B., Jollivet M. et P. Lacombe (dir.), 1990, Les Agriculteurs et la politique. Paris : Presses de la Fondation nationale des sciences politiques, $594 \mathrm{p}$.

Dabat M.H., Gastineau B., Jenn-Treyer O., Roland J.-P., Martignac C. et A. Pierre-Bernard, 2008, "L'agriculture malgache peut-elle sortir de l'impasse démo-économique?", Autrepart, $46: 189-202$. 
Daniels R., 2003, Guarding the Golden Door - American Immigration Policy and Immigrants since 1882. New York: Hill and Wang, 328 p.

Davis B., Reardon T., Stamoulis K.G. and P. Winters (eds.), 2002, Promoting farm/non-farm linkages for rural development. Case studies from Africa and Latin America. Rome: FAO.

Davis B., Winters P., Carletto G., Covarrubias K., Quinones E., Zezza A., Stamoulis K., Bonomi G. and S. DiGiuseppe, 2007, Rural Income Generating Activities: A Cross Country Comparison. Washington DC, World Bank - FAO. Background Paper for the World Development Report 2008.

Davis M., 2006. The planet of slums. New York - London: Verso.

Deaton A. and S. Zaldi, 2002, Guidelines for Constructing Consumption Aggregates for Welfare Analysis. LSMS Working Paper, 135. Washington DC: World Bank, 104 p.

De Ferrandi D., Perry E. P., Foster W., Lederman D. and A. Valdés, 2005, Beyond the City. The Rural Contribution to Development. Washington DC: World Bank.

Deininger K. and L. Squire, 1996, "A New Data Set Measuring Income Inequality", The World Bank Economic Review, 10(3): 565-91.

Deininger K. and L. Squire, 1998, "New Ways of Looking at Old Issues: Inequality and Growth", Journal of Development Economics, 57(2): 259-287.

De Ita A., 2008, Fourteen years of NAFTA and the tortilla crisis. Americas Program Special Report, Washington DC: Center for International Policy.

De Janvry A., 2009, Agriculture for development: New paradigm and options for success. Elmhirst Lecture, IAAE Conference, Beijing, August 16-22, 2009.

De Janvry A. and E. Sadoulet, 2001, "Income strategies among rural households in Mexico: The role of off-farm activities". World Development, 29(3): 467-480.

Delgado C., Hopkins J. and V. Kelly, 1998, Agricultural Growth Linkages in Sub-Saharan Africa. Research Report 107. Washington DC: IFPRI.

Del Rey Poveda A., 2008, "Determinants and consequences of internal and international migration: The case of rural populations in the south of Veracruz, Mexico", Demographic Research, 16: 288-313.

Denis E. and F. Moriconi-Ebrard (eds.), 2009, Africapolis. Urbanization trends 1950-2020: A geo-statistical approach. West Africa. Paris: Agence Française de Développement.

Dercon S. and P. Krishnan 1998, Changes in Poverty in Rural Ethiopia 1989-1995: Measurement, Robustness Tests and Decomposition. Leuven: Centre for the Study of African Economies - Katholieke Universiteit, Discussion Paper Series DPS 98.19.

Devèze J.-C. (ed.), 2010, Challenges for African Agriculture. Washington DC: World Bank - Agence Française de Développement, 267 p.

Diao X., Hazell P., Resnick D. and J. Thurlow, 2007, The Role of Agriculture in Development: Implications for Sub-Saharan Africa. Research Report 153. Washington DC: IFPRI.

Djurfeldt G., Holmén H., Jirström M. and R. Larsson (eds.), 2005, The African Food Crisis: Lessons from the Asian Green Revolution. CABI, $285 \mathrm{p}$.

DNSI, 2004, Enquête malienne sur l'évaluation de la pauvreté (EMEP), 2001. Principaux résultats. Bamako : ministère du Plan et de l'Aménagement du territoire - Banque mondiale.

Dolan C. and J. Humphrey, 2000, "Governance and trade in fresh vegetables: the impact of UK supermarkets on the African horticulture Industry". Journal of Development Studies, 37(2): 147-176. 
Echanove F. and C. Steffen, 2005, "Agribusiness and Farmers in Mexico: the importance of contractual relations". The Geographical Journal, 171(2): 166-176.

e-Geopolis, Population of Urban Areas of 10,000 habitants or more. http://e-geopolis.eu/.

El Hadad F., 1995, "Enjeux et perspectives de la filière agrumes du Maroc. Les agricultures maghrébines à l'aube de l'an 2000”. Ciheam, Options méditerranéennes, 14 B: 16.

Ellis F., 1998, "Household Strategies and Rural Livelihood Diversification". The Journal of Development Studies. 35(1): 1-38.

Ellis F., 2000, Rural Livelihoods and Diversity in Developing Countries. Oxford: Oxford University Press.

Ellis F., 2004, Occupational Diversification in Developing Countries and Implications for Agricultural Policy. Programme of Advisory and Support Services to DFID (PASS). Project $\mathrm{N}^{\circ}$. WB0207.

English P., Jaffee S. and J. Okello, 2006, "Exporting Out of Africa. The Kenyan Horticulture Success Story”. In Fox L. and R. Liebenthal (eds.), Attacking Africa's Poverty. Experience from the Ground, p. 117-147.

Evans P., 1995, Embedded Autonomy. States and Industrial Transformation. Princeton: Princeton University Press.

Fafchamps M., 2004, Market Institutions in Sub-Saharan Africa. Cambridge, MA: MIT Press.

Faivre Dupaigre B., 2007, APE et dynamiques des flux régionaux : une application aux pays de la CEDEAO. Paris : AFD, coll. "Documents de travail".

FAO, 2001, Food Balance Sheets. A Handbook. Rome: FAO.

FAO, 2004, The State of Food Insecurity in the World. Rome: FAO.

FAO, 2007, The Roles of Agriculture in Development: The Policy Implications and Guidance. Research Programme Summary Report. Rome: FAO, 27 p.

Fan S. (ed.), 2008, Public Expenditures, Growth, and Poverty: Lessons from Developing Countries. Baltimore: Johns Hopkins University Press-IFPRI.

Fay M. and C. Opal, 2000, Urbanization without Growth. A Not-So-Uncommon Phenomenon. Policy Research Working Paper \# 2412,Washington DC: World Bank.

Ferry B. (eds.), 2007, L'Afrique face à ses défis démographiques. Paris : Karthala-CEPED - AFD.

Fréguin-Gresh S., Ba C. O., Bélières J.-F., Losch B. et L. Randrianarison, 2010, “Pauvreté, diversification rurale et transitions africaines : état des lieux et perspectives à partir d'analyses croisées de situations régionales dans quatre pays". Colloque international sur l'agriculture africaine, CEDRES, Ouagadougou, du 6 au 8 décembre 2010.

Friedmann H. and P. McMichael, 1989, "Agriculture and the state system: the rise and fall of national agricultures, 1870 to present”. Sociologia Ruralis, 29(2): 93-117.

Fuglie K.O., 2009, Agricultural Productivity in Sub-Saharan Africa. Symposium on The Food and Financial Crises and Their Impacts on the Achievement of the Millenium Development Goals, Cornell University, Ithaca, May 1-2, 2009.

Foster V. and C. Briceño-Garmendia (eds.), 2010, Africa's infrastructure: a time for transformation. Washington DC: World Bank - Agence Française de Développement, 355 p.

Fox L. and R. Liebenthal (eds.), 2006, Attacking Africa's Poverty. Experience from the Ground. Washington DC: World Bank, 389 p. 
Gabas J.-J. and B. Losch, 2008, "Fabrication and Illusions of Emergence". In C. Jaffrelot (ed.), Emerging States: The Wellspring of a New World Order, p. 13-27.

Galtier F., 2009, How to manage food price instability in developing countries? Montpellier: Cirad, Working Paper Moisa \# 4.

Gastellu J.-M. et J.-Y. Marchal (eds.), 1997, La Ruralité dans les pays du Sud à la fin du $x x^{e}$ siècle. Montpellier : ORSTOM.

Gazel H., Harre D. et F. Moriconi-Ebrard, 2010, Africapolis II, l’urbanisation en Afrique centrale et orientale. Paris : Agence Française de Développement - e-Geopolis.

Gendreau F, 2010, “The Demographic Challenges”. In J.-C. Devèze (ed.), Challenges for African Agriculture, p. 9-33.

Gibbon P. and S. Ponte, 2005, Trading down: Africa, value chains, and the global economy. Philadelphia: Temple University Press, 251p.

Giordano T. et B. Losch, 2007, “Transition : risques d'impasse ?", Courrier de la planète, $(81-82): 22-26$.

Giovannucci D., Josling T., Kerr W., O’Connor B. and M.T. Yeung, 2009, Guide to Geographical Indications. Linking Products and their Origins. Geneva: International Trade Centre, $207 \mathrm{p}$.

Giraud J.-N., 1996, L'Inégalité du monde. Économie du monde contemporain. Paris : Gallimard.

Goody J., 2006, The Theft of History. Cambridge: Cambridge University Press.

Gore C., 2003, Globalization, the International Poverty Trap and Chronic Poverty in the Least Develop Countries. Geneva: UNCTAD, CPRC Working Paper Nº 30.

Groupe Polanyi, 2008, La multifonctionnalité de l'agriculture. Une dialectique entre marché et identité. Versailles : Éditions Quae, 360 p.

Guengant J.-P., 2007, "La démographie africaine entre convergence et divergence". In B. Ferry (eds.), L’Afrique face à ses défis démographiques, p. 27-121.

Guengant J.-P. and J.F. May, 2009, Proximate Determinants of Fertility in Sub-Saharan Africa and their Possible Use in Fertility Projection. United Nations Expert Group Meeting, Population Division, United Nations, New York, 2-4 December 2009.

Gutman P., 2007, “Ecosystem services: Foundations for a new rural-urban compact". Ecological Economics, 62: 382-387.

Haggblade S., 2007, "Alternative Perceptions of the Rural Nonfarm Economy”. In Haggblade S., Hazell P. and T. Reardon (eds.), Transforming the Rural Nonfarm Economy, p. 25-54.

Haggblade S., Hazell P. and T. Reardon (eds.), 2007, Transforming the Rural Nonfarm Economy. Opportunities and Threats in the Developing World. IFPRI, Washington, DC - The John Hopkins University Press, Baltimore, 490 p.

Habbglade S., Hazell P. and T. Reardon, 2010, “The Rural Nonfarm Economy: Prospects for Growth and Poverty Reduction". World Development, 38(10): 1429-1441.

Harre D., Moriconi-Ebrard F. et H. Gazel, 2010, “Fiche République du Kenya”. In H. Gazel et al., Africapolis II, L'urbanisation en Afrique centrale et orientale.

Harris S., 1992, Kenya horticultural subsector survey. Nairobi: Kenya Export Development support project.

Hart G., 2010, "Redrawing the Map of the World? Reflections on the World Development Report 2009”. Economic Geography, 86(4): 341-350. 
Hatton T. J. and G. W. Williamson, 2005, Global Migration and the World Economy. Two centuries of Policy and Performance. Cambridge: The MIT Press.

Hausmann R., Rodrik D. and A. Velasco, 2005, Growth Diagnostics. Harvard: John F. Kennedy School of Government, Harvard University, $35 \mathrm{p}$.

Hazell P., Haggblade S. and T. Reardon, 2007a, "Structural Transformation of the Rural NonFarm Economy”. In Haggblade S., Hazell P., T. Reardon (eds.), Transforming the Rural Nonfarm Economy, p. 83-98.

Hazell P., Poulton C., Wiggins S. and A. Dorward, 2007b, The Future of Small Farms for Poverty Reduction and Growth. Washington: IFPRI, 2020 Discussion Paper 42.

Headey D., Bezemer D. and P. Hazell, 2010, "Agricultural Employment Trends in Asia and Africa: Too Fast or Too Slow?” The World Bank Research Observer, 25(1): 57-89.

Hoekman B. and L.A. Winters, 2005, Trade and Employment: Stylized Facts and Research Findings. World Bank Policy Research Paper 3676. Washington DC: World Bank, 36 p.

INSTAT (Institut national de la statistique), 2009, $4^{e}$ recensement général de la population et de l'habitat du Mali. Bamako : Instat.

IAASTD (International Assessment of Agricultural Knowledge, Science and Technology for Development), 2009, Agriculture at Crossroads. Synthesis Report. Washington DC: IAASTD, $95 \mathrm{p}$.

IFAD (International Fund for Agricultural Development), 2010, Rural Poverty Report 2011. New realities, new challenges: new opportunities for tomorrow's generation. Rome: IFAD, $319 \mathrm{p}$.

ILC (International Land Coalition), 2009, Increasing commercial pressure on land: Building a coordinated response. Rome: ILC.

Jaffee S., 1994, "Contract Farming in the Shadow of Competitive Markets: The Experience of Kenyan Horticulture." In Little P. and M. Watts (eds.), Living Under Contract: Contract Farming and Agrarian Transformation in Sub-Saharan Africa, p. 97-139.

Jaffee S., 1995, "The Many Faces of Success: The Development of Kenyan Horticultural Exports.” In Jaffee S. and J. Morton (eds.), Marketing Africa's High Value Foods. Washington DC: World Bank.

Jaffee S., 2003, From Challenge to Opportunity: The Transformation of the Kenyan Fresh Vegetable Trade in the Context of Emerging Food Safety and Other Standards. ARD Discussion Paper 1. Washington DC: World Bank.

Jaffrelot C. (ed.), Emerging States: The Wellspring of a New World Order. London: C. Hurst \& Co.

Johnston B.F., Kilby P., 1975, Agriculture and Structural Transformation: Economic Strategies in Late-Developing Countries. Oxford: Oxford University Press.

Jütting J. and J.R. De Laiglesia, 2009, Is Informal Normal? Towards More and Better Jobs in Developing Countries. Paris: OECD.

Kirsten J. and K. Sartorius, 2002, "Linking Agribusiness and Small-scale Farmers in Developing Countries: Is there a new role for contract farming?" Development Southern Africa. 17(4): 503-529.

Kydd J., 2002, "Agriculture and Rural Livelihoods: Is Globalization Opening or Blocking Paths out of Rural Poverty?" AgREN Network Paper, 121. London: ODI.

Lamarche H., 1991, L'Agriculture familiale. Tome 1 : Une réalité polymorphe. Paris : L'Harmattan. 
Larson D. and Y. Mundlak, 1997, "On the Intersectoral Migration of Agricultural Labor". Economic Development and Cultural Change, 42(2): 295-319.

Lederman D., Maloney W.F. and L. Servén, 2005, Lessons from NAFTA for Latin America and the Caribbean, Palo Alto: Stanford University Press - Washington DC: World Bank, $407 \mathrm{p}$.

Léonard E., 2010, Libéralisation et régulation privée du secteur agroalimentaire du maïs au Mexique au temps de l'ALENA. Note pour le programme RuralStruc. Washington DC: multigr., 8 p.

Léonard E. et R. Palma, 2002, "Désagrarisation de l'économie paysanne et 'refonctionnalisation' de la localité rurale au Mexique”. Cahiers des Amériques latines (39): 155-173.

Léonard E., Quesnel A. y A. del Rey, 2004, “De la comunidad territorial al archipiélago familiar: Movilidad, contractualizacion de la relaciones intergeneracionales y desarrollo local en el sur del estado de Veracruz". Estudios sociologicos, XXII (66): 557-589.

Léonard E. y B. Losch, 2009, "La inserción de la agricultura mexicana en el mercado norteamericano: cambios estructurales, mutaciones de la acción pública y recomposición de la economía rural y regional". Foro internacional, 49(1): 5-46.

Lewis A. W., 1954, "Economic Development with Unlimited Supplies of Labour". The Manchester School, 28(2): 139-91.

Lin J., 2010, New Structural Economics. A Framework for Rethinking Development. Policy Research Paper 5197, Washington DC: World Bank.

Lin J. and H-J. Chang, 2009, "DPR Debate: Should Industrial Policy in Developing Countries Conform to Comparative Advantage or Defy it? A Debate Between Justin Lin and Ha-Joon Chang”. Development Policy Review, 2009, 27(5): 483-502.

Lipchitz A., Torre C. and P. Chedanne, 2010, “Toward a Regional Food Market Priority”. In J.-C. Devèze, Challenges for African Agriculture. Washington DC: World Bank - Agence Française de Développement , p. 153-178.

Lipton M., 1977, Why Poor People Stay Poor: A Study of Urban Bias in World Development. London: Temple Smith.

Little P. and M. Watts (eds.), 1994, Living Under Contract: Contract Farming and Agrarian Transformation in Sub-Saharan Africa. Madison: University of Wisconsin Press, 285 p.

Losch B., 2004, "Debating the multifunctionality of agriculture: from trade negotiations to development policies by the south". Journal of Agrarian Change, 4(3): 336-360.

Losch B., 2006, "Les limites des discussions internationales sur la libéralisation de l'agriculture : les oublis du débat et les 'oubliés de l'histoire”, OCL, vol. 13(4) : 272-277.

Losch B., 2007, "Quel statut pour l'instabilité des prix dans les changements structurels des agricultures des Suds?" In Boussard J.-M. et H. Delorme (eds.), La Régulation des marchés agricoles internationaux : un enjeu décisif pour le développement. Paris : L'Harmattan, p. 113-131.

Losch B., 2008, "Migrations and the challenge of demographic and economic transitions in the new globalization era". Social Science Research Council, Migration and Development: Future for Research and Policy, New York, February 28-March 1 ${ }^{\text {st }}, 2008,15$ p.

Losch B., 2010a, "The Need for Inclusive Agricultural Growth at the Heart of Africa's Economic Transition”. In J.-C. Devèze (ed.), Challenges for African Agriculture, p. 35-58.

Losch B., 2010b, "Beyond trade: economic transition in the globalization era and prospects for poverty and environment". In Cook J. et al. Vulnerable Places, Vulnerable People. Trade Liberalization, Rural Poverty and the Environment, p. 198-209. 
Losch B., 2010c, “An urgent need for structural policies”. Spore, 146:16.

Losch B., Laudié N., Varlet F. et F. Ruf, 1997, Politiques publiques et agriculture : une mise en perspective des cas mexicains, camerounais et indonésien. Montpellier : CIRAD, coll. "Repères".

Losch B., Fréguin-Gresh S. and T. Giordano, 2008, Structural Dimensions of Liberalization on Agriculture and Rural Development. Background, positioning and results of the first phase. Washington DC: World Bank, processed, $187 \mathrm{p}$.

Losch B. et S. Fréguin-Gresh, forthcoming, "Quels rôles pour quelles agricultures face aux défis des transitions africaines? Le small-scale versus large-scale en débat". Cahiers Agricultures.

Lucas R. E. B., 2005, International Migration and Economic Development: Lessons from Low-Income Countries. Northampton and Cheltenham: Edward Elgar.

Lucas R. E. B., 2008, International Labor Migration in a Globalizing Economy. Washington DC: Carnegie Endowment for International Peace, Carnegie Paper \# 92.

Maertens M., Dries L., Dedehouanou F. A. and J. F. M. Swinnen, 2006, High-value supply chains, food standards and rural households in Senegal. Leuven: Interdisciplinary Research Group on International Agreements and Sustainable Development, Working Paper $\mathrm{N}^{\circ} .9$.

Maertens M. and J. F. M. Swinnen, 2007, Trade, Standards, and Poverty: Evidence from Senegal. Leuven: University of Leuven, LICOS Centre for Institutions and Economic Performance \& Department of Economics, Discussion Paper 177, 35 p.

Mahoney J., 2001, "Path-Dependent Explanations of Regime Change: Central America in Comparative Perspective". Studies in Comparative International Development, 36(1): 111-141.

Maimbo S.M. and D. Ratha, 2005, Remittances. Development Impact and Future Prospects. Washington DC: World Bank, 378 p.

Mazoyer M., 2001, Protecting small Farmers and the rural poor in the context of globalization. Rome: FAO.

Mazoyer M. et L. Roudart, 1997, Histoire des agricultures du monde. Paris : Le Seuil.

McCulloch N. and M. Ota, 2002, Export Horticulture and Poverty in Kenya. Brighton: IDS, Working Paper 174, 40 p.

McKinsey Global Institute, 2010, Lions on the Move: The Progress and Potential of African Economies. McKinsey\&Co.

McMichael P., 1996, Development and Social Change. A Global Perspective. London: Thousand Oaks - New Delhi: Pine Forge Press.

McMichael P., 2009, "A food regime genealogy". The Journal of Peasant Studies, 36(1): 139-169.

Mesplé-Somps S., Robilliard A.-S., Gräb J., Cogneau D. et M. Grimm, 2008, Impact de la culture du coton sur les conditions de vie des ménages. Étude sur le Mali et le Burkina Faso. Paris : DIAL.

Mortimer M., 2003, The future of family farms in West Africa. What can we learn from longterm data? London: IIED, Issue paper $\mathrm{N}^{\circ} .119,72 \mathrm{p}$.

Muchnik J., Requier-Desjardins D., Sautier D. et J.-M. Touzard, 2007, "Les systèmes agroalimentaires localisés (SYAL) : Introduction". Économies et Sociétés, série "Systèmes agroalimentaires", AG 29(9) : 1465-1484. 
Muendo K.M. and D. Tschirley, 2004, Improving Kenya's domestic horticultural production and marketing system: current competitiveness, forces of change, and challenges for the future. Working Paper No. 08C/2004. Nairobi: Tegemeo Institute of Agricultural Policy and Development, Egerton University.

Ndulu B., 2006, "Infrastructure, Regional Integration and Growth in Sub-Saharan Africa: Dealing with the disadvantages of Geography and Sovereign Fragmentation", Journal of African Economies, 15, SUPP/2: 212-244.

Neven D. and T. Reardon, 2004, "The Rise of Kenyan Supermarkets and the Evolution of Their Horticulture Product Procurement Systems", Development Policy Review, 22(6): 669-99.

Oudin X., 2003, “Transition démographique et transformations sociales dans les pays en développement". Économies et sociétés, série "Développement”, 5(41).

O'Rourke K. H. and J. G. Williamson, 1999, Globalization and History: The Evolution of the Nineteenth-Century Atlantic Economy. Cambridge: MIT Press.

Oya C., 2009, “The World Development Report 2008: inconsistencies, silences, and the myth of 'win-win' scenarios". The Journal of Peasant Studies, 36(3): 593-601.

Pachauri R.K. and A. Reisinger (eds.), 2007, Contribution of Working Groups I, II and III to the Fourth Assessment Report of the Intergovernmental Panel on Climate Change. Geneva: IPCC, $104 \mathrm{p}$.

Paulais T., 2010, “Financing African Cities: Changing Scale and Changing Paradigm”, Villes en développement, 88.

Paulais T., forthcoming, Financing Africa's Cities. Washington DC - Paris: AFD - Cities Alliance - World Bank.

Pérez C. E. y M.A. Farah Q. (comp.), 2004, Desarollo Rural y Nueva Ruralidad en América Latina y la Unión Europea. Bogota: Pontificia Universidad Javeriana - Paris: Cirad.

Pérez C. E., Farah Q. M.A. y H. Carton Grammont (eds.), 2008, La nueva ruralidad en América Latina: avances teóricos y evidencias empíricas. Bogotá: Pontificia Universidad Javeriana, $380 \mathrm{p}$.

Peréz M., Schlesinger S. and T. Wise, 2008, The Promise and Perils of Agricultural Trade Liberalization. Lessons from Latin America, WOLA (Washington Office on Latin America) - GDAE at Tuffs University, $36 \mathrm{p}$.

Pierson P., 2000, "Increasing Returns, Path Dependence, and the Study of Politics". The American Political Science Review, 94(2): 251-267.

Polaski S., 2006, Winners and losers: Impact of The Doha Round on Developing Countries. Washington DC: Carnegie Endowment for International Peace.

Pomeranz K., 2000, The Great Divergence. Princeton: Princeton University Press.

Poulton C., Kydd J., Wiggins S. and A. Dorward, 2006, "State intervention for price stabilization in Africa: Can it work?” Food Policy, 31: 342-356.

Poulton C., Tyler G., Hazell P., Dorward A., Kydd J. and M. Stockbridge, 2008, All-Africa Review of Experiences with Commercial Agriculture. Lessons from Success and Failure. Background paper for the Competitive Commercial Agriculture in SSA. Washington DC: World Bank.

Pritchett L., 2006, Let Their People Come - Breaking the Gridlock on Global Labor Mobility. Washington DC: Center for Global Development.

Puricelli S., 2010, El movimiento El campo no aguanta más. Auge, contradicciones y declive (México 2002-2004). México: Plaza y Valdés, 248 p. 
Quesnel A. y A. del Rey, 2005, "La construcción de una economía familiar de archipiélago. Movilidad y recomposición de las relaciones intergeneracionales en el medio rural mexicano". Estudios Demográficos y Urbanos, Vol. 20, 2(59): 197-228.

Radelet S., 2010, Emerging Africa: How 17 Countries Are Leading the Way. Washington DC: Center for Global Development.

Ranis G. and F. Stewart, 1999, "V-Goods and the Role of the Urban Informal Sector in Development." Economic Development and Cultural Change, 47(2): 259-288.

Ratha D. and W. Shaw, 2007, South-South Migration and Remittances. Washington DC: World Bank, Development Prospect Group, 38 p.

Ravallion M., Datt G. and D. van de Walle, 1991, "Quantifying Absolute Poverty in the Developing World”. Review of Income and Wealth, 37: 345-361.

Reardon, T., Berdegue J. and G. Escobar, 2001, "Rural Nonfarm Employment and Incomes in Latin America: Overview and Policy implications". World Development, 29(3): 395-409.

Reardon T, Berdegué J., Barret C. and K. Stamoulis, 2007, "Household Income Diversification into Rural NonFarm Activities”. In Haggblade S., Hazell P. and T. Reardon (eds.), Transforming the Rural Nonfarm Economy, p. 115-140.

Reardon T. and C.P. Timmer, 2007, “Transformation of Markets for Agricultural Output. In Developing Countries Since 1950: How Has Thinking Changed?” In Evenson R. and P. Pingali (eds.), 2007, Handbook of Agricultural Economics, Elsevier, édition 1, 3(1): 2807-2855.

Reardon T. and J. Huang, 2008, Keys to Inclusion of Small-Scale Producers in Dynamic Markets. Synthesis report - meso-study: Meso-Level Restructuring of the Food Industry in Developing Countries, Regoverning Markets Programme.

Rello F. and M. Morales, 2002, "The rural non-farm economy and farm/non-farm linkages in Querétaro, Mexico". In Davis B. et al., Promoting farm/non-farm linkages for rural development. Case studies from Africa and Latin America, p. 61-95.

Republic of Kenya (2005), Nakuru District Strategic Plan 2005-2010. Nairobi: National Coordinating Agency for Population and Development.

Rist G., 1996, Le Développement. Histoire d'une croyance occidentale. Paris : Presses de Sciences-Po.

Rolland J.-P. et A. Alpha, 2010, Étude sur la cohérence des politiques commerciales en Afrique de l'Ouest. Paris - Nogent-sur-Marne : AFD - GRET.

Rygiel P., 2007, Le Temps des migrations blanches. Migrer en Occident (1850-1950). Paris : Aux Lieux d'être, 208 p.

Sadoulet E., de Janvry A. and B. Davis, 2001, "Cash Transfer Programs With Income Multipliers: PROCAMPO in Mexico”. World Development, 29(6): 1043-1056.

Sagarpa, 2007, Situación actual y perspectivas del maíz en México, 1996-2012. SIAP, México DF: Sagarpa, 208 p.

Sala-i-Martin X. and M. Pinkovskiy, 2010, African poverty is falling... much faster than you think! Cambridge: NBER, Working Paper 15775.

Sánchez Albarrán A., 2007, El Campo no aguanta más. México: Universidad Autónoma Metropolitana, Unidad Azcapotzalco (UAM), 312 p.

Satterthwaite D. and C. Tacoli, 2003, The urban part of rural development: the role of small and intermediate urban centres in rural and regional development and poverty reduction. Human Settlements Working Paper Series No. 9, London: IIED. 
Schwentesius R. and M. A. Gomez, 2002, "Supermarkets in Mexico: Impacts on Horticulture Systems”, Development Policy Review, 20(4): 487-502.

Simler K. and N. Dudwick, 2010, Urbanization and Rural-Urban Welfare Inequalities. Draft report, Washington DC: World Bank.

Stern N., 2007, The Stern Review on the Economics of Climate Change. New York: Cambridge University Press, $712 \mathrm{p}$.

Stiglitz J.E, 1998, Towards a New Paradigm for Development: Strategies, Policies and Process. $9^{\text {th }}$ Raul Prebisch Lecture, October 19, 1998, UNCTAD, Geneva.

Timmer C. P., 1988, “The agricultural transformation”. In Chenery H. and T.N. Srinivasan (eds.) Handbook of development economics, Vol. 1. Amsterdam: North Holland.

Timmer C. P., 1989, "Food price policy: the rationale for government intervention". Food Policy, 14(1): 17-42.

Timmer C. P., 2009, A World without Agriculture: The Structural Transformation in Historical Perspective. Washington DC: The American Enterprise Institute Press, 83 p.

Timmer C. P., 2010, “Reflections on food crises past”. Food Policy, (35): 1-11.

Timmer C. P. and S. Akkus, 2008, The Structural Transformation as a Pathway out of Poverty: Analytics, Empirics and Politics. Washington DC: Center for Global Development, Working paper, No. 150.

Todaro M.P., 1971, “Income Expectations, Rural-Urban Expectations and Employment in Africa”. International Labour Review, 104(5): 387-413.

Toulmin C. and B. Guèye, 2003, Transformations in West African agriculture and the role of family farms. London: IIED, Issue paper $\mathrm{N}^{\circ} .123,84 \mathrm{p}$.

UNCTAD, 2006, Developing Productive Capacities. Report on the Least Developed Countries, New York and Geneva: United Nations.

UN-Habitat, 2003, The Challenge of the Slums: Global Report on Human Settlements. Nairobi - London - Sterling: UN-Habitat.

Unido (United Nations Industrial Development Organization), 2008, Breaking In and Moving UP: New Industrial Challenges for the Bottom Billion and the Middle-Income Countries. Industrial Development Report 2009, Vienna: UNIDO.

United Nations, 2010, The Global Partnership for Development at a Critical Juncture. MDG Gap Task Force Report 2010. New York: United Nations, 80 p.

UNRISD (United Nations Research Institute for Social Development), 2010, Combating Poverty and Inequality. Structural Change, Social Policy and Politics. Geneva: UNRISD, $360 \mathrm{p}$.

Van Buren M., 1996, "Rethinking the Vertical Archipelago: Ethnicity, Exchange, and History in the South Central Andes". American Anthropologist, 98(2):338-351.

Veltz P., 1996, Mondialisation, villes et territoires. L'économie d'archipel. Paris : PUF, 288 p.

Viard J., 1998, La Société d'archipel ou les territoires du village global. La Tour-d'Aigues : Éditions de l'Aube, $126 \mathrm{p}$.

Vorley B., 2003, Food, Inc. Corporate Concentration from Farm to Consumer. London: UK Food Group, $89 \mathrm{p}$.

Vorley B., Fearne A. and D. Ray, 2007, Regoverning Markets: A Place for Small-Scale Producers in Modern Agrifood Chains? Aldershot: IIED - Gower, 220 p.

Wallerstein I., 1989, The Modern World-System. San Diego: Academic Press, 3 volumes. 
Webber C. M. and P. Labaste, 2010, Building Competitiveness in Africa's Agriculture. A Guide to Value Chain Concepts and Applications. Washington DC: World Bank.

Wiggins S., 2009, "Can the Smallholder Model Deliver Poverty Reduction and Food Security for a Rapidly Growing Population in Africa?” FAO Expert Meeting on How to Feed the World in 2050, ODI - FAO.

Wiggins S. and J. R. Davis, 2003, Types of RNFE activities and their Returns: Framework and findings. Natural Resources Institute - DFID - World Bank. NRI Report 2754.

Winters L. A., Walmsley T.L., Zhen Kun W. and R. Grynberg, 2003, "Liberalising Temporary Movement of Natural Persons: An Agenda for the Development Round". World Economy, (26): 1137-1161.

Winters L.A., McCulloch N. and A. McKay, 2004, “Trade Liberalization and Poverty: The Evidence So Far?" Journal of Economic Literature, Vol. XLII (March): 72-115.

Wise T. A., 2009, Agricultural Dumping Under NAFTA: Estimating the Costs of U.S. Agricultural Policies to Mexican Producers. Global Development and Environment Institute, Working Paper 09-08. Medford: Tufts University, 38 p.

Wodon Q., Briand V., Labaste P., Nouve K. and Y. Sangho, 2005, Cotton and poverty in Mali. Washington DC: World Bank.

World Bank, 2005, Pro-poor Growth. Country Experiences in the 1990's. Washington DC: World Bank.

World Bank, 2007, Agriculture for Development. World Development Report 2008, Washington DC: World Bank.

World Bank, 2008a, Reshaping Economic Geography. World Development Report 2009, Washington DC: World Bank.

World Bank, 2008b, Regional Trade in Food Staples: Prospects for Stimulating Agricultural Growth and Moderating Short-Term Food Security Crises in Eastern and Southern Africa. Washington DC: World Bank.

World Bank, 2009a, Development and Climate Change. World Development Report 2010, Washington DC: World Bank.

World Bank, 2009b, Awakening Africa's Sleeping Giant. Prospects for Commercial Agriculture in the Guinea Savannah Zone and Beyond. Washington DC: FAO - World Bank, 218 p.

World Bank, 2009c, Youth and Employment in Africa: The Potential, the Problem, the Promise. Africa Development Indicators 2008/09. Washington DC: World Bank.

World Bank, 2010a, Missing Food: The case of postharvest grain losses in Sub-Saharan Africa. Washington, DC: NRI - FAO - World Bank.

World Bank, 2010b, Rising Global Interest in Farmland. Can it Yield Sustainable and Equitable Benefits? Washington DC: World Bank.

Yunes Naude A., 2003, "The dismantling of CONASUPO, a Mexican State Trader in Agriculture". The World Economy, 26: 97-122.

Zahniser S. and W. Coyle, 2004, U.S.-Mexico Corn Trade During the NAFTA Era: New Twists to an Old Story. USDA Outlook, FDS-04D-01. Washington DC: USDA, 20 p.

Zepeda E., Chemingui M., Bchir E., Karingui S., Onynago C. and B. Wanjala, 2009, The Impact of the Doha Round in Kenya. Washington DC: Carnegie Endowment for International Peace, $83 \mathrm{p}$.

Ziébé R., Thys E. and R. De Deken, 2005, "Analysis Method of an Animal Production System in Cameroon", Revue d'élevage et de médecine vétérinaire des pays tropicaux, 58(3): 159-165. 
Dans les régions en développement, les pays les plus pauvres sont confrontés à des défis considérables. Ils doivent affronter simultanément leur transition démographique et leur transition économique dans un monde globalisé tout en tenant compte du changement climatique. S'appuyant sur de nouveaux résultats issus d'enquêtes de terrain (8 000 ménages ruraux dans sept pays d'Afrique et d'Amérique latine), Transformations rurales et développement analyse la situation très particulière de régions encore profondément ancrées dans l'agriculture. L'ouvrage examine la réalité de leur intégration aux marchés globaux et la diversité de leur économie rurale. II s'intéresse ensuite aux mécanismes de diversification des économies agricoles et aboutit à des recommandations pour faciliter les processus de changement structurel. Les propositions portent sur la nécessité de réinvestir clairement le champ des stratégies de développement, avec des politiques ciblées et des approches régionalisées, sur un appui prioritaire aux agricultures familiales et aux cultures vivrières et, enfin, sur un développement territorial fondé sur la densification des liaisons villes - campagnes grâce à un renforcement des fonctions économiques des petites villes et des bourgs ruraux.

" Le grand intérêt de cet ouvrage est de nous rappeler l'importance des processus de changement structurel et le rôle central qu'y joue l'agriculture, mais aussi de nous alerter sur les risques auxquels nous serons confrontés si nous nions les réalités de la démographie, de la marginalisation des petits producteurs et de politiques publiques qui sont insuffisamment inclusives. Le futur immédiat de l'Afrique ne connaîtra la stabilité que si nous combattons l'expropriation politique de la majeure partie de ses habitants, qui est rurale, et si nous savons tirer parti de ce qui fait la force de sa population : sa jeunesse. Transformations rurales et développement nous aide à réfléchir à ces défis et à agir plus intelligemment. »

Ibrahim Assane Mayaki, secrétaire exécutif de l'agence du NEPAD (Nouveau partenariat pour le développement de I'Afrique) 